Draft VERSiOn MAY 20, 2021

Typeset using $\mathrm{LAT}_{\mathrm{E}} \mathrm{X}$ twocolumn style in AASTeX63

\title{
Targeting Bright Metal-poor Stars in the Disk and Halo Systems of the Galaxy
}

\author{
Guilherme Limberg (iD, ${ }^{1}$ Rafael M. Santucci (D), ${ }^{2,3}$ Silvia Rossi (iD, ${ }^{1}$ Derek Shank (D), ${ }^{4}$ Vinicius M. Placco (D), ${ }^{5}$ \\ Timothy C. Beers (D), ${ }^{4}$ Kevin C. Schlaufman (D), ${ }^{6}$ Andrew R. Casey (D) ${ }^{7,8}$ Hélio D. Perottoni (D), ${ }^{1}$ and \\ Young Sun LEE (D) 9 \\ ${ }^{1}$ Universidade de São Paulo, Instituto de Astronomia, Geofísica e Ciências Atmosféricas, Departamento de Astronomia, \\ SP 05508-090, São Paulo, Brazil \\ ${ }^{2}$ Universidade Federal de Goiás, Instituto de Estudos Socioambientais, Planetário, Goiânia, GO 74055-140, Brazil \\ ${ }^{3}$ Universidade Federal de Goiás, Campus Samambaia, Instituto de Física, Goiânia, GO 74001-970, Brazil \\ ${ }^{4}$ Department of Physics and JINA Center for the Evolution of the Elements, University of Notre Dame, Notre Dame, IN 46556, USA \\ ${ }^{5}$ Community Science and Data Center/NSF's NOIRLab, 950 N. Cherry Ave., Tucson, AZ 85719, USA \\ ${ }^{6}$ Department of Physics and Astronomy Johns Hopkins University 3400 North Charles Street Baltimore, MD 21218, USA \\ ${ }^{7}$ School of Physics 83 Astronomy, Monash University, Wellington Road, Clayton 3800, Victoria, Australia \\ ${ }^{8}$ ARC Centre of Excellence for All Sky Astrophysics in 3 Dimensions (ASTRO 3D), Canberra, ACT 2611, Australia \\ ${ }^{9}$ Department of Astronomy and Space Science, Chungnam National University, Daejeon 34134, Republic of Korea
}

\begin{abstract}
We present the results of spectroscopic follow-up for 1897 low-metallicity star candidates, selected from the Best \& Brightest (B\&B) Survey, carried out with the GMOS-N/S (Gemini North/South telescopes) and Goodman (SOAR Telescope) spectrographs. From these low-resolution $(R \sim 2000)$ spectra, we estimate stellar atmospheric parameters, as well as carbon and magnesium abundance ratios. We confirm that $56 \%$ of our program stars are metal-poor $([\mathrm{Fe} / \mathrm{H}]<-1.0), 30 \%$ are very metal-poor $(\mathrm{VMP} ;[\mathrm{Fe} / \mathrm{H}]<-2.0)$ and $2 \%$ are extremely metal-poor $(\mathrm{EMP} ;[\mathrm{Fe} / \mathrm{H}]<-3.0)$. There are 191 carbon-enhanced metal-poor (CEMP) stars, resulting in CEMP fractions of $19 \%$ and $43 \%$ for the VMP and EMP regimes, respectively. A total of 94 confirmed CEMP stars belong to Group I $(A(\mathrm{C}) \gtrsim$ $7.25)$ and 97 to Group II $(A(\mathrm{C}) \lesssim 7.25)$ in the Yoon-Beers $A(\mathrm{C})-[\mathrm{Fe} / \mathrm{H}]$ diagram. Moreover, we combine these data with Gaia EDR3 astrometric information to delineate new target-selection criteria, which have been applied to the Goodman/SOAR candidates, to more than double the efficiency for identification of bona-fide VMP and EMP stars in comparison to random draws from the B\&B catalog. We demonstrate that this target-selection approach can achieve success rates of $96 \%, 76 \%, 28 \%$ and $4 \%$ for $[\mathrm{Fe} / \mathrm{H}] \leq-1.5, \leq-2.0, \leq-2.5$ and $\leq-3.0$, respectively. Finally, we investigate the presence of dynamically interesting stars in our sample. We find that several VMP/EMP $([\mathrm{Fe} / \mathrm{H}] \leq-2.5)$ stars can be associated with either the disk system or halo substructures like Gaia-Sausage/Enceladus and Sequoia.
\end{abstract}

Keywords: Galaxy: halo - Galaxy: kinematics and dynamics - stars: atmospheres - stars: carbon stars: Population II - techniques: spectroscopy

\section{INTRODUCTION}

Very metal-poor (VMP; $\left.[\mathrm{Fe} / \mathrm{H}]^{11}<-2.0\right)$ and extremely metal-poor $(\mathrm{EMP} ;[\mathrm{Fe} / \mathrm{H}]<-3.0)$ stars are relics of the formation and evolution of the Galaxy, providing clues on the nucleosynthesis processes operating

Corresponding author: Guilherme Limberg

guilherme.limberg@usp.br

11 Definition of elemental abundances for a star $(\star)$ relative to the Sun $(\odot):[\mathrm{A} / \mathrm{B}]=\log \left(N_{\mathrm{A}} / N_{\mathrm{B}}\right)_{\star}-\log \left(N_{\mathrm{A}} / N_{\mathrm{B}}\right)_{\odot}$, where $N_{\mathrm{A}}\left(N_{\mathrm{B}}\right)$ is the number density of atoms of element A (B). The adopted composition of the Sun is from Asplund et al. (2009). throughout its early history (Beers \& Christlieb 2005). Seminal efforts, focused towards discovering VMP and EMP stars in the past (e.g., the HK survey; Beers et al. 1985, 1992, and the Hamburg/ESO survey; Christlieb 2003; Christlieb et al. 2008), provided the majority of targets observed at high spectroscopic resolution over the last few decades (e.g., Norris et al. 1996; Hill et al. 2002; Cayrel et al. 2004; Aoki et al. 2007; Cohen et al. 2008; Yong et al. 2013a; Roederer et al. 2014). Studies of these ancient stars have allowed stellar archaeologists to constrain the conditions for the chemical enrichment of the star-forming environments that existed in the nascent Milky Way (Frebel \& Norris 2015). 
Beers et al. (1992) first noted the presence of a surprisingly large number of carbon-enhanced stars among their sample at the lowest metallicities. As the sample sizes increased, it was recognized that the fraction of carbon-enhanced metal-poor (CEMP; $[\mathrm{C} / \mathrm{Fe}]>+0.7$ and $[\mathrm{Fe} / \mathrm{H}]<-1.0)$ stars indeed rapidly increases with decreasing $[\mathrm{Fe} / \mathrm{H}]$ (Norris et al. 1997; Rossi et al. 1999, 2005; Lucatello et al. 2006; Lee et al. 2013; Yong et al. 2013b; Placco et al. 2014b; Yoon et al. 2018) and at greater distances from the Galactic plane $\left(|Z|_{\text {Gal }}\right.$; Frebel et al. 2006; Carollo et al. 2012; Lee et al. 2017, 2019; Yoon et al. 2018). This behavior has been proposed to be related to the dual nature of the stellar halo (hereafter "halo"; Carollo et al. 2007, 2010; Beers et al. 2012). It has been suggested that the majority of the CEMP stars in the $[\mathrm{Fe} / \mathrm{H}] \lesssim-2.5$ regime belong to the CEMP-no subclass, showing no enhancements in neutron-capture elements $([\mathrm{Ba} / \mathrm{Fe}]<0.0$; see Yoon et al. 2016, 2019). These observations support the hypothesis that the CEMP-no stars are the direct descendants of massive Pop III stars that are now long vanished (Ryan et al. 2005; Aoki et al. 2007; Ito et al. 2013; Spite et al. 2013; Tominaga et al. 2014; Keller et al. 2014; Frebel et al. 2015; Roederer et al. 2016; Placco et al. 2016a,b; Aguado et al. 2018; Ezzeddine et al. 2019).

Another peculiarity found in the chemical-abundance profiles of some metal-poor stars is their enhancement in $r$-process (rapid neutron capture) elements (see Sneden et al. 2008 and Frebel 2018 for reviews on the topic). The qualitative aspects of the formation of these neutron-rich nuclei have been known for many decades (e.g., Burbidge et al. 1957; Cameron 1957). However, the astrophysical site(s) in which the $r$-process occurs remained speculative up until the photometric and spectroscopic observations of the electromagnetic counterpart AT2017gfo (Arcavi et al. 2017; Drout et al. 2017; Pian et al. 2017; Shappee et al. 2017; Smartt et al. 2017) of the gravitational wave event GW170817 (Abbott et al. $2017 \mathrm{a}, \mathrm{b}, \mathrm{c}$ ) of a neutron star merger. These authors concluded that this transient (kilonova) was powered by the radioactive decay of large amounts of $r$-process elements, in agreement with early theoretical predictions (Lattimer \& Schramm 1974). It has been suggested that neutron star mergers are the primary (perhaps the only) source of $r$-process enrichment in the Galaxy (e.g., Côté et al. 2018; Safarzadeh et al. 2019a; Banerjee et al. 2020; Dvorkin et al. 2020), but other studies (Belczynski et al. 2018; Ji \& Frebel 2018; Ji et al. 2019; Côté et al. 2019; Safarzadeh et al. 2019b; Haynes \& Kobayashi 2019; Kobayashi et al. 2020) have provided evidence that additional sources may be involved.

In the Gaia era, proper motions (PMs) and parallaxes of exquisite quality have been made available for more than a billion stars (Gaia Collaboration et al. 2016a). The phase-space information, particularly from Gaia Data Release 2 (DR2; Gaia Collaboration et al. 2018), combined with previously available high- resolution spectroscopic data, has enabled the exploration of the chemo-dynamical properties of these lowmetallicity stars. It has been revealed that a large population of EMP and ultra metal-poor (UMP; $[\mathrm{Fe} / \mathrm{H}]$ $<-4.0)$ stars are apparently kinematically connected (similar $|Z|_{\text {Gal }}$ and rotational motion around the Galactic center) to the disk system (Sestito et al. 2019, 2020; Cordoni et al. 2020), providing constraints on the assembly of the newborn Milky Way (redshift $z \gtrsim 2$ ) through its primordial building blocks (Di Matteo et al. 2020; Sestito et al. 2021).

Considering all of the above-mentioned advances, it is clear that numerous VMP and EMP stars are necessary to advance our understanding of the formation and evolution of the Galaxy. The pioneering objective-prism surveys (Bond 1970, 1980; Bidelman \& MacConnell 1973; Beers et al. 1985, 1992; Christlieb 2003; Christlieb et al. 2008) have been responsible for the identification of thousands of VMP stars and several hundred EMP stars. Large spectroscopic surveys such as the Sloan Digital Sky Survey (SDSS; York et al. 2000) and its stellarspecific sub-survey Sloan Extension for Galactic Understanding and Exploration (SEGUE; Yanny et al. 2009), the Large Sky Area Multi-object Fiber Spectroscopic Telescope (LAMOST; Cui et al. 2012; Zhao et al. 2012; see also Li et al. 2018b), and the Radial Velocity Experiment (RAVE; Steinmetz et al. 2006) have increased these numbers to tens of thousands of VMP and several thousand EMP stars. Ongoing and planned spectroscopic surveys in the near future (WEAVE: Dalton et al. 2012; 4MOST: de Jong et al. 2012, 2014, Pristine: Starkenburg et al. 2017b; Aguado et al. 2019; Youakim et al. 2020; H3: Conroy et al. 2019a,b; SDSS-V/MWM: Kollmeier et al. 2017) are expected to expand these numbers further.

More recently, the Best \& Brightest (B\&B; Schlaufman \& Casey 2014, and see also Casey \& Schlaufman 2015; Reggiani et al. 2020) initiative has taken advantage of mid-infrared photometry from the Wide-field Infrared Survey Explorer (WISE; Wright et al. 2010) mission, in combination with near-infrared photometry from the Two Micron All Sky Survey (2MASS; Skrutskie et al. 2006), to select almost 12000 low-metallicity candidates. From follow-up spectroscopy of $\sim 200$ objects in this list, these authors obtained efficiencies of $33 \%$ and $4 \%$ in finding VMP and EMP stars, respectively. Placco et al. (2019, hereafter P19) have incorporated magnitude (in the $V$-band), reddening $(E(B-V))$ and PM cuts in the target selection of their own follow-up of stars from the B\&B catalog. The more restrictive criteria yielded similar success rates, $42 \%$ (VMP) and $2 \%$ (EMP), for their much larger sample of 800 candidates, but including fainter targets. The VMP and EMP stars uncovered through the B\&B selection have served as targets for high-resolution spectroscopy conducted by the $R$-Process Alliance (e.g., Holmbeck et al. 2020 and references therein), as they are all brighter than $V=14$ 


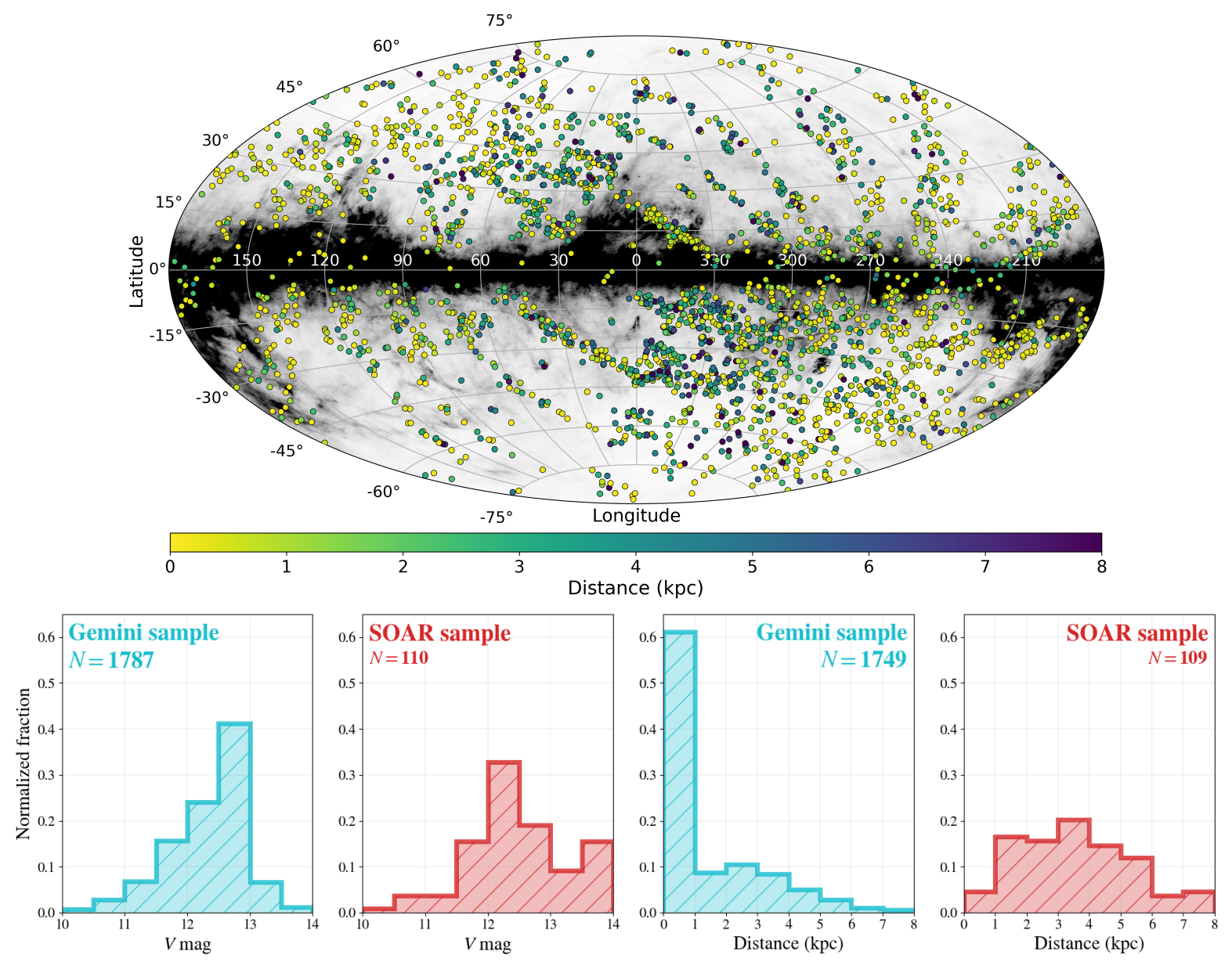

Figure 1. Top panel: Distribution of the Gemini (Section 2.1), SOAR (Section 2.2), and P19 samples in the Galactic coordinate system, color-coded by heliocentric distances (Section 5). The background all-sky distribution of the Galactic reddening comes from the Schlegel et al. (1998) dust map, as re-calibrated by Schlafly \& Finkbeiner (2011). The different gray scales represent $E(B-V)$ values from 0.0 (white) to 0.5 (black). Bottom panels: Distributions of $V$-band magnitudes (Section 2) and heliocentric distance estimates (Section 5) for the Gemini (cyan) and SOAR samples (red). The total number of stars represented in each histogram is also shown in their respective panels.

and can be readily observed with $2.5-\mathrm{m}$ to $6.5-\mathrm{m}$ class telescopes from the ground.

With the advent of Gaia, we have the opportunity to combine astrometric (as discussed in Placco et al. 2018, hereafter P18) and photometric (WISE+2MASS/B\&B) information to increase the efficiency in identifying VMP and EMP stars. This should allow us to populate the carbon- and $r$-process-enhanced classes of metal-poor stars with bright objects much faster, enabling studies of their origins, constraining supernovae nucleosynthesis and chemical evolution models, and probing rare, chemically peculiar targets for future investigations.

The goal of the present work is to identify VMP and EMP stars from the B\&B catalog, confirming (or not) their metal-poor nature via low-resolution $(R \sim 2000)$ spectroscopy. We also seek to determine carbon and magnesium (representative of the $\alpha$ elements) abundances. Crucially, we incorporate Gaia Early Data Release 3 (EDR3; Gaia Collaboration et al. 2020) astrom- etry to investigate the effect of kinematic-based target selection on the success rates of finding low-metallicity stars. These criteria can be taken into account for ongoing and future searches for metal-poor stars in the Galaxy. The most interesting VMP and EMP stars vetted by this approach will serve as targets for ongoing and forthcoming high-resolution spectroscopic campaigns. Finally, we revisit the behaviors of VMP star fractions, as functions of both $|Z|_{\text {Gal }}$ and velocities, and the increase of CEMP star fractions with declining metallicity. We also investigate the dynamically interesting VMP/EMP stars with either disk- or halo-like orbits.

This paper is outlined as follows. In Section 2, details of target selection, observations, and data reduction are provided. Section 3 is dedicated to the estimation of stellar atmospheric parameters: effective temperature $\left(T_{\text {eff }}\right)$, surface gravity $(\log g)$, and metallicity (as represented by $[\mathrm{Fe} / \mathrm{H}]$ ), and also elemental abundances of 
Table 1. Coordinates and Observing Details

\begin{tabular}{ccccccccc}
\hline $\begin{array}{c}\text { Star Name } \\
(2 \mathrm{MASS})\end{array}$ & $\begin{array}{c}\text { Star Name } \\
\text { (Gaia EDR3) }\end{array}$ & $\begin{array}{c}\text { R.A. } \\
(\mathrm{deg})\end{array}$ & $\begin{array}{c}\text { Decl. } \\
(\mathrm{deg})\end{array}$ & $\begin{array}{c}l \\
(\mathrm{deg})\end{array}$ & $\begin{array}{c}b \\
(\mathrm{deg})\end{array}$ & Telescope & Instrument & Program ID \\
\hline \hline $00003305-7953389$ & 4634573766005607552 & 0.1375 & -79.8942 & 305.7037 & -36.9587 & SOAR & Goodman & SO-2019B-013 \\
$00020162-4430117$ & 4994519032163925632 & 0.5067 & -44.5033 & 329.4221 & -69.9972 & SOAR & Goodman & SO-2019B-013 \\
$00040305-6106367$ & 4905632480654004608 & 1.0127 & -61.1102 & 312.9121 & -55.0903 & Gemini South & GMOS-S & GS-2016A-Q-76 \\
$00043646+4124062$ & 384060304935385984 & 1.1519 & 41.4017 & 113.5728 & -20.6174 & Gemini North & GMOS-N & GN-2017B-Q-75 \\
$00045403+3524010$ & 2876804519751163008 & 1.2251 & 35.4003 & 112.3464 & -26.5131 & Gemini North & GMOS-N & GN-2016A-Q-75 \\
\hline
\end{tabular}

This table is available in its entirety in machine-readable form.

Table 2. Colors, Magnitudes, and Reddening Estimates

\begin{tabular}{ccccccccc}
\hline $\begin{array}{c}\text { Star Name } \\
(2 \mathrm{MASS})\end{array}$ & $\begin{array}{c}\text { Star Name } \\
\text { (Gaia EDR3) }\end{array}$ & $V$ & $(B-V)$ & $G$ & $\left(G_{\mathrm{BP}}-G_{\mathrm{RP}}\right)$ & $J$ & $(J-K)$ & $E(B-V)$ \\
\hline \hline $00003305-7953389$ & 4634573766005607552 & 12.411 & 0.775 & 12.184 & 1.069 & 10.804 & 0.535 & 0.074 \\
$00020162-4430117$ & 4994519032163925632 & 12.631 & 0.807 & 12.397 & 1.067 & 11.007 & 0.567 & 0.011 \\
$00040305-6106367$ & 4905632480654004608 & 12.818 & 1.075 & 12.417 & 1.466 & 10.546 & 0.745 & 0.010 \\
$00043646+4124062$ & 384060304935385984 & 12.612 & 1.025 & 12.371 & 1.082 & 10.896 & 0.643 & 0.073 \\
$00045403+3524010$ & 2876804519751163008 & 12.174 & 0.698 & 11.925 & 1.011 & 10.576 & 0.513 & 0.063 \\
\hline
\end{tabular}

This table is available in its entirety in machine-readable form.

interest $([\mathrm{C} / \mathrm{Fe}]$ and $[\mathrm{Mg} / \mathrm{Fe}])$. We explore the behaviors of these abundance ratios as functions of $[\mathrm{Fe} / \mathrm{H}]$ in Section 4. In Section 5, we investigate the kinematics of the selected low-metallicity candidates and examine the improvements in the efficiency of finding VMP stars in the Galaxy. We also analyze the orbits of VMP/EMP in Section 5 in the context of the recent literature. Finally, Section 6 presents a summary of our conclusions.

\section{TARGET SELECTION, OBSERVATIONS, AND DATA REDUCTION}

All of our targets have been selected as metal-poor candidates by Schlaufman \& Casey (2014) as part of the B\&B Survey. A total of 1897 stars have been observed with either the Gemini Multi-Object Spectrographs (GMOS-N/S; Davies et al. 1997; Gimeno et al. 2016) or Goodman spectrograph (Clemens et al. 2004) at the Gemini (North/South; $8.1 \mathrm{~m}$ ) and the Southern Astrophysical Research (SOAR; $4.1 \mathrm{~m}$ ) telescopes, respectively. By design, all candidates are significantly bright $(V \lesssim 14.0$; bottom left panels of Figure 1 ), which makes them excellent for high-resolution follow-up. The typical signal-to-noise ratio (SNR) of our spectra is $\gtrsim 30$ per pixel at the wavelength region of the $\mathrm{Ca}$ II $\mathrm{K} / \mathrm{H}$ lines (3900-4000 A). Since the target-selection criteria were different for observations with each instrument, we divide our stars into a "Gemini sample" (Section 2.1) and a "SOAR sample" (Section 2.2). The calibrations in- cluded bias frames, quartz flats, and arc-lamp exposures. The background subtraction, definition of aperture, extraction of the one-dimensional spectra, and wavelength calibrations for each spectrum have been conducted with standard IRAF (Tody 1986, 1993) packages.

Pertinent observational information for the stars in our samples are presented in Table 1, including 2MASS names and Gaia EDR3 (Gaia Collaboration et al. 2020) IDs and coordinates. The different telescopes and instruments are also listed. Table 2 includes relevant photometric information: $V, G$ and $J$ magnitudes and $B-V, G_{\mathrm{BP}}-G_{\mathrm{RP}}$ and $J-K$ colors from AAVSO Photometric All Sky Survey (APASS; Henden \& Munari 2014) DR9, Gaia EDR3 (Riello et al. 2020), and 2MASS, respectively. The reddening values (Table 2) have been estimated with the Schlegel et al. (1998) dust maps (top panel of Figure 1). These can be easily recalibrated into other $E(B-V)$ systems (e.g., Schlafly \& Finkbeiner 2011). 


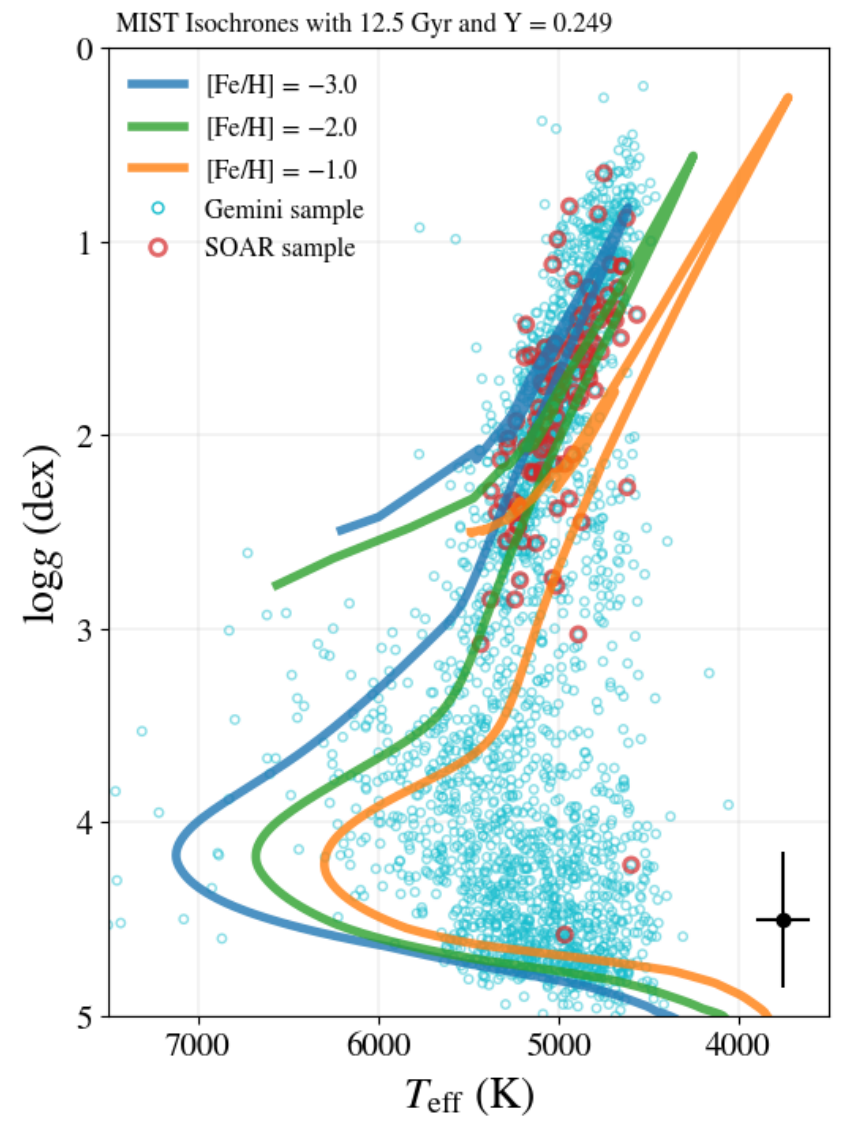

Figure 2. $T_{\text {eff }}$ vs. $\log g$ diagram of the Gemini (cyan circles; Section 2.1) and SOAR (red symbols; Section 2.2) samples. Typical uncertainties for these atmospheric parameters ( $\pm 150 \mathrm{~K}$ for $T_{\text {eff }}$ and \pm 0.35 dex for $\log g$; Section 3 ) are represented by the black dot with error bars in the bottom right corner. The colored lines are MESA Isochrones and Stellar Tracks (MIST; Dotter 2016; Choi et al. 2016) isochrones with varying metallicities. The orange, green, and blue isochrones represent $[\mathrm{Fe} / \mathrm{H}]=-1.0,-2.0$, and -3.0 , respectively. At the top, we list the rest of the conditions employed to generate the MIST stellar population models: Age $=12.5$ Gyr and $Y=0.249$ (primordial He abundance; Planck Collaboration et al. 2016).

\subsection{The Gemini Sample}

The majority $(1787 / 1897 ; 94 \%)$ of the spectra were acquired with the GMOS-N and GMOS-S spectrographs at Gemini North and South, respectively. The observations in this program were obtained between 2014A and 2019B; the various proposal IDs are listed in Table 1. All observations used $0.5^{\prime \prime}$ slits. During the semesters 2014A, 2015A, and 2015B, the spectra were obtained with the B1200 $\mathrm{l} \mathrm{mm}^{-1}$ G5301 (North) and G5321 (South) gratings, which led to a resolving power of $R \sim 2400-2800$. For all other observing runs, we have employed the B600 $\mathrm{l} \mathrm{mm}^{-1}$ G5307 (North) and
G5323 (South) gratings, resulting in $R \sim 2000-2600$. The typical wavelength coverage of GMOS-N/S spectra is $3200-5800 \AA$. We have been able to obtain stellarparameter estimates for all but five stars in this sample (Section 3). The cases for which we have not been able to estimate $T_{\text {eff }}$ and $\log g$ were due to low-SNR $(\lesssim 10$ per pixel at $\sim 4000 \AA$ ) spectra and/or large mismatches between the color-based temperatures and the spectroscopic calibrations, which is expected for stars outside the $4000 \leq T_{\text {eff }}(\mathrm{K}) \leq 7000$ range.

\subsection{The SOAR Sample}

Unlike the Gemini sample, stars in the SOAR sample took into account phase-space information in the target selection. In P18, PMs from Gaia DR1 (Gaia Collaboration et al. 2016b) and line-of-sight ${ }^{12}$ velocities ( $\left.V_{\text {los }}\right)$ from the RAVE DR5 (Kunder et al. 2017) had already been used to explore this possibility. The success rate in finding low-metallicity $([\mathrm{Fe} / \mathrm{H}] \lesssim-1.5)$ stars was higher for both larger $|Z|_{\text {Gal }}$ and transverse velocities relative to the Sun $\left(V_{\mathrm{T}}\right)$. Here, we develop this idea further, and propose an improved, more robust set of criteria. In order to take full advantage of both PMs and $V_{\text {los }}$ from Gaia's past and future DRs, we introduce the quantity "total available velocity" ( $\left.V_{\text {TAV }}\right)$, where:

$V_{\mathrm{TAV}}=\left\{\begin{array}{l}\left(V_{\mathrm{los}}^{2}+V_{\mathrm{T}}^{2}\right)^{1 / 2} \text { if } V_{\mathrm{los}} \text { and } V_{\mathrm{T}} \text { are available; } \\ V_{\mathrm{T}} \text { if only proper motions are available; } \\ V_{\mathrm{los}} \text { if only this component is available. }\end{array}\right.$

A detailed description, along with the advantages and limitations of the $V_{\text {TAV }}$ parameter, is given in Section 5.1. From this definition, the candidates in the SOAR sample have been selected according to: $|Z|_{\text {Gal }}>0.5$ $\mathrm{kpc}$ and $V_{\text {TAV }}>100 \mathrm{~km} \mathrm{~s}^{-1}$, derived from the Gaia DR2 data available at the time of observations. In Section 5.2 , we provide an in-depth exploration of the $Z_{\mathrm{Gal}}$ vs. $V_{\text {TAV }}$ diagram, justifying these choices.

With Goodman/SOAR, 110 (6\% of the complete sample) metal-poor candidates were observed over the course of the $2018 \mathrm{~B}$ and $2019 \mathrm{~B}$ semesters (Table 1 ). The instrumental setup was similar to the one described in Section 2.1, including a $600 \mathrm{lmm}^{-1}$ grating and a $1.0^{\prime \prime}$ long slit. The typical wavelength coverage for Goodman/SOAR spectra is $3600-6200 \AA$, and the achieved resolution is $R \sim 1300$. For the SOAR sample, stellar parameters have been derived for all but two stars.

12 Throughout this work, we employ the "line-of-sight" terminology instead of the more usual "radial velocity" one. We reserve the latter for the radial component of the Galactocentric velocities in the cylindrical coordinate frame (see Section 5.3). 
Table 3. Stellar Atmospheric Parameters and Abundances

\begin{tabular}{ccccccccc}
\hline $\begin{array}{c}\text { Star Name } \\
(2 \mathrm{MASS})\end{array}$ & $\begin{array}{c}\text { Star Name } \\
\text { (Gaia EDR3) }\end{array}$ & $\begin{array}{c}T_{\text {eff }} \\
(\mathrm{K})\end{array}$ & $\begin{array}{c}\log g \\
(\mathrm{cgs})\end{array}$ & {$[\mathrm{Fe} / \mathrm{H}]$} & {$[\mathrm{C} / \mathrm{Fe}]$} & {$[\mathrm{C} / \mathrm{Fe}]_{c}$} & $A(\mathrm{C})_{c}$ & {$[\mathrm{Mg} / \mathrm{Fe}]$} \\
\hline \hline $00003305-7953389$ & 4634573766005607552 & 5280 & 2.06 & -1.93 & +0.13 & +0.17 & 6.67 & +0.38 \\
$00020162-4430117$ & 4994519032163925632 & 5036 & 2.00 & -1.81 & +0.30 & +0.37 & 6.99 & $\ldots$ \\
$00040305-6106367$ & 4905632480654004608 & 4648 & 4.13 & -1.27 & +0.15 & +0.15 & 7.31 & -0.19 \\
$00043646+4124062$ & 384060304935385984 & 6068 & 3.51 & -1.29 & +0.95 & +0.95 & 8.09 & +0.14 \\
$00045403+3524010$ & 2876804519751163008 & 5455 & 3.52 & -2.59 & +0.50 & +0.50 & 6.34 & +0.06 \\
\hline
\end{tabular}

$[\mathrm{C} / \mathrm{Fe}]_{c}$ and $A(\mathrm{C})_{c}$ values have been corrected for evolutionary status (Placco et al. 2014b).

This table is available in its entirety in machine-readable form.

\section{STELLAR PARAMETERS AND ABUNDANCES}

In order to determine the stellar atmosphericparameter values $\left(T_{\text {eff }}, \log g\right.$, and $\left.[\mathrm{Fe} / \mathrm{H}]\right)$, we have followed the same approach as P18, P19, and Limberg et al. (2021), where the fundamental proxy for metallicity is the Ca II K line ( $3933 \AA)$. We have employed the n-SSPP (Beers et al. 2014, 2017), a customized version of the Segue Stellar Parameter Pipeline (SSPP; Lee et al. 2008a,b, 2011, 2013). This methodology consists of several routines (e.g., photometric calibrations and spectral-line indices) to derive estimates of the stellar parameters. It also compares the input spectra with a dense grid of synthetic ones in a $\chi^{2}$ minimization framework. The best set of values is then adopted taking into account the wavelength coverage of the analyzed spectrum, its SNR, and calculated uncertainties.

Considering both samples, the atmospheric parameters have been determined for all but seven stars (out of 1897; see Table 3), as mentioned in Section 2. Based on empirical comparisons with high-resolution spectroscopic analyses (Placco et al. 2014a; Beers et al. 2014, $2017)$, the typical errors for $T_{\text {eff }}, \log g$, and $[\mathrm{Fe} / \mathrm{H}]$ are $\pm 150 \mathrm{~K}, \pm 0.35 \mathrm{dex}$ and $\pm 0.20 \mathrm{dex}$, respectively, for $\mathrm{SNR} \sim 30$ per pixel at $\sim 4000 \AA$. These estimated atmospheric parameters can be visualized in Figure 2, where the $\log g$ vs. $T_{\text {eff }}$ distribution is overlapped by MESA Isochrones and Stellar Tracks (MIST; Dotter 2016; Choi et al. 2016) models with varying metallicities. Overall, we have confirmed that $1064\left(56_{-2}^{+2} \%{ }^{13}\right)$ of the newly observed stars are metal-poor $([\mathrm{Fe} / \mathrm{H}]<-1.0), 566$ $\left(30_{-2}^{+2} \%\right)$ are VMP, and $35\left(2_{-1}^{+1} \%\right)$ are EMP. These are very similar proportions to what had been previously achieved by both Schlaufman \& Casey (2014) and P19 who performed spectroscopic follow-up with similar selection functions, targets from the B\&B Catalog. We

13 Uncertainties in the fractions are given by the Wilson (1927) score approximation, which provides an estimate of the binomial proportion confidence intervals. This approximation is commonly used for small numbers statistics $(n \lesssim 40)$, but is also comparable to other metrics when the analyzed sample is larger. reinforce that, although the targets observed at SOAR were chosen from kinematic criteria, these represent only a small portion of the complete (Gemini+SOAR) sample, hence these comparisons remain valid.

With the n-SSPP pipeline application, we have also obtained $[\mathrm{C} / \mathrm{Fe}]$ estimates for most stars from both samples. The carbon-to-iron ratios are estimated from the strength of the $\mathrm{CH} G$-band molecular feature $(\sim 4300 \AA)$. We have also calculated corrections for the $[\mathrm{C} / \mathrm{Fe}]$ (and $A(\mathrm{C})^{14}$ ) following the prescriptions of Placco et al. (2014b). These authors employed stellar evolution models (Stancliffe et al. 2009) to account for the intrinsic carbon depletion (with consequent enhancement in nitrogen) in the atmospheres of metal-poor stars due to mixing with internal layers of material enriched by the $\mathrm{CN}$ cycle during the red giant branch phase (Charbonnel 1995). The corrected carbon-abundance values are indicated by $[\mathrm{C} / \mathrm{Fe}]_{c}\left(\right.$ or $\left.A(\mathrm{C})_{c}\right)$ throughout this work, and are listed in Table 3 . We also note that metallicities and carbon abundances of cooler $\left(T_{\text {eff }} \lesssim 4500\right.$ $\mathrm{K}$ ) CEMP stars found in this work (and other lowresolution efforts) might be severely affected by the presence of "carbon-veiling", which depresses the continuum in the wavelength region of the $\mathrm{Ca}$ II $\mathrm{K} / \mathrm{H}$ lines, hindering the metallicity determination (see discussion in Yoon et al. 2020). The accuracy for $[\mathrm{C} / \mathrm{Fe}]$ (and $[\mathrm{C} / \mathrm{Fe}]_{c}$ ) is 0.20 dex (Lee et al. 2013; Beers et al. 2014, 2017).

The n-SSPP derives the abundances of magnesium from the $\mathrm{Mg}$ I triplet absorption lines (located in the range of 5150-5200 ̊). For the Gemini sample, Mg-toiron ratios have been obtained for $1104 / 1787(62 \%)$ of the stars. For the SOAR sample, 85/110 (77\%) stars had $[\alpha / \mathrm{Fe}]$ estimated. The cases for which these abundances are not determined are due to low-quality $(\mathrm{SNR} \lesssim 10)$ spectra, or absorption features that are too weak to be distinguished from the underlying noise. The typical er-

14 Absolute abundance. $A(\mathrm{X})=\log \epsilon(\mathrm{X})=\log \left(N_{\mathrm{X}} / N_{\mathrm{H}}\right)+12$, where $N_{\mathrm{X}}$ and $N_{\mathrm{H}}$ are the number density of atoms of the given element and of hydrogen, respectively. 
rors for $[\mathrm{Mg} / \mathrm{Fe}]$ are also 0.20 dex (Lee et al. 2011; P18; $\mathrm{P} 19)$. The $[\mathrm{Mg} / \mathrm{Fe}]$ ratios are listed in Table 3.

\section{CARBON AND $\alpha$-ELEMENTS}

Carbon-to-iron ratios in low-metallicity stars can constrain their different formation scenarios (see, e.g., Aoki et al. 2002, 2007; Nomoto et al. 2013; Hansen et al. 2016). The $\alpha$-element abundances are crucial for our classical understanding of Galactic chemical evolution (e.g., Chiappini et al. 1997). Furthermore, the Mgto-carbon $([\mathrm{Mg} / \mathrm{C}])$ ratio serves as a diagnostic for the so-called "mono-enriched" population $([\mathrm{Fe} / \mathrm{H}] \lesssim-2.5$ and $[\mathrm{Mg} / \mathrm{C}] \lesssim-1.0$; Hartwig et al. 2018, see also Rasmussen et al. 2020) which are, potentially, genuine second-generation stars. Figure 3 provides plots of the $[\mathrm{Mg} / \mathrm{Fe}]$ and $A(\mathrm{C})_{c}$ distributions as functions of $[\mathrm{Fe} / \mathrm{H}]$ (left and right panels, respectively).

We first discuss the $\alpha$-element abundances. Within the wavelength coverage and resolution of our spectra, the estimated $[\mathrm{Mg} / \mathrm{Fe}]$ is the main representative of the $\alpha$ process. In the metal-rich regime, there is an accumulation of stars at $[\mathrm{Mg} / \mathrm{Fe}] \sim 0.0$ and $[\mathrm{Fe} / \mathrm{H}] \gtrsim-0.5$, associated with the (low- $\alpha$ ) thin disk. We also note the presence of stars related to the (high- $\alpha$ ) thick disk at $[\mathrm{Mg} / \mathrm{Fe}] \sim+0.3$ and $[\mathrm{Fe} / \mathrm{H}] \gtrsim-1.0$. Since we focus on metal-poor stars, further exploration of these components is beyond the scope of the present work. All estimated magnesium-to-iron ratios are in the range $-0.2<[\mathrm{Mg} / \mathrm{Fe}]<+0.6$, which is expected from Galactic chemical-evolution models based on high-precision spectroscopy of $\alpha$ elements (Zhao et al. 2016; Reggiani et al. 2017).

Regarding the carbon abundances, we have explored the Yoon-Beers $A(\mathrm{C})$ vs. $[\mathrm{Fe} / \mathrm{H}]$ diagram following the suggested classification of Yoon et al. (2016, 2019). For consistency, we have applied the criteria outlined by $\mathrm{P} 18$, also employed in $\mathrm{P} 19$, presented in Figure 3 (dashed lines). Besides the $[\mathrm{Fe} / \mathrm{H}]<-1.0$ and $[\mathrm{C} / \mathrm{Fe}]_{c}>$ +0.7 conditions, we consider CEMP stars to be members of the so-called Group I if $A(\mathrm{C})_{c} \gtrsim 7.25$. Since the carbon enrichment of these stars' atmospheres are usually accompanied by enhancement in their abundances of slow neutron-capture ( $s$-process) elements (Spite et al. 2013; Bonifacio et al. 2015; Hansen et al. 2015; Cruz et al. 2018), they are generally associated with the CEMP-s $([\mathrm{Ba} / \mathrm{Fe}]>+1.0$ and $[\mathrm{Ba} / \mathrm{Eu}]>+0.5$; Beers \& Christlieb 2005) subclass of CEMP stars. Such a chemical profile is thought to be the result of mass transfer from a binary companion in the asymptotic giant branch (AGB) phase (Suda et al. 2004; Ryan et al. 2005; Lucatello et al. 2005; Bisterzo et al. 2011; Allen et al. 2012; Placco et al. 2013; Starkenburg et al. 2014; Hansen et al. 2016). On the other hand, CEMP stars with $A(\mathrm{C})_{c}$ $\lesssim 7.25$ are classified as Group II. Unlike the CEMP-s category, these low- $A(\mathrm{C})$ objects do not present overabundances of $s$-process elements $([\mathrm{Ba} / \mathrm{Fe}]<0.0$; Beers \& Christlieb 2005), and are known as CEMP-no stars.
This abundance pattern is the result of pollution of these stars' birth environments through a single EMP (perhaps metal-free; Heger \& Woosley 2010) core-collapse supernovae episode (see Nomoto et al. 2013 for a review). Typically, the $A(\mathrm{C})$ vs. $[\mathrm{Fe} / \mathrm{H}]$ diagram is further partitioned with a Group III, analogous to Group II, but with $[\mathrm{C} / \mathrm{Fe}]_{c} \gtrsim+1.5$ (Yoon et al. 2016, 2019). These same authors argued that CEMP- $s$ and CEMPno stars could be distinguished from $A(\mathrm{C})$ alone with $\sim 90 \%$ purity. We also note that the Group III stars are generally found at the lowest metallicities $([\mathrm{Fe} / \mathrm{H}]$ $\lesssim-3.0$ ), for which we have only a handful of stars in our samples (Section 3). Hence, for simplicity, we have not considered the Group III classification in this work.

We have confirmed that a total of 191 stars in the Gemini+SOAR sample are CEMP stars ${ }^{15}$. Out of these, 94 belong to the (Group I) CEMP- $s$ subclass according to the criteria described above. The remaining 97 of our CEMP stars can be classified as Group II (CEMPno). From Figure 3, not only the overall fraction of CEMP stars increases with decreasing metallicity, but also the CEMP-no class dominates the $[\mathrm{Fe} / \mathrm{H}] \lesssim-2.3$ regime, similar to Yoon et al. (2018), which is in line with the expectation that the emergence of CEMP-s stars in the Galaxy is driven by AGB evolution (Herwig 2005) timescales. On the other hand, all CEMP stars above $[\mathrm{Fe} / \mathrm{H}] \gtrsim-1.8$ are CEMP-s (Group I).

Finally, we have confirmed the trend of increasing CEMP star fractions as a function of declining metallicity. For $[\mathrm{Fe} / \mathrm{H}]<-2.5$, we have found a CEMP star fraction of $32_{-6}^{+6} \%$, compatible $(1 \sigma)$ with both P19 and Schlaufman \& Casey (2014). We have also found excellent agreement between our results, those from P19, and the high-resolution effort of Placco et al. (2014b). For $[\mathrm{Fe} / \mathrm{H}]<-2.0$, we have obtained $19_{-3}^{+3} \%$. In the $[\mathrm{Fe} / \mathrm{H}]<-3.0$ regime, for which we have very few stars, we have calculated $43_{-15}^{+16 \%}$.

\section{KINEMATICS OF THE METAL-POOR CANDIDATES}

Astrometry can be used to improve the efficiency in the search for VMP stars in the Galaxy. In P18, the Tycho-Gaia Astrometric Solution database (Lindegren et al. 2016) was combined with distances from Astraatmadja \& Bailer-Jones (2016) to calculate the Cartesian Galactic positions $\left(X_{\mathrm{Gal}}, Y_{\mathrm{Gal}}\right.$, and $\left.Z_{\mathrm{Gal}}\right)$ and velocities (particularly $V_{\mathrm{T}}$ ). For $V_{\mathrm{T}} \geq 75 \mathrm{~km} \mathrm{~s}^{-1}$, the authors noticed a considerable increase in the fractions of metalpoor and VMP stars in their own validation sample of low-metallicity stars from RAVE DR5. However, the full

15 Note that CEMP stars can be further divided into the CEMP- $r$ $([\mathrm{Eu} / \mathrm{Fe}]>+0.3$ and $[\mathrm{Ba} / \mathrm{Eu}]<0.0)$ and $\mathrm{CEMP}-r / s$ or CEMP- $i$ $(0.0<[\mathrm{Ba} / \mathrm{Eu}]<+0.5)$ categories (Hansen et al. 2018; Ezzeddine et al. 2020). However, these are not considered in this work given the impossibility of classifying them without knowledge of their $\mathrm{Ba}$ and $\mathrm{Eu}$ abundances. 

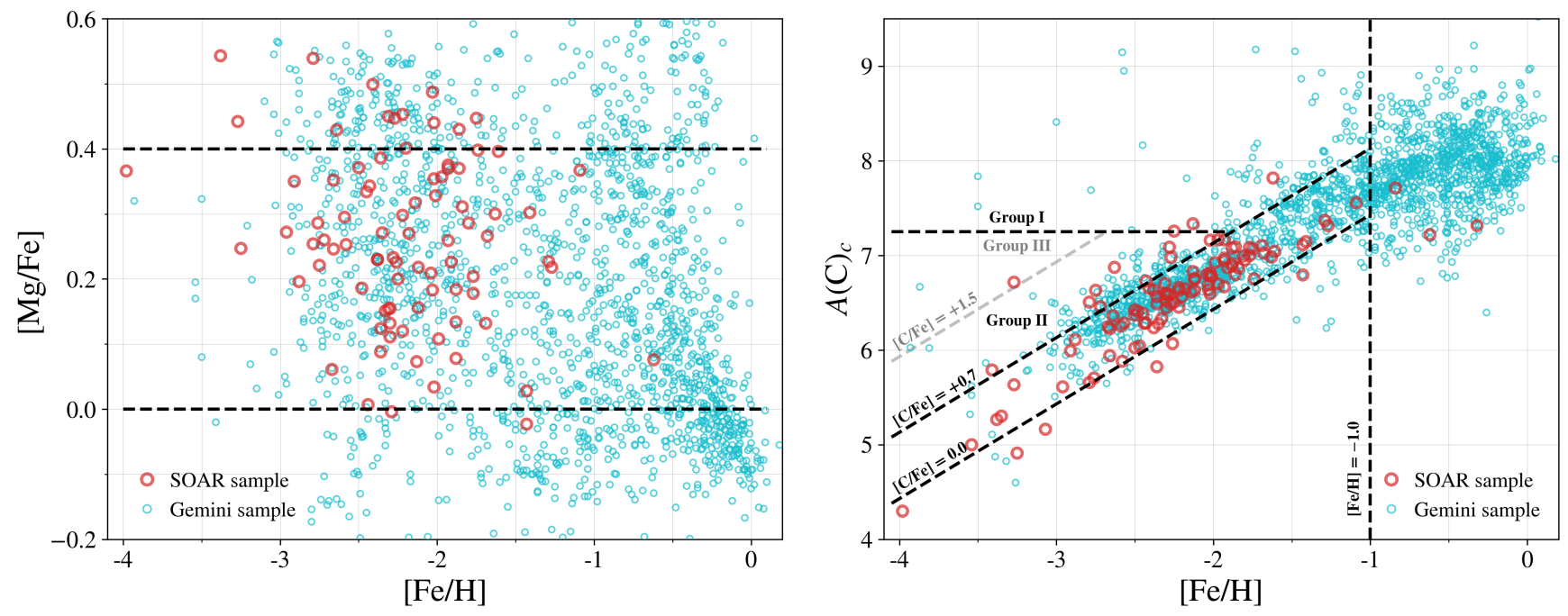

Figure 3. Left panel: Mg-to-iron ratio ([Mg/Fe]; Section 4) vs. metallicity $([\mathrm{Fe} / \mathrm{H}]$; Section 3). Dashed lines mark different levels of Mg enrichment. Cyan and red open circles correspond to stars in the Gemini (Section 2.1) and SOAR (Section 2.2) samples, respectively, in a color scheme similar to Figures 1 and 2. Right panel: Absolute carbon abundance $(A(\mathrm{C}))_{c}$; Section 4), corrected for evolutionary effects (Placco et al. 2014b), vs. [Fe/H] diagram. Black dashed lines mark the classification for different levels of carbon enhancement according to Yoon et al. (2016, 2019) and considered in this work; see text for details. For convenience, we mark the characteristic region of Group III with a gray line. The vertical line marks the metal-poor limit at $[\mathrm{Fe} / \mathrm{H}]=-1.0$, for reference.

potential of this approach was still not clear at the time, because $(i)$ those targets were selected with prior information of their metallicities from RAVE's moderateresolution $(R \sim 7500)$ spectroscopy and $(i i)$ the limited coverage and quality of Gaia DR1 data did not allow for a confident (with small errors) investigation of the majority of their studied stars. In P19, despite this knowledge, the better astrometry from Gaia DR2 was still not available at the time of observations, hence only a simple PM (plus $V$-band magnitude and reddening) cut was applied. In this work, we seek to explore more fully the influence of kinematic-based target selection in finding VMP and EMP stars, taking advantage of Gaia EDR3 PMs and $V_{\text {los. }}$. The results of these analyses will be (and are being) employed in future (and ongoing) observational campaigns.

We have cross-matched both the Gemini and SOAR samples with Gaia EDR3 to acquire accurate $V_{\text {los }}$, parallaxes, and PMs (Table 4). These measured parameters have been converted to the Cartesian Galactic phase-space positions and velocities using the Astropy package (Astropy Collaboration et al. 2013, 2018). The assumed in-plane distance from the Sun to the Galactic center is $R_{\odot}=8.2 \mathrm{kpc}$ (Bland-Hawthorn \& Gerhard 2016), which is compatible with Gravity Collaboration et al. (2019, 2020). We recall that stars in the SOAR sample have been observed with prior knowledge of their kinematics, derived from Gaia DR2 data. For the Gemini sample, matches with Gaia EDR3 have been found for all but two stars (Section 2). Out of these, 1766/1787 (99\%) have non-negative parallaxes. The distances have been calculated through the inversion of these parallaxes ${ }^{16}$ after re-calibration $\left(+0.017\right.$ mas $^{17}$; Lindegren et al. 2020a). For the purpose of our investigation, we have applied a relative error cut, keeping stars with parallax_over_error $\geq 1$ (see Lindegren et al. 2020b). Despite being very permissive in comparison to the recent literature, this uncertainty cut is consistent with P18, so our study can be directly comparable to theirs, and has the objective of not removing too many candidates. However, we note that, thanks to the improved astrometry of Gaia EDR3 in comparison to DR2, only a handful $(\sim 80)$ stars in the full (Gemini+SOAR+P19) sample have parallax_over_error $\leq 5$ (or missing/negative values). Furthermore, $\sim 91 \%$ of all stars observed have parallax_over_error $\geq 10$, providing reliability to our kinematic/dynamical calculations.

16 During the reviewing process of this paper, distances derived from a probabilistic approach have been made available by BailerJones et al. (2021) for sources in Gaia EDR3. Since our sample is fairly local ( $\sim 80 \%$ within $4 \mathrm{kpc}$; Figure 1), the differences between these authors' calculated distances and those adopted here are negligible ( $>10 \%$ for only a couple of stars). In fact, even considering the full sample (up to $\sim 8 \mathrm{kpc}$ ), only $\sim 5 \%$ of the stars with parallax_over_error $\geq 5$ show differences $>10 \%$ in their distance estimates.

17 The parallax offset depends in a non-linear, non-trivial way on the $(G$-band $)$ magnitude and color $\left(G_{\mathrm{BP}}-G_{\mathrm{RP}}\right)$ of the source. For simplicity, we adopt a global correction equal to the median of quasar's parallaxes in Gaia EDR3 (Lindegren et al. 2020a). 


\subsection{Why $V_{\mathrm{TAV}}$ ?}

In this work, we build on the results presented by P18. One of the refinements that we have made is the introduction of the $V_{\text {TAV }}$ quantity (Section 2.2), which demands either PMs or $V_{\text {los }}$, but makes use of their combination whenever both are available. With the goal of performing a comprehensive analysis, we have divided the $Z_{\text {Gal }}$ vs. velocity diagrams into four different regions, represented with distinct grayish and white colors in Figure 4. We find that high efficiencies of VMP and EMP detections are achieved even if only one velocity component is known. The $\pm 0.5 \mathrm{kpc}$ stripe at the center of all panels is motivated by the characteristic scale height of thin-disk stars around the Galactic plane (Recio-Blanco et al. 2014; Li et al. 2018a), which is also consistent with the analysis of P18. The separation at $100 \mathrm{~km} \mathrm{~s}^{-1}$ approximately marks the end of the metalrich-dominated $([\mathrm{Fe} / \mathrm{H}]>-2.0)$ portion of the diagrams and can be considered a velocity limit between thin-disk $\left(\lesssim 100 \mathrm{~km} \mathrm{~s}^{-1}\right)$ and thick disk/halo populations, which is visible from the large concentrations of black dots in Figure 4.

Indeed, it is expected that higher values of velocity should yield greater fractions of genuine halo stars (see Koppelman et al. 2018 and Posti et al. 2018 for recent discussions). However, as is made clear below, the chosen boundaries already produce quite high success rates. The combination of these cuts at $\pm 0.5 \mathrm{kpc}$ and $100 \mathrm{~km}$ $\mathrm{s}^{-1}$ defines the various areas in Figure 4 . Then, we calculate the fractions of stars showing $[\mathrm{Fe} / \mathrm{H}] \leq-2.0$ $\left(f_{\mathrm{VMP}}\right)$ in each of these regions. We take these fractions as representative of the efficiency of finding VMP stars in each different portion of the $Z_{\text {Gal }}$ vs. velocity diagrams for stars originally identified as possibly metal-poor on the basis of the B\&B photometric selection.

In the top panel of Figure 4, only $V_{\text {los }}$ is employed, in combination with $Z_{\mathrm{Gal}}$, to explore the $f_{\mathrm{VMP}}$ in the P19 sample. Although high success rates already appear for $|Z|_{\text {Gal }} \geq 0.5 \mathrm{kpc}$, the number of available candidates in the P19 sample is reduced to approximately half of its total. In the middle panel of Figure 4 , the $\left(Z_{\mathrm{Gal}}, V_{\mathrm{T}}\right)$ space is displayed, similar to the figures presented by P18. We note that almost the entire P19 sample has suitable PMs and parallaxes from Gaia EDR3, allowing us to calculate accurate $V_{\mathrm{T}}$ for these stars. The $f_{\mathrm{VMP}}$ in the white regions of the $\left(Z_{\mathrm{Gal}}, V_{\mathrm{T}}\right)$ diagram remains statistically the same as the $\left(Z_{\mathrm{Gal}}, V_{\mathrm{los}}\right)$ one, but the number of stars has more than tripled, which is advantageous for planning an observational campaign.

The bottom panel of Figure 4 shows the $Z_{\mathrm{Gal}}$ vs. $V_{\mathrm{TAV}}$ diagram for the $\mathrm{P} 19$ sample. Once more, the $f_{\mathrm{VMP}}$ in each area of the diagram is similar to previous panels, but there is an increase of $\sim 13 \%$ in the number of stars in the $|Z|_{\text {Gal }} \geq 0.5 \mathrm{kpc}$ and $V_{\mathrm{TAV}} \geq 100 \mathrm{~km} \mathrm{~s}^{-1}$ region, while maintaining high efficiency, almost double the $f_{\mathrm{VMP}}$ achieved by P19. This finding inspired
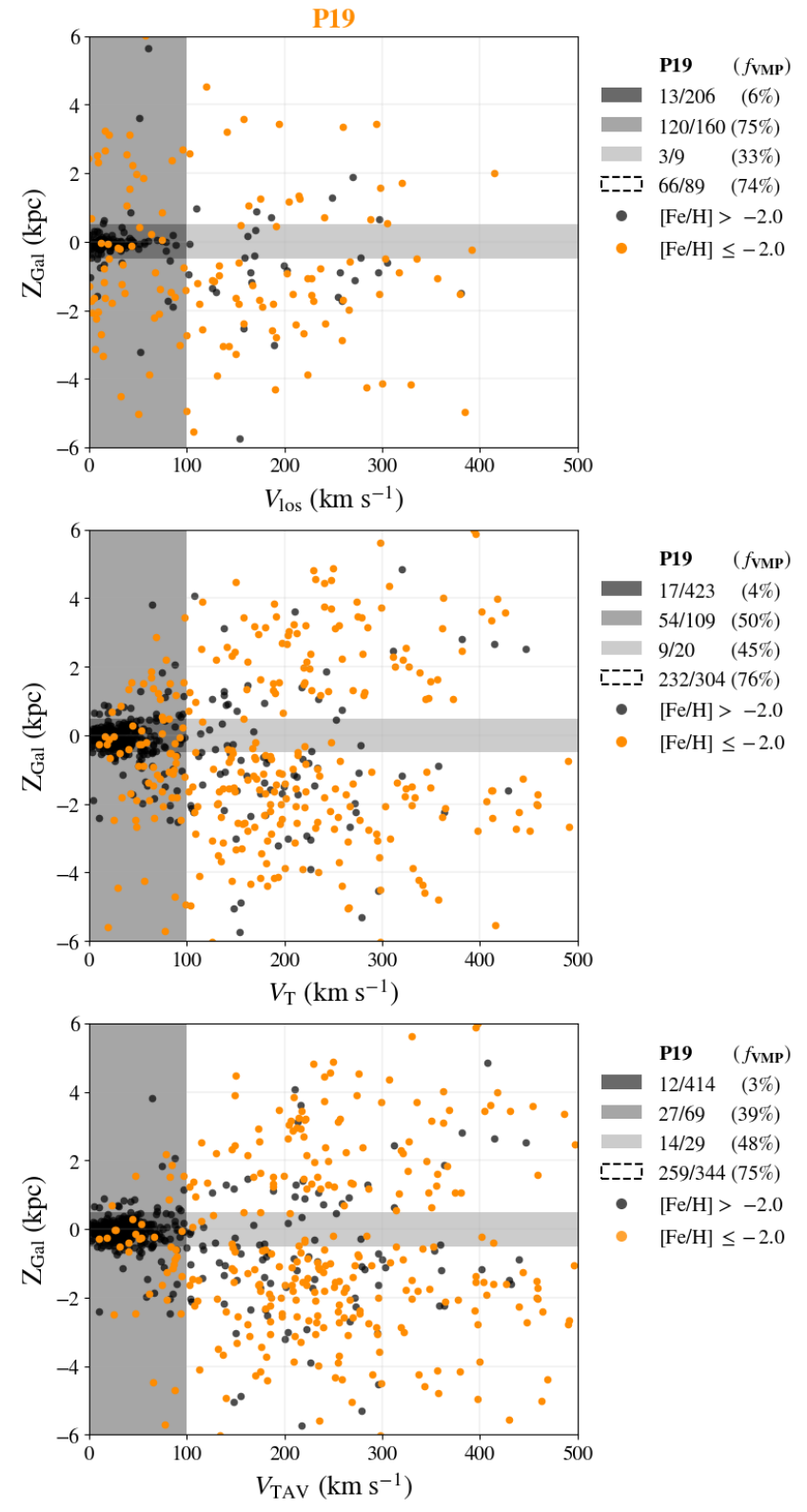

Figure 4. Diagrams of the P19 sample for $Z_{\mathrm{Gal}}$ vs. different components of velocity: $V_{\mathrm{los}}$ (top panel), $V_{\mathrm{T}}$ (middle), and $V_{\text {TAV }}$ (bottom panel). To the right of each panel, the fractions of VMP stars for each gray-shaded and white regions are presented. The black and orange dots represent stars with $[\mathrm{Fe} / \mathrm{H}]>-2.0$ and VMP stars, respectively

the target-selection criteria for observations with Goodman/SOAR. Even though we had the prospect of improvements, we were surprised by these results, since the $\mathrm{B} \& \mathrm{~B}$ catalog is by-design biased towards metal-poor stars. In general, from top to bottom, one can notice the increasing number of points in each diagram of Figure 4 , but maintaining statistically equivalent success rates for the different regions, demonstrating the advantages of employing the $V_{\mathrm{TAV}}$ parameter. 
Table 4. Phase-space Information from Gaia EDR3

\begin{tabular}{ccrrrrrrr}
\hline $\begin{array}{c}\text { Star Name } \\
(2 \mathrm{MASS})\end{array}$ & $\begin{array}{c}\text { Star Name } \\
(\text { Gaia EDR3 })\end{array}$ & $\begin{array}{c}V_{\text {los }} \\
\left(\mathrm{km} \mathrm{s}^{-1}\right)\end{array}$ & $\begin{array}{c}d_{\text {helio }} \\
(\mathrm{kpc})\end{array}$ & $\begin{array}{c}\sigma_{d} \\
(\mathrm{kpc})\end{array}$ & $\begin{array}{c}\mathrm{PM}_{\text {R.A. }} \\
\left(\mathrm{mas} \mathrm{yr}^{-1}\right)\end{array}$ & $\begin{array}{c}\mathrm{PM}_{\text {Decl. }} \\
\left(\mathrm{mas} \mathrm{yr}^{-1}\right)\end{array}$ & $\begin{array}{c}Z_{\text {Gal }} \\
(\mathrm{kpc})\end{array}$ & $\begin{array}{c}V_{\text {TAV }} \\
\left(\mathrm{km} \mathrm{s}^{-1}\right)\end{array}$ \\
\hline \hline $00003305-7953389$ & 4634573766005607552 & 269.3 & 2.51 & 0.06 & -4.538 & -4.538 & -1.48 & 274.3 \\
$00020162-4430117$ & 4994519032163925632 & 58.5 & 3.13 & 0.19 & 14.656 & 14.656 & -2.92 & 202.6 \\
$00040305-6106367$ & 4905632480654004608 & -6.0 & 0.12 & 0.00 & 53.448 & 53.448 & -0.07 & 15.7 \\
$00043646+4124062$ & 384060304935385984 & -13.7 & 1.01 & 0.01 & -7.058 & -7.058 & -0.33 & 28.8 \\
$00045403+3524010$ & 2876804519751163008 & -91.5 & 1.06 & 0.02 & 9.525 & 9.525 & -0.45 & 154.2 \\
\hline
\end{tabular}

This table is available in its entirety in machine-readable form.
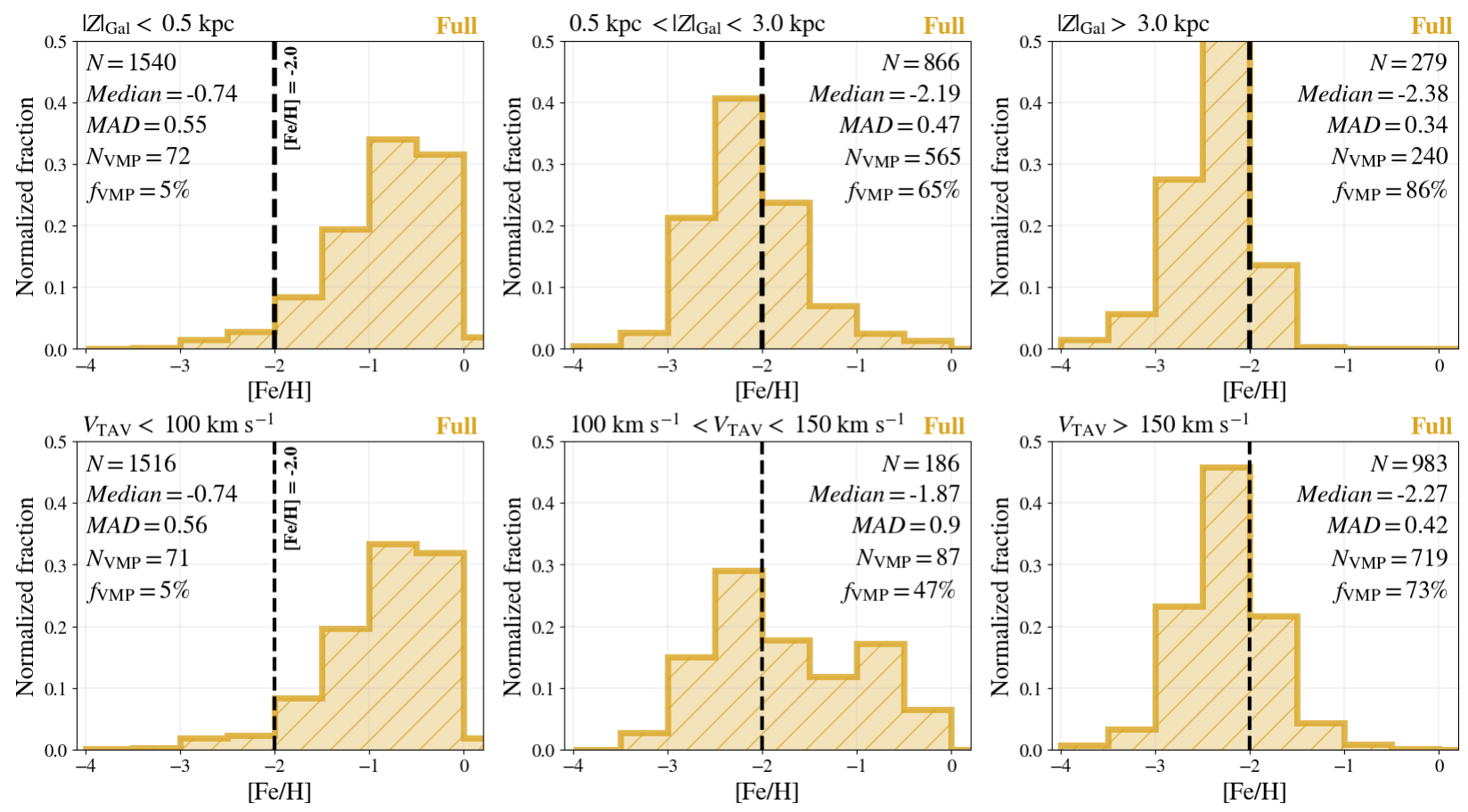

Figure 5. Metallicity distribution functions (MDFs) of stars observed from the B\&B catalog. The histograms present the normalized fractions; the total number of stars in each panel $(N)$ is provided. Top panels: MDFs of the full (Gemini+SOAR+P19) sample for the slices: $|Z|_{\text {Gal }}<0.5 \mathrm{kpc}, 0.5<|Z|_{\text {Gal }}(\mathrm{kpc})<3.0$, and $|Z|_{\text {Gal }}>3.0 \mathrm{kpc}$ (left, middle, and right, respectively). Bottom panels: MDFs of the full sample, but for various ranges of $V_{\mathrm{TAV}}: V_{\mathrm{TAV}}<100 \mathrm{~km} \mathrm{~s}^{-1}, 100<V_{\mathrm{TAV}}\left(\mathrm{km} \mathrm{s}^{-1}\right)<150$ and $V_{\mathrm{TAV}}>150 \mathrm{~km} \mathrm{~s}^{-1}$ (left, middle, and right, respectively). The median [Fe/H], median absolute deviation (MAD), the total number $\left(N_{\mathrm{VMP}}\right)$, and the fraction of VMP stars $\left(f_{\mathrm{VMP}}\right)$ are shown in each panel. The dashed vertical lines mark the VMP limit at $[\mathrm{Fe} / \mathrm{H}]=-2.0$, for reference.

\section{2. $Z_{\text {Gal }}$ vs. $V_{\mathrm{TAV}}$ Diagrams and Final Success Rates}

The individual impact of variations in $|Z|_{\text {Gal }}$ and $V_{\mathrm{TAV}}$ in the metallicity distribution function (MDF) of the full B\&B sample are presented from the top and bottom rows of Figure 5, respectively. In the thin-disklike regions $\left(|Z|_{\text {Gal }}<0.5 \mathrm{kpc}\right.$ and $V_{\text {TAV }}<100 \mathrm{~km}$ $\mathrm{s}^{-1}$; left column), the MDFs peak at $[\mathrm{Fe} / \mathrm{H}] \sim-0.7$ and the $f_{\mathrm{VMP}}$ is only $\sim 5 \%$. However, as we move to higher values of $|Z|_{\mathrm{Gal}}$, the peak of the MDF is shifted to the VMP regime already at $|Z|_{\text {Gal }}>0.5 \mathrm{kpc}$ (mid- dle). The $f_{\mathrm{VMP}}$ reaches $86 \%$ for $|Z|_{\mathrm{Gal}}>3.0 \mathrm{kpc}$ (right), which reflects the lower metallicity of the Galactic halo in comparison to the canonical disk system, despite the already low-metallicity-biased B\&B selection. For the $V_{\text {TAV }}$ quantity, this transition is smoother, noticeable from the extended MDF within the range $100<V_{\text {TAV }}\left(\mathrm{km} \mathrm{s}^{-1}\right)<150$ (middle column). However, the $f_{\mathrm{VMP}}$ significantly increases at $V_{\mathrm{TAV}}>150 \mathrm{~km} \mathrm{~s}^{-1}$ (right).

Figure 6 presents the $Z_{\mathrm{Gal}}$ vs. $V_{\mathrm{TAV}}$ diagrams for the Gemini and SOAR samples. Colored symbols are VMP 

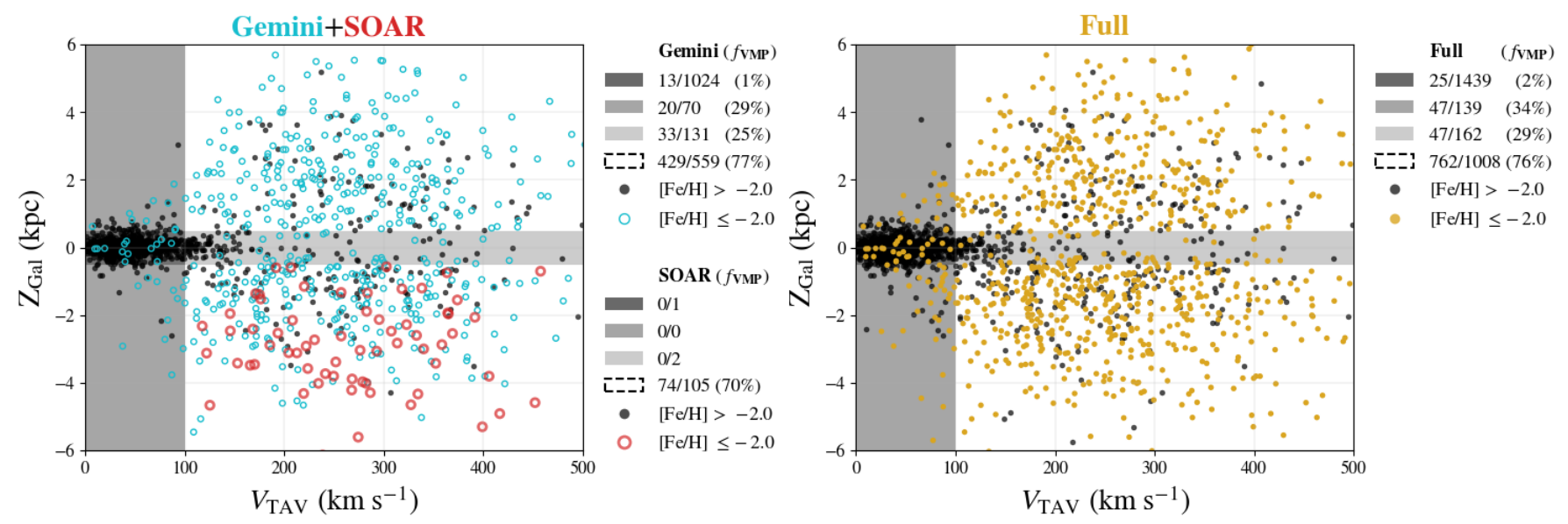

Figure 6. $Z_{\text {Gal }}$ vs. $V_{\mathrm{TAV}}$ diagrams. Left: Distribution of stars from Gemini (Section 2.1) and SOAR (Section 2.2) samples. Right: Distribution of stars from the full (Gemini+SOAR+P19) sample. Colored symbols are VMP stars, while the black ones have $[\mathrm{Fe} / \mathrm{H}]>-2.0$. Both panels are shown with a color scheme similar to Figures 1, 2, 3, and 5. Specific VMP fractions are also provided for each gray-shaded and white regions (see text and Table 5 for details) to the right of each panel.

Table 5. Regions of the $Z_{\text {Gal vs. }} V_{\mathrm{TAV}}$ Diagrams and Final Success Rates for Different Metallicities

\begin{tabular}{|c|c|c|c|c|c|c|}
\hline $\begin{array}{l}\text { Region } \\
(\text { Color }) \\
\end{array}$ & $\begin{array}{r}|Z|_{\text {Gal }} \\
(\mathrm{kpc}) \\
\end{array}$ & $\begin{array}{c}V_{\mathrm{TAV}} \\
\left(\mathrm{km} \mathrm{s}^{-1}\right) \\
\end{array}$ & $\begin{array}{l}{[\mathrm{Fe} / \mathrm{H}]} \\
\leq-1.5 \\
\end{array}$ & $\begin{array}{l}{[\mathrm{Fe} / \mathrm{H}]} \\
\leq-2.0 \\
\end{array}$ & $\begin{array}{l}{[\mathrm{Fe} / \mathrm{H}]} \\
\leq-2.5 \\
\end{array}$ & $\begin{array}{l}{[\mathrm{Fe} / \mathrm{H}]} \\
\leq-3.0 \\
\end{array}$ \\
\hline Dark gray & $<0.5$ & $<100$ & $8_{-1}^{+2} \%$ & $2_{-1}^{+1} \%$ & $1_{-0}^{+1} \%$ & $0_{-0}^{+0} \%$ \\
\hline Medium gray & $\geq 0.5$ & $<100$ & $58_{-8}^{+8} \%$ & $34_{-7}^{+8 \%} \%$ & $17_{-5}^{+7} \%$ & $3_{-2}^{+4} \%$ \\
\hline Light gray & $<0.5$ & $\geq 100$ & $52_{-8}^{+8} \%$ & $29_{-6}^{+7} \%$ & $9_{-4}^{+6} \%$ & $1_{-1}^{+3} \%$ \\
\hline White & $\geq 0.5$ & $\geq 100$ & $96_{-1}^{+1} \%$ & $76_{-3}^{+3} \%$ & $28_{-3}^{+3} \%$ & $4_{-1}^{+1} \%$ \\
\hline
\end{tabular}

stars spectroscopically confirmed by the present work, following the same color scheme as Figures 1, 2, 3, and 5, while the black dots are those with $[\mathrm{Fe} / \mathrm{H}]>-2.0$. The left panel shows the distributions of stars from Gemini and SOAR samples in this parameter space. Given the target selection described in Section 2.2, the majority of stars observed from SOAR occupy the most interesting regions of the $Z_{\mathrm{Gal}}$ vs. $V_{\mathrm{TAV}}$ diagram. Interestingly, the few cases in which they are outside the VMP regions originate from differences between the Gaia DR2 and EDR3 parameters. The right panel presents the $\left(Z_{\mathrm{Gal}}\right.$, $\left.V_{\text {TAV }}\right)$ space of the full sample, including that from P19.

The $f_{\mathrm{VMP}}$ in each of the gray-shaded and white regions of the $Z_{\mathrm{Gal}}$ vs. $V_{\mathrm{TAV}}$ diagrams are also provided in Figure 6 . Table 5 lists the final success rates for each region and for various metallicity regimes $([\mathrm{Fe} / \mathrm{H}] \leq-1.5$, $\leq-2.0, \leq-2.5$, and $\leq-3.0$ ), along with their respective $|Z|_{\text {Gal }}$ and $V_{\mathrm{TAV}}$ boundaries. These final efficiencies are drawn from the full sample for better statistics. Particularly in the white regions $\left(|Z|_{\text {Gal }} \geq 0.5\right.$ $\mathrm{kpc}$ and $V_{\mathrm{TAV}} \geq 100 \mathrm{~km} \mathrm{~s}^{-1}$ ), the thick disk/halolike ones, the fractions of stars within $[\mathrm{Fe} / \mathrm{H}] \leq-1.5$, $\leq-2.0, \leq-2.5$, and $\leq-3.0$ are $96 \%, 76 \%, 28 \%$, and
$4 \%$, respectively. Such effectiveness in the search for low-metallicity stars in the Galaxy are only rivaled by those with prior narrow-band photometry at the wavelength of the Ca II K line (e.g., Youakim et al. 2017; Aguado et al. 2019; Da Costa et al. 2019). In the near future, we expect to be able to combine the astrometric information from Gaia's DRs with narrow-band photometry from, e.g., S-PLUS (Southern-Photometric Local Universe Survey; Mendes de Oliveira et al. 2019), JPLUS (Javalambre-Photometric Local Universe Survey; Cenarro et al. 2019), and J-PAS (Javalambre-Physics of the Accelerating Universe Astrophysical Survey; Bonoli et al. 2020) to obtain even better success rates, particularly in the EMP regime.

\subsection{Toomre Diagrams}

For comparison, we have also investigated the success rates in finding low-metallicity stars using a kinematicbased target selection within different regions of the Toomre diagram (Figure 7). The peculiar motion of the Sun with respect to the local standard of rest (LSR) is $(U, V, W)_{\odot}=(11.10,12.24,7.25) \mathrm{km} \mathrm{s}^{-1}$ (Schönrich et al. 2010). The adopted velocity of the LSR is $V_{\mathrm{LSR}}=(0.0,232.8,0.0) \mathrm{km} \mathrm{s}^{-1}$ (McMillan 2017). Re- 

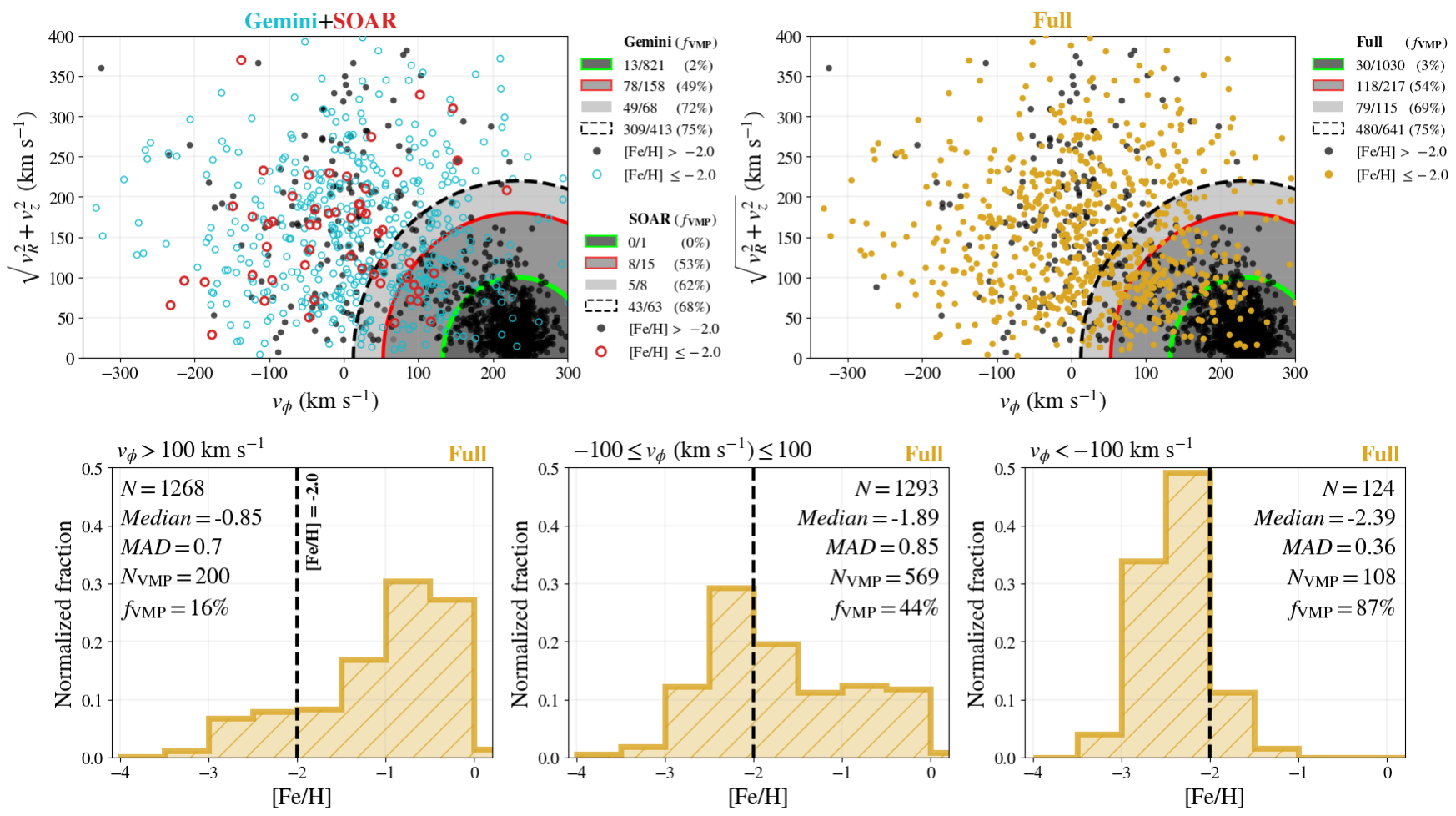

Figure 7. Top panels: Toomre diagrams $\left(\sqrt{v_{R}^{2}+v_{z}^{2}}\right.$ vs. $\left.v_{\phi}\right)$. Top left: Gemini (Section 2.1) and SOAR (Section 2.2) samples. Top right: Full (Gemini+SOAR+P19) sample. Stars that are VMP are represented with colored symbols in the same color scheme as Figures 1, 2, 3, 5, and 6. Black dots are stars with $[\mathrm{Fe} / \mathrm{H}]>-2.0$. The green and red solid lines mark the velocity boundaries for the thin $\left(\left|V_{\text {total }}-V_{\mathrm{LSR}}\right|<100 \mathrm{~km} \mathrm{~s}^{-1}\right.$; dark gray $)$ and thick disks $\left(100 \mathrm{~km} \mathrm{~s}^{-1} \leq\left|V_{\text {total }}-V_{\mathrm{LSR}}\right|<180 \mathrm{~km} \mathrm{~s}{ }^{-1}\right.$; medium gray), respectively (see text). The dashed line marks the transition region $\left(180 \mathrm{~km} \mathrm{~s}^{-1} \leq\left|V_{\mathrm{total}}-V_{\mathrm{LSR}}\right|<220 \mathrm{~km} \mathrm{~s}^{-1}\right.$; light gray stripe) between the thick disk and halo. Finally, the halo is defined by $\left|V_{\text {total }}-V_{\mathrm{LSR}}\right| \geq 220 \mathrm{~km} \mathrm{~s}{ }^{-1}$ (white region). To the right of each panel, the specific VMP fractions $\left(f_{\mathrm{VMP}}\right)$ in each of the described areas in $\left(\sqrt{v_{R}^{2}+v_{z}^{2}}, v_{\phi}\right)$ space are provided. Bottom panels: Metallicity distribution functions (MDFs) of the full sample for various intervals of $v_{\phi}$. Bottom left: $v_{\phi}>100$ $\mathrm{km} \mathrm{s}^{-1}$. Bottom middle: $-100 \leq v_{\phi}\left(\mathrm{km} \mathrm{s}^{-1}\right) \leq 100$. Bottom right: $v_{\phi}<-100 \mathrm{~km} \mathrm{~s}^{-1}$. The total numbers of stars $(N)$, the medians, median absolute deviations (MAD), the numbers $\left(N_{\mathrm{VMP}}\right)$, and the fractions $\left(f_{\mathrm{VMP}}\right)$ of VMP stars are also displayed in the corresponding panels. The dashed vertical lines mark the VMP limit at $[\mathrm{Fe} / \mathrm{H}]=-2.0$, for reference.

cently, purely acceleration-based estimates of the Galactic fundamental parameters have been made available, thanks to the direct measurement of the Solar System's acceleration from Gaia EDR3 astrometry. Nevertheless, the values adopted here have been chosen due to their consistency with the Galactic model of McMillan (2017), which will be employed for orbit integration in Section 5.4. Figure 7 shows the $\left(\sqrt{v_{R}^{2}+v_{z}^{2}}, v_{\phi}\right)$ space, where $\left(v_{R}, v_{\phi}, v_{z}\right)$ compose the velocity vector of each star in the cylindrical coordinate system (radial, azimuthal, and vertical directions, respectively). Stars in prograde motion have $v_{\phi}>0$. Note that it is necessary to have measured $V_{\text {los }}$ in order to calculate the complete set of $\left(v_{R}, v_{\phi}, v_{z}\right)$. This is a clear disadvantage in utilizing the Toomre diagram for selecting targets for systematic searches of low-metallicity stars, since many targets would be ignored due to lack of available $V_{\text {los }}$. Therefore, we wish to evaluate whether or not the efficiency in finding VMP stars is hindered by the application of $Z_{\text {Gal }}$ vs. $V_{\mathrm{TAV}}$ diagram in comparison to $\sqrt{v_{R}^{2}+v_{z}^{2}}$ vs. $v_{\phi}$, where the complete phase-space vector needs to be available. In this context, we also study changes in the full sample's MDF with $v_{\phi}$ (see Figure 7).

For consistency with the analysis presented in Section 5.2 , we have divided the Toomre diagrams into four regions, similar to what has been done for the $\left(Z_{\mathrm{Gal}}, V_{\mathrm{TAV}}\right)$ space. In Figure 7, these are displayed in an arrangement similar to Figure 6, with a gray scale tentatively representing different Galactic components. Stars that are VMP are shown with colored symbols in the same color scheme as Figures 1, 2, 3, 5, and 6. The dark gray area with a green contour contains stars within $\left|V_{\text {total }}-V_{\mathrm{LSR}}\right|<100 \mathrm{~km} \mathrm{~s}^{-1}$, where $V_{\text {total }}$ is the complete velocity vector of a given star. This is the most crowded zone in both top panels of Figure 7, which is noticeable from the accumulation of black points around $v_{\phi} \sim V_{\mathrm{LSR}}$. This region in velocity space is representative of the thin disk (e.g., Venn et al. 2004; Bensby et al. 2014 ), and is also recognizable from its $f_{\mathrm{VMP}} \lesssim 3 \%$ in both diagrams of Figure 7, despite the B\&B selection. 
The predominance of this component is also perceptible from the MDF of stars with $v_{\phi}>100 \mathrm{~km} \mathrm{~s}^{-1}$ (bottom left panel of Figure 7). This MDF peaks at $[\mathrm{Fe} / \mathrm{H}]$ $\sim-0.8$, but also shows an extended VMP tail associated with the thick disk and prograde portion of the halo.

Moving radially outwards from the thin-disk region of the $\left(\sqrt{v_{R}^{2}+v_{z}^{2}}, v_{\phi}\right)$ space, the medium gray area in between the green and red lines is defined by $100 \mathrm{~km} \mathrm{~s}^{-1} \leq$ $\left|V_{\text {total }}-V_{\mathrm{LSR}}\right|<180 \mathrm{~km} \mathrm{~s}^{-1}$, and is characteristic of the thick disk (see, e.g., Amarante et al. 2020b for a recent discussion). A noteworthy feature of the Toomre diagrams in Figure 7 is the presence of high fractions $(\gtrsim 50 \%)$ of VMP stars in this thick-disk-like slice of the velocity space. We speculate that this feature is also due to the underlying $\mathrm{B} \& \mathrm{~B}$ selection. This finding has led us to scrutinize the presence of VMP and EMP stars associated with the Galactic disks further in Section 5.4.

The light gray stripe, defined by the dashed and red solid lines in Figure $7\left(180 \mathrm{~km} \mathrm{~s}^{-1} \leq\left|V_{\text {total }}-V_{\mathrm{LSR}}\right|<\right.$ $220 \mathrm{~km} \mathrm{~s}^{-1}$ ), comprises the tail of the velocity distribution of the Galactic disk (e.g., Bonaca et al. 2017 and Koppelman et al. 2018). This transition region between thick disk and halo also contains stars from the "splashed disk" (Belokurov et al. 2020; Amarante et al. 2020a; An \& Beers 2020), considered to be the metalrich, in-situ counterpart of the halo (Bonaca et al. 2017, 2020; Haywood et al. 2018; Gallart et al. 2019; Di Matteo et al. 2019). The $f_{\mathrm{VMP}}$ in this area of the $\left(\sqrt{v_{R}^{2}+v_{z}^{2}}, v_{\phi}\right)$ space is statistically equivalent to the halo selection.

We note that the concentration of stars within $-50 \lesssim$ $v_{\phi}\left(\mathrm{km} \mathrm{s}^{-1}\right) \lesssim+50$, permeating all values of $\sqrt{v_{R}^{2}+v_{z}^{2}}$, is reminiscent of the Gaia-Sausage/Enceladus (GSE) merging event (Belokurov et al. 2018; Haywood et al. 2018; Helmi et al. 2018). However, considering $v_{\phi}$ alone, we also note the extended MDF shown in the bottom middle panel of Figure $7\left(-100 \leq v_{\phi}\left(\mathrm{km} \mathrm{s}^{-1}\right) \leq 100\right)$, which peaks between $-2.5<[\mathrm{Fe} / \mathrm{H}]<-2.0$, but has a prominent metal-rich tail linked to the thick and splashed disks. On the other hand, the MDF in the $v_{\phi}<-100 \mathrm{~km} \mathrm{~s}^{-1}$ panel of Figure 7 (bottom right) is almost entirely comprised by VMP stars. These stars might also be associated with previously identified halo substructures, e.g., Sequoia (Myeong et al. 2019) and Thamnos (Koppelman et al. 2019). Indeed, independent efforts have suggested that these retrograde groups are more metal-poor than GSE (Matsuno et al. 2019; Monty et al. 2020; Dietz et al. 2020; Kordopatis et al. 2020). Nevertheless, these supposed remnants of nowdestroyed dwarf galaxies are also of interest to this work, particularly because, recently, large numbers of VMP stars, including those exhibiting $r$-process-element enhancement (Gudin et al. 2021), have been demonstrated to be associated with them (Yuan et al. 2020; Limberg et al. 2021; see Section 5.4).

Finally, the black dashed line delineates the $\mid V_{\text {total }}-$ $V_{\mathrm{LSR}} \mid \geq 220 \mathrm{~km} \mathrm{~s}^{-1}$ zone of the Toomre diagrams. These are the white areas in both top panels of Fig- ure 7. Stars inhabiting this part of the $\left(\sqrt{v_{R}^{2}+v_{z}^{2}}, v_{\phi}\right)$ space are from either the in-situ or accreted components of the halo. The $f_{\mathrm{VMP}}$ of the full sample in this white region is similar to the one found for the halo-like portions of the $Z_{\text {Gal }}$ vs. $V_{\text {TAV }}$ diagram in Section 5.2. Nonetheless, we have found no statistical differences in $f_{\mathrm{VMP}}$ between the prograde and retrograde components of the halo. To conclude, kinematic criteria based on the $\left(Z_{\text {Gal }}\right.$, $\left.V_{\mathrm{TAV}}\right)$ space can be as efficient as the traditional Toomre diagram in finding low-metallicity stars, with the additional advantage of not needing prior measurement of $V_{\text {los }}$, thus enabling access to a larger number of candidates, while making optimal usage of this parameter whenever it is available. Thus, we recommend consideration of the $V_{\mathrm{TAV}}$ quantity for the target selection of observational campaigns aiming to find VMP and EMP stars in the Galaxy.

\subsection{Orbits of VMP/EMP Stars}

We now examine the presence of dynamically interesting stars in all samples, which will become priority targets for high-resolution follow-up. First, we check for VMP and EMP stars residing in the kinematically defined thick disk (Section 5.3). In order to confirm whether or not these stars are truly confined to the disk system, we have integrated their orbits with the publicly available library AGAMA (Vasiliev 2019) forward in time for $\sim 5$ Gyr. The Galactic model employed is from McMillan (2017), and includes stellar and gaseous disks, a flattened bulge, and a spherical dark matter halo. The assumptions regarding the Galactic fundamental parameters are the same as Sections 5 and 5.3. The $V_{\text {los }}$ have been acquired from Gaia EDR3, where available, but we have only considered stars with parallax_over_error $\geq 5$ for this exercise. We have performed 1000 realizations of each star's orbit according to its uncertainties in the astrometric quantities, assuming Gaussian distributions for them. The medians of each dynamical parameter have been taken as our nominal values. Our investigation is focused on the maximum vertical distance achieved during a star's orbit $\left(Z_{\max }\right)$, the perigalactic distance $\left(r_{\min }\right)$, the apogalactic distance $\left(r_{\max }\right)$, eccentricity $\left(e=\left(r_{\max }-r_{\min }\right) /\left(r_{\max }+r_{\min }\right)\right)$, orbital energy $(E)$, and the vertical component of angular momentum $L_{z}=R_{\text {Gal }} \times v_{\phi}$, where $R_{\text {Gal }}=\sqrt{X_{\text {Gal }}^{2}+Y_{\text {Gal }}^{2}}$ is the plane-projected distance of a given star from the Galactic center.

The top panel of Figure 8 shows the distribution of the full sample in the $Z_{\max }$ vs. $r_{\max }$ space. A star is considered to be on a planar orbit if its $Z_{\max } \leq 3$ kpc. The transition between the thick disk and halo is smooth in this diagram, as already noticed from the full sample's MDFs for different slices of both $|Z|_{\text {Gal }}$ (Figure 5 ) and $v_{\phi}$ (Figure 7). However, the $Z_{\max }$ limit at $3 \mathrm{kpc}$ reflects the recently estimated scale height of the thick disk by Li \& Zhao (2017). Crucially, it is also consistent with what has been considered in the analyses of 

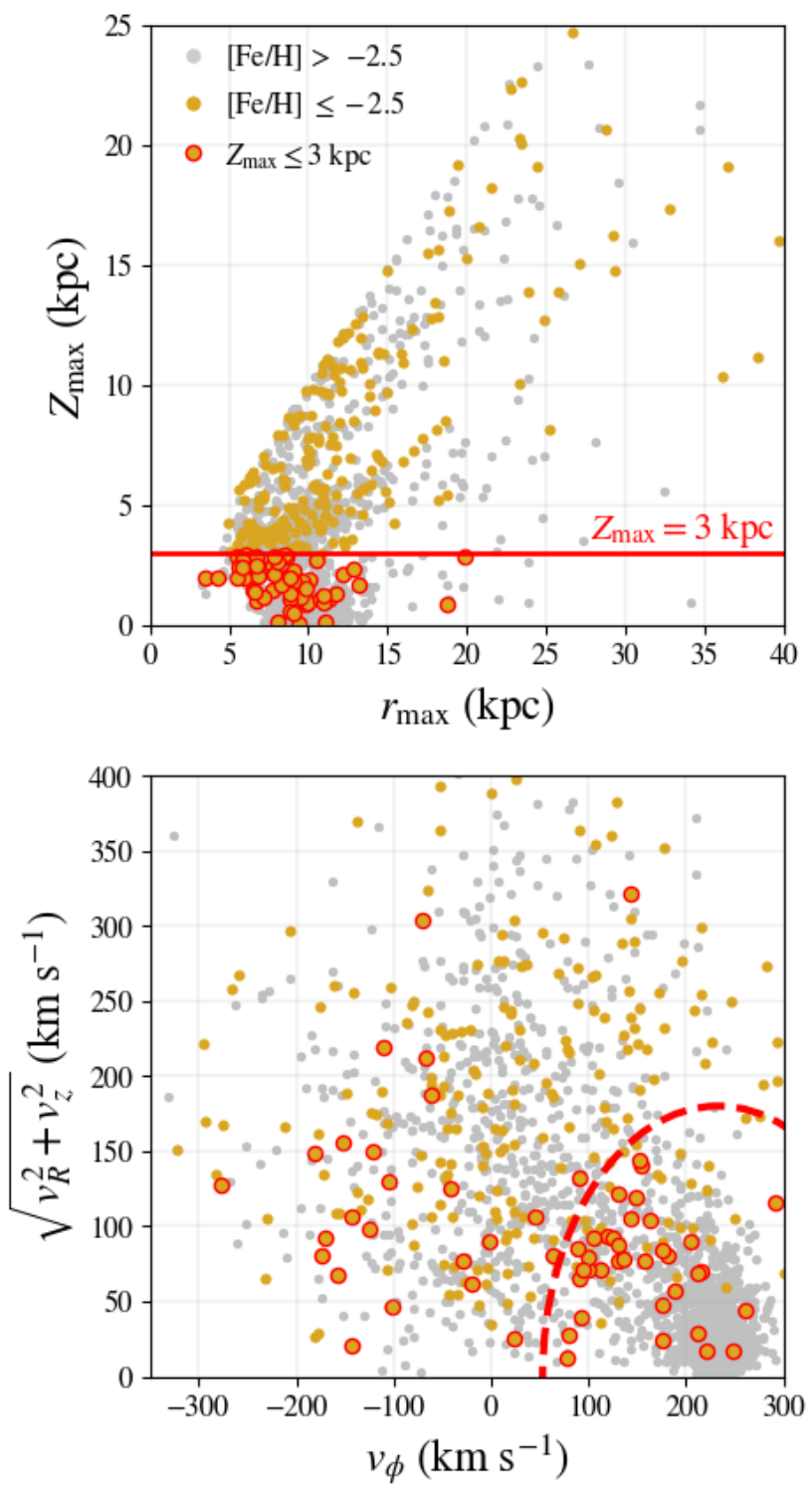

Figure 8. Top panel: $Z_{\max }$ vs. $r_{\max }$. The red horizontal line at $Z_{\max }=3 \mathrm{kpc}$ marks the limit for stars considered to be on planar or halo-like orbits in our analysis (see text). Bottom panel: Toomre diagram, similar to Figure 7. The red dashed line marks the $\left|V_{\text {total }}-V_{\mathrm{LSR}}\right|=180 \mathrm{~km} \mathrm{~s}^{-1}$ boundary between the thick disk and the transition region as in Section 5.3. In both panels, gray dots represent stars with metallicities $[\mathrm{Fe} / \mathrm{H}]>-2.5$. Stars with $[\mathrm{Fe} / \mathrm{H}] \leq-2.5$ are displayed as yellow dots. Among these, the planar ones are plotted with red edges.

Sestito et al. 2019, 2020. Interestingly, a separation between thick disk and halo at $Z_{\max } \sim 3 \mathrm{kpc}$ has also been achieved by Pérez-Villegas et al. (2020) in their independent analysis of orbits of Galactic globular clusters. We have paid particular attention to stars with $[\mathrm{Fe} / \mathrm{H}]$ $\leq-2.5$, as these are potential members of the aforemen- tioned mono-enriched class (Hartwig et al. 2018). Hence, this metallicity cutoff is, apparently, more physically significant than the classic definitions of VMP and EMP stars, and is also consistent with the analyses of Sestito et al. (2020, 2021). For convenience, throughout this section, we refer to a star as "VMP/EMP" when $[\mathrm{Fe} / \mathrm{H}]$ $\leq-2.5$. A total of 275 stars in the full sample are consistent with this metallicity regime. These are shown as yellow dots in Figure 8, while those with $[\mathrm{Fe} / \mathrm{H}]>-2.5$ are represented in gray. All VMP/EMP stars on planar orbits are plotted with red edges, and correspond to a fraction of $22_{-4}^{+5} \%(60 / 275)$, consistent with both Sestito et al. (2020) and Cordoni et al. (2020), despite our small biases towards halo kinematics (Section 2).

The bottom panel of Figure 8 presents the Toomre diagram for the full sample, divided according to the prescription above. One can see that there is a substantial number of VMP/EMP stars on low- $Z_{\max }$ orbits occupying the region of the $\left(\sqrt{v_{R}^{2}+v_{z}^{2}}, v_{\phi}\right)$ space characteristic of the thin and thick disks, as conjectured in Sections 5.2 and 5.3. These stars can be considered part of the third stellar population that resides in the Galactic disk, known as the metal-weak thick disk (MWTD; Norris et al. 1985; Morrison et al. 1990; Beers \& Sommer-Larsen 1995; Bonifacio et al. 1999; Chiba \& Beers 2000; Beers et al. 2002, 2014; Brown et al. 2008; Reddy \& Lambert 2008; Kordopatis et al. 2013; Carollo et al. 2014, 2019; Hawkins et al. 2015; Li \& Zhao 2017; Li et al. 2018a; An \& Beers 2020; Di Matteo et al. 2020). Despite early suggestions that the MDF of the MWTD might extend down to the VMP/EMP regime (Beers \& SommerLarsen 1995; Bonifacio et al. 1999), confirmation with large numbers of VMP/EMP stars was only possible thanks to the powerful combination of Gaia DR2 with large-scale spectroscopic surveys (e.g., LAMOST; Sestito et al. 2020). In fact, there is increasing evidence for the existence of an "ultra"-MWTD, reaching metallicities $<-4.0$ (Sestito et al. 2019; Cordoni et al. 2020; Di Matteo et al. 2020). An UMP star has even been found in the thin disk $\left(Z_{\max }<0.5 \mathrm{kpc} ; e<0.2\right)$ (Schlaufman et al. 2018). On the other hand, about a third of the planar VMP/EMP stars are rotating counter to the Galactic disks, some of them with $v_{\phi} \lesssim-100 \mathrm{~km} \mathrm{~s}^{-1}$ ).

The VMP/EMP stars on planar orbits are of crucial importance to constrain the early formation of the Galaxy. Recent cosmological zoom-in simulations have been pointing to the majority of $\mathrm{VMP} / \mathrm{EMP}$ stars being born very early on $(z \gtrsim 5)$, but mostly ex-situ, in primordial galaxies that have been accreted by the main progenitor through hierarchical assembly up until $z \sim 1$ (Starkenburg et al. 2017a; El-Badry et al. 2018). Hence, these objects would be deposited into the main halo with a variety of different orbits, either prograde or retrograde (Sestito et al. 2021), and become phase-space mixed with those of in-situ origin. However, given the presence of the ultra-MWTD, indistinguishable from the canonical thick disk from kinematics alone, Di Matteo et al. 

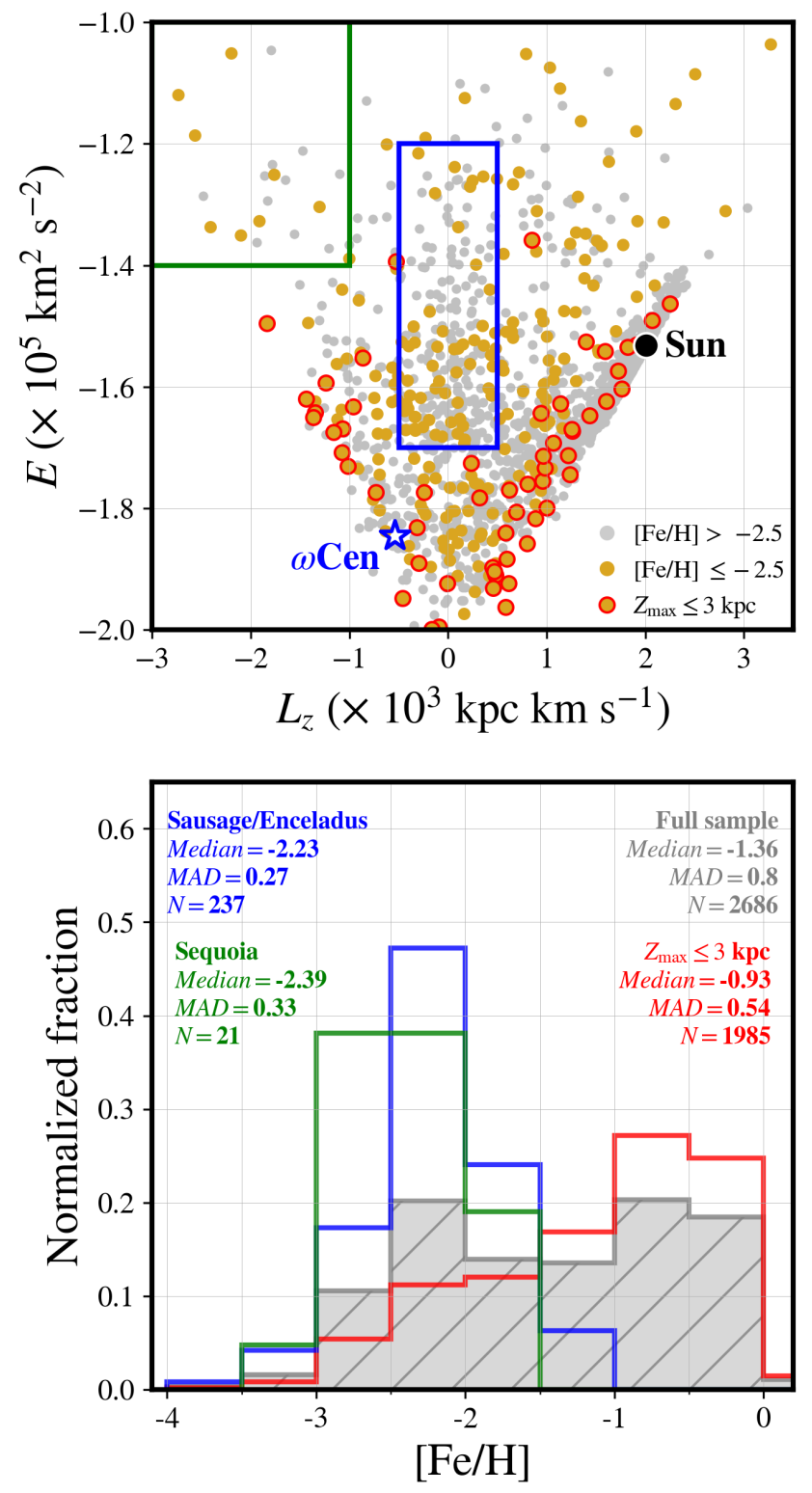

Figure 9. Top panel: $E$ vs. $L_{z}$. The position of the Sun is indicated with a black circle: $\left(E, L_{z}\right) \odot=(-1.5 \times$ $\left.10^{5} \mathrm{~km}^{2} \mathrm{~s}^{-2},+2.0 \times 10^{3} \mathrm{kpc} \mathrm{km} \mathrm{s}^{-1}\right)$. The blue solid lines delineate our selection box for Gaia-Sausage/Enceladus (GSE; see text) stars. The blue star shows the position of the $\omega$ Centauri globular cluster: $\left(E, L_{z}\right)_{\omega \mathrm{Cen}}=(-1.8 \times$ $\left.10^{5} \mathrm{~km}^{2} \mathrm{~s}^{-2},-0.5 \times 10^{3} \mathrm{kpc} \mathrm{km} \mathrm{s}{ }^{-1}\right)$. The green box represents the selection of Sequoia stars (see text). Gray dots represent stars with $[\mathrm{Fe} / \mathrm{H}]>-2.5$. Stars with $[\mathrm{Fe} / \mathrm{H}] \leq-2.5$ are displayed as yellow dots. Among these, the disk-like stars are plotted with red edges. Bottom panel: Metallicity distribution functions of the full (gray), the planar (red), GSE (blue), and Sequoia (green) samples. The medians, the median absolute deviations (MAD), and the total numbers of stars $(N)$ are also shown.
(2020) emphasize that the simplest deduction would be that these VMP/EMP stars were born in the Galaxy itself. Hence, these stars would have suffered from the same heating mechanism that operated to form both the splashed disk and the thick disk, i.e., the GSE event (Di Matteo et al. 2019; Gallart et al. 2019; Belokurov et al. 2020; Bonaca et al. 2020), although the exact role that merging events play in dynamical heating remains under debate (see, e.g., Jean-Baptiste et al. 2017 and Amarante et al. 2020a). Nevertheless, these scenarios are not necessarily mutually exclusive, although the retrograde fraction of the VMP/EMP stars on planar orbits might be difficult to reconcile with the latter. In fact, Sestito et al. (2021) has noted that the Galaxy apparently hosts an excess of retrograde, low- $Z_{\max }$, low-metallicity stars in comparison to their simulations. The authors have further suggested that this aspect might be intrinsically related to a Milky Way-specific evolution, particularly the accretion of GSE's progenitor system. Such features of VMP/EMP stars on planar orbits in the Galaxy, especially the prograde/retrograde ratio, might help us understand this puzzling assembly history.

We continue our exploration for dynamically interesting stars in Figure 9, where the $E$ vs. $L_{z}$ diagram is presented (top panel). This parameter space has been extensively utilized in the search for substructures in the Galaxy, especially in the halo (e.g., Chiba \& Beers 2000 and numerous subsequent authors; see Helmi 2020 for a recent review). Unsurprisingly, we notice the spread of these objects on planar orbits throughout the locus, in $\left(E, L_{z}\right)$ space, associated with either the disks or the low- $E\left(\lesssim-1.6 \times 10^{5} \mathrm{~km}^{2} \mathrm{~s}^{-2}\right)$, retrograde halo. $\mathrm{Cu}-$ riously, the latter is also commonly attributed to the remnant of the proposed Thamnos event (Koppelman et al. 2019). Indeed, we had already noticed that VMP stars from this substructure are unequivocally contained within $Z_{\max } \lesssim 3.5 \mathrm{kpc}$ in Limberg et al. (2021). One might even speculate that the accretion of a small system like the Thamnos progenitor could have been the responsible for the apparent excess of retrograde low$Z_{\max }$ stars reported by Sestito et al. (2021). However, this should be taken with caution, since both the specific chemical profile of this substructure (Monty et al. 2020), as well as its independence from GSE (Kordopatis et al. 2020), are still under active study (see also discussions about the consequences of massive mergers in Jean-Baptiste et al. 2017 and Koppelman et al. 2020).

From the $\left(E, L_{z}\right)$ space, we have also tentatively isolated stars from both GSE and Sequoia debris. We have placed characteristic selection boxes for each of these substructures (blue and green lines, respectively, in the top panel of Figure 9), in an approach similar to both Matsuno et al. (2019, who analyzed the MDFs and $\alpha$-to-iron ratios of their member stars) and Massari et al. (2019, who studied possible dynamical associations between globular clusters and various Galactic components). For reference, we have also lo- 
cated the $\omega$ Centauri globular cluster in $\left(E, L_{z}\right)$ space, which has been proposed to be the remnant nuclear star cluster of GSE (Pfeffer et al. 2021). The GSE selection is: $-1.7 \leq E\left(\times 10^{5} \mathrm{~km}^{2} \mathrm{~s}^{-2}\right) \leq-1.2$, and $-0.5 \leq L_{z}\left(\times 10^{3} \mathrm{kpc} \mathrm{km} \mathrm{s}{ }^{-1}\right) \leq+0.5$. For Sequoia: $-1.4 \leq E\left(\times 10^{5} \mathrm{~km}^{2} \mathrm{~s}^{-2}\right) \leq-1.0$, and $L_{z} \leq-1.0 \times 10^{3}$ $\mathrm{kpc} \mathrm{km} \mathrm{s}{ }^{-1}$. The MDFs resulting from these cuts are presented in the bottom panel of Figure 9 with the same colors as their respective selection boxes. For comparison, we have also plotted the MDFs for the full sample (gray) and for those stars on planar orbits (red). The MDFs from both GSE and Sequoia mostly occupy the metallicity range of $-3.0<[\mathrm{Fe} / \mathrm{H}]<-1.5$, with medians of $[\mathrm{Fe} / \mathrm{H}] \sim-2.2$ and $\sim-2.4$, respectively, but median absolute deviations that make them compatible with each other. Despite some overlap, these are much more metal poor than the typical values found for these substructures in the literature (e.g., Helmi et al. 2018; Conroy et al. 2019a; Vincenzo et al. 2019; Amarante et al. 2020b; Feuillet et al. 2020 for GSE and Myeong et al. 2019; Matsuno et al. 2019; Monty et al. 2020; Dietz et al. 2020 for both). We have experimented with other selections in velocity space, as recommended by Feuillet et al. (2020), but the results are equivalent. We conclude that this low-metallicity bias is an effect of the underlying B\&B selection.

From the bottom panel of Figure 9, we note that the MDF of GSE is reminiscent of those presented in Figure 5 (for $|Z|_{\text {Gal }}>3 \mathrm{kpc}$ and $V_{\mathrm{TAV}}>150 \mathrm{~km} \mathrm{~s}^{-1}$ ), commensurate with this substructure being the predominant population in the nearby, accreted halo. The presence of this component is also noticeable from the behavior of the full sample's MDF (Figure 9), which has two peaks, with the lowest-metallicity one being in the same location as GSE's $(-2.5 \leq[\mathrm{Fe} / \mathrm{H}] \leq-2.0)$. The other peak, at $-1.0 \leq[\mathrm{Fe} / \mathrm{H}] \leq-0.5$, is associated with metal-rich, thin-disk stars, mistakenly selected as VMP candidates and followed-up during our program. We note, however, the extended low-metallicity tail of the MDF representing the low $-Z_{\max }$ portion of the full sample, related to the previously discussed VMP/EMP stars with thickdisk-like kinematics.

Finally, we stress that not only the VMP/EMP stars with $Z_{\max }>3 \mathrm{kpc}$, but also those confined to the Galactic plane, exhibit a wide variety of orbital behaviors, as seen in Figures 8 and 9. Both of these populations span the full range of orbital $e$, a feature highlighted by Cordoni et al. (2020) in their analysis, from very low $(e<0.2)$, disk-like, to very high $(e>0.8)$, typical of GSE debris. The mild-e $(0.4<e<0.6)$ stars might also be associated with substructures of ex-situ nature (see Helmi 2020), but most of them apparently belong to the in-situ counterpart of the halo. Cordoni et al. (2020) highlights that VMP/EMP stars presenting low-to-mild- $e$, low- $Z_{\max }$ orbits follow the $e$ distributions of simulated thick disks constructed via heating mechanisms by Sales et al. (2009). The retrograde counterpart of such a population would likely be an accreted one according to these authors. This presented "continuity" (in the words of Di Matteo et al. 2020) in the dynamical properties of the most metal-poor stars will certainly need to be taken into consideration in future simulation efforts trying to reproduce the birth and evolution of the Milky Way.

\section{CONCLUSIONS}

In this paper, we have presented a low-resolution $(R \sim 2000)$ spectroscopic study of 1897 metal-poor star candidates selected in the Best \& Brightest Survey (Schlaufman \& Casey 2014). The observations have been conducted with either the GMOS/Gemini (North and South) or Goodman/SOAR combinations between semesters 2014A and 2019B. We have obtained the atmospheric parameters $\left(T_{\text {eff }}, \log g\right.$, and $\left.[\mathrm{Fe} / \mathrm{H}]\right)$ for these stars, as well as carbon and magnesium abundance ratios for most of them. Furthermore, we have utilized the phase-space information provided by Gaia EDR3 to perform an in-depth investigation on the influence of kinematic-based target selection criteria in the efficiency of finding VMP stars. Finally, we have explored the presence of dynamically interesting VMP/EMP stars in our sample. The main results can be summarized as follows.

- Overall, $56 \%$ (1064) of the newly observed stars have $[\mathrm{Fe} / \mathrm{H}]<-1.0,30 \%$ (566) are $\mathrm{VMP}$, and 2\% (35) are EMP. Combined with the previously published data of P19, the full B\&B sample is now one the largest homogeneously-analyzed compilations of bright $(V \lesssim$ 14) VMP and EMP stars available.

- There are 191 CEMP stars in the Gemini+SOAR sample (94 from Group I and 97 from Group II in the Yoon-Beers $A(\mathrm{C})-[\mathrm{Fe} / \mathrm{H}]$ diagram).

- The fraction of CEMP stars increases with decreasing metallicity. In the VMP and EMP regimes, we have found $19 \%$ and $43 \%$, respectively.

- We have introduced the $V_{\text {TAV }}$ quantity, which demands either PMs or $V_{\text {los }}$, but makes optimal use of their combination when both are available. We have explored the $Z_{\text {Gal vs. }} V_{\text {TAV }}$ diagrams. and confirmed that the $f_{\mathrm{VMP}}$ grows for greater distances from the Galactic plane and velocities within the full B\&B sample.

- The final success rates that have been achieved for $Z_{\mathrm{Gal}} \geq 0.5 \mathrm{kpc}$ and $V_{\mathrm{TAV}} \geq 100 \mathrm{~km} \mathrm{~s}^{-1}$ are $96 \%, 76 \%$, $28 \%$, and $4 \%$ for $[\mathrm{Fe} / \mathrm{H}] \leq-1.5, \leq-2.0, \leq-2.5$, and $\leq-3.0$, respectively.

- Using the Toomre diagram, the $f_{\mathrm{VMP}}$ in the kinematically-defined halo is equivalent (at the $1 \sigma$ level) to what has been found for the most interesting regions of the $\left(Z_{\mathrm{Gal}}, V_{\mathrm{TAV}}\right)$ space.

- After integrating their orbits, $22 \%$ of the stars with $[\mathrm{Fe} / \mathrm{H}] \leq-2.5$ have been found to be confined within $Z_{\max } \leq 3 \mathrm{kpc}$. However, the $\mathrm{VMP} / \mathrm{EMP}$ stars vetted here exhibit a wide variety of orbital behaviors, spanning 
all values of $L_{z}$ and orbital eccentricity, in keeping with the post-Gaia literature.

- Most of the VMP/EMP stars on planar orbits can be kinematically $\left(100 \mathrm{~km} \mathrm{~s}^{-1} \leq\left|V_{\text {total }}-V_{\mathrm{LSR}}\right|<\right.$ $180 \mathrm{~km} \mathrm{~s}^{-1}$ ) and dynamically $(e \lesssim 0.6)$ attributed to the Galactic metal-weak thick disk. Moreover, a third of these planar, mild-e stars are retrograde (some with $\left.v_{\phi} \lesssim-100 \mathrm{~km} \mathrm{~s}^{-1}\right)$, and their origin remains unclear.

- Stars on halo-like orbits $\left(Z_{\max }>3 \mathrm{kpc}\right)$ with $e \gtrsim 0.8$ are generally associated with the GSE merging event. On the other hand, those with mild values of $e$ might be linked to either the in-situ counterpart of the halo or other accreted substructures (e.g., Sequoia).

We note that there remain many thousands of candidate metal-poor stars originally identified in the B\&B survey, as well as in the HK and Hamburg/ESO surveys, which have not yet been vetted with low-resolution spectroscopy. Given the high success rates for the identification of VMP stars demonstrated by our kinematicselection approach, it is feasible to go directly to highresolution follow-up for many of these stars in future campaigns.

\section{ACKNOWLEDGMENTS}

The authors thank Angeles Pérez-Villegas for insightful discussions at the early stages of this work. We also thank the anonymous referee for a useful report. G.L. acknowledges CAPES (PROEX; Proc. 88887.481172/2020-00) and CNPq (PIBIC; Proc. 144638/2018-5). R.M.S. acknowledges CNPq (Proc. 436696/2018-5 and 306667/2020-7). S.R. would like to acknowledge support from FAPESP (Proc. 2015/503740 and 2014/18100-4), CAPES, and CNPq. H.D.P. thanks FAPESP Proc. 2018/21250-9. D.S., V.M.P., and T.C.B. acknowledge partial support from grant $\mathrm{PHY}$ 14-30152, Physics Frontier Center/JINA Center for the Evolution of the Elements (JINA-CEE), awarded by the US National Science Foundation. The work of V.M.P. is supported by NOIRLab, which is managed by the Association of Universities for Research in Astronomy (AURA) under a cooperative agreement with the National Science Foundation. Y.S.L. acknowledges support from the National Research Foundation (NRF) of Korea grant funded by the Ministry of Science and ICT (NRF-2018R1A2B6003961).

This work is partially based on observations obtained under the programs SO-2018B-010 and SO-2019B-013 at the Southern Astrophysical Research (SOAR) telescope, which is a joint project of the Ministério da Ciência, Tecnologia e Inovações (MCTI/LNA) do Brasil, the US National Science Foundation's NOIRLab, the University of North Carolina at Chapel Hill (UNC), and Michigan State University (MSU). This research is also partially based on observations obtained under programs GN-2015A-Q-76, GN-2015B-Q-86, GN-2016A-
DD-3, GN-2016A-Q-75, GN-2016B-Q-77, GN-2017AQ-82, GN-2017B-Q-75, GN-2017B-Q-79, GN-2018A-Q403, GN-2018B-Q-316, GN-2019A-Q-309, GN-2019BQ-403, GS-2014A-Q-74, GS-2014A-Q-8, GS-2015A-Q77, GS-2015A-Q-92, GS-2015B-Q-71, GS-2016A-Q-76, GS-2016B-Q-81, GS-2017A-Q-86, GS-2017B-Q-75, GS2017B-Q-84, GS-2018A-Q-406, and GS-2018B-Q-315 at the international Gemini Observatory, a program of NSF's NOIRLab, which is managed by the Association of Universities for Research in Astronomy (AURA) under a cooperative agreement with the National Science Foundation on behalf of the Gemini Observatory partnership: the National Science Foundation (United States), National Research Council (Canada), Agencia Nacional de Investigación y Desarrollo (Chile), Ministerio de Ciencia, Tecnología e Innovación (Argentina), Ministério da Ciência, Tecnologia e Inovações (Brazil), and Korea Astronomy and Space Science Institute (Republic of Korea).

This work has made use of data from the European Space Agency (ESA) mission Gaia (https://www. cosmos.esa.int/gaia), processed by the Gaia Data Processing and Analysis Consortium (DPAC, https://www. cosmos.esa.int/web/gaia/dpac/consortium. Funding for the DPAC has been provided by national institutions, in particular the institutions participating in the Gaia Multilateral Agreement. This research has made use of the SIMBAD database and VizieR catalogue access tool, operated at CDS, Strasbourg, France. This research was made possible through the use of the AAVSO Photometric All-Sky Survey (APASS), funded by the Robert Martin Ayers Sciences Fund and NSF AST-1412587. This publication makes use of data products from the Two Micron All Sky Survey, which is a joint project of the University of Massachusetts and the Infrared Processing and Analysis Center/California Institute of Technology, funded by the National Aeronautics and Space Administration and the National Science Foundation.

This research has been conducted despite the ongoing dismantling of the Brazilian scientific system.

Facilities: Gemini North (8.1 m): GMOS-N, Gemini South $(8.1 \mathrm{~m})$ : GMOS-S, SOAR $(4.1 \mathrm{~m})$ : Goodman

Software: matplotlib (Hunter 2007), Numpy (van der Walt et al. 2011), scipy (Virtanen et al. 2020). 


\section{REFERENCES}

Abbott, B. P., Abbott, R., Abbott, T. D., et al. 2017a, ApJL, 848, L12, doi: 10.3847/2041-8213/aa91c9

—. 2017b, ApJL, 848, L13, doi: 10.3847/2041-8213/aa920c

-. 2017c, PhRvL, 119, 161101, doi: 10.1103/PhysRevLett.119.161101

Aguado, D. S., González Hernández, J. I., Allende Prieto, C., \& Rebolo, R. 2018, ApJL, 852, L20, doi: 10.3847/2041-8213/aaa23a

Aguado, D. S., Youakim, K., González Hernández, J. I., et al. 2019, MNRAS, 490, 2241, doi: 10.1093/mnras/stz2643

Allen, D. M., Ryan, S. G., Rossi, S., Beers, T. C., \& Tsangarides, S. A. 2012, A\&A, 548, A34, doi: 10.1051/0004-6361/201015615

Amarante, J. A. S., Beraldo e Silva, L., Debattista, V. P., \& Smith, M. C. 2020a, ApJL, 891, L30, doi: 10.3847/2041-8213/ab78a4

Amarante, J. A. S., Smith, M. C., \& Boeche, C. 2020b, MNRAS, 492, 3816, doi: 10.1093/mnras/staa077

An, D., \& Beers, T. C. 2020, ApJ, 897, 39, doi: $10.3847 / 1538-4357 / a b 8 d 39$

Aoki, W., Beers, T. C., Christlieb, N., et al. 2007, ApJ, 655, 492, doi: 10.1086/509817

Aoki, W., Norris, J. E., Ryan, S. G., Beers, T. C., \& Ando, H. 2002, ApJ, 567, 1166, doi: 10.1086/338756

Arcavi, I., Hosseinzadeh, G., Howell, D. A., et al. 2017, Nature, 551, 64, doi: 10.1038/nature24291

Asplund, M., Grevesse, N., Sauval, A. J., \& Scott, P. 2009, ARA\&A, 47, 481, doi: 10.1146/annurev.astro.46.060407.145222

Astraatmadja, T. L., \& Bailer-Jones, C. A. L. 2016, ApJ, 833, 119, doi: 10.3847/1538-4357/833/1/119

Astropy Collaboration, Robitaille, T. P., Tollerud, E. J., et al. 2013, A\&A, 558, A33, doi: 10.1051/0004-6361/201322068

Astropy Collaboration, Price-Whelan, A. M., Sipőcz, B. M., et al. 2018, AJ, 156, 123, doi: 10.3847/1538-3881/aabc4f

Bailer-Jones, C. A. L., Rybizki, J., Fouesneau, M., Demleitner, M., \& Andrae, R. 2021, AJ, 161, 147, doi: $10.3847 / 1538-3881 / a b d 806$

Banerjee, P., Wu, M.-R., \& Yuan, Z. 2020, ApJL, 902, L34, doi: $10.3847 / 2041-8213 /$ abbc0d

Beers, T. C., \& Christlieb, N. 2005, ARA\&A, 43, 531, doi: 10.1146/annurev.astro.42.053102.134057

Beers, T. C., Drilling, J. S., Rossi, S., et al. 2002, AJ, 124, 931, doi: 10.1086/341377

Beers, T. C., Norris, J. E., Placco, V. M., et al. 2014, ApJ, 794, 58, doi: 10.1088/0004-637X/794/1/58
Beers, T. C., Preston, G. W., \& Shectman, S. A. 1985, AJ, 90, 2089, doi: 10.1086/113917

—. 1992, AJ, 103, 1987

Beers, T. C., \& Sommer-Larsen, J. 1995, ApJS, 96, 175, doi: $10.1086 / 192117$

Beers, T. C., Carollo, D., Ivezić, Ž., et al. 2012, ApJ, 746, 34, doi: 10.1088/0004-637X/746/1/34

Beers, T. C., Placco, V. M., Carollo, D., et al. 2017, ApJ, 835, 81, doi: 10.3847/1538-4357/835/1/81

Belczynski, K., Askar, A., Arca-Sedda, M., et al. 2018, A\&A, 615, A91, doi: 10.1051/0004-6361/201732428

Belokurov, V., Erkal, D., Evans, N. W., Koposov, S. E., \& Deason, A. J. 2018, MNRAS, 478, 611, doi: $10.1093 / \mathrm{mnras} /$ sty982

Belokurov, V., Sanders, J. L., Fattahi, A., et al. 2020, MNRAS, 494, 3880, doi: 10.1093/mnras/staa876

Bensby, T., Feltzing, S., \& Oey, M. S. 2014, A\&A, 562, A71, doi: 10.1051/0004-6361/201322631

Bidelman, W. P., \& MacConnell, D. J. 1973, AJ, 78, 687, doi: $10.1086 / 111475$

Bisterzo, S., Gallino, R., Straniero, O., Cristallo, S., \& Käppeler, F. 2011, MNRAS, 418, 284, doi: 10.1111/j.1365-2966.2011.19484.x

Bland-Hawthorn, J., \& Gerhard, O. 2016, ARA\&A, 54, 529, doi: 10.1146/annurev-astro-081915-023441

Bonaca, A., Conroy, C., Wetzel, A., Hopkins, P. F., \& Kereš, D. 2017, ApJ, 845, 101, doi: $10.3847 / 1538-4357 / \mathrm{aa} 7 \mathrm{~d} 0 \mathrm{c}$

Bonaca, A., Conroy, C., Cargile, P. A., et al. 2020, ApJL, 897, L18, doi: 10.3847/2041-8213/ab9caa

Bond, H. E. 1970, ApJS, 22, 117, doi: 10.1086/190220

—. 1980, ApJS, 44, 517, doi: 10.1086/190703

Bonifacio, P., Centurion, M., \& Molaro, P. 1999, MNRAS, 309, 533, doi: 10.1046/j.1365-8711.1999.02866.x

Bonifacio, P., Caffau, E., Spite, M., et al. 2015, A\&A, 579, A28, doi: 10.1051/0004-6361/201425266

Bonoli, S., Marín-Franch, A., Varela, J., et al. 2020, arXiv e-prints, arXiv:2007.01910.

https://arxiv.org/abs/2007.01910

Brown, W. R., Beers, T. C., Wilhelm, R., et al. 2008, AJ, 135, 564, doi: 10.1088/0004-6256/135/2/564

Burbidge, E. M., Burbidge, G. R., Fowler, W. A., \& Hoyle, F. 1957, Reviews of Modern Physics, 29, 547, doi: 10.1103/RevModPhys.29.547

Cameron, A. G. W. 1957, PASP, 69, 201, doi: $10.1086 / 127051$ 
Carollo, D., Freeman, K., Beers, T., et al. 2014, Carbon-Enhanced Metal-Poor Stars: CEMP-s and CEMP-no Sub-Classes in the Halo System of the Milky Way. https://arxiv.org/abs/1401.0574

Carollo, D., Beers, T. C., Lee, Y. S., et al. 2007, Nature, 450, 1020, doi: 10.1038/nature06460

Carollo, D., Beers, T. C., Chiba, M., et al. 2010, ApJ, 712, 692, doi: 10.1088/0004-637X/712/1/692

Carollo, D., Beers, T. C., Bovy, J., et al. 2012, ApJ, 744, 195, doi: 10.1088/0004-637X/744/2/195

Carollo, D., Chiba, M., Ishigaki, M., et al. 2019, ApJ, 887, 22, doi: $10.3847 / 1538-4357 /$ ab517c

Casey, A. R., \& Schlaufman, K. C. 2015, ApJ, 809, 110, doi: 10.1088/0004-637X/809/2/110

Cayrel, R., Depagne, E., Spite, M., et al. 2004, A\&A, 416, 1117, doi: 10.1051/0004-6361:20034074

Cenarro, A. J., Moles, M., Cristóbal-Hornillos, D., et al. 2019, A\&A, 622, A176, doi: 10.1051/0004-6361/201833036

Charbonnel, C. 1995, ApJL, 453, L41, doi: 10.1086/309744

Chiappini, C., Matteucci, F., \& Gratton, R. 1997, ApJ, 477, 765, doi: $10.1086 / 303726$

Chiba, M., \& Beers, T. C. 2000, AJ, 119, 2843, doi: 10.1086/301409

Choi, J., Dotter, A., Conroy, C., et al. 2016, ApJ, 823, 102, doi: 10.3847/0004-637X/823/2/102

Christlieb, N. 2003, Reviews in Modern Astronomy, 16, 191, doi: 10.1002/9783527617647.ch8

Christlieb, N., Schörck, T., Frebel, A., et al. 2008, A\&A, 484, 721, doi: 10.1051/0004-6361:20078748

Clemens, J. C., Crain, J. A., \& Anderson, R. 2004, in Society of Photo-Optical Instrumentation Engineers (SPIE) Conference Series, Vol. 5492, Ground-based Instrumentation for Astronomy, ed. A. F. M. Moorwood \& M. Iye, 331-340, doi: 10.1117/12.550069

Cohen, J. G., Christlieb, N., McWilliam, A., et al. 2008, ApJ, 672, 320, doi: 10.1086/523638

Conroy, C., Naidu, R. P., Zaritsky, D., et al. 2019a, ApJ, 887, 237, doi: 10.3847/1538-4357/ab5710

Conroy, C., Bonaca, A., Cargile, P., et al. 2019b, ApJ, 883, 107, doi: 10.3847/1538-4357/ab38b8

Cordoni, G., Da Costa, G. S., Yong, D., et al. 2020, MNRAS, doi: 10.1093/mnras/staa3417

Côté, B., Fryer, C. L., Belczynski, K., et al. 2018, ApJ, 855, 99, doi: 10.3847/1538-4357/aaad67

Côté, B., Eichler, M., Arcones, A., et al. 2019, ApJ, 875, 106, doi: 10.3847/1538-4357/ab10db

Cruz, M. A., Cogo-Moreira, H., \& Rossi, S. 2018, MNRAS, 475, 4781, doi: 10.1093/mnras/stx3330
Cui, X.-Q., Zhao, Y.-H., Chu, Y.-Q., et al. 2012, Research in Astronomy and Astrophysics, 12, 1197, doi: 10.1088/1674-4527/12/9/003

Da Costa, G. S., Bessell, M. S., Mackey, A. D., et al. 2019, MNRAS, 489, 5900, doi: 10.1093/mnras/stz2550

Dalton, G., Trager, S. C., Abrams, D. C., et al. 2012, in Society of Photo-Optical Instrumentation Engineers (SPIE) Conference Series, Vol. 8446, Ground-based and Airborne Instrumentation for Astronomy IV, ed. I. S. McLean, S. K. Ramsay, \& H. Takami, 84460P, doi: $10.1117 / 12.925950$

Davies, R. L., Allington-Smith, J. R., Bettess, P., et al. 1997, in Society of Photo-Optical Instrumentation Engineers (SPIE) Conference Series, Vol. 2871, Optical Telescopes of Today and Tomorrow, ed. A. L. Ardeberg, 1099-1106, doi: 10.1117/12.268996

de Jong, R. S., Bellido-Tirado, O., Chiappini, C., et al. 2012, in Society of Photo-Optical Instrumentation Engineers (SPIE) Conference Series, Vol. 8446, Ground-based and Airborne Instrumentation for Astronomy IV, ed. I. S. McLean, S. K. Ramsay, \& H. Takami, 84460T, doi: 10.1117/12.926239

de Jong, R. S., Barden, S., Bellido-Tirado, O., et al. 2014, in Society of Photo-Optical Instrumentation Engineers (SPIE) Conference Series, Vol. 9147, Ground-based and Airborne Instrumentation for Astronomy V, ed. S. K.

Ramsay, I. S. McLean, \& H. Takami, 91470M, doi: $10.1117 / 12.2055826$

Di Matteo, P., Haywood, M., Lehnert, M. D., et al. 2019, A\&A, 632, A4, doi: 10.1051/0004-6361/201834929

Di Matteo, P., Spite, M., Haywood, M., et al. 2020, A\&A, 636, A115, doi: 10.1051/0004-6361/201937016

Dietz, S. E., Yoon, J., Beers, T. C., \& Placco, V. M. 2020, ApJ, 894, 34, doi: 10.3847/1538-4357/ab7fa4

Dotter, A. 2016, ApJS, 222, 8, doi: $10.3847 / 0067-0049 / 222 / 1 / 8$

Drout, M. R., Piro, A. L., Shappee, B. J., et al. 2017, Science, 358, 1570, doi: 10.1126/science.aaq0049

Dvorkin, I., Daigne, F., Goriely, S., Vangioni, E., \& Silk, J. 2020, arXiv e-prints, arXiv:2010.00625.

https://arxiv.org/abs/2010.00625

El-Badry, K., Bland-Hawthorn, J., Wetzel, A., et al. 2018, MNRAS, 480, 652, doi: 10.1093/mnras/sty1864

Ezzeddine, R., Frebel, A., Roederer, I. U., et al. 2019, ApJ, 876, 97, doi: 10.3847/1538-4357/ab14e7

Ezzeddine, R., Rasmussen, K., Frebel, A., et al. 2020, ApJ, 898, 150, doi: 10.3847/1538-4357/ab9d1a

Feuillet, D. K., Feltzing, S., Sahlholdt, C. L., \& Casagrande, L. 2020, MNRAS, 497, 109, doi: 10.1093/mnras/staa1888 
Frebel, A. 2018, Annual Review of Nuclear and Particle Science, 68, 237, doi: 10.1146/annurev-nucl-101917-021141

Frebel, A., Chiti, A., Ji, A. P., Jacobson, H. R., \& Placco, V. M. 2015, ApJL, 810, L27, doi: 10.1088/2041-8205/810/2/L27

Frebel, A., \& Norris, J. E. 2015, ARA\&A, 53, 631, doi: 10.1146/annurev-astro-082214-122423

Frebel, A., Christlieb, N., Norris, J. E., et al. 2006, ApJ, 652, 1585, doi: 10.1086/508506

Gaia Collaboration, Brown, A. G. A., Vallenari, A., et al. 2020, arXiv e-prints, arXiv:2012.01533.

https://arxiv.org/abs/2012.01533

Gaia Collaboration, Prusti, T., de Bruijne, J. H. J., et al. 2016a, A\&A, 595, A1, doi: 10.1051/0004-6361/201629272

Gaia Collaboration, Brown, A. G. A., Vallenari, A., et al. 2016b, A\&A, 595, A2, doi: 10.1051/0004-6361/201629512

—. 2018, A\&A, 616, A1, doi: 10.1051/0004-6361/201833051

Gallart, C., Bernard, E. J., Brook, C. B., et al. 2019, Nature Astronomy, 3, 932, doi: 10.1038/s41550-019-0829-5

Gimeno, G., Roth, K., Chiboucas, K., et al. 2016, in Society of Photo-Optical Instrumentation Engineers (SPIE) Conference Series, Vol. 9908, Ground-based and Airborne Instrumentation for Astronomy VI, ed. C. J. Evans, L. Simard, \& H. Takami, 99082S, doi: 10.1117/12.2233883

Gravity Collaboration, Abuter, R., Amorim, A., et al. 2019, A\&A, 625, L10, doi: 10.1051/0004-6361/201935656

—. 2020, A\&A, 636, L5, doi: 10.1051/0004-6361/202037813

Gudin, D., Shank, D., Beers, T. C., et al. 2021, ApJ, 908, 79, doi: 10.3847/1538-4357/abd7ed

Hansen, T., Hansen, C. J., Christlieb, N., et al. 2015, ApJ, 807, 173, doi: 10.1088/0004-637X/807/2/173

Hansen, T. T., Andersen, J., Nordström, B., et al. 2016, A\&A, 588, A3, doi: 10.1051/0004-6361/201527409

Hansen, T. T., Holmbeck, E. M., Beers, T. C., et al. 2018, ApJ, 858, 92, doi: 10.3847/1538-4357/aabacc

Hartwig, T., Yoshida, N., Magg, M., et al. 2018, MNRAS, 478, 1795, doi: 10.1093/mnras/sty1176

Hawkins, K., Jofré, P., Masseron, T., \& Gilmore, G. 2015, MNRAS, 453, 758, doi: 10.1093/mnras/stv1586

Haynes, C. J., \& Kobayashi, C. 2019, MNRAS, 483, 5123, doi: 10.1093/mnras/sty3389

Haywood, M., Di Matteo, P., Lehnert, M. D., et al. 2018, ApJ, 863, 113, doi: 10.3847/1538-4357/aad235

Heger, A., \& Woosley, S. E. 2010, ApJ, 724, 341, doi: 10.1088/0004-637X/724/1/341

Helmi, A. 2020, ARA\&A, 58, 205, doi: 10.1146/annurev-astro-032620-021917

Helmi, A., Babusiaux, C., Koppelman, H. H., et al. 2018, Nature, 563, 85, doi: 10.1038/s41586-018-0625-x
Henden, A., \& Munari, U. 2014, Contributions of the Astronomical Observatory Skalnate Pleso, 43, 518

Herwig, F. 2005, ARA\&A, 43, 435, doi: 10.1146/annurev.astro.43.072103.150600

Hill, V., Plez, B., Cayrel, R., et al. 2002, A\&A, 387, 560, doi: 10.1051/0004-6361:20020434

Holmbeck, E. M., Hansen, T. T., Beers, T. C., et al. 2020, ApJS, 249, 30, doi: 10.3847/1538-4365/ab9c19

Hunter, J. D. 2007, Computing in Science and Engineering, 9, 90, doi: 10.1109/MCSE.2007.55

Ito, H., Aoki, W., Beers, T. C., et al. 2013, ApJ, 773, 33, doi: 10.1088/0004-637X/773/1/33

Jean-Baptiste, I., Di Matteo, P., Haywood, M., et al. 2017, A\&A, 604, A106, doi: 10.1051/0004-6361/201629691

Ji, A. P., Drout, M. R., \& Hansen, T. T. 2019, ApJ, 882, 40, doi: 10.3847/1538-4357/ab3291

Ji, A. P., \& Frebel, A. 2018, ApJ, 856, 138, doi: $10.3847 / 1538-4357 /$ aab14a

Keller, S. C., Bessell, M. S., Frebel, A., et al. 2014, Nature, 506, 463, doi: 10.1038/nature12990

Kobayashi, C., Karakas, A. I., \& Lugaro, M. 2020, ApJ, 900, 179, doi: 10.3847/1538-4357/abae65

Kollmeier, J. A., Zasowski, G., Rix, H.-W., et al. 2017, arXiv e-prints, arXiv:1711.03234. https://arxiv.org/abs/1711.03234

Koppelman, H., Helmi, A., \& Veljanoski, J. 2018, ApJL, 860, L11, doi: 10.3847/2041-8213/aac882

Koppelman, H. H., Bos, R. O. Y., \& Helmi, A. 2020, A\&A, 642, L18, doi: 10.1051/0004-6361/202038652

Koppelman, H. H., Helmi, A., Massari, D., Price-Whelan, A. M., \& Starkenburg, T. K. 2019, A\&A, 631, L9, doi: 10.1051/0004-6361/201936738

Kordopatis, G., Recio-Blanco, A., Schultheis, M., \& Hill, V. 2020, A\&A, 643, A69, doi: 10.1051/0004-6361/202038686

Kordopatis, G., Gilmore, G., Wyse, R. F. G., et al. 2013, MNRAS, 436, 3231, doi: 10.1093/mnras/stt1804

Kunder, A., Kordopatis, G., Steinmetz, M., et al. 2017, AJ, 153, 75, doi: 10.3847/1538-3881/153/2/75

Lattimer, J. M., \& Schramm, D. N. 1974, ApJL, 192, L145, doi: 10.1086/181612

Lee, Y. S., Beers, T. C., \& Kim, Y. K. 2019, ApJ, 885, 102, doi: 10.3847/1538-4357/ab4791

Lee, Y. S., Beers, T. C., Kim, Y. K., et al. 2017, ApJ, 836, 91, doi: 10.3847/1538-4357/836/1/91

Lee, Y. S., Beers, T. C., Sivarani, T., et al. 2008a, AJ, 136, 2022, doi: 10.1088/0004-6256/136/5/2022

-. 2008b, AJ, 136, 2050, doi: 10.1088/0004-6256/136/5/2050

Lee, Y. S., Beers, T. C., Allende Prieto, C., et al. 2011, AJ, 141, 90, doi: 10.1088/0004-6256/141/3/90 
Lee, Y. S., Beers, T. C., Masseron, T., et al. 2013, AJ, 146, 132, doi: 10.1088/0004-6256/146/5/132

Li, C., \& Zhao, G. 2017, ApJ, 850, 25, doi: 10.3847/1538-4357/aa93f4

Li, C., Zhao, G., Zhai, M., \& Jia, Y. 2018a, ApJ, 860, 53, doi: $10.3847 / 1538-4357 /$ aac50f

Li, H., Tan, K., \& Zhao, G. 2018b, ApJS, 238, 16, doi: 10.3847/1538-4365/aada4a

Limberg, G., Rossi, S., Beers, T. C., et al. 2021, ApJ, 907, 10, doi: 10.3847/1538-4357/abcb87

Lindegren, L., Lammers, U., Bastian, U., et al. 2016, A\&A, 595, A4, doi: 10.1051/0004-6361/201628714

Lindegren, L., Bastian, U., Biermann, M., et al. 2020a, arXiv e-prints, arXiv:2012.01742. https://arxiv.org/abs/2012.01742

Lindegren, L., Klioner, S. A., Hernández, J., et al. 2020b, arXiv e-prints, arXiv:2012.03380.

https://arxiv.org/abs/2012.03380

Lucatello, S., Beers, T. C., Christlieb, N., et al. 2006, ApJL, 652, L37, doi: 10.1086/509780

Lucatello, S., Tsangarides, S., Beers, T. C., et al. 2005, ApJ, 625, 825, doi: 10.1086/428104

Massari, D., Koppelman, H. H., \& Helmi, A. 2019, A\&A, 630, L4, doi: 10.1051/0004-6361/201936135

Matsuno, T., Aoki, W., \& Suda, T. 2019, ApJL, 874, L35, doi: 10.3847/2041-8213/ab0ec0

McMillan, P. J. 2017, MNRAS, 465, 76, doi: 10.1093/mnras/stw2759

Mendes de Oliveira, C., Ribeiro, T., Schoenell, W., et al. 2019, MNRAS, 489, 241, doi: 10.1093/mnras/stz1985

Monty, S., Venn, K. A., Lane, J. M. M., Lokhorst, D., \& Yong, D. 2020, MNRAS, 497, 1236, doi: $10.1093 / \mathrm{mnras} / \mathrm{staa} 1995$

Morrison, H. L., Flynn, C., \& Freeman, K. C. 1990, AJ, 100, 1191, doi: 10.1086/115587

Myeong, G. C., Vasiliev, E., Iorio, G., Evans, N. W., \& Belokurov, V. 2019, MNRAS, 488, 1235, doi: $10.1093 / \mathrm{mnras} / \mathrm{stz} 1770$

Nomoto, K., Kobayashi, C., \& Tominaga, N. 2013, ARA\&A, 51, 457, doi: 10.1146/annurev-astro-082812-140956

Norris, J., Bessell, M. S., \& Pickles, A. J. 1985, ApJS, 58, 463, doi: 10.1086/191049

Norris, J. E., Ryan, S. G., \& Beers, T. C. 1996, ApJS, 107, 391, doi: 10.1086/192368

—. 1997, ApJ, 488, 350, doi: 10.1086/304695

Pérez-Villegas, A., Barbuy, B., Kerber, L. O., et al. 2020, MNRAS, 491, 3251, doi: 10.1093/mnras/stz3162
Pfeffer, J., Lardo, C., Bastian, N., Saracino, S., \& Kamann, S. 2021, MNRAS, 500, 2514, doi: $10.1093 / \mathrm{mnras} /$ staa3407

Pian, E., D'Avanzo, P., Benetti, S., et al. 2017, Nature, 551, 67, doi: 10.1038/nature24298

Placco, V. M., Beers, T. C., Reggiani, H., \& Meléndez, J. 2016a, ApJL, 829, L24, doi: 10.3847/2041-8205/829/2/L24

Placco, V. M., Frebel, A., Beers, T. C., et al. 2014a, ApJ, 781, 40, doi: 10.1088/0004-637X/781/1/40

—. 2013, ApJ, 770, 104, doi: 10.1088/0004-637X/770/2/104

Placco, V. M., Frebel, A., Beers, T. C., \& Stancliffe, R. J. 2014b, ApJ, 797, 21, doi: 10.1088/0004-637X/797/1/21

Placco, V. M., Frebel, A., Beers, T. C., et al. 2016b, ApJ, 833, 21, doi: 10.3847/0004-637X/833/1/21

Placco, V. M., Beers, T. C., Santucci, R. M., et al. 2018, AJ, 155, 256, doi: 10.3847/1538-3881/aac20c

Placco, V. M., Santucci, R. M., Beers, T. C., et al. 2019, ApJ, 870, 122, doi: 10.3847/1538-4357/aaf3b9

Planck Collaboration, Ade, P. A. R., Aghanim, N., et al. 2016, A\&A, 594, A13, doi: 10.1051/0004-6361/201525830

Posti, L., Helmi, A., Veljanoski, J., \& Breddels, M. A. 2018, A\&A, 615, A70, doi: 10.1051/0004-6361/201732277

Rasmussen, K. C., Zepeda, J., Beers, T. C., et al. 2020, ApJ, 905, 20, doi: 10.3847/1538-4357/abc005

Recio-Blanco, A., de Laverny, P., Kordopatis, G., et al. 2014, A\&A, 567, A5, doi: 10.1051/0004-6361/201322944

Reddy, B. E., \& Lambert, D. L. 2008, MNRAS, 391, 95, doi: 10.1111/j.1365-2966.2008.13905.x

Reggiani, H., Meléndez, J., Kobayashi, C., Karakas, A., \& Placco, V. 2017, A\&A, 608, A46, doi: 10.1051/0004-6361/201730750

Reggiani, H., Schlaufman, K. C., Casey, A. R., \& Ji, A. P. 2020, AJ, 160, 173, doi: 10.3847/1538-3881/aba948

Riello, M., De Angeli, F., Evans, D. W., et al. 2020, arXiv e-prints, arXiv:2012.01916.

https://arxiv.org/abs/2012.01916

Roederer, I. U., Placco, V. M., \& Beers, T. C. 2016, ApJL, 824, L19, doi: 10.3847/2041-8205/824/2/L19

Roederer, I. U., Preston, G. W., Thompson, I. B., et al. 2014, AJ, 147, 136, doi: 10.1088/0004-6256/147/6/136

Rossi, S., Beers, T. C., \& Sneden, C. 1999, in Astronomical Society of the Pacific Conference Series, Vol. 165, The Third Stromlo Symposium: The Galactic Halo, ed. B. K. Gibson, R. S. Axelrod, \& M. E. Putman, 264

Rossi, S., Beers, T. C., Sneden, C., et al. 2005, AJ, 130, 2804, doi: 10.1086/497164

Ryan, S. G., Aoki, W., Norris, J. E., \& Beers, T. C. 2005, ApJ, 635, 349, doi: 10.1086/497282 
Safarzadeh, M., Ramirez-Ruiz, E., Andrews, J. J., et al. 2019a, ApJ, 872, 105, doi: 10.3847/1538-4357/aafe0e

Safarzadeh, M., Sarmento, R., \& Scannapieco, E. 2019b, ApJ, 876, 28, doi: 10.3847/1538-4357/ab1341

Sales, L. V., Helmi, A., Abadi, M. G., et al. 2009, MNRAS, 400, L61, doi: 10.1111/j.1745-3933.2009.00763.x

Schlafly, E. F., \& Finkbeiner, D. P. 2011, ApJ, 737, 103, doi: $10.1088 / 0004-637 \mathrm{X} / 737 / 2 / 103$

Schlaufman, K. C., \& Casey, A. R. 2014, ApJ, 797, 13, doi: 10.1088/0004-637X/797/1/13

Schlaufman, K. C., Thompson, I. B., \& Casey, A. R. 2018, ApJ, 867, 98, doi: 10.3847/1538-4357/aadd97

Schlegel, D. J., Finkbeiner, D. P., \& Davis, M. 1998, ApJ, 500, 525, doi: 10.1086/305772

Schönrich, R., Binney, J., \& Dehnen, W. 2010, MNRAS, 403, 1829, doi: 10.1111/j.1365-2966.2010.16253.x

Sestito, F., Longeard, N., Martin, N. F., et al. 2019, MNRAS, 484, 2166, doi: 10.1093/mnras/stz043

Sestito, F., Martin, N. F., Starkenburg, E., et al. 2020, MNRAS, 497, L7, doi: 10.1093/mnrasl/slaa022

Sestito, F., Buck, T., Starkenburg, E., et al. 2021, MNRAS, 500, 3750, doi: 10.1093/mnras/staa3479

Shappee, B. J., Simon, J. D., Drout, M. R., et al. 2017, Science, 358, 1574, doi: 10.1126/science.aaq0186

Skrutskie, M. F., Cutri, R. M., Stiening, R., et al. 2006, AJ, 131, 1163, doi: 10.1086/498708

Smartt, S. J., Chen, T. W., Jerkstrand, A., et al. 2017, Nature, 551, 75, doi: 10.1038/nature24303

Sneden, C., Cowan, J. J., \& Gallino, R. 2008, ARA\&A, 46, 241, doi: 10.1146/annurev.astro.46.060407.145207

Spite, M., Caffau, E., Bonifacio, P., et al. 2013, A\&A, 552, A107, doi: 10.1051/0004-6361/201220989

Stancliffe, R. J., Church, R. P., Angelou, G. C., \& Lattanzio, J. C. 2009, MNRAS, 396, 2313, doi: 10.1111/j.1365-2966.2009.14900.x

Starkenburg, E., Oman, K. A., Navarro, J. F., et al. 2017a, MNRAS, 465, 2212, doi: 10.1093/mnras/stw2873

Starkenburg, E., Shetrone, M. D., McConnachie, A. W., \& Venn, K. A. 2014, MNRAS, 441, 1217, doi: $10.1093 / \mathrm{mnras} / \mathrm{stu} 623$

Starkenburg, E., Martin, N., Youakim, K., et al. 2017b, MNRAS, 471, 2587, doi: 10.1093/mnras/stx1068

Steinmetz, M., Zwitter, T., Siebert, A., et al. 2006, The Astronomical Journal, 132, 1645, doi: 10.1086/506564

Suda, T., Aikawa, M., Machida, M. N., Fujimoto, M. Y., \& Iben, Icko, J. 2004, ApJ, 611, 476, doi: 10.1086/422135
Tody, D. 1986, in Society of Photo-Optical Instrumentation Engineers (SPIE) Conference Series, Vol. 627, Instrumentation in astronomy VI, ed. D. L. Crawford, 733, doi: $10.1117 / 12.968154$

Tody, D. 1993, in Astronomical Society of the Pacific Conference Series, Vol. 52, Astronomical Data Analysis Software and Systems II, ed. R. J. Hanisch, R. J. V. Brissenden, \& J. Barnes, 173

Tominaga, N., Iwamoto, N., \& Nomoto, K. 2014, ApJ, 785, 98, doi: 10.1088/0004-637X/785/2/98

van der Walt, S., Colbert, S. C., \& Varoquaux, G. 2011, Computing in Science and Engineering, 13, 22, doi: 10.1109/MCSE.2011.37

Vasiliev, E. 2019, MNRAS, 482, 1525, doi: 10.1093/mnras/sty2672

Venn, K. A., Irwin, M., Shetrone, M. D., et al. 2004, AJ, 128, 1177, doi: 10.1086/422734

Vincenzo, F., Spitoni, E., Calura, F., et al. 2019, MNRAS, 487, L47, doi: 10.1093/mnrasl/slz070

Virtanen, P., Gommers, R., Oliphant, T. E., et al. 2020, Nature Methods, 17, 261, doi: https://doi.org/10.1038/s41592-019-0686-2

Wilson, E. B. 1927, Journal of the American Statistical Association, 22, 209, doi: 10.1080/01621459.1927.10502953

Wright, E. L., Eisenhardt, P. R. M., Mainzer, A. K., et al. 2010, AJ, 140, 1868, doi: 10.1088/0004-6256/140/6/1868

Yanny, B., Rockosi, C., Newberg, H. J., et al. 2009, AJ, 137, 4377, doi: 10.1088/0004-6256/137/5/4377

Yong, D., Norris, J. E., Bessell, M. S., et al. 2013a, ApJ, 762, 26, doi: 10.1088/0004-637X/762/1/26

—. 2013b, ApJ, 762, 27, doi: 10.1088/0004-637X/762/1/27

Yoon, J., Beers, T. C., Tian, D., \& Whitten, D. D. 2019, ApJ, 878, 97, doi: 10.3847/1538-4357/ablead

Yoon, J., Whitten, D. D., Beers, T. C., et al. 2020, ApJ, 894, 7, doi: 10.3847/1538-4357/ab7daf

Yoon, J., Beers, T. C., Placco, V. M., et al. 2016, ApJ, 833, 20, doi: 10.3847/0004-637X/833/1/20

Yoon, J., Beers, T. C., Dietz, S., et al. 2018, ApJ, 861, 146, doi: 10.3847/1538-4357/aaccea

York, D. G., Adelman, J., Anderson, John E., J., et al. 2000, AJ, 120, 1579, doi: 10.1086/301513

Youakim, K., Starkenburg, E., Aguado, D. S., et al. 2017, MNRAS, 472, 2963, doi: 10.1093/mnras/stx2005

Youakim, K., Starkenburg, E., Martin, N. F., et al. 2020, MNRAS, 492, 4986, doi: 10.1093/mnras/stz3619

Yuan, Z., Myeong, G. C., Beers, T. C., et al. 2020, ApJ, 891, 39, doi: 10.3847/1538-4357/ab6ef7

Zhao, G., Zhao, Y.-H., Chu, Y.-Q., Jing, Y.-P., \& Deng, L.-C. 2012, Research in Astronomy and Astrophysics, 12, 723, doi: 10.1088/1674-4527/12/7/002

Zhao, G., Mashonkina, L., Yan, H. L., et al. 2016, ApJ, 833, 225, doi: 10.3847/1538-4357/833/2/225 
Table 1. Coordinates and Observing Details

\begin{tabular}{|c|c|c|c|c|c|c|c|c|}
\hline $\begin{array}{c}\text { Star Name } \\
\text { (2MASS) }\end{array}$ & $\begin{array}{c}\text { Star Name } \\
\text { (Gaia EDR3) }\end{array}$ & $\begin{array}{l}\text { R.A. } \\
(\operatorname{deg})\end{array}$ & $\begin{array}{l}\text { Decl. } \\
(\mathrm{deg})\end{array}$ & $\begin{array}{c}l \\
(\mathrm{deg})\end{array}$ & $\begin{array}{c}b \\
(\mathrm{deg})\end{array}$ & Telescope & Instrument & Program ID \\
\hline 00003305-7953389 & 4634573766005607552 & 0.1375 & -79.8942 & 305.7037 & -36.9587 & SOAR & Goodman & SO-2019B-013 \\
\hline 00020162-4430117 & 4994519032163925632 & 0.5067 & -44.5033 & 329.4221 & -69.9972 & SOAR & Goodman & SO-2019B-013 \\
\hline 00040305-6106367 & 4905632480654004608 & 1.0127 & -61.1102 & 312.9121 & -55.0903 & Gemini South & GMOS-S & GS-2016A-Q-76 \\
\hline $00043646+4124062$ & 384060304935385984 & 1.1519 & 41.4017 & 113.5728 & -20.6174 & Gemini North & GMOS-N & GN-2017B-Q-75 \\
\hline $00045403+3524010$ & 2876804519751163008 & 1.2251 & 35.4003 & 112.3464 & -26.5131 & Gemini North & GMOS-N & GN-2016A-Q-75 \\
\hline $00052731+0025510$ & 2546113345293028608 & 1.3638 & 0.4309 & 99.2097 & -60.3059 & Gemini North & GMOS-N & GN-2015B-Q-86 \\
\hline 00060902-6238019 & 4904480471642380928 & 1.5376 & -62.6339 & 311.7034 & -53.7163 & Gemini South & GMOS-S & GS-2015B-Q-71 \\
\hline $00071189+4724466$ & 393031258963415936 & 1.8005 & 47.4133 & 115.2160 & -14.7946 & Gemini North & GMOS-N & GN-2015B-Q-86 \\
\hline $00111339+0152512$ & 2546752127188959232 & 2.8058 & 1.8810 & 102.8772 & -59.4159 & Gemini North & GMOS-N & GN-2017B-Q-75 \\
\hline $00140089+3148167$ & 2861747777456341120 & 3.5037 & 31.8047 & 113.7150 & -30.3965 & Gemini North & GMOS-N & GN-2015B-Q-86 \\
\hline 00144636-2246093 & 2361100512255883904 & 3.6932 & -22.7693 & 58.6142 & -80.6197 & Gemini South & GMOS-S & GS-2016A-Q-76 \\
\hline 00150914-3736048 & 2308246644711975680 & 3.7881 & -37.6014 & 336.7743 & -77.0386 & Gemini South & GMO & GS-2017A- \\
\hline 00152923-2436237 & 2336022438732700672 & 3.8719 & -24.6066 & 48.2514 & -81.5320 & Gemini South & GMOS-S & GS-2016A-Q-76 \\
\hline $00152952-6121258$ & 4904881106191486592 & 3.8730 & -61.3572 & 310.4784 & -55.2413 & Gemini South & & \\
\hline 00154806-6253207 & 4901504815220315648 & 3.9503 & -62.8891 & 309.7887 & -53.7622 & Gemini South & GMOS-S & GS-2015B-Q-71 \\
\hline 00162809-0505519 & 2443891577459590016 & 4.1171 & -5.0978 & 100.6696 & -66.4462 & SOAR & Good & 013 \\
\hline $00163655+3538314$ & 2876439211309388672 & 4.1523 & 35.6421 & 115.0170 & -26.6939 & Gemini North & GMOS-N & GN-2015B-Q-86 \\
\hline 00163809-4912369 & 4977077135617904640 & 4.1587 & -49.2102 & 317.5332 & -66.9196 & SOAR & Goodman & SO-2019B-013 \\
\hline $00165353+3642326$ & 2876647328245159552 & 4.2231 & 36.7090 & 115.2577 & -25.6474 & Gemini North & GMOS-N & GN-2017B-Q-75 \\
\hline $00170767+4614488$ & 392109932642310272 & 4.2820 & 46.2469 & 116.7657 & -16.2137 & Gemini North & GMOS-N & GN-2017B-Q-75 \\
\hline 00175076-6819295 & 4706413931618489600 & 4.4614 & -68.3249 & 307.6012 & -48.4976 & Gemini South & GMOS-S & GS-2016A-Q-76 \\
\hline 00182947-7829325 & 4635466706886248448 & 4.6230 & -78.4923 & 305.0249 & -38.5016 & Gemini South & GMOS-S & GS-2017A-Q-86 \\
\hline $00192284+4431554$ & 385734487485292928 & 4.8452 & 44.5320 & 116.9346 & -17.9662 & Gemini North & GMOS-N & GN-2015B-Q-86 \\
\hline 00202312-3950260 & 4997141779713479680 & 5.0964 & -39.8406 & 327.8488 & -75.7481 & Gemini South & GMOS-S & GS-2015B-Q-71 \\
\hline $00213396+0008219$ & 2545302077511395968 & 5.3920 & 1391 & 106.9664 & -61.8031 & Gemini North & GMOS-N & GN-2017B-Q-75 \\
\hline 00223511-4231148 & 4992150679821873664 & 5.6463 & -42.5208 & 321.9763 & -73.5240 & SOAR & Goodman & SO-2019B-013 \\
\hline 00234358-1117357 & 2424691974134738816 & 5.9316 & -11.2933 & 99.2429 & -72.8781 & Gemini South & GMOS-S & GS-2017A-Q-86 \\
\hline 00235345-6649211 & 4707128682896207872 & 5.9727 & -66.8225 & 307.1492 & -50.0796 & Gemini South & GMOS-S & GS-2015B-Q-71 \\
\hline $00241677+2941440$ & 2858965188404148480 & 6.0699 & 29.6955 & 115.9138 & -32.8059 & Gemini North & GMOS-N & GN-2017B-Q-75 \\
\hline $00275509+3458068$ & 365999795656991616 & 6.9796 & 34.9685 & 117.4935 & -27.6547 & Gemini North & GMOS-N & GN-2017B-Q-75 \\
\hline 00281899-6820268 & 4703742084003326080 & 7.0791 & -68.3408 & 306.1569 & -48.6424 & SOAR & Goodman & SO-2019B-013 \\
\hline $00293797+2103054$ & 2796582811359131392 & 7.4083 & 21.0515 & 116.1304 & -41.5322 & Gemini North & GMOS-N & GN-2015B-Q-86 \\
\hline $00301775+2957334$ & 2858881625520606848 & 7.5740 & 29.9592 & 117.4904 & -32.6891 & Gemini North & GMOS-N & GN-2017B-Q-75 \\
\hline $00303445+2816193$ & 2857835710788936832 & 7.6436 & 28.2720 & 117.3655 & -34.3741 & Gemini North & GMOS-N & GN-2017B-Q-75 \\
\hline $00311900+4957158$ & 391597358363860608 & 7.8292 & 49.9544 & 119.6155 & -12.7878 & Gemini North & GMOS-N & GN-2017B-Q-75 \\
\hline $00325147+4107490$ & 381063654713703040 & 8.2145 & 41.1303 & 119.1702 & -21.6057 & Gemini North & GMOS-N & GN-2015B-Q-86 \\
\hline $00331311+2033190$ & 2796309861892160384 & 8.3049 & 20.5551 & 117.1793 & -42.1131 & Gemini North & GMOS-N & GN-2017B-Q-75 \\
\hline 00341417-3943068 & 4994368227272214784 & 8.5591 & -39.7186 & 317.6889 & -76.9128 & Gemini South & GMOS-S & GS-2015B-Q-71 \\
\hline 00342795-2413107 & 2348080164279166208 & 8.6165 & -24.2197 & 69.2506 & -85.1960 & Gemini South & GMOS-S & GS-2016A-Q-76 \\
\hline 00351869-2854190 & 2319002587146050304 & 8.8279 & -28.9053 & 5.4597 & -86.0226 & Gemini South & GMOS-S & GS-2017A-Q-86 \\
\hline $00354136+1618228$ & 2780880960680375808 & 8.9224 & 16.3063 & 117.4487 & -46.3978 & Gemini North & GMOS-N & GN-2017B-Q-75 \\
\hline
\end{tabular}


Table 1 (continued)

\begin{tabular}{|c|c|c|c|c|c|c|c|c|}
\hline $\begin{array}{c}\text { Star Name } \\
\text { (2MASS) }\end{array}$ & $\begin{array}{c}\text { Star Name } \\
\text { (Gaia EDR3) }\end{array}$ & $\begin{array}{l}\text { R.A. } \\
\text { (deg) }\end{array}$ & $\begin{array}{l}\text { Decl. } \\
\text { (deg) }\end{array}$ & $\begin{array}{c}l \\
(\operatorname{deg})\end{array}$ & $\begin{array}{c}b \\
(\mathrm{deg})\end{array}$ & Telescope & Instrument & Program ID \\
\hline $00370433+4341208$ & 387703815888550656 & 9.2681 & 43.6891 & 120.1843 & -19.1060 & Gemini North & GMOS-N & GN-2015B-Q-86 \\
\hline 00370966-4224102 & 4992914501101243264 & 9.2903 & -42.4029 & 312.8062 & -74.4501 & Gemini South & GMOS-S & GS-2015B-Q-71 \\
\hline $00374809-2751193$ & 2343095974991737600 & 9.4505 & -27.8554 & 18.6251 & -86.8901 & Gemini South & GMOS-S & GS-2016A-Q-76 \\
\hline $00400685-4325183$ & 4980747129334018944 & 10.0286 & -43.4218 & 310.2078 & -73.5462 & SOAR & Goodman & SO-2019B-013 \\
\hline $00410467+1649478$ & 2782313903504860032 & 10.2695 & 16.8299 & 119.3640 & -45.9702 & Gemini North & GMOS-N & GN-2017B-Q-75 \\
\hline $00425182+1925361$ & 2795241995584048000 & 10.7160 & 19.4267 & 120.1494 & -43.3988 & Gemini North & GMOS-N & GN-2017B-Q-75 \\
\hline 00433651-2719379 & 2343181981712123520 & 10.9022 & -27.3272 & 25.9649 & -88.2487 & Gemini South & GMOS-S & GS-2017A-Q-86 \\
\hline 00435264-2132285 & 2350389894612060544 & 10.9694 & -21.5413 & 105.4023 & -84.1539 & Gemini South & GMOS-S & GS-2017A-Q-86 \\
\hline $00482715-8224023$ & 4629945985987660416 & 12.1131 & -82.4007 & 303.0520 & -34.7269 & SOAR & Goodman & SO-2019B-013 \\
\hline 00504527-6351504 & 4709709305405862656 & 12.6887 & -63.8640 & 303.0577 & -53.2641 & SOAR & Goodman & SO-2019B-013 \\
\hline 00510748-7809118 & 4635149768362820096 & 12.7813 & -78.1533 & 302.9524 & -38.9749 & Gemini South & GMOS-S & GS-2016A-Q-76 \\
\hline 00521010-6006097 & 4903551070783622528 & 13.0422 & -60.1027 & 302.7647 & -57.0253 & Gemini South & GMOS-S & GS-2015B-Q-71 \\
\hline 00522310-5804087 & 4907332914042242304 & 13.0963 & -58.0691 & 302.6883 & -59.0587 & Gemini South & GMOS-S & GS-2015B-Q-71 \\
\hline $00523111+3758286$ & 367656523458156544 & 13.1297 & 37.9746 & 123.1667 & -24.8967 & Gemini North & GMOS-N & GN-2017B-Q-75 \\
\hline $00582707+0633561$ & 2553447805108682496 & 14.6128 & 6.5656 & 126.0693 & -56.2634 & Gemini North & GMOS-N & GN-2017B-Q-75 \\
\hline $00594615+1223173$ & 2584343227151274752 & 14.9423 & 12.3882 & 126.1264 & -50.4320 & Gemini North & GMOS-N & GN-2017B-Q-75 \\
\hline $01021265+0428241$ & 2551971405806340096 & 15.5528 & 4.4734 & 128.0453 & -58.2914 & SOAR & Goodman & SO-2019B-013 \\
\hline 01051545-0041328 & 2533223147709485312 & 16.3145 & -0.6924 & 130.6547 & -63.3569 & Gemini South & GMOS-S & GS-2018B-Q-315 \\
\hline 01065190-5244105 & 4927175937828177280 & 16.7163 & -52.7363 & 297.5567 & -64.2307 & SOAR & Goodman & SO-2019B-013 \\
\hline 01132198-6139522 & 4710458244623085184 & 18.3416 & -61.6645 & 298.3670 & -55.2690 & Gemini South & GMOS-S & GS-2018B-Q-315 \\
\hline 01200289-0158201 & 2533312006288169600 & 20.0121 & -1.9723 & 139.3789 & -63.9267 & Gemini South & GMOS-S & GS-2015B-Q-71 \\
\hline $01250922-5614027$ & 4910744423745801472 & 21.2884 & -56.2341 & 293.4760 & -60.2709 & SOAR & Goodman & SO-2019B-013 \\
\hline 01253364-4148345 & 4984027698370924672 & 21.3902 & -41.8096 & 279.6701 & -73.7420 & Gemini South & GMOS-S & GS-2015B-Q-71 \\
\hline $01253802-2911025$ & & 21.4106 & -29.1842 & 230.1786 & -82.1877 & Gemini South & GMOS-S & GS-2018B-Q-315 \\
\hline 01291742-7139220 & 4687775246726470144 & 22.3226 & -71.6561 & 298.7235 & -45.1615 & Gemini South & GMOS-S & GS-2018B-Q-315 \\
\hline 01311599-4016510 & 5008222486100643200 & 22.8166 & -40.2808 & 273.3931 & -74.4808 & SOAR & Goodman & SO-2019B-013 \\
\hline 01315199-6547540 & 4710799574264331904 & 22.9666 & -65.7984 & 296.3937 & -50.8133 & Gemini South & GMOS-S & GS-2018B-Q-315 \\
\hline 01323787-1530302 & 2451901038631956224 & 23.1578 & -15.5085 & 164.4872 & -74.9483 & Gemini South & GMOS-S & GS-2018B-Q-315 \\
\hline $01363655+5451319$ & 409152813849385216 & 24.1524 & 54.8588 & 129.4589 & -7.4395 & Gemini North & GMOS-N & GN-2017B-Q-75 \\
\hline 01372246-4611110 & 4931138955692103424 & 24.3436 & -46.1864 & 280.4367 & -68.8839 & SOAR & Goodman & SO-2019B-013 \\
\hline 01373378-6921368 & 4691261969896942848 & 24.3907 & -69.3602 & 296.9761 & -47.2310 & Gemini South & GMOS-S & GS-2018B-Q-315 \\
\hline 01382048-7637319 & 4636801853303548416 & 24.5853 & -76.6256 & 299.4044 & -40.1797 & Gemini South & GMOS-S & GS-2018B-Q-315 \\
\hline
\end{tabular}


Table 1 (continued)

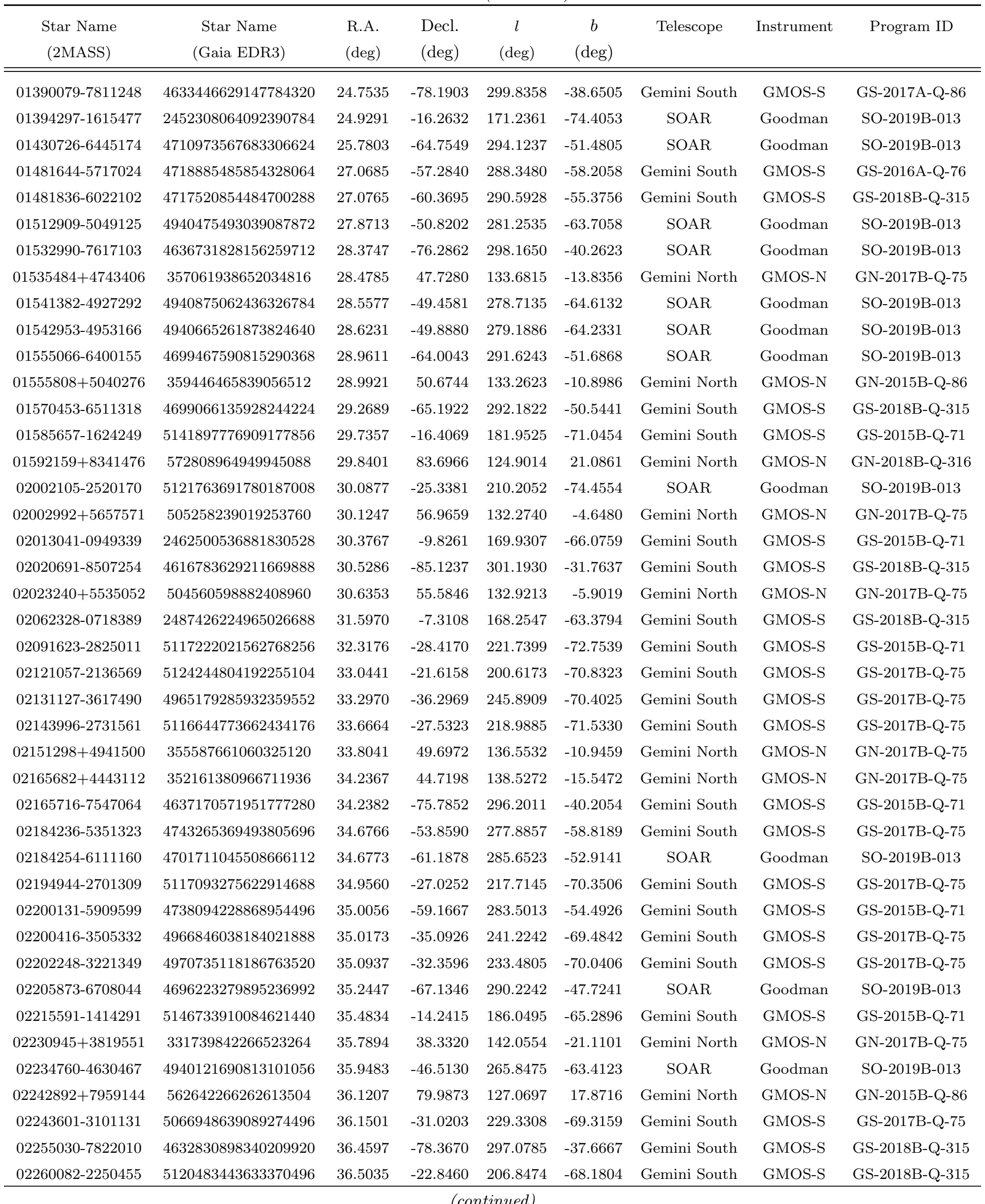


Table 1 (continued)

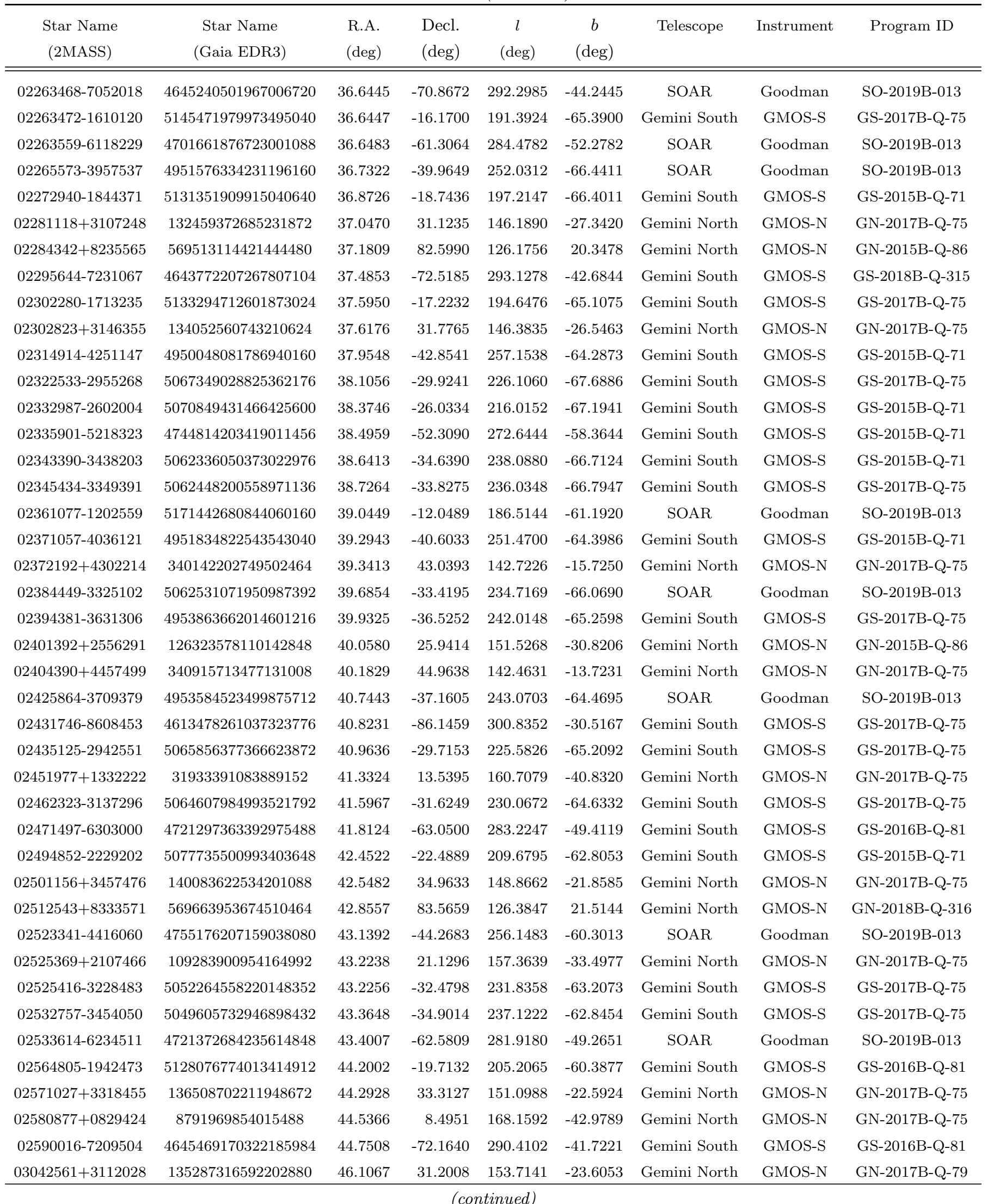


Table 1 (continued)

\begin{tabular}{|c|c|c|c|c|c|c|c|c|}
\hline $\begin{array}{l}\text { Star Name } \\
\text { (2MASS) }\end{array}$ & $\begin{array}{c}\text { Star Name } \\
\text { (Gaia EDR3) }\end{array}$ & $\begin{array}{l}\text { R.A. } \\
\text { (deg) }\end{array}$ & $\begin{array}{l}\text { Decl. } \\
\text { (deg) }\end{array}$ & $\begin{array}{c}l \\
(\operatorname{deg})\end{array}$ & $\begin{array}{c}b \\
(\mathrm{deg})\end{array}$ & Telescope & Instrument & Program ID \\
\hline 03042843-3406046 & 5050991770433282688 & 46.1185 & -34.1013 & 234.9072 & -60.6791 & SOAR & Goodman & SO-2019B-013 \\
\hline 03044130-3635405 & 5047482782152551808 & 46.1721 & -36.5946 & 239.9268 & -60.3394 & Gemini South & GMOS-S & GS-2015B-Q-71 \\
\hline $03053573+2834227$ & 115967213665888000 & 46.3989 & 28.5730 & 155.5073 & -25.6821 & Gemini North & GMOS-N & GN-2015B-Q-86 \\
\hline 03060595-5251430 & 4746980344406420736 & 46.5248 & -52.8620 & 268.0662 & -54.1651 & Gemini South & GMOS-S & GS-2017B-Q-75 \\
\hline 03091996-5853578 & 4724087928200048768 & 47.3332 & -58.8994 & 275.6901 & -50.3023 & Gemini South & GMOS-S & GS-2017B-Q-75 \\
\hline 03095592-0459281 & 5182694120770155264 & 47.4830 & -4.9912 & 185.2953 & -50.2884 & Gemini South & GMOS-S & GS-2015B-Q-71 \\
\hline $03104602+4514503$ & 433115020506112512 & 47.6919 & 45.2473 & 147.1148 & -10.9824 & Gemini North & GMOS-N & GN-2017B-Q-79 \\
\hline 03121034-5703094 & 4727345712434297984 & 48.0431 & -57.0526 & 272.9948 & -51.1012 & SOAR & Goodman & SO-2019B-013 \\
\hline 03134048-8045218 & 4619419089865071744 & 48.4186 & -80.7560 & 296.4234 & -34.4973 & Gemini South & GMOS-S & GS-2017B-Q-75 \\
\hline $03135196+4230102$ & 240424332130561024 & 48.4666 & 42.5028 & 149.0726 & -13.0255 & Gemini North & GMOS-N & GN-2017B-Q-79 \\
\hline $03152783+3353586$ & 137535813574478592 & 48.8659 & 33.8997 & 154.2316 & -20.0755 & Gemini North & GMOS-N & GN-2015B-Q-86 \\
\hline $03155572+3357169$ & 125528253246150656 & 48.9822 & 33.9547 & 154.2839 & -19.9760 & Gemini North & GMOS-N & GN-2015B-Q-86 \\
\hline 03155933-7432577 & 4639776204054863232 & 48.9972 & -74.5494 & 291.2478 & -39.1230 & Gemini South & GMOS-S & GS-2017B-Q-75 \\
\hline $03163710+2332211$ & 110907432953980800 & 49.1547 & 23.5392 & 161.0217 & -28.3927 & Gemini North & GMOS-N & GN-2015B-Q-86 \\
\hline 03170396-3740469 & 4854289415101796608 & 49.2665 & -37.6798 & 241.2914 & -57.7376 & SOAR & Goodman & SO-2019B-013 \\
\hline 03171573-3747479 & 4854284497363037696 & 49.3155 & -37.7967 & 241.4981 & -57.6823 & Gemini South & GMOS-S & GS-2016B-Q-81 \\
\hline 03173348-3705188 & 4854694001021568896 & 49.3901 & -37.0885 & 240.1693 & -57.7204 & Gemini South & GMOS-S & GS-2015B-Q-71 \\
\hline 03242169-3515217 & 4860920810247328256 & 51.0904 & -35.2560 & 236.5766 & -56.5207 & SOAR & Goodman & SO-2019B-013 \\
\hline 03242519-1550054 & 5106733402188456320 & 51.1050 & -15.8348 & 203.2431 & -52.8317 & Gemini South & GMOS-S & GS-2015B-Q-71 \\
\hline $03252266+8009505$ & 568038153934104320 & 51.3446 & 80.1640 & 129.3988 & 19.2853 & Gemini North & GMOS-N & GN-2015B-Q-86 \\
\hline 03260086-4126000 & 4849648891917127424 & 51.5036 & -41.4334 & 247.4454 & -55.3754 & Gemini South & GMOS-S & GS-2017B-Q-75 \\
\hline 03260534-2006507 & 5101544016542831232 & 51.5223 & -20.1141 & 210.1724 & -54.0333 & Gemini South & GMOS-S & GS-2017B-Q-75 \\
\hline $03263031+0616326$ & 9265348264761984 & 51.6263 & 6.2757 & 177.0232 & -39.7860 & Gemini North & GMOS-N & GN-2017B-Q-75 \\
\hline 03265306-0053348 & 3264007336293442048 & 51.7211 & -0.8930 & 184.3959 & -44.4288 & Gemini South & GMOS-S & GS-2017B-Q-75 \\
\hline $03265389+0202281$ & 3268028903151246720 & 51.7245 & 2.0412 & 181.2838 & -42.5542 & SOAR & Goodman & SO-2019B-013 \\
\hline 03275664-4544078 & 4846530329047408384 & 51.9860 & -45.7356 & 254.4884 & -53.9429 & Gemini South & GMOS-S & GS-2016B-Q-81 \\
\hline 03283529-4000252 & 4853194778260569856 & 52.1471 & -40.0070 & 244.8265 & -55.1614 & Gemini South & GMOS-S & GS-2017B-Q-75 \\
\hline $03283748+1856359$ & 57481853722629888 & 52.1562 & 18.9433 & 166.8230 & -30.2260 & Gemini North & GMOS-N & GN-2017B-Q-75 \\
\hline 03292423-6057094 & 4722654951015751680 & 52.3509 & -60.9526 & 275.9767 & -47.0630 & SOAR & Goodman & SO-2019B-013 \\
\hline 03304035-0321071 & 3249179288121898752 & 52.6682 & -3.3520 & 187.9605 & -45.1673 & Gemini South & GMOS-S & GS-2017B-Q-75 \\
\hline 03305484-0304088 & 3249204710032811648 & 52.7285 & -3.0692 & 187.6878 & -44.9517 & Gemini South & GMOS-S & GS-2015B-Q-71 \\
\hline $03312821+0749469$ & 11354660875621376 & 52.8676 & 7.8297 & 176.6676 & -37.8221 & Gemini North & GMOS-N & GN-2016A-Q-75 \\
\hline
\end{tabular}


Table 1 (continued)

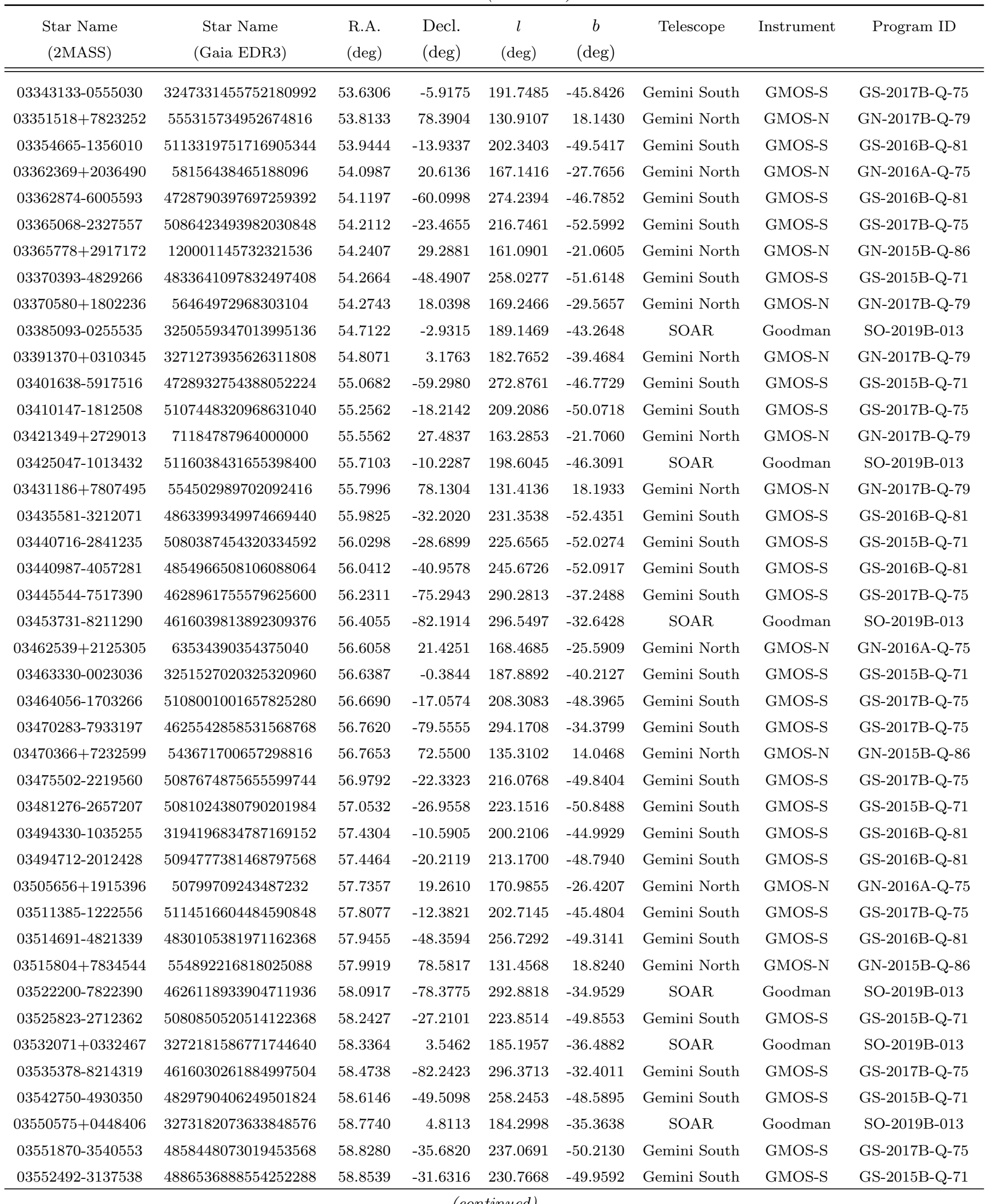


Table 1 (continued)

\begin{tabular}{|c|c|c|c|c|c|c|c|c|}
\hline $\begin{array}{c}\text { Star Name } \\
\text { (2MASS) }\end{array}$ & $\begin{array}{c}\text { Star Name } \\
\text { (Gaia EDR3) }\end{array}$ & $\begin{array}{l}\text { R.A. } \\
\text { (deg) }\end{array}$ & $\begin{array}{l}\text { Decl. } \\
\text { (deg) }\end{array}$ & $\begin{array}{c}l \\
(\operatorname{deg})\end{array}$ & $\begin{array}{c}b \\
(\operatorname{deg})\end{array}$ & Telescope & Instrument & Program ID \\
\hline $03570714+1150353$ & 3304873572159327104 & 59.2798 & 11.8432 & 178.2663 & -30.4563 & Gemini North & GMOS-N & GN-2016A-Q-75 \\
\hline 03575259-3507496 & 4858859741341036032 & 59.4691 & -35.1304 & 236.2315 & -49.6774 & Gemini South & GMOS-S & GS-2017B-Q-75 \\
\hline 03580690-4125514 & 4842342190474113920 & 59.5288 & -41.4310 & 245.9431 & -49.4306 & Gemini South & GMOS-S & GS-2015B-Q-71 \\
\hline $03582857+0851479$ & 3301417188637954304 & 59.6191 & 8.8633 & 181.1615 & -32.1514 & Gemini North & GMOS-N & GN-2017B-Q-79 \\
\hline 03583609-3024329 & 4887268613542620160 & 59.6504 & -30.4092 & 229.0155 & -49.1411 & SOAR & Goodman & SO-2019B-013 \\
\hline $03585372-2512589$ & 5083157811305565184 & 59.7239 & -25.2164 & 221.2993 & -48.1409 & Gemini South & GMOS-S & GS-2016B-Q-81 \\
\hline 03590972-7052417 & 4665955133531907840 & 59.7905 & -70.8783 & 284.9861 & -39.0435 & SOAR & Goodman & SO-2019B-013 \\
\hline 04021386-2714330 & 4890018148526236416 & 60.5578 & -27.2426 & 224.4957 & -47.8406 & SOAR & Goodman & SO-2019B-013 \\
\hline 04051129-3610404 & 4857845781166112384 & 61.2971 & -36.1779 & 237.8955 & -48.2251 & Gemini South & GMOS-S & GS-2017B-Q-84 \\
\hline 04062658-1417389 & 5110102993011576192 & 61.6107 & -14.2942 & 207.3194 & -42.9383 & SOAR & Goodman & SO-2019B-013 \\
\hline 04064080-2822221 & 4889098510129080064 & 61.6700 & -28.3728 & 226.4113 & -47.0866 & Gemini South & GMOS-S & GS-2017B-Q-84 \\
\hline 04065230-1132234 & 3190316692611552000 & 61.7180 & -11.5399 & 204.0054 & -41.6766 & Gemini South & GMOS-S & GS-2016B-Q-81 \\
\hline $04065870-5405560$ & 4779874227616110592 & 61.7446 & -54.0989 & 263.9083 & -45.4414 & Gemini South & GMOS-S & GS-2016B-Q-81 \\
\hline $04071847-3844270$ & 4844183765074837760 & 61.8270 & -38.7409 & 241.7322 & -47.8230 & Gemini South & GMOS-S & GS-2017B-Q-84 \\
\hline 04073220-1508180 & 5097968885766682240 & 61.8842 & -15.1384 & 208.5218 & -43.0334 & Gemini South & GMOS-S & GS-2017B-Q-84 \\
\hline 04081065-4723248 & 4831180463825371776 & 62.0443 & -47.3901 & 254.4277 & -46.8376 & Gemini South & GMOS-S & GS-2017B-Q-84 \\
\hline 04082208-8054055 & 4622225700669724160 & 62.0919 & -80.9015 & 294.7378 & -32.8298 & SOAR & Goodman & SO-2019B-013 \\
\hline 04161576-0521188 & 3202575628785925888 & 64.0657 & -5.3552 & 198.3846 & -36.7066 & Gemini South & GMOS-S & GS-2015B-Q-71 \\
\hline 04162853-6636540 & 4668867224438266368 & 64.1189 & -66.6150 & 279.1833 & -39.7610 & Gemini South & GMOS-S & GS-2017B-Q-84 \\
\hline 04163240-0602269 & 3202394930922308096 & 64.1350 & -6.0408 & 199.1708 & -36.9880 & Gemini South & GMOS-S & GS-2015B-Q-71 \\
\hline $04174265-5558407$ & 4778645523371812480 & 64.4278 & -55.9779 & 265.8096 & -43.4287 & Gemini South & GMOS-S & GS-2017B-Q-84 \\
\hline 04211598-4852031 & 4782730209069106432 & 65.3166 & -48.8675 & 256.0015 & -44.4416 & SOAR & Goodman & SO-2019B-013 \\
\hline 04212925-5219577 & 4780985799511960576 & 65.3718 & -52.3327 & 260.7408 & -43.7828 & Gemini South & GMOS-S & GS-2017B-Q-84 \\
\hline 04213031-2030287 & 5091573542023760384 & 65.3763 & -20.5080 & 216.9266 & -41.8366 & Gemini South & GMOS-S & GS-2017B-Q-84 \\
\hline 04215801-2739310 & 4892329974803162752 & 65.4917 & -27.6586 & 226.3554 & -43.6383 & Gemini South & GMOS-S & GS-2015B-Q-71 \\
\hline 04225741-8308401 & 4615741777521531136 & 65.7392 & -83.1445 & 296.5485 & -31.1202 & Gemini South & GMOS-S & GS-2017B-Q-84 \\
\hline $04242725-8157493$ & 4621912305496245248 & 66.1137 & -81.9637 & 295.3635 & -31.7317 & SOAR & Goodman & SO-2019B-013 \\
\hline 04251520-6213209 & 4675852078212908416 & 66.3134 & -62.2225 & 273.3995 & -40.5733 & Gemini South & GMOS-S & GS-2017B-Q-84 \\
\hline 04262201-3615167 & 4869018507227798400 & 66.5917 & -36.2547 & 238.3376 & -43.9642 & Gemini South & GMOS-S & GS-2015B-Q-71 \\
\hline 04282895-3353452 & 4871041608622321664 & 67.1206 & -33.8959 & 235.1545 & -43.2975 & Gemini South & GMOS-S & GS-2015B-Q-71 \\
\hline 04291257-4921466 & 4787721991859465728 & 67.3024 & -49.3630 & 256.4256 & -43.0847 & Gemini South & GMOS-S & GS-2015B-Q-71 \\
\hline $04293837-5448372$ & 4776030816001572736 & 67.4099 & -54.8103 & 263.6985 & -42.0761 & Gemini South & GMOS-S & GS-2015B-Q-71 \\
\hline
\end{tabular}


Table 1 (continued)

\begin{tabular}{|c|c|c|c|c|c|c|c|c|}
\hline $\begin{array}{c}\text { Star Name } \\
\text { (2MASS) }\end{array}$ & $\begin{array}{c}\text { Star Name } \\
\text { (Gaia EDR3) }\end{array}$ & $\begin{array}{l}\text { R.A. } \\
\text { (deg) }\end{array}$ & $\begin{array}{l}\text { Decl. } \\
\text { (deg) }\end{array}$ & $\begin{array}{c}l \\
(\operatorname{deg})\end{array}$ & $\begin{array}{c}b \\
(\operatorname{deg})\end{array}$ & Telescope & Instrument & Program ID \\
\hline $04311275-2258353$ & 4898098833139598464 & 67.8031 & -22.9765 & 220.9589 & -40.4386 & Gemini South & GMOS-S & GS-2017B-Q-84 \\
\hline 04313335-7337168 & 4652567991051879168 & 67.8891 & -73.6213 & 286.4338 & -35.5434 & SOAR & Goodman & SO-2019B-013 \\
\hline 04325046-3205240 & 4871721965802188160 & 68.2102 & -32.0900 & 232.8953 & -42.1392 & Gemini South & GMOS-S & GS-2015B-Q-71 \\
\hline 04330582-1605256 & 3172371116657963008 & 68.2743 & -16.0905 & 212.7851 & -37.7178 & Gemini South & GMOS-S & GS-2015B-Q-71 \\
\hline 04332826-0431033 & 3201331222141772928 & 68.3678 & -4.5176 & 200.0894 & -32.5706 & SOAR & Goodman & SO-2019B-013 \\
\hline $04334315+8752515$ & 575291322904958208 & 68.4305 & 87.8810 & 124.8749 & 25.9169 & Gemini North & GMOS-N & GN-2018B-Q-316 \\
\hline $04354147-4750031$ & 4788319503413123200 & 68.9228 & -47.8342 & 254.2029 & -42.1835 & Gemini South & GMOS-S & GS-2015B-Q-71 \\
\hline 04354314-5355093 & 4777636927611794432 & 68.9298 & -53.9192 & 262.2959 & -41.3823 & Gemini South & GMOS-S & GS-2017B-Q-84 \\
\hline 04392855-3957548 & 4816386358778340608 & 69.8690 & -39.9653 & 243.6100 & -41.6288 & Gemini South & GMOS-S & GS-2017B-Q-84 \\
\hline 04400253-1922270 & 2978208560171576448 & 70.0106 & -19.3742 & 217.3987 & -37.3499 & Gemini South & GMOS-S & GS-2015B-Q-71 \\
\hline 04404386-2741005 & 4879633570439853440 & 70.1828 & -27.6835 & 227.6267 & -39.5939 & Gemini South & GMOS-S & GS-2015B-Q-71 \\
\hline $04404847-4214219$ & 4814898930000598272 & 70.2020 & -42.2395 & 246.6587 & -41.4585 & Gemini South & GMOS-S & GS-2017B-Q-84 \\
\hline 04404877-3530531 & 4867833886528092288 & 70.2032 & -35.5148 & 237.7374 & -40.9790 & SOAR & Goodman & SO-2019B-013 \\
\hline 04414545-1703252 & 2980627661847718016 & 70.4394 & -17.0570 & 214.8726 & -36.1503 & Gemini South & GMOS-S & GS-2016B-Q-81 \\
\hline 04452944-4656234 & 4786751256234998272 & 71.3726 & -46.9399 & 252.8797 & -40.5822 & Gemini South & GMOS-S & GS-2017B-Q-84 \\
\hline $04455221+8238074$ & 569892445935443200 & 71.4677 & 82.6354 & 129.7655 & 23.1288 & Gemini North & GMOS-N & GN-2018B-Q-316 \\
\hline $04460040-5244239$ & 4777958977143271936 & 71.5017 & -52.7400 & 260.4558 & -40.0392 & Gemini South & GMOS-S & GS-2017B-Q-84 \\
\hline 04514248-3210438 & 4874664052759778304 & 72.9270 & -32.1788 & 233.9209 & -38.2219 & Gemini South & GMOS-S & GS-2017B-Q-84 \\
\hline $04520803-2837181$ & 4879979504285453184 & 73.0335 & -28.6218 & 229.5477 & -37.3718 & Gemini South & GMOS-S & GS-2016B-Q-81 \\
\hline 04522717-3451392 & 4873001625538322688 & 73.1133 & -34.8609 & 237.3246 & -38.5330 & Gemini South & GMOS-S & GS-2015B-Q-71 \\
\hline 04525913-4008568 & 4816836269489019904 & 73.2464 & -40.1491 & 244.0793 & -39.0621 & Gemini South & GMOS-S & GS-2017B-Q-84 \\
\hline $04542625-4241431$ & 4811990171989821824 & 73.6094 & -42.6953 & 247.3725 & -38.9630 & SOAR & Goodman & SO-2019B-013 \\
\hline 04562421-3124177 & 4874779806424378496 & 74.1009 & -31.4049 & 233.2135 & -37.0897 & Gemini South & GMOS-S & GS-2017B-Q-84 \\
\hline 04563790-5602511 & 4776246457719437440 & 74.1579 & -56.0476 & 264.4353 & -38.0939 & Gemini South & GMOS-S & GS-2016B-Q-81 \\
\hline $04582722-4611154$ & 4810231400062085504 & 74.6134 & -46.1876 & 251.8735 & -38.3626 & Gemini South & GMOS-S & GS-2015B-Q-71 \\
\hline 04583735-0449416 & 3212422747548345216 & 74.6556 & -4.8282 & 203.8847 & -27.2262 & Gemini South & GMOS-S & GS-2017B-Q-84 \\
\hline $05000638+0109473$ & 3228880619846899712 & 75.0266 & 1.1631 & 198.2944 & -23.9657 & Gemini North & GMOS-N & GN-2017A-Q-82 \\
\hline $05011881+0803047$ & 3290174403068832640 & 75.3284 & 8.0513 & 192.1425 & -20.0878 & Gemini North & GMOS-N & GN-2015B-Q-86 \\
\hline 05015088-4139079 & 4813429157832439168 & 75.4621 & -41.6522 & 246.1685 & -37.5210 & Gemini South & GMOS-S & GS-2015B-Q-71 \\
\hline $05015244+6745243$ & 483790308838071424 & 75.4678 & 67.7565 & 143.3439 & 15.4893 & Gemini North & GMOS-N & GN-2017A-Q-82 \\
\hline 05021896-5152056 & 4783532062283301120 & 75.5790 & -51.8682 & 259.0683 & -37.6296 & Gemini South & GMOS-S & GS-2017A-Q-86 \\
\hline 05022161-4603414 & 4810297405118590592 & 75.5903 & -46.0606 & 251.7315 & -37.6828 & Gemini South & GMOS-S & GS-2016B-Q-81 \\
\hline
\end{tabular}


Table 1 (continued)

\begin{tabular}{|c|c|c|c|c|c|c|c|c|}
\hline $\begin{array}{c}\text { Star Name } \\
\text { (2MASS) }\end{array}$ & $\begin{array}{c}\text { Star Name } \\
\text { (Gaia EDR3) }\end{array}$ & $\begin{array}{l}\text { R.A. } \\
\text { (deg) }\end{array}$ & $\begin{array}{l}\text { Decl. } \\
\text { (deg) }\end{array}$ & $\begin{array}{c}l \\
(\operatorname{deg})\end{array}$ & $\begin{array}{c}b \\
(\operatorname{deg})\end{array}$ & Telescope & Instrument & Program ID \\
\hline $05024868+0053487$ & 3228646389509963008 & 75.7028 & 0.8969 & 198.9190 & -23.5172 & Gemini North & GMOS-N & GN-2017A-Q-82 \\
\hline 05025851-3909387 & 4814331680783356800 & 75.7438 & -39.1608 & 243.0822 & -37.0478 & Gemini South & GMOS-S & GS-2015B-Q-71 \\
\hline 05042173-6614399 & 4662031938654411008 & 76.0907 & -66.2443 & 276.7597 & -35.3683 & Gemini South & GMOS-S & GS-2015B-Q-71 \\
\hline $05043110+0151252$ & 3229000397895064960 & 76.1296 & 1.8570 & 198.2535 & -22.6635 & Gemini North & GMOS-N & GN-2015B-Q-86 \\
\hline 05070417-0543063 & 3211326195154437760 & 76.7674 & -5.7184 & 205.8593 & -25.7801 & Gemini South & GMOS-S & GS-2016B-Q-81 \\
\hline $05071155+3428162$ & 184903053675527552 & 76.7981 & 34.4712 & 170.8444 & -3.6845 & Gemini North & GMOS-N & GN-2015B-Q-86 \\
\hline $05071933+0950093$ & 3290839985560775552 & 76.8306 & 9.8359 & 191.4076 & -17.8734 & Gemini North & GMOS-N & GN-2016B-Q-77 \\
\hline $05071949+2233222$ & 3415364831369329024 & 76.8312 & 22.5562 & 180.5544 & -10.7051 & Gemini North & GMOS-N & GN-2017A-Q-82 \\
\hline 05115864-0512205 & 3211775654892063360 & 77.9944 & -5.2057 & 205.9788 & -24.4598 & Gemini South & GMOS-S & GS-2016B-Q-81 \\
\hline $05120490+0354513$ & 3235687077858885248 & 78.0204 & 3.9143 & 197.3867 & -19.9902 & SOAR & Goodman & SO-2019B-013 \\
\hline 05124167-4059465 & 4818944995352554496 & 78.1736 & -40.9962 & 245.6219 & -35.4271 & Gemini South & GMOS-S & GS-2017A-Q-86 \\
\hline $05130026-2540113$ & 2956447060393795200 & 78.2511 & -25.6698 & 227.5934 & -32.1105 & Gemini South & GMOS-S & GS-2017A-Q-86 \\
\hline 05134078-0629405 & 3208349473581491968 & 78.4200 & -6.4946 & 207.4520 & -24.6725 & SOAR & Goodman & SO-2019B-013 \\
\hline $05144882+7604501$ & 503757371280728448 & 78.7034 & 76.0806 & 136.5107 & 20.7923 & Gemini North & GMOS-N & GN-2017A-Q-82 \\
\hline $05145383+3147370$ & 180592968094361472 & 78.7243 & 31.7936 & 173.9649 & -3.9603 & Gemini North & GMOS-N & GN-2015B-Q-86 \\
\hline 05160558-1418474 & 2985144932353707264 & 79.0233 & -14.3132 & 215.6108 & -27.4664 & Gemini South & GMOS-S & GS-2016B-Q-81 \\
\hline 05161882-0759103 & 3207022255670687488 & 79.0785 & -7.9862 & 209.2422 & -24.7559 & Gemini South & GMOS-S & GS-2016B-Q-81 \\
\hline 05180022-2418191 & 2958140484397025792 & 79.5010 & -24.3053 & 226.4763 & -30.6153 & Gemini South & GMOS-S & GS-2017B-Q-84 \\
\hline $05181193+1750335$ & 3395305135071832448 & 79.5497 & 17.8427 & 185.9829 & -11.2749 & Gemini North & GMOS-N & GN-2016B-Q-77 \\
\hline 05181299-0356513 & 3213469349475577472 & 79.5542 & -3.9476 & 205.5501 & -22.4988 & Gemini South & GMOS-S & GS-2017B-Q-84 \\
\hline 05183018-4217511 & 4806766701483968640 & 79.6258 & -42.2976 & 247.3613 & -34.5101 & Gemini South & GMOS-S & GS-2016B-Q-81 \\
\hline 05183839-0208303 & 3214278143356960256 & 79.6600 & -2.1417 & 203.8853 & -21.5549 & Gemini South & GMOS-S & GS-2017A-Q-86 \\
\hline 05191853-0625013 & 3207648530621533056 & 79.8273 & -6.4170 & 208.0685 & -23.3898 & Gemini South & GMOS-S & GS-2016B-Q-81 \\
\hline 05202360-2522274 & 2957773415014463872 & 80.0984 & -25.3742 & 227.8508 & -30.4312 & Gemini South & GMOS-S & GS-2017A-Q-86 \\
\hline 05204433-0757598 & 3207154098284136064 & 80.1847 & -7.9666 & 209.7540 & -23.7644 & Gemini South & GMOS-S & GS-2017A-Q-86 \\
\hline 05211432-1032144 & 3014044878233010304 & 80.3097 & -10.5374 & 212.3543 & -24.7672 & Gemini South & GMOS-S & GS-2016B-Q-81 \\
\hline $05221674+1850154$ & 3401393307050179968 & 80.5698 & 18.8376 & 185.6805 & -9.9188 & Gemini North & GMOS-N & GN-2017B-Q-79 \\
\hline 05224909-0008545 & 3221372600399707520 & 80.7045 & -0.1485 & 202.5518 & -19.6824 & Gemini South & GMOS-S & GS-2016B-Q-81 \\
\hline $05232882+6904581$ & 485426657021670784 & 80.8700 & 69.0828 & 143.2871 & 17.8761 & Gemini North & GMOS-N & GN-2015B-Q-86 \\
\hline $05234159+0001547$ & 3221399469715891712 & 80.9233 & 0.0319 & 202.4966 & -19.4037 & Gemini North & GMOS-N & GN-2017B-Q-79 \\
\hline $05234178+0140465$ & 3222180191690519296 & 80.9241 & 1.6796 & 200.9753 & -18.5999 & Gemini North & GMOS-N & GN-2015B-Q-86 \\
\hline $05240221+0037377$ & 3221857420604615040 & 81.0092 & 0.6272 & 201.9894 & -19.0399 & Gemini North & GMOS-N & GN-2015B-Q-86 \\
\hline
\end{tabular}


Table 1 (continued)

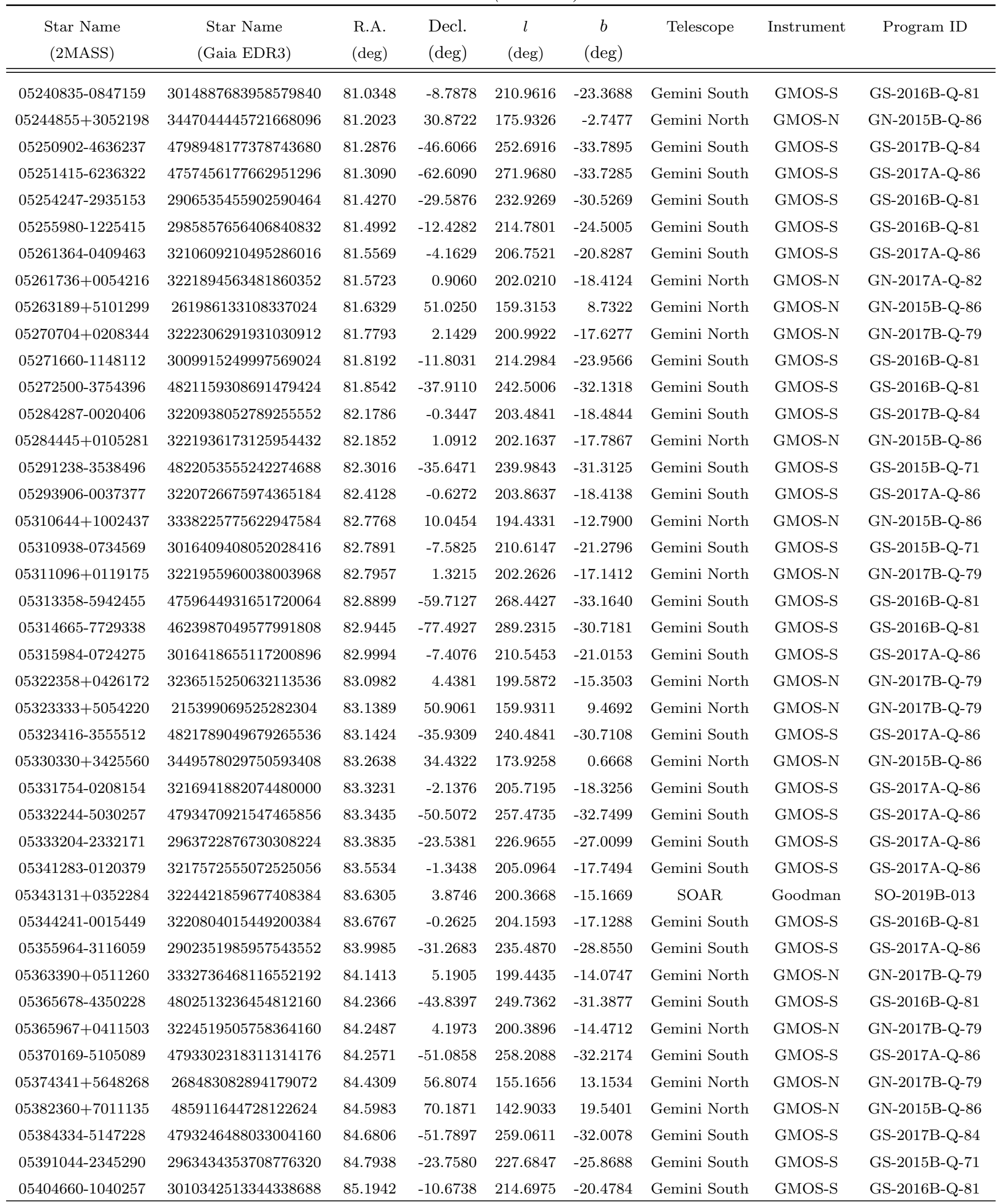


Table 1 (continued)

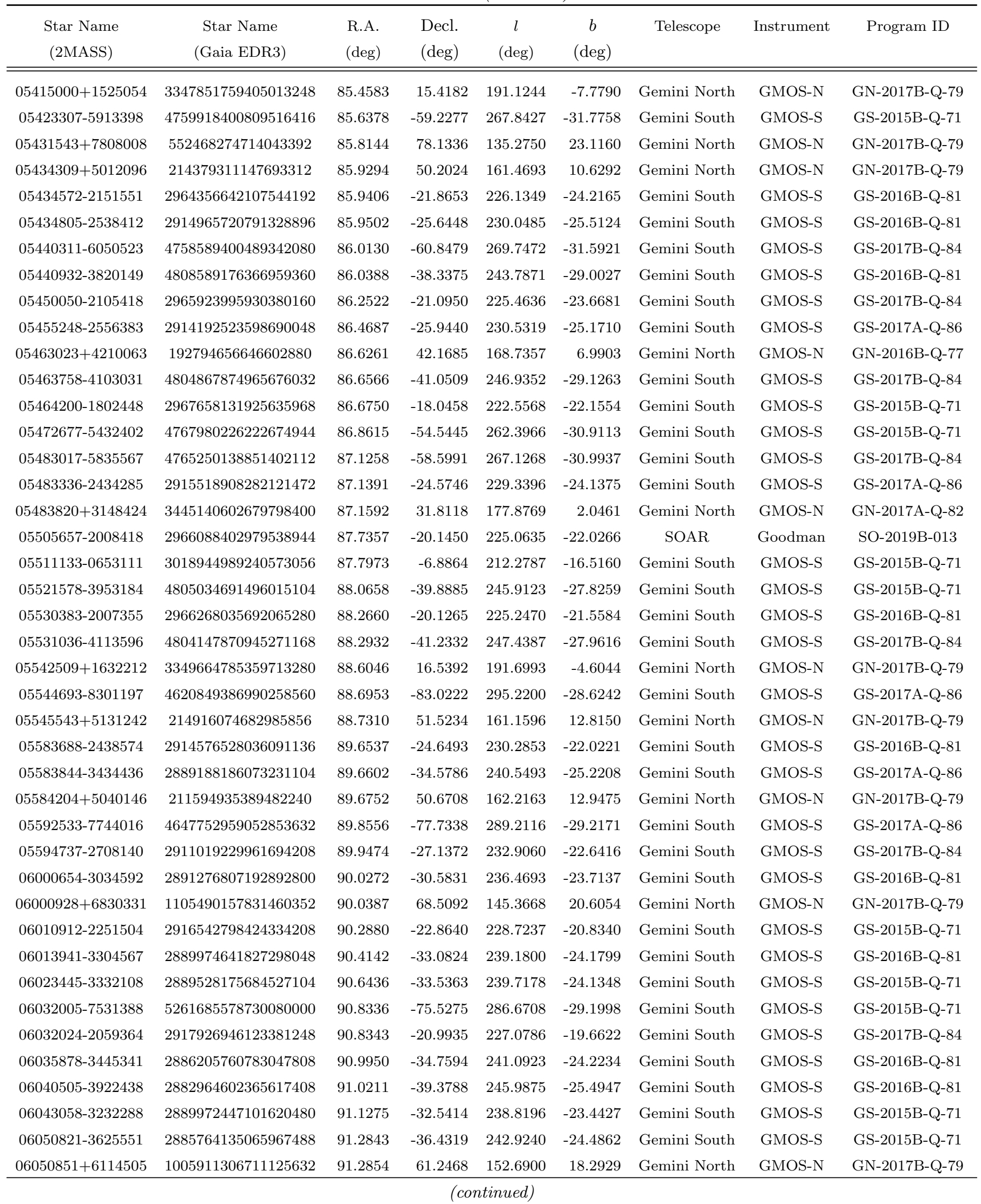


Table 1 (continued)

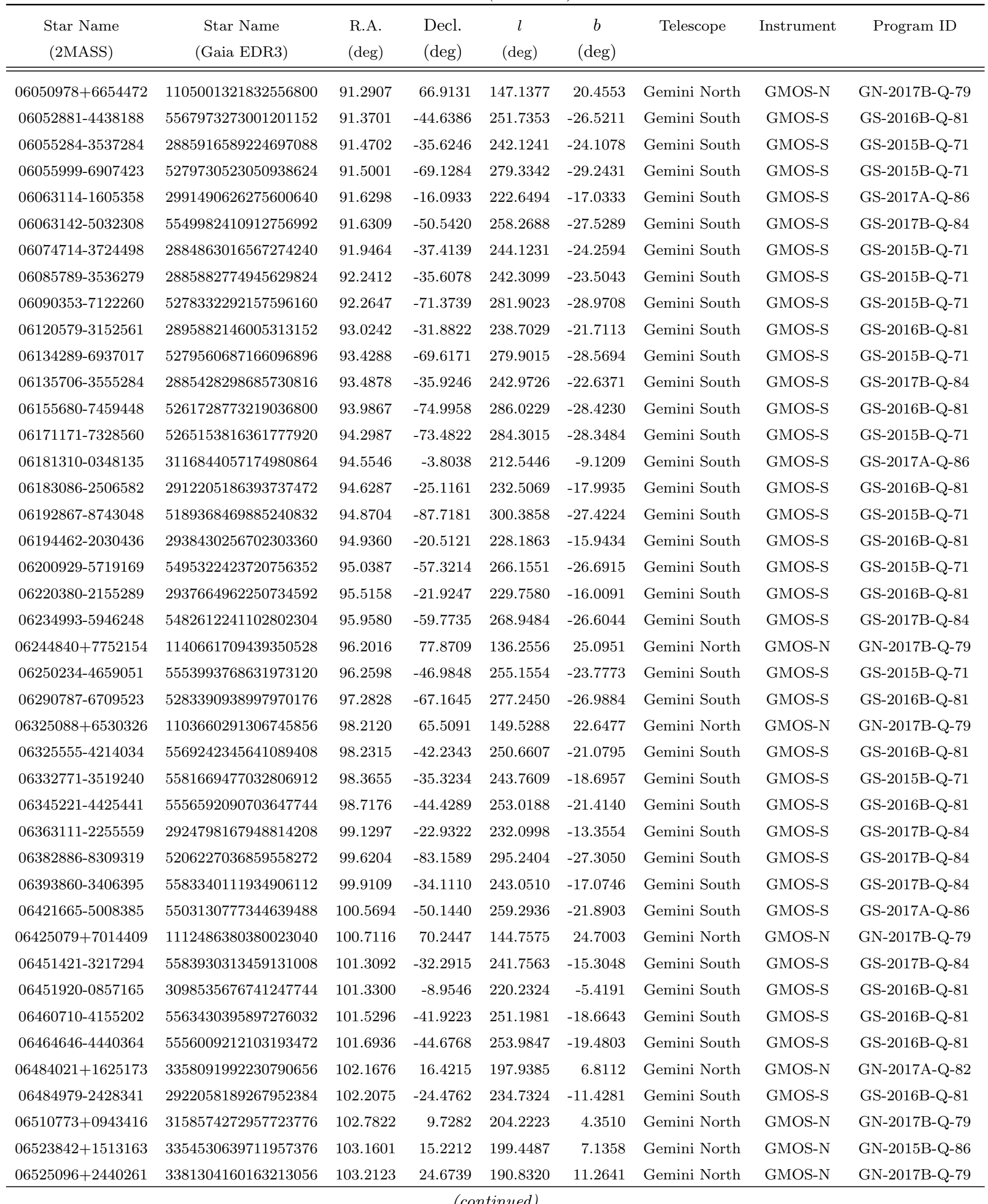


Table 1 (continued)

\begin{tabular}{|c|c|c|c|c|c|c|c|c|}
\hline $\begin{array}{c}\text { Star Name } \\
\text { (2MASS) }\end{array}$ & $\begin{array}{c}\text { Star Name } \\
\text { (Gaia EDR3) }\end{array}$ & $\begin{array}{l}\text { R.A. } \\
\text { (deg) }\end{array}$ & $\begin{array}{l}\text { Decl. } \\
\text { (deg) }\end{array}$ & $\begin{array}{c}l \\
(\mathrm{deg})\end{array}$ & $\begin{array}{c}b \\
(\operatorname{deg})\end{array}$ & Telescope & Instrument & Program ID \\
\hline $06525451+1446083$ & 3354323111187785216 & 103.2271 & 14.7690 & 199.8862 & 6.9938 & Gemini North & GMOS-N & GN-2017B-Q-79 \\
\hline $06533075+1226225$ & 3351515542604966912 & 103.3782 & 12.4396 & 202.0523 & 6.0900 & Gemini North & GMOS-N & GN-2017B-Q-79 \\
\hline $06533083+6836050$ & 1103319172123035136 & 103.3785 & 68.6014 & 146.7338 & 25.3085 & Gemini North & MOS-N & GN-2017B-Q-79 \\
\hline 06554143-4313578 & 5562195201959849984 & 103.9227 & -43.2328 & 253.1404 & -17.4757 & Gemini South & GMOS-S & GS-2017A-Q-86 \\
\hline $06560363+2846500$ & 887636359876055424 & 104.0151 & 28.7806 & 187.3011 & 13.6074 & Gemini North & GMOS-N & GN-2017B-Q-79 \\
\hline 06570150-6751413 & 5280814817608368000 & 104.2562 & -67.8614 & 278.4813 & -24.4684 & SOAR & Goodman & SO-2019B-013 \\
\hline 06571276-5526234 & 5485259315346971136 & 104.3032 & -55.4398 & 265.4469 & -21.2721 & Gemini South & MOS-S & GS-2017A-Q-86 \\
\hline 06580901-8334199 & 5194228654877524096 & 104.5372 & -83.5722 & 295.7325 & -26.7565 & Gemini South & GMOS-S & GS-2017B-Q-84 \\
\hline $06585640+0850479$ & 3157301205994883840 & 104.7350 & 8.8466 & 205.8823 & 5.6762 & Gemini North & $\mathrm{S}-\mathrm{N}$ & GN-2015B-Q-86 \\
\hline $06593152-5853177$ & 5480647173305937280 & 104.8814 & -58.8883 & 269.0946 & -21.9731 & Gemini South & GMOS-S & GS-2016B-Q-81 \\
\hline $06593924+2056439$ & 3366133201801121536 & 104.9135 & 20.9455 & 194.9522 & 11.1160 & Gemini North & & Q-79 \\
\hline 07001955-3637535 & 5578346443656712960 & 105.0815 & -36.6316 & 247.1443 & -14.1452 & Gemini South & GMOS-S & GS-2015A-Q-77 \\
\hline $07003916+2701430$ & 884148846432586240 & 105.1632 & 27.0286 & 189.3721 & 13.8324 & Gemini North & GMOS-N & GN-2016B-Q-77 \\
\hline $07004613+2933178$ & 887873162895952768 & 105.1922 & 29.5550 & 186.9889 & 14.8582 & Gemini North & GMOS-N & GN-2017B-Q-79 \\
\hline $07012363+1813452$ & 3364377384810729088 & 105.3485 & 18.2293 & 197.6317 & 10.3319 & Gemini North & GMOS-N & GN-2017B-Q-79 \\
\hline 07013933-5842103 & 5480743208774977024 & 105.4139 & -58.7029 & 268.9887 & -21.6555 & Gemini South & GMOS-S & GS-2017B-Q-84 \\
\hline $07023862+0659243$ & 3153768440772179584 & 105.6609 & 6.9901 & 207.9632 & 5.6624 & Gemini North & GMOS-N & GN-2016B-Q-77 \\
\hline $07030318+4006286$ & 947804380629842816 & 105.7633 & 40.1080 & 176.8966 & 19.1621 & Gemini North & GMOS-N & GN-2016A-Q-75 \\
\hline $07033238+2225136$ & 3368024709696717824 & 105.8849 & 22.4204 & 193.9687 & 12.5525 & Gemini North & GMOS-N & GN-2016A-Q-75 \\
\hline $07035093+1955164$ & 3365212055873231104 & 105.9622 & 19.9212 & 196.3221 & 11.5753 & Gemini North & GMOS-N & GN-2016A-Q-75 \\
\hline $07041471+1901123$ & 3364898931280232448 & 106.0613 & 19.0201 & 197.1957 & 11.2800 & Gemini North & GMOS-N & GN-2017B-Q-79 \\
\hline 07041738-1908113 & 2932674107172462592 & 106.0725 & -19.1365 & 231.4478 & -5.8748 & Gemini South & GMOS-S & GS-2015A-Q-77 \\
\hline $07041880+0246488$ & 3115642187883221632 & 106.0783 & 2.7802 & 211.9211 & 4.1306 & Gemini North & GMOS-N & GN-2017A-Q-82 \\
\hline $07041957+2340564$ & 3368538353422137344 & 106.0816 & 23.6824 & 192.8648 & 13.2360 & Gemini North & GMOS-N & GN-2017B-Q-79 \\
\hline $07054097+1733350$ & 3361464812868710144 & 106.4207 & 17.5597 & 198.6911 & 10.9689 & Gemini North & GMOS-N & GN-2016A-Q-75 \\
\hline $07070364+2657035$ & 883254423785454080 & 106.7652 & 26.9510 & 190.0311 & 15.1118 & Gemini North & GMOS-N & GN-2017B-Q-79 \\
\hline $07073503+2356476$ & 3368586663216905984 & 106.8960 & 23.9465 & 192.9275 & 14.0241 & Gemini North & GMOS-N & GN-2017B-Q-79 \\
\hline 07081426-3557115 & 5566430245872289408 & 107.0592 & -35.9533 & 247.1761 & -12.4129 & Gemini South & GMOS-S & GS-2017A-Q-86 \\
\hline $07083867-4621128$ & 5510018805377253632 & 107.1612 & -46.3536 & 257.0594 & -16.4919 & Gemini South & GMOS-S & GS-2016A-Q-76 \\
\hline $07085997-5424564$ & 5491233099459990528 & 107.2498 & -54.4157 & 264.9876 & -19.3409 & Gemini South & GMOS-S & GS-2017B-Q-84 \\
\hline $07090577+2142312$ & 3367308652748446464 & 107.2741 & 21.7087 & 195.1780 & 13.4330 & Gemini North & GMOS-N & GN-2017B-Q-79 \\
\hline $07093534+2210136$ & 3367373077257509120 & 107.3973 & 22.1705 & 194.7925 & 13.7259 & Gemini North & GMOS-N & GN-2015A-Q-76 \\
\hline 07093868-0419118 & 3107527762016884096 & 107.4112 & -4.3199 & 218.8548 & 2.0676 & Gemini South & GMOS-S & GS-2016A-Q-76 \\
\hline 07101117-2219008 & 2928210850185413120 & 107.5466 & -22.3169 & 234.9271 & -6.0775 & Gemini South & GMOS-S & GS-2017B-Q-84 \\
\hline $07102845+1245411$ & 3161108024483929600 & 107.6186 & 12.7614 & 203.6013 & 9.9470 & Gemini North & GMOS-N & GN-2017B-Q-79 \\
\hline 07103372-0244311 & 3108335765621350656 & 107.6405 & -2.7420 & 217.5577 & 2.9968 & Gemini South & GMOS-S & GS-2016A-Q-76 \\
\hline $07104970+0826444$ & 3154409902728232320 & 107.7071 & 8.4457 & 207.5648 & 8.1268 & Gemini North & GMOS-N & GN-2017B-Q-79 \\
\hline 07110190-6301262 & 5285981079149837184 & 107.7579 & -63.0239 & 273.7955 & -21.8324 & Gemini South & GMOS-S & GS-2015A-Q-77 \\
\hline $07113988+8541053$ & 1150345383223043712 & 107.9161 & 85.6848 & 127.7758 & 27.4254 & Gemini North & GMOS-N & GN-2018B-Q-316 \\
\hline $07121704+1402591$ & 3167279926849037568 & 108.0710 & 14.0498 & 202.6140 & 10.9014 & Gemini North & GMOS-N & GN-2017B-Q-79 \\
\hline $07122036+4221574$ & 949584631690298368 & 108.0848 & 42.3660 & 175.2141 & 21.5404 & Gemini North & GMOS-N & GN-2017B-Q-79 \\
\hline $07122182-8422429$ & 5193956796331371264 & 108.0909 & -84.3786 & 296.6754 & -26.4773 & Gemini South & GMOS-S & GS-2017A-Q-86 \\
\hline
\end{tabular}


Table 1 (continued)

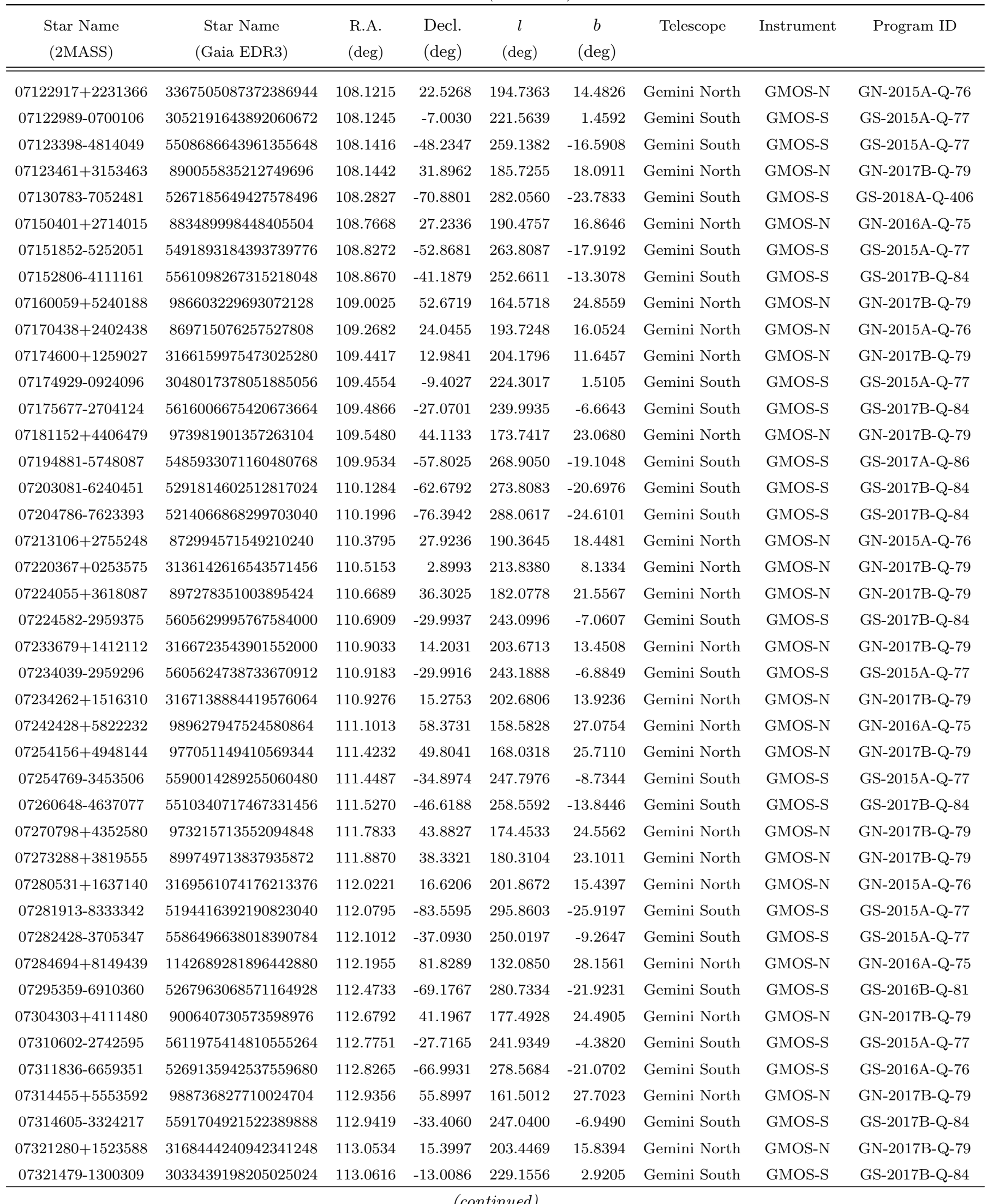


Table 1 (continued)

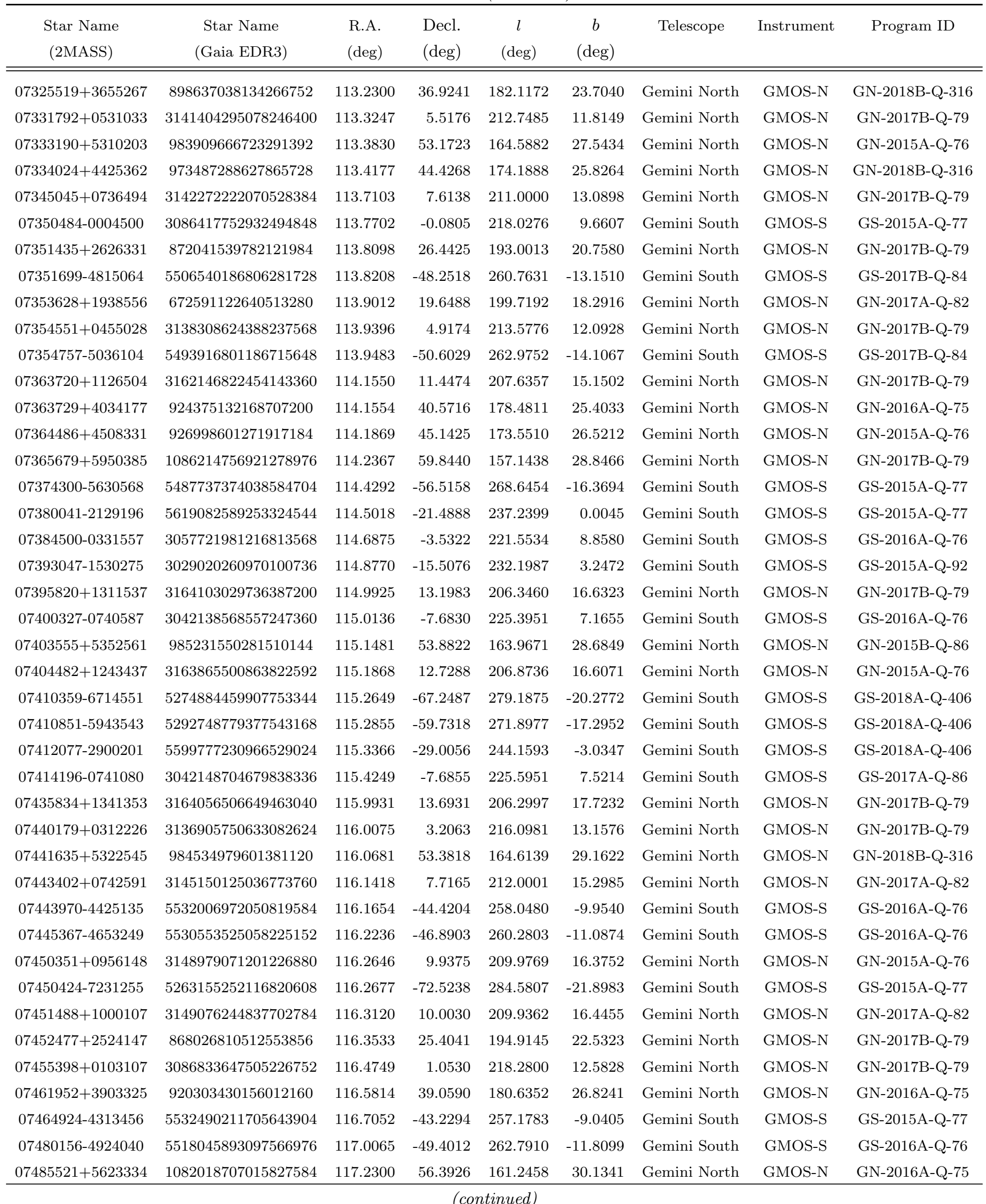


Table 1 (continued)

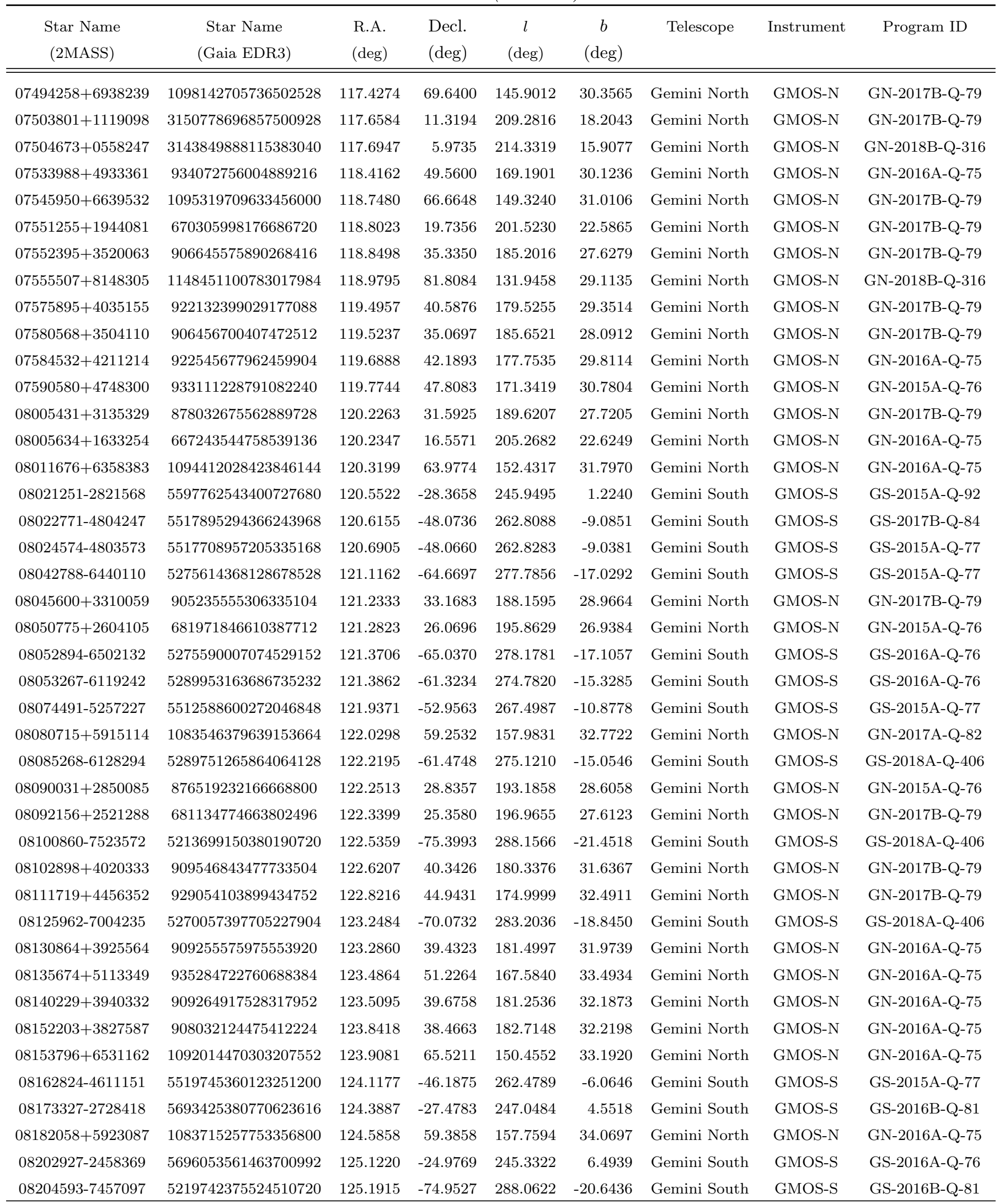


Table 1 (continued)

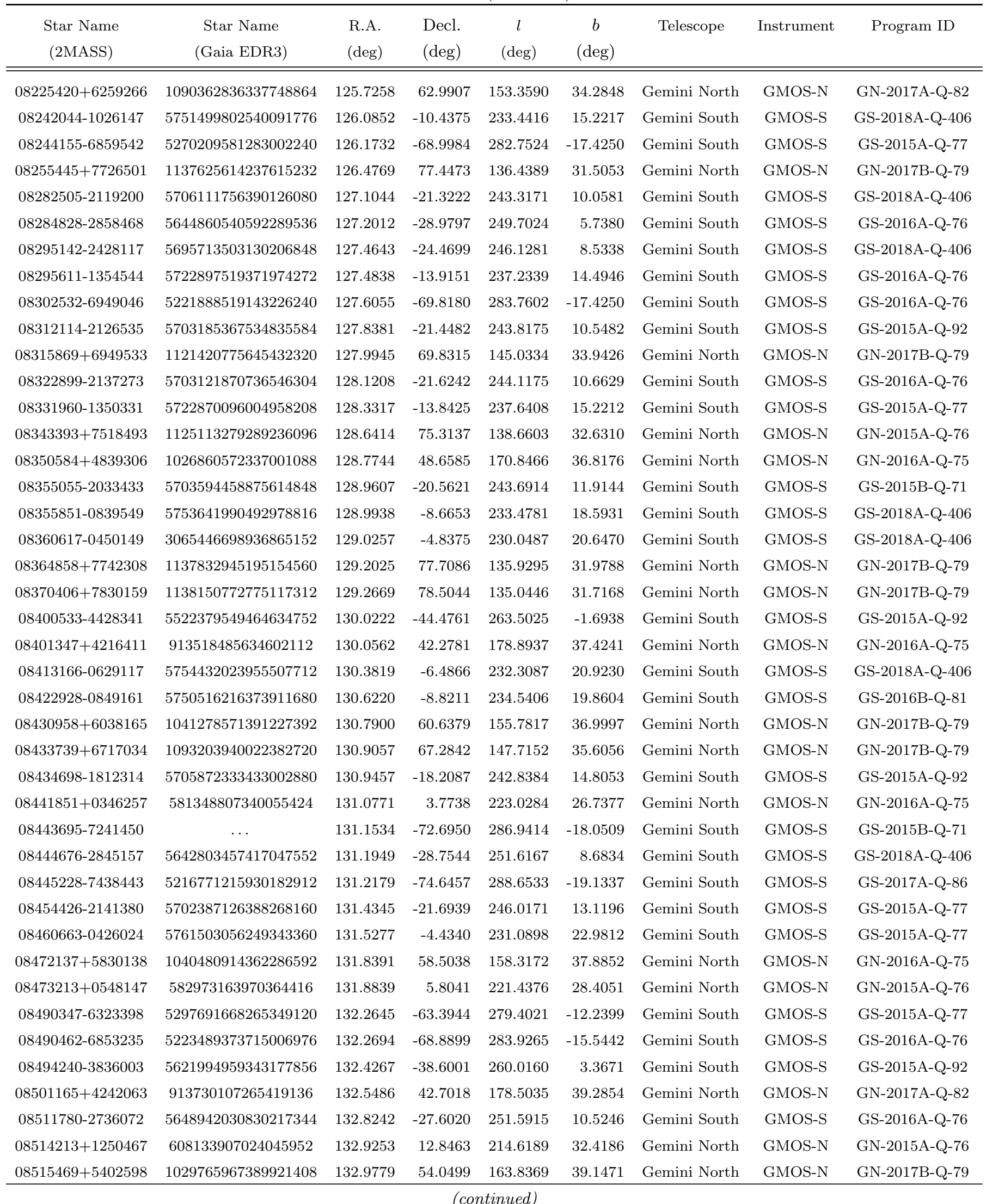


Table 1 (continued)

\begin{tabular}{|c|c|c|c|c|c|c|c|c|}
\hline $\begin{array}{c}\text { Star Name } \\
\text { (2MASS) }\end{array}$ & $\begin{array}{c}\text { Star Name } \\
\text { (Gaia EDR3) }\end{array}$ & $\begin{array}{l}\text { R.A. } \\
\text { (deg) }\end{array}$ & $\begin{array}{l}\text { Decl. } \\
\text { (deg) }\end{array}$ & $\begin{array}{c}l \\
(\operatorname{deg})\end{array}$ & $\begin{array}{c}b \\
(\operatorname{deg})\end{array}$ & Telescope & & Program ID \\
\hline 08523133-0804579 & 5750792365590211328 & 133.1306 & -8.0827 & 235.3475 & 22.3377 & Gemini South & GMOS-S & GS-2016A-Q-76 \\
\hline $08524186+6924318$ & 1118241228535948672 & 133.1744 & 69.4088 & 144.9128 & 35.7919 & Gemini North & GMOS-N & GN-2016A-Q-75 \\
\hline $08525722+6423125$ & 1044196095432573952 & 133.2385 & 64.3868 & 150.8830 & 37.2824 & Gemini North & GMOS-N & GN-2017B-Q-79 \\
\hline $08533398+1644112$ & 611494731817426560 & 133.3915 & 16.7364 & 210.5401 & 34.3529 & Gemini North & GMOS-N & GN-2017B-Q-79 \\
\hline 08543649-4744205 & 5328756032854574592 & 133.6520 & -47.7391 & 267.6232 & -1.7802 & Gemini South & GMOS-S & GS-2015A-Q-92 \\
\hline 08544986-1159089 & 5736694942894396672 & 133.7077 & -11.9858 & 239.1484 & 20.5921 & Gemini South & GMOS-S & GS-2017A-Q-86 \\
\hline 08554225-6955045 & 5222449162695490432 & 133.9261 & -69.9179 & 285.1430 & -15.6979 & Gemini South & GMOS-S & GS-2016A-Q-76 \\
\hline 08565578-7603590 & 5215675105917524608 & 134.2325 & -76.0664 & 290.3478 & -19.3293 & Gemini South & GMOS-S & GS-2018A-Q-406 \\
\hline $08571123+0040261$ & 576743880843785088 & 134.2968 & 0.6739 & 227.8532 & 28.0031 & Gemini North & GMOS-N & GN-2015A-Q-76 \\
\hline 08571600-6556117 & 5296305287178801280 & 134.3166 & -65.9366 & 282.0070 & -13.1191 & Gemini South & GMOS-S & GS-2015A-Q-77 \\
\hline 09002061-2220490 & 5654811563195280512 & 135.0859 & -22.3470 & 248.6832 & 15.4017 & Gemini South & & GS-2015A \\
\hline 09002174-3342353 & 5627698985144472448 & 135.0906 & -33.7098 & 257.6274 & 8.1513 & Gemini South & GMOS-S & GS-2018B-Q-315 \\
\hline 09012132-7055369 & 5222157448519411584 & 135.3389 & -70.9269 & 286.2717 & -15.9489 & Gemini South & & GS-2016 \\
\hline 09023125-0810363 & 5755853112670730112 & 135.6302 & -8.1768 & 236.9506 & 24.3290 & Gemini South & GMOS-S & GS-2016A- \\
\hline 09030506-2047415 & 5656156368995636608 & 135.7711 & -20.7949 & 247.8360 & 16.8689 & Gemini South & & GS-2015A \\
\hline 09030861-0427115 & 5759379998080221824 & 135.7859 & -4.4532 & 233.6317 & 26.5448 & Gemini South & GMOS-S & GS-2016A \\
\hline 09040262-0523492 & 5759068836288541696 & 136.0109 & -5.3970 & 234.6475 & 26.2090 & emini South & & Q-77 \\
\hline $09043992+0733498$ & 584226980887837824 & 136.1664 & 7.5638 & 221.9216 & 32.9892 & Gemini North & GMOS-N & GN-2015A- \\
\hline 09050894-2050426 & 5656143484093610368 & 136.2873 & -20.8452 & 248.1932 & 17.2134 & emini South & & GS-2018B \\
\hline $09065688+5836407$ & 1036942720302654080 & 136.7370 & 58.6113 & 157.5092 & 40.3638 & Gemini North & GMOS-N & GN-2017B-Q-79 \\
\hline 09073395-0340147 & 5759902820154063744 & 136.8914 & -3.6708 & 233.5797 & 27.8960 & Gemini South & MOS-S & GS-2017A-Q-86 \\
\hline 09090697-0324211 & 5759936900718820864 & 137.2791 & -3.4059 & 233.5711 & 28.3650 & Gemini South & GMOS-S & GS-2018B-Q-315 \\
\hline 09093944-2006527 & 5679552155368264704 & 137.4144 & -20.1147 & 248.2990 & 18.4933 & Gemini South & GMOS-S & GS-2018B-Q-315 \\
\hline $09102690+6033156$ & 1039441978952648064 & 137.6121 & 60.5544 & 154.8979 & 40.2767 & Gemini North & GMOS-N & GN-2015A-Q-76 \\
\hline $09103058+6419220$ & 1043675820274039040 & 137.6275 & 64.3228 & 150.2386 & 39.1139 & Gemini North & MOS-N & GN-2017A-Q-82 \\
\hline 09104309-1444185 & 5731383034718059520 & 137.6796 & -14.7385 & 243.9942 & 22.0526 & Gemini South & GMOS-S & GS-2016A-Q-76 \\
\hline 09110582-2014595 & 5679493090978018304 & 137.7742 & -20.2498 & 248.6357 & 18.6680 & Gemini South & GMOS-S & GS-2015A-Q-77 \\
\hline $09110758+0217287$ & 3843821216808928512 & 137.7816 & 2.2914 & 228.3045 & 31.8237 & Gemini North & GMOS-N & GN-2017B-Q-79 \\
\hline 09114163+1017524 & 591768294919763072 & 137.9235 & 10.2978 & 219.9151 & 35.7725 & Gemini North & GMOS-N & GN-2016A-Q-75 \\
\hline 09122631-0037340 & 3842047846288227712 & 138.1097 & -0.6262 & 231.4101 & 30.5733 & Gemini South & GMOS-S & GS-2015A-Q-77 \\
\hline $09123863+6218061$ & 1040178033268005504 & 138.1610 & 62.3017 & 152.6153 & 40.0001 & Gemini North & GMOS-N & GN-2016A-Q-75 \\
\hline 09131573-1716172 & 5682721055254568576 & 138.3156 & -17.2714 & 246.5333 & 20.9531 & Gemini South & GMOS-S & GS-2015A-Q-92 \\
\hline 09152673-0018170 & 3842238950857112064 & 138.8614 & -0.3047 & 231.5644 & 31.3802 & Gemini South & GMOS-S & GS-2018B-Q-315 \\
\hline $09155397+1212314$ & 593696288558989312 & 138.9749 & 12.2087 & 218.3204 & 37.5270 & Gemini North & GMOS-N & GN-2015A-Q-76 \\
\hline $09161100+0140496$ & 3845027557158758144 & 139.0458 & 1.6805 & 229.6951 & 32.5890 & Gemini North & GMOS-N & GN-2015A-Q-76 \\
\hline 09164357-0512382 & 5758526021141799552 & 139.1816 & -5.2106 & 236.4872 & 28.9189 & Gemini South & GMOS-S & GS-2018B-Q-315 \\
\hline 09171078-6147067 & 5298830659236242688 & 139.2949 & -61.7852 & 280.2988 & -8.7373 & Gemini South & GMOS-S & GS-2015A-Q-77 \\
\hline 09172903-1021252 & 5742681753643854464 & 139.3710 & -10.3570 & 241.2975 & 26.0352 & Gemini South & GMOS-S & GS-2016A-Q-76 \\
\hline 09180855-0852126 & 5743138252832504576 & 139.5357 & -8.8702 & 240.0791 & 27.0594 & Gemini South & GMOS-S & GS-2016A-Q-76 \\
\hline $09184848+0713360$ & 586821454667385344 & 139.7020 & 7.2267 & 224.2767 & 35.9284 & Gemini North & GMOS-N & GN-2017B-Q-79 \\
\hline $09185431+1211488$ & 593613206711676800 & 139.7263 & 12.1969 & 218.7275 & 38.1876 & Gemini North & GMOS-N & GN-2017B-Q-79 \\
\hline 09201726-0554052 & 5746372328846303360 & 140.0718 & -5.9015 & 237.7186 & 29.2423 & Gemini South & GMOS-S & GS-2017A-Q-86 \\
\hline
\end{tabular}


Table 1 (continued)

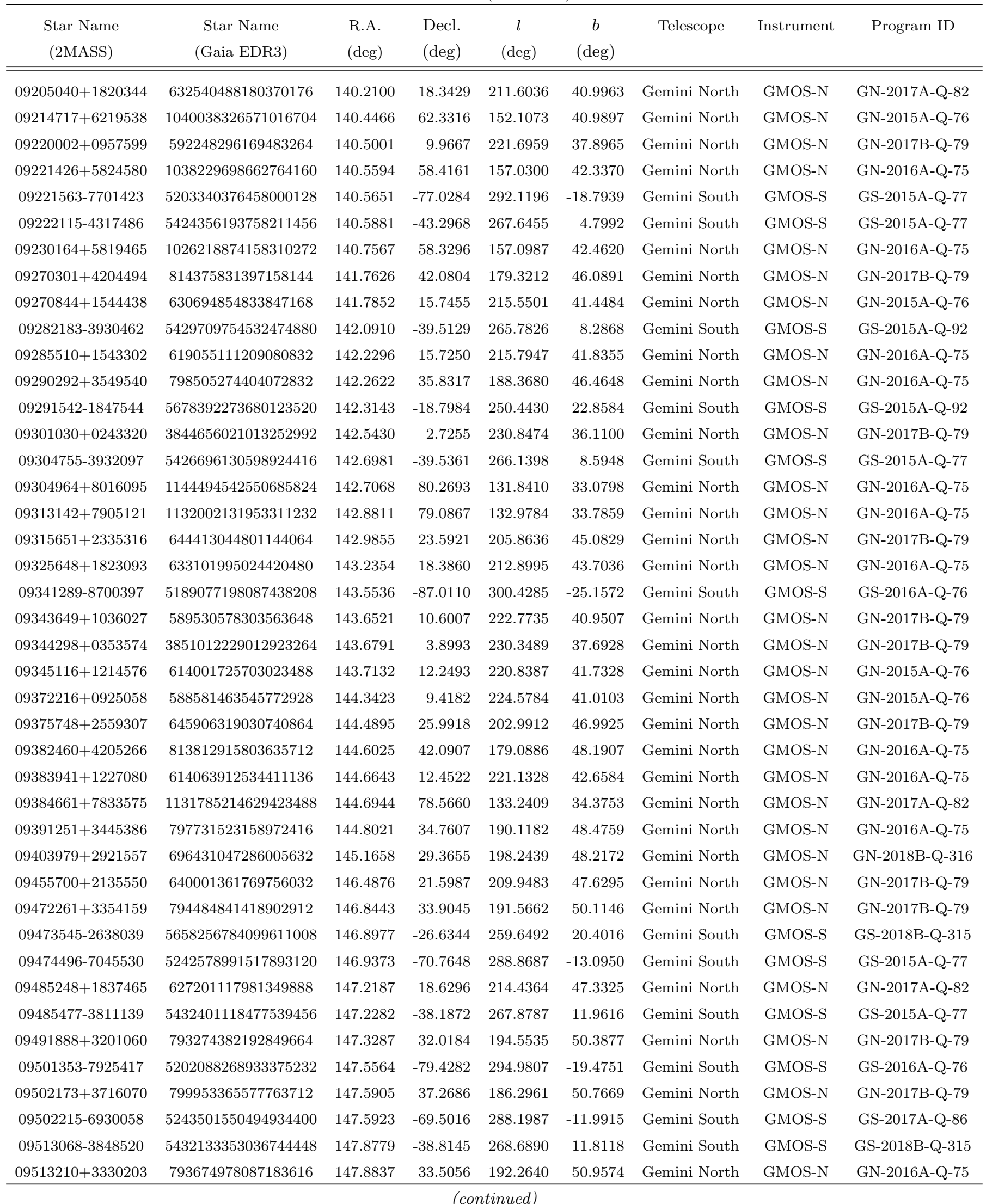


Table 1 (continued)

\begin{tabular}{|c|c|c|c|c|c|c|c|c|}
\hline $\begin{array}{c}\text { Star Name } \\
\text { (2MASS) }\end{array}$ & $\begin{array}{c}\text { Star Name } \\
\text { (Gaia EDR3) }\end{array}$ & $\begin{array}{l}\text { R.A. } \\
\text { (deg) }\end{array}$ & $\begin{array}{l}\text { Decl. } \\
\text { (deg) }\end{array}$ & $\begin{array}{c}l \\
(\mathrm{deg})\end{array}$ & $\begin{array}{c}b \\
(\mathrm{deg})\end{array}$ & Telescope & Instrument & Program ID \\
\hline 09532759-2717101 & 5657259969431486336 & 148.3650 & -27.2861 & 261.1500 & 20.8035 & Gemini South & GMOS-S & GS-2018B-Q-315 \\
\hline $09535089+3329284$ & 794978139884594560 & 148.4621 & 33.4912 & 192.3250 & 51.4383 & Gemini North & GMOS-N & GN-2017B-Q-79 \\
\hline $09541478+2735210$ & 647086477259746176 & 148.5615 & 27.5891 & 201.6990 & 50.8711 & Gemini North & GMOS-N & GN-2017B-Q-79 \\
\hline $09545564+6756128$ & 1069803755602231168 & 148.7319 & 67.9368 & 143.3686 & 41.4950 & Gemini North & GMOS-N & GN-2016A-Q-75 \\
\hline 09555639-2403072 & 5660496214405409152 & 148.9849 & -24.0520 & 259.2573 & 23.5787 & Gemini South & GMOS-S & GS-2015A-Q-77 \\
\hline 09560894-5924282 & 5257538328273646336 & 149.0373 & -59.4079 & 282.2515 & -3.7686 & Gemini South & GMOS-S & GS-2015A-Q-92 \\
\hline $09574469+1400136$ & 615228711960558464 & 149.4362 & 14.0038 & 221.9602 & 47.5210 & Gemini North & GMOS-N & GN-2017B-Q-79 \\
\hline 09580008-4318227 & 5418253457547492096 & 149.5004 & -43.3063 & 272.5354 & 9.0977 & Gemini South & GMOS-S & GS-2015A-Q-92 \\
\hline $10003325-2537597$ & 5658983939239396352 & 150.1386 & -25.6333 & 261.2538 & 23.0933 & Gemini South & GMOS-S & GS-2016A-Q-76 \\
\hline $10020621-1554291$ & 5673966327061493760 & 150.5259 & -15.9081 & 254.1886 & 30.5128 & Gemini South & GMOS-S & GS-2015A-Q-77 \\
\hline $10030615+7054115$ & 1071492949060164992 & 150.7757 & 70.9033 & 139.6443 & 40.3149 & Gemini North & GMOS-N & GN-2018B-Q-316 \\
\hline $10033379-2329108$ & 5665883610926876672 & 150.8908 & -23.4864 & 260.2736 & 25.1616 & Gemini South & GMOS-S & GS-2017A-Q-86 \\
\hline $10045623-4228179$ & 5418445670219076224 & 151.2343 & -42.4717 & 273.0402 & 10.5340 & Gemini South & GMOS-S & GS-2015A-Q-77 \\
\hline $10052247+3945408$ & 803621576887555840 & 151.3436 & 39.7614 & 181.8813 & 53.5055 & Gemini North & GMOS-N & GN-2018B-Q-316 \\
\hline 10053264-1827501 & 5672581221584085504 & 151.3860 & -18.4639 & 256.8987 & 29.2154 & Gemini South & GMOS-S & GS-2016A-Q-76 \\
\hline $10063882+2403476$ & 630450488374469120 & 151.6618 & 24.0632 & 208.2170 & 52.8818 & Gemini North & GMOS-N & GN-2016A-Q-75 \\
\hline 10075999-2736413 & 5465659970825328896 & 152.0000 & -27.6115 & 264.0179 & 22.6509 & Gemini South & GMOS-S & GS-2016A-Q-76 \\
\hline $10124027-2249482$ & 5666123373181152000 & 153.1678 & -22.8301 & 261.5760 & 27.0178 & Gemini South & GMOS-S & GS-2016B-Q-81 \\
\hline $10124666+4624575$ & 810408381126766592 & 153.1944 & 46.4160 & 170.4712 & 53.3811 & Gemini North & GMOS-N & GN-2018B-Q-316 \\
\hline $10143521+2324515$ & 725459455633859840 & 153.6467 & 23.4142 & 210.0263 & 54.4867 & Gemini North & GMOS-N & GN-2015A-Q-76 \\
\hline $10151784+5551061$ & 853205526912187264 & 153.8243 & 55.8516 & 156.2551 & 49.9446 & Gemini North & GMOS-N & GN-2018B-Q-316 \\
\hline 10152469-1057352 & 3767574556484311424 & 153.8529 & -10.9598 & 252.8540 & 36.3190 & Gemini South & GMOS-S & GS-2015A-Q-92 \\
\hline $10152705+6456284$ & 1053650967717923072 & 153.8627 & 64.9413 & 145.0401 & 44.9123 & Gemini North & GMOS-N & GN-2016A-Q-75 \\
\hline $10160573+6533551$ & 1065697491989952896 & 154.0240 & 65.5653 & 144.2896 & 44.5806 & Gemini North & GMOS-N & GN-2015A-Q-76 \\
\hline $10171294+4654224$ & 810350480671638784 & 154.3040 & 46.9062 & 169.2765 & 53.9459 & Gemini North & GMOS-N & GN-2016A-Q-75 \\
\hline $10193367+7257464$ & 1077961238527864704 & 154.8900 & 72.9629 & 136.5230 & 39.9068 & Gemini North & GMOS-N & GN-2018B-Q-316 \\
\hline $10203763+7221398$ & 1077708213414112128 & 155.1569 & 72.3611 & 137.0126 & 40.3847 & Gemini North & GMOS-N & GN-2016A-Q-75 \\
\hline $10211155+4557314$ & 809444964126986240 & 155.2982 & 45.9588 & 170.4210 & 54.9071 & Gemini North & GMOS-N & GN-2015A-Q-76 \\
\hline $10213675+7058455$ & 1076692848785487104 & 155.4031 & 70.9793 & 138.2536 & 41.4023 & Gemini North & GMOS-N & GN-2015A-Q-76 \\
\hline $10221281-4123416$ & 5416543996142159488 & 155.5534 & -41.3949 & 275.0838 & 13.2694 & Gemini South & GMOS-S & GS-2016A-Q-76 \\
\hline $10244576-7355423$ & 5229049672736749184 & 156.1906 & -73.9285 & 293.1251 & -13.9184 & Gemini South & GMOS-S & GS-2016A-Q-76 \\
\hline $10250250+7723238$ & 1128041244394048000 & 156.2605 & 77.3900 & 132.3043 & 36.9396 & Gemini North & GMOS-N & GN-2018B-Q-316 \\
\hline
\end{tabular}


Table 1 (continued)

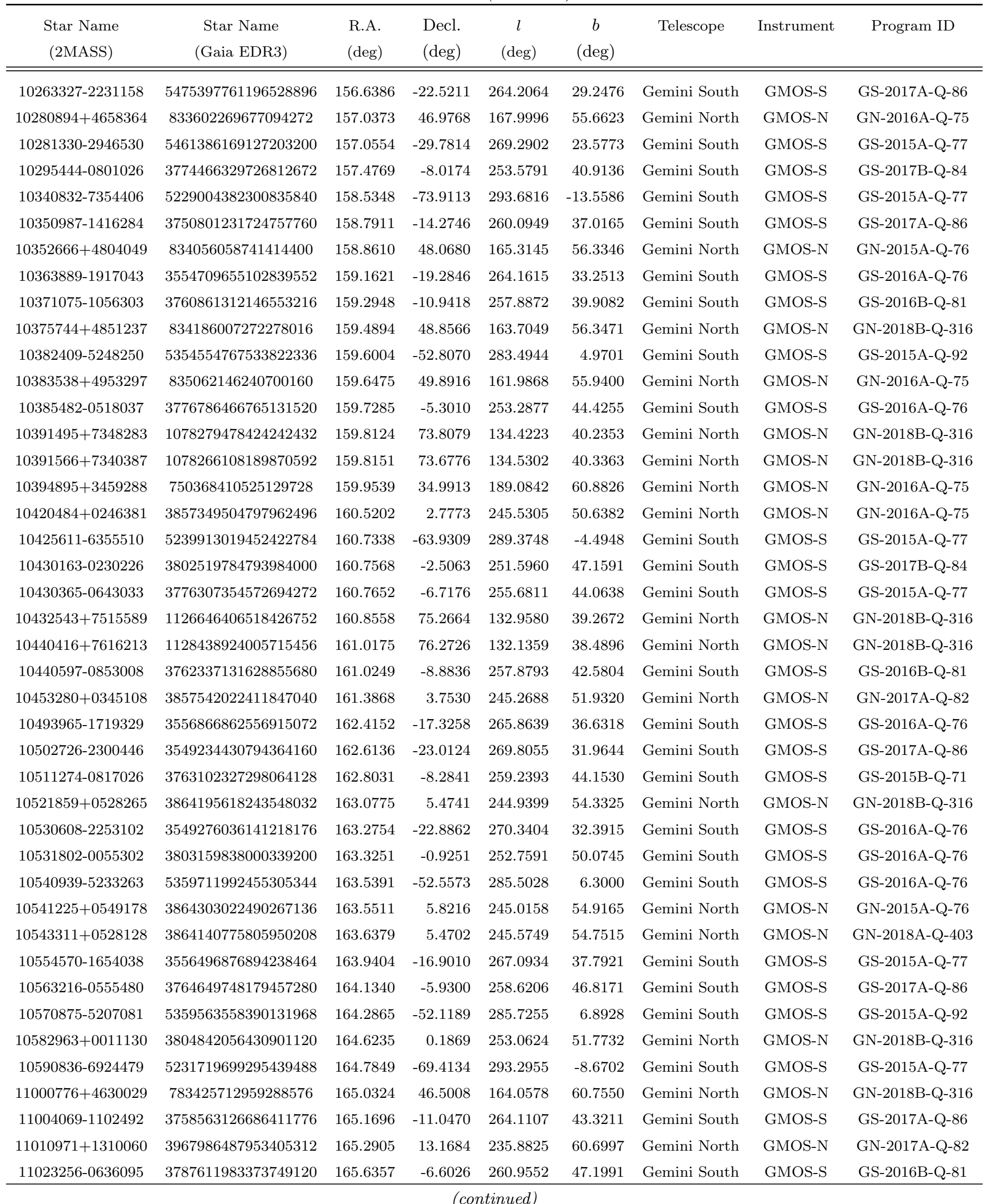


Table 1 (continued)

\begin{tabular}{|c|c|c|c|c|c|c|c|c|}
\hline $\begin{array}{c}\text { Star Name } \\
\text { (2MASS) }\end{array}$ & $\begin{array}{c}\text { Star Name } \\
\text { (Gaia EDR3) }\end{array}$ & $\begin{array}{l}\text { R.A. } \\
\text { (deg) }\end{array}$ & $\begin{array}{l}\text { Decl. } \\
\text { (deg) }\end{array}$ & $\begin{array}{c}l \\
(\operatorname{deg})\end{array}$ & $\begin{array}{c}b \\
(\operatorname{deg})\end{array}$ & Telescope & Instrument & Program ID \\
\hline $11023598+0102539$ & 3805298933576500480 & 165.6500 & 1.0483 & 253.3387 & 53.1025 & Gemini North & GMOS-N & GN-2017A-Q-82 \\
\hline $11051574-4752557$ & 5362354531210538624 & 166.3156 & -47.8821 & 285.1366 & 11.2873 & Gemini South & GMOS-S & GS-2015A-Q-92 \\
\hline 11051929-1329347 & 3564247918473953792 & 166.3305 & -13.4930 & 267.2291 & 41.9058 & Gemini South & GMOS-S & GS-2016A-Q-76 \\
\hline $11064962-5323068$ & 5347385401964090240 & 166.7068 & -53.3853 & 287.5943 & 6.3425 & Gemini South & GMOS-S & GS-2015A-Q-92 \\
\hline $11065939-4920517$ & 5362109546275295872 & 166.7474 & -49.3477 & 286.0017 & 10.0599 & Gemini South & GMOS-S & GS-2015A-Q-77 \\
\hline $11090121+0754418$ & 3818459160048340352 & 167.2551 & 7.9116 & 246.5690 & 59.0610 & Gemini North & GMOS-N & GN-2016A-Q-75 \\
\hline $11092807-0242021$ & 3791025112280181248 & 167.3670 & -2.7006 & 259.4333 & 51.3490 & Gemini South & GMOS-S & GS-2015A-Q-92 \\
\hline $11093391+2327348$ & 3995259156621469440 & 167.3913 & 23.4597 & 216.3178 & 66.7243 & Gemini North & GMOS-N & GN-2016A-Q-75 \\
\hline $11144345-1133225$ & 3566319673258222208 & 168.6811 & -11.5563 & 268.5224 & 44.7575 & Gemini South & GMOS-S & GS-2016A-Q-76 \\
\hline $11172319-5030507$ & 5349769001420934784 & 169.3467 & -50.5141 & 288.0217 & 9.6149 & Gemini South & GMOS-S & GS-2016A-Q-76 \\
\hline $11213850+3100109$ & 4023379441379492096 & 170.4104 & 31.0030 & 196.3934 & 70.0356 & Gemini North & GMOS-N & GN-2015A-Q-76 \\
\hline $11215118-4555200$ & 5376213596097250176 & 170.4633 & -45.9222 & 287.0673 & 14.1715 & Gemini South & GMOS-S & GS-2016A-Q-76 \\
\hline $11232110+6118098$ & 862722319742958592 & 170.8381 & 61.3028 & 140.2424 & 52.7642 & Gemini North & GMOS-N & GN-2016A-Q-75 \\
\hline $11235327+0025363$ & 3797935886458318336 & 170.9720 & 0.4267 & 261.1012 & 56.0175 & Gemini North & GMOS-N & GN-2016A-Q-75 \\
\hline $11242364-0642128$ & 3785352182036872320 & 171.0985 & -6.7036 & 267.8622 & 50.1467 & Gemini South & GMOS-S & GS-2018B-Q-315 \\
\hline 11245109-0118132 & 3796858154608999424 & 171.2129 & -1.3037 & 263.2168 & 54.7493 & Gemini South & GMOS-S & GS-2016A-Q-76 \\
\hline $11245186+8132096$ & 1133615145575572992 & 171.2161 & 81.5360 & 126.7292 & 34.9441 & Gemini North & GMOS-N & GN-2018B-Q-316 \\
\hline $11293922+6718355$ & 1057741219692489856 & 172.4134 & 67.3099 & 134.5346 & 47.9342 & Gemini North & GMOS-N & GN-2018B-Q-316 \\
\hline $11320178+4540156$ & 784645621097082624 & 173.0074 & 45.6709 & 158.0878 & 65.6618 & Gemini North & GMOS-N & GN-2018B-Q-316 \\
\hline $11322424+7217439$ & 1075031310621259776 & 173.1009 & 72.2955 & 131.0878 & 43.5594 & Gemini North & GMOS-N & GN-2018B-Q-316 \\
\hline $11332470+1251440$ & 3917497017084939776 & 173.3529 & 12.8622 & 247.0928 & 66.8328 & Gemini North & GMOS-N & GN-2016A-Q-75 \\
\hline $11343570+1932184$ & 3977488952772409856 & 173.6488 & 19.5385 & 232.1589 & 70.8274 & Gemini North & GMOS-N & GN-2016A-Q-75 \\
\hline $11344124+5314269$ & 841082728317714048 & 173.6718 & 53.2408 & 146.2966 & 60.2666 & Gemini North & GMOS-N & GN-2018B-Q-316 \\
\hline $11351928-3430096$ & 3477077051780069632 & 173.8303 & -34.5027 & 285.5691 & 25.7895 & Gemini South & GMOS-S & GS-2017A-Q-86 \\
\hline $11364104+3220057$ & 4024512758694334336 & 174.1711 & 32.3348 & 190.6288 & 72.9844 & Gemini North & GMOS-N & GN-2015A-Q-76 \\
\hline $11372004+1845309$ & 3974320228980706816 & 174.3335 & 18.7586 & 235.1985 & 71.0284 & Gemini North & GMOS-N & GN-2015A-Q-76 \\
\hline $11373800-5202251$ & 5345681227653768064 & 174.4084 & -52.0403 & 291.5579 & 9.1941 & Gemini South & GMOS-S & GS-2015A-Q-92 \\
\hline $11381456+1534117$ & 3972403604119095808 & 174.5607 & 15.5699 & 243.4314 & 69.4503 & Gemini North & GMOS-N & GN-2018B-Q-316 \\
\hline $11403298+6340440$ & 864134573709331584 & 175.1373 & 63.6789 & 135.5058 & 51.6840 & Gemini North & GMOS-N & GN-2018B-Q-316 \\
\hline $11415054+5035361$ & 790901636102569472 & 175.4606 & 50.5934 & 147.6995 & 63.0563 & Gemini North & GMOS-N & GN-2015A-Q-76 \\
\hline $11423344+0800282$ & 3910233647567741056 & 175.6394 & 8.0078 & 259.2826 & 64.8674 & Gemini North & GMOS-N & GN-2016A-Q-75 \\
\hline $11444533-4644384$ & 5372404612941931392 & 176.1889 & -46.7440 & 291.2111 & 14.6047 & Gemini South & GMOS-S & GS-2017A-Q-86 \\
\hline
\end{tabular}


Table 1 (continued)

\begin{tabular}{|c|c|c|c|c|c|c|c|c|}
\hline $\begin{array}{c}\text { Star Name } \\
\text { (2MASS) }\end{array}$ & $\begin{array}{c}\text { Star Name } \\
\text { (Gaia EDR3) }\end{array}$ & $\begin{array}{l}\text { R.A. } \\
\text { (deg) }\end{array}$ & $\begin{array}{l}\text { Decl. } \\
\text { (deg) }\end{array}$ & $\begin{array}{c}l \\
(\operatorname{deg})\end{array}$ & $\begin{array}{c}b \\
(\mathrm{deg})\end{array}$ & Telescope & Instrument & Program ID \\
\hline $11465844+4835156$ & 787263141676539648 & 176.7435 & 48.5877 & 148.8585 & 65.1678 & Gemini North & GMOS-N & GN-2016A-Q-75 \\
\hline $11471027+0341265$ & 3895958344506982784 & 176.7927 & 3.6907 & 266.9204 & 61.9823 & Gemini North & GMOS-N & GN-2017A-Q-82 \\
\hline $11483814+1952557$ & 3976143459777843968 & 177.1589 & 19.8821 & 236.3338 & 73.9015 & Gemini North & GMOS-N & GN-2016A-Q-75 \\
\hline $11492027+3132066$ & 4021576517188258688 & 177.3345 & 31.5352 & 191.1803 & 75.7817 & Gemini North & GMOS-N & GN-2018B-Q-316 \\
\hline $11515346+8151152$ & 1133680021057772544 & 177.9728 & 81.8542 & 125.4783 & 34.9775 & Gemini North & GMOS-N & GN-2016A-Q-75 \\
\hline $11515556-4738558$ & 5371554866553160960 & 177.9816 & -47.6489 & 292.6615 & 14.0327 & Gemini South & GMOS-S & GS-2015A-Q-77 \\
\hline $11524486-4709547$ & 5377584485241041408 & 178.1869 & -47.1652 & 292.6840 & 14.5354 & Gemini South & GMOS-S & GS-2017A-Q-86 \\
\hline $11541040-5208317$ & 5368942804889403520 & 178.5433 & -52.1421 & 294.0745 & 9.7421 & Gemini South & GMOS-S & GS-2016A-Q-76 \\
\hline $11593802+2922115$ & 4008146360612154112 & 179.9084 & 29.3699 & 198.7872 & 78.3800 & Gemini North & GMOS-N & GN-2016A-Q-75 \\
\hline $11595151-3905280$ & 3459312659023799552 & 179.9647 & -39.0911 & 292.1107 & 22.6945 & Gemini South & GMOS-S & GS-2017A-Q-86 \\
\hline $12001449+1641370$ & 3925430066494830336 & 180.0604 & 16.6936 & 251.6743 & 74.2129 & Gemini North & GMOS-N & GN-2018B-Q-316 \\
\hline $12004306+2949116$ & 4008186046109996672 & 180.1794 & 29.8199 & 196.3776 & 78.5403 & Gemini North & GMOS-N & GN-2016A-Q-75 \\
\hline $12005811-3929233$ & 3459242084121356288 & 180.2422 & -39.4898 & 292.4301 & 22.3505 & Gemini South & GMOS-S & GS-2015A-Q-92 \\
\hline $12022430+6255274$ & 1582997852929561600 & 180.6012 & 62.9242 & 132.2401 & 53.3089 & Gemini North & GMOS-N & GN-2016A-Q-75 \\
\hline $12034594+3035029$ & 4014343925404883328 & 180.9414 & 30.5842 & 191.7929 & 79.0115 & Gemini North & GMOS-N & GN-2016A-Q-75 \\
\hline $12052554-4408587$ & 6147464275054037120 & 181.3565 & -44.1496 & 294.2815 & 17.9479 & Gemini South & GMOS-S & GS-2015A-Q-77 \\
\hline $12055188+1735548$ & 3925930962760630656 & 181.4661 & 17.5985 & 252.7511 & 75.8085 & Gemini North & GMOS-N & GN-2018B-Q-316 \\
\hline $12104009+7032545$ & 1684079324125218944 & 182.6669 & 70.5486 & 127.8158 & 46.1914 & Gemini North & GMOS-N & GN-2015A-Q-76 \\
\hline $12111339+2220415$ & 4001468785978496768 & 182.8058 & 22.3449 & 238.4024 & 79.6971 & Gemini North & GMOS-N & GN-2018B-Q-316 \\
\hline $12132563-3800203$ & 3461061226110222080 & 183.3568 & -38.0057 & 294.7283 & 24.2595 & Gemini South & GMOS-S & GS-2014A-Q-8 \\
\hline $12143659-1517191$ & 3569366385619378432 & 183.6524 & -15.2887 & 289.9377 & 46.6518 & Gemini South & GMOS-S & GS-2015A-Q-77 \\
\hline $12155841-3547529$ & 3461905891557813248 & 183.9934 & -35.7981 & 294.9008 & 26.5200 & Gemini South & GMOS-S & GS-2017A-Q-86 \\
\hline $12161019+4524243$ & 1538985944361745536 & 184.0425 & 45.4068 & 141.6600 & 70.4175 & Gemini North & GMOS-N & GN-2016A-Q-75 \\
\hline $12181234-3721065$ & 6151711177372668928 & 184.5514 & -37.3518 & 295.6478 & 25.0495 & Gemini South & GMOS-S & GS-2014A-Q-8 \\
\hline $12214951+4244520$ & 1538031641283723008 & 185.4563 & 42.7479 & 142.1107 & 73.2601 & Gemini North & GMOS-N & GN-2018B-Q-316 \\
\hline $12221869-3853368$ & 6150224495918526848 & 185.5779 & -38.8936 & 296.7506 & 23.6283 & Gemini South & GMOS-S & GS-2016A-Q-76 \\
\hline $12240228-7202466$ & 5842561802238077952 & 186.0095 & -72.0463 & 300.7970 & -9.2882 & Gemini South & GMOS-S & GS-2015A-Q-77 \\
\hline $12245683+7007242$ & 1683832243246834304 & 186.2368 & 70.1234 & 126.2179 & 46.8355 & Gemini North & GMOS-N & GN-2016A-Q-75 \\
\hline $12261008+3926273$ & 1532300402563759360 & 186.5421 & 39.4409 & 144.4637 & 76.6121 & Gemini North & GMOS-N & GN-2016A-Q-75 \\
\hline $12280652-3319179$ & 3468446817511036160 & 187.0272 & -33.3216 & 297.3445 & 29.2969 & Gemini South & GMOS-S & GS-2017A-Q-86 \\
\hline $12284069+1942295$ & 3948963287525544576 & 187.1696 & 19.7082 & 266.6246 & 80.9305 & Gemini North & GMOS-N & GN-2016A-Q-75 \\
\hline $12293447-3233073$ & 3468579201286803200 & 187.3937 & -32.5521 & 297.6074 & 30.0936 & Gemini South & GMOS-S & GS-2014A-Q-74 \\
\hline
\end{tabular}


Table 1 (continued)

\begin{tabular}{|c|c|c|c|c|c|c|c|c|}
\hline $\begin{array}{c}\text { Star Name } \\
\text { (2MASS) }\end{array}$ & $\begin{array}{c}\text { Star Name } \\
\text { (Gaia EDR3) }\end{array}$ & $\begin{array}{l}\text { R.A. } \\
(\operatorname{deg})\end{array}$ & $\begin{array}{l}\text { Decl. } \\
\text { (deg) }\end{array}$ & $\begin{array}{c}l \\
(\operatorname{deg})\end{array}$ & $\begin{array}{c}b \\
(\operatorname{deg})\end{array}$ & & Instrument & n ID \\
\hline 12334194+1952177 & 3949121587136124160 & 188.4247 & 19.8716 & 272.7490 & 81.6835 & Gemini North & GMOS-N & GN-2018B-Q-316 \\
\hline $12335935-3246361$ & 6160173770478603520 & 188.4973 & -32.7767 & 298.6990 & 29.9516 & Gemini South & MOS-S & GS-2016A-Q-76 \\
\hline 12335980-2944042 & 3471719754389184384 & 188.4992 & -29.7345 & 298.4178 & 32.9843 & Gemini South & MOS-S & GS-2016A-Q-76 \\
\hline 12340964-3453438 & 6158168604865990016 & 188.5401 & -34.8955 & 298.9259 & 27.8418 & Gemini South & MOS-S & GS-2015 \\
\hline $12342913+4826025$ & 1543862488889757568 & 188.6214 & 48.4340 & 130.6002 & 68.4411 & Gemini North & GMOS-N & GN-2018B-Q-316 \\
\hline $12353682+4854057$ & 1544253571429564800 & 188.9034 & 48.9016 & 129.8899 & 68.0123 & Gemini North & GMOS-N & GN-2016A-Q-75 \\
\hline 12353793-4301524 & 6145744883683553408 & 188.9081 & -43.0312 & 299.8638 & 19.7464 & Gemini South & GMOS-S & GS-2015A-Q-77 \\
\hline 12360829-7040522 & 5843251466600455808 & 189.0345 & -70.6811 & 301.6554 & -7.8473 & Gemini South & MOS-S & GS-201 \\
\hline $12363406+4334497$ & 1540600409684268032 & 189.1420 & 43.5805 & 132.3259 & 73.2756 & Gemini North & GMOS-N & GN-2016A-Q-75 \\
\hline $12382743-3400315$ & 6158315870705750912 & 189.6144 & -34.0087 & 299.8627 & 28.7857 & Gemini South & S-S & GS- 20 \\
\hline $12383506+2039384$ & 3949279882451077888 & 189.6468 & 20.6598 & 277.8373 & 82.8971 & Gemini North & GMOS-N & GN-2016A \\
\hline $12392838+5038459$ & 1568524878294905088 & 189.8683 & 50.6461 & 127.6678 & 66.3719 & Gemini North & GMOS-N & GN-2015A-Q-76 \\
\hline 12395834-3131208 & 6160731124090635520 & 189.9931 & -31.5225 & 300.0725 & 31.2856 & Gemini South & GMOS-S & GS-2015A \\
\hline 12401578-7012252 & 5843372485918189440 & 190.0654 & -70.2071 & 301.9784 & -7.3559 & Gemini South & GMC & GS-201: \\
\hline $12413756-5412247$ & 6074478212169867904 & 190.4065 & -54.2069 & 301.4810 & 8.6375 & Gemini South & GMOS-S & GS-2015A-Q-92 \\
\hline $12432213-5955445$ & 6056631317380990208 & 190.8422 & -59.9290 & 301.9200 & 2.9269 & Gemini South & & GS-201 \\
\hline 12433114-8753309 & 5765813554148627328 & 190.8799 & -87.8919 & 302.8516 & -25.0214 & Gemini South & MOS-S & GS-2016A \\
\hline 12433200-2408376 & 3501624164266183552 & 190.8833 & -24.1438 & 300.6213 & 38.6924 & mini South & & GS-2 \\
\hline $12435927+7222303$ & 1689741705929430016 & 190.9970 & 72.3751 & 123.7260 & 44.7418 & Gemini North & MOS-N & GN-2015A-Q-76 \\
\hline $12450496-1907283$ & 3521841434322113152 & 191.2707 & -19.1246 & 300.8546 & 43.7215 & mini South & & GS-2 \\
\hline $12493821+6415038$ & 1676938094886271104 & 192.4092 & 64.2510 & 123.2559 & 52.8762 & Gemini North & GMOS-N & GN-2016A-Q-75 \\
\hline $12503487+4033500$ & 1527854729160394496 & 192.6454 & 40.5639 & 123.6322 & 76.5633 & mini North & & GN-2015 \\
\hline $12505600+5649548$ & 1576792896497757440 & 192.7333 & 56.8319 & 123.0718 & 60.2964 & Gemini North & GMOS-N & GN-2015A-Q-76 \\
\hline $12510042-1942157$ & 3509552399017285632 & 192.7518 & -19.7044 & 302.7930 & 43.1671 & mini South & & $2-86$ \\
\hline $12511944+6940558$ & 1688859141689049984 & 192.8311 & 69.6822 & 122.9468 & 47.4462 & Gemini North & GMOS-N & GN-2018B-Q-316 \\
\hline $12534156+4512260$ & 1530523729214664192 & 193.4232 & 45.2073 & 1.6527 & 71.9152 & Gemini North & & $2-76$ \\
\hline $12540492-1445540$ & 3525348811990850560 & 193.5205 & -14.7650 & 303.8888 & 48.1021 & Gemini South & GMOS-S & GS-2016A-Q-76 \\
\hline $12541483-6940177$ & 5844853661205358208 & 193.5618 & -69.6716 & 303.1776 & -6.8011 & Gemini South & & $2-77$ \\
\hline $12550382+4640597$ & 1530993770436582784 & 193.7659 & 46.6833 & 121.0752 & 70.4320 & Gemini North & GMOS-N & GN-2015A-Q-76 \\
\hline 12552381-8428164 & 5770402155472131712 & 193.8493 & -84.4712 & 303.0345 & -21.6002 & Gemini South & GMOS-S & GS-2016 \\
\hline $12553388+6801121$ & 1679621251151114624 & 193.8911 & 68.0201 & 122.3420 & 49.1036 & Gemini North & GMOS-N & GN-2018B-Q-316 \\
\hline $12572833-5125217$ & 6081346139752486784 & 194.3680 & -51.4227 & 303.8916 & 11.4378 & Gemini South & GMOS-S & GS-2015A-Q-92 \\
\hline $12592188+5338040$ & 1558199669539397376 & 194.8412 & 53.6344 & 120.3024 & 63.4534 & Gemini North & GMOS-N & GN-2018B-Q-316 \\
\hline $12595962-0829171$ & 3627268897025456000 & 194.9984 & -8.4881 & 306.5606 & 54.3234 & Gemini South & GMOS-S & GS-2017A-Q-86 \\
\hline $13003028+6135085$ & 1579614380772959104 & 195.1262 & 61.5857 & 121.0277 & 55.5092 & Gemini North & GMOS-N & GN-2015A-Q-76 \\
\hline $13022750+5836042$ & 1578633165428231552 & 195.6146 & 58.6012 & 120.1874 & 58.4683 & Gemini North & GMOS-N & GN-2016A-Q-75 \\
\hline $13031323+4812574$ & 1555059567409267328 & 195.8051 & 48.2159 & 117.5012 & 68.7880 & Gemini North & GMOS-N & GN-2018B-Q-316 \\
\hline 13044809-8623048 & 5769157650045097984 & 196.2007 & -86.3847 & 303.1616 & -23.5189 & Gemini South & GMOS-S & GS-2017A-Q-86 \\
\hline 13070832-5007271 & 6081477913650691456 & 196.7847 & -50.1242 & 305.5100 & 12.6689 & Gemini South & GMOS-S & GS-2017A-Q-86 \\
\hline $13073032+4948349$ & 1556370498804511360 & 196.8763 & 49.8097 & 116.2582 & 67.1099 & Gemini North & GMOS-N & GN-2016A-Q-75 \\
\hline $13091593+4640598$ & 1553980847721855616 & 197.3163 & 46.6833 & 113.908 & 70.1316 & Gemini North & GMOS-N & GN-2016A-Q-75 \\
\hline $13091772+4524279$ & 1529793344255574016 & 197.3238 & 45.4077 & 113.0784 & 71.3774 & Gemini North & GMOS-N & GN-2016A-Q-75 \\
\hline
\end{tabular}


Table 1 (continued)

\begin{tabular}{|c|c|c|c|c|c|c|c|c|}
\hline $\begin{array}{l}\text { Star Name } \\
\text { (2MASS) }\end{array}$ & $\begin{array}{c}\text { Star Name } \\
\text { (Gaia EDR3) }\end{array}$ & $\begin{array}{l}\text { R.A. } \\
\text { (deg) }\end{array}$ & $\begin{array}{l}\text { Decl. } \\
\text { (deg) }\end{array}$ & $\begin{array}{c}l \\
(\operatorname{deg})\end{array}$ & $\begin{array}{c}b \\
(\operatorname{deg})\end{array}$ & Telescope & Instrument & Program ID \\
\hline $13123590+7259464$ & 1688176585486569600 & 198.1497 & 72.9962 & 120.7828 & 44.0435 & Gemini North & GMOS-N & GN-2018B-Q-316 \\
\hline 13133130-6138303 & 5863379783621954944 & 198.3805 & -61.6418 & 305.5516 & 1.1176 & Gemini South & GMOS-S & GS-2015A-Q-77 \\
\hline $13134248+3405127$ & 1473046861950780032 & 198.4270 & 34.0869 & 89.7628 & 81.5548 & Gemini North & GMOS-N & GN-2017A-Q-82 \\
\hline $13140302-0929340$ & 3624508096342874240 & 198.5126 & -9.4928 & 312.2159 & 52.9709 & Gemini South & GMOS-S & GS-2017A-Q-86 \\
\hline $13151765+5708386$ & 1566249645139278720 & 198.8236 & 57.1441 & 116.5195 & 59.6861 & Gemini North & GMOS-N & GN-2018B-Q-316 \\
\hline $13175280+3411307$ & 1473007485690704128 & 199.4700 & 34.1919 & 85.7376 & 80.9371 & Gemini North & GMOS-N & GN-2018B-Q-316 \\
\hline $13222653+7020284$ & 1685430349037732864 & 200.6105 & 70.3413 & 119.1484 & 46.5586 & Gemini North & GMOS-N & GN-2016A-Q-75 \\
\hline $13222932+3936562$ & 1476579283573419264 & 200.6222 & 39.6156 & 97.5638 & 75.9445 & Gemini North & GMOS-N & GN-2016A-Q-75 \\
\hline 13240005-1748099 & 3604036456848334720 & 201.0003 & -17.8028 & 313.8055 & 44.3804 & Gemini South & GMOS-S & GS-2016A-Q-76 \\
\hline $13241831+4610307$ & 1551088548024093952 & 201.0763 & 46.1752 & 106.2184 & 69.8718 & Gemini North & GMOS-N & GN-2016A-Q-75 \\
\hline $13242995-4551146$ & 6087082811610225536 & 201.1248 & -45.8541 & 308.9301 & 16.6323 & Gemini South & GMOS-S & GS-2015A-Q-92 \\
\hline $13244275-1607008$ & 3604537731071348992 & 201.1782 & -16.1169 & 314.4756 & 46.0077 & Gemini South & GMOS-S & GS-2016A-Q-76 \\
\hline $13253853-1412517$ & 3608192335923918208 & 201.4106 & -14.2144 & 315.3307 & 47.8322 & Gemini South & GMOS-S & GS-2016A-Q-76 \\
\hline $13282335+7427122$ & 1712581998412032768 & 202.0974 & 74.4534 & 119.5899 & 42.4342 & Gemini North & GMOS-N & GN-2018B-Q-316 \\
\hline $13294999+0115237$ & 3711389512304903168 & 202.4583 & 1.2566 & 324.1240 & 62.5379 & Gemini North & GMOS-N & GN-2016A-Q-75 \\
\hline $13303726-4125545$ & 6160938171579414016 & 202.6552 & -41.4318 & 310.7768 & 20.8423 & Gemini South & GMOS-S & GS-2017A-Q-86 \\
\hline 13351401-0110524 & 3638534188221336960 & 203.8085 & -1.1812 & 325.1055 & 59.7917 & Gemini South & GMOS-S & GS-2016A-Q-76 \\
\hline $13404811+0717285$ & 3724481569055443712 & 205.2004 & 7.2912 & 335.7372 & 66.9644 & Gemini North & GMOS-N & GN-2019A-Q-309 \\
\hline 13425404-0717005 & 3620124270467822848 & 205.7252 & -7.2835 & 324.6761 & 53.4016 & Gemini South & GMOS-S & GS-2016A-Q-76 \\
\hline $13433867+4844266$ & 1558284370590734336 & 205.9111 & 48.7408 & 101.3291 & 66.1398 & Gemini North & GMOS-N & GN-2016A-DD-3 \\
\hline $13434635-0806060$ & 3618345883425109632 & 205.9432 & -8.1016 & 324.5634 & 52.5582 & Gemini South & GMOS-S & GS-2016A-Q-76 \\
\hline $13443166+1523410$ & 3742083100949072640 & 206.1319 & 15.3948 & 352.0486 & 72.9768 & Gemini North & GMOS-N & GN-2017A-Q-82 \\
\hline $13443667-4143163$ & 6112289699794699264 & 206.1528 & -41.7212 & 313.4593 & 20.0610 & Gemini South & GMOS-S & GS-2015A-Q-77 \\
\hline 13452111-0730545 & 3619873547457548160 & 206.3380 & -7.5152 & 325.4995 & 52.9780 & Gemini South & GMOS-S & GS-2016A-Q-76 \\
\hline $13454247-7355568$ & 5791382177358811264 & 206.4272 & -73.9325 & 306.7305 & -11.4624 & Gemini South & GMOS-S & GS-2015A-Q-77 \\
\hline $13455046+0513062$ & 3714276142644562432 & 206.4603 & 5.2184 & 335.9458 & 64.5444 & Gemini North & GMOS-N & GN-2016A-Q-75 \\
\hline $13455076+1426436$ & 3741132710585731840 & 206.4616 & 14.4455 & 350.6401 & 72.0691 & Gemini North & GMOS-N & GN-2019A-Q-309 \\
\hline $13460278-6854556$ & 5850193237592455552 & 206.5116 & -68.9155 & 307.8349 & -6.5651 & Gemini South & GMOS-S & GS-2015A-Q-92 \\
\hline $13461713-4155246$ & 6112273791235652352 & 206.5714 & -41.9235 & 313.7354 & 19.7947 & Gemini South & GMOS-S & GS-2015A-Q-77 \\
\hline $13465223-4334387$ & 6108883889504179072 & 206.7176 & -43.5774 & 313.4538 & 18.1589 & Gemini South & GMOS-S & GS-2017A-Q-86 \\
\hline 13481581-7052139 & 5840796639405915648 & 207.0658 & -70.8705 & 307.5960 & -8.5153 & Gemini South & GMOS-S & GS-2017A-Q-86 \\
\hline $13510697+5853470$ & 1659341789968532736 & 207.7790 & 58.8964 & 108.9568 & 56.5832 & Gemini North & GMOS-N & GN-2017A-Q-82 \\
\hline
\end{tabular}


Table 1 (continued)

\begin{tabular}{|c|c|c|c|c|c|c|c|c|}
\hline $\begin{array}{c}\text { Star Name } \\
\text { (2MASS) }\end{array}$ & $\begin{array}{c}\text { Star Name } \\
\text { (Gaia EDR3) }\end{array}$ & $\begin{array}{l}\text { R.A. } \\
\text { (deg) }\end{array}$ & $\begin{array}{l}\text { Decl. } \\
\text { (deg) }\end{array}$ & $\begin{array}{c}l \\
(\operatorname{deg})\end{array}$ & $\begin{array}{c}b \\
(\operatorname{deg})\end{array}$ & Telescope & Instrument & Program ID \\
\hline $13512378+0741110$ & 3721736844435125760 & 207.8491 & 7.6864 & 341.8480 & 65.9188 & Gemini North & GMOS-N & GN-2016A-Q-75 \\
\hline $13521659-3554258$ & 6115884072018761728 & 208.0692 & -35.9072 & 316.5320 & 25.3527 & Gemini South & GMOS-S & GS-2014A-Q-8 \\
\hline $13533557+7552137$ & 1712349073745277056 & 208.3985 & 75.8704 & 117.9866 & 40.6554 & Gemini North & GMOS-N & GN-2019A-Q-309 \\
\hline $13584157-3151091$ & 6171032134996732416 & 209.6732 & -31.8526 & 319.2277 & 28.8819 & Gemini South & GMOS-S & GS-2014A-Q-74 \\
\hline $13592706+1141130$ & 3727361838547995648 & 209.8628 & 11.6870 & 352.1834 & 67.7904 & Gemini North & GMOS-N & GN-2016A-Q-75 \\
\hline $14002121+5538479$ & 1657538212943126400 & 210.0884 & 55.6466 & 104.0608 & 58.8848 & Gemini North & GMOS-N & GN-2019A-Q-309 \\
\hline $14004596+1215235$ & 3727546045400955136 & 210.1915 & 12.2566 & 353.8509 & 67.9740 & Gemini North & GMOS-N & GN-2019A-Q-309 \\
\hline $14053819+7503388$ & 1712061237921283456 & 211.4093 & 75.0609 & 116.6787 & 41.1540 & Gemini North & GMOS-N & GN-2019A-Q-309 \\
\hline $14063229+4115356$ & 1498298211635183744 & 211.6345 & 41.2599 & 80.2537 & 69.0896 & Gemini North & GMOS-N & GN-2016A-Q-75 \\
\hline $14070810+6936102$ & 1674781746425953792 & 211.7837 & 69.6028 & 113.5464 & 46.1215 & Gemini North & GMOS-N & GN-2016A-Q-75 \\
\hline $14071697+1212488$ & 1226501033191248640 & 211.8207 & 12.2135 & 356.6407 & 66.7967 & Gemini North & GMOS-N & GN-2019A-Q-309 \\
\hline $14095514-2844258$ & 6269381255374610176 & 212.4798 & -28.7405 & 323.0317 & 31.0498 & Gemini South & GMOS-S & GS-2016A-Q-76 \\
\hline $14103285-2816339$ & 6270148611411493888 & 212.6369 & -28.2761 & 323.3736 & 31.4362 & Gemini South & GMOS-S & GS-2016A-Q-76 \\
\hline $14164140+6136563$ & 1666637942972460672 & 214.1724 & 61.6156 & 106.3717 & 52.6850 & Gemini North & GMOS-N & GN-2016A-Q-75 \\
\hline $14173352-2745144$ & 6269861295279466752 & 214.3897 & -27.7540 & 325.2843 & 31.3526 & Gemini South & GMOS-S & GS-2014A-Q-8 \\
\hline $14192484-2307370$ & 6275972209107871872 & 214.8535 & -23.1270 & 327.9382 & 35.4367 & Gemini South & GMOS-S & GS-2014A-Q-8 \\
\hline $14255416+2648176$ & 1256475262057930112 & 216.4756 & 26.8049 & 37.4795 & 68.9802 & Gemini North & GMOS-N & GN-2016A-Q-75 \\
\hline $14261661+8102449$ & 1721820606208706176 & 216.5692 & 81.0458 & 118.5362 & 35.2578 & Gemini North & GMOS-N & GN-2016A-Q-75 \\
\hline $14280573-1353174$ & 6300021547768039808 & 217.0239 & -13.8882 & 335.6955 & 42.7512 & Gemini South & GMOS-S & GS-2016A-Q-76 \\
\hline $14294135+6525010$ & 1669746193625166976 & 217.4223 & 65.4169 & 107.7386 & 48.7113 & Gemini North & GMOS-N & GN-2017B-Q-75 \\
\hline $14295841+4502025$ & 1494491496221024512 & 217.4934 & 45.0341 & 81.7578 & 63.4216 & Gemini North & GMOS-N & GN-2017B-Q-75 \\
\hline $14324019+3238042$ & 1287120579569866496 & 218.1675 & 32.6345 & 53.1817 & 67.4362 & Gemini North & GMOS-N & GN-2016A-Q-75 \\
\hline $14341379-6707026$ & 5848443708440147072 & 218.5575 & -67.1174 & 312.6971 & -6.2152 & Gemini South & GMOS-S & GS-2015A-Q-77 \\
\hline $14364248-0715099$ & 6331121238455373696 & 219.1770 & -7.2528 & 343.2746 & 47.2051 & Gemini South & GMOS-S & GS-2016A-Q-76 \\
\hline $14385266+3937037$ & 1487940434144428416 & 219.7195 & 39.6177 & 69.0708 & 64.4716 & Gemini North & GMOS-N & GN-2018A-Q-403 \\
\hline $14402612+0655539$ & 1171807334801223808 & 220.1088 & 6.9316 & 359.9458 & 57.1894 & Gemini North & GMOS-N & GN-2016A-Q-75 \\
\hline $14410823+4223420$ & 1490063071404892544 & 220.2843 & 42.3950 & 74.4527 & 62.9795 & Gemini North & GMOS-N & GN-2015A-Q-76 \\
\hline $14411830+1044242$ & 1177885611173494016 & 220.3263 & 10.7401 & 5.8566 & 59.4075 & Gemini North & GMOS-N & GN-2016A-Q-75 \\
\hline $14421990+5654506$ & 1607745454489656960 & 220.5829 & 56.9140 & 97.1322 & 54.3031 & Gemini North & GMOS-N & GN-2015A-Q-76 \\
\hline $14431485-0206178$ & 3648915222959836800 & 220.8119 & -2.1050 & 350.0369 & 50.2535 & Gemini South & GMOS-S & GS-2016A-Q-76 \\
\hline $14442119+4758464$ & 1591067649802389632 & 221.0883 & 47.9796 & 84.0216 & 59.7291 & Gemini North & GMOS-N & GN-2019A-Q-309 \\
\hline
\end{tabular}


Table 1 (continued)

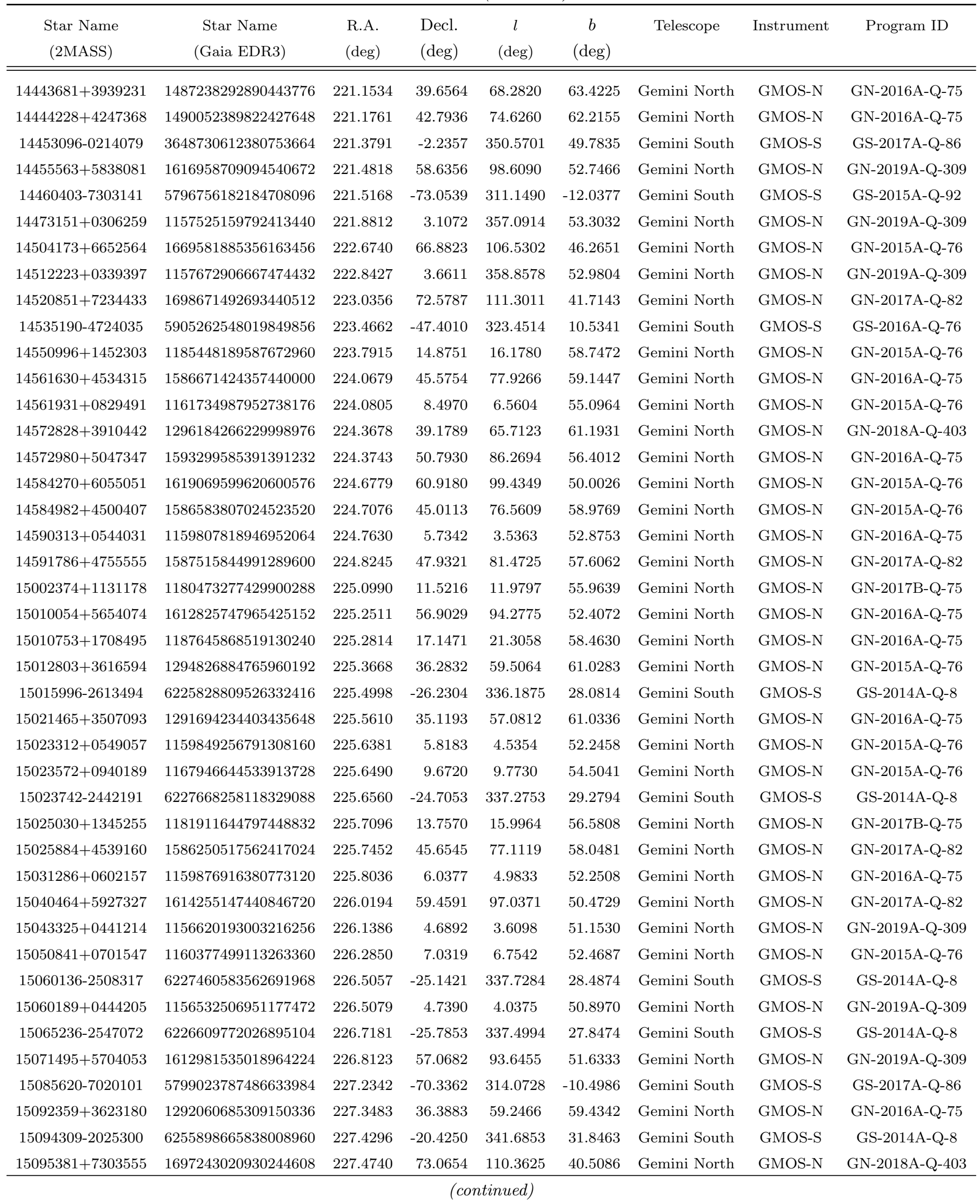


Table 1 (continued)

\begin{tabular}{|c|c|c|c|c|c|c|c|c|}
\hline $\begin{array}{c}\text { Star Name } \\
\text { (2MASS) }\end{array}$ & $\begin{array}{c}\text { Star Name } \\
\text { (Gaia EDR3) }\end{array}$ & $\begin{array}{l}\text { R.A. } \\
\text { (deg) }\end{array}$ & $\begin{array}{l}\text { Decl. } \\
\text { (deg) }\end{array}$ & $\begin{array}{c}l \\
(\operatorname{deg})\end{array}$ & $\begin{array}{c}b \\
(\operatorname{deg})\end{array}$ & Telescope & Instrument & Program ID \\
\hline $15102643+0809151$ & 1166563287107603328 & 227.6101 & 8.1542 & 9.4922 & 52.0486 & Gemini North & GMOS-N & GN-2016A-Q-75 \\
\hline $15114226+2557063$ & 1265182569515722880 & 227.9261 & 25.9517 & 38.8871 & 58.7082 & Gemini North & GMOS-N & GN-2019A-Q-309 \\
\hline $15122018-6551473$ & 5824755211457838848 & 228.0841 & -65.8632 & 316.6733 & -6.8242 & Gemini South & GMOS-S & GS-2017A-Q-86 \\
\hline $15140783+1824326$ & 1211721397891599872 & 228.5326 & 18.4091 & 25.6801 & 56.0976 & Gemini North & GMOS-N & GN-2015A-Q-76 \\
\hline $15174310-6858306$ & 5799218602888406272 & 229.4296 & -68.9752 & 315.4580 & -9.7405 & Gemini South & GMOS-S & GS-2016A-Q-76 \\
\hline $15185830+2620276$ & 1270426797601163008 & 229.7430 & 26.3410 & 40.1419 & 57.1693 & Gemini North & GMOS-N & GN-2016A-Q-75 \\
\hline $15193496+4256501$ & 1394164216985937152 & 229.8957 & 42.9472 & 70.5652 & 56.1708 & Gemini North & GMOS-N & GN-2017A-Q-82 \\
\hline $15193617+2304315$ & 1215718152722386816 & 229.9007 & 23.0754 & 34.3768 & 56.3328 & Gemini North & GMOS-N & GN-2015A-Q-76 \\
\hline $15244942+6935080$ & 1695610791623488128 & 231.2058 & 69.5856 & 105.9664 & 42.1297 & Gemini North & GMOS-N & GN-2019A-Q-309 \\
\hline $15252134+6351159$ & 1640849688017226496 & 231.3389 & 63.8545 & 99.7938 & 45.7530 & Gemini North & GMOS-N & GN-2019A-Q-309 \\
\hline $15255933+5026270$ & 1594780288252588032 & 231.4973 & 50.4407 & 82.0462 & 52.5882 & Gemini North & GMOS-N & GN-2016A-Q-75 \\
\hline $15264322-7017087$ & 5796025453632860544 & 231.6801 & -70.2858 & 315.3838 & -11.2653 & Gemini South & GMOS-S & GS-2017A-Q-86 \\
\hline $15272716+2937502$ & 1273518040182639872 & 231.8632 & 29.6306 & 46.4979 & 55.7525 & Gemini North & GMOS-N & GN-2016A-Q-75 \\
\hline $15282654+6737516$ & 1645668263365914368 & 232.1106 & 67.6310 & 103.6595 & 43.1537 & Gemini North & GMOS-N & GN-2019A-Q-309 \\
\hline $15304031+2345045$ & 1220893455176256512 & 232.6680 & 23.7512 & 36.6269 & 54.0401 & Gemini North & GMOS-N & GN-2015A-Q-76 \\
\hline $15312758+2528504$ & 1222449092327405056 & 232.8648 & 25.4807 & 39.5737 & 54.2501 & Gemini North & GMOS-N & GN-2016A-Q-75 \\
\hline $15330912+8158562$ & 1721465807550041856 & 233.2877 & 81.9823 & 116.7339 & 33.0944 & Gemini North & GMOS-N & GN-2019A-Q-309 \\
\hline $15392367+4037228$ & 1389938076542244992 & 234.8487 & 40.6231 & 65.3294 & 53.0318 & Gemini North & GMOS-N & GN-2015A-Q-76 \\
\hline $15392783+5557319$ & 1601593274615155712 & 234.8660 & 55.9589 & 88.5655 & 48.4213 & Gemini North & GMOS-N & GN-2017B-Q-75 \\
\hline $15393011+7252178$ & 1696583898069432704 & 234.8755 & 72.8716 & 108.2194 & 39.0869 & Gemini North & GMOS-N & GN-2017B-Q-75 \\
\hline $15394006+4426174$ & 1397344691808122624 & 234.9170 & 44.4381 & 71.5001 & 52.2849 & Gemini North & GMOS-N & GN-2016A-Q-75 \\
\hline $15414028+8245352$ & 1723003508921638656 & 235.4183 & 82.7598 & 117.1416 & 32.3417 & Gemini North & GMOS-N & GN-2019A-Q-309 \\
\hline $15420239+0515389$ & 4427879525740941056 & 235.5100 & 5.2608 & 12.4234 & 43.9188 & Gemini North & GMOS-N & GN-2016A-Q-75 \\
\hline $15431257+6424109$ & 1641066429247008128 & 235.8024 & 64.4030 & 98.8461 & 43.8446 & Gemini North & GMOS-N & GN-2016A-Q-75 \\
\hline $15433525+3739578$ & 1376344024013884800 & 235.8968 & 37.6660 & 60.3300 & 52.5193 & Gemini North & GMOS-N & GN-2015A-Q-76 \\
\hline $15441676+0459116$ & 4429308311037974272 & 236.0698 & 4.9866 & 12.5277 & 43.3020 & Gemini North & GMOS-N & GN-2016A-Q-75 \\
\hline $15452566-4444040$ & 5988917073480792704 & 236.3569 & -44.7345 & 332.5139 & 7.9142 & Gemini South & GMOS-S & GS-2015A-Q-77 \\
\hline $15460715+0559221$ & 4429522058673688192 & 236.5299 & 5.9895 & 14.0293 & 43.4575 & Gemini North & GMOS-N & GN-2018A-Q-403 \\
\hline $15470901+0505149$ & 4426320800506688384 & 236.7876 & 5.0875 & 13.1753 & 42.7569 & Gemini North & GMOS-N & GN-2018A-Q-403 \\
\hline $15471083-4404210$ & 5989331898604674176 & 236.7951 & -44.0725 & 333.1771 & 8.2384 & Gemini South & GMOS-S & GS-2015A-Q-77 \\
\hline $15475041+6321134$ & 1640197196585984896 & 236.9601 & 63.3538 & 97.2355 & 43.9625 & Gemini North & GMOS-N & GN-2015A-Q-76 \\
\hline $15482592-3959257$ & 6008057268799998848 & 237.1080 & -39.9905 & 335.9638 & 11.2675 & Gemini South & GMOS-S & GS-2014A-Q-74 \\
\hline
\end{tabular}


Table 1 (continued)

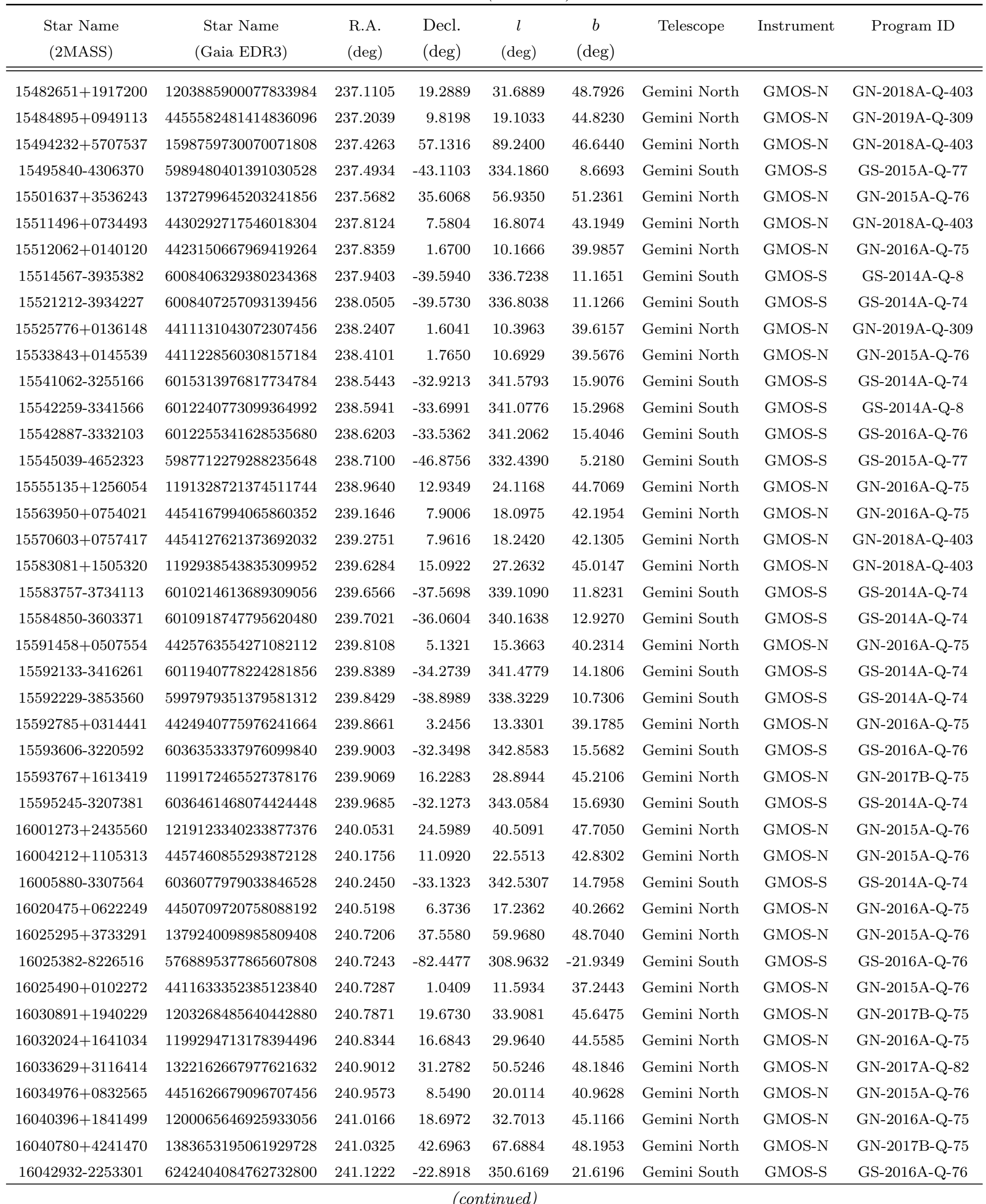


Table 1 (continued)

\begin{tabular}{|c|c|c|c|c|c|c|c|c|}
\hline $\begin{array}{c}\text { Star Name } \\
\text { (2MASS) }\end{array}$ & $\begin{array}{c}\text { Star Name } \\
\text { (Gaia EDR3) }\end{array}$ & $\begin{array}{l}\text { R.A. } \\
\text { (deg) }\end{array}$ & $\begin{array}{l}\text { Decl. } \\
\text { (deg) }\end{array}$ & $\begin{array}{c}l \\
(\operatorname{deg})\end{array}$ & $\begin{array}{c}b \\
(\mathrm{deg})\end{array}$ & Telescope & Instrument & Program ID \\
\hline $16045324-2005064$ & 6244194055324808576 & 241.2218 & -20.0851 & 352.8743 & 23.5013 & Gemini South & GMOS-S & GS-2015A-Q-77 \\
\hline $16051879+1359570$ & 1191908675099304704 & 241.3283 & 13.9991 & 26.7985 & 43.0681 & Gemini North & GMOS-N & GN-2015A-Q-76 \\
\hline $16052710+0024235$ & 4409850528641208192 & 241.3629 & 0.4066 & 11.3808 & 36.3634 & Gemini North & GMOS-N & GN-2015A-Q-76 \\
\hline $16052946-2543348$ & 6043663818991262208 & 241.3728 & -25.7263 & 348.6370 & 19.4631 & Gemini South & GMOS-S & GS-2014A-Q-8 \\
\hline $16055339+1944171$ & 1203234576873824384 & 241.4725 & 19.7381 & 34.2955 & 45.0589 & Gemini North & GMOS-N & GN-2015A-Q-76 \\
\hline $16060454-3508190$ & 6034898993336955008 & 241.5189 & -35.1386 & 341.9189 & 12.6086 & Gemini South & GMOS-S & GS-2014A-Q-74 \\
\hline 16061119-3620355 & 5998751552140270976 & 241.5466 & -36.3432 & 341.0933 & 11.7142 & Gemini South & GMOS-S & GS-2014A-Q-74 \\
\hline $16061643-3237103$ & 6036155219728437760 & 241.5685 & -32.6195 & 343.7311 & 14.4096 & Gemini South & GMOS-S & GS-2014A-Q-8 \\
\hline $16073532-3208471$ & 6036547126907295616 & 241.8972 & -32.1465 & 344.2762 & 14.5588 & Gemini South & GMOS-S & GS-2014A-Q-74 \\
\hline $16074138-3314575$ & 6035358520474835456 & 241.9224 & -33.2494 & 343.5035 & 13.7492 & Gemini South & GMOS-S & GS-2014A-Q-8 \\
\hline $16074907+1420135$ & 4458558752013944960 & 241.9545 & 14.3371 & 27.5567 & 42.6512 & Gemini North & GMOS-N & GN-2016A-Q-75 \\
\hline 16080889-2943209 & 6041589418507890688 & 242.0371 & -29.7225 & 346.1198 & 16.2113 & Gemini South & GMOS-S & GS-2014A-Q-8 \\
\hline 16081391-2017182 & 6244117639267067008 & 242.0580 & -20.2884 & 353.2985 & 22.7907 & Gemini South & GMOS-S & GS-2014A-Q-8 \\
\hline $16083267-3438595$ & 6034940156304474112 & 242.1362 & -34.6499 & 342.6401 & 12.6151 & Gemini South & GMOS-S & GS-2014A-Q-8 \\
\hline $16084292-2820180$ & 6041824610913270656 & 242.1789 & -28.3383 & 347.2285 & 17.1073 & Gemini South & GMOS-S & GS-2014A-Q-8 \\
\hline $16091164+3008425$ & 1318809707269021184 & 242.2985 & 30.1451 & 49.1055 & 46.8398 & Gemini North & GMOS-N & GN-2015A-Q-76 \\
\hline $16101125-3446225$ & 6034969392126840320 & 242.5469 & -34.7729 & 342.8021 & 12.2924 & Gemini South & GMOS-S & GS-2014A-Q-8 \\
\hline $16111495-3204491$ & 6035900167382119808 & 242.8123 & -32.0803 & 344.8963 & 14.0648 & Gemini South & GMOS-S & GS-2014A-Q-8 \\
\hline $16113577-1909453$ & 6245765566678852608 & 242.8991 & -19.1626 & 354.7795 & 22.9703 & Gemini South & GMOS-S & GS-2015A-Q-77 \\
\hline $16114668-2427053$ & 6049806927886876928 & 242.9445 & -24.4515 & 350.6585 & 19.3357 & Gemini South & GMOS-S & GS-2014A-Q-8 \\
\hline $16121101-2818128$ & 6042164669240420864 & 243.0459 & -28.3036 & 347.8161 & 16.5902 & Gemini South & GMOS-S & GS-2014A-Q-8 \\
\hline $16123013+4456046$ & 1385790890480677376 & 243.1255 & 44.9347 & 70.7306 & 46.4518 & Gemini North & GMOS-N & GN-2017B-Q-75 \\
\hline 16132830-2013405 & 6245397122905040000 & 243.3679 & -20.2279 & 354.2467 & 21.9276 & Gemini South & GMOS-S & GS-2014A-Q-74 \\
\hline $16134375-2517381$ & 6049518065566679040 & 243.4323 & -25.2940 & 350.3364 & 18.4341 & Gemini South & GMOS-S & GS-2014A-Q-74 \\
\hline 16135700-2757099 & 6042198238705334144 & 243.4876 & -27.9528 & 348.3618 & 16.5565 & Gemini South & GMOS-S & GS-2014A-Q-8 \\
\hline $16141120-2033379$ & 6244625270041451648 & 243.5467 & -20.5605 & 354.1028 & 21.5799 & Gemini South & GMOS-S & GS-2014A-Q-8 \\
\hline $16150157-2339354$ & 6050327233103808768 & 243.7566 & -23.6599 & 351.8080 & 19.3390 & Gemini South & GMOS-S & GS-2014A-Q-8 \\
\hline $16150863-0734143$ & 4348947961102169344 & 243.7860 & -7.5706 & 5.3411 & 29.7363 & Gemini South & GMOS-S & GS-2017A-Q-86 \\
\hline $16154084+3326445$ & 1322837974275568384 & 243.9202 & 33.4457 & 54.1051 & 45.8933 & Gemini North & GMOS-N & GN-2017B-Q-75 \\
\hline $16154634-1423377$ & 4329332948541070976 & 243.9431 & -14.3939 & 359.4389 & 25.3518 & Gemini South & GMOS-S & GS-2015A-Q-77 \\
\hline $16154808-2355376$ & 6050304972294952064 & 243.9503 & -23.9271 & 351.7286 & 19.0272 & Gemini South & GMOS-S & GS-2014A-Q-74 \\
\hline 16154986-0751166 & 4345832009572003840 & 243.9578 & -7.8546 & 5.2000 & 29.4288 & Gemini South & GMOS-S & GS-2017A-Q-86 \\
\hline
\end{tabular}


Table 1 (continued)

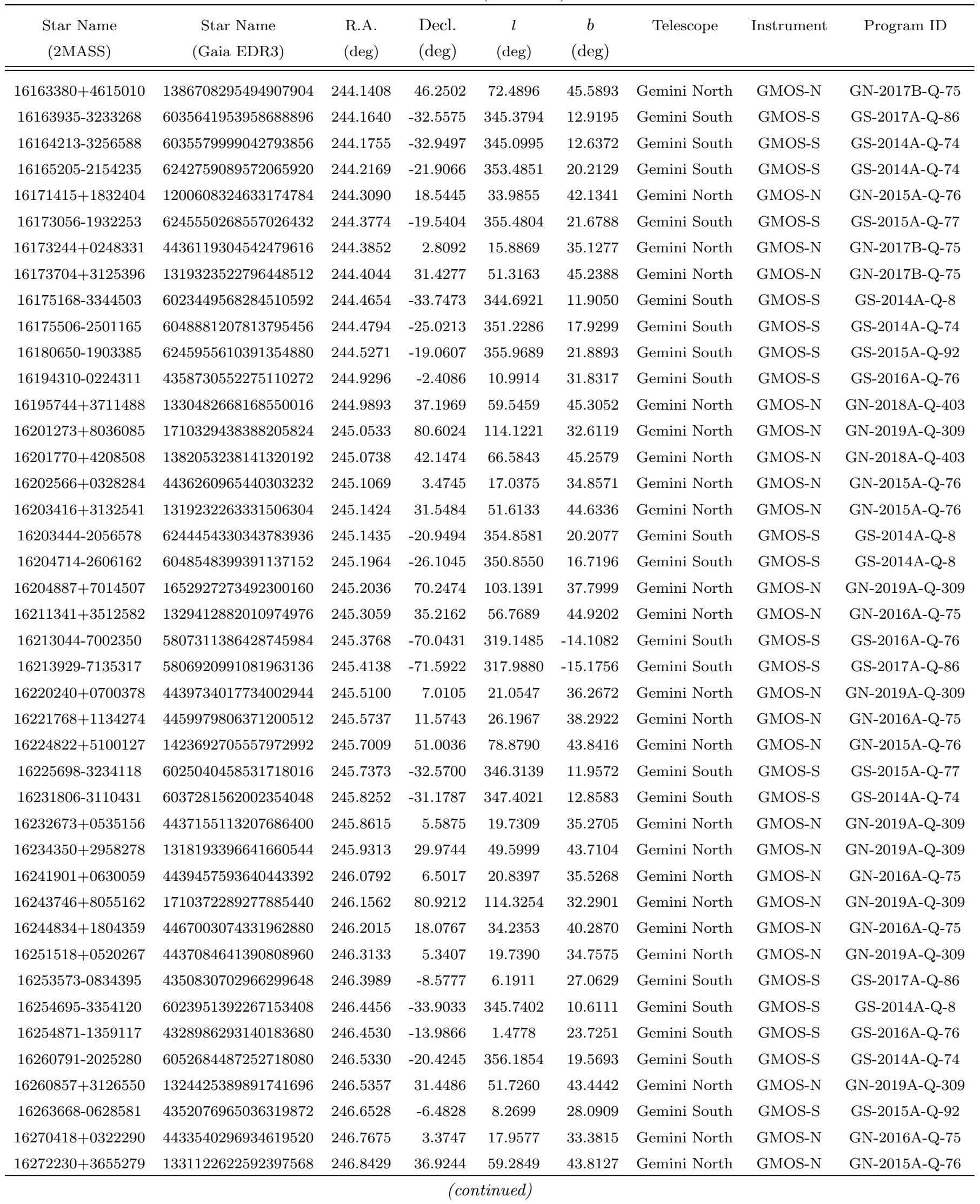


Table 1 (continued)

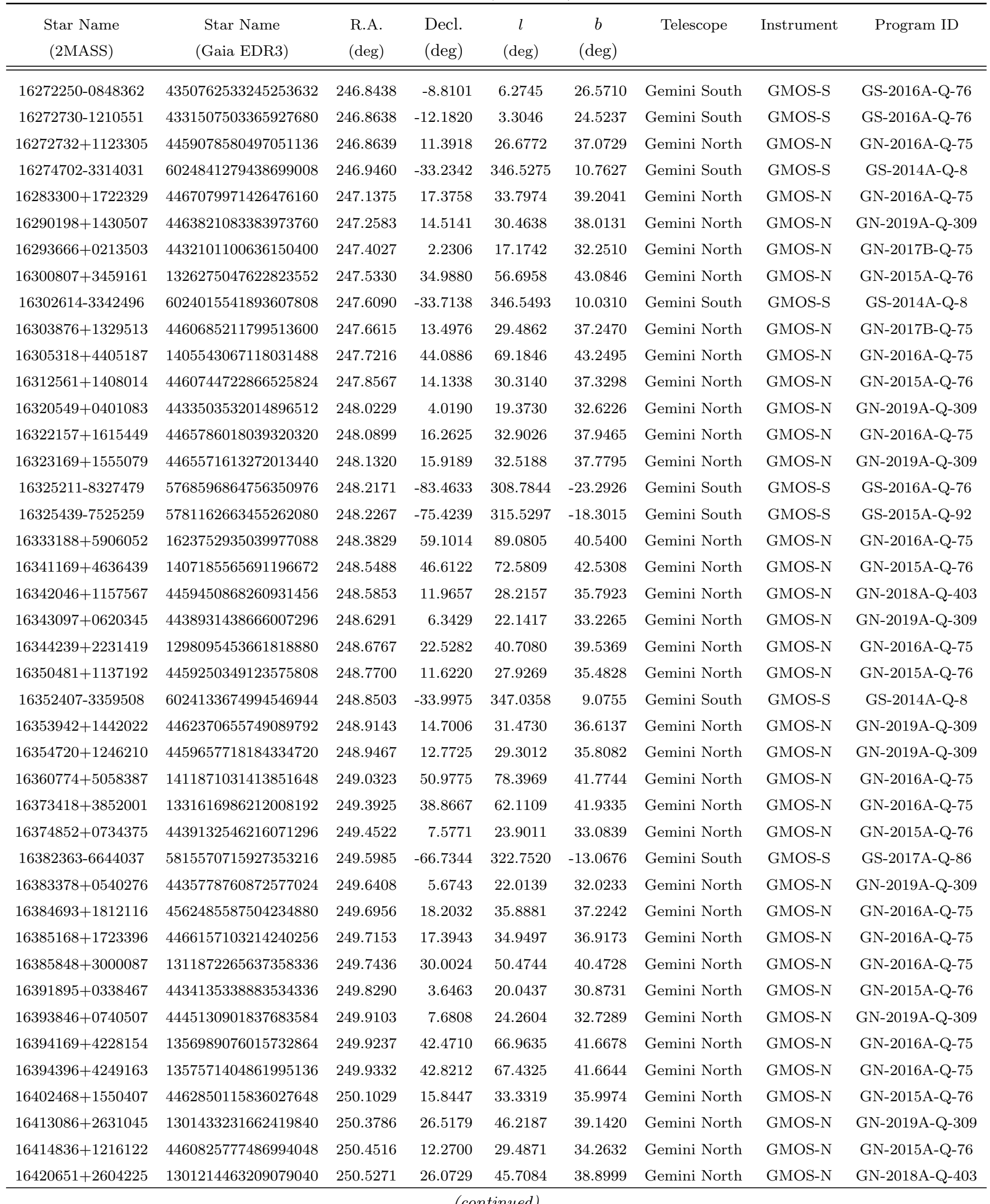


Table 1 (continued)

\begin{tabular}{|c|c|c|c|c|c|c|c|c|}
\hline $\begin{array}{c}\text { Star Name } \\
\text { (2MASS) }\end{array}$ & $\begin{array}{c}\text { Star Name } \\
\text { (Gaia EDR3) }\end{array}$ & $\begin{array}{l}\text { R.A. } \\
(\operatorname{deg})\end{array}$ & $\begin{array}{l}\text { Decl. } \\
\text { (deg) }\end{array}$ & $\begin{array}{c}l \\
(\operatorname{deg})\end{array}$ & $\begin{array}{c}b \\
(\operatorname{deg})\end{array}$ & & Instrument & n ID \\
\hline $16421928+2344032$ & 1299750291677336960 & 250.5803 & 23.7342 & 42.8477 & 38.2158 & Gemini North & GMOS-N & GN-2019A-Q-309 \\
\hline $16423938-8306366$ & 5768700008393198464 & 250.6643 & 33.1102 & 309.2785 & -23.3163 & Gemini South & GMOS-S & GS-2016A-Q-76 \\
\hline $16430853+0426005$ & 4434657430811775232 & 250.7856 & 4.4335 & 21.3845 & 30.4262 & Gemini North & GMOS-N & GN-2019A-Q-309 \\
\hline $16432853+1024103$ & 4446900634521443584 & 250.8689 & 10.4029 & 27.6652 & 33.0988 & Gemini North & GMOS-N & GN-2019A-Q-309 \\
\hline $16435657+3232386$ & 1314234398867571072 & 250.9857 & 32.5441 & 54.0014 & 39.9008 & Gemini North & GMOS-N & GN-2019A-Q-309 \\
\hline $16440315+1154212$ & 4448745095340450944 & 251.0132 & 11.9059 & 29.3649 & 33.6119 & Gemini North & MOS-N & GN-2015A-Q-76 \\
\hline 16443996-7951474 & 5775442729091999616 & 251.1664 & -79.8632 & 312.1943 & -21.5044 & Gemini South & GMOS-S & GS-2016A-Q-76 \\
\hline $16461012+3554551$ & 1327313154102801664 & 251.5422 & 35.9153 & 58.4382 & 39.9504 & Gemini North & GMOS-N & GN-20 \\
\hline $16462075+1719330$ & 4559215674287937536 & 251.5865 & 17.3259 & 35.6738 & 35.2285 & Gemini North & GMOS-N & GN-2019A-Q-309 \\
\hline $16462090+0333477$ & 4433981853933019264 & 251.5871 & 3.5633 & 20.9569 & 29.3042 & Gemini North & $\mathrm{S}-\mathrm{N}$ & Q-76 \\
\hline $16464088+0311203$ & 4385915672332609280 & 251.6703 & 3.1890 & 20.6299 & 29.0484 & Gemini North & GMOS-N & GN-2019A-Q-309 \\
\hline $16471020+5015587$ & 1412373954904373760 & 251.7925 & 50.2663 & 77.2155 & 40.1085 & Gemini North & GMOS-N & GN-2015A-Q-76 \\
\hline $16472419+1507161$ & 4461858837385161088 & 251.8508 & 15.1212 & 33.3048 & 34.1646 & Gemini North & GMOS-N & GN-2016A-Q-75 \\
\hline $16472795+2336019$ & 1299862235704972928 & 251.8665 & 23.6006 & 43.1186 & 37.0499 & Gemini North & GMOS-N & GN-2016A-Q-75 \\
\hline $16474061+1340279$ & 4461395668115705344 & 251.9192 & 13.6744 & 31.7358 & 33.5324 & Gemini North & GMOS-N & GN-2016A-Q-75 \\
\hline $16482585+4245290$ & 1356678949313407360 & 252.1077 & 42.7581 & 67.3926 & 40.0678 & Gemini North & GMOS-N & GN-2015A-Q-76 \\
\hline $16483067+5232124$ & 1413923647824211456 & 252.1278 & 52.5368 & 80.1359 & 39.6851 & Gemini North & MOS-N & GN-2019A-Q-309 \\
\hline $16485963+2050021$ & 4564767112786411648 & 252.2484 & 20.8339 & 39.9853 & 35.8549 & Gemini North & MOS-N & GN-2016A-Q-75 \\
\hline $16491646+1922215$ & 4562883030895304960 & 252.3186 & 19.3726 & 38.3194 & 35.3028 & Gemini North & MOS-N & GN-2019A-Q-309 \\
\hline & 4559694756410004480 & 252.3507 & 18.1805 & 36.9680 & 34.8573 & mini North & & GN-2019A-C \\
\hline $16501693+3227048$ & 1314079917484186880 & 252.5706 & 32.4514 & 54.1905 & 38.5689 & Gemini North & GMOS-N & GN-2018A-Q-403 \\
\hline $16501966+2135346$ & 4565674033785572096 & 252.5819 & 21.5930 & 40.9952 & 35.8058 & mini North & & Q-75 \\
\hline $16505552+5951110$ & 1438058374730240256 & 252.7313 & 59.8530 & 89.3675 & 38.2182 & Gemini North & GMOS-N & GN-2015A-Q-76 \\
\hline $16510088+3648458$ & 1351373148578890624 & 252.7536 & 36.8128 & 59.7525 & 39.0964 & mini North & & GN-2016A-Q-75 \\
\hline $16511460+5352298$ & 1426065314211359488 & 252.8108 & 53.8749 & 81.7968 & 39.1273 & Gemini North & GMOS-N & GN-2019B-Q-403 \\
\hline $16514772+3844288$ & 1352397519754683136 & 252.9488 & 38.7413 & 62.2453 & 39.1613 & emini North & & $2-75$ \\
\hline $16523005+0311543$ & 4386055756985792640 & 253.1252 & 3.1985 & 21.4471 & 27.7859 & Gemini North & GMOS-N & GN-2015A-Q-76 \\
\hline $16534583+2531166$ & 1306113268546500352 & 253.4410 & 25.5213 & 45.9217 & 36.2246 & Gemini North & MOS-N & GN-2016A-Q-75 \\
\hline $16534773+3049290$ & 1312862856894855040 & 253.4489 & 30.8247 & 52.3535 & 37.5142 & Gemini North & GMOS-N & GN-2018A-Q-403 \\
\hline $16545744+2603567$ & 1306294683667797248 & 253.7393 & 26.0658 & 46.6613 & 36.1152 & Gemini North & GMOS-N & GN-2015A-Q-76 \\
\hline 16553977-8810318 & 5765193738828393600 & 253.9147 & -88.1756 & 304.7118 & -26.2341 & Gemini South & GMOS-S & GS-2017A-Q-86 \\
\hline $16563138+3921373$ & 1352143528272068864 & 254.1308 & 39.3604 & 63.1548 & 38.3113 & Gemini North & GMOS-N & GN-2019B-Q-403 \\
\hline $16563406+1516544$ & 4545782356611145728 & 254.1419 & 15.2818 & 34.5042 & 32.1877 & Gemini North & GMOS-N & GN-2015A-Q-76 \\
\hline $16574714+4326284$ & 1358072923898101120 & 254.4461 & 43.4411 & 68.3670 & 38.3907 & Gemini North & GMOS-N & GN-2015A-Q-76 \\
\hline $16581141+4516190$ & 1359217446784103296 & 254.5475 & 45.2719 & 70.7044 & 38.3895 & Gemini North & GMOS-N & GN-2019B-Q-403 \\
\hline $16583722+0153432$ & 4384878175734959744 & 254.6551 & 1.8953 & 21.0105 & 25.8156 & Gemini North & GMOS-N & GN-2015A-Q-76 \\
\hline $16590140+2521558$ & 4572761867992849920 & 254.7559 & 25.3655 & 46.1472 & 35.0413 & Gemini Nor & & A-Q-75 \\
\hline $16591231-5353249$ & 5935760874615991808 & 254.8016 & -53.8907 & 334.5301 & -6.9891 & Gemini South & GMOS-S & GS-2015A-Q-77 \\
\hline 17001311-7651059 & 5777695868934489600 & 255.0535 & -76.8510 & 315.3336 & -20.4624 & Gemini South & GMOS-S & GS-2015A-Q-77 \\
\hline $17024781+2559130$ & 4573174150493770368 & 255.6992 & 25.9870 & 47.1646 & 34.4012 & Gemini North & GMOS-N & GN-2017B-Q-75 \\
\hline $17034238+4012376$ & 1353472361090529536 & 255.9266 & 40.2104 & 64.4086 & 37.0299 & Gemini North & GMOS-N & GN-2017B-Q-75 \\
\hline $17034776+2709455$ & 4573403428730280064 & 255.9490 & 27.1627 & 48.6099 & 34.5099 & Gemini North & GMOS-N & GN-2018A-Q-403 \\
\hline
\end{tabular}


Table 1 (continued)

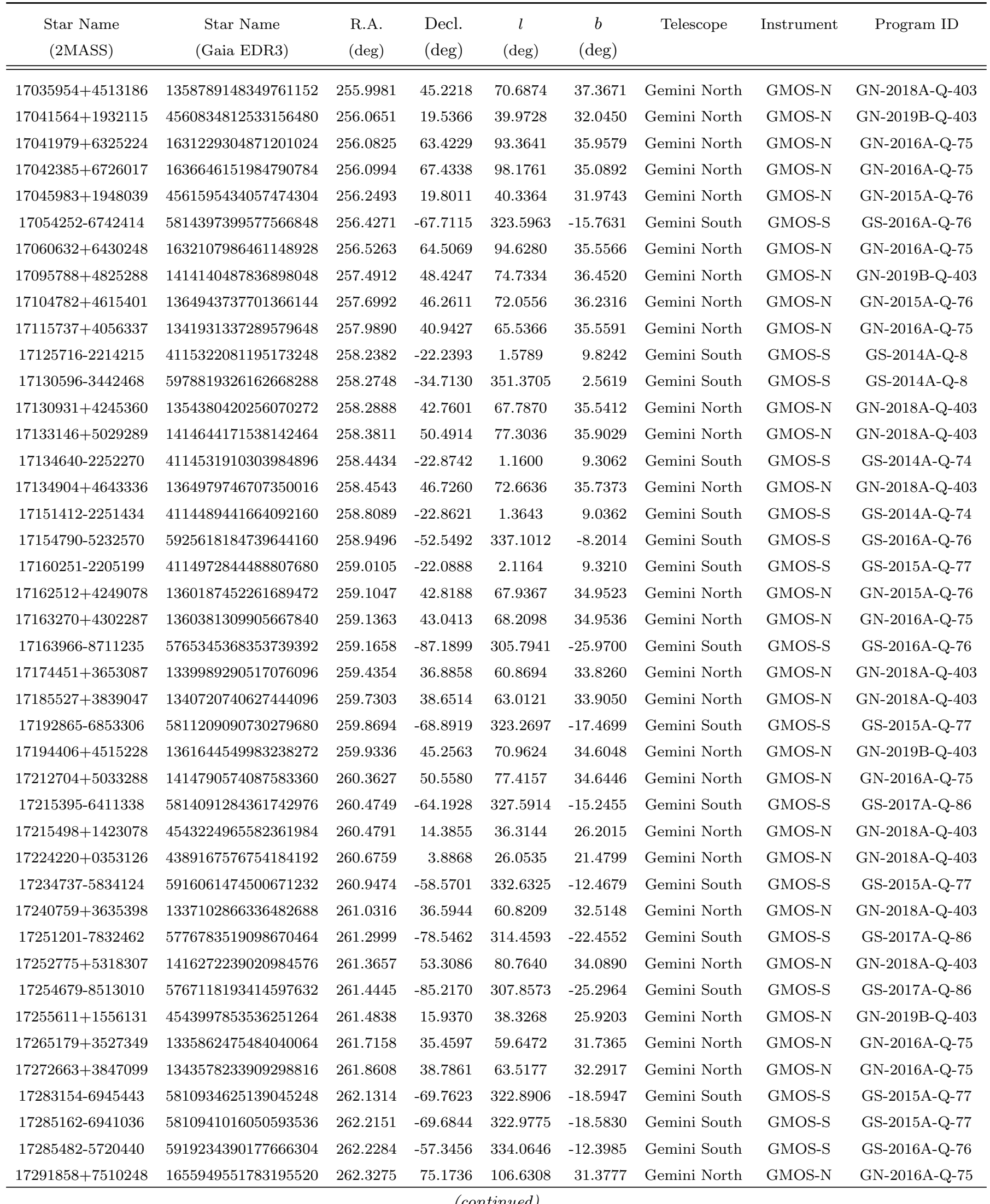


Table 1 (continued)

\begin{tabular}{|c|c|c|c|c|c|c|c|c|}
\hline $\begin{array}{c}\text { Star Name } \\
\text { (2MASS) }\end{array}$ & $\begin{array}{c}\text { Star Name } \\
\text { (Gaia EDR3) }\end{array}$ & $\begin{array}{l}\text { R.A. } \\
(\operatorname{deg})\end{array}$ & $\begin{array}{l}\text { Decl. } \\
\text { (deg) }\end{array}$ & $\begin{array}{c}l \\
(\operatorname{deg})\end{array}$ & $\begin{array}{c}b \\
(\operatorname{deg})\end{array}$ & Telescope & & Program ID \\
\hline $17292254+0305472$ & 4388901086920961024 & 262.3439 & .0964 & 26.1330 & 19.6383 & Gemini North & GMOS-N & GN-2015A-Q-76 \\
\hline $17293008+5534560$ & 1421238419509501568 & 262.3753 & 55.5822 & 83.5052 & 33.5179 & Gemini North & GMOS-N & GN-2017B-Q-75 \\
\hline $17303697+0421414$ & 4389421362079616512 & 262.6541 & 4.3615 & 27.4774 & 19.9486 & Gemini North & GMOS-N & GN-2017B-Q-75 \\
\hline $17314553+2843156$ & 4598739479466205568 & 262.9397 & 28.7210 & 52.3989 & 29.0150 & Gemini North & $\mathrm{S}-\mathrm{N}$ & GN-2016A-Q-75 \\
\hline 17315874-5342306 & 5921618165752908928 & 262.9948 & -53.7085 & 337.4620 & -10.8670 & Gemini South & -S & GS-2015A-Q-92 \\
\hline $17330791+2548590$ & 4594030404311114240 & 263.2830 & 25.8164 & 49.3580 & 27.8411 & Gemini North & GMOS-N & GN-2016A-Q-75 \\
\hline $17331311+1713463$ & 4550007577936164864 & 263.3047 & 17.2295 & 40.3911 & 24.8082 & Gemini North & MOS-N & GN-2018A-Q-403 \\
\hline $17331323+3152308$ & 4601590066440260096 & 263.3052 & 31.8752 & 55.9743 & 29.5875 & Gemini North & GMOS-N & GN-2019B-Q-403 \\
\hline $17332654+2633432$ & 4594840366419966208 & 263.3606 & 26.5620 & 50.1837 & 28.0092 & Gemini North & GMOS-N & GN-2016A-Q-75 \\
\hline $17333170+5456025$ & 1420926742322768640 & 263.3821 & 54.9340 & 82.7385 & 32.9365 & Gemini North & GMOS-N & GN-2016A-Q-75 \\
\hline 17333194-5022586 & 5946394938738915584 & 263.3831 & -50.3830 & 340.4648 & -9.3252 & Gemini South & & GS-2016 \\
\hline $17334423+1911331$ & 4553866722012938496 & 263.4344 & 19.1925 & 42.4515 & 25.4319 & Gemini North & GMOS-N & GN-2016A-Q-75 \\
\hline 17334679-6744028 & 5813027335062608384 & 263.4450 & -67.7341 & 325.0095 & -18.0527 & Gemini South & & GS-201? \\
\hline $17335350+1815568$ & 4550692088345948672 & 263.4729 & 18.2658 & 41.5156 & 25.0529 & Gemini North & GMOS-N & GN-2018A-Q-403 \\
\hline $17335522+1719221$ & 4550032381370693376 & 263.4801 & 17.3228 & 40.5567 & 24.6891 & emini North & & $\mathrm{F}-2016 \mathrm{f}$ \\
\hline $17355093+1411487$ & 4542596697766095232 & 263.9622 & 14.1969 & 37.6130 & 23.0336 & Gemini North & GMOS-N & GN-2019B-Q-403 \\
\hline $17370392-7408233$ & 5803200381170398336 & 264.2663 & 4.1398 & 319.0587 & -21.1662 & mini South & & -86 \\
\hline $17371472+1603347$ & 4549079418323779712 & 264.3113 & 16.0597 & 39.6189 & 23.4657 & Gemini North & GMOS-N & GN-2015A-Q-76 \\
\hline $17373165+0109351$ & 4375387436181302912 & 264.3819 & 1598 & 25.3350 & 16.9322 & Gemini North & & GN-2C \\
\hline $17374593-4903259$ & 5946896105559051392 & 264.4413 & -49.0572 & 341.9724 & -9.2166 & Gemini South & GMOS-S & GS-2017A-Q-86 \\
\hline $17381613+1806130$ & 4550553412442483456 & 264.5672 & 18.1037 & 41.7822 & 24.0289 & Gemini North & DS-N & GN-2019B-Q-403 \\
\hline $17382795+8319481$ & 1711261033974731648 & 264.6165 & 83.3301 & 115.6847 & 29.0293 & Gemini North & GMOS-N & GN-2016A-Q-75 \\
\hline 17390078-7342041 & 5803233817494625536 & 264.7534 & -73.7012 & 319.5460 & -21.1023 & Gemini South & GMOS-S & GS-2017A-Q-86 \\
\hline $17390590+1504553$ & 4548685209048009600 & 264.7746 & 15.0820 & 38.8351 & 22.6690 & Gemini North & GMOS-N & GN-2015A-Q-76 \\
\hline 17393096-5334586 & 5921761544627396352 & 264.8790 & -53.5830 & 338.1559 & -11.7617 & Gemini South & S-S & GS-2016A- \\
\hline 17412217-6605479 & 5812858972344007680 & 265.3424 & -66.0966 & 326.8979 & -17.9587 & Gemini South & GMO & GS-2015A-Q-77 \\
\hline $17412978+2128403$ & 4556052314917665920 & 265.3741 & 21.4779 & 45.5304 & 24.5694 & Gemini North & GMOS-N & GN-2016A-Q-75 \\
\hline $17415044+2448137$ & 4581633552441283328 & 265.4602 & 24.8038 & 49.0054 & 25.6500 & Gemini North & GMC & GN-2018A-Q-403 \\
\hline 17421570-5343061 & 5921703652781168768 & 265.5654 & -53.7184 & 338.2431 & -12.1819 & Gemini South & GMOS-S & GS-2015A-Q-77 \\
\hline $17422649+3217551$ & 4601113221991774976 & 265.6104 & 32.2987 & 57.0410 & 27.8215 & Gemini North & GMOS-N & GN-2018A-Q-403 \\
\hline $17425789+0417381$ & 4473307880166709248 & 265.7412 & 4.2939 & 28.8983 & 17.1797 & Gemini North & GMOS-N & GN-2019B-Q-403 \\
\hline $17431049+5633516$ & 1421354113045323904 & 265.7937 & 56.5644 & 84.7158 & 31.6302 & Gemini North & GMOS-N & GN-2019B-Q-403 \\
\hline 17435113-5359333 & 5921684136450078592 & 265.9631 & -53.9926 & 338.1167 & -12.5209 & Gemini South & GMOS-S & GS-2015A-Q-77 \\
\hline $17442011+0827068$ & 4488216467565808512 & 266.0838 & 8.4519 & 32.9700 & 18.7297 & Gemini North & GMOS-N & GN-2018A-Q-403 \\
\hline $17442313+1055585$ & 4489909058337283584 & 266.0964 & 10.9329 & 35.3481 & 19.7861 & Gemini North & GMOS-N & GN-2016A-Q-75 \\
\hline $17451045+1600587$ & 4501194587422903936 & 266.2935 & 16.0163 & 40.3894 & 21.6979 & Gemini North & GMOS-N & GN-2019B-Q-403 \\
\hline $17452465+0613284$ & 4474168965270090368 & 266.3527 & 6.2246 & 30.9912 & 17.5069 & Gemini North & GMOS-N & GN-2018A-Q-403 \\
\hline $17452911+0549214$ & 4474076782390335616 & 266.3713 & 5.8227 & 30.6231 & 17.3105 & Gemini North & GMOS-N & GN-2015A-Q-76 \\
\hline $17460256+1657355$ & 4549397894441550848 & 266.5107 & 16.9599 & 41.4117 & 21.8781 & Gemini North & GMOS-N & GN-2018A-Q-403 \\
\hline $17465957+1519406$ & 4501077351995658496 & 266.7482 & 15.3279 & 39.8990 & 21.0220 & Gemini North & GMOS-N & GN-2016A-Q-75 \\
\hline $17470290-50$ & 5945699978662588416 & 266.7621 & -50.9706 & 341.0550 & -11.4681 & Gemini South & GMOS-S & GS-2016A-Q-76 \\
\hline $17471725-6723320$ & 5812356121877182080 & 266.8219 & -67.3922 & 325.9405 & -19.0520 & Gemini South & GMOS-S & GS-2016A-Q-76 \\
\hline
\end{tabular}


Table 1 (continued)

\begin{tabular}{|c|c|c|c|c|c|c|c|c|}
\hline $\begin{array}{c}\text { Star Name } \\
\text { (2MASS) }\end{array}$ & $\begin{array}{c}\text { Star Name } \\
\text { (Gaia EDR3) }\end{array}$ & $\begin{array}{l}\text { R.A. } \\
\text { (deg) }\end{array}$ & $\begin{array}{l}\text { Decl. } \\
\text { (deg) }\end{array}$ & $\begin{array}{c}l \\
(\operatorname{deg})\end{array}$ & $\begin{array}{c}b \\
(\mathrm{deg})\end{array}$ & Telescope & Instrument & Program ID \\
\hline 17471835-0035363 & 4371256532336413056 & 266.8265 & -0.5935 & 24.9271 & 13.9498 & Gemini South & GMOS-S & GS-2016A-Q-76 \\
\hline $17472373+5750230$ & 1422587593292960640 & 266.8489 & 57.8397 & 86.2346 & 31.1099 & Gemini North & GMOS-N & GN-2016A-Q-75 \\
\hline $17473698+5229473$ & 1368840406910596992 & 266.9042 & 52.4965 & 80.0233 & 30.7093 & Gemini North & GMOS-N & GN-2016A-Q-75 \\
\hline 17485598-7415094 & 5803007691756413056 & 267.2333 & -74.2526 & 319.2992 & -21.9506 & Gemini South & GMOS-S & GS-2015A-Q-77 \\
\hline $17493192-5314272$ & 5921036111776622592 & 267.3830 & -53.2409 & 339.2080 & -12.8965 & Gemini South & GMOS-S & GS-2017A-Q-86 \\
\hline $17505960+1845110$ & 4551354475384137600 & 267.7484 & 18.7531 & 43.6819 & 21.4870 & Gemini North & GMOS-N & GN-2018A-Q-403 \\
\hline $17512033+4313226$ & 1346347658884884608 & 267.8345 & 43.2230 & 69.5685 & 28.7120 & Gemini North & GMOS-N & GN-2016A-Q-75 \\
\hline $17513368+0735474$ & 4475867611955393280 & 267.8903 & 7.5965 & 32.9860 & 16.7468 & Gemini North & GMOS-N & GN-2018A-Q-403 \\
\hline $17531820-5828173$ & 5918003040226875648 & 268.3258 & -58.4715 & 334.6678 & -15.7762 & Gemini South & GMOS-S & GS-2016A-Q-76 \\
\hline $17553087+1344549$ & 4499700449901916672 & 268.8786 & 13.7486 & 39.2587 & 18.4954 & Gemini North & GMOS-N & GN-2016A-Q-75 \\
\hline $17563856-6259025$ & 5911002106095737472 & 269.1607 & -62.9840 & 330.5943 & -18.0888 & Gemini South & GMOS-S & GS-2016A-Q-76 \\
\hline $17570985+6104567$ & 1435424254107968896 & 269.2910 & 61.0824 & 90.0569 & 30.0062 & Gemini North & GMOS-N & GN-2016A-Q-75 \\
\hline $17580876-5017025$ & 5947067839841987840 & 269.5365 & -50.2840 & 342.5270 & -12.6917 & Gemini South & GMOS-S & GS-2016A-Q-76 \\
\hline $17582442-5200036$ & 5945364387093681024 & 269.6018 & -52.0010 & 340.9784 & -13.5132 & Gemini South & GMOS-S & GS-2015A-Q-77 \\
\hline $17583367+0851134$ & 4476514330950969216 & 269.6403 & 8.8537 & 34.9532 & 15.7402 & Gemini North & GMOS-N & GN-2018A-Q-403 \\
\hline $17590214+0226114$ & 4468887736043966848 & 269.7589 & 2.4365 & 29.0855 & 12.7636 & Gemini North & GMOS-N & GN-2017B-Q-75 \\
\hline $17593067+0547081$ & 4474462912835838336 & 269.8779 & 5.7855 & 32.2112 & 14.1744 & Gemini North & GMOS-N & GN-2015A-Q-76 \\
\hline $18025682-7550189$ & 6414540257944747008 & 270.7368 & -75.8386 & 318.0342 & -23.3533 & Gemini South & GMOS-S & GS-2015A-Q-77 \\
\hline $18033495+4211510$ & 2113756098755468288 & 270.8956 & 42.1975 & 68.9946 & 26.2925 & Gemini North & GMOS-N & GN-2016A-Q-75 \\
\hline $18042621-7447453$ & 6417661629719563264 & 271.1092 & -74.7960 & 319.1422 & -23.1096 & Gemini South & GMOS-S & GS-2016A-Q-76 \\
\hline $18042842-7051331$ & 6431507302910109952 & 271.1185 & -70.8592 & 323.1601 & -21.8016 & Gemini South & GMOS-S & GS-2017A-Q-86 \\
\hline $18045298-5543550$ & 6652206220339855104 & 271.2208 & -55.7319 & 337.9426 & -16.0058 & Gemini South & GMOS-S & GS-2017A-Q-86 \\
\hline $18045953+0826177$ & 4476206639494994944 & 271.2481 & 8.4383 & 35.2851 & 14.1289 & Gemini North & GMOS-N & GN-2016A-Q-75 \\
\hline $18052892+0754210$ & 4475421897432191616 & 271.3705 & 7.9058 & 34.8468 & 13.7863 & Gemini North & GMOS-N & GN-2015B-Q-86 \\
\hline $18062441-6457049$ & 6629637992566177664 & 271.6026 & -64.9518 & 329.1292 & -19.8488 & Gemini South & GMOS-S & GS-2016A-Q-76 \\
\hline $18063457+0547198$ & 4471868065390279680 & 271.6441 & 5.7888 & 33.0216 & 12.6039 & Gemini North & GMOS-N & GN-2016A-Q-75 \\
\hline $18072175+5952412$ & 2158636960809774336 & 271.8407 & 59.8781 & 88.7772 & 28.6706 & Gemini North & GMOS-N & GN-2017B-Q-75 \\
\hline $18073369+5849547$ & 2152484604840257280 & 271.8905 & 58.8319 & 87.5943 & 28.5423 & Gemini North & GMOS-N & GN-2016B-Q-77 \\
\hline $18082002-5104378$ & 6702907209758894848 & 272.0835 & -51.0772 & 342.5353 & -14.4897 & Gemini South & GMOS-S & GS-2016B-Q-80 \\
\hline $18083624-6750220$ & 6435985682492232320 & 272.1511 & -67.8395 & 326.3388 & -21.0953 & Gemini South & GMOS-S & GS-2015A-Q-77 \\
\hline $18085682-2106299$ & 4094063947258804608 & 272.2368 & -21.1083 & 9.4190 & -0.6395 & Gemini South & GMOS-S & GS-2014A-Q-8 \\
\hline $18095421-6630506$ & 6437097494904121600 & 272.4759 & -66.5141 & 327.7151 & -20.7494 & Gemini South & GMOS-S & GS-2016A-Q-76 \\
\hline
\end{tabular}


Table 1 (continued)

\begin{tabular}{|c|c|c|c|c|c|c|c|c|}
\hline $\begin{array}{l}\text { Star Name } \\
\text { (2MASS) }\end{array}$ & $\begin{array}{c}\text { Star Name } \\
\text { (Gaia EDR3) }\end{array}$ & $\begin{array}{l}\text { R.A. } \\
\text { (deg) }\end{array}$ & $\begin{array}{l}\text { Decl. } \\
\text { (deg) }\end{array}$ & $\begin{array}{c}l \\
(\operatorname{deg})\end{array}$ & $\begin{array}{c}b \\
(\operatorname{deg})\end{array}$ & Telescope & Instrument & Program ID \\
\hline $18100815-6731119$ & 6436045915114732672 & 272.5340 & -67.5200 & 326.7140 & -21.1210 & Gemini South & GMOS-S & GS-2017A-Q-86 \\
\hline $18102490-7119083$ & 6431254307857238784 & 272.6037 & -71.3190 & 322.8651 & -22.4086 & Gemini South & GMOS-S & GS-2016A-Q-76 \\
\hline $18123519-6033510$ & 6635102251817906304 & 273.1465 & -60.5648 & 333.7220 & -18.8644 & Gemini South & GMOS-S & GS-2015A-Q-77 \\
\hline $18124977+5854462$ & 2152380494834185984 & 273.2075 & 58.9129 & 87.7729 & 27.8750 & Gemini North & GMOS-N & GN-2016B-Q-77 \\
\hline $18141754+1612114$ & 4498809506188122624 & 273.5731 & 16.2032 & 43.5513 & 15.3760 & Gemini North & GMOS-N & GN-2016A-Q-75 \\
\hline $18145902+5852559$ & 2152342188021376384 & 273.7459 & 58.8822 & 87.7782 & 27.5953 & Gemini North & GMOS-N & GN-2018A-Q-403 \\
\hline $18150365-3751206$ & 6727766450449365248 & 273.7652 & -37.8557 & 355.2089 & -9.7156 & Gemini South & GMOS-S & GS-2014A-Q-74 \\
\hline $18162072-4725281$ & 6707603223921252608 & 274.0863 & -47.4245 & 346.5204 & -14.1192 & Gemini South & GMOS-S & GS-2015A-Q-77 \\
\hline 18191919-2029254 & 4091364130814956032 & 274.8300 & -20.4904 & 11.1207 & -2.4740 & Gemini South & GMOS-S & GS-2014A-Q-8 \\
\hline $18192007-5552473$ & 6649365494611393920 & 274.8337 & -55.8798 & 338.6485 & -17.9261 & Gemini South & GMOS-S & GS-2016A-Q-76 \\
\hline $18193127-3713134$ & 6728130968571404800 & 274.8804 & -37.2204 & 356.1883 & -10.2206 & Gemini South & GMOS-S & GS-2014A-Q-74 \\
\hline $18194826-5310564$ & 6653631805885843200 & 274.9511 & -53.1823 & 341.2882 & -16.9569 & Gemini South & GMOS-S & GS-2016A-Q-76 \\
\hline 18203066-2016012 & 4091455699522188416 & 275.1278 & -20.2670 & 11.4494 & -2.6152 & Gemini South & GMOS-S & GS-2014A-Q-8 \\
\hline $18204921-3419480$ & 4044557100560972800 & 275.2051 & -34.3300 & 358.9308 & -9.1641 & Gemini South & GMOS-S & GS-2014A-Q-74 \\
\hline $18211549+5653298$ & 2150956936513301632 & 275.3146 & 56.8916 & 85.7084 & 26.4733 & Gemini North & GMOS-N & GN-2016A-Q-75 \\
\hline $18220774-7608090$ & 6413638383531665664 & 275.5322 & -76.1359 & 318.0782 & -24.5509 & Gemini South & GMOS-S & GS-2015A-Q-77 \\
\hline $18223327-5258169$ & 6653722004497397120 & 275.6386 & -52.9714 & 341.6581 & -17.2570 & Gemini South & GMOS-S & GS-2016A-Q-76 \\
\hline $18301354-4555101$ & 6708532208165979392 & 277.5564 & -45.9195 & 348.9436 & -15.6845 & Gemini South & GMOS-S & GS-2015A-Q-92 \\
\hline $18302241-3955233$ & 6723716880376076928 & 277.5934 & -39.9232 & 354.6252 & -13.2964 & Gemini South & GMOS-S & GS-2017A-Q-86 \\
\hline $18305087-6953306$ & 6431994249123338240 & 277.7120 & -69.8919 & 324.8775 & -23.6243 & Gemini South & GMOS-S & GS-2017A-Q-86 \\
\hline $18311217+4103160$ & 2110452925308798080 & 277.8007 & 41.0545 & 69.3521 & 21.0178 & Gemini North & GMOS-N & GN-2017B-Q-75 \\
\hline 18315993-6920161 & 6432068225640386432 & 277.9997 & -69.3378 & 325.4903 & -23.5812 & Gemini South & GMOS-S & GS-2015A-Q-77 \\
\hline $18321423-3829407$ & 6726896461847358848 & 278.0593 & -38.4946 & 356.1143 & -13.0343 & Gemini South & GMOS-S & GS-2014A-Q-8 \\
\hline $18324025-6202554$ & 6631161743881174400 & 278.1677 & -62.0488 & 333.1007 & -21.6336 & Gemini South & GMOS-S & GS-2017A-Q-86 \\
\hline $18334251+4115292$ & 2110471101610527616 & 278.4272 & 41.2581 & 69.7179 & 20.6345 & Gemini North & GMOS-N & GN-2016A-Q-75 \\
\hline $18340800-5627514$ & 6649667246135478144 & 278.5334 & -56.4643 & 338.8379 & -20.0593 & Gemini South & GMOS-S & GS-2015A-Q-92 \\
\hline $18352484-3639135$ & 6733341416736455936 & 278.8535 & -36.6538 & 358.1010 & -12.8460 & Gemini South & GMOS-S & GS-2015A-Q-77 \\
\hline $18352710-4928306$ & 6703851728906337152 & 278.8629 & -49.4752 & 345.8479 & -17.8363 & Gemini South & GMOS-S & GS-2017A-Q-86 \\
\hline $18354018-3948416$ & 6723580609650147712 & 278.9174 & -39.8116 & 355.1563 & -14.1789 & Gemini South & GMOS-S & GS-2015A-Q-92 \\
\hline $18360814+1919587$ & 4524742759245230592 & 279.0339 & 19.3330 & 48.7042 & 11.9459 & Gemini North & GMOS-N & GN-2016A-Q-75 \\
\hline 18361214-7333443 & 6418205892271978624 & 279.0506 & -73.5623 & 321.0645 & -24.8735 & Gemini South & GMOS-S & GS-2015A-Q-92 \\
\hline 18371329-3141091 & 4046640705089371136 & 279.3054 & -31.6859 & 2.8720 & -11.1024 & Gemini South & GMOS-S & GS-2014A-Q-74 \\
\hline
\end{tabular}


Table 1 (continued)

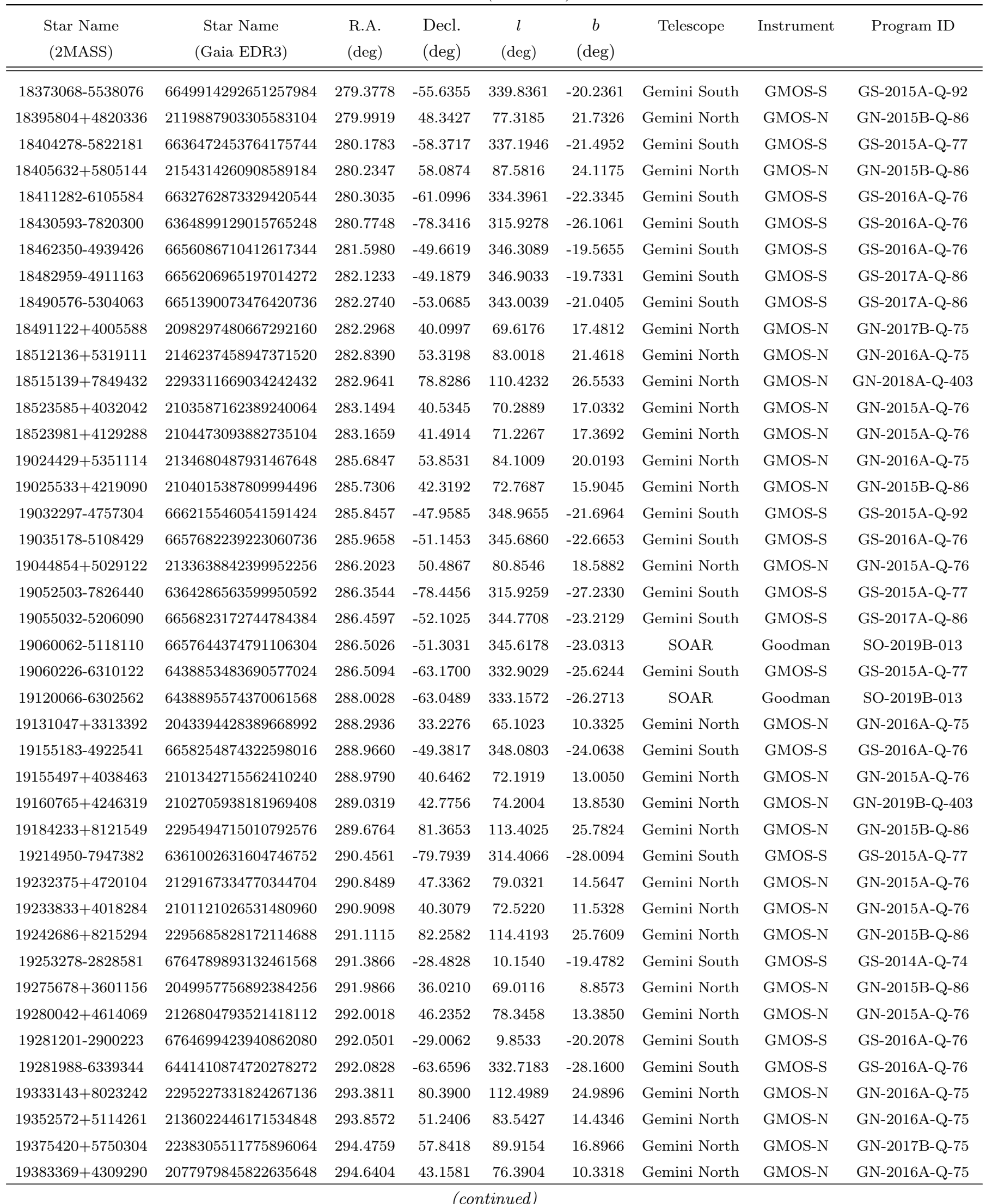


Table 1 (continued)

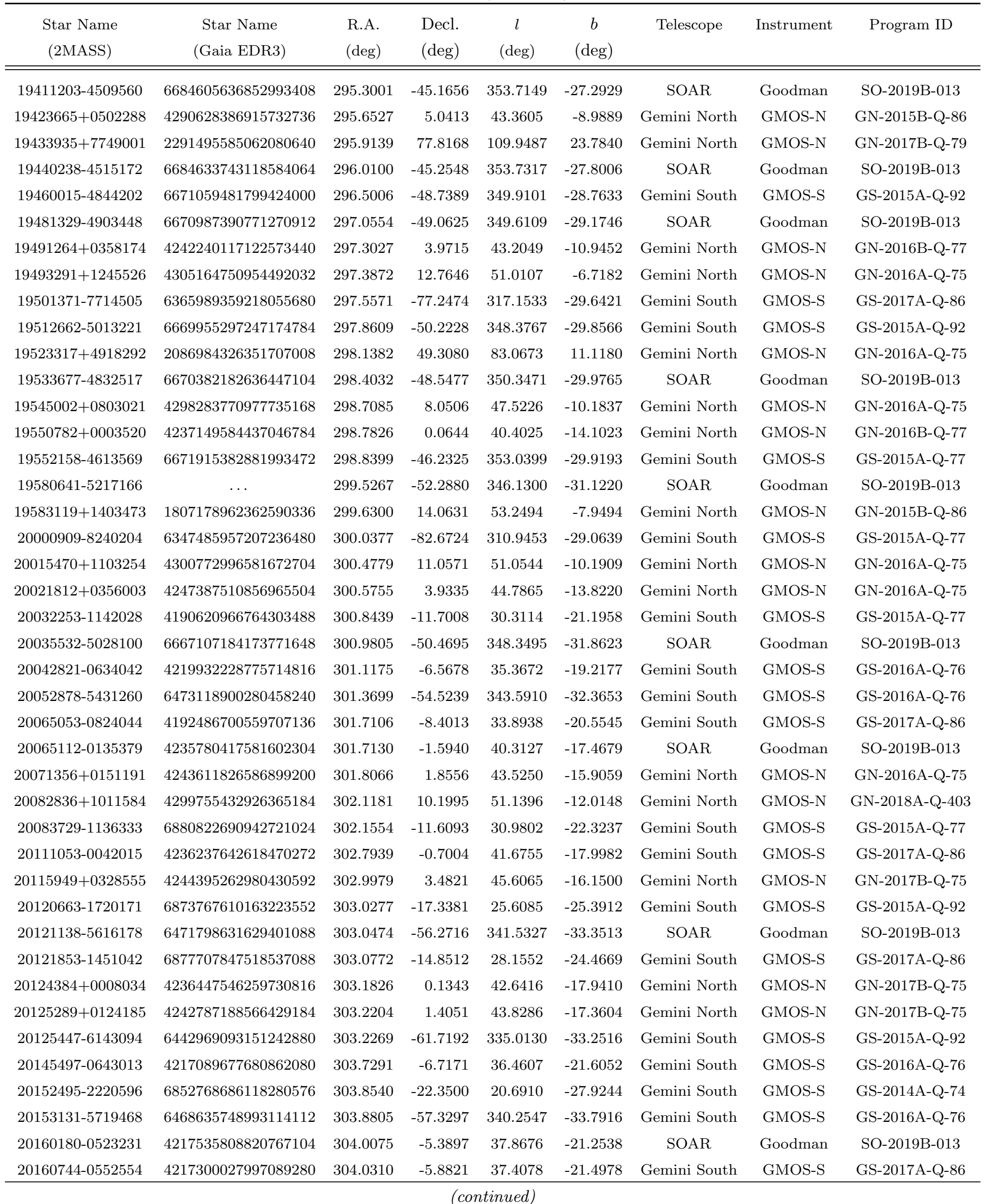


Table 1 (continued)

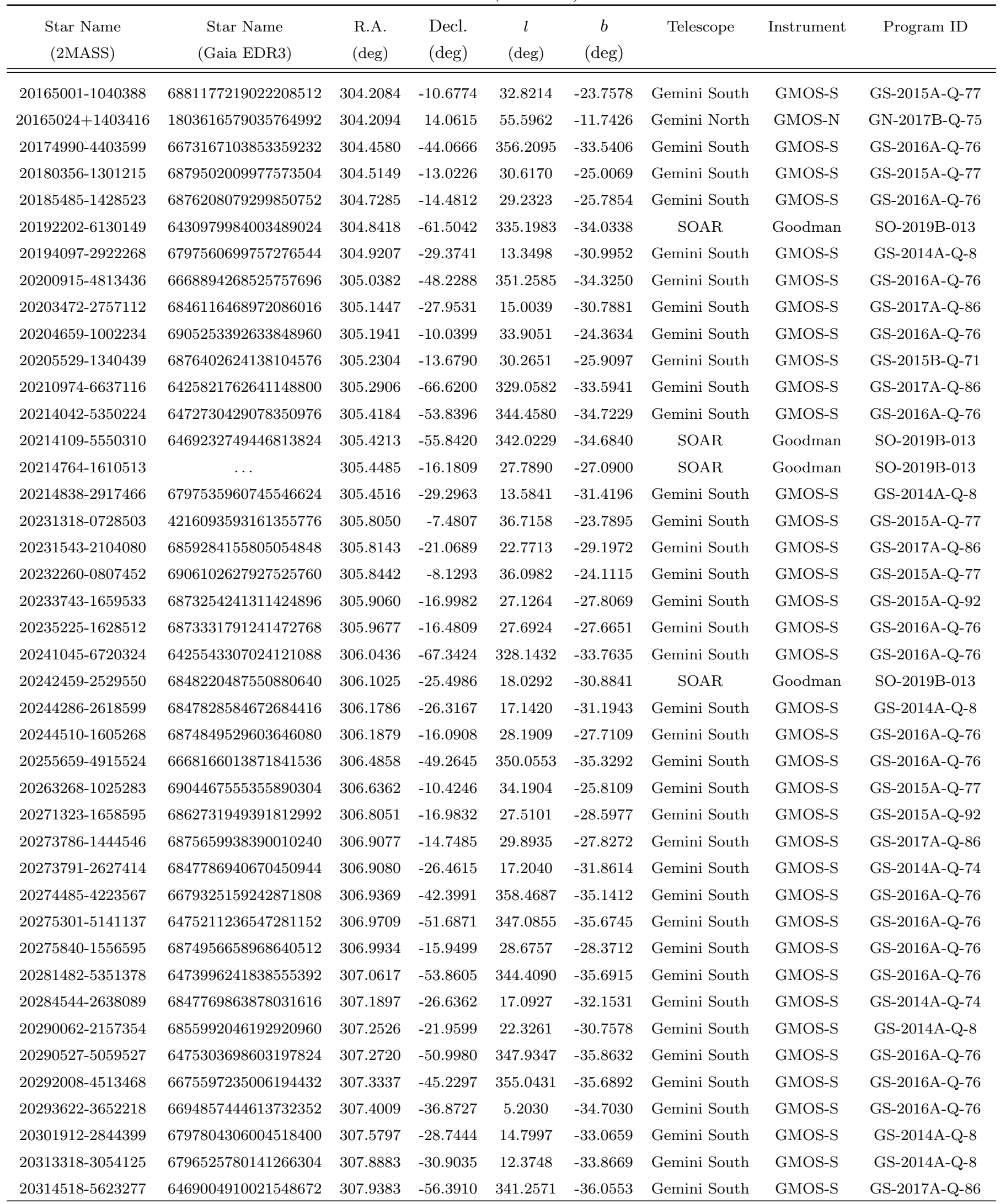


Table 1 (continued)

\begin{tabular}{|c|c|c|c|c|c|c|c|c|}
\hline $\begin{array}{c}\text { Star Name } \\
\text { (2MASS) }\end{array}$ & $\begin{array}{c}\text { Star Name } \\
\text { (Gaia EDR3) }\end{array}$ & $\begin{array}{l}\text { R.A. } \\
\text { (deg) }\end{array}$ & $\begin{array}{l}\text { Decl. } \\
\text { (deg) }\end{array}$ & $\begin{array}{c}l \\
(\operatorname{deg})\end{array}$ & $\begin{array}{c}b \\
(\mathrm{deg})\end{array}$ & Telescope & Instrument & Program ID \\
\hline $20331212-4200270$ & 6679615950005226240 & 308.3006 & -42.0075 & 359.0814 & -36.1034 & SOAR & Goodman & SO-2019B-013 \\
\hline $20331607-5047126$ & 6475629909958997376 & 308.3170 & -50.7869 & 348.1927 & -36.5233 & Gemini South & GMOS-S & GS-2016A-Q-76 \\
\hline $20351407-4625056$ & 6674758921813508736 & 308.8087 & -46.4183 & 353.6442 & -36.7828 & Gemini South & GMOS-S & GS-2016A-Q-76 \\
\hline $20354257-0714557$ & 6907591641550947072 & 308.9274 & -7.2488 & 38.4748 & -26.4555 & Gemini South & GMOS-S & GS-2015A-Q-77 \\
\hline 20375779-2518252 & 6800352497219577728 & 309.4909 & -25.3070 & 19.3213 & -33.7499 & Gemini South & GMOS-S & GS-2014A-Q-74 \\
\hline $20381947-2750475$ & 6798666258699212672 & 309.5811 & -27.8466 & 16.3996 & -34.5301 & Gemini South & GMOS-S & GS-2014A-Q-8 \\
\hline $20385050-2657542$ & 6799133551141107712 & 309.7104 & -26.9651 & 17.4671 & -34.4053 & Gemini South & GMOS-S & GS-2015A-Q-77 \\
\hline 20404939-5843259 & 6455696764877344896 & 310.2058 & -58.7239 & 338.2068 & -37.0110 & SOAR & Goodman & SO-2019B-013 \\
\hline 20440973-2940093 & 6795239665071836160 & 311.0406 & -29.6693 & 14.6231 & -36.2216 & Gemini South & GMOS-S & GS-2017A-Q-86 \\
\hline $20442721-7405038$ & 6369772538209241216 & 311.1133 & -74.0844 & 319.7990 & -33.6442 & Gemini South & GMOS-S & GS-2016A-Q-76 \\
\hline 20443065-2936534 & 6795263648167892992 & 311.1278 & -29.6148 & 14.7107 & -36.2828 & Gemini South & GMOS-S & GS-2014A-Q-8 \\
\hline 20445501-3732403 & 6778221802053181824 & 311.2292 & -37.5445 & 4.9575 & -37.8187 & Gemini South & GMOS-S & GS-2017A-Q-86 \\
\hline 20451053-2934207 & 6795077693263710080 & 311.2939 & -29.5724 & 14.8036 & -36.4133 & Gemini South & GMOS-S & GS-2015A-Q-77 \\
\hline $20460102+1506250$ & 1762690140608564480 & 311.5043 & 15.1069 & 60.5339 & -17.0411 & Gemini North & GMOS-N & GN-2018A-Q-403 \\
\hline 20461039-3940468 & 6678691295087392640 & 311.5433 & -39.6797 & 2.2997 & -38.3093 & Gemini South & GMOS-S & GS-2017A-Q-86 \\
\hline 20461191-3833114 & 6681901765961577344 & 311.5496 & -38.5532 & 3.7265 & -38.1932 & Gemini South & GMOS-S & GS-2014A-Q-8 \\
\hline $20465470-3948150$ & 6678675249089470464 & 311.7280 & -39.8042 & 2.1594 & -38.4628 & Gemini South & GMOS-S & GS-2015A-Q-77 \\
\hline $20504877+1007551$ & 1751382561168000256 & 312.7031 & 10.1320 & 56.9193 & -20.8407 & Gemini North & GMOS-N & GN-2016A-Q-75 \\
\hline $20512785-4843325$ & 6481344866460154880 & 312.8661 & -48.7257 & 350.7058 & -39.5168 & Gemini South & GMOS-S & GS-2016A-Q-76 \\
\hline $20523629-5241033$ & 6477303675894157824 & 313.1512 & -52.6843 & 345.5746 & -39.4037 & Gemini South & GMOS-S & GS-2017A-Q-86 \\
\hline $20530472-3836380$ & 6774883405575766400 & 313.2697 & -38.6106 & 3.8368 & -39.5364 & SOAR & Goodman & SO-2019B-013 \\
\hline 20531334-4520139 & 6484187516335125760 & 313.3056 & -45.3372 & 355.1009 & -39.8911 & Gemini South & GMOS-S & GS-2015A-Q-92 \\
\hline $20531555+1147415$ & 1757847139781935616 & 313.3148 & 11.7949 & 58.7453 & -20.3878 & Gemini North & GMOS-N & GN-2017B-Q-75 \\
\hline $20541462-4811494$ & 6481473440600905472 & 313.5609 & -48.1971 & 351.3660 & -40.0007 & Gemini South & GMOS-S & GS-2017A-Q-86 \\
\hline 20545308-4710289 & 6481731516596154368 & 313.7212 & -47.1747 & 352.6956 & -40.1483 & Gemini South & GMOS-S & GS-2015A-Q-92 \\
\hline 20555702-3912091 & 6774608463246378880 & 313.9875 & -39.2025 & 3.1341 & -40.1459 & Gemini South & GMOS-S & GS-2015A-Q-77 \\
\hline $20565365-5609461$ & 6457897089506392064 & 314.2236 & -56.1629 & 340.9995 & -39.5451 & Gemini South & GMOS-S & GS-2015A-Q-92 \\
\hline $20571292-4958553$ & 6478163463924318208 & 314.3038 & -49.9820 & 348.9937 & -40.3727 & Gemini South & GMOS-S & GS-2017A-Q-86 \\
\hline 20574855-4154444 & 6677344182887180800 & 314.4524 & -41.9122 & 359.6092 & -40.6592 & Gemini South & GMOS-S & GS-2017A-Q-86 \\
\hline $20575772-5637258$ & 6457695677015968384 & 314.4905 & -56.6239 & 340.3770 & -39.6099 & Gemini South & GMOS-S & GS-2015A-Q-92 \\
\hline $20580267+1427040$ & 1761667216837966848 & 314.5112 & 14.4511 & 61.7658 & -19.7828 & Gemini North & GMOS-N & GN-2016A-Q-75 \\
\hline 20585673-4013142 & 6773745346616608000 & 314.7365 & -40.2206 & 1.8524 & -40.7926 & Gemini South & GMOS-S & GS-2015A-Q-77 \\
\hline
\end{tabular}


Table 1 (continued)

\begin{tabular}{|c|c|c|c|c|c|c|c|c|}
\hline $\begin{array}{c}\text { Star Name } \\
\text { (2MASS) }\end{array}$ & $\begin{array}{c}\text { Star Name } \\
\text { (Gaia EDR3) }\end{array}$ & $\begin{array}{l}\text { R.A. } \\
(\operatorname{deg})\end{array}$ & $\begin{array}{l}\text { Decl. } \\
\text { (deg) }\end{array}$ & $\begin{array}{c}l \\
(\operatorname{deg})\end{array}$ & $\begin{array}{c}b \\
(\operatorname{deg})\end{array}$ & Telescope & Instrument & Program ID \\
\hline $20590249+1847021$ & 1765354016765575680 & 314.7604 & 18.7839 & 65.5668 & -17.3639 & Gemini North & GMOS-N & GN-2015B-Q-86 \\
\hline 20592599-4724182 & 6483013375355411200 & 314.8583 & -47.4051 & 352.3504 & -40.9093 & Gemini South & GMOS-S & GS-2017A-Q-86 \\
\hline 20595162-4937337 & 6478288460356702464 & 314.9651 & -49.6260 & 349.4139 & -40.8279 & Gemini South & GMOS-S & GS-2017A-Q-86 \\
\hline 21011036-4537322 & 6483420263376508032 & 315.2932 & -45.6256 & 354.6937 & -41.2803 & Gemini South & GMOS-S & GS-2016A-Q-76 \\
\hline 21011096-4148546 & 6581257453716372352 & 315.2957 & -41.8152 & 359.7650 & -41.2839 & Gemini South & GMOS-S & GS-2016A-Q-76 \\
\hline 21014399-4913588 & 6478323339786839168 & 315.4333 & -49.2331 & 349.8978 & -41.1650 & Gemini South & GMOS-S & GS-2016A-Q-76 \\
\hline $21032352-4211400$ & 6581180590980731520 & 315.8480 & -42.1945 & 359.2713 & -41.7026 & Gemini South & GMOS-S & GS-2015A-Q-77 \\
\hline 21032466-7254213 & 6370267975574142592 & 315.8528 & -72.9059 & 320.5214 & -35.3307 & SOAR & Goodman & SO-2019B-013 \\
\hline 21032961-7321324 & 6370238112664426624 & 315.8734 & -73.3590 & 320.0114 & -35.1522 & Gemini South & GMOS-S & GS-2016A-Q-76 \\
\hline 21042794-4710017 & 6480078194704569088 & 316.1165 & -47.1671 & 352.6012 & -41.7751 & Gemini South & GMOS-S & GS-2017A-Q-86 \\
\hline 21051884-6831537 & 6376679571472808064 & 316.3285 & -68.5316 & 325.4555 & -37.1555 & Gemini South & GMOS-S & GS-2016A-Q-76 \\
\hline $21054066-4520565$ & 6483621714522704128 & 316.4194 & -45.3491 & 355.0269 & -42.0790 & Gemini South & GMOS-S & GS-2016A-Q-76 \\
\hline $21080234+1835409$ & 1788340995265967488 & 317.0097 & 18.5947 & 66.7980 & -19.1553 & Gemini North & GMOS-N & GN-2016A-Q-75 \\
\hline 21091442-4721520 & 6480023214826621696 & 317.3101 & -47.3645 & 352.2549 & -42.5683 & Gemini South & GMOS-S & GS-2016A-Q-76 \\
\hline 21092218-4250491 & 6580163542725955840 & 317.3424 & -42.8470 & 358.3936 & -42.8059 & Gemini South & GMOS-S & GS-2016A-Q-76 \\
\hline 21094841-5600060 & 6463024322681098368 & 317.4517 & -56.0017 & 340.7479 & -41.3433 & SOAR & Goodman & SO-2019B-013 \\
\hline $21095801+1725439$ & 1788003032879354752 & 317.4917 & 17.4289 & 66.1366 & -20.2422 & Gemini North & MOS-N & GN-2015B-Q-86 \\
\hline $21102133+3016061$ & 1852687405024593024 & 317.5888 & 30.2683 & 76.4046 & -12.0093 & Gemini North & S-N & GN-2015B-Q-86 \\
\hline $21105535+2140380$ & 1790165875330692352 & 317.7299 & 21.6772 & 69.7624 & -17.7230 & Gemini North & MOS-N & GN-2016B-Q-77 \\
\hline 21110533-4239222 & 6580263048527430400 & 317.7722 & -42.6562 & 358.6489 & -43.1238 & Gemini South & $-\mathrm{S}$ & GS-20 \\
\hline 21111175-4126536 & 6581456534040000128 & 317.7990 & -41.4482 & 0.3041 & -43.1482 & Gemini South & GMOS-S & GS-2015A-Q-77 \\
\hline 21114008-5138220 & 6476892256683323008 & 317.9170 & -51.6395 & 346.4333 & -42.4391 & SOAR & Goo & 013 \\
\hline $21115127-5257071$ & 6464688708407259008 & 317.9636 & -52.9520 & 344.6766 & -42.2436 & Gemini South & GMOS-S & GS-2016A-Q-76 \\
\hline $21120163+2520001$ & 1841468911788506112 & 318.0068 & 25.3334 & 72.8379 & -15.5436 & Gemini North & GMOS-N & GN-2015B-Q-86 \\
\hline $21125173+2110327$ & 1790077841384173312 & 318.2156 & 21.1758 & 69.6644 & -18.3915 & Gemini North & GMOS-N & GN-2016A-Q-75 \\
\hline 21134390-6802355 & 6399985335331112064 & 318.4330 & -68.0432 & 325.6540 & -38.0609 & Gemini South & GMOS-S & GS-2016A-Q-76 \\
\hline $21145602+2112242$ & 1791382721170477824 & 318.7334 & 21.2067 & 70.0184 & -18.7400 & Gemini North & GMOS-N & GN-2015B-Q-86 \\
\hline $21150824+2631245$ & 1847680160351457024 & 318.7844 & 26.5235 & 74.2443 & -15.2833 & Gemini North & GMOS-N & GN-2015B-Q-86 \\
\hline 21151790-4333404 & 6579952677010742272 & 318.8246 & -43.5612 & 357.3729 & -43.8671 & Gemini South & GMOS-S & GS-2017A-Q-86 \\
\hline 21154971-6848520 & 6375872461218303488 & 318.9572 & -68.8145 & 324.6596 & -37.9272 & Gemini South & GMOS-S & GS-2017A-Q-86 \\
\hline 21171659-4115323 & 6580773015762728704 & 319.3191 & -41.2590 & 0.5642 & -44.2895 & Gemini South & GMOS-S & GS-2016A-Q-76 \\
\hline $21190273+3318462$ & 1854629726683249024 & 319.7614 & 33.3128 & 79.9704 & -11.3120 & Gemini North & GMOS-N & GN-2016A-Q-75 \\
\hline 21192932-7715553 & 6356252535213950592 & 319.8722 & -77.2654 & 315.2274 & -34.2544 & Gemini South & GMOS-S & GS-2015A-Q-77 \\
\hline 21203573-5321426 & 6463751271665659264 & 320.1488 & -53.3619 & 343.7702 & -43.4450 & SOAR & Goodman & SO-2019B-013 \\
\hline $21211669+2032551$ & 1790500809763786624 & 320.3195 & 20.5487 & 70.5165 & -20.2922 & Gemini North & GMOS-N & GN-2016A-Q-75 \\
\hline $21214670+1916532$ & 1785668323017152384 & 320.4446 & 19.2815 & 69.5759 & -21.2120 & Gemini North & GMOS-N & GN-2017B-Q-79 \\
\hline 21232828-5328287 & 6463713682111820160 & 320.8679 & -53.4746 & 343.4834 & -43.8366 & Gemini South & GMOS-S & GS-2016A-Q-76 \\
\hline $21240060-5241520$ & 6465286018099447680 & 321.0026 & -52.6978 & 344.5084 & -44.0933 & Gemini South & GMOS-S & GS-2016A-Q-76 \\
\hline $21243758-6400120$ & 6403279953204895360 & 321.1566 & -64.0033 & 329.8995 & -40.6628 & Gemini South & GMOS-S & GS-2015A-Q-92 \\
\hline 21254398-6753045 & 6399850542078013568 & 321.4332 & -67.8846 & 325.2363 & -39.1502 & Gemini South & GMOS-S & GS-2015B-Q-71 \\
\hline $21262879+1749436$ & 1784515446419862656 & 321.6200 & 17.8288 & 69.1801 & -23.0057 & Gemini North & GMOS-N & GN-2017B-Q-79 \\
\hline $21263180+2031469$ & 1790358392944627712 & 321.6325 & 20.5297 & 71.3727 & -21.2253 & Gemini North & GMOS-N & GN-2015B-Q-86 \\
\hline
\end{tabular}


Table 1 (continued)

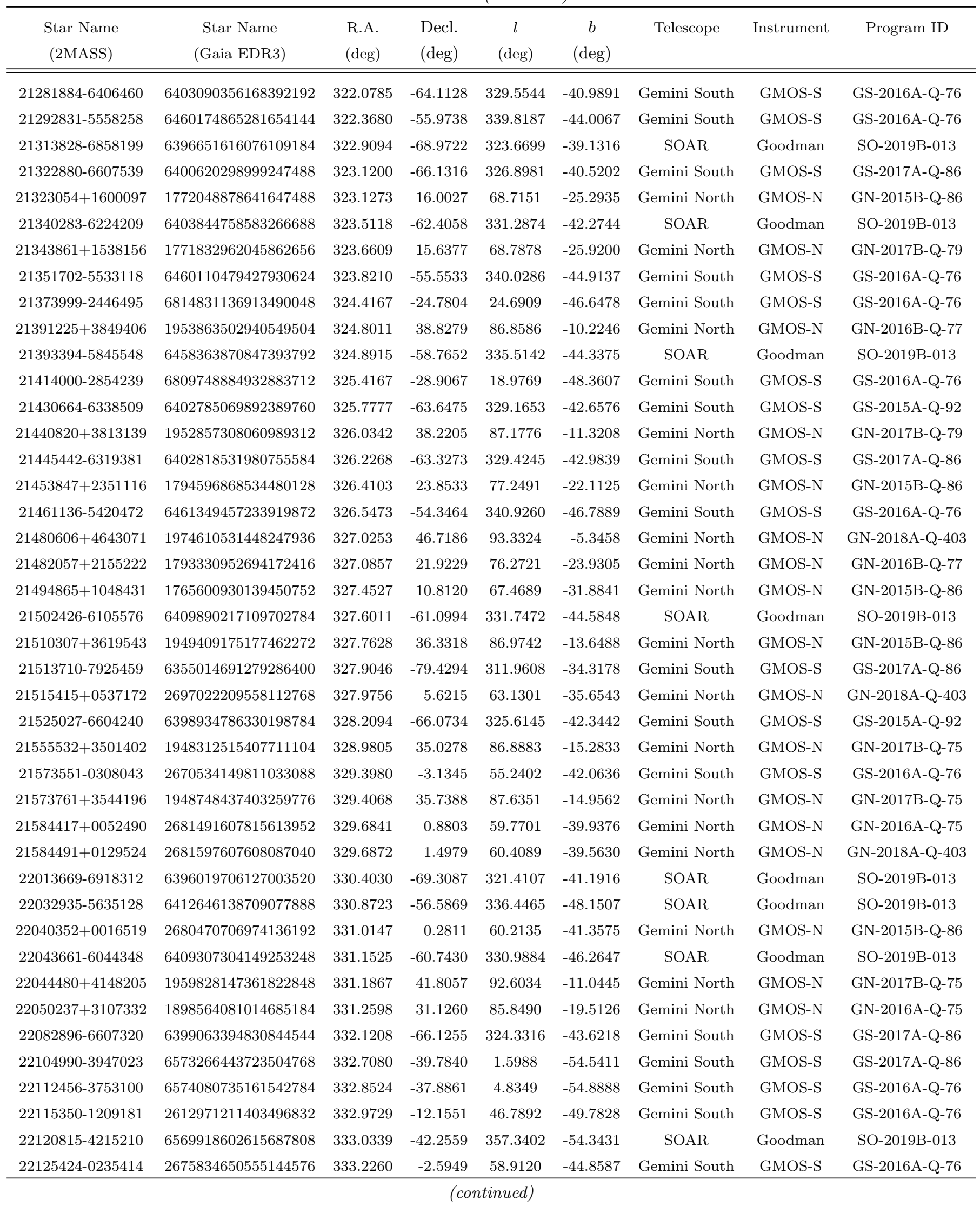


Table 1 (continued)

\begin{tabular}{|c|c|c|c|c|c|c|c|c|}
\hline $\begin{array}{l}\text { Star Name } \\
\text { (2MASS) }\end{array}$ & $\begin{array}{c}\text { Star Name } \\
\text { (Gaia EDR3) }\end{array}$ & $\begin{array}{l}\text { R.A. } \\
\text { (deg) }\end{array}$ & $\begin{array}{l}\text { Decl. } \\
\text { (deg) }\end{array}$ & $\begin{array}{c}l \\
(\mathrm{deg})\end{array}$ & $\begin{array}{c}b \\
(\operatorname{deg})\end{array}$ & Telescope & Instrument & Program ID \\
\hline 22140658-0016065 & 2678634350759886336 & 333.5274 & -0.2685 & 61.7379 & -43.6714 & Gemini South & GMOS-S & GS-2015A-Q-77 \\
\hline $22165631-1154207$ & 2612857755547023232 & 334.2346 & -11.9058 & 48.0461 & -50.7522 & Gemini South & GMOS-S & GS-2015A-Q-77 \\
\hline $22211976-4111021$ & 6569481855982624640 & 335.3324 & -41.1839 & 358.5334 & -56.2484 & Gemini South & GMOS-S & GS-2015A-Q-92 \\
\hline $22221878-7406283$ & 6357952517629300352 & 335.5782 & -74.1079 & 315.2965 & -39.2321 & SOAR & Goodman & SO-2019B-013 \\
\hline $22224538+3555471$ & 1905936405260048384 & 335.6891 & 35.9298 & 92.0012 & -17.8594 & Gemini North & GMOS-N & GN-2017A-Q-82 \\
\hline $22224859+2804264$ & 1881899328730042368 & 335.7025 & 28.0740 & 87.1525 & -24.2839 & Gemini North & GMOS-N & GN-2015B-Q-86 \\
\hline $22225487+2954084$ & 1894651090929156096 & 335.7286 & 29.9024 & 88.3423 & -22.8160 & Gemini North & GMOS-N & GN-2017A-Q-82 \\
\hline $22241984+2430382$ & 1878702846566493440 & 336.0827 & 24.5106 & 85.1026 & -27.3515 & Gemini North & GMOS-N & GN-2015B-Q-86 \\
\hline $22244368+3236401$ & 1901779602770732032 & 336.1820 & 32.6112 & 90.3632 & -20.8244 & Gemini North & GMOS-N & GN-2017B-Q-75 \\
\hline $22251594+2911332$ & 1894379923873649536 & 336.3164 & 29.1926 & 88.3463 & -23.6909 & Gemini North & GMOS-N & GN-2015B-Q-86 \\
\hline $22280812+3546524$ & 1905786459361887488 & 337.0338 & 35.7812 & 92.8667 & -18.5901 & Gemini North & GMOS-N & GN-2016A-Q-75 \\
\hline $22284549-4124119$ & 6593293356532115712 & 337.1895 & -41.4033 & 357.5392 & -57.5526 & Gemini South & GMOS-S & GS-2017A-Q-86 \\
\hline $22290341+3954145$ & 1909092729485051776 & 337.2642 & 39.9040 & 95.3670 & -15.2270 & Gemini North & GMOS-N & GN-2015B-Q-86 \\
\hline $22293323+4332048$ & 1981952760850101376 & 337.3885 & 43.5347 & 97.4382 & -12.2034 & Gemini North & GMOS-N & GN-2018A-Q-403 \\
\hline 22294083-3305402 & 6600574876582217344 & 337.4201 & -33.0945 & 13.1498 & -58.9197 & Gemini South & GMOS-S & GS-2016A-Q-76 \\
\hline 22303946-1809055 & 2594309161890648320 & 337.6645 & -18.1516 & 40.9165 & -56.4245 & Gemini South & GMOS-S & GS-2016A-Q-76 \\
\hline $22355721-2434108$ & 6623904314300239488 & 338.9886 & -24.5701 & 30.0249 & -59.5016 & Gemini South & GMOS-S & GS-2016A-Q-76 \\
\hline $22360602+3930413$ & 1908318669000212480 & 339.0251 & 39.5115 & 96.3488 & -16.2740 & Gemini North & GMOS-N & GN-2016A-Q-75 \\
\hline 22364074-7026524 & 6384994623861077376 & 339.1698 & -70.4480 & 317.5510 & -42.6499 & Gemini South & GMOS-S & GS-2017A-Q-86 \\
\hline 22373316-4341181 & 6520826714109323392 & 339.3882 & -43.6884 & 352.5076 & -58.4074 & Gemini South & GMOS-S & GS-2016A-Q-76 \\
\hline $22401067-3738259$ & 6595625729931613568 & 340.0444 & -37.6406 & 3.7082 & -60.5466 & Gemini South & GMOS-S & GS-2016A-Q-76 \\
\hline $22412632-3627304$ & 6596002145160945664 & 340.3597 & -36.4584 & 5.9980 & -61.0060 & Gemini South & GMOS-S & GS-2016A-Q-76 \\
\hline $22413573+2931038$ & 1887539965117822464 & 340.3989 & 29.5177 & 91.8009 & -25.3921 & Gemini North & GMOS-N & GN-2015B-Q-86 \\
\hline $22432022-7547340$ & 6357547759911189120 & 340.8343 & -75.7927 & 312.5515 & -38.8457 & Gemini South & GMOS-S & GS-2015A-Q-77 \\
\hline $22451263+3532066$ & 1903656636619168768 & 341.3026 & 35.5352 & 95.8658 & -20.6169 & Gemini North & GMOS-N & GN-2016A-Q-75 \\
\hline $22453503+1301210$ & 2731609959149919360 & 341.3960 & 13.0225 & 81.6630 & -39.5584 & Gemini North & GMOS-N & GN-2016A-Q-75 \\
\hline $22454796+2826260$ & 1887186850086385152 & 341.4498 & 28.4406 & 92.0438 & -26.7912 & Gemini North & GMOS-N & GN-2017B-Q-75 \\
\hline $22471965-7201440$ & 6382433144021702656 & 341.8319 & -72.0289 & 315.2876 & -41.9933 & Gemini South & GMOS-S & GS-2015A-Q-77 \\
\hline $22472643+3532411$ & 1903562838830927744 & 341.8601 & 35.5447 & 96.2925 & -20.8328 & Gemini North & GMOS-N & GN-2016A-Q-75 \\
\hline $22473447-5511534$ & 6505905757201226368 & 341.8936 & -55.1982 & 332.9805 & -54.0938 & Gemini South & GMOS-S & GS-2016A-Q-76 \\
\hline $22474545+2517113$ & 1876790894628571264 & 341.9394 & 25.2865 & 90.5938 & -29.7079 & Gemini North & GMOS-N & GN-2017B-Q-75 \\
\hline
\end{tabular}


Table 1 (continued)

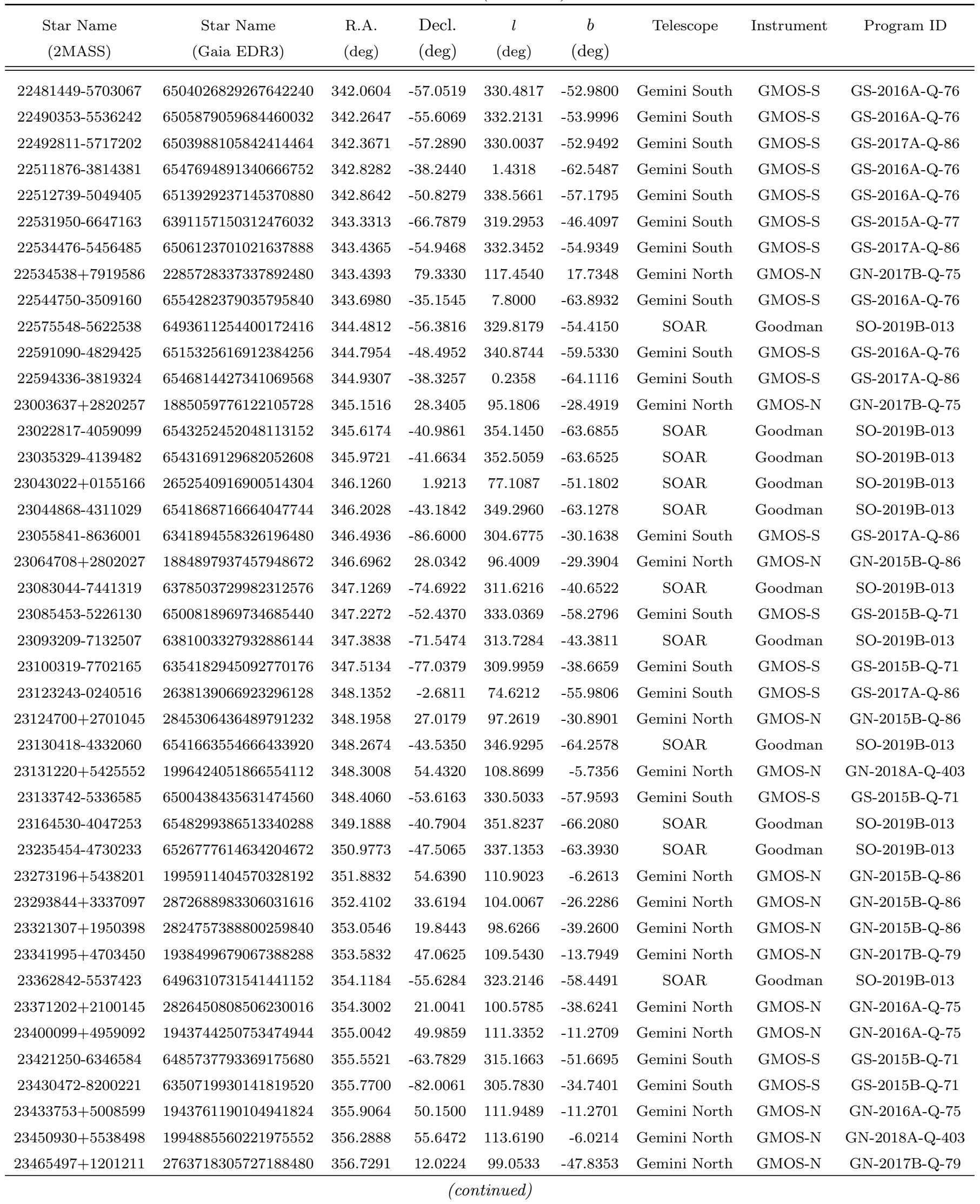


Table 1 (continued)

\begin{tabular}{ccccccccc}
\hline $\begin{array}{c}\text { Star Name } \\
(2 \mathrm{MASS})\end{array}$ & $\begin{array}{c}\text { Star Name } \\
\text { (Gaia EDR3) }\end{array}$ & $\begin{array}{c}\text { R.A. } \\
(\mathrm{deg})\end{array}$ & $\begin{array}{c}\text { Decl. } \\
(\mathrm{deg})\end{array}$ & $\begin{array}{c}l \\
(\mathrm{deg})\end{array}$ & $\begin{array}{c}b \\
(\mathrm{deg})\end{array}$ & Telescope & Instrument & Program ID \\
\hline \hline $23472384+4835469$ & 1940559103007605120 & 356.8494 & 48.5964 & 112.1453 & -12.9295 & Gemini North & GMOS-N & GN-2018A-Q-403 \\
$23505349+1142348$ & 2766637307235175296 & 357.7229 & 11.7097 & 100.2448 & -48.4769 & Gemini North & GMOS-N & GN-2016A-Q-75 \\
$23550005+1255039$ & 2766874664308017408 & 358.7503 & 12.9178 & 102.2681 & -47.6768 & Gemini North & GMOS-N & GN-2017B-Q-79 \\
$23555398+5702462$ & 1998062118035485056 & 358.9749 & 57.0462 & 115.4026 & -5.0114 & Gemini North & GMOS-N & GN-2016A-Q-75 \\
$23560245+1109200$ & 2765621221052354816 & 359.0102 & 11.1555 & 101.7665 & -49.4263 & Gemini North & GMOS-N & GN-2015B-Q-86 \\
$23562635+0651168$ & 2746230337064331776 & 359.1098 & 6.8547 & 99.5568 & -53.5025 & Gemini North & GMOS-N & GN-2017B-Q-79 \\
$23563722+4615436$ & 1927134439687586560 & 359.1551 & 46.2621 & 113.1422 & -15.5654 & Gemini North & GMOS-N & GN-2015B-Q-86 \\
$23564530-4429484$ & 6532542491534291968 & 359.1887 & -44.4968 & 331.6470 & -69.4587 & Gemini South & GMOS-S & GS-2017A-Q-86 \\
\hline
\end{tabular}


Table 2. Colors, Magnitudes and Reddening Estimates

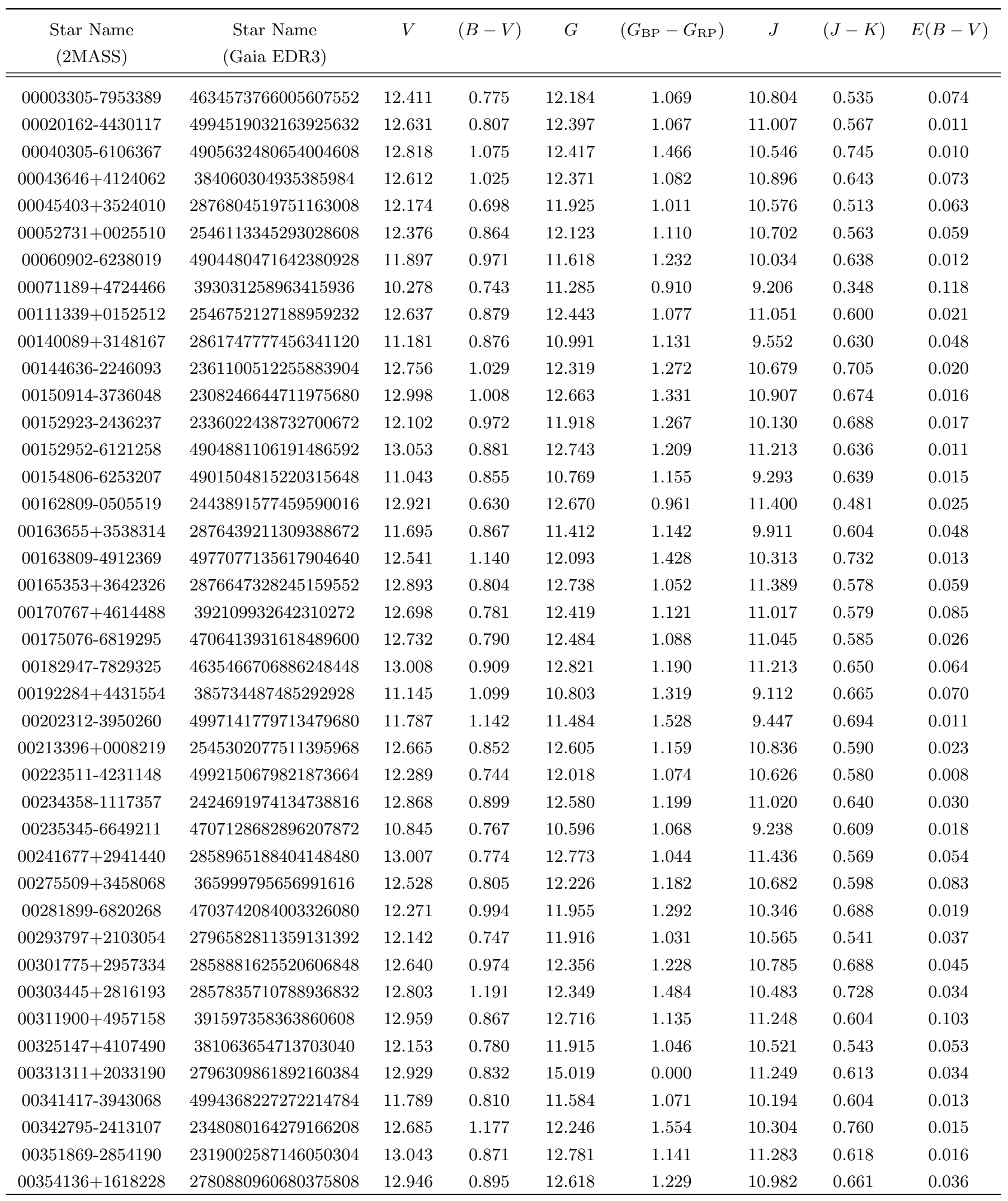


Table 2 (continued)

\begin{tabular}{|c|c|c|c|c|c|c|c|c|}
\hline $\begin{array}{l}\text { Star Name } \\
\text { (2MASS) }\end{array}$ & $\begin{array}{c}\text { Star Name } \\
\text { (Gaia EDR3) }\end{array}$ & $V$ & $(B-V)$ & G & $\left(G_{\mathrm{BP}}-G_{\mathrm{RP}}\right)$ & $J$ & $(J-K)$ & $E(B-V)$ \\
\hline $00370433+4341208$ & 387703815888550656 & 11.746 & 1.152 & 11.393 & 1.434 & 9.607 & 0.721 & 0.056 \\
\hline 00370966-4224102 & 4992914501101243264 & 11.800 & 0.872 & 11.584 & 1.125 & 10.109 & 0.589 & 0.009 \\
\hline 00374809-2751193 & 2343095974991737600 & 12.673 & 1.189 & 12.327 & 1.488 & 10.462 & 0.760 & 0.013 \\
\hline 00400685-4325183 & 4980747129334018944 & 12.363 & 1.092 & 11.923 & 1.430 & 10.179 & 0.720 & 0.007 \\
\hline $00410089+3633397$ & 365840847508048384 & 12.363 & 0.740 & 12.147 & 1.021 & 10.803 & 0.509 & 0.041 \\
\hline $00410467+1649478$ & 2782313903504860032 & 12.866 & 0.909 & 12.328 & 1.342 & 10.522 & 0.702 & 0.040 \\
\hline $00425182+1925361$ & 2795241995584048000 & 12.917 & 0.819 & 12.630 & 1.142 & 11.115 & 0.563 & 0.055 \\
\hline 00433651-2719379 & 2343181981712123520 & 12.806 & 0.989 & 12.490 & 1.252 & 10.896 & 0.634 & 0.008 \\
\hline 00435264-2132285 & 2350389894612060544 & 12.831 & 0.794 & 12.605 & 1.047 & 11.238 & 0.558 & 0.014 \\
\hline 00452879-5846450 & 4906911251332870144 & 10.615 & 0.912 & 10.405 & 1.164 & 8.934 & 0.604 & 0.012 \\
\hline 00463619-3739335 & 5000753194373767424 & 11.929 & 0.804 & 11.685 & 1.117 & 10.279 & 0.631 & 0.010 \\
\hline 00482546-7441092 & 4685477782827620992 & 12.023 & 0.713 & 11.904 & 0.984 & 10.633 & 0.513 & 0.040 \\
\hline 00482715-8224023 & 4629945985987660416 & 12.494 & 1.058 & 12.163 & 1.330 & 10.458 & 0.672 & 0.113 \\
\hline 00504527-6351504 & 4709709305405862656 & 12.528 & 0.996 & 12.128 & 1.344 & 10.444 & 0.641 & 0.019 \\
\hline 00510748-7809118 & 4635149768362820096 & 12.784 & 0.992 & 12.447 & 1.294 & 10.803 & 0.648 & 0.052 \\
\hline 00521010-6006097 & 4903551070783622528 & 11.995 & 1.021 & 11.651 & 1.316 & 10.033 & 0.671 & 0.014 \\
\hline 00522310-5804087 & 4907332914042242304 & 11.960 & 0.783 & 11.670 & 1.079 & 10.324 & 0.663 & 0.015 \\
\hline $00523111+3758286$ & 367656523458156544 & 12.869 & 0.698 & 12.692 & 0.970 & 11.406 & 0.522 & 0.039 \\
\hline $00582707+0633561$ & 2553447805108682496 & 12.512 & 0.895 & 12.251 & 1.149 & 10.743 & 0.603 & 0.051 \\
\hline $00594615+1223173$ & 2584343227151274752 & 12.972 & 0.968 & 12.654 & 1.260 & 11.123 & 0.700 & 0.060 \\
\hline $01021265+0428241$ & 2551971405806340096 & 11.608 & 0.956 & 11.315 & 1.241 & 9.772 & 0.697 & 0.016 \\
\hline $01024809+4300486$ & 376344555103575808 & 11.487 & 1.063 & 11.217 & 1.280 & 9.520 & 0.753 & 0.073 \\
\hline $01031767+0908145$ & 2581189278047060224 & 11.959 & 0.757 & 11.712 & 1.069 & 10.326 & 0.598 & 0.032 \\
\hline 01033338-7410471 & 4684860888081427840 & 12.091 & 0.766 & 11.937 & 1.039 & 10.512 & 0.578 & 0.038 \\
\hline $01040440+0504477$ & 2552119633717667584 & 11.498 & 0.823 & 11.257 & 1.059 & 9.952 & 0.563 & 0.018 \\
\hline $01042513+4011391$ & 371347102956265728 & 12.846 & 0.935 & 12.570 & 1.234 & 11.011 & 0.634 & 0.041 \\
\hline $01042908+0755213$ & 2578034366869468800 & 12.792 & 0.905 & 12.559 & 1.198 & 10.960 & 0.614 & 0.034 \\
\hline 01051545-0041328 & 2533223147709485312 & 12.693 & 1.046 & 12.308 & 1.359 & 10.620 & 0.685 & 0.030 \\
\hline 01065190-5244105 & 4927175937828177280 & 13.642 & 0.805 & 13.364 & 1.130 & 11.908 & 0.619 & 0.011 \\
\hline 01132198-6139522 & 4710458244623085184 & 12.819 & 0.921 & 12.596 & 1.201 & 11.090 & 0.697 & 0.015 \\
\hline 01200289-0158201 & 2533312006288169600 & 11.233 & 0.755 & 10.998 & 1.032 & 9.673 & 0.581 & 0.042 \\
\hline 01250922-5614027 & 4910744423745801472 & 13.799 & 0.900 & 13.550 & 1.159 & 12.063 & 0.642 & 0.022 \\
\hline 01253364-4148345 & 4984027698370924672 & 10.475 & 0.701 & 10.314 & 0.995 & 8.830 & 0.608 & 0.014 \\
\hline 01253802-2911025 & $\ldots$ & 13.971 & 1.476 & $\ldots$ & $\ldots$ & 10.390 & 0.761 & 0.011 \\
\hline 01291742-7139220 & 4687775246726470144 & 12.563 & 0.959 & 12.290 & 1.231 & 10.713 & 0.616 & 0.038 \\
\hline 01311599-4016510 & 5008222486100643200 & 11.110 & 0.802 & 10.862 & 1.088 & 9.526 & 0.668 & 0.012 \\
\hline 01315199-6547540 & 4710799574264331904 & 12.519 & 0.844 & 12.367 & 1.128 & 10.807 & 0.617 & 0.016 \\
\hline 01323787-1530302 & 2451901038631956224 & 12.692 & 0.858 & 12.428 & 1.071 & 11.095 & 0.600 & 0.015 \\
\hline $01363655+5451319$ & 409152813849385216 & 12.990 & 1.059 & 12.764 & 1.401 & 10.813 & 0.711 & 0.226 \\
\hline 01372246-4611110 & 4931138955692103424 & 12.059 & 0.842 & 11.865 & 1.074 & 10.469 & 0.593 & 0.015 \\
\hline 01373378-6921368 & 4691261969896942848 & 12.621 & 0.988 & 12.282 & 1.304 & 10.627 & 0.674 & 0.021 \\
\hline 01382048-7637319 & 4636801853303548416 & 12.701 & 0.663 & 12.490 & 0.959 & 11.233 & 0.516 & 0.044 \\
\hline
\end{tabular}


Table 2 (continued)

\begin{tabular}{|c|c|c|c|c|c|c|c|c|}
\hline $\begin{array}{l}\text { Star Name } \\
\text { (2MASS) }\end{array}$ & $\begin{array}{c}\text { Star Name } \\
\text { (Gaia EDR3) }\end{array}$ & $V$ & $(B-V)$ & $G$ & $\left(G_{\mathrm{BP}}-G_{\mathrm{RP}}\right)$ & $J$ & $(J-K)$ & $E(B-V)$ \\
\hline 01390079-7811248 & 4633446629147784320 & 13.024 & 0.958 & 12.721 & 1.263 & 11.096 & 0.661 & 0.053 \\
\hline 01394297-1615477 & 2452308064092390784 & 12.154 & 0.974 & 11.841 & 1.228 & 10.266 & 0.630 & 0.012 \\
\hline 01430726-6445174 & 4710973567683306624 & 11.980 & 0.867 & 11.725 & 1.150 & 10.241 & 0.635 & 0.019 \\
\hline 01481644-5717024 & 4718885485854328064 & 12.640 & 0.690 & 12.456 & 0.956 & 11.199 & 0.502 & 0.020 \\
\hline 01481836-6022102 & 4717520854484700288 & 12.534 & 0.749 & 12.357 & 1.019 & 11.015 & 0.515 & 0.018 \\
\hline 01512909-5049125 & 4940475493039087872 & 13.842 & 0.906 & 13.558 & 1.191 & 12.013 & 0.657 & 0.014 \\
\hline 01532990-7617103 & 4636731828156259712 & 12.221 & 0.865 & 11.955 & 1.143 & 10.494 & 0.600 & 0.048 \\
\hline $01535484+4743406$ & 357061938652034816 & 12.928 & 0.825 & 12.742 & 1.100 & 11.289 & 0.629 & 0.121 \\
\hline 01541382-4927292 & 4940875062436326784 & 12.483 & 0.786 & 12.247 & 1.062 & 10.837 & 0.564 & 0.018 \\
\hline 01542953-4953166 & 4940665261873824640 & 12.401 & 1.076 & 12.063 & 1.332 & 10.387 & 0.662 & 0.015 \\
\hline 01555066-6400155 & 4699467590815290368 & 12.668 & 0.841 & 12.392 & 1.146 & 10.872 & 0.558 & 0.023 \\
\hline $01555808+5040276$ & 359446465839056512 & 12.130 & 0.915 & 11.790 & 1.217 & 10.324 & 0.705 & 0.234 \\
\hline 01570453-6511318 & 4699066135928244224 & 12.892 & 0.799 & 12.648 & 1.122 & 10.981 & 0.672 & 0.023 \\
\hline 01585657-1624249 & 5141897776909177856 & 11.991 & 0.767 & 11.796 & 0.979 & 10.537 & 0.531 & 0.025 \\
\hline $01592159+8341476$ & 572808964949945088 & 12.687 & 0.895 & 12.435 & 1.199 & 10.912 & 0.671 & 0.153 \\
\hline 02002105-2520170 & 5121763691780187008 & 11.839 & 0.981 & 11.438 & 1.282 & 9.776 & 0.721 & 0.012 \\
\hline $02002992+5657571$ & 505258239019253760 & 12.684 & 0.902 & 12.323 & 1.261 & 10.706 & 0.670 & 0.297 \\
\hline 02013041-0949339 & 2462500536881830528 & 11.970 & 0.822 & 11.727 & 1.063 & 10.326 & 0.558 & 0.019 \\
\hline 02020691-8507254 & 4616783629211669888 & 12.910 & 1.101 & 12.510 & 1.455 & 10.672 & 0.714 & 0.111 \\
\hline $02023240+5535052$ & 504560598882408960 & 12.946 & 0.847 & 15.132 & 1.119 & 11.134 & 0.657 & 0.276 \\
\hline 02062328-0718389 & 2487426224965026688 & 12.886 & 0.915 & 12.585 & 1.186 & 11.139 & 0.667 & 0.018 \\
\hline 02091623-2825011 & 5117222021562768256 & 11.211 & 1.105 & 10.857 & 1.370 & 9.139 & 0.708 & 0.011 \\
\hline 02121057-2136569 & 5124244804192255104 & 12.878 & 0.734 & 12.648 & 1.014 & 11.319 & 0.570 & 0.013 \\
\hline 02131127-3617490 & 4965179285932359552 & 12.942 & 1.016 & 12.663 & 1.327 & 10.940 & 0.665 & 0.014 \\
\hline 02143996-2731561 & 5116644773662434176 & 12.877 & 1.009 & 12.346 & 1.461 & 10.550 & 0.744 & 0.011 \\
\hline $02151298+4941500$ & 355587661060325120 & 12.854 & 0.917 & 12.561 & 1.151 & 11.156 & 0.639 & 0.172 \\
\hline $02165682+4443112$ & 352161380966711936 & 12.583 & 1.104 & 12.224 & 1.410 & 10.485 & 0.711 & 0.072 \\
\hline 02165716-7547064 & 4637170571951777280 & 11.894 & 1.064 & 11.573 & 1.345 & 9.886 & 0.683 & 0.041 \\
\hline 02184236-5351323 & 4743265369493805696 & 12.964 & 0.927 & 12.649 & 1.239 & 11.052 & 0.624 & 0.025 \\
\hline 02184254-6111160 & 4701711045508666112 & 12.167 & 0.751 & 11.847 & 1.118 & 10.376 & 0.570 & 0.044 \\
\hline 02194944-2701309 & 5117093275622914688 & 12.934 & 0.598 & 12.804 & 0.756 & 12.021 & 0.526 & 0.011 \\
\hline 02200131-5909599 & 4738094228868954496 & 11.649 & 1.070 & 11.219 & 1.432 & 9.430 & 0.709 & 0.024 \\
\hline 02200416-3505332 & 4966846038184021888 & 12.908 & 0.834 & 12.674 & 1.080 & 11.276 & 0.573 & 0.014 \\
\hline 02202248-3221349 & 4970735118186763520 & 12.745 & 1.048 & 12.371 & 1.390 & 10.582 & 0.697 & 0.016 \\
\hline 02205873-6708044 & 4696223279895236992 & 11.790 & 0.968 & 11.509 & 1.230 & 9.953 & 0.660 & 0.026 \\
\hline 02215591-1414291 & 5146733910084621440 & 11.685 & 0.872 & 13.681 & 0.000 & 10.033 & 0.581 & 0.017 \\
\hline $02230945+3819551$ & 331739842266523264 & 12.688 & 1.163 & 12.351 & 1.107 & 11.037 & 0.652 & 0.041 \\
\hline 02234760-4630467 & 4940121690813101056 & 12.498 & 0.839 & 12.294 & 1.066 & 10.894 & 0.584 & 0.014 \\
\hline $02242892+7959144$ & 562642266262613504 & 11.413 & 0.832 & 11.268 & 1.080 & 9.838 & 0.554 & 0.161 \\
\hline 02243601-3101131 & 5066948639089274496 & 12.942 & 0.795 & 12.725 & 1.083 & 11.277 & 0.589 & 0.015 \\
\hline 02255030-7822010 & 4632830898340209920 & 12.564 & 0.755 & 12.326 & 1.062 & 10.914 & 0.532 & 0.066 \\
\hline 02260082-2250455 & 5120483443633370496 & 12.773 & 0.947 & 12.499 & 1.233 & 10.844 & 0.640 & 0.016 \\
\hline
\end{tabular}


Table 2 (continued)

\begin{tabular}{|c|c|c|c|c|c|c|c|c|}
\hline $\begin{array}{l}\text { Star Name } \\
\text { (2MASS) }\end{array}$ & $\begin{array}{c}\text { Star Name } \\
\text { (Gaia EDR3) }\end{array}$ & $V$ & $(B-V)$ & $G$ & $\left(G_{\mathrm{BP}}-G_{\mathrm{RP}}\right)$ & $J$ & $(J-K)$ & $E(B-V)$ \\
\hline 02263468-7052018 & 4645240501967006720 & 13.722 & 0.831 & 13.479 & 1.121 & 12.006 & 0.585 & 0.037 \\
\hline 02263472-1610120 & 5145471979973495040 & 12.670 & 1.159 & 12.304 & 1.469 & 10.418 & 0.776 & 0.022 \\
\hline 02263559-6118229 & 4701661876723001088 & 13.029 & 1.046 & 12.692 & 1.315 & 11.027 & 0.688 & 0.029 \\
\hline 02265573-3957537 & 4951576334231196160 & 12.222 & 0.803 & 11.966 & 1.117 & 10.543 & 0.629 & 0.017 \\
\hline 02272940-1844371 & 5131351909915040640 & 11.904 & 0.917 & 11.513 & 1.184 & 10.030 & 0.700 & 0.026 \\
\hline $02281118+3107248$ & 132459372685231872 & 13.016 & 0.992 & 15.066 & 0.000 & 10.905 & 0.651 & 0.080 \\
\hline $02284342+8235565$ & 569513114421444480 & 11.582 & 0.818 & 11.337 & 1.219 & 9.573 & 0.708 & 0.231 \\
\hline 02295644-7231067 & 4643772207267807104 & 12.959 & 0.753 & 12.789 & 1.007 & 11.406 & 0.556 & 0.028 \\
\hline 02302280-1713235 & 5133294712601873024 & 12.692 & 0.973 & 12.294 & 1.286 & 10.581 & 0.663 & 0.020 \\
\hline $02302823+3146355$ & 134052560743210624 & 12.685 & 0.985 & 12.491 & 1.249 & 10.813 & 0.644 & 0.075 \\
\hline 02314914-4251147 & 4950048081786940160 & 11.567 & 1.009 & 11.297 & 1.276 & 9.663 & 0.603 & 0.018 \\
\hline 02322533-2955268 & 5067349028825362176 & 12.655 & 0.835 & 12.453 & 1.099 & 11.016 & 0.603 & 0.014 \\
\hline 02332987-2602004 & 5070849431466425600 & 11.620 & 0.928 & 11.397 & 1.170 & 9.918 & 0.632 & 0.018 \\
\hline 02335901-5218323 & 4744814203419011456 & 11.178 & 0.860 & 10.988 & 1.138 & 9.486 & 0.607 & 0.030 \\
\hline 02343390-3438203 & 5062336050373022976 & 11.374 & 0.711 & 11.133 & 1.002 & 10.061 & 0.716 & 0.020 \\
\hline 02345434-3349391 & 5062448200558971136 & 12.831 & 0.978 & 12.475 & 1.291 & 10.833 & 0.657 & 0.014 \\
\hline 02361077-1202559 & 5171442680844060160 & 12.212 & 0.782 & 11.982 & 1.037 & 10.624 & 0.564 & 0.022 \\
\hline 02371057-4036121 & 4951834822543543040 & 10.727 & 0.599 & 10.578 & 0.779 & 9.257 & 0.397 & 0.019 \\
\hline $02372192+4302214$ & 340142202749502464 & 12.990 & 0.901 & 12.877 & 1.157 & 11.409 & 0.646 & 0.075 \\
\hline 02384449-3325102 & 5062531071950987392 & 12.013 & 0.736 & 11.802 & 1.023 & 10.472 & 0.550 & 0.015 \\
\hline 02394381-3631306 & 4953863662014601216 & 12.968 & 0.590 & 12.806 & 0.806 & 11.923 & 0.371 & 0.029 \\
\hline $02401392+2556291$ & 126323578110142848 & 12.385 & 0.896 & 12.148 & 1.232 & 10.492 & 0.644 & 0.129 \\
\hline $02404390+4457499$ & 340915713477131008 & 13.009 & 1.312 & 12.653 & 1.405 & 10.797 & 0.734 & 0.095 \\
\hline 02425864-3709379 & 4953584523499875712 & 12.458 & 0.721 & 12.254 & 0.989 & 10.947 & 0.544 & 0.018 \\
\hline 02431746-8608453 & 4613478261037323776 & 12.948 & 1.198 & 12.537 & 1.504 & 10.635 & 0.724 & 0.085 \\
\hline 02435125-2942551 & 5065856377366623872 & 12.824 & 1.228 & 12.386 & 1.514 & 10.553 & 0.714 & 0.017 \\
\hline $02451977+1332222$ & 31933391083889152 & 13.027 & 0.758 & 12.719 & 1.128 & 11.228 & 0.576 & 0.086 \\
\hline 02462323-3137296 & 5064607984993521792 & 12.590 & 0.900 & 12.345 & 1.110 & 10.971 & 0.595 & 0.021 \\
\hline 02471497-6303000 & 4721297363392975488 & 12.201 & 0.975 & 11.885 & 1.237 & 10.251 & 0.663 & 0.021 \\
\hline 02494852-2229202 & 5077735500993403648 & 11.173 & 0.796 & 11.036 & 1.095 & 9.516 & 0.613 & 0.029 \\
\hline $02501156+3457476$ & 140083622534201088 & 12.653 & 1.009 & 12.262 & 1.355 & 10.558 & 0.692 & 0.068 \\
\hline $02512543+8333571$ & 569663953674510464 & 12.962 & 1.033 & 12.652 & 1.396 & 11.011 & 0.741 & 0.259 \\
\hline 02523341-4416060 & 4755176207159038080 & 13.373 & 1.094 & 12.997 & 1.365 & 11.293 & 0.675 & 0.009 \\
\hline $02525369+2107466$ & 109283900954164992 & 12.899 & 1.035 & 12.512 & 1.429 & 10.628 & 0.688 & 0.300 \\
\hline $02525416-3228483$ & 5052264558220148352 & 12.594 & 1.165 & 13.924 & 0.000 & 10.364 & 0.742 & 0.024 \\
\hline $02532757-3454050$ & 5049605732946898432 & 13.017 & 0.848 & 12.790 & 1.097 & 11.367 & 0.592 & 0.017 \\
\hline 02533614-6234511 & 4721372684235614848 & 12.766 & 0.752 & 12.533 & 1.006 & 11.229 & 0.540 & 0.025 \\
\hline 02564805-1942473 & 5128076774013414912 & 12.202 & 0.908 & 11.995 & 1.175 & 10.439 & 0.644 & 0.017 \\
\hline $02571027+3318455$ & 136508702211948672 & 12.905 & 0.931 & 12.553 & 1.268 & 10.919 & 0.624 & 0.105 \\
\hline $02580877+0829424$ & 8791969854015488 & 12.794 & 0.770 & 12.538 & 1.066 & 11.218 & 0.573 & 0.249 \\
\hline 02590016-7209504 & 4645469170322185984 & 12.470 & 0.864 & 12.123 & 1.150 & 10.632 & 0.618 & 0.023 \\
\hline $03042561+3112028$ & 135287316592202880 & 13.014 & 0.991 & 12.625 & 1.302 & 11.042 & 0.700 & 0.191 \\
\hline
\end{tabular}


Table 2 (continued)

\begin{tabular}{|c|c|c|c|c|c|c|c|c|}
\hline $\begin{array}{l}\text { Star Name } \\
\text { (2MASS) }\end{array}$ & $\begin{array}{c}\text { Star Name } \\
\text { (Gaia EDR3) }\end{array}$ & $V$ & $(B-V)$ & $G$ & $\left(G_{\mathrm{BP}}-G_{\mathrm{RP}}\right)$ & $J$ & $(J-K)$ & $E(B-V)$ \\
\hline 03042843-3406046 & 5050991770433282688 & 10.884 & 0.918 & 10.600 & 1.223 & 9.078 & 0.642 & 0.018 \\
\hline 03044130-3635405 & 5047482782152551808 & 11.953 & 0.731 & 11.699 & 1.008 & 10.363 & 0.545 & 0.016 \\
\hline $03053573+2834227$ & 115967213665888000 & 11.946 & 1.038 & 11.577 & 1.266 & 9.918 & 0.659 & 0.156 \\
\hline 03060595-5251430 & 4746980344406420736 & 12.480 & 0.666 & 12.275 & 0.976 & 10.999 & 0.558 & 0.015 \\
\hline $03080797+3836489$ & 142648478221664256 & 11.960 & 0.651 & 11.799 & 0.852 & 10.854 & 0.472 & 0.127 \\
\hline 03091996-5853578 & 4724087928200048768 & 13.027 & 0.874 & 12.800 & 1.192 & 11.306 & 0.652 & 0.017 \\
\hline 03095592-0459281 & 5182694120770155264 & 11.961 & 1.051 & 11.659 & 1.357 & 9.862 & 0.669 & 0.063 \\
\hline $03104602+4514503$ & 433115020506112512 & 12.523 & 1.003 & 12.134 & 1.345 & 10.454 & 0.693 & 0.220 \\
\hline 03121034-5703094 & 4727345712434297984 & 13.740 & 1.027 & 13.427 & 1.272 & 11.806 & 0.626 & 0.016 \\
\hline 03123270-2849566 & 5059444369153859200 & 12.969 & 0.800 & 12.726 & 1.087 & 11.311 & 0.564 & 0.014 \\
\hline 03123346-5234570 & 4735321737284031232 & 12.981 & 0.837 & 12.729 & 1.120 & 11.265 & 0.579 & 0.018 \\
\hline 03131491-8107109 & 4619392843320436608 & 13.091 & 0.874 & 12.819 & 1.151 & 11.331 & 0.591 & 0.060 \\
\hline 03134048-8045218 & 4619419089865071744 & 13.018 & 0.842 & 12.629 & 1.032 & 11.584 & 0.582 & 0.057 \\
\hline $03135196+4230102$ & 240424332130561024 & 12.624 & 0.724 & 12.426 & 1.004 & 10.897 & 0.574 & 0.157 \\
\hline $03152783+3353586$ & 137535813574478592 & 11.562 & 0.869 & 11.354 & 1.073 & 9.917 & 0.512 & 0.138 \\
\hline $03155572+3357169$ & 125528253246150656 & 12.397 & 0.981 & 12.036 & 1.304 & 10.348 & 0.704 & 0.162 \\
\hline 03155933-7432577 & 4639776204054863232 & 12.619 & 1.093 & 12.108 & 1.356 & 10.446 & 0.759 & 0.057 \\
\hline $03163710+2332211$ & 110907432953980800 & 11.506 & 0.950 & 11.215 & 1.255 & 9.609 & 0.666 & 0.178 \\
\hline 03170396-3740469 & 4854289415101796608 & 12.072 & 0.914 & 11.788 & 1.189 & 10.264 & 0.635 & 0.022 \\
\hline 03171573-3747479 & 4854284497363037696 & 12.305 & 1.085 & 11.882 & 1.436 & 10.080 & 0.683 & 0.020 \\
\hline 03173348-3705188 & 4854694001021568896 & 11.175 & 1.011 & 13.836 & 0.000 & 9.274 & 0.646 & 0.022 \\
\hline $03180842+1814447$ & 56110625281842816 & 11.276 & 0.593 & 10.658 & 1.240 & 9.040 & 0.639 & 0.114 \\
\hline 03190720-5245069 & 4735169768457507968 & 12.790 & 0.796 & 12.558 & 1.087 & 11.150 & 0.566 & 0.019 \\
\hline 03214149-5553303 & 4733473496892938368 & 12.785 & 0.985 & 12.531 & 1.257 & 10.936 & 0.675 & 0.024 \\
\hline 03220165-0020329 & 3262842880464520576 & 12.311 & 0.858 & 12.058 & 1.114 & 10.693 & 0.644 & 0.060 \\
\hline 03222245-3731294 & 4854443793406263040 & 12.643 & 0.789 & 12.420 & 1.032 & 11.163 & 0.645 & 0.015 \\
\hline $03223653+0859382$ & 11176917949244800 & 12.909 & 1.150 & 12.429 & 1.539 & 10.515 & 0.758 & 0.234 \\
\hline 03242169-3515217 & 4860920810247328256 & 10.724 & 0.691 & 10.488 & 1.024 & 9.169 & 0.536 & 0.011 \\
\hline 03242519-1550054 & 5106733402188456320 & 11.926 & 0.967 & 11.529 & 1.266 & 9.882 & 0.713 & 0.032 \\
\hline $03252266+8009505$ & 568038153934104320 & 11.696 & 0.993 & 11.388 & 1.352 & 9.645 & 0.644 & 0.199 \\
\hline 03260086-4126000 & 4849648891917127424 & 13.010 & 0.855 & 12.630 & 1.197 & 11.099 & 0.693 & 0.010 \\
\hline 03260534-2006507 & 5101544016542831232 & 12.887 & 0.989 & 12.513 & 1.252 & 10.966 & 0.706 & 0.027 \\
\hline $03263031+0616326$ & 9265348264761984 & 12.969 & 1.027 & 12.574 & 1.336 & 10.853 & 0.717 & 0.209 \\
\hline 03265306-0053348 & 3264007336293442048 & 12.942 & 1.108 & 12.348 & 1.474 & 10.521 & 0.731 & 0.084 \\
\hline $03265389+0202281$ & 3268028903151246720 & 11.861 & 0.764 & 11.567 & 1.123 & 10.110 & 0.624 & 0.102 \\
\hline 03275664-4544078 & 4846530329047408384 & 12.269 & 1.036 & 11.957 & 1.309 & 10.365 & 0.693 & 0.009 \\
\hline 03283529-4000252 & 4853194778260569856 & 12.876 & 0.802 & 12.652 & 1.047 & 11.319 & 0.552 & 0.007 \\
\hline $03283748+1856359$ & 57481853722629888 & 12.681 & 0.879 & 12.232 & 1.303 & 10.508 & 0.664 & 0.138 \\
\hline 03292423-6057094 & 4722654951015751680 & 12.424 & 0.793 & 12.084 & 1.158 & 10.559 & 0.628 & 0.043 \\
\hline 03304035-0321071 & 3249179288121898752 & 12.606 & 0.721 & 12.451 & 1.009 & 11.171 & 0.593 & 0.050 \\
\hline 03305484-0304088 & 3249204710032811648 & 12.059 & 0.872 & 11.762 & 1.119 & 10.314 & 0.642 & 0.046 \\
\hline $03312821+0749469$ & 11354660875621376 & 12.183 & 1.071 & 11.775 & 1.428 & 9.995 & 0.691 & 0.248 \\
\hline
\end{tabular}


Table 2 (continued)

\begin{tabular}{|c|c|c|c|c|c|c|c|c|}
\hline $\begin{array}{l}\text { Star Name } \\
\text { (2MASS) }\end{array}$ & $\begin{array}{c}\text { Star Name } \\
\text { (Gaia EDR3) }\end{array}$ & $V$ & $(B-V)$ & $G$ & $\left(G_{\mathrm{BP}}-G_{\mathrm{RP}}\right)$ & $J$ & $(J-K)$ & $E(B-V)$ \\
\hline 03343133-0555030 & 3247331455752180992 & 12.639 & 1.135 & 12.285 & 1.557 & 10.257 & 0.776 & 0.032 \\
\hline $03351518+7823252$ & 555315734952674816 & 12.669 & 0.973 & 12.392 & 1.296 & 10.733 & 0.699 & 0.141 \\
\hline 03354665-1356010 & 5113319751716905344 & 12.298 & 0.984 & 11.989 & 1.271 & 10.277 & 0.703 & 0.031 \\
\hline $03362369+2036490$ & 58156438465188096 & 12.851 & 0.873 & 12.568 & 1.161 & 11.075 & 0.609 & 0.161 \\
\hline 03362874-6005593 & 4728790397697259392 & 12.223 & 0.868 & 12.004 & 1.197 & 10.445 & 0.680 & 0.031 \\
\hline 03365068-2327557 & 5086423493982030848 & 12.574 & 0.882 & 12.248 & 1.183 & 10.731 & 0.632 & 0.020 \\
\hline $03365778+2917172$ & 120001145732321536 & 12.384 & 0.940 & 12.057 & 1.306 & 10.343 & 0.690 & 0.217 \\
\hline 03370393-4829266 & 4833641097832497408 & 11.865 & 0.941 & 11.485 & 1.204 & 9.955 & 0.658 & 0.009 \\
\hline $03370580+1802236$ & 56464972968303104 & 12.659 & 0.541 & 13.161 & 0.800 & 11.890 & 0.383 & 0.116 \\
\hline 03385093-0255535 & 3250559347013995136 & 13.114 & 0.948 & 12.839 & 1.199 & 11.280 & 0.642 & 0.074 \\
\hline $03391370+0310345$ & 3271273935626311808 & 12.694 & 0.932 & 12.342 & 1.253 & 10.746 & 0.667 & 0.146 \\
\hline 03401638-5917516 & 4728932754388052224 & 11.504 & 0.846 & 14.063 & 0.000 & 9.951 & 0.583 & 0.038 \\
\hline 03410147-1812508 & 5107448320968631040 & 12.891 & 0.986 & 12.545 & 1.310 & 10.886 & 0.659 & 0.061 \\
\hline $03421349+2729013$ & 71184787964000000 & 12.687 & 0.981 & 12.428 & 1.225 & 10.801 & 0.583 & 0.140 \\
\hline 03425047-1013432 & 5116038431655398400 & 13.143 & 0.825 & 12.879 & 1.098 & 11.435 & 0.549 & 0.058 \\
\hline $03431186+7807495$ & 554502989702092416 & 12.534 & 0.873 & 12.308 & 1.114 & 10.931 & 0.604 & 0.159 \\
\hline 03435581-3212071 & 4863399349974669440 & 12.439 & 0.713 & 12.213 & 0.997 & 10.935 & 0.558 & 0.007 \\
\hline 03440716-2841235 & 5080387454320334592 & 11.932 & 0.687 & 11.719 & 0.965 & 10.426 & 0.535 & 0.010 \\
\hline 03440987-4057281 & 4854966508106088064 & 12.506 & 0.827 & 12.199 & 1.151 & 10.736 & 0.627 & 0.012 \\
\hline 03445544-7517390 & 4628961755579625600 & 13.036 & 0.956 & 12.763 & 1.289 & 11.012 & 0.659 & 0.126 \\
\hline 03453731-8211290 & 4616039813892309376 & 12.238 & 0.806 & 11.968 & 1.116 & 10.508 & 0.597 & 0.052 \\
\hline $03462539+2125305$ & 63534390354375040 & 12.581 & 0.995 & 12.262 & 1.376 & 10.453 & 0.668 & 0.166 \\
\hline 03463330-0023036 & 3251527020325320960 & 11.489 & 1.222 & 10.909 & 1.637 & 8.988 & 0.783 & 0.189 \\
\hline 03464056-1703266 & 5108001001657825280 & 12.844 & 0.759 & 12.585 & 1.067 & 11.199 & 0.599 & 0.053 \\
\hline 03470283-7933197 & 4625542858531568768 & 12.726 & 0.912 & 12.471 & 1.150 & 10.974 & 0.629 & 0.070 \\
\hline $03470366+7232599$ & 543671700657298816 & 11.912 & 0.985 & 12.086 & 1.360 & 9.952 & 0.662 & 0.291 \\
\hline 03475502-2219560 & 5087674875655599744 & 12.761 & 0.946 & 12.571 & 1.166 & 11.038 & 0.610 & 0.036 \\
\hline 03481276-2657207 & 5081024380790201984 & 12.064 & 0.681 & 11.850 & 0.975 & 10.607 & 0.552 & 0.011 \\
\hline 03494330-1035255 & 3194196834787169152 & 12.287 & 1.138 & 11.853 & 1.451 & 10.063 & 0.728 & 0.033 \\
\hline 03494712-2012428 & 5094777381468797568 & 12.466 & 0.982 & 12.113 & 1.276 & 10.487 & 0.700 & 0.051 \\
\hline $03505656+1915396$ & 50799709243487232 & 12.642 & 1.023 & 12.200 & 1.513 & 10.214 & 0.751 & 0.254 \\
\hline 03511385-1222556 & 5114516604484590848 & 12.557 & 0.972 & 12.198 & 1.240 & 10.597 & 0.670 & 0.037 \\
\hline 03514691-4821339 & 4830105381971162368 & 12.354 & 0.955 & 12.085 & 1.215 & 10.582 & 0.667 & 0.006 \\
\hline $03515804+7834544$ & 554892216818025088 & 11.804 & 0.990 & 11.647 & 1.275 & 9.896 & 0.636 & 0.121 \\
\hline 03522200-7822390 & 4626118933904711936 & 12.931 & 0.861 & 12.613 & 1.195 & 11.076 & 0.646 & 0.073 \\
\hline 03525823-2712362 & 5080850520514122368 & 11.963 & 0.963 & 11.800 & 1.221 & 10.155 & 0.654 & 0.008 \\
\hline $03532071+0332467$ & 3272181586771744640 & 12.522 & 1.016 & 12.162 & 1.319 & 10.432 & 0.648 & 0.260 \\
\hline 03535378-8214319 & 4616030261884997504 & 12.808 & 0.845 & 12.559 & 1.114 & 11.098 & 0.579 & 0.052 \\
\hline 03542750-4930350 & 4829790406249501824 & 11.653 & 1.021 & 11.262 & 1.279 & 9.655 & 0.691 & 0.006 \\
\hline $03550575+0448406$ & 3273182073633848576 & 12.868 & 0.964 & 12.523 & 1.274 & 10.846 & 0.625 & 0.221 \\
\hline 03551870-3540553 & 4858448073019453568 & 12.525 & 0.765 & 12.300 & 1.042 & 10.969 & 0.592 & 0.007 \\
\hline 03552492-3137538 & 4886536888554252288 & 11.589 & 0.809 & 11.377 & 1.056 & 10.022 & 0.616 & 0.009 \\
\hline
\end{tabular}


Table 2 (continued)

\begin{tabular}{|c|c|c|c|c|c|c|c|c|}
\hline $\begin{array}{l}\text { Star Name } \\
\text { (2MASS) }\end{array}$ & $\begin{array}{c}\text { Star Name } \\
\text { (Gaia EDR3) }\end{array}$ & $V$ & $(B-V)$ & $G$ & $\left(G_{\mathrm{BP}}-G_{\mathrm{RP}}\right)$ & $J$ & $(J-K)$ & $E(B-V)$ \\
\hline $03570714+1150353$ & 3304873572159327104 & 12.682 & 0.955 & 12.313 & 1.293 & 10.602 & 0.690 & 0.233 \\
\hline 03575259-3507496 & 4858859741341036032 & 13.004 & 0.812 & 12.896 & 1.084 & 11.374 & 0.624 & 0.007 \\
\hline 03580690-4125514 & 4842342190474113920 & 11.138 & 0.708 & 10.852 & 0.954 & 9.630 & 0.558 & 0.008 \\
\hline $03582857+0851479$ & 3301417188637954304 & 12.952 & 0.956 & 12.601 & 1.288 & 10.951 & 0.677 & 0.154 \\
\hline 03582979-0646599 & 3196842083667299968 & 12.946 & 1.151 & 12.802 & 1.428 & 10.806 & 0.649 & 0.072 \\
\hline 03583609-3024329 & 4887268613542620160 & 10.472 & 0.693 & 10.276 & 0.952 & 9.069 & 0.489 & 0.005 \\
\hline 03585372-2512589 & 5083157811305565184 & 12.348 & 0.833 & 12.124 & 1.053 & 10.784 & 0.555 & 0.033 \\
\hline 03590972-7052417 & 4665955133531907840 & 11.913 & 0.687 & 11.729 & 0.992 & 10.401 & 0.537 & 0.075 \\
\hline 04021386-2714330 & 4890018148526236416 & 11.049 & 0.888 & 10.807 & 1.127 & 9.395 & 0.630 & 0.017 \\
\hline 04030414-2123136 & 5090671530172203648 & 11.520 & 0.772 & 11.316 & 1.031 & 10.034 & 0.581 & 0.033 \\
\hline 04032141-5057006 & 4828667358199528064 & 12.274 & 0.910 & 16.234 & 0.000 & 10.636 & 0.665 & 0.010 \\
\hline $04034276+0516322$ & 3272889569179614464 & 12.495 & 1.062 & 12.266 & 1.346 & 10.433 & 0.685 & 0.192 \\
\hline 04051129-3610404 & 4857845781166112384 & 12.892 & 1.066 & 12.510 & 1.387 & 10.798 & 0.710 & 0.003 \\
\hline 04062658-1417389 & 5110102993011576192 & 12.150 & 0.783 & 11.902 & 1.019 & 10.608 & 0.549 & 0.037 \\
\hline 04064080-2822221 & 4889098510129080064 & 13.004 & 0.717 & 12.815 & 0.999 & 11.545 & 0.577 & 0.018 \\
\hline 04065230-1132234 & 3190316692611552000 & 12.331 & 0.786 & 12.161 & 0.938 & 11.000 & 0.503 & 0.045 \\
\hline 04065870-5405560 & 4779874227616110592 & 12.210 & 0.715 & 12.042 & 0.973 & 10.767 & 0.515 & 0.008 \\
\hline $04071847-3844270$ & 4844183765074837760 & 12.732 & 0.981 & 12.537 & 1.257 & 10.851 & 0.639 & 0.007 \\
\hline 04073220-1508180 & 5097968885766682240 & 12.941 & 1.127 & 12.575 & 1.388 & 10.828 & 0.745 & 0.030 \\
\hline 04081065-4723248 & 4831180463825371776 & 12.584 & 1.212 & 12.287 & 1.616 & 10.089 & 0.793 & 0.013 \\
\hline 04082208-8054055 & 4622225700669724160 & 13.052 & 0.900 & 12.779 & 1.179 & 11.228 & 0.630 & 0.077 \\
\hline 04082291-3306502 & 4882442375971513216 & 13.103 & 0.869 & 12.744 & 1.252 & 11.140 & 0.647 & 0.009 \\
\hline 04095634-2018250 & 5091033814957326464 & 13.014 & 0.799 & 12.744 & 1.019 & 11.522 & 0.587 & 0.025 \\
\hline 04123785-0354082 & 3203733925629461376 & 12.780 & 0.922 & 12.383 & 1.190 & 10.805 & 0.661 & 0.040 \\
\hline 04133760-2855548 & 4885822480873873408 & 12.592 & 0.708 & 12.381 & 0.996 & 11.080 & 0.537 & 0.024 \\
\hline 04144546-4431586 & 4837831066064068480 & 13.980 & -1.200 & 13.584 & 1.493 & 11.727 & 0.745 & 0.014 \\
\hline 04152012-4554089 & 4837327760319848576 & 12.415 & 0.944 & 12.124 & 1.208 & 10.605 & 0.651 & 0.011 \\
\hline 04161576-0521188 & 3202575628785925888 & 9.559 & 0.534 & 9.458 & 0.697 & 8.769 & 0.556 & 0.067 \\
\hline 04162853-6636540 & 4668867224438266368 & 12.733 & 0.830 & 12.482 & 1.105 & 11.040 & 0.602 & 0.028 \\
\hline 04163240-0602269 & 3202394930922308096 & 12.073 & 0.815 & 11.993 & 1.094 & 10.399 & 0.597 & 0.053 \\
\hline 04174265-5558407 & 4778645523371812480 & 12.989 & 0.983 & 12.758 & 1.224 & 11.078 & 0.638 & 0.013 \\
\hline 04211598-4852031 & 4782730209069106432 & 12.155 & 0.777 & 11.909 & 1.058 & 10.512 & 0.581 & 0.009 \\
\hline 04212925-5219577 & 4780985799511960576 & 12.954 & 1.027 & 12.626 & 1.287 & 10.995 & 0.699 & 0.010 \\
\hline 04213031-2030287 & 5091573542023760384 & 12.685 & 0.912 & 12.411 & 1.163 & 10.946 & 0.670 & 0.032 \\
\hline 04215801-2739310 & 4892329974803162752 & 11.013 & 0.989 & 10.650 & 1.285 & 9.085 & 0.678 & 0.044 \\
\hline 04225741-8308401 & 4615741777521531136 & 13.002 & 0.979 & 12.687 & 1.266 & 11.071 & 0.703 & 0.089 \\
\hline 04242725-8157493 & 4621912305496245248 & 12.847 & 0.741 & 12.637 & 1.011 & 11.303 & 0.526 & 0.063 \\
\hline 04251520-6213209 & 4675852078212908416 & 13.049 & 0.829 & 12.781 & 1.123 & 11.338 & 0.594 & 0.025 \\
\hline 04262201-3615167 & 4869018507227798400 & 11.518 & 0.805 & 11.294 & 1.068 & 9.904 & 0.580 & 0.021 \\
\hline 04282895-3353452 & 4871041608622321664 & 11.872 & 0.930 & 11.627 & 1.179 & 10.126 & 0.600 & 0.020 \\
\hline 04291257-4921466 & 4787721991859465728 & 12.017 & 0.806 & 11.813 & 1.078 & 10.447 & 0.655 & 0.013 \\
\hline $04293837-5448372$ & 4776030816001572736 & 11.356 & 0.961 & 11.019 & 1.262 & 9.475 & 0.652 & 0.005 \\
\hline
\end{tabular}


Table 2 (continued)

\begin{tabular}{|c|c|c|c|c|c|c|c|c|}
\hline $\begin{array}{l}\text { Star Name } \\
\text { (2MASS) }\end{array}$ & $\begin{array}{c}\text { Star Name } \\
\text { (Gaia EDR3) }\end{array}$ & $V$ & $(B-V)$ & G & $\left(G_{\mathrm{BP}}-G_{\mathrm{RP}}\right)$ & $J$ & $(J-K)$ & $E(B-V)$ \\
\hline $04311275-2258353$ & 4898098833139598464 & 12.938 & 0.891 & 12.602 & 1.237 & 10.998 & 0.636 & 0.048 \\
\hline 04313335-7337168 & 4652567991051879168 & 12.641 & 0.873 & 12.387 & 1.132 & 10.875 & 0.555 & 0.102 \\
\hline 04325046-3205240 & 4871721965802188160 & 10.366 & 0.726 & 10.175 & 0.911 & 9.048 & 0.533 & 0.016 \\
\hline 04330582-1605256 & 3172371116657963008 & 11.721 & 0.684 & 11.957 & 0.957 & 10.213 & 0.561 & 0.036 \\
\hline $04332408+7049217$ & 500082940861162240 & 12.251 & 0.780 & 12.151 & 1.109 & 10.764 & 0.596 & 0.189 \\
\hline 04332826-0431033 & 3201331222141772928 & 12.176 & 0.960 & 11.870 & 1.218 & 10.303 & 0.642 & 0.049 \\
\hline $04334315+8752515$ & 575291322904958208 & 12.989 & 1.022 & 12.598 & 1.425 & 10.753 & 0.732 & 0.300 \\
\hline $04354147-4750031$ & 4788319503413123200 & 11.313 & 0.672 & 11.154 & 0.885 & 10.097 & 0.481 & 0.009 \\
\hline 04354314-5355093 & 4777636927611794432 & 12.490 & 0.863 & 12.235 & 1.154 & 10.739 & 0.590 & 0.004 \\
\hline $04363802+7949143$ & 556538739776801664 & 12.939 & 0.867 & 12.732 & 1.153 & 11.275 & 0.629 & 0.096 \\
\hline 04391892-3404504 & 4868176796716806272 & 12.232 & 0.496 & 12.986 & 0.699 & 11.405 & 0.183 & 0.017 \\
\hline 04392332-2434534 & 4894484605277323648 & 10.378 & 0.616 & 10.144 & 0.933 & 8.944 & 0.516 & 0.039 \\
\hline 04392855-3957548 & 4816386358778340608 & 12.871 & 1.065 & 12.525 & 1.335 & 10.848 & 0.675 & 0.022 \\
\hline 04400253-1922270 & 2978208560171576448 & 11.835 & -0.025 & 15.859 & 1.237 & 14.194 & 0.701 & 0.034 \\
\hline 04404386-2741005 & 4879633570439853440 & 11.668 & 0.806 & 11.429 & 1.085 & 9.987 & 0.587 & 0.038 \\
\hline 04404847-4214219 & 4814898930000598272 & 12.832 & 0.807 & 12.567 & 1.116 & 11.106 & 0.567 & 0.015 \\
\hline 04404877-3530531 & 4867833886528092288 & 11.564 & 0.970 & 11.294 & 1.224 & 9.732 & 0.646 & 0.015 \\
\hline 04414545-1703252 & 2980627661847718016 & 12.286 & 0.787 & 12.033 & 1.112 & 10.587 & 0.614 & 0.041 \\
\hline 04452944-4656234 & 4786751256234998272 & 12.671 & 0.907 & 12.409 & 1.190 & 10.909 & 0.722 & 0.009 \\
\hline $04455221+8238074$ & 569892445935443200 & 12.958 & 1.092 & 12.571 & 1.416 & 10.798 & 0.705 & 0.080 \\
\hline 04460040-5244239 & 4777958977143271936 & 12.704 & 0.917 & 12.514 & 1.219 & 10.820 & 0.599 & 0.005 \\
\hline 04460379-4440454 & 4790350855440186752 & 11.878 & 0.928 & 11.566 & 1.231 & 9.992 & 0.679 & 0.011 \\
\hline 04480413-5133186 & 4784117101252301056 & 12.117 & 1.049 & 11.726 & 1.391 & 10.005 & 0.684 & 0.007 \\
\hline 04481274-3428106 & 4873248568978164608 & 12.342 & 1.101 & 11.958 & 1.409 & 10.211 & 0.700 & 0.013 \\
\hline 04482945-5127292 & 4784118686097335808 & 12.752 & 0.936 & 12.449 & 1.232 & 10.758 & 0.680 & 0.008 \\
\hline 04504247-4831497 & 4786320148893885568 & 12.334 & 0.906 & 12.053 & 1.189 & 10.555 & 0.606 & 0.016 \\
\hline $04505386+1803498$ & 3406732187621193600 & 12.927 & 0.520 & 12.714 & 0.869 & 12.075 & 0.736 & 0.300 \\
\hline 04514248-3210438 & 4874664052759778304 & 12.764 & 0.882 & 12.518 & 1.136 & 11.028 & 0.611 & 0.015 \\
\hline 04520803-2837181 & 4879979504285453184 & 12.353 & 0.982 & 12.075 & 1.222 & 10.653 & 0.709 & 0.017 \\
\hline 04522717-3451392 & 4873001625538322688 & 11.920 & 1.157 & 11.543 & 1.470 & 9.713 & 0.750 & 0.010 \\
\hline 04525913-4008568 & 4816836269489019904 & 12.948 & 0.947 & 12.685 & 1.200 & 11.151 & 0.670 & 0.014 \\
\hline $04542625-4241431$ & 4811990171989821824 & 12.043 & 1.018 & 11.743 & 1.311 & 10.123 & 0.673 & 0.008 \\
\hline 04562421-3124177 & 4874779806424378496 & 12.879 & 1.184 & 12.529 & 1.530 & 10.521 & 0.734 & 0.012 \\
\hline 04563790-5602511 & 4776246457719437440 & 12.341 & 1.002 & 12.017 & 1.275 & 10.369 & 0.645 & 0.012 \\
\hline 04582722-4611154 & 4810231400062085504 & 11.951 & 0.874 & 11.722 & 1.155 & 10.194 & 0.658 & 0.013 \\
\hline 04583735-0449416 & 3212422747548345216 & 12.846 & 1.044 & 12.496 & 1.282 & 10.882 & 0.747 & 0.043 \\
\hline $05000638+0109473$ & 3228880619846899712 & 12.824 & 1.139 & 12.454 & 1.436 & 10.636 & 0.717 & 0.071 \\
\hline $05011881+0803047$ & 3290174403068832640 & 10.775 & 0.685 & 10.371 & 1.076 & 8.984 & 0.606 & 0.114 \\
\hline 05015088-4139079 & 4813429157832439168 & 11.761 & 0.914 & 11.549 & 1.216 & 10.039 & 0.664 & 0.017 \\
\hline $05015244+6745243$ & 483790308838071424 & 12.826 & 0.828 & 13.690 & 1.308 & 11.429 & 0.472 & 0.136 \\
\hline 05021896-5152056 & 4783532062283301120 & 13.040 & 0.830 & 12.826 & 1.065 & 11.430 & 0.557 & 0.011 \\
\hline 05022161-4603414 & 4810297405118590592 & 12.380 & 1.203 & 13.960 & 0.000 & 10.059 & 0.768 & 0.013 \\
\hline
\end{tabular}


Table 2 (continued)

\begin{tabular}{|c|c|c|c|c|c|c|c|c|}
\hline $\begin{array}{l}\text { Star Name } \\
\text { (2MASS) }\end{array}$ & $\begin{array}{c}\text { Star Name } \\
\text { (Gaia EDR3) }\end{array}$ & $V$ & $(B-V)$ & $G$ & $\left(G_{\mathrm{BP}}-G_{\mathrm{RP}}\right)$ & $J$ & $(J-K)$ & $E(B-V)$ \\
\hline $05024868+0053487$ & 3228646389509963008 & 12.935 & 0.933 & 12.906 & 1.145 & 11.371 & 0.617 & 0.100 \\
\hline 05025851-3909387 & 4814331680783356800 & 12.059 & 0.564 & 11.953 & 0.748 & 11.181 & 0.503 & 0.022 \\
\hline 05042173-6614399 & 4662031938654411008 & 11.623 & 1.198 & 11.236 & 1.462 & 9.315 & 0.678 & 0.095 \\
\hline $05043110+0151252$ & 3229000397895064960 & 12.559 & 0.683 & 12.442 & 1.047 & 11.131 & 0.571 & 0.062 \\
\hline 05065541-1056146 & 3181964218172190464 & 11.319 & 1.087 & 10.952 & 1.365 & 9.197 & 0.634 & 0.150 \\
\hline 05070417-0543063 & 3211326195154437760 & 12.447 & 1.045 & 12.031 & 1.451 & 10.206 & 0.746 & 0.077 \\
\hline $05071155+3428162$ & 184903053675527552 & 12.016 & 1.013 & 11.584 & 1.449 & 9.709 & 0.659 & 0.300 \\
\hline $05071933+0950093$ & 3290839985560775552 & 13.910 & -0.390 & 13.706 & 1.342 & 11.886 & 0.682 & 0.162 \\
\hline $05071949+2233222$ & 3415364831369329024 & 12.921 & 0.920 & 12.784 & 1.271 & 10.802 & 0.646 & 0.300 \\
\hline $05085713+0345040$ & 3238657198066863616 & 12.106 & 0.936 & 11.804 & 1.224 & 10.144 & 0.634 & 0.081 \\
\hline $05090948+0758512$ & 3242001298259483776 & 12.780 & 1.040 & 12.434 & 1.334 & 10.698 & 0.659 & 0.181 \\
\hline 05104812-0827001 & 3206923024747054080 & 12.345 & 0.895 & 11.981 & 1.131 & 10.583 & 0.611 & 0.084 \\
\hline 05115864-0512205 & 3211775654892063360 & 12.181 & 0.844 & 11.830 & 1.135 & 10.339 & 0.643 & 0.119 \\
\hline $05120490+0354513$ & 3235687077858885248 & 12.163 & 0.825 & 11.903 & 1.094 & 10.478 & 0.546 & 0.084 \\
\hline 05124167-4059465 & 4818944995352554496 & 12.776 & 0.908 & 12.485 & 1.179 & 10.979 & 0.599 & 0.025 \\
\hline 05130026-2540113 & 2956447060393795200 & 12.943 & 1.058 & 12.609 & 1.354 & 10.872 & 0.673 & 0.017 \\
\hline 05134078-0629405 & 3208349473581491968 & 11.724 & 1.137 & 11.353 & 1.407 & 9.601 & 0.764 & 0.114 \\
\hline $05144882+7604501$ & 503757371280728448 & 12.959 & 0.804 & 12.610 & 1.201 & 10.985 & 0.640 & 0.157 \\
\hline $05145383+3147370$ & 180592968094361472 & 11.931 & 0.974 & 11.575 & 1.304 & 9.892 & 0.756 & 0.300 \\
\hline 05160558-1418474 & 2985144932353707264 & 12.529 & 1.101 & 12.069 & 1.503 & 10.193 & 0.740 & 0.124 \\
\hline 05161882-0759103 & 3207022255670687488 & 12.479 & 0.891 & 12.135 & 1.263 & 10.552 & 0.681 & 0.118 \\
\hline 05163919-0740502 & 3207049193707252992 & 12.934 & 0.802 & 13.003 & 1.132 & 11.140 & 0.603 & 0.158 \\
\hline 05165318-1711429 & 2982252980551829760 & 11.776 & 1.234 & 11.371 & 1.514 & 9.494 & 0.733 & 0.067 \\
\hline $05170982+0306456$ & 3235326060087791232 & 12.913 & 0.989 & 12.616 & 1.237 & 11.055 & 0.696 & 0.078 \\
\hline $05171185+7649087$ & 551880864924665088 & 12.341 & 0.972 & 11.949 & 1.300 & 10.294 & 0.669 & 0.121 \\
\hline 05172978-4253358 & 4800721449115828352 & 11.652 & 1.010 & 11.335 & 1.282 & 9.707 & 0.647 & 0.019 \\
\hline $05175177-1441274$ & 2985070199923468416 & 12.443 & 1.226 & 12.005 & 1.582 & 10.012 & 0.738 & 0.119 \\
\hline 05180022-2418191 & 2958140484397025792 & 12.687 & 0.804 & 12.422 & 1.110 & 10.965 & 0.604 & 0.023 \\
\hline $05181193+1750335$ & 3395305135071832448 & 12.540 & 0.813 & 12.244 & 1.175 & 10.655 & 0.641 & 0.300 \\
\hline 05181299-0356513 & 3213469349475577472 & 12.781 & 1.076 & 12.231 & 1.371 & 10.537 & 0.737 & 0.249 \\
\hline 05183018-4217511 & 4806766701483968640 & 12.350 & 0.683 & 12.065 & 0.977 & 10.838 & 0.665 & 0.017 \\
\hline 05183839-0208303 & 3214278143356960256 & 12.982 & 1.038 & 12.700 & 1.369 & 10.955 & 0.754 & 0.187 \\
\hline 05191853-0625013 & 3207648530621533056 & 12.297 & 0.799 & 12.068 & 1.056 & 10.728 & 0.541 & 0.141 \\
\hline 05202360-2522274 & 2957773415014463872 & 12.808 & 1.065 & 12.534 & 1.397 & 10.679 & 0.700 & 0.024 \\
\hline 05204433-0757598 & 3207154098284136064 & 12.829 & 0.724 & 12.539 & 1.103 & 11.040 & 0.600 & 0.123 \\
\hline 05211432-1032144 & 3014044878233010304 & 12.516 & 0.922 & 12.109 & 1.141 & 10.697 & 0.638 & 0.119 \\
\hline $05221674+1850154$ & 3401393307050179968 & 12.963 & 0.927 & 12.636 & 1.327 & 11.011 & 0.772 & 0.300 \\
\hline 05224909-0008545 & 3221372600399707520 & 12.279 & 0.823 & 12.139 & 1.149 & 10.561 & 0.559 & 0.130 \\
\hline $05232882+6904581$ & 485426657021670784 & 10.751 & 0.582 & 10.643 & 0.837 & 9.426 & 0.696 & 0.096 \\
\hline $05234159+0001547$ & 3221399469715891712 & 12.626 & 0.824 & 12.507 & 1.120 & 11.072 & 0.634 & 0.130 \\
\hline $05234178+0140465$ & 3222180191690519296 & 12.475 & 0.873 & 12.238 & 1.086 & 10.846 & 0.535 & 0.131 \\
\hline $05240221+0037377$ & 3221857420604615040 & 11.534 & 0.718 & 11.300 & 0.983 & 9.858 & 0.572 & 0.104 \\
\hline
\end{tabular}


Table 2 (continued)

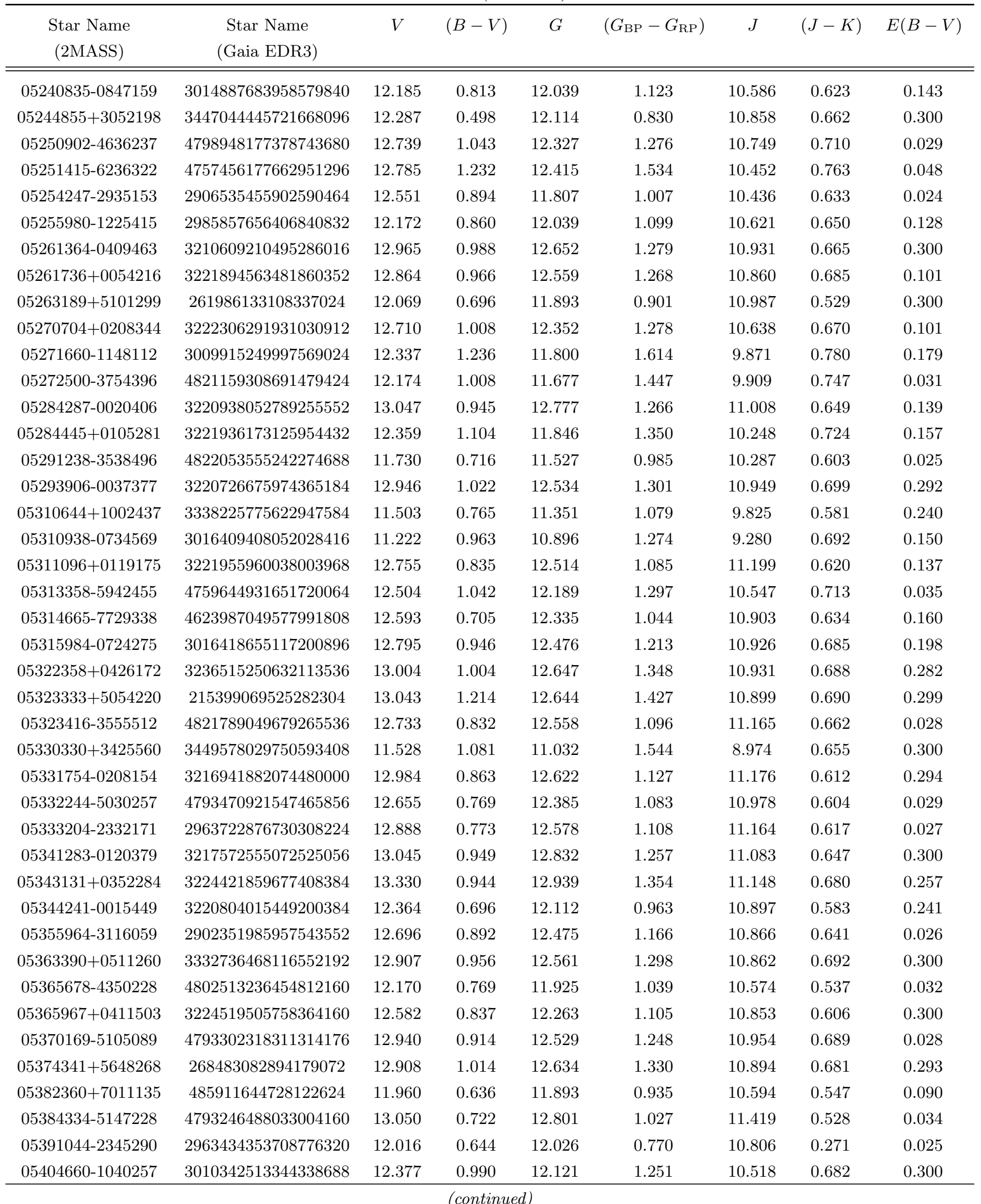


Table 2 (continued)

\begin{tabular}{|c|c|c|c|c|c|c|c|c|}
\hline $\begin{array}{l}\text { Star Name } \\
\text { (2MASS) }\end{array}$ & $\begin{array}{c}\text { Star Name } \\
\text { (Gaia EDR3) }\end{array}$ & $V$ & $(B-V)$ & $G$ & $\left(G_{\mathrm{BP}}-G_{\mathrm{RP}}\right)$ & $J$ & $(J-K)$ & $E(B-V)$ \\
\hline $05415000+1525054$ & 3347851759405013248 & 12.570 & 0.974 & 12.209 & 1.348 & 10.512 & 0.698 & 0.300 \\
\hline 05423307-5913398 & 4759918400809516416 & 11.932 & 0.781 & 11.662 & 1.098 & 10.243 & 0.608 & 0.056 \\
\hline $05431543+7808008$ & 552468274714043392 & 12.929 & 1.022 & 12.556 & 1.349 & 10.859 & 0.679 & 0.092 \\
\hline $05434309+5012096$ & 214379311147693312 & 12.821 & 0.930 & 13.390 & 1.370 & 11.047 & 0.636 & 0.218 \\
\hline 05434572-2151551 & 2964356642107544192 & 12.130 & 1.048 & 11.775 & 1.366 & 10.043 & 0.712 & 0.025 \\
\hline 05434805-2538412 & 2914965720791328896 & 12.215 & 0.729 & 12.021 & 0.989 & 10.717 & 0.517 & 0.017 \\
\hline 05440311-6050523 & 4758589400489342080 & 12.874 & 0.998 & 12.727 & 1.216 & 10.990 & 0.688 & 0.055 \\
\hline 05440932-3820149 & 4808589176366959360 & 12.491 & 0.845 & 12.287 & 1.061 & 10.882 & 0.562 & 0.037 \\
\hline 05450050-2105418 & 2965923995930380160 & 12.721 & 1.233 & 12.326 & 1.579 & 10.348 & 0.769 & 0.036 \\
\hline $05455248-2556383$ & 29141925235986900048 & 12.879 & 0.741 & 12.656 & 1.013 & 11.334 & 0.578 & 0.022 \\
\hline $05463023+4210063$ & 192794656646602880 & 12.562 & 1.174 & 12.499 & 1.514 & 10.251 & 0.744 & 0.300 \\
\hline 05463758-4103031 & 4804867874965676032 & 12.959 & 1.081 & 12.702 & 1.387 & 10.857 & 0.699 & 0.039 \\
\hline 05464200-1802448 & 2967658131925635968 & 12.043 & 1.126 & 11.595 & 1.462 & 9.760 & 0.674 & 0.063 \\
\hline 05472677-5432402 & 4767980226222674944 & 12.043 & 0.931 & 11.776 & 1.201 & 10.191 & 0.636 & 0.077 \\
\hline 05483017-5835567 & 4765250138851402112 & 12.640 & 0.962 & 12.299 & 1.256 & 10.769 & 0.674 & 0.088 \\
\hline 05483336-2434285 & 2915518908282121472 & 12.787 & 0.695 & 12.458 & 1.089 & 11.123 & 0.646 & 0.023 \\
\hline $05483820+3148424$ & 3445140602679798400 & 12.801 & 0.412 & 12.581 & 0.765 & 11.373 & 0.626 & 0.300 \\
\hline 05505657-2008418 & 2966088402979538944 & 12.163 & 0.811 & 11.912 & 1.099 & 10.460 & 0.545 & 0.050 \\
\hline 05511133-0653111 & 3018944989240573056 & 11.291 & 0.760 & 10.979 & 1.091 & 9.572 & 0.598 & 0.300 \\
\hline 05521578-3953184 & 4805034691496015104 & 11.511 & 0.896 & 11.268 & 1.182 & 9.710 & 0.635 & 0.052 \\
\hline 05530383-2007355 & 2966268035692065280 & 12.568 & 0.788 & 12.347 & 1.056 & 10.955 & 0.639 & 0.054 \\
\hline 05531036-4113596 & 4804147870945271168 & 12.788 & 1.058 & 12.531 & 1.324 & 10.795 & 0.741 & 0.044 \\
\hline $05542509+1632212$ & 3349664785359713280 & 12.828 & 0.874 & 12.903 & 1.160 & 10.989 & 0.605 & 0.275 \\
\hline 05544693-8301197 & 4620849386990258560 & 12.770 & 0.926 & 12.364 & 1.330 & 10.633 & 0.628 & 0.131 \\
\hline $05545543+5131242$ & 214916074682985856 & 12.915 & 1.269 & 12.627 & 1.294 & 10.862 & 0.673 & 0.166 \\
\hline 05583688-2438574 & 2914576528036091136 & 12.300 & 0.827 & 11.999 & 1.207 & 10.348 & 0.625 & 0.036 \\
\hline 05583844-3434436 & 2889188186073231104 & 13.005 & 0.878 & 12.775 & 1.214 & 11.182 & 0.633 & 0.029 \\
\hline $05584204+5040146$ & 211594935389482240 & 12.825 & 0.848 & 12.494 & 1.199 & 10.964 & 0.671 & 0.300 \\
\hline 05592533-7744016 & 4647752959052853632 & 13.038 & 0.713 & 12.797 & 1.065 & 11.360 & 0.534 & 0.102 \\
\hline $05594737-2708140$ & 2911019229961694208 & 12.889 & 1.076 & 12.578 & 1.301 & 10.934 & 0.682 & 0.024 \\
\hline 06000654-3034592 & 2891276807192892800 & 12.471 & 0.861 & 12.247 & 1.138 & 10.738 & 0.611 & 0.028 \\
\hline $06000928+6830331$ & 1105490157831460352 & 12.785 & 0.974 & 12.553 & 1.131 & 11.068 & 0.640 & 0.093 \\
\hline 06010912-2251504 & 2916542798424334208 & 12.061 & 1.029 & 11.726 & 1.338 & 10.027 & 0.661 & 0.033 \\
\hline 06013941-3304567 & 2889974641827298048 & 12.488 & 1.016 & 12.203 & 1.356 & 10.356 & 0.710 & 0.035 \\
\hline 06023445-3332108 & 2889528175684527104 & 10.581 & 0.740 & 10.346 & 1.020 & 8.969 & 0.579 & 0.038 \\
\hline 06032005-7531388 & 5261685578730080000 & 11.935 & 0.762 & 11.643 & 1.139 & 9.983 & 0.704 & 0.076 \\
\hline 06032024-2059364 & 2917926946123381248 & 13.013 & 0.802 & 12.800 & 1.040 & 11.448 & 0.542 & 0.046 \\
\hline 06035878-3445341 & 2886205760783047808 & 12.163 & 0.822 & 11.934 & 1.078 & 10.578 & 0.578 & 0.040 \\
\hline 06040505-3922438 & 2882964602365617408 & 12.231 & 0.934 & 11.937 & 1.179 & 10.476 & 0.648 & 0.048 \\
\hline 06043058-3232288 & 2889972447101620480 & 11.843 & 0.979 & 11.597 & 1.252 & 9.893 & 0.673 & 0.032 \\
\hline 06050821-3625551 & 2885764135065967488 & 11.155 & 0.725 & 11.194 & 0.955 & 9.732 & 0.538 & 0.053 \\
\hline $06050851+6114505$ & 1005911306711125632 & 13.026 & 1.100 & 15.643 & 2.066 & 10.841 & 0.772 & 0.122 \\
\hline
\end{tabular}


Table 2 (continued)

\begin{tabular}{|c|c|c|c|c|c|c|c|c|}
\hline $\begin{array}{l}\text { Star Name } \\
\text { (2MASS) }\end{array}$ & $\begin{array}{c}\text { Star Name } \\
\text { (Gaia EDR3) }\end{array}$ & $V$ & $(B-V)$ & G & $\left(G_{\mathrm{BP}}-G_{\mathrm{RP}}\right)$ & $J$ & $(J-K)$ & $E(B-V)$ \\
\hline $06050978+6654472$ & 1105001321832556800 & 12.999 & 0.691 & 12.736 & 1.149 & 11.279 & 0.592 & 0.096 \\
\hline 06052881-4438188 & 5567973273001201152 & 12.167 & 0.693 & 11.980 & 0.931 & 10.808 & 0.572 & 0.059 \\
\hline 06055284-3537284 & 2885916589224697088 & 10.531 & 0.803 & 10.278 & 1.122 & 8.819 & 0.586 & 0.063 \\
\hline 06055999-6907423 & 5279730523050938624 & 11.915 & 0.823 & 11.683 & 1.103 & 10.211 & 0.572 & 0.066 \\
\hline 06063114-1605358 & 2991490626275600640 & 12.935 & 0.921 & 12.576 & 1.200 & 11.102 & 0.656 & 0.211 \\
\hline 06063142-5032308 & 5549982410912756992 & 13.340 & 1.010 & 13.584 & 1.184 & 11.741 & 0.634 & 0.043 \\
\hline 06074714-3724498 & 2884863016567274240 & 11.619 & 0.692 & 11.364 & 1.012 & 9.996 & 0.620 & 0.071 \\
\hline 06085789-3536279 & 2885882774945629824 & 11.462 & 0.716 & 11.282 & 0.927 & 10.117 & 0.538 & 0.054 \\
\hline 06090353-7122260 & 5278332292157596160 & 11.157 & 0.822 & 10.913 & 1.131 & 9.480 & 0.654 & 0.076 \\
\hline 06120579-3152561 & 2895882146005313152 & 12.576 & 0.881 & 12.209 & 1.237 & 10.661 & 0.653 & 0.045 \\
\hline 06134289-6937017 & 5279560687166096896 & 12.062 & 0.878 & 11.803 & 1.148 & 10.293 & 0.615 & 0.069 \\
\hline 06135706-3555284 & 2885428298685730816 & 13.013 & 0.854 & 12.900 & 1.107 & 11.333 & 0.591 & 0.045 \\
\hline 06155680-7459448 & 5261728773219036800 & 12.192 & 0.846 & 11.898 & 1.166 & 10.347 & 0.578 & 0.113 \\
\hline 06171171-7328560 & 5265153816361777920 & 11.471 & 0.615 & 11.480 & 0.997 & 10.034 & 0.630 & 0.095 \\
\hline 06181310-0348135 & 3116844057174980864 & 13.470 & -0.510 & 13.719 & 1.759 & 11.667 & 0.799 & 0.300 \\
\hline 06183086-2506582 & 2912205186393737472 & 12.145 & 1.009 & 11.939 & 1.261 & 10.204 & 0.658 & 0.033 \\
\hline 06192867-8743048 & 5189368469885240832 & 11.739 & 0.874 & 11.443 & 1.197 & 9.856 & 0.603 & 0.107 \\
\hline 06194462-2030436 & 2938430256702303360 & 12.208 & 1.010 & 11.886 & 1.341 & 10.152 & 0.670 & 0.112 \\
\hline 06200929-5719169 & 5495322423720756352 & 11.166 & 0.785 & 10.947 & 1.052 & 9.602 & 0.587 & 0.042 \\
\hline 06220380-2155289 & 2937664962250734592 & 12.552 & 0.774 & 12.357 & 1.061 & 10.897 & 0.582 & 0.073 \\
\hline 06234993-5946248 & 5482612241102802304 & 12.976 & 0.850 & 14.070 & 1.209 & 12.316 & 0.585 & 0.041 \\
\hline $06244840+7752154$ & 1140661709439350528 & 12.923 & 1.136 & 12.585 & 1.444 & 10.809 & 0.715 & 0.084 \\
\hline 06250234-4659051 & 5553993768631973120 & 10.324 & 0.702 & 10.034 & 1.017 & 8.747 & 0.502 & 0.039 \\
\hline 06290787-6709523 & 5283390938997970176 & 12.441 & 0.979 & 12.102 & 1.295 & 10.425 & 0.669 & 0.050 \\
\hline $06325088+6530326$ & 1103660291306745856 & 12.709 & 1.136 & 12.558 & 1.402 & 10.609 & 0.717 & 0.070 \\
\hline 06325555-4214034 & 5569242345641089408 & 12.379 & 1.055 & 12.033 & 1.362 & 10.352 & 0.710 & 0.059 \\
\hline 06332771-3519240 & 5581669477032806912 & 11.507 & 0.672 & 11.303 & 0.964 & 10.083 & 0.587 & 0.074 \\
\hline 06345221-4425441 & 5556592090703647744 & 12.228 & 1.087 & 11.956 & 1.430 & 10.130 & 0.729 & 0.056 \\
\hline 06363111-2255559 & 2924798167948814208 & 13.690 & 1.050 & 13.253 & 1.316 & 11.627 & 0.722 & 0.085 \\
\hline 06382886-8309319 & 5206227036859558272 & 12.766 & 0.900 & 12.413 & 1.243 & 10.792 & 0.614 & 0.173 \\
\hline 06393860-3406395 & 5583340111934906112 & 13.030 & 1.045 & 12.634 & 1.384 & 10.896 & 0.717 & 0.084 \\
\hline 06421665-5008385 & 5503130777344639488 & 12.834 & 0.915 & 12.489 & 1.159 & 11.060 & 0.636 & 0.049 \\
\hline $06425079+7014409$ & 1112486380380023040 & 13.039 & 0.692 & 12.968 & 1.055 & 11.427 & 0.600 & 0.104 \\
\hline 06451421-3217294 & 5583930313459131008 & 12.934 & 0.854 & 12.658 & 1.147 & 11.160 & 0.557 & 0.085 \\
\hline 06451920-0857165 & 3098535676741247744 & 12.427 & 0.852 & 12.163 & 1.192 & 10.588 & 0.697 & 0.300 \\
\hline 06460710-4155202 & 5563430395897276032 & 12.456 & 0.907 & 12.212 & 1.182 & 10.748 & 0.653 & 0.093 \\
\hline 06464646-4440364 & 5556009212103193472 & 12.217 & 0.898 & 11.957 & 1.182 & 10.352 & 0.634 & 0.069 \\
\hline $06484021+1625173$ & 3358091992230790656 & 12.714 & 1.067 & 12.391 & 1.291 & 10.697 & 0.748 & 0.150 \\
\hline 06484979-2428341 & 2922058189267952384 & 12.830 & -0.120 & 12.933 & 1.146 & 11.455 & 0.604 & 0.097 \\
\hline $06510773+0943416$ & 3158574272957723776 & 12.733 & 0.978 & 12.377 & 1.328 & 10.716 & 0.720 & 0.187 \\
\hline $06523842+1513163$ & 3354530639711957376 & 12.440 & 0.657 & 12.456 & 0.941 & 11.303 & 0.563 & 0.109 \\
\hline $06525096+2440261$ & 3381304160163213056 & 13.028 & 1.045 & 12.726 & 1.328 & 11.005 & 0.700 & 0.087 \\
\hline
\end{tabular}


Table 2 (continued)

\begin{tabular}{|c|c|c|c|c|c|c|c|c|}
\hline $\begin{array}{c}\text { Star Name } \\
\text { (2MASS) }\end{array}$ & $\begin{array}{c}\text { Star Name } \\
\text { (Gaia EDR3) }\end{array}$ & $V$ & $(B-V)$ & $G$ & $\left(G_{\mathrm{BP}}-G_{\mathrm{RP}}\right)$ & $J$ & $(J-K)$ & $E(B-V)$ \\
\hline $06525451+1446083$ & 3354323111187785216 & 13.029 & 0.874 & 12.733 & 1.172 & 11.191 & 0.668 & 0.120 \\
\hline $06533075+1226225$ & 3351515542604966912 & 12.978 & 0.421 & 12.770 & 0.805 & 11.553 & 0.586 & 0.172 \\
\hline $06533083+6836050$ & 1103319172123035136 & 12.729 & 0.867 & 12.554 & 1.146 & 11.067 & 0.600 & 0.031 \\
\hline 06554143-4313578 & 5562195201959849984 & 12.774 & 0.992 & 12.506 & 1.294 & 10.833 & 0.696 & 0.083 \\
\hline $06560363+2846500$ & 887636359876055424 & 12.823 & 0.915 & 12.596 & 1.155 & 11.108 & 0.637 & 0.063 \\
\hline 06570150-6751413 & 5280814817608368000 & 10.818 & 0.932 & 10.520 & 1.228 & 8.918 & 0.610 & 0.110 \\
\hline 06571276-5526234 & 5485259315346971136 & 12.680 & 0.815 & 12.446 & 1.128 & 10.943 & 0.569 & 0.080 \\
\hline 06580901-8334199 & 5194228654877524096 & 12.858 & 0.927 & 12.487 & 1.295 & 10.862 & 0.687 & 0.159 \\
\hline $06585640+0850479$ & 3157301205994883840 & 12.181 & 0.724 & 12.542 & 1.164 & 11.037 & 0.604 & 0.115 \\
\hline 06593152-5853177 & 5480647173305937280 & 12.560 & 0.949 & 12.234 & 1.275 & 10.574 & 0.677 & 0.074 \\
\hline $06593924+2056439$ & 3366133201801121536 & 12.716 & 0.747 & 12.489 & 1.033 & 11.118 & 0.547 & 0.081 \\
\hline 07001955-3637535 & 5578346443656712960 & 11.727 & 0.803 & 11.401 & 0.973 & 10.314 & 0.561 & 0.200 \\
\hline $07003916+2701430$ & 884148846432586240 & 12.117 & 0.708 & 11.847 & 1.045 & 10.545 & 0.610 & 0.061 \\
\hline $07004613+2933178$ & 887873162895952768 & 12.959 & 0.958 & 12.667 & 1.235 & 11.084 & 0.671 & 0.075 \\
\hline $07012363+1813452$ & 3364377384810729088 & 12.746 & 0.648 & 12.499 & 1.000 & 11.169 & 0.541 & 0.061 \\
\hline 07013933-5842103 & 5480743208774977024 & 12.969 & 0.751 & 12.706 & 1.101 & 11.250 & 0.576 & 0.077 \\
\hline $07023862+0659243$ & 3153768440772179584 & 12.393 & 0.636 & 12.249 & 0.934 & 10.898 & 0.607 & 0.153 \\
\hline $07030318+4006286$ & 947804380629842816 & 12.404 & 0.731 & 12.169 & 1.061 & 10.770 & 0.551 & 0.073 \\
\hline $07033238+2225136$ & 3368024709696717824 & 12.461 & 0.984 & 12.121 & 1.261 & 10.490 & 0.648 & 0.044 \\
\hline $07035093+1955164$ & 3365212055873231104 & 12.492 & 0.808 & 12.215 & 1.008 & 10.820 & 0.637 & 0.067 \\
\hline $07041471+1901123$ & 3364898931280232448 & 12.995 & 1.049 & 12.586 & 1.407 & 10.854 & 0.740 & 0.054 \\
\hline 07041738-1908113 & 2932674107172462592 & 11.702 & 0.863 & 11.577 & 1.584 & 9.547 & 0.712 & 0.300 \\
\hline $07041880+0246488$ & 3115642187883221632 & 12.888 & 0.733 & 12.623 & 1.109 & 11.055 & 0.688 & 0.295 \\
\hline $07041957+2340564$ & 3368538353422137344 & 12.837 & 0.642 & 13.435 & 1.288 & 11.383 & 0.601 & 0.040 \\
\hline $07054097+1733350$ & 3361464812868710144 & 12.260 & 0.902 & 11.985 & 1.153 & 10.482 & 0.583 & 0.048 \\
\hline $07070364+2657035$ & 883254423785454080 & 12.659 & 0.748 & 12.437 & 1.090 & 10.971 & 0.547 & 0.051 \\
\hline $07073503+2356476$ & 3368586663216905984 & 12.837 & 1.196 & 12.425 & 1.383 & 10.670 & 0.680 & 0.049 \\
\hline 07081426-3557115 & 5566430245872289408 & 12.784 & 0.809 & 13.548 & 0.000 & 11.204 & 0.548 & 0.300 \\
\hline 07083867-4621128 & 5510018805377253632 & 12.211 & 1.051 & 11.654 & 1.337 & 10.009 & 0.764 & 0.101 \\
\hline $07085997-5424564$ & 5491233099459990528 & 12.703 & 0.674 & 12.487 & 0.998 & 11.164 & 0.538 & 0.106 \\
\hline $07090577+2142312$ & 3367308652748446464 & 12.976 & 0.917 & 12.747 & 1.195 & 11.182 & 0.655 & 0.048 \\
\hline $07093534+2210136$ & 3367373077257509120 & 11.956 & 0.638 & 11.824 & 0.789 & 10.969 & 0.536 & 0.050 \\
\hline 07093868-0419118 & 3107527762016884096 & 12.269 & 0.534 & 12.039 & 0.889 & 10.692 & 0.625 & 0.300 \\
\hline 07101117-2219008 & 2928210850185413120 & 12.886 & 0.785 & 12.543 & 1.122 & 11.087 & 0.692 & 0.300 \\
\hline $07102845+1245411$ & 3161108024483929600 & 12.687 & 0.773 & 12.420 & 1.104 & 10.962 & 0.587 & 0.045 \\
\hline 07103372-0244311 & 3108335765621350656 & 12.491 & 0.686 & 12.263 & 0.956 & 10.883 & 0.541 & 0.235 \\
\hline $07104970+0826444$ & 3154409902728232320 & 12.977 & 0.926 & 12.670 & 1.229 & 11.102 & 0.650 & 0.088 \\
\hline 07110190-6301262 & 5285981079149837184 & 11.470 & 0.700 & 11.241 & 1.002 & 9.954 & 0.586 & 0.104 \\
\hline $07113988+8541053$ & 1150345383223043712 & 12.825 & 0.852 & 12.623 & 1.107 & 11.209 & 0.563 & 0.082 \\
\hline $07121704+1402591$ & 3167279926849037568 & 12.940 & 1.041 & 12.593 & 1.316 & 10.913 & 0.684 & 0.059 \\
\hline $07122036+4221574$ & 949584631690298368 & 12.850 & 0.916 & 12.516 & 1.273 & 10.891 & 0.650 & 0.062 \\
\hline 07122182-8422429 & 5193956796331371264 & 12.745 & 0.799 & 12.461 & 1.142 & 10.968 & 0.608 & 0.149 \\
\hline
\end{tabular}


Table 2 (continued)

\begin{tabular}{|c|c|c|c|c|c|c|c|c|}
\hline $\begin{array}{c}\text { Star Name } \\
\text { (2MASS) }\end{array}$ & $\begin{array}{c}\text { Star Name } \\
\text { (Gaia EDR3) }\end{array}$ & $V$ & $(B-V)$ & $G$ & $\left(G_{\mathrm{BP}}-G_{\mathrm{RP}}\right)$ & $J$ & $(J-K)$ & $E(B-V)$ \\
\hline $07122917+2231366$ & 3367505087372386944 & 11.100 & 1.046 & 10.704 & 1.348 & 9.070 & 0.587 & 0.054 \\
\hline 07122989-0700106 & 3052191643892060672 & 11.589 & 1.057 & 11.166 & 1.475 & 9.229 & 0.626 & 0.300 \\
\hline 07123398-4814049 & 5508686643961355648 & 11.566 & 1.061 & 11.206 & 1.356 & 9.490 & 0.710 & 0.097 \\
\hline $07123461+3153463$ & 890055835212749696 & 12.508 & 0.928 & 13.236 & 1.361 & 11.557 & 0.720 & 0.055 \\
\hline 07130783-7052481 & 5267185649427578496 & 12.918 & 0.976 & 12.668 & 1.258 & 11.005 & 0.617 & 0.162 \\
\hline $07150401+2714015$ & 883489998448405504 & 12.412 & 0.786 & 12.481 & 1.012 & 10.852 & 0.587 & 0.055 \\
\hline $07151852-5252051$ & 5491893184393739776 & 12.093 & 0.865 & 11.784 & 1.233 & 10.177 & 0.649 & 0.132 \\
\hline 07152806-4111161 & 5561098267315218048 & 12.660 & 1.039 & 12.550 & 1.377 & 10.593 & 0.721 & 0.162 \\
\hline $07160059+5240188$ & 986603229693072128 & 12.762 & 0.734 & 12.480 & 0.982 & 11.178 & 0.510 & 0.067 \\
\hline $07170438+2402438$ & 869715076257527808 & 11.874 & 0.655 & 11.639 & 0.958 & 10.221 & 0.595 & 0.046 \\
\hline $07174600+1259027$ & 3166159975473025280 & 12.945 & 0.739 & 12.899 & 1.228 & 11.282 & 0.614 & 0.075 \\
\hline 07174929-0924096 & 3048017378051885056 & 11.804 & 0.786 & 11.592 & 1.047 & 10.190 & 0.536 & 0.300 \\
\hline 07175677-2704124 & 5616006675420673664 & 12.756 & 0.828 & 12.624 & 1.087 & 11.375 & 0.674 & 0.300 \\
\hline $07181152+4406479$ & 973981901357263104 & 12.923 & 0.928 & 12.515 & 1.161 & 11.177 & 0.669 & 0.071 \\
\hline 07194881-5748087 & 5485933071160480768 & 13.020 & 0.994 & 12.478 & 1.345 & 10.703 & 0.696 & 0.112 \\
\hline 07203081-6240451 & 5291814602512817024 & 12.955 & 0.834 & 12.614 & 1.268 & 10.984 & 0.662 & 0.110 \\
\hline 07204786-7623393 & 5214066868299703040 & 12.797 & 0.804 & 12.543 & 1.095 & 11.108 & 0.562 & 0.195 \\
\hline $07213106+2755248$ & 872994571549210240 & 10.559 & 0.756 & 10.376 & 0.953 & 9.060 & 0.554 & 0.049 \\
\hline $07220367+0253575$ & 3136142616543571456 & 12.806 & 1.060 & 12.474 & 1.303 & 10.830 & 0.709 & 0.080 \\
\hline $07224055+3618087$ & 897278351003895424 & 12.813 & 0.892 & 12.740 & 1.182 & 10.918 & 0.635 & 0.046 \\
\hline 07224582-2959375 & 5605629995767584000 & 13.510 & 0.070 & 12.936 & 1.009 & 11.569 & 0.599 & 0.196 \\
\hline $07233679+1412112$ & 3166723543901552000 & 12.922 & 0.957 & 12.554 & 1.301 & 10.882 & 0.704 & 0.102 \\
\hline 07234039-2959296 & 5605624738733670912 & 11.877 & 0.350 & 15.434 & 1.236 & 13.730 & 0.567 & 0.198 \\
\hline $07234262+1516310$ & 3167138884419576064 & 12.795 & 0.869 & 12.578 & 1.149 & 11.094 & 0.588 & 0.079 \\
\hline $07242428+5822232$ & 989627947524580864 & 10.960 & 1.053 & 10.556 & 1.344 & 8.830 & 0.656 & 0.050 \\
\hline $07254156+4948144$ & 977051149410569344 & 12.991 & 0.814 & 12.868 & 1.065 & 11.549 & 0.632 & 0.071 \\
\hline 07254769-3453506 & 5590014289255060480 & 11.036 & 0.790 & 10.889 & 1.090 & 9.439 & 0.633 & 0.300 \\
\hline 07260648-4637077 & 5510340717467331456 & 12.657 & 1.031 & 12.306 & 1.395 & 10.551 & 0.765 & 0.191 \\
\hline $07270798+4352580$ & 973215713552094848 & 12.907 & 0.983 & 12.633 & 1.273 & 10.978 & 0.636 & 0.066 \\
\hline $07273288+3819555$ & 899749713837935872 & 12.958 & 1.034 & 12.650 & 1.259 & 11.034 & 0.646 & 0.051 \\
\hline $07280531+1637140$ & 3169561074176213376 & 11.969 & 0.794 & 11.821 & 1.036 & 10.485 & 0.548 & 0.074 \\
\hline 07281913-8333342 & 5194416392190823040 & 11.951 & 0.706 & 11.608 & 1.103 & 10.215 & 0.596 & 0.122 \\
\hline 07282428-3705347 & 5586496638018390784 & 12.046 & 0.858 & 11.752 & 1.085 & 10.483 & 0.628 & 0.284 \\
\hline $07284694+8149439$ & 1142689281896442880 & 11.671 & 0.792 & 11.459 & 1.076 & 10.024 & 0.568 & 0.037 \\
\hline 07295359-6910360 & 5267963068571164928 & 12.524 & 0.940 & 12.186 & 1.255 & 10.632 & 0.666 & 0.232 \\
\hline $07304303+4111480$ & 900640730573598976 & 12.861 & 1.071 & 12.406 & 1.488 & 10.535 & 0.723 & 0.055 \\
\hline 07310602-2742595 & 5611975414810555264 & 11.367 & 0.874 & 11.147 & 1.102 & 9.786 & 0.601 & 0.300 \\
\hline 07311836-6659351 & 5269135942537559680 & 12.388 & 1.119 & 11.932 & 1.485 & 10.122 & 0.712 & 0.115 \\
\hline $07314455+5553592$ & 988736827710024704 & 12.938 & 1.057 & 12.520 & 1.411 & 10.751 & 0.727 & 0.059 \\
\hline 07314605-3324217 & 5591704921522389888 & 12.662 & 0.359 & 12.472 & 0.762 & 11.170 & 0.738 & 0.298 \\
\hline $07321280+1523588$ & 3168444240942341248 & 12.809 & 0.905 & 12.657 & 1.193 & 10.907 & 0.626 & 0.045 \\
\hline 07321479-1300309 & 3033439198205025024 & 12.868 & 0.893 & 12.841 & 1.461 & 10.920 & 0.746 & 0.297 \\
\hline
\end{tabular}


Table 2 (continued)

\begin{tabular}{|c|c|c|c|c|c|c|c|c|}
\hline $\begin{array}{c}\text { Star Name } \\
\text { (2MASS) }\end{array}$ & $\begin{array}{c}\text { Star Name } \\
\text { (Gaia EDR3) }\end{array}$ & $V$ & $(B-V)$ & $G$ & $\left(G_{\mathrm{BP}}-G_{\mathrm{RP}}\right)$ & $J$ & $(J-K)$ & $E(B-V)$ \\
\hline $07325519+3655267$ & 898637038134266752 & 12.695 & 0.966 & 12.343 & 1.272 & 10.705 & 0.665 & 0.048 \\
\hline $07331792+0531033$ & 3141404295078246400 & 12.977 & 0.982 & 12.696 & 1.269 & 11.014 & 0.654 & 0.046 \\
\hline $07333190+5310203$ & 983909666723291392 & 10.432 & 0.246 & 10.322 & 0.564 & 9.304 & 0.709 & 0.050 \\
\hline $07334024+4425362$ & 973487288627865728 & 12.697 & 0.933 & 12.518 & 1.192 & 10.867 & 0.605 & 0.052 \\
\hline $07345045+0736494$ & 3142272222070528384 & 12.622 & 1.149 & 12.653 & 1.558 & 10.267 & 0.715 & 0.043 \\
\hline 07350484-0004500 & 3086417752932494848 & 11.957 & 1.075 & 11.541 & 1.400 & 9.800 & 0.708 & 0.112 \\
\hline $07351435+2626331$ & 872041539782121984 & 13.006 & 0.962 & 12.595 & 1.256 & 10.951 & 0.678 & 0.045 \\
\hline 07351699-4815064 & 5506540186806281728 & 12.979 & 0.930 & 12.671 & 1.185 & 11.217 & 0.646 & 0.175 \\
\hline $07353628+1938556$ & 672591122640513280 & 12.553 & 0.857 & 12.371 & 1.034 & 10.928 & 0.563 & 0.046 \\
\hline $07354551+0455028$ & 3138308624388237568 & 12.852 & 0.792 & 12.426 & 1.181 & 10.880 & 0.718 & 0.062 \\
\hline 07354757-5036104 & 5493916801186715648 & 12.851 & 0.780 & 12.808 & 1.111 & 11.198 & 0.620 & 0.175 \\
\hline $07363720+1126504$ & 3162146822454143360 & 12.530 & 0.977 & 12.225 & 1.259 & 10.686 & 0.730 & 0.029 \\
\hline $07363729+4034177$ & 924375132168707200 & 12.343 & 1.120 & 11.945 & 1.433 & 10.182 & 0.708 & 0.048 \\
\hline $07364486+4508331$ & 926998601271917184 & 11.560 & 0.774 & 11.371 & 1.010 & 10.061 & 0.588 & 0.053 \\
\hline $07365679+5950385$ & 1086214756921278976 & 12.505 & 1.203 & 11.986 & 1.530 & 10.101 & 0.730 & 0.060 \\
\hline 07374300-5630568 & 54877373740385847704 & 11.927 & 0.818 & 11.655 & 1.159 & 10.155 & 0.643 & 0.154 \\
\hline 07380041-2129196 & 5619082589253324544 & 12.078 & 1.159 & 11.877 & 1.455 & 9.869 & 0.685 & 0.300 \\
\hline 07384500-0331557 & 3057721981216813568 & 11.469 & 0.738 & 11.223 & 1.036 & 9.880 & 0.557 & 0.083 \\
\hline 07393047-1530275 & 3029020260970100736 & 11.309 & 0.995 & 10.929 & 1.286 & 9.268 & 0.726 & 0.300 \\
\hline $07395820+1311537$ & 3164103029736387200 & 12.973 & 0.872 & 12.720 & 1.122 & 11.284 & 0.659 & 0.047 \\
\hline 07400327-0740587 & 3042138568557247360 & 11.511 & 0.807 & 11.223 & 1.115 & 9.751 & 0.599 & 0.117 \\
\hline $07403555+5352561$ & 985231550281510144 & 11.858 & 1.119 & 11.508 & 1.477 & 9.587 & 0.751 & 0.044 \\
\hline $07404482+1243437$ & 3163865500863822592 & 11.773 & 1.017 & 11.385 & 1.368 & 9.646 & 0.677 & 0.040 \\
\hline 07410359-6714551 & 5274884459907753344 & 12.851 & 1.067 & 12.486 & 1.340 & 10.714 & 0.692 & 0.116 \\
\hline 07410851-5943543 & 5292748779377543168 & 12.816 & 0.906 & 12.449 & 1.213 & 10.989 & 0.662 & 0.206 \\
\hline 07412077-2900201 & 5599777230966529024 & 12.749 & 0.859 & 12.477 & 1.165 & 10.978 & 0.612 & 0.300 \\
\hline 07414196-0741080 & 3042148704679838336 & 13.007 & 0.774 & 13.045 & 1.154 & 11.345 & 0.568 & 0.100 \\
\hline $07435834+1341353$ & 3164056506649463040 & 12.873 & 0.829 & 12.623 & 1.063 & 11.223 & 0.607 & 0.055 \\
\hline $07440179+0312226$ & 3136905750633082624 & 13.032 & 0.916 & 12.631 & 1.137 & 11.251 & 0.629 & 0.048 \\
\hline $07441635+5322545$ & 984534979601381120 & 11.773 & 1.002 & 11.420 & 1.252 & 9.854 & 0.649 & 0.036 \\
\hline $07443402+0742591$ & 3145150125036773760 & 12.590 & 0.498 & 12.491 & 0.675 & 11.875 & 0.499 & 0.034 \\
\hline 07443970-4425135 & 5532006972050819584 & 12.412 & 0.994 & 12.021 & 1.380 & 10.208 & 0.713 & 0.300 \\
\hline 07445367-4653249 & 5530553525058225152 & 12.106 & 0.880 & 11.797 & 1.130 & 10.379 & 0.627 & 0.169 \\
\hline $07450351+0956148$ & 3148979071201226880 & 11.373 & 0.959 & 11.041 & 1.279 & 9.454 & 0.703 & 0.023 \\
\hline 07450424-7231255 & 5263155252116820608 & 11.621 & 0.294 & 11.467 & 0.665 & 10.402 & 0.601 & 0.183 \\
\hline $07451488+1000107$ & 3149076244837702784 & 12.941 & 0.710 & 12.661 & 1.019 & 11.370 & 0.611 & 0.022 \\
\hline $07452477+2524147$ & 868026810512553856 & 12.828 & 0.983 & 12.615 & 1.197 & 11.003 & 0.645 & 0.039 \\
\hline $07455398+0103107$ & 3086833647505226752 & 12.837 & 0.619 & 12.691 & 0.785 & 11.845 & 0.529 & 0.070 \\
\hline $07461952+3903325$ & 920303430156012160 & 12.293 & 0.916 & 11.975 & 1.206 & 10.403 & 0.627 & 0.045 \\
\hline 07464924-4313456 & 5532490211705643904 & 11.826 & 0.725 & 11.611 & 0.957 & 10.454 & 0.547 & 0.284 \\
\hline 07480156-4924040 & 5518045893097566976 & 12.262 & 0.909 & 11.927 & 1.272 & 10.270 & 0.676 & 0.202 \\
\hline $07485521+5623334$ & 1082018707015827584 & 12.452 & 0.933 & 12.130 & 1.256 & 10.438 & 0.711 & 0.071 \\
\hline
\end{tabular}


Table 2 (continued)

\begin{tabular}{|c|c|c|c|c|c|c|c|c|}
\hline $\begin{array}{l}\text { Star Name } \\
\text { (2MASS) }\end{array}$ & $\begin{array}{c}\text { Star Name } \\
\text { (Gaia EDR3) }\end{array}$ & $V$ & $(B-V)$ & $G$ & $\left(G_{\mathrm{BP}}-G_{\mathrm{RP}}\right)$ & $J$ & $(J-K)$ & $E(B-V)$ \\
\hline $07494258+6938239$ & 1098142705736502528 & 12.964 & 0.951 & 12.717 & 1.238 & 11.115 & 0.697 & 0.030 \\
\hline $07503801+1119098$ & 3150778696857500928 & 12.548 & 0.910 & 12.270 & 1.194 & 10.726 & 0.602 & 0.024 \\
\hline $07504673+0558247$ & 3143849888115383040 & 11.534 & 0.850 & 11.286 & 1.082 & 9.894 & 0.566 & 0.020 \\
\hline $07533988+4933361$ & 934072756004889216 & 12.418 & 0.674 & 12.106 & 0.983 & 10.799 & 0.528 & 0.043 \\
\hline $07545950+6639532$ & 1095319709633456000 & 13.017 & 0.819 & 12.800 & 1.076 & 11.409 & 0.588 & 0.035 \\
\hline $07551255+1944081$ & 670305998176686720 & 12.556 & 0.894 & 12.292 & 1.195 & 10.756 & 0.644 & 0.049 \\
\hline $07552395+3520063$ & 906645575890268416 & 12.507 & 1.108 & 12.233 & 1.451 & 10.268 & 0.726 & 0.044 \\
\hline $07555507+8148305$ & 1148451100783017984 & 12.778 & 1.013 & 12.424 & 1.366 & 10.735 & 0.693 & 0.027 \\
\hline $07575895+4035155$ & 922132399029177088 & 12.864 & 0.973 & 12.511 & 1.280 & 10.858 & 0.645 & 0.041 \\
\hline $07580568+3504110$ & 906456700407472512 & 12.810 & 0.781 & 13.873 & 1.219 & 12.313 & 0.622 & 0.047 \\
\hline $07584532+4211214$ & 922545677962459904 & 12.401 & 0.814 & 12.057 & 1.131 & 10.512 & 0.649 & 0.037 \\
\hline $07590580+4748300$ & 933111228791082240 & 12.081 & 0.965 & 11.898 & 1.246 & 10.136 & 0.712 & 0.037 \\
\hline $08005431+3135329$ & 878032675562889728 & 12.932 & 0.775 & 14.086 & 1.508 & 12.221 & 0.733 & 0.046 \\
\hline $08005634+1633254$ & 667243544758539136 & 12.465 & 0.753 & 12.095 & 1.148 & 10.581 & 0.581 & 0.031 \\
\hline $08011676+6358383$ & 1094412028423846144 & 12.264 & 0.993 & 12.000 & 1.219 & 10.471 & 0.668 & 0.043 \\
\hline 08021251-2821568 & 5597762543400727680 & 12.014 & 0.140 & 15.980 & 1.435 & 13.905 & 0.604 & 0.300 \\
\hline 08022771-4804247 & 5517895294366243968 & 12.952 & 0.865 & 12.637 & 1.148 & 11.151 & 0.627 & 0.236 \\
\hline 08024574-4803573 & 5517708957205335168 & 11.977 & 0.894 & 11.872 & 1.201 & 10.135 & 0.618 & 0.240 \\
\hline 08042788-6440110 & 5275614368128678528 & 11.911 & 0.749 & 11.670 & 1.070 & 10.258 & 0.570 & 0.141 \\
\hline $08045600+3310059$ & 905235555306335104 & 12.982 & 0.949 & 12.689 & 1.180 & 11.159 & 0.626 & 0.050 \\
\hline $08050775+2604105$ & 681971846610387712 & 11.856 & 0.743 & 11.576 & 1.050 & 10.071 & 0.651 & 0.032 \\
\hline 08052894-6502132 & 5275590007074529152 & 12.456 & 0.879 & 12.141 & 1.243 & 10.483 & 0.609 & 0.172 \\
\hline 08053267-6119242 & 5289953163686735232 & 12.467 & 0.869 & 12.245 & 1.094 & 10.760 & 0.634 & 0.151 \\
\hline 08074491-5257227 & 5512588600272046848 & 11.823 & 0.878 & 11.682 & 1.133 & 10.163 & 0.582 & 0.300 \\
\hline $08080715+5915114$ & 1083546379639153664 & 12.654 & 0.902 & 12.323 & 1.264 & 10.739 & 0.637 & 0.045 \\
\hline 08085268-6128294 & 5289751265864064128 & 12.833 & 0.770 & 12.570 & 1.112 & 11.080 & 0.533 & 0.146 \\
\hline $08090031+2850085$ & 876519232166668800 & 11.747 & 0.790 & 11.491 & 1.095 & 10.049 & 0.594 & 0.035 \\
\hline $08092156+2521288$ & 681134774663802496 & 12.725 & 0.892 & 12.428 & 1.154 & 10.931 & 0.627 & 0.038 \\
\hline 08100860-7523572 & 5213699150380190720 & 13.017 & 0.902 & 12.696 & 1.250 & 11.057 & 0.674 & 0.119 \\
\hline $08102898+4020333$ & 909546843477733504 & 12.726 & 0.646 & 12.443 & 1.220 & 10.692 & 0.692 & 0.043 \\
\hline $08111719+4456352$ & 929054103899434752 & 12.996 & 0.925 & 12.708 & 1.194 & 11.190 & 0.618 & 0.034 \\
\hline 08125962-7004235 & 5270057397705227904 & 12.902 & 1.070 & 12.483 & 1.332 & 10.900 & 0.711 & 0.133 \\
\hline $08130864+3925564$ & 909255575975553920 & 12.445 & 0.766 & 12.196 & 1.151 & 10.708 & 0.623 & 0.033 \\
\hline $08135674+5113349$ & 935284722760688384 & 11.897 & 0.692 & 11.889 & 0.970 & 10.434 & 0.557 & 0.048 \\
\hline $08140229+3940332$ & 909264917528317952 & 12.106 & 0.808 & 11.816 & 1.230 & 10.274 & 0.684 & 0.033 \\
\hline $08152203+3827587$ & 908032124475412224 & 12.441 & 0.795 & 12.265 & 1.066 & 10.819 & 0.594 & 0.035 \\
\hline $08153796+6531162$ & 1092014470303207552 & 12.335 & 1.157 & 11.990 & 1.351 & 10.275 & 0.670 & 0.042 \\
\hline 08162824-4611151 & 5519745360123251200 & 11.715 & 0.880 & 11.438 & 1.078 & 10.084 & 0.587 & 0.300 \\
\hline $08173327-2728418$ & 5693425380770623616 & 12.591 & 0.870 & 12.464 & 1.160 & 10.892 & 0.586 & 0.187 \\
\hline $08182058+5923087$ & 1083715257753356800 & 12.184 & 0.872 & 11.876 & 1.284 & 10.112 & 0.721 & 0.061 \\
\hline 08202927-2458369 & 5696053561463700992 & 12.244 & 0.922 & 11.938 & 1.140 & 10.559 & 0.618 & 0.116 \\
\hline 08204593-7457097 & 5219742375524510720 & 12.547 & 0.738 & 12.478 & 1.054 & 10.925 & 0.605 & 0.114 \\
\hline
\end{tabular}


Table 2 (continued)

\begin{tabular}{|c|c|c|c|c|c|c|c|c|}
\hline $\begin{array}{l}\text { Star Name } \\
\text { (2MASS) }\end{array}$ & $\begin{array}{c}\text { Star Name } \\
\text { (Gaia EDR3) }\end{array}$ & $V$ & $(B-V)$ & $G$ & $\left(G_{\mathrm{BP}}-G_{\mathrm{RP}}\right)$ & $J$ & $(J-K)$ & $E(B-V)$ \\
\hline $08225420+6259266$ & 1090362836337748864 & 12.783 & 1.104 & 12.472 & 1.302 & 10.825 & 0.689 & 0.034 \\
\hline 08242044-1026147 & 5751499802540091776 & 13.047 & 1.054 & 12.691 & 1.371 & 10.879 & 0.735 & 0.033 \\
\hline 08244155-6859542 & 5270209581283002240 & 11.994 & 0.732 & 11.808 & 0.987 & 10.575 & 0.572 & 0.113 \\
\hline $08255445+7726501$ & 1137625614237615232 & 12.980 & 0.830 & 12.753 & 1.109 & 11.364 & 0.625 & 0.026 \\
\hline 08282505-2119200 & 5706111756390126080 & 12.989 & 0.611 & 12.644 & 1.131 & 11.016 & 0.601 & 0.089 \\
\hline 08284828-2858468 & 5644860540592289536 & 12.711 & 0.764 & 12.554 & 1.070 & 11.050 & 0.694 & 0.153 \\
\hline 08295142-2428117 & 5695713503130206848 & 12.814 & 0.864 & 12.607 & 1.141 & 11.092 & 0.593 & 0.092 \\
\hline 08295611-1354544 & 5722897519371974272 & 12.216 & 0.925 & 11.925 & 1.217 & 10.285 & 0.587 & 0.048 \\
\hline 08302532-6949046 & 5221888519143226240 & 12.209 & 0.982 & 11.849 & 1.358 & 10.106 & 0.675 & 0.112 \\
\hline 08312114-2126535 & 5703185367534835584 & 12.154 & 0.839 & 11.789 & 1.170 & 10.312 & 0.606 & 0.085 \\
\hline $08315869+6949533$ & 1121420775645432320 & 13.021 & 0.702 & 12.776 & 0.960 & 11.528 & 0.527 & 0.032 \\
\hline 08322899-2137273 & 5703121870736546304 & 12.228 & 0.997 & 12.032 & 1.294 & 10.202 & 0.699 & 0.087 \\
\hline 08331960-1350331 & 5722870096004958208 & 12.078 & 0.670 & 11.889 & 0.999 & 10.412 & 0.605 & 0.049 \\
\hline $08343393+7518493$ & 1125113279289236096 & 11.158 & 0.741 & 10.977 & 1.087 & 9.518 & 0.642 & 0.019 \\
\hline $08350584+4839306$ & 1026860572337001088 & 12.287 & 0.719 & 12.047 & 1.096 & 10.634 & 0.600 & 0.028 \\
\hline 08355055-2033433 & 5703594458875614848 & 11.411 & 1.178 & 10.943 & 1.525 & 9.126 & 0.772 & 0.077 \\
\hline 08355851-0839549 & 5753641990492978816 & 12.938 & 0.808 & 12.673 & 1.091 & 11.231 & 0.581 & 0.037 \\
\hline 08360617-0450149 & 3065446698936865152 & 12.610 & 0.990 & 12.138 & 1.336 & 10.424 & 0.663 & 0.030 \\
\hline $08364858+7742308$ & 1137832945195154560 & 12.697 & 0.742 & 12.489 & 1.045 & 11.161 & 0.578 & 0.017 \\
\hline $08370406+7830159$ & 1138150772775117312 & 12.553 & 0.752 & 12.345 & 1.058 & 10.944 & 0.581 & 0.018 \\
\hline 08400533-4428341 & 5522379549464634752 & 11.201 & 1.167 & 10.778 & 1.492 & 8.855 & 0.654 & 0.300 \\
\hline $08401347+4216411$ & 913518485634602112 & 12.151 & 1.011 & 11.631 & 1.314 & 10.049 & 0.684 & 0.020 \\
\hline 08413166-0629117 & 5754432023955507712 & 12.784 & 0.712 & 12.602 & 1.022 & 11.034 & 0.623 & 0.023 \\
\hline 08422928-0849161 & 5750516216373911680 & 12.176 & 0.847 & 11.986 & 1.110 & 10.494 & 0.597 & 0.028 \\
\hline $08430958+6038165$ & 1041278571391227392 & 12.858 & 1.002 & 12.476 & 1.306 & 10.902 & 0.732 & 0.075 \\
\hline $08433739+6717034$ & 1093203940022382720 & 12.603 & 1.150 & 12.157 & 1.600 & 10.183 & 0.747 & 0.029 \\
\hline 08434698-1812314 & 5705872333433002880 & 11.974 & 0.897 & 11.687 & 1.168 & 10.173 & 0.599 & 0.068 \\
\hline $08441851+0346257$ & 581348807340055424 & 12.468 & 1.184 & 12.025 & 1.539 & 10.055 & 0.734 & 0.033 \\
\hline 08443695-7241450 & $\cdots$ & 11.497 & 0.929 & $\ldots$ & $\ldots$ & 9.440 & 0.675 & 0.118 \\
\hline 08444676-2845157 & 5642803457417047552 & 12.763 & 0.872 & 12.515 & 1.190 & 10.849 & 0.711 & 0.147 \\
\hline 08445228-7438443 & 5216771215930182912 & 12.975 & 0.841 & 12.634 & 1.121 & 11.189 & 0.586 & 0.121 \\
\hline 08454426-2141380 & 5702387126388268160 & 11.438 & 0.995 & 11.126 & 1.254 & 9.481 & 0.675 & 0.139 \\
\hline 08460663-0426024 & 5761503056249343360 & 11.639 & 0.786 & 11.239 & 1.122 & 9.558 & 0.684 & 0.020 \\
\hline $08472137+5830138$ & 1040480914362286592 & 12.326 & 0.852 & 11.996 & 1.161 & 10.492 & 0.618 & 0.055 \\
\hline $08473213+0548147$ & 582973163970364416 & 11.616 & 0.773 & 11.337 & 1.072 & 9.961 & 0.568 & 0.041 \\
\hline 08490347-6323398 & 5297691668265349120 & 10.503 & 0.558 & 10.358 & 0.793 & 9.683 & 0.672 & 0.159 \\
\hline 08490462-6853235 & 5223489373715006976 & 12.246 & 0.870 & 12.032 & 1.092 & 10.626 & 0.548 & 0.102 \\
\hline 08494240-3836003 & 5621994959343177856 & 11.410 & 1.019 & 11.110 & 1.266 & 9.370 & 0.706 & 0.300 \\
\hline $08501165+4242063$ & 913730107265419136 & 12.504 & 0.836 & 12.166 & 1.365 & 10.472 & 0.698 & 0.023 \\
\hline 08511780-2736072 & 5648942030830217344 & 12.453 & 0.835 & 12.204 & 1.132 & 10.747 & 0.618 & 0.099 \\
\hline $08514213+1250467$ & 608133907024045952 & 10.987 & 0.865 & 10.752 & 1.159 & 9.200 & 0.619 & 0.027 \\
\hline $08515469+5402598$ & 1029765967389921408 & 12.648 & 0.675 & 12.587 & 0.993 & 11.159 & 0.556 & 0.020 \\
\hline
\end{tabular}


Table 2 (continued)

\begin{tabular}{|c|c|c|c|c|c|c|c|c|}
\hline $\begin{array}{l}\text { Star Name } \\
\text { (2MASS) }\end{array}$ & $\begin{array}{c}\text { Star Name } \\
\text { (Gaia EDR3) }\end{array}$ & $V$ & $(B-V)$ & $G$ & $\left(G_{\mathrm{BP}}-G_{\mathrm{RP}}\right)$ & $J$ & $(J-K)$ & $E(B-V)$ \\
\hline 08523133-0804579 & 5750792365590211328 & 12.177 & 0.928 & 11.860 & 1.237 & 10.292 & 0.697 & 0.032 \\
\hline $08524186+6924318$ & 1118241228535948672 & 12.099 & 0.838 & 11.771 & 1.111 & 10.373 & 0.584 & 0.031 \\
\hline $08525722+6423125$ & 1044196095432573952 & 12.852 & 0.807 & 12.572 & 1.178 & 11.081 & 0.577 & 0.070 \\
\hline $08533398+1644112$ & 611494731817426560 & 12.944 & 0.826 & 12.641 & 1.203 & 11.004 & 0.626 & 0.019 \\
\hline 08543649-4744205 & 5328756032854574592 & 11.770 & 1.115 & 11.271 & 1.611 & 9.208 & 0.722 & 0.300 \\
\hline 08544986-1159089 & 5736694942894396672 & 13.016 & 0.855 & 12.834 & 1.158 & 11.203 & 0.673 & 0.043 \\
\hline 08554225-6955045 & 5222449162695490432 & 12.469 & 0.729 & 12.211 & 1.073 & 10.762 & 0.549 & 0.078 \\
\hline 08565578-7603590 & 5215675105917524608 & 12.759 & 1.109 & 12.624 & 1.491 & 10.347 & 0.786 & 0.104 \\
\hline $08571123+0040261$ & 576743880843785088 & 11.615 & 0.982 & 11.370 & 1.237 & 9.790 & 0.699 & 0.033 \\
\hline 08571600-6556117 & 5296305287178801280 & 11.803 & 0.737 & 11.578 & 1.051 & 10.173 & 0.537 & 0.127 \\
\hline 09002061-2220490 & 5654811563195280512 & 11.955 & 0.821 & 11.733 & 1.051 & 10.337 & 0.576 & 0.204 \\
\hline 09002174-3342353 & 5627698985144472448 & 12.858 & 0.758 & 12.559 & 1.084 & 11.075 & 0.683 & 0.256 \\
\hline 09012132-7055369 & 5222157448519411584 & 12.492 & 0.839 & 12.334 & 1.079 & 10.875 & 0.562 & 0.082 \\
\hline 09023125-0810363 & 5755853112670730112 & 11.186 & 0.971 & 10.880 & 1.235 & 9.349 & 0.656 & 0.036 \\
\hline 09030506-2047415 & 5656156368995636608 & 11.327 & 0.956 & 11.024 & 1.223 & 9.426 & 0.646 & 0.151 \\
\hline 09030861-0427115 & 5759379998080221824 & 12.279 & 0.702 & 12.029 & 1.004 & 10.612 & 0.591 & 0.018 \\
\hline 09040262-0523492 & 5759068836288541696 & 12.095 & 0.958 & 11.793 & 1.200 & 10.260 & 0.679 & 0.017 \\
\hline $09043992+0733498$ & 584226980887837824 & 11.406 & 0.789 & 11.128 & 1.094 & 9.728 & 0.579 & 0.049 \\
\hline 09050894-2050426 & 5656143484093610368 & 12.597 & 1.030 & 12.418 & 1.298 & 10.637 & 0.686 & 0.131 \\
\hline $09065688+5836407$ & 1036942720302654080 & 12.745 & 0.669 & 12.677 & 1.046 & 11.365 & 0.592 & 0.029 \\
\hline 09073395-0340147 & 5759902820154063744 & 13.027 & 1.032 & 12.636 & 1.330 & 10.990 & 0.745 & 0.018 \\
\hline 09090697-0324211 & 5759936900718820864 & 12.938 & 0.790 & 12.682 & 1.081 & 11.273 & 0.581 & 0.018 \\
\hline 09093944-2006527 & 5679552155368264704 & 12.953 & 0.870 & 12.708 & 1.109 & 11.261 & 0.548 & 0.129 \\
\hline $09102690+6033156$ & 1039441978952648064 & 11.630 & 1.218 & 11.500 & 1.278 & 9.826 & 0.684 & 0.039 \\
\hline $09103058+6419220$ & 1043675820274039040 & 12.681 & 0.884 & 12.379 & 1.325 & 10.685 & 0.678 & 0.100 \\
\hline 09104309-1444185 & 5731383034718059520 & 12.118 & 1.008 & 11.779 & 1.299 & 10.132 & 0.649 & 0.044 \\
\hline 09110582-2014595 & 5679493090978018304 & 11.381 & 0.948 & 11.092 & 1.214 & 9.597 & 0.642 & 0.122 \\
\hline $09110758+0217287$ & 3843821216808928512 & 12.946 & 0.853 & 12.741 & 1.131 & 11.207 & 0.604 & 0.030 \\
\hline $09114163+1017524$ & 591768294919763072 & 12.091 & 0.961 & 11.794 & 1.237 & 10.211 & 0.673 & 0.049 \\
\hline 09122631-0037340 & 3842047846288227712 & 12.098 & 0.946 & 11.855 & 1.271 & 10.133 & 0.643 & 0.027 \\
\hline $09123863+6218061$ & 1040178033268005504 & 12.458 & 0.632 & 12.303 & 1.035 & 10.911 & 0.563 & 0.037 \\
\hline 09131573-1716172 & 5682721055254568576 & 11.553 & 0.881 & 11.259 & 1.169 & 9.792 & 0.670 & 0.040 \\
\hline 09152673-0018170 & 3842238950857112064 & 12.998 & 0.832 & 12.676 & 1.185 & 11.080 & 0.693 & 0.026 \\
\hline $09155397+1212314$ & 593696288558989312 & 11.329 & 0.704 & 11.109 & 0.956 & 9.811 & 0.548 & 0.023 \\
\hline $09161100+0140496$ & 3845027557158758144 & 11.954 & 1.163 & 11.428 & 1.530 & 9.574 & 0.727 & 0.021 \\
\hline 09164357-0512382 & 5758526021141799552 & 12.727 & 0.915 & 12.428 & 1.220 & 10.875 & 0.667 & 0.031 \\
\hline 09171078-6147067 & 5298830659236242688 & 11.059 & 0.874 & 10.803 & 1.189 & 9.263 & 0.625 & 0.157 \\
\hline 09172903-1021252 & 5742681753643854464 & 12.118 & 1.124 & 11.602 & 1.521 & 9.741 & 0.737 & 0.047 \\
\hline 09180855-0852126 & 5743138252832504576 & 12.184 & 0.825 & 11.910 & 1.130 & 10.418 & 0.573 & 0.037 \\
\hline $09184848+0713360$ & 586821454667385344 & 13.005 & 0.759 & 12.741 & 1.060 & 11.362 & 0.566 & 0.035 \\
\hline $09185431+1211488$ & 593613206711676800 & 13.016 & 1.051 & 12.569 & 1.413 & 10.839 & 0.700 & 0.025 \\
\hline 09201726-0554052 & 5746372328846303360 & 12.956 & 1.148 & 12.760 & 1.445 & 10.785 & 0.743 & 0.026 \\
\hline
\end{tabular}


Table 2 (continued)

\begin{tabular}{|c|c|c|c|c|c|c|c|c|}
\hline $\begin{array}{l}\text { Star Name } \\
\text { (2MASS) }\end{array}$ & $\begin{array}{c}\text { Star Name } \\
\text { (Gaia EDR3) }\end{array}$ & $V$ & $(B-V)$ & $G$ & $\left(G_{\mathrm{BP}}-G_{\mathrm{RP}}\right)$ & $J$ & $(J-K)$ & $E(B-V)$ \\
\hline $09205040+1820344$ & 632540488180370176 & 13.046 & 0.854 & 12.901 & 1.185 & 11.172 & 0.627 & 0.024 \\
\hline $09214717+6219538$ & 1040038326571016704 & 11.446 & 0.842 & 11.220 & 1.174 & 9.725 & 0.612 & 0.034 \\
\hline $09220002+0957599$ & 592248296169483264 & 12.714 & 1.084 & 12.225 & 1.373 & 10.495 & 0.720 & 0.034 \\
\hline $09221426+5824580$ & 1038229698662764160 & 12.175 & 0.679 & 12.048 & 1.026 & 10.705 & 0.536 & 0.026 \\
\hline 09221563-7701423 & 5203340376458000128 & 11.493 & 1.020 & 11.165 & 1.332 & 9.542 & 0.718 & 0.262 \\
\hline 09222115-4317486 & 5424356193758211456 & 11.999 & 0.760 & 11.532 & 1.154 & 9.970 & 0.701 & 0.300 \\
\hline $09230164+5819465$ & 1026218874158310272 & 12.348 & 1.106 & 12.516 & 1.587 & 10.012 & 0.771 & 0.026 \\
\hline $09270301+4204494$ & 814375831397158144 & 12.841 & 0.874 & 12.596 & 1.116 & 11.178 & 0.632 & 0.014 \\
\hline $09270844+1544438$ & 630694854833847168 & 11.902 & 0.798 & 11.632 & 1.119 & 10.126 & 0.549 & 0.026 \\
\hline 09282183-3930462 & 5429709754532474880 & 11.963 & 0.828 & 11.654 & 1.119 & 10.273 & 0.661 & 0.300 \\
\hline $09285510+1543302$ & 619055111209080832 & 12.443 & 0.790 & 12.221 & 1.059 & 10.821 & 0.561 & 0.025 \\
\hline $09290292+3549540$ & 798505274404072832 & 12.186 & 0.753 & 11.992 & 1.049 & 10.453 & 0.640 & 0.014 \\
\hline 09291542-1847544 & 5678392273680123520 & 12.099 & 0.794 & 11.841 & 1.080 & 10.409 & 0.647 & 0.052 \\
\hline $09301030+0243320$ & 3844656021013252992 & 12.875 & 0.828 & 12.576 & 1.155 & 11.064 & 0.596 & 0.047 \\
\hline 09304755-3932097 & 5426696130598924416 & 11.896 & 0.926 & 11.557 & 1.279 & 9.917 & 0.663 & 0.300 \\
\hline $09304964+8016095$ & 1144494542550685824 & 12.010 & 1.092 & 11.631 & 1.405 & 9.870 & 0.692 & 0.025 \\
\hline $09313142+7905121$ & 1132002131953311232 & 12.468 & 0.881 & 12.251 & 1.125 & 10.845 & 0.656 & 0.019 \\
\hline $09315651+2335316$ & 644413044801144064 & 12.579 & 1.104 & 12.337 & 1.137 & 10.889 & 0.636 & 0.029 \\
\hline $09325648+1823093$ & 633101995024420480 & 11.036 & 0.760 & 10.792 & 1.070 & 9.389 & 0.579 & 0.028 \\
\hline 09341289-8700397 & 5189077198087438208 & 11.608 & 0.832 & 11.354 & 1.120 & 9.952 & 0.630 & 0.169 \\
\hline $09343649+1036027$ & 589530578303563648 & 12.699 & 0.721 & 12.447 & 0.983 & 11.156 & 0.547 & 0.028 \\
\hline $09344298+0353574$ & 3851012229012923264 & 12.869 & 1.032 & 12.529 & 1.315 & 10.856 & 0.685 & 0.031 \\
\hline $09345116+1214576$ & 614001725703023488 & 11.919 & 1.108 & 12.151 & 1.392 & 9.792 & 0.711 & 0.020 \\
\hline $09372216+0925058$ & 588581463545772928 & 11.335 & 0.839 & 11.073 & 1.096 & 9.674 & 0.648 & 0.031 \\
\hline $09375748+2559307$ & 645906319030740864 & 12.783 & 0.990 & 12.447 & 1.264 & 10.879 & 0.677 & 0.017 \\
\hline $09382460+4205266$ & 813812915803635712 & 12.100 & 1.114 & 11.826 & 1.214 & 10.286 & 0.706 & 0.011 \\
\hline $09383941+1227080$ & 614063912534411136 & 12.431 & 0.807 & 12.141 & 1.103 & 10.693 & 0.594 & 0.017 \\
\hline $09384661+7833575$ & 1131785214629423488 & 12.866 & 0.724 & 12.673 & 1.013 & 11.335 & 0.548 & 0.019 \\
\hline $09391251+3445386$ & 797731523158972416 & 12.333 & 0.951 & 11.964 & 1.187 & 10.383 & 0.667 & 0.010 \\
\hline $09403979+2921557$ & 696431047286005632 & 12.074 & 0.649 & 11.872 & 1.054 & 10.480 & 0.527 & 0.019 \\
\hline $09455700+2135550$ & 640001361769756032 & 13.047 & 0.936 & 13.108 & 1.266 & 11.120 & 0.691 & 0.024 \\
\hline $09472261+3354159$ & 794484841418902912 & 13.030 & 0.732 & 12.712 & 1.091 & 11.315 & 0.587 & 0.011 \\
\hline 09473545-2638039 & 5658256784099611008 & 12.683 & 0.596 & 12.792 & 0.797 & 11.644 & 0.517 & 0.080 \\
\hline 09474496-7045530 & 5242578991517893120 & 12.040 & 0.890 & 11.755 & 1.208 & 10.161 & 0.640 & 0.144 \\
\hline $09485248+1837465$ & 627201117981349888 & 12.590 & 0.779 & 12.299 & 1.104 & 10.846 & 0.597 & 0.022 \\
\hline 09485477-3811139 & 5432401118477539456 & 11.964 & 0.392 & 11.496 & 0.614 & 10.678 & 0.548 & 0.139 \\
\hline $09491888+3201060$ & 793274382192849664 & 12.827 & 1.016 & 12.502 & 1.221 & 10.942 & 0.621 & 0.016 \\
\hline 09501353-7925417 & 5202088268933375232 & 12.475 & 1.140 & 12.036 & 1.497 & 10.145 & 0.712 & 0.220 \\
\hline $09502173+3716070$ & 799953365577763712 & 12.637 & 0.944 & 12.307 & 1.190 & 10.769 & 0.689 & 0.012 \\
\hline 09502215-6930058 & 5243501550494934400 & 13.021 & 0.874 & 12.652 & 1.129 & 11.187 & 0.625 & 0.163 \\
\hline 09513068-3848520 & 5432133353036744448 & 12.826 & 0.686 & 13.945 & 1.323 & 12.221 & 0.732 & 0.193 \\
\hline $09513210+3330203$ & 793674978087183616 & 12.502 & 0.939 & 12.106 & 1.302 & 10.486 & 0.672 & 0.012 \\
\hline
\end{tabular}


Table 2 (continued)

\begin{tabular}{|c|c|c|c|c|c|c|c|c|}
\hline $\begin{array}{l}\text { Star Name } \\
\text { (2MASS) }\end{array}$ & $\begin{array}{c}\text { Star Name } \\
\text { (Gaia EDR3) }\end{array}$ & $V$ & $(B-V)$ & $G$ & $\left(G_{\mathrm{BP}}-G_{\mathrm{RP}}\right)$ & $J$ & $(J-K)$ & $E(B-V)$ \\
\hline 09532759-2717101 & 5657259969431486336 & 12.979 & 1.013 & 12.591 & 1.314 & 10.956 & 0.689 & 0.068 \\
\hline $09535089+3329284$ & 794978139884594560 & 12.995 & 0.711 & 12.781 & 1.004 & 11.473 & 0.535 & 0.013 \\
\hline $09541478+2735210$ & 647086477259746176 & 12.656 & 0.931 & 12.427 & 1.149 & 10.848 & 0.616 & 0.016 \\
\hline $09545564+6756128$ & 1069803755602231168 & 10.770 & 0.989 & 10.461 & 1.285 & 8.838 & 0.658 & 0.083 \\
\hline $09553934+4319179$ & 808168980883073792 & 12.336 & 0.746 & 12.086 & 1.034 & 10.722 & 0.636 & 0.010 \\
\hline 09555639-2403072 & 5660496214405409152 & 11.502 & 0.771 & 11.148 & 1.024 & 9.823 & 0.588 & 0.049 \\
\hline 09560894-5924282 & 5257538328273646336 & 11.466 & 0.912 & 11.175 & 1.136 & 9.733 & 0.650 & 0.300 \\
\hline $09574469+1400136$ & 615228711960558464 & 13.047 & 1.031 & 12.697 & 1.305 & 11.078 & 0.672 & 0.018 \\
\hline 09580008-4318227 & 5418253457547492096 & 12.027 & 0.819 & 11.588 & 1.173 & 9.928 & 0.694 & 0.237 \\
\hline $09580109+2933087$ & 743582087319504640 & 12.974 & 0.957 & 12.558 & 1.338 & 10.834 & 0.629 & 0.016 \\
\hline $09593411+3708378$ & 796772718958610304 & 12.694 & 1.112 & 12.276 & 1.415 & 10.480 & 0.693 & 0.011 \\
\hline $09594843+7246373$ & 1125921282896321792 & 12.993 & 0.532 & 12.797 & 0.933 & 11.604 & 0.500 & 0.030 \\
\hline $10003325-2537597$ & 5658983939239396352 & 12.333 & 0.859 & 12.216 & 1.132 & 10.632 & 0.612 & 0.055 \\
\hline 10020621-1554291 & 5673966327061493760 & 11.905 & 1.120 & 11.445 & 1.483 & 9.623 & 0.701 & 0.042 \\
\hline $10030615+7054115$ & 1071492949060164992 & 12.657 & 0.784 & 12.365 & 1.140 & 10.866 & 0.568 & 0.081 \\
\hline 10033379-2329108 & 5665883610926876672 & 12.670 & 0.961 & 12.351 & 1.240 & 10.837 & 0.659 & 0.052 \\
\hline $10045623-4228179$ & 5418445670219076224 & 10.895 & 0.704 & 10.692 & 1.030 & 9.361 & 0.528 & 0.142 \\
\hline $10052247+3945408$ & 803621576887555840 & 12.758 & 0.774 & 12.471 & 1.113 & 11.001 & 0.617 & 0.013 \\
\hline 10053264-1827501 & 5672581221584085504 & 12.482 & 0.958 & 12.169 & 1.261 & 10.587 & 0.720 & 0.035 \\
\hline $10063882+2403476$ & 630450488374469120 & 12.251 & 0.937 & 11.989 & 1.202 & 10.410 & 0.610 & 0.037 \\
\hline 10075999-2736413 & 5465659970825328896 & 12.078 & 1.013 & 11.773 & 1.354 & 10.047 & 0.751 & 0.069 \\
\hline $10085157-3236041$ & 5459310600414841856 & 12.076 & 0.954 & 11.737 & 1.318 & 10.020 & 0.691 & 0.072 \\
\hline $10100365+2227088$ & 629233049829242368 & 13.026 & 1.118 & 12.648 & 1.501 & 10.732 & 0.740 & 0.025 \\
\hline 10111521-6620282 & 5245373292941453952 & 12.244 & 0.825 & 12.178 & 1.100 & 10.680 & 0.607 & 0.188 \\
\hline 10115056-4309343 & 5415268253418659200 & 11.792 & 0.771 & 11.542 & 1.001 & 10.320 & 0.588 & 0.134 \\
\hline $10115917+5502205$ & 852773418843119616 & 12.343 & 1.073 & 12.157 & 1.306 & 10.411 & 0.694 & 0.008 \\
\hline 10121964-3221347 & 5459373341297312384 & 11.095 & 1.033 & 10.752 & 1.327 & 9.133 & 0.692 & 0.079 \\
\hline $10124027-2249482$ & 5666123373181152000 & 12.506 & 1.037 & 12.210 & 1.337 & 10.487 & 0.705 & 0.060 \\
\hline $10124666+4624575$ & 810408381126766592 & 12.926 & 0.725 & 12.636 & 1.026 & 11.302 & 0.558 & 0.007 \\
\hline $10143521+2324515$ & 725459455633859840 & 11.535 & 0.836 & 11.344 & 1.119 & 9.986 & 0.652 & 0.018 \\
\hline $10151784+5551061$ & 853205526912187264 & 12.837 & 1.004 & 12.481 & 1.321 & 10.914 & 0.706 & 0.007 \\
\hline 10152469-1057352 & 3767574556484311424 & 11.831 & 1.199 & 11.437 & 1.424 & 9.671 & 0.720 & 0.052 \\
\hline $10152705+6456284$ & 1053650967717923072 & 12.204 & 0.976 & 11.926 & 1.298 & 10.255 & 0.668 & 0.025 \\
\hline $10160573+6533551$ & 1065697491989952896 & 10.843 & 0.876 & 11.045 & 1.033 & 9.273 & 0.633 & 0.033 \\
\hline $10171294+4654224$ & 810350480671638784 & 12.356 & 0.936 & 12.153 & 1.071 & 10.613 & 0.617 & 0.008 \\
\hline $10193367+7257464$ & 1077961238527864704 & 12.645 & 0.906 & 12.585 & 1.297 & 10.671 & 0.664 & 0.064 \\
\hline $10203763+7221398$ & 1077708213414112128 & 12.334 & 0.799 & 12.020 & 1.147 & 10.481 & 0.604 & 0.057 \\
\hline $10211155+4557314$ & 809444964126986240 & 12.049 & 0.965 & 11.794 & 1.194 & 10.214 & 0.619 & 0.010 \\
\hline $10213675+7058455$ & 1076692848785487104 & 11.920 & 0.752 & 11.727 & 1.086 & 10.250 & 0.598 & 0.088 \\
\hline 10221281-4123416 & 5416543996142159488 & 12.152 & 0.829 & 11.876 & 1.140 & 10.372 & 0.579 & 0.098 \\
\hline 10244576-7355423 & 5229049672736749184 & 12.370 & 0.942 & 12.105 & 1.170 & 10.721 & 0.687 & 0.189 \\
\hline $10250250+7723238$ & 1128041244394048000 & 12.907 & 0.802 & 12.698 & 1.104 & 11.260 & 0.574 & 0.024 \\
\hline
\end{tabular}


Table 2 (continued)

\begin{tabular}{|c|c|c|c|c|c|c|c|c|}
\hline $\begin{array}{l}\text { Star Name } \\
\text { (2MASS) }\end{array}$ & $\begin{array}{c}\text { Star Name } \\
\text { (Gaia EDR3) }\end{array}$ & $V$ & $(B-V)$ & $G$ & $\left(G_{\mathrm{BP}}-G_{\mathrm{RP}}\right)$ & $J$ & $(J-K)$ & $E(B-V)$ \\
\hline 10263327-2231158 & 5475397761196528896 & 12.884 & 0.984 & 12.560 & 1.245 & 10.938 & 0.669 & 0.080 \\
\hline $10280894+4658364$ & 833602269677094272 & 12.257 & 1.170 & 11.877 & 1.307 & 10.244 & 0.677 & 0.011 \\
\hline 10281330-2946530 & 5461386169127203200 & 11.255 & 1.032 & 10.995 & 1.278 & 9.269 & 0.706 & 0.038 \\
\hline 10295444-0801026 & 3774466329726812672 & 12.999 & 0.714 & 12.727 & 1.059 & 11.333 & 0.579 & 0.050 \\
\hline $10340832-7354406$ & 5229004382300835840 & 11.526 & 0.963 & 11.219 & 1.240 & 9.658 & 0.656 & 0.180 \\
\hline 10350987-1416284 & 3750801231724757760 & 12.915 & 0.779 & 12.677 & 1.065 & 11.263 & 0.536 & 0.056 \\
\hline $10352666+4804049$ & 834056058741414400 & 12.049 & 0.648 & 11.801 & 0.942 & 10.552 & 0.510 & 0.013 \\
\hline 10363889-1917043 & 3554709655102839552 & 12.192 & 0.646 & 12.005 & 0.999 & 10.580 & 0.593 & 0.044 \\
\hline 10371075-1056303 & 3760861312146553216 & 12.564 & 0.955 & 12.230 & 1.213 & 10.627 & 0.656 & 0.048 \\
\hline $10375744+4851237$ & 834186007272278016 & 12.960 & 0.991 & 12.690 & 1.289 & 10.967 & 0.670 & 0.009 \\
\hline $10382409-5248250$ & 5354554767533822336 & 10.977 & 1.040 & 10.614 & 1.360 & 8.883 & 0.672 & 0.300 \\
\hline $10383538+4953297$ & 835062146240700160 & 11.800 & 0.945 & 11.530 & 1.126 & 10.075 & 0.613 & 0.012 \\
\hline $10385482-0518037$ & 3776786466765131520 & 12.410 & 0.883 & 12.070 & 1.201 & 10.544 & 0.669 & 0.042 \\
\hline $10391495+7348283$ & 1078279478424242432 & 12.970 & 0.729 & 12.771 & 0.981 & 11.567 & 0.529 & 0.083 \\
\hline $10391566+7340387$ & 1078266108189870592 & 12.504 & 0.777 & 12.524 & 1.024 & 10.998 & 0.618 & 0.114 \\
\hline $10394895+3459288$ & 750368410525129728 & 11.721 & 1.037 & 11.435 & 1.271 & 9.816 & 0.683 & 0.021 \\
\hline $10420484+0246381$ & 3857349504797962496 & 12.485 & 0.854 & 12.214 & 1.122 & 10.740 & 0.604 & 0.034 \\
\hline $10425611-6355510$ & 5239913019452422784 & 11.899 & 0.867 & 11.619 & 1.124 & 10.179 & 0.642 & 0.300 \\
\hline 10430163-0230226 & 3802519784793984000 & 12.909 & 0.960 & 12.609 & 1.300 & 10.879 & 0.663 & 0.058 \\
\hline 10430365-0643033 & 3776307354572694272 & 11.952 & 0.572 & 11.363 & 1.078 & 9.793 & 0.604 & 0.040 \\
\hline $10432543+7515589$ & 1126646406518426752 & 12.519 & 1.083 & 12.096 & 1.461 & 10.321 & 0.712 & 0.065 \\
\hline $10440416+7616213$ & 1128438924005715456 & 12.923 & 0.716 & 12.673 & 1.059 & 11.334 & 0.566 & 0.026 \\
\hline $10440597-0853008$ & 3762337131628855680 & 12.607 & 0.981 & 12.302 & 1.259 & 10.627 & 0.625 & 0.034 \\
\hline $10453280+0345108$ & 3857542022411847040 & 12.557 & 0.955 & 12.248 & 1.224 & 10.703 & 0.660 & 0.038 \\
\hline 10493965-1719329 & 3556866862556915072 & 12.389 & 0.779 & 12.112 & 1.092 & 10.706 & 0.596 & 0.038 \\
\hline 10502726-2300446 & 3549234430794364160 & 12.809 & 0.765 & 12.582 & 0.998 & 11.265 & 0.539 & 0.064 \\
\hline 10511274-0817026 & 3763102327298064128 & 11.640 & 1.104 & 11.283 & 1.339 & 9.639 & 0.654 & 0.030 \\
\hline $10521859+0528265$ & 3864195618243548032 & 12.831 & 1.089 & 12.473 & 1.365 & 10.795 & 0.705 & 0.025 \\
\hline 10530608-2253102 & 3549276036141218176 & 12.388 & 1.014 & 12.041 & 1.309 & 10.380 & 0.707 & 0.060 \\
\hline 10531802-0055302 & 3803159838000339200 & 12.329 & 1.026 & 11.986 & 1.285 & 10.336 & 0.676 & 0.043 \\
\hline 10540939-5233263 & 5359711992455305344 & 12.296 & 0.990 & 11.935 & 1.282 & 10.340 & 0.698 & 0.300 \\
\hline $10541225+0549178$ & 3864303022490267136 & 11.707 & 0.729 & 11.463 & 1.047 & 10.103 & 0.599 & 0.028 \\
\hline $10543311+0528128$ & 3864140775805950208 & 12.915 & 0.733 & 12.657 & 1.050 & 11.220 & 0.533 & 0.027 \\
\hline 10554570-1654038 & 3556496876894238464 & 11.881 & 0.870 & 11.617 & 1.121 & 10.152 & 0.586 & 0.046 \\
\hline 10563216-0555480 & 3764649748179457280 & 12.859 & 0.983 & 12.556 & 1.324 & 10.888 & 0.759 & 0.028 \\
\hline $10570875-5207081$ & 5359563558390131968 & 11.983 & 1.150 & 11.517 & 1.478 & 9.732 & 0.745 & 0.300 \\
\hline $10582963+0011130$ & 3804842056430901120 & 12.776 & 0.632 & 12.484 & 0.999 & 11.162 & 0.513 & 0.037 \\
\hline 10590836-6924479 & 5231719699295439488 & 11.022 & 0.929 & 10.743 & 1.152 & 9.285 & 0.580 & 0.181 \\
\hline $11000776+4630029$ & 783425712959288576 & 13.010 & 0.852 & 12.723 & 1.088 & 11.327 & 0.598 & 0.009 \\
\hline 11004069-1102492 & 3758563126686411776 & 12.984 & 0.687 & 12.358 & 1.195 & 10.793 & 0.617 & 0.024 \\
\hline $11010971+1310060$ & 3967986487953405312 & 12.961 & 0.772 & 12.712 & 1.050 & 11.354 & 0.604 & 0.012 \\
\hline 11023256-0636095 & 3787611983373749120 & 12.562 & 1.169 & 12.165 & 1.399 & 10.461 & 0.746 & 0.034 \\
\hline
\end{tabular}


Table 2 (continued)

\begin{tabular}{|c|c|c|c|c|c|c|c|c|}
\hline $\begin{array}{l}\text { Star Name } \\
\text { (2MASS) }\end{array}$ & $\begin{array}{c}\text { Star Name } \\
\text { (Gaia EDR3) }\end{array}$ & $V$ & $(B-V)$ & G & $\left(G_{\mathrm{BP}}-G_{\mathrm{RP}}\right)$ & $J$ & $(J-K)$ & $E(B-V)$ \\
\hline $11023598+0102539$ & 3805298933576500480 & 12.638 & 0.742 & 12.362 & 1.058 & 10.965 & 0.549 & 0.026 \\
\hline $11051574-4752557$ & 5362354531210538624 & 12.040 & 0.733 & 11.785 & 1.058 & 10.278 & 0.602 & 0.143 \\
\hline 11051929-1329347 & 3564247918473953792 & 12.142 & 1.057 & 11.752 & 1.346 & 10.096 & 0.674 & 0.034 \\
\hline 11064962-5323068 & 5347385401964090240 & 11.795 & 0.285 & 11.689 & 0.587 & 10.671 & 0.597 & 0.247 \\
\hline $11065789-4643237$ & 5386525267120422912 & 12.850 & 0.936 & 12.630 & 1.235 & 10.970 & 0.629 & 0.110 \\
\hline 11065939-4920517 & 5362109546275295872 & 10.797 & 0.680 & 10.669 & 1.027 & 8.920 & 0.620 & 0.162 \\
\hline $11090121+0754418$ & 3818459160048340352 & 12.400 & 0.958 & 12.057 & 1.270 & 10.443 & 0.660 & 0.025 \\
\hline $11092807-0242021$ & 3791025112280181248 & 10.486 & 0.710 & 10.356 & 0.923 & 9.201 & 0.542 & 0.048 \\
\hline $11093391+2327348$ & 3995259156621469440 & 12.492 & 0.903 & 12.214 & 1.249 & 10.525 & 0.648 & 0.014 \\
\hline $11112337+6943386$ & 1074121743923049344 & 12.633 & 1.180 & 12.201 & 1.578 & 10.280 & 0.740 & 0.013 \\
\hline 11132240-0910000 & 3783099076553128064 & 12.966 & 0.876 & 12.755 & 1.137 & 11.300 & 0.606 & 0.030 \\
\hline $11133490+1015249$ & 3963264914441096320 & 12.197 & 0.724 & 11.933 & 1.023 & 10.593 & 0.579 & 0.014 \\
\hline $11144345-1133225$ & 3566319673258222208 & 11.874 & 0.942 & 11.577 & 1.226 & 10.039 & 0.698 & 0.046 \\
\hline 11172319-5030507 & 5349769001420934784 & 12.413 & 1.006 & 12.063 & 1.306 & 10.371 & 0.639 & 0.197 \\
\hline $11213850+3100109$ & 4023379441379492096 & 11.489 & 1.071 & 11.304 & 1.324 & 9.640 & 0.749 & 0.015 \\
\hline $11215118-4555200$ & 5376213596097250176 & 12.563 & 0.854 & 12.325 & 1.107 & 10.936 & 0.636 & 0.122 \\
\hline $11232110+6118098$ & 862722319742958592 & 12.083 & 0.729 & 11.865 & 1.064 & 10.467 & 0.568 & 0.009 \\
\hline $11235327+0025363$ & 3797935886458318336 & 11.025 & 1.042 & 10.698 & 1.296 & 9.070 & 0.701 & 0.029 \\
\hline 11242364-0642128 & 3785352182036872320 & 13.027 & 0.826 & 12.716 & 1.167 & 11.223 & 0.610 & 0.031 \\
\hline 11245109-0118132 & 3796858154608999424 & 12.546 & 0.961 & 12.217 & 1.263 & 10.589 & 0.660 & 0.038 \\
\hline $11245186+8132096$ & 1133615145575572992 & 12.589 & 0.842 & 12.378 & 1.128 & 10.969 & 0.618 & 0.040 \\
\hline $11251752+4810412$ & 789033325338645888 & 12.631 & 0.600 & 12.423 & 0.938 & 11.233 & 0.519 & 0.015 \\
\hline $11252946-0035203$ & 3797041055791986048 & 12.769 & 0.833 & 12.456 & 1.161 & 11.012 & 0.681 & 0.025 \\
\hline $11255206+5922341$ & 859132616140394880 & 12.670 & 0.935 & 12.429 & 1.179 & 10.867 & 0.610 & 0.012 \\
\hline 11260849-0718091 & 3591618782978505472 & 12.537 & 0.717 & 12.317 & 1.020 & 11.039 & 0.536 & 0.027 \\
\hline $11264720+2321085$ & 3992675231281619584 & 12.967 & 1.026 & 12.568 & 1.246 & 11.012 & 0.685 & 0.015 \\
\hline $11284462+1159546$ & 3917369061419260928 & 12.784 & 0.961 & 12.527 & 1.197 & 10.972 & 0.658 & 0.028 \\
\hline $11293922+6718355$ & 1057741219692489856 & 12.798 & 0.826 & 12.555 & 1.158 & 11.075 & 0.609 & 0.009 \\
\hline $11320178+4540156$ & 784645621097082624 & 12.632 & 0.626 & 12.354 & 1.007 & 11.020 & 0.532 & 0.017 \\
\hline $11322424+7217439$ & 1075031310621259776 & 12.715 & 1.055 & 12.338 & 1.390 & 10.586 & 0.675 & 0.023 \\
\hline $11332470+1251440$ & 3917497017084939776 & 12.264 & 0.998 & 11.952 & 1.273 & 10.326 & 0.677 & 0.036 \\
\hline $11343570+1932184$ & 3977488952772409856 & 12.353 & 0.939 & 12.105 & 1.256 & 10.471 & 0.692 & 0.022 \\
\hline $11344124+5314269$ & 841082728317714048 & 12.838 & 0.782 & 12.624 & 0.946 & 11.377 & 0.507 & 0.008 \\
\hline $11351928-3430096$ & 3477077051780069632 & 12.992 & 1.069 & 12.567 & 1.412 & 10.754 & 0.716 & 0.069 \\
\hline $11364104+3220057$ & 4024512758694334336 & 11.965 & 1.155 & 11.500 & 1.505 & 9.632 & 0.699 & 0.021 \\
\hline $11372004+1845309$ & 3974320228980706816 & 11.964 & 0.859 & 11.692 & 1.159 & 10.186 & 0.611 & 0.024 \\
\hline $11373800-5202251$ & 5345681227653768064 & 11.785 & 0.878 & 11.487 & 1.216 & 9.891 & 0.597 & 0.180 \\
\hline $11381456+1534117$ & 3972403604119095808 & 12.751 & 0.895 & 12.483 & 1.119 & 11.119 & 0.656 & 0.026 \\
\hline $11403298+6340440$ & 864134573709331584 & 13.046 & 1.013 & 12.828 & 1.189 & 11.286 & 0.734 & 0.027 \\
\hline $11415054+5035361$ & 790901636102569472 & 11.967 & 0.832 & 11.674 & 1.163 & 10.204 & 0.602 & 0.018 \\
\hline $11423344+0800282$ & 3910233647567741056 & 12.187 & 0.776 & 11.940 & 1.066 & 10.543 & 0.579 & 0.020 \\
\hline $11444533-4644384$ & 5372404612941931392 & 12.875 & 0.861 & 12.607 & 1.112 & 11.125 & 0.562 & 0.103 \\
\hline
\end{tabular}


Table 2 (continued)

\begin{tabular}{|c|c|c|c|c|c|c|c|c|}
\hline $\begin{array}{l}\text { Star Name } \\
\text { (2MASS) }\end{array}$ & $\begin{array}{c}\text { Star Name } \\
\text { (Gaia EDR3) }\end{array}$ & $V$ & $(B-V)$ & $G$ & $\left(G_{\mathrm{BP}}-G_{\mathrm{RP}}\right)$ & $J$ & $(J-K)$ & $E(B-V)$ \\
\hline $11465844+4835156$ & 787263141676539648 & 12.416 & 0.890 & 12.287 & 1.191 & 10.659 & 0.637 & 0.016 \\
\hline $11471027+0341265$ & 3895958344506982784 & 12.642 & 0.845 & 12.393 & 1.084 & 10.988 & 0.582 & 0.020 \\
\hline $11483814+1952557$ & 3976143459777843968 & 12.295 & 0.855 & 11.989 & 1.170 & 10.472 & 0.599 & 0.031 \\
\hline $11492027+3132066$ & 4021576517188258688 & 12.957 & 0.894 & 12.638 & 1.204 & 11.151 & 0.675 & 0.019 \\
\hline $11505337-8036338$ & 5199459645869304704 & 12.301 & 0.772 & 12.052 & 1.105 & 10.602 & 0.578 & 0.300 \\
\hline $11515346+8151152$ & 1133680021057772544 & 12.463 & 0.783 & 12.230 & 1.087 & 10.792 & 0.563 & 0.069 \\
\hline $11515556-4738558$ & 5371554866553160960 & 11.587 & 0.915 & 11.291 & 1.206 & 9.724 & 0.633 & 0.086 \\
\hline $11524486-4709547$ & 5377584485241041408 & 12.947 & 0.860 & 12.935 & 1.162 & 11.365 & 0.592 & 0.080 \\
\hline $11541040-5208317$ & 5368942804889403520 & 12.432 & 0.689 & 12.263 & 1.326 & 10.587 & 0.686 & 0.129 \\
\hline $11543955+7023508$ & 1061694681253422336 & 12.631 & 0.763 & 12.341 & 1.096 & 10.838 & 0.590 & 0.015 \\
\hline $11545004+4749009$ & 786606630149557248 & 11.929 & 0.703 & 11.676 & 1.100 & 10.284 & 0.615 & 0.020 \\
\hline $11564526+3145522$ & 4026626810316850048 & 12.743 & 0.941 & 12.392 & 1.269 & 10.765 & 0.692 & 0.018 \\
\hline $11593802+2922115$ & 4008146360612154112 & 12.415 & 1.074 & 12.065 & 1.279 & 10.444 & 0.675 & 0.016 \\
\hline $11595151-3905280$ & 3459312659023799552 & 12.951 & 1.089 & 12.579 & 1.411 & 10.744 & 0.713 & 0.136 \\
\hline $12001449+1641370$ & 3925430066494830336 & 13.018 & 1.115 & 12.561 & 1.518 & 10.657 & 0.726 & 0.038 \\
\hline $12004306+2949116$ & 4008186046109996672 & 12.344 & 0.813 & 12.069 & 1.047 & 10.688 & 0.556 & 0.017 \\
\hline 12005811-3929233 & 3459242084121356288 & 10.800 & 1.000 & 10.471 & 1.295 & 8.822 & 0.668 & 0.138 \\
\hline $12022430+6255274$ & 1582997852929561600 & 12.625 & 1.106 & 12.453 & 1.328 & 10.656 & 0.706 & 0.021 \\
\hline $12034594+3035029$ & 4014343925404883328 & 12.520 & 1.086 & 12.214 & 1.330 & 10.440 & 0.671 & 0.016 \\
\hline $12052554-4408587$ & 6147464275054037120 & 12.019 & 0.773 & 11.906 & 0.976 & 10.554 & 0.556 & 0.135 \\
\hline $12055188+1735548$ & 3925930962760630656 & 12.853 & 0.707 & 12.651 & 0.936 & 11.419 & 0.486 & 0.032 \\
\hline $12070423-5345229$ & 6076898344653529344 & 12.318 & 0.893 & 12.020 & 1.236 & 10.383 & 0.604 & 0.173 \\
\hline $12070444+7600223$ & 1692730414755815552 & 12.187 & 0.848 & 11.995 & 1.147 & 10.484 & 0.608 & 0.057 \\
\hline $12074090+3519591$ & 40297607663488666944 & 12.522 & 0.778 & 12.300 & 1.080 & 10.864 & 0.613 & 0.015 \\
\hline $12083074+3954084$ & 1536035581002935424 & 12.984 & 0.599 & 12.746 & 1.176 & 11.226 & 0.625 & 0.024 \\
\hline $12094665+6450371$ & 1585030811273958400 & 12.575 & 1.079 & 12.326 & 1.310 & 10.668 & 0.695 & 0.015 \\
\hline $12100140+4520564$ & 1539687570219614080 & 12.871 & 0.609 & 12.648 & 1.029 & 11.291 & 0.565 & 0.011 \\
\hline $12104009+7032545$ & 1684079324125218944 & 11.542 & 0.563 & 11.468 & 0.804 & 10.227 & 0.496 & 0.014 \\
\hline $12111339+2220415$ & 4001468785978496768 & 13.061 & 0.947 & 12.774 & 1.213 & 11.254 & 0.656 & 0.027 \\
\hline $12132563-3800203$ & 3461061226110222080 & 11.265 & 0.814 & 11.086 & 0.958 & 9.917 & 0.488 & 0.074 \\
\hline $12143659-1517191$ & 3569366385619378432 & 11.652 & 1.043 & 11.301 & 1.308 & 9.677 & 0.664 & 0.048 \\
\hline $12155841-3547529$ & 3461905891557813248 & 13.500 & 0.650 & 13.255 & 1.221 & 11.669 & 0.595 & 0.068 \\
\hline $12161019+4524243$ & 1538985944361745536 & 12.483 & 0.783 & 12.168 & 1.325 & 10.447 & 0.657 & 0.010 \\
\hline $12181234-3721065$ & 6151711177372668928 & 14.909 & 1.094 & 14.540 & 1.337 & 12.825 & 0.714 & 0.095 \\
\hline $12214951+4244520$ & 1538031641283723008 & 12.867 & 0.824 & 12.527 & 1.286 & 10.895 & 0.643 & 0.016 \\
\hline $12221869-3853368$ & 6150224495918526848 & 12.459 & 0.791 & 12.173 & 1.100 & 10.732 & 0.598 & 0.069 \\
\hline $12240228-7202466$ & 5842561802238077952 & 11.904 & 1.120 & 11.509 & 1.537 & 9.514 & 0.659 & 0.300 \\
\hline $12245683+7007242$ & 1683832243246834304 & 12.609 & 0.912 & 12.153 & 1.187 & 10.647 & 0.717 & 0.018 \\
\hline $12261008+3926273$ & 1532300402563759360 & 12.216 & 0.600 & 12.315 & 1.088 & 10.522 & 0.628 & 0.016 \\
\hline $12280652-3319179$ & 3468446817511036160 & 12.967 & 0.829 & 12.674 & 1.126 & 11.190 & 0.574 & 0.071 \\
\hline $12284069+1942295$ & 39489632875255444576 & 12.587 & 0.818 & 12.301 & 1.160 & 10.779 & 0.615 & 0.032 \\
\hline $12293447-3233073$ & 3468579201286803200 & 12.589 & 0.974 & 12.273 & 1.244 & 10.616 & 0.642 & 0.083 \\
\hline
\end{tabular}


Table 2 (continued)

\begin{tabular}{|c|c|c|c|c|c|c|c|c|}
\hline $\begin{array}{l}\text { Star Name } \\
\text { (2MASS) }\end{array}$ & $\begin{array}{c}\text { Star Name } \\
\text { (Gaia EDR3) }\end{array}$ & $V$ & $(B-V)$ & G & $\left(G_{\mathrm{BP}}-G_{\mathrm{RP}}\right)$ & $J$ & $(J-K)$ & $E(B-V)$ \\
\hline $12334194+1952177$ & 3949121587136124160 & 13.001 & 1.095 & 12.631 & 1.391 & 10.894 & 0.701 & 0.025 \\
\hline $12335935-3246361$ & 6160173770478603520 & 12.684 & 0.772 & 12.399 & 1.022 & 11.162 & 0.611 & 0.069 \\
\hline 12335980-2944042 & 3471719754389184384 & 11.216 & 0.788 & 10.962 & 1.094 & 9.549 & 0.583 & 0.067 \\
\hline 12340964-3453438 & 6158168604865990016 & 12.000 & 0.904 & 11.687 & 1.207 & 10.112 & 0.608 & 0.053 \\
\hline $12342913+4826025$ & 1543862488889757568 & 12.799 & 0.867 & 12.527 & 1.117 & 11.081 & 0.604 & 0.013 \\
\hline $12353682+4854057$ & 1544253571429564800 & 12.646 & 1.150 & 12.247 & 1.394 & 10.523 & 0.708 & 0.013 \\
\hline 12353793-4301524 & 6145744883683553408 & 11.745 & 0.907 & 11.473 & 1.151 & 10.010 & 0.592 & 0.061 \\
\hline 12360829-7040522 & 5843251466600455808 & 12.234 & 1.026 & 11.830 & 1.501 & 9.874 & 0.659 & 0.300 \\
\hline $12363406+4334497$ & 1540600409684268032 & 12.623 & 0.960 & 12.291 & 1.184 & 10.883 & 0.557 & 0.019 \\
\hline $12382743-3400315$ & 6158315870705750912 & 12.795 & 0.856 & 12.536 & 1.124 & 11.048 & 0.588 & 0.061 \\
\hline $12383506+2039384$ & 3949279882451077888 & 12.448 & 1.127 & 11.958 & 1.528 & 10.082 & 0.769 & 0.026 \\
\hline $12392838+5038459$ & 1568524878294905088 & 12.429 & 0.944 & 12.069 & 1.323 & 10.382 & 0.667 & 0.013 \\
\hline 12395834-3131208 & 6160731124090635520 & 10.996 & 0.907 & 10.778 & 1.202 & 9.169 & 0.655 & 0.065 \\
\hline 12401578-7012252 & 5843372485918189440 & 11.237 & 0.928 & 11.449 & 1.150 & 9.816 & 0.568 & 0.300 \\
\hline 12413756-5412247 & 6074478212169867904 & 12.095 & 0.930 & 11.723 & 1.276 & 10.169 & 0.713 & 0.244 \\
\hline $12432213-5955445$ & 6056631317380990208 & 11.638 & 0.853 & 11.403 & 1.188 & 9.818 & 0.683 & 0.300 \\
\hline 12433114-8753309 & 5765813554148627328 & 12.111 & 1.060 & 11.680 & 1.367 & 9.932 & 0.729 & 0.116 \\
\hline 12433200-2408376 & 3501624164266183552 & 11.788 & 0.916 & 11.489 & 1.197 & 9.924 & 0.647 & 0.076 \\
\hline $12435927+7222303$ & 1689741705929430016 & 10.893 & 0.655 & 10.822 & 0.961 & 9.571 & 0.527 & 0.024 \\
\hline $12450496-1907283$ & 3521841434322113152 & 13.005 & 0.757 & 12.745 & 1.038 & 11.371 & 0.541 & 0.055 \\
\hline $12493821+6415038$ & 1676938094886271104 & 12.593 & 0.852 & 12.329 & 1.179 & 10.866 & 0.612 & 0.017 \\
\hline $12503487+4033500$ & 1527854729160394496 & 12.219 & 0.753 & 12.034 & 1.102 & 10.540 & 0.574 & 0.010 \\
\hline $12505600+5649548$ & 1576792896497757440 & 11.591 & 1.008 & 11.321 & 1.347 & 9.607 & 0.694 & 0.008 \\
\hline $12510042-1942157$ & 3509552399017285632 & 12.886 & 0.986 & 12.494 & 1.302 & 10.882 & 0.712 & 0.046 \\
\hline $12511944+6940558$ & 1688859141689049984 & 12.833 & 0.530 & 12.782 & 0.853 & 11.703 & 0.540 & 0.011 \\
\hline $12534156+4512260$ & 1530523729214664192 & 12.449 & 0.841 & 12.108 & 1.219 & 10.528 & 0.600 & 0.021 \\
\hline $12540492-1445540$ & 3525348811990850560 & 10.511 & 0.819 & 10.315 & 0.991 & 9.042 & 0.500 & 0.041 \\
\hline $12541483-6940177$ & 5844853661205358208 & 14.510 & 1.280 & 14.547 & 1.099 & 13.131 & 0.558 & 0.284 \\
\hline $12550382+4640597$ & 1530993770436582784 & 11.835 & 1.012 & 11.545 & 1.241 & 9.960 & 0.680 & 0.014 \\
\hline $12552381-8428164$ & 5770402155472131712 & 12.190 & 0.827 & 11.937 & 1.158 & 10.450 & 0.635 & 0.102 \\
\hline $12553388+6801121$ & 1679621251151114624 & 12.950 & 1.077 & 12.810 & 1.296 & 11.143 & 0.671 & 0.020 \\
\hline $12572833-5125217$ & 6081346139752486784 & 11.920 & 0.999 & 11.556 & 1.346 & 9.806 & 0.637 & 0.198 \\
\hline $12592188+5338040$ & 1558199669539397376 & 12.896 & 0.749 & 12.679 & 1.081 & 11.257 & 0.563 & 0.013 \\
\hline $12595962-0829171$ & 3627268897025456000 & 12.678 & 0.847 & 12.394 & 1.039 & 11.177 & 0.583 & 0.040 \\
\hline $13003028+6135085$ & 1579614380772959104 & 10.527 & 0.500 & 10.288 & 0.997 & 8.984 & 0.564 & 0.010 \\
\hline $13022750+5836042$ & 1578633165428231552 & 12.662 & 0.919 & 12.484 & 1.244 & 10.747 & 0.716 & 0.009 \\
\hline $13031323+4812574$ & 1555059567409267328 & 12.895 & 0.938 & 12.497 & 1.321 & 10.857 & 0.691 & 0.012 \\
\hline $13044809-8623048$ & 5769157650045097984 & 12.962 & 0.767 & 12.668 & 1.143 & 11.160 & 0.590 & 0.101 \\
\hline $13070832-5007271$ & 6081477913650691456 & 12.880 & 0.772 & 12.663 & 1.043 & 11.357 & 0.552 & 0.185 \\
\hline $13073032+4948349$ & 1556370498804511360 & 12.574 & 0.872 & 12.335 & 1.101 & 10.891 & 0.605 & 0.009 \\
\hline $13091593+4640598$ & 1553980847721855616 & 12.217 & 1.037 & 11.946 & 1.275 & 10.327 & 0.701 & 0.009 \\
\hline $13091772+4524279$ & 1529793344255574016 & 12.248 & 0.950 & 11.820 & 1.303 & 10.126 & 0.691 & 0.012 \\
\hline
\end{tabular}


Table 2 (continued)

\begin{tabular}{|c|c|c|c|c|c|c|c|c|}
\hline $\begin{array}{c}\text { Star Name } \\
\text { (2MASS) }\end{array}$ & $\begin{array}{c}\text { Star Name } \\
\text { (Gaia EDR3) }\end{array}$ & $V$ & $(B-V)$ & $G$ & $\left(G_{\mathrm{BP}}-G_{\mathrm{RP}}\right)$ & $J$ & $(J-K)$ & $E(B-V)$ \\
\hline $13123590+7259464$ & 1688176585486569600 & 12.967 & 0.857 & 12.781 & 1.201 & 11.271 & 0.658 & 0.013 \\
\hline 13133130-6138303 & 5863379783621954944 & 11.476 & 1.143 & 11.047 & 1.524 & 9.106 & 0.670 & 0.300 \\
\hline $13134248+3405127$ & 1473046861950780032 & 13.040 & 0.758 & 12.781 & 1.051 & 11.418 & 0.602 & 0.009 \\
\hline $13140302-0929340$ & 3624508096342874240 & 12.674 & 0.823 & 12.446 & 1.061 & 11.115 & 0.601 & 0.033 \\
\hline $13145513+3825569$ & 1522993032339838848 & 12.828 & 0.670 & 12.693 & 1.049 & 11.290 & 0.549 & 0.011 \\
\hline $13151765+5708386$ & 1566249645139278720 & 12.998 & 0.917 & 12.863 & 1.005 & 11.586 & 0.532 & 0.011 \\
\hline $13175280+3411307$ & 1473007485690704128 & 13.035 & 0.775 & 12.782 & 1.045 & 11.431 & 0.584 & 0.009 \\
\hline $13222653+7020284$ & 1685430349037732864 & 12.400 & 1.089 & 12.279 & 1.306 & 10.648 & 0.682 & 0.011 \\
\hline $13222932+3936562$ & 1476579283573419264 & 12.614 & 0.718 & 12.364 & 1.233 & 10.680 & 0.656 & 0.011 \\
\hline $13230288-1704404$ & 3604328999956748032 & 13.019 & 0.764 & 12.758 & 1.081 & 11.327 & 0.562 & 0.075 \\
\hline 13231009-0531428 & 3635041726974665600 & 11.179 & 0.783 & 10.931 & 1.066 & 9.456 & 0.621 & 0.031 \\
\hline $13231283+4320557$ & 1549603412755666944 & 12.495 & 0.824 & 12.297 & 1.104 & 10.851 & 0.573 & 0.014 \\
\hline 13240005-1748099 & 3604036456848334720 & 12.402 & 0.859 & 12.152 & 1.138 & 10.637 & 0.581 & 0.082 \\
\hline $13241831+4610307$ & 1551088548024093952 & 12.570 & 1.119 & 12.192 & 1.341 & 10.519 & 0.673 & 0.021 \\
\hline $13242995-4551146$ & 6087082811610225536 & 11.833 & 0.766 & 11.539 & 0.994 & 10.295 & 0.586 & 0.077 \\
\hline $13244275-1607008$ & 3604537731071348992 & 12.220 & 0.869 & 11.923 & 1.193 & 10.392 & 0.672 & 0.056 \\
\hline $13253853-1412517$ & 3608192335923918208 & 12.695 & 0.848 & 12.448 & 1.112 & 10.983 & 0.589 & 0.048 \\
\hline $13282335+7427122$ & 1712581998412032768 & 12.866 & 0.840 & 12.706 & 1.102 & 11.357 & 0.611 & 0.015 \\
\hline $13294999+0115237$ & 3711389512304903168 & 12.310 & 0.874 & 12.029 & 1.154 & 10.619 & 0.695 & 0.024 \\
\hline $13303726-4125545$ & 6160938171579414016 & 12.970 & 0.903 & 12.685 & 1.162 & 11.277 & 0.665 & 0.081 \\
\hline 13351401-0110524 & 3638534188221336960 & 11.932 & 0.985 & 11.555 & 1.289 & 9.905 & 0.670 & 0.024 \\
\hline $13360938-4408536$ & 6111481627465700352 & 11.492 & 0.763 & 11.233 & 1.083 & 9.801 & 0.561 & 0.101 \\
\hline $13373017-7717500$ & 5789490093947866624 & 12.668 & 0.943 & 12.324 & 1.301 & 10.606 & 0.646 & 0.203 \\
\hline $13373672+5902017$ & 1662055552169232896 & 12.156 & 0.688 & 11.940 & 1.090 & 10.530 & 0.586 & 0.009 \\
\hline $13375051+4742311$ & 1552172322891498240 & 11.362 & 0.761 & 11.093 & 1.178 & 9.566 & 0.606 & 0.026 \\
\hline $13394721+6824054$ & 1672939136736222464 & 12.413 & 1.079 & 12.323 & 1.161 & 10.600 & 0.661 & 0.014 \\
\hline $13401321+7237543$ & 1687511071713939712 & 13.037 & 0.708 & 12.862 & 1.058 & 11.460 & 0.580 & 0.014 \\
\hline $13404811+0717285$ & 3724481569055443712 & 12.820 & 0.873 & 12.526 & 1.140 & 11.067 & 0.599 & 0.022 \\
\hline 13425404-0717005 & 3620124270467822848 & 12.030 & 1.106 & 11.676 & 1.368 & 10.033 & 0.729 & 0.033 \\
\hline $13433867+4844266$ & 1558284370590734336 & 12.147 & 0.538 & 12.034 & 0.744 & 11.031 & 0.422 & 0.009 \\
\hline $13434635-0806060$ & 3618345883425109632 & 12.089 & 1.019 & 11.837 & 1.241 & 10.262 & 0.689 & 0.042 \\
\hline $13443166+1523410$ & 3742083100949072640 & 12.691 & 1.150 & 12.149 & 1.589 & 10.202 & 0.763 & 0.023 \\
\hline $13443667-4143163$ & 6112289699794699264 & 10.621 & 0.815 & 10.393 & 1.073 & 8.989 & 0.601 & 0.073 \\
\hline 13452111-0730545 & 3619873547457548160 & 12.455 & 1.074 & 12.120 & 1.338 & 10.448 & 0.696 & 0.034 \\
\hline $13454247-7355568$ & 5791382177358811264 & 12.032 & 0.997 & 11.848 & 1.233 & 10.163 & 0.669 & 0.210 \\
\hline $13455046+0513062$ & 3714276142644562432 & 11.768 & 0.861 & 11.452 & 1.166 & 9.938 & 0.622 & 0.021 \\
\hline $13455076+1426436$ & 3741132710585731840 & 12.913 & 0.980 & 12.582 & 1.269 & 10.888 & 0.649 & 0.022 \\
\hline $13460278-6854556$ & 5850193237592455552 & 11.193 & 1.036 & 10.779 & 1.212 & 9.160 & 0.776 & 0.267 \\
\hline $13461713-4155246$ & 6112273791235652352 & 11.337 & 0.777 & 11.068 & 1.074 & 9.641 & 0.543 & 0.075 \\
\hline $13465223-4334387$ & 6108883889504179072 & 12.969 & 0.997 & 12.613 & 1.286 & 10.951 & 0.673 & 0.074 \\
\hline $13481581-7052139$ & 5840796639405915648 & 12.852 & 1.055 & 12.586 & 1.420 & 10.764 & 0.717 & 0.300 \\
\hline $13510697+5853470$ & 1659341789968532736 & 13.024 & 0.951 & 12.782 & 1.248 & 11.201 & 0.671 & 0.011 \\
\hline
\end{tabular}


Table 2 (continued)

\begin{tabular}{|c|c|c|c|c|c|c|c|c|}
\hline $\begin{array}{l}\text { Star Name } \\
\text { (2MASS) }\end{array}$ & $\begin{array}{c}\text { Star Name } \\
\text { (Gaia EDR3) }\end{array}$ & $V$ & $(B-V)$ & G & $\left(G_{\mathrm{BP}}-G_{\mathrm{RP}}\right)$ & $J$ & $(J-K)$ & $E(B-V)$ \\
\hline $13512378+0741110$ & 3721736844435125760 & 12.466 & 0.930 & 12.185 & 1.220 & 10.582 & 0.609 & 0.022 \\
\hline $13521659-3554258$ & 6115884072018761728 & 14.545 & 0.788 & 14.299 & 1.052 & 12.938 & 0.564 & 0.058 \\
\hline $13533557+7552137$ & 1712349073745277056 & 12.974 & 0.934 & 12.804 & 1.129 & 11.306 & 0.630 & 0.035 \\
\hline $13550687+1707259$ & 1243948839616012160 & 11.843 & 0.992 & 11.335 & 1.411 & 9.584 & 0.727 & 0.025 \\
\hline $13580646+1002348$ & 3723801761632006784 & 11.525 & 0.972 & 11.233 & 1.282 & 9.585 & 0.663 & 0.025 \\
\hline 13584157-3151091 & 6171032134996732416 & 11.269 & 0.860 & 11.055 & 1.042 & 9.747 & 0.525 & 0.065 \\
\hline $13592706+1141130$ & 3727361838547995648 & 12.192 & 0.815 & 11.938 & 1.070 & 10.573 & 0.592 & 0.019 \\
\hline $14002121+5538479$ & 1657538212943126400 & 12.787 & 0.972 & 12.728 & 1.259 & 10.853 & 0.672 & 0.009 \\
\hline $14004596+1215235$ & 3727546045400955136 & 12.761 & 0.720 & 12.447 & 1.041 & 11.075 & 0.537 & 0.018 \\
\hline $14012934+1126361$ & 3724429582771428352 & 12.364 & 0.968 & 12.011 & 1.256 & 10.432 & 0.663 & 0.019 \\
\hline $14020262-6530100$ & 5851263646573358848 & 11.088 & 0.735 & 10.886 & 1.046 & 9.403 & 0.589 & 0.300 \\
\hline $14043744+1255144$ & 1229600350311933952 & 12.199 & 1.179 & 11.747 & 1.464 & 9.929 & 0.740 & 0.022 \\
\hline $14053819+7503388$ & 1712061237921283456 & 12.695 & 1.009 & 12.348 & 1.388 & 10.595 & 0.660 & 0.026 \\
\hline $14063229+4115356$ & 1498298211635183744 & 12.271 & 0.915 & 12.116 & 1.131 & 10.663 & 0.639 & 0.012 \\
\hline $14070810+6936102$ & 1674781746425953792 & 12.222 & 0.778 & 12.003 & 1.151 & 10.514 & 0.607 & 0.021 \\
\hline $14071697+1212488$ & 1226501033191248640 & 12.626 & 0.950 & 12.275 & 1.275 & 10.651 & 0.673 & 0.021 \\
\hline 14095514-2844258 & 6269381255374610176 & 11.857 & 0.733 & 11.593 & 1.037 & 10.221 & 0.552 & 0.045 \\
\hline 14103285-2816339 & 6270148611411493888 & 12.260 & 1.128 & 11.872 & 1.385 & 10.124 & 0.719 & 0.041 \\
\hline $14164140+6136563$ & 1666637942972460672 & 12.219 & 1.020 & 11.889 & 1.387 & 10.138 & 0.676 & 0.018 \\
\hline 14173352-2745144 & 6269861295279466752 & 13.526 & 1.031 & 13.233 & 1.215 & 11.704 & 0.594 & 0.058 \\
\hline 14192484-2307370 & 6275972209107871872 & 13.237 & 1.062 & 13.031 & 1.340 & 11.196 & 0.663 & 0.090 \\
\hline $14193074+4035121$ & 1491839749053036160 & 11.530 & 0.838 & 11.329 & 1.129 & 9.830 & 0.604 & 0.007 \\
\hline $14200302+5936293$ & 1660185630782829952 & 12.587 & 1.057 & 12.226 & 1.375 & 10.388 & 0.681 & 0.008 \\
\hline 14203031-2729455 & 6269707565513913088 & 12.092 & 0.870 & 11.848 & 1.109 & 10.438 & 0.589 & 0.063 \\
\hline 14211081-5015172 & 6091186773125289728 & 13.048 & 0.796 & 12.787 & 1.102 & 11.355 & 0.554 & 0.300 \\
\hline $14223584+4045568$ & 1491104691170130944 & 12.678 & 0.755 & 12.464 & 1.016 & 11.136 & 0.573 & 0.004 \\
\hline $14243397+5624475$ & 1610452860137779584 & 12.889 & 0.563 & 12.731 & 0.978 & 11.418 & 0.503 & 0.013 \\
\hline $14255416+2648176$ & 1256475262057930112 & 12.346 & 1.094 & 11.945 & 1.403 & 10.225 & 0.714 & 0.018 \\
\hline $14261661+8102449$ & 1721820606208706176 & 12.324 & 1.003 & 12.084 & 1.280 & 10.446 & 0.685 & 0.033 \\
\hline 14280573-1353174 & 6300021547768039808 & 12.052 & 0.922 & 11.730 & 1.220 & 10.121 & 0.620 & 0.074 \\
\hline $14294135+6525010$ & 1669746193625166976 & 12.638 & 0.687 & 12.525 & 0.877 & 11.330 & 0.564 & 0.014 \\
\hline $14295841+4502025$ & 1494491496221024512 & 12.985 & 0.698 & 12.748 & 1.027 & 11.404 & 0.535 & 0.011 \\
\hline $14324019+3238042$ & 1287120579569866496 & 12.333 & 0.985 & 12.040 & 1.159 & 10.562 & 0.657 & 0.007 \\
\hline $14341379-6707026$ & 5848443708440147072 & 12.025 & 1.123 & 11.566 & 1.469 & 9.773 & 0.718 & 0.300 \\
\hline 14364248-0715099 & 6331121238455373696 & 11.933 & 0.792 & 11.671 & 1.082 & 10.269 & 0.563 & 0.060 \\
\hline $14385266+3937037$ & 1487940434144428416 & 12.741 & 1.001 & 12.399 & 1.306 & 10.723 & 0.679 & 0.010 \\
\hline $14402612+0655539$ & 1171807334801223808 & 12.672 & 0.690 & 12.465 & 0.989 & 11.165 & 0.524 & 0.026 \\
\hline $14410823+4223420$ & 1490063071404892544 & 11.729 & 0.653 & 11.500 & 0.969 & 10.236 & 0.546 & 0.011 \\
\hline $14411830+1044242$ & 1177885611173494016 & 12.305 & 0.883 & 11.986 & 1.205 & 10.441 & 0.616 & 0.026 \\
\hline $14421990+5654506$ & 1607745454489656960 & 11.631 & 0.854 & 11.386 & 1.126 & 9.980 & 0.593 & 0.012 \\
\hline 14431485-0206178 & 3648915222959836800 & 12.066 & 1.132 & 11.647 & 1.449 & 9.881 & 0.701 & 0.046 \\
\hline $14442119+4758464$ & 1591067649802389632 & 12.943 & 0.790 & 12.715 & 1.149 & 11.234 & 0.631 & 0.020 \\
\hline
\end{tabular}


Table 2 (continued)

\begin{tabular}{|c|c|c|c|c|c|c|c|c|}
\hline $\begin{array}{l}\text { Star Name } \\
\text { (2MASS) }\end{array}$ & $\begin{array}{c}\text { Star Name } \\
\text { (Gaia EDR3) }\end{array}$ & $V$ & $(B-V)$ & $G$ & $\left(G_{\mathrm{BP}}-G_{\mathrm{RP}}\right)$ & $J$ & $(J-K)$ & $E(B-V)$ \\
\hline $14443681+3939231$ & 1487238292890443776 & 12.550 & 1.006 & 12.056 & 1.427 & 10.278 & 0.696 & 0.006 \\
\hline $14444228+4247368$ & 1490052389822427648 & 12.480 & 1.015 & 12.108 & 1.330 & 10.445 & 0.682 & 0.012 \\
\hline $14453096-0214079$ & 3648730612380753664 & 12.961 & 0.679 & 13.289 & 0.893 & 11.841 & 0.493 & 0.053 \\
\hline $14455563+5838081$ & 1616958709094540672 & 12.742 & 1.081 & 12.359 & 1.451 & 10.497 & 0.709 & 0.008 \\
\hline 14460403-7303141 & 5796756182184708096 & 12.046 & 1.052 & 11.677 & 1.365 & 9.908 & 0.696 & 0.135 \\
\hline $14473151+0306259$ & 1157525159792413440 & 12.811 & 0.794 & 12.538 & 1.055 & 11.209 & 0.561 & 0.030 \\
\hline $14504173+6652564$ & 1669581885356163456 & 11.025 & 0.815 & 10.802 & 1.205 & 9.249 & 0.641 & 0.011 \\
\hline $14512223+0339397$ & 1157672906667474432 & 12.925 & 1.070 & 12.480 & 1.424 & 10.690 & 0.734 & 0.033 \\
\hline $14520851+7234433$ & 1698671492693440512 & 13.048 & 0.680 & 12.729 & 1.263 & 11.123 & 0.625 & 0.021 \\
\hline $14535190-4724035$ & 5905262548019849856 & 12.322 & 1.070 & 11.867 & 1.486 & 10.010 & 0.756 & 0.191 \\
\hline $14550996+1452303$ & 1185448189587672960 & 10.981 & 0.925 & 10.812 & 1.185 & 9.191 & 0.647 & 0.028 \\
\hline $14561630+4534315$ & 1586671424357440000 & 12.210 & 0.629 & 12.043 & 0.962 & 10.815 & 0.520 & 0.015 \\
\hline $14561931+0829491$ & 1161734987952738176 & 11.064 & 0.856 & 10.758 & 1.163 & 9.259 & 0.647 & 0.027 \\
\hline $14572828+3910442$ & 1296184266229998976 & 12.860 & 1.123 & 12.514 & 1.409 & 10.747 & 0.700 & 0.009 \\
\hline $14572980+5047347$ & 1593299585391391232 & 12.554 & 0.823 & 12.210 & 1.333 & 10.426 & 0.617 & 0.012 \\
\hline $14584270+6055051$ & 1619069599620600576 & 11.913 & 1.058 & 11.741 & 1.259 & 9.980 & 0.653 & 0.010 \\
\hline $14584982+4500407$ & 1586583807024523520 & 11.426 & 0.770 & 11.218 & 1.025 & 9.883 & 0.523 & 0.016 \\
\hline $14590313+0544031$ & 1159807818946952064 & 12.507 & 0.993 & 12.457 & 1.306 & 10.499 & 0.668 & 0.036 \\
\hline $14591786+4755555$ & 1587515844991289600 & 12.634 & 0.728 & 12.534 & 1.081 & 11.108 & 0.561 & 0.024 \\
\hline $15002374+1131178$ & 1180473277429900288 & 12.990 & 0.800 & 12.710 & 1.071 & 11.388 & 0.577 & 0.028 \\
\hline $15010054+5654074$ & 1612825747965425152 & 11.264 & 0.888 & 14.413 & 0.000 & 9.528 & 0.601 & 0.013 \\
\hline $15010753+1708495$ & 1187645868519130240 & 12.565 & 0.729 & 12.279 & 1.063 & 10.873 & 0.549 & 0.034 \\
\hline $15012803+3616594$ & 1294826884765960192 & 11.835 & 0.899 & 11.655 & 1.161 & 10.130 & 0.667 & 0.013 \\
\hline 15015996-2613494 & 6225828809526332416 & 11.506 & 0.984 & 11.175 & 1.287 & 9.480 & 0.656 & 0.144 \\
\hline $15021465+3507093$ & 1291694234403435648 & 12.456 & 0.975 & 12.187 & 1.262 & 10.525 & 0.663 & 0.012 \\
\hline $15023312+0549057$ & 1159849256791308160 & 11.326 & 0.856 & 11.073 & 1.142 & 9.541 & 0.711 & 0.032 \\
\hline $15023572+0940189$ & 1167946644533913728 & 11.764 & 1.039 & 11.393 & 1.300 & 9.723 & 0.710 & 0.026 \\
\hline $15023742-2442191$ & 6227668258118329088 & 11.604 & 0.934 & 11.368 & 1.152 & 9.884 & 0.585 & 0.135 \\
\hline $15025030+1345255$ & 1181911644797448832 & 12.680 & 1.176 & 12.167 & 1.651 & 10.164 & 0.765 & 0.025 \\
\hline $15025884+4539160$ & 1586250517562417024 & 12.962 & 0.748 & 12.681 & 1.112 & 11.197 & 0.562 & 0.016 \\
\hline $15031286+0602157$ & 1159876916380773120 & 11.048 & 0.728 & 10.635 & 1.143 & 9.194 & 0.654 & 0.036 \\
\hline $15040464+5927327$ & 1614255147440846720 & 12.840 & 0.839 & 12.658 & 1.062 & 11.257 & 0.588 & 0.009 \\
\hline $15043325+0441214$ & 1156620193003216256 & 12.808 & 0.941 & 12.461 & 1.209 & 10.877 & 0.620 & 0.043 \\
\hline $15050841+0701547$ & 1160377499113263360 & 11.967 & 0.921 & 11.569 & 1.251 & 9.950 & 0.672 & 0.026 \\
\hline $15060136-2508317$ & 6227460583562691968 & 12.452 & 0.985 & 12.179 & 1.257 & 10.502 & 0.636 & 0.135 \\
\hline $15060189+0444205$ & 1156532506951177472 & 12.659 & 0.872 & 12.321 & 1.168 & 10.797 & 0.610 & 0.040 \\
\hline $15065236-2547072$ & 6226609772026895104 & 13.139 & 1.166 & 14.067 & 1.359 & 11.151 & 0.730 & 0.141 \\
\hline $15071495+5704053$ & 1612981535018964224 & 12.931 & 1.130 & 12.607 & 1.356 & 10.988 & 0.704 & 0.012 \\
\hline 15085620-7020101 & 5799023787486633984 & 13.043 & 0.961 & 12.777 & 1.249 & 11.199 & 0.661 & 0.088 \\
\hline $15092359+3623180$ & 1292060685309150336 & 12.255 & 0.884 & 12.039 & 1.158 & 10.576 & 0.648 & 0.016 \\
\hline 15094309-2025300 & 6255898665838008960 & 13.535 & 0.881 & 13.196 & 1.177 & 11.677 & 0.594 & 0.097 \\
\hline $15095381+7303555$ & 1697243020930244608 & 12.946 & 1.136 & 12.628 & 1.462 & 10.795 & 0.720 & 0.018 \\
\hline
\end{tabular}


Table 2 (continued)

\begin{tabular}{|c|c|c|c|c|c|c|c|c|}
\hline $\begin{array}{l}\text { Star Name } \\
\text { (2MASS) }\end{array}$ & $\begin{array}{c}\text { Star Name } \\
\text { (Gaia EDR3) }\end{array}$ & $V$ & $(B-V)$ & $G$ & $\left(G_{\mathrm{BP}}-G_{\mathrm{RP}}\right)$ & $J$ & $(J-K)$ & $E(B-V)$ \\
\hline $15102643+0809151$ & 1166563287107603328 & 12.174 & 0.766 & 11.911 & 1.067 & 10.500 & 0.581 & 0.025 \\
\hline $15114226+2557063$ & 1265182569515722880 & 13.047 & 1.063 & 12.731 & 1.074 & 11.318 & 0.578 & 0.048 \\
\hline $15122018-6551473$ & 5824755211457838848 & 12.832 & 1.036 & 12.418 & 1.414 & 10.457 & 0.744 & 0.283 \\
\hline $15140783+1824326$ & 1211721397891599872 & 11.495 & 0.798 & 11.202 & 1.125 & 9.719 & 0.597 & 0.036 \\
\hline $15142395+2613341$ & 1265208133161061888 & 12.535 & 0.803 & 12.271 & 1.112 & 10.805 & 0.583 & 0.053 \\
\hline 15174310-6858306 & 5799218602888406272 & 12.574 & 0.998 & 12.338 & 1.340 & 10.513 & 0.703 & 0.092 \\
\hline $15185830+2620276$ & 1270426797601163008 & 12.575 & 0.848 & 12.328 & 1.108 & 10.890 & 0.572 & 0.032 \\
\hline $15193496+4256501$ & 1394164216985937152 & 12.859 & 0.883 & 12.554 & 1.266 & 10.869 & 0.666 & 0.019 \\
\hline $15193617+2304315$ & 1215718152722386816 & 11.935 & 0.977 & 11.695 & 1.213 & 10.131 & 0.642 & 0.044 \\
\hline 15200891-6701472 & 5823675417983108096 & 12.792 & 1.023 & 12.427 & 1.314 & 10.742 & 0.670 & 0.160 \\
\hline $15210142+7816076$ & 1708318332837616256 & 12.875 & 0.775 & 12.647 & 1.157 & 11.134 & 0.591 & 0.024 \\
\hline $15243997-8015248$ & 5772460033220024576 & 11.584 & 0.918 & 11.343 & 1.175 & 9.844 & 0.615 & 0.158 \\
\hline $15244942+6935080$ & 1695610791623488128 & 12.980 & 0.913 & 12.824 & 1.150 & 11.364 & 0.627 & 0.018 \\
\hline $15252134+6351159$ & 1640849688017226496 & 12.985 & 1.068 & 12.589 & 1.418 & 10.796 & 0.674 & 0.014 \\
\hline $15255933+5026270$ & 1594780288252588032 & 12.254 & 1.036 & 11.855 & 1.372 & 10.148 & 0.689 & 0.017 \\
\hline $15264322-7017087$ & 5796025453632860544 & 12.840 & 1.048 & 12.523 & 1.297 & 10.850 & 0.653 & 0.087 \\
\hline $15272716+2937502$ & 1273518040182639872 & 12.571 & 0.875 & 12.278 & 1.149 & 10.712 & 0.622 & 0.023 \\
\hline $15282654+6737516$ & 1645668263365914368 & 12.557 & 0.877 & 12.396 & 1.103 & 10.990 & 0.602 & 0.023 \\
\hline $15304031+2345045$ & 1220893455176256512 & 11.899 & 0.903 & 11.609 & 1.183 & 10.067 & 0.610 & 0.046 \\
\hline $15312758+2528504$ & 1222449092327405056 & 12.127 & 0.887 & 11.895 & 1.118 & 10.326 & 0.609 & 0.042 \\
\hline $15330912+8158562$ & 1721465807550041856 & 12.515 & 0.905 & 12.282 & 1.184 & 10.658 & 0.634 & 0.044 \\
\hline $15345353+5610250$ & 1601294344891081472 & 11.717 & 0.460 & 11.818 & 0.718 & 10.769 & 0.510 & 0.009 \\
\hline $15351593+0251226$ & 4427077397649058944 & 12.449 & 0.904 & 12.144 & 1.208 & 10.570 & 0.647 & 0.043 \\
\hline $15352535+2843009$ & 1272505179518285696 & 12.455 & 1.163 & 11.974 & 1.523 & 10.126 & 0.730 & 0.022 \\
\hline $15371627+3318345$ & 1370624708124173312 & 11.868 & 0.974 & 11.568 & 1.214 & 9.999 & 0.646 & 0.030 \\
\hline $15381851+4804330$ & 1401181854246440704 & 12.973 & 0.877 & 12.599 & 1.314 & 10.944 & 0.710 & 0.013 \\
\hline $15391788+5403318$ & 1597864040410848896 & 12.587 & 0.849 & 12.363 & 1.116 & 10.870 & 0.583 & 0.014 \\
\hline $15392367+4037228$ & 1389938076542244992 & 10.592 & 0.770 & 10.351 & 1.098 & 8.936 & 0.549 & 0.025 \\
\hline $15392783+5557319$ & 1601593274615155712 & 12.710 & 0.870 & 12.530 & 1.310 & 10.693 & 0.680 & 0.013 \\
\hline $15393011+7252178$ & 1696583898069432704 & 12.828 & 0.809 & 12.673 & 1.159 & 11.151 & 0.616 & 0.029 \\
\hline $15394006+4426174$ & 1397344691808122624 & 12.395 & 0.963 & 12.086 & 1.189 & 10.541 & 0.656 & 0.015 \\
\hline $15414028+8245352$ & 1723003508921638656 & 12.954 & 0.832 & 12.745 & 1.044 & 11.296 & 0.581 & 0.065 \\
\hline $15420239+0515389$ & 4427879525740941056 & 12.501 & 0.785 & 12.248 & 1.061 & 10.836 & 0.554 & 0.043 \\
\hline $15431257+6424109$ & 1641066429247008128 & 12.522 & 0.577 & 12.342 & 1.058 & 10.929 & 0.535 & 0.018 \\
\hline $15433525+3739578$ & 1376344024013884800 & 12.050 & 0.937 & 11.753 & 1.215 & 10.198 & 0.626 & 0.010 \\
\hline $15441676+0459116$ & 4429308311037974272 & 12.232 & 1.015 & 11.862 & 1.327 & 10.148 & 0.675 & 0.049 \\
\hline $15452566-4444040$ & 5988917073480792704 & 11.866 & 0.925 & 11.528 & 1.266 & 9.888 & 0.635 & 0.259 \\
\hline $15460715+0559221$ & 4429522058673688192 & 12.659 & 0.764 & 12.396 & 1.076 & 10.987 & 0.579 & 0.054 \\
\hline $15470901+0505149$ & 4426320800506688384 & 12.926 & 0.877 & 12.738 & 1.213 & 11.133 & 0.643 & 0.053 \\
\hline $15471083-4404210$ & 5989331898604674176 & 11.761 & 0.938 & 11.526 & 1.232 & 9.999 & 0.661 & 0.237 \\
\hline $15475041+6321134$ & 1640197196585984896 & 11.888 & 1.032 & 11.492 & 1.394 & 9.788 & 0.741 & 0.018 \\
\hline $15482592-3959257$ & 6008057268799998848 & 13.564 & 1.111 & 13.221 & 1.456 & 11.348 & 0.561 & 0.300 \\
\hline
\end{tabular}


Table 2 (continued)

\begin{tabular}{|c|c|c|c|c|c|c|c|c|}
\hline $\begin{array}{l}\text { Star Name } \\
\text { (2MASS) }\end{array}$ & $\begin{array}{c}\text { Star Name } \\
\text { (Gaia EDR3) }\end{array}$ & $V$ & $(B-V)$ & $G$ & $\left(G_{\mathrm{BP}}-G_{\mathrm{RP}}\right)$ & $J$ & $(J-K)$ & $E(B-V)$ \\
\hline $15482651+1917200$ & 1203885900077833984 & 12.669 & 1.003 & 12.349 & 1.283 & 10.699 & 0.661 & 0.035 \\
\hline $15484895+0949113$ & 4455582481414836096 & 12.720 & 0.920 & 12.411 & 1.218 & 10.861 & 0.646 & 0.040 \\
\hline $15494232+5707537$ & 1598759730070071808 & 12.623 & 0.961 & 12.318 & 1.248 & 10.716 & 0.638 & 0.009 \\
\hline $15495840-4306370$ & 5989480401391030528 & 12.074 & 1.010 & 11.633 & 1.272 & 10.053 & 0.686 & 0.286 \\
\hline $15501637+3536243$ & 1372799645203241856 & 11.273 & 0.737 & 11.058 & 0.982 & 9.782 & 0.531 & 0.019 \\
\hline $15511496+0734493$ & 4430292717546018304 & 12.770 & 0.759 & 12.485 & 1.060 & 11.088 & 0.551 & 0.035 \\
\hline $15512062+0140120$ & 4423150667969419264 & 12.228 & 0.865 & 12.014 & 1.226 & 10.292 & 0.629 & 0.121 \\
\hline $15514567-3935382$ & 6008406329380234368 & 12.956 & 1.101 & 12.567 & 1.417 & 10.776 & 0.579 & 0.300 \\
\hline $15521212-3934227$ & 6008407257093139456 & 13.630 & 0.866 & 13.370 & 1.136 & 11.923 & 0.508 & 0.300 \\
\hline $15525776+0136148$ & 4411131043072307456 & 12.979 & 0.905 & 12.641 & 1.261 & 10.949 & 0.640 & 0.091 \\
\hline $15533843+0145539$ & 4411228560308157184 & 11.257 & 0.971 & 10.905 & 1.313 & 9.281 & 0.690 & 0.085 \\
\hline $15541062-3255166$ & 6015313976817734784 & 13.774 & 1.051 & 13.444 & 1.353 & 11.664 & 0.688 & 0.182 \\
\hline $15542259-3341566$ & 6012240773099364992 & 11.390 & 0.975 & 11.085 & 1.236 & 9.510 & 0.572 & 0.246 \\
\hline $15542887-3332103$ & 6012255341628535680 & 12.715 & 0.968 & 12.371 & 1.328 & 10.621 & 0.667 & 0.213 \\
\hline $15545039-4652323$ & 5987712279288235648 & 11.052 & 0.888 & 10.825 & 1.125 & 9.383 & 0.583 & 0.300 \\
\hline $15555135+1256054$ & 1191328721374511744 & 12.526 & 0.915 & 12.216 & 1.224 & 10.635 & 0.637 & 0.044 \\
\hline $15563950+0754021$ & 4454167994065860352 & 12.485 & 0.982 & 12.125 & 1.273 & 10.510 & 0.643 & 0.037 \\
\hline $15570603+0757417$ & 4454127621373692032 & 12.876 & 1.186 & 12.438 & 1.534 & 10.524 & 0.688 & 0.037 \\
\hline $15583081+1505320$ & 1192938543835309952 & 12.721 & 0.961 & 12.394 & 1.246 & 10.811 & 0.647 & 0.033 \\
\hline $15583757-3734113$ & 6010214613689309056 & 10.769 & 0.647 & 10.545 & 1.000 & 9.245 & 0.454 & 0.300 \\
\hline $15584850-3603371$ & 6010918747795620480 & 12.965 & 1.080 & 12.816 & 1.343 & 10.925 & 0.648 & 0.300 \\
\hline $15591458+0507554$ & 4425763554271082112 & 11.811 & 1.054 & 11.467 & 1.325 & 9.777 & 0.714 & 0.047 \\
\hline 15592133-3416261 & 6011940778224281856 & 12.521 & 0.848 & 12.275 & 1.085 & 10.860 & 0.466 & 0.265 \\
\hline $15592229-3853560$ & 5997979351379581312 & 12.920 & 1.092 & 12.556 & 1.370 & 10.812 & 0.637 & 0.300 \\
\hline $15592785+0314441$ & 4424940775976241664 & 12.436 & 0.748 & 12.144 & 1.113 & 10.692 & 0.558 & 0.161 \\
\hline 15593606-3220592 & 6036353337976099840 & 12.421 & 1.010 & 12.039 & 1.320 & 10.356 & 0.692 & 0.265 \\
\hline $15593767+1613419$ & 1199172465527378176 & 12.728 & 0.871 & 12.432 & 1.141 & 10.965 & 0.625 & 0.030 \\
\hline $15595245-3207381$ & 6036461468074424448 & 14.060 & 1.090 & 14.226 & 1.102 & 12.779 & 0.501 & 0.189 \\
\hline $16001273+2435560$ & 1219123340233877376 & 12.372 & 0.978 & 12.125 & 1.172 & 10.570 & 0.588 & 0.058 \\
\hline $16004212+1105313$ & 4457460855293872128 & 11.149 & 0.848 & 10.917 & 1.139 & 9.433 & 0.636 & 0.046 \\
\hline 16005880-3307564 & 6036077979033846528 & 13.466 & 0.870 & 13.174 & 1.166 & 11.699 & 0.447 & 0.285 \\
\hline $16020475+0622249$ & 4450709720758088192 & 10.952 & 0.890 & 10.700 & 1.117 & 9.341 & 0.622 & 0.043 \\
\hline $16025295+3733291$ & 1379240098985809408 & 12.341 & 0.938 & 12.046 & 1.181 & 10.528 & 0.637 & 0.015 \\
\hline $16025382-8226516$ & 5768895377865607808 & 12.353 & 0.929 & 12.051 & 1.216 & 10.446 & 0.619 & 0.105 \\
\hline $16025490+0102272$ & 4411633352385123840 & 12.258 & 1.115 & 11.931 & 1.390 & 10.110 & 0.693 & 0.090 \\
\hline $16030891+1940229$ & 1203268485640442880 & 12.832 & 0.776 & 12.547 & 1.098 & 11.106 & 0.592 & 0.037 \\
\hline $16032024+1641034$ & 1199294713178394496 & 12.503 & 0.936 & 12.192 & 1.275 & 10.499 & 0.641 & 0.030 \\
\hline $16033629+3116414$ & 1322162667977621632 & 12.726 & 0.855 & 12.525 & 1.054 & 11.183 & 0.553 & 0.025 \\
\hline $16034976+0832565$ & 4451626679096707456 & 12.283 & 0.888 & 11.994 & 1.166 & 10.465 & 0.596 & 0.049 \\
\hline $16040396+1841499$ & 1200065646925933056 & 12.510 & 0.889 & 12.160 & 1.227 & 10.617 & 0.662 & 0.046 \\
\hline $16040780+4241470$ & 1383653195061929728 & 12.693 & 0.623 & 12.380 & 1.018 & 11.048 & 0.535 & 0.011 \\
\hline $16042932-2253301$ & 6242404084762732800 & 12.670 & 0.945 & 12.375 & 1.203 & 10.819 & 0.596 & 0.149 \\
\hline
\end{tabular}


Table 2 (continued)

\begin{tabular}{|c|c|c|c|c|c|c|c|c|}
\hline $\begin{array}{l}\text { Star Name } \\
\text { (2MASS) }\end{array}$ & $\begin{array}{c}\text { Star Name } \\
\text { (Gaia EDR3) }\end{array}$ & $V$ & $(B-V)$ & $G$ & $\left(G_{\mathrm{BP}}-G_{\mathrm{RP}}\right)$ & $J$ & $(J-K)$ & $E(B-V)$ \\
\hline 16045324-2005064 & 6244194055324808576 & 11.956 & 0.840 & 11.674 & 1.142 & 10.180 & 0.564 & 0.254 \\
\hline $16051879+1359570$ & 1191908675099304704 & 12.238 & 0.693 & 12.170 & 0.946 & 10.789 & 0.510 & 0.044 \\
\hline $16052710+0024235$ & 4409850528641208192 & 11.798 & 1.173 & 11.284 & 1.606 & 9.270 & 0.693 & 0.269 \\
\hline 16052946-2543348 & 6043663818991262208 & 12.034 & 1.572 & 11.588 & 1.491 & 9.719 & 0.737 & 0.130 \\
\hline $16053749+1843052$ & 1200145322861774080 & 12.453 & 1.023 & 12.085 & 1.338 & 10.351 & 0.697 & 0.037 \\
\hline $16055339+1944171$ & 1203234576873824384 & 11.835 & 1.070 & 11.538 & 1.429 & 9.648 & 0.721 & 0.044 \\
\hline 16060454-3508190 & 6034898993336955008 & 13.528 & 1.064 & 13.227 & 1.291 & 11.557 & 0.648 & 0.300 \\
\hline 16061119-3620355 & 5998751552140270976 & 13.453 & 1.237 & 13.080 & 1.521 & 11.170 & 0.701 & 0.300 \\
\hline $16061643-3237103$ & 6036155219728437760 & 12.231 & 0.792 & 12.043 & 1.073 & 10.624 & 0.461 & 0.249 \\
\hline $16064542-3216179$ & 6036585644175848576 & 13.858 & 0.772 & 13.637 & 1.055 & 12.278 & 0.454 & 0.207 \\
\hline $16065604-3707315$ & 5998563982328033920 & 11.667 & 0.695 & 11.469 & 0.956 & 10.313 & 0.452 & 0.300 \\
\hline $16073239-2250378$ & 6242439784523836416 & 11.662 & 0.667 & 11.442 & 0.928 & 10.258 & 0.431 & 0.175 \\
\hline $16073532-3208471$ & 6036547126907295616 & 14.150 & -0.160 & 14.099 & 1.237 & 12.582 & 0.668 & 0.155 \\
\hline $16074138-3314575$ & 6035358520474835456 & 11.031 & 0.684 & 10.871 & 0.949 & 9.627 & 0.502 & 0.200 \\
\hline $16074907+1420135$ & 4458558752013944960 & 12.387 & 1.084 & 11.967 & 1.374 & 10.200 & 0.748 & 0.033 \\
\hline 16080889-2943209 & 6041589418507890688 & 13.093 & 0.925 & 12.950 & 1.084 & 11.617 & 0.522 & 0.198 \\
\hline 16081391-2017182 & 6244117639267067008 & 12.786 & 0.826 & 12.499 & 1.118 & 11.018 & 0.472 & 0.271 \\
\hline $16083267-3438595$ & 6034940156304474112 & 14.602 & 0.873 & 14.459 & 1.130 & 12.986 & 0.554 & 0.300 \\
\hline $16084292-2820180$ & 6041824610913270656 & 12.224 & 0.696 & 11.978 & 0.976 & 10.664 & 0.476 & 0.198 \\
\hline $16091164+3008425$ & 1318809707269021184 & 11.607 & 1.077 & 11.288 & 1.310 & 9.639 & 0.716 & 0.028 \\
\hline $16101125-3446225$ & 6034969392126840320 & 13.360 & 0.980 & 13.016 & 1.300 & 11.328 & 0.625 & 0.300 \\
\hline $16102112+0023264$ & 4408313235288945024 & 12.498 & 0.833 & 12.211 & 1.152 & 10.678 & 0.595 & 0.114 \\
\hline $16102340+3952510$ & 1379957603338177280 & 12.774 & 0.932 & 12.337 & 1.508 & 10.495 & 0.732 & 0.009 \\
\hline $16102544+1810108$ & 1199821898940802944 & 11.897 & 0.922 & 11.603 & 1.210 & 10.032 & 0.650 & 0.045 \\
\hline $16103301+0031597$ & 4408318943304571776 & 12.197 & 0.782 & 11.938 & 1.079 & 10.521 & 0.546 & 0.128 \\
\hline 16103970-2443561 & 6049772323329029632 & 13.386 & 1.067 & 13.066 & 1.314 & 11.330 & 0.662 & 0.152 \\
\hline $16105877-2811435$ & 6041842237458491520 & 13.986 & 1.077 & 13.635 & 1.264 & 11.967 & 0.633 & 0.225 \\
\hline 16111495-3204491 & 6035900167382119808 & 10.844 & 0.908 & 10.579 & 1.169 & 9.053 & 0.533 & 0.206 \\
\hline $16113577-1909453$ & 6245765566678852608 & 11.839 & 0.898 & 11.525 & 1.236 & 9.919 & 0.594 & 0.300 \\
\hline $16114668-2427053$ & 6049806927886876928 & 14.594 & 0.790 & 14.359 & 1.059 & 12.927 & 0.479 & 0.225 \\
\hline $16121101-2818128$ & 6042164669240420864 & 10.301 & 0.719 & 10.177 & 0.803 & 9.162 & 0.508 & 0.241 \\
\hline $16123013+4456046$ & 1385790890480677376 & 12.688 & 0.776 & 12.432 & 1.040 & 11.067 & 0.580 & 0.009 \\
\hline 16132830-2013405 & 6245397122905040000 & 12.458 & 0.871 & 12.269 & 1.083 & 10.839 & 0.429 & 0.300 \\
\hline $16134375-2517381$ & 6049518065566679040 & 11.082 & 0.807 & 10.787 & 1.161 & 9.299 & 0.528 & 0.300 \\
\hline 16135700-2757099 & 6042198238705334144 & 12.687 & 0.906 & 12.451 & 1.071 & 11.028 & 0.484 & 0.293 \\
\hline 16141120-2033379 & 6244625270041451648 & 13.297 & 0.703 & 13.162 & 0.962 & 11.875 & 0.432 & 0.247 \\
\hline $16150157-2339354$ & 6050327233103808768 & 12.306 & 0.955 & 11.980 & 1.275 & 10.277 & 0.669 & 0.197 \\
\hline 16150863-0734143 & 4348947961102169344 & 12.845 & 0.964 & 12.511 & 1.287 & 10.815 & 0.669 & 0.199 \\
\hline $16154084+3326445$ & 13228379742755688384 & 12.840 & 0.759 & 12.586 & 1.005 & 11.263 & 0.525 & 0.020 \\
\hline 16154634-1423377 & 4329332948541070976 & 11.815 & 0.782 & 11.517 & 1.101 & 10.098 & 0.583 & 0.298 \\
\hline $16154808-2355376$ & 6050304972294952064 & 11.163 & 0.764 & 10.932 & 1.061 & 9.540 & 0.470 & 0.210 \\
\hline 16154986-0751166 & 4345832009572003840 & 12.937 & 1.105 & 12.525 & 1.454 & 10.644 & 0.741 & 0.193 \\
\hline
\end{tabular}


Table 2 (continued)

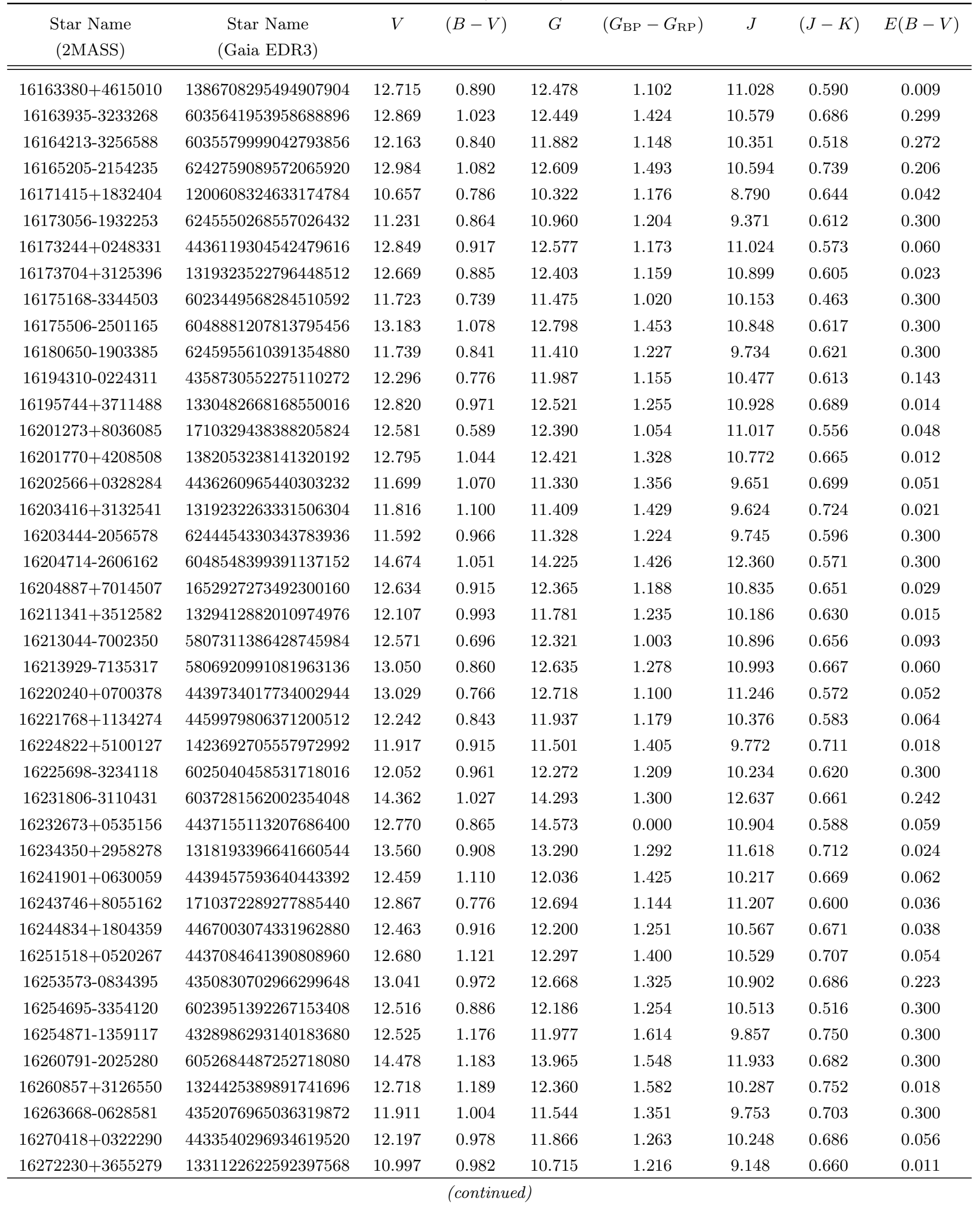


Table 2 (continued)

\begin{tabular}{|c|c|c|c|c|c|c|c|c|}
\hline $\begin{array}{l}\text { Star Name } \\
\text { (2MASS) }\end{array}$ & $\begin{array}{c}\text { Star Name } \\
\text { (Gaia EDR3) }\end{array}$ & $V$ & $(B-V)$ & $G$ & $\left(G_{\mathrm{BP}}-G_{\mathrm{RP}}\right)$ & $J$ & $(J-K)$ & $E(B-V)$ \\
\hline $16272250-0848362$ & 4350762533245253632 & 12.773 & 1.170 & 12.330 & 1.499 & 10.352 & 0.723 & 0.210 \\
\hline $16272730-1210551$ & 4331507503365927680 & 12.577 & 0.971 & 12.263 & 1.369 & 10.470 & 0.730 & 0.272 \\
\hline $16272732+1123305$ & 4459078580497051136 & 12.340 & 0.826 & 12.054 & 1.105 & 10.596 & 0.546 & 0.060 \\
\hline $16274702-3314031$ & 6024841279438699008 & 12.806 & 1.436 & 12.262 & 1.710 & 10.110 & 0.761 & 0.300 \\
\hline $16283300+1722329$ & 4467079971426476160 & 11.786 & 0.992 & 11.415 & 1.326 & 9.731 & 0.676 & 0.044 \\
\hline $16290198+1430507$ & 4463821083383973760 & 12.688 & 0.927 & 12.345 & 1.269 & 10.727 & 0.651 & 0.036 \\
\hline $16293666+0213503$ & 4432101100636150400 & 12.699 & 1.019 & 12.406 & 1.290 & 10.758 & 0.660 & 0.055 \\
\hline $16300807+3459161$ & 1326275047622823552 & 11.183 & 0.836 & 14.638 & 0.000 & 9.571 & 0.537 & 0.018 \\
\hline 16302614-3342496 & 6024015541893607808 & 12.690 & 0.775 & 12.407 & 1.103 & 10.993 & 0.450 & 0.300 \\
\hline $16303876+1329513$ & 4460685211799513600 & 12.960 & 0.885 & 12.671 & 1.177 & 11.126 & 0.610 & 0.044 \\
\hline $16305318+4405187$ & 1405543067118031488 & 12.484 & 1.074 & 12.171 & 1.273 & 10.596 & 0.679 & 0.006 \\
\hline $16312561+1408014$ & 4460744722866525824 & 12.013 & 0.872 & 11.773 & 1.257 & 9.968 & 0.639 & 0.043 \\
\hline $16320549+0401083$ & 4433503532014896512 & 12.780 & 0.589 & 12.500 & 0.942 & 11.250 & 0.489 & 0.056 \\
\hline $16322157+1615449$ & 4465786018039320320 & 12.161 & 0.928 & 11.827 & 1.235 & 10.224 & 0.626 & 0.054 \\
\hline $16323169+1555079$ & 4465571613272013440 & 12.608 & 0.681 & 12.310 & 1.047 & 10.932 & 0.539 & 0.052 \\
\hline $16325211-8327479$ & 5768596864756350976 & 12.511 & 0.937 & 12.149 & 1.281 & 10.433 & 0.733 & 0.086 \\
\hline 16325439-7525259 & 5781162663455262080 & 12.055 & 1.031 & 11.753 & 1.291 & 10.060 & 0.669 & 0.065 \\
\hline $16333188+5906052$ & 1623752935039977088 & 12.821 & 0.636 & 12.618 & 0.977 & 11.274 & 0.485 & 0.013 \\
\hline $16341169+4636439$ & 1407185565691196672 & 11.977 & 1.143 & 11.670 & 1.328 & 9.858 & 0.723 & 0.017 \\
\hline $16342046+1157567$ & 4459450868260931456 & 12.584 & 0.701 & 12.322 & 1.015 & 10.993 & 0.546 & 0.055 \\
\hline $16343097+0620345$ & 4438931438666007296 & 12.930 & 0.956 & 12.619 & 1.228 & 11.025 & 0.653 & 0.068 \\
\hline $16344239+2231419$ & 1298095453661818880 & 12.412 & 1.116 & 12.112 & 1.334 & 10.373 & 0.685 & 0.041 \\
\hline $16350481+1137192$ & 4459250349123575808 & 11.668 & 1.132 & 11.229 & 1.448 & 9.467 & 0.745 & 0.058 \\
\hline $16352407-3359508$ & 6024133674994546944 & 13.522 & 0.857 & 13.244 & 1.113 & 11.781 & 0.526 & 0.300 \\
\hline $16353942+1442022$ & 4462370655749089792 & 12.614 & 0.915 & 12.260 & 1.243 & 10.695 & 0.648 & 0.048 \\
\hline $16354720+1246210$ & 4459657718184334720 & 12.868 & 0.912 & 12.486 & 1.266 & 10.883 & 0.634 & 0.040 \\
\hline $16360774+5058387$ & 1411871031413851648 & 12.469 & 0.729 & 12.350 & 0.944 & 11.134 & 0.537 & 0.020 \\
\hline $16373418+3852001$ & 1331616986212008192 & 12.594 & 0.751 & 12.378 & 1.018 & 11.010 & 0.540 & 0.008 \\
\hline $16374852+0734375$ & 4439132546216071296 & 11.648 & 0.499 & 11.992 & 0.784 & 10.950 & 0.327 & 0.093 \\
\hline $16382363-6644037$ & 5815570715927353216 & 12.687 & 0.903 & 12.533 & 1.213 & 10.960 & 0.647 & 0.071 \\
\hline $16383378+0540276$ & 4435778760872577024 & 12.722 & 1.039 & 12.401 & 1.363 & 10.603 & 0.673 & 0.055 \\
\hline $16384693+1812116$ & 4562485587504234880 & 12.478 & 0.750 & 12.111 & 1.179 & 10.590 & 0.607 & 0.071 \\
\hline $16385168+1723396$ & 4466157103214240256 & 12.327 & 0.818 & 12.054 & 1.116 & 10.594 & 0.590 & 0.057 \\
\hline $16385848+3000087$ & 1311872265637358336 & 12.477 & 1.047 & 12.217 & 1.301 & 10.478 & 0.657 & 0.027 \\
\hline $16391895+0338467$ & 4434135338883534336 & 11.250 & 0.760 & 10.995 & 1.028 & 9.716 & 0.584 & 0.060 \\
\hline $16393846+0740507$ & 4445130901837683584 & 12.948 & 1.002 & 12.499 & 1.323 & 10.914 & 0.697 & 0.099 \\
\hline $16394169+4228154$ & 1356989076015732864 & 12.464 & 0.943 & 12.089 & 1.249 & 10.500 & 0.670 & 0.009 \\
\hline $16394396+4249163$ & 1357571404861995136 & 12.462 & 1.065 & 12.057 & 1.343 & 10.328 & 0.688 & 0.012 \\
\hline $16402468+1550407$ & 4462850115836027648 & 11.984 & 0.826 & 11.695 & 1.155 & 10.163 & 0.623 & 0.054 \\
\hline $16413086+2631045$ & 1301433231662419840 & 13.046 & 0.868 & 12.765 & 1.154 & 11.238 & 0.589 & 0.053 \\
\hline $16414836+1216122$ & 4460825777486994048 & 11.564 & 0.790 & 11.295 & 1.110 & 9.803 & 0.594 & 0.037 \\
\hline $16420651+2604225$ & 1301214463209079040 & 12.636 & 1.116 & 12.415 & 1.414 & 10.498 & 0.720 & 0.045 \\
\hline
\end{tabular}


Table 2 (continued)

\begin{tabular}{|c|c|c|c|c|c|c|c|c|}
\hline $\begin{array}{l}\text { Star Name } \\
\text { (2MASS) }\end{array}$ & $\begin{array}{c}\text { Star Name } \\
\text { (Gaia EDR3) }\end{array}$ & $V$ & $(B-V)$ & $G$ & $\left(G_{\mathrm{BP}}-G_{\mathrm{RP}}\right)$ & $J$ & $(J-K)$ & $E(B-V)$ \\
\hline $16421928+2344032$ & 1299750291677336960 & 12.739 & 1.026 & 12.444 & 1.261 & 10.854 & 0.641 & 0.040 \\
\hline 16423938-8306366 & 5768700008393198464 & 12.217 & 0.846 & 11.851 & 1.229 & 10.251 & 0.642 & 0.124 \\
\hline $16430853+0426005$ & 4434657430811775232 & 12.985 & 1.025 & 12.627 & 1.368 & 10.870 & 0.716 & 0.067 \\
\hline $16432853+1024103$ & 4446900634521443584 & 12.724 & 0.935 & 12.392 & 1.227 & 10.826 & 0.680 & 0.043 \\
\hline $16435657+3232386$ & 1314234398867571072 & 12.653 & 0.890 & 12.354 & 1.178 & 10.805 & 0.621 & 0.026 \\
\hline $16440315+1154212$ & 4448745095340450944 & 12.059 & 0.890 & 11.795 & 1.152 & 10.336 & 0.600 & 0.049 \\
\hline 16443996-7951474 & 5775442729091999616 & 12.470 & 1.013 & 12.138 & 1.296 & 10.459 & 0.645 & 0.123 \\
\hline $16461012+3554551$ & 1327313154102801664 & 12.224 & 0.806 & 11.924 & 1.075 & 10.514 & 0.583 & 0.019 \\
\hline $16462075+1719330$ & 4559215674287937536 & 12.619 & 0.904 & 12.369 & 1.186 & 10.818 & 0.599 & 0.077 \\
\hline $16462090+0333477$ & 4433981853933019264 & 12.092 & 0.893 & 11.753 & 1.222 & 10.163 & 0.623 & 0.071 \\
\hline $16464088+0311203$ & 4385915672332609280 & 12.764 & 1.151 & 12.381 & 1.403 & 10.554 & 0.638 & 0.071 \\
\hline $16471020+5015587$ & 1412373954904373760 & 12.044 & 0.815 & 11.801 & 1.069 & 10.396 & 0.575 & 0.016 \\
\hline $16472419+1507161$ & 4461858837385161088 & 12.150 & 0.735 & 11.931 & 0.991 & 10.687 & 0.518 & 0.062 \\
\hline $16472795+2336019$ & 1299862235704972928 & 12.497 & 0.872 & 12.280 & 1.133 & 10.822 & 0.633 & 0.048 \\
\hline $16474061+1340279$ & 4461395668115705344 & 12.476 & 0.997 & 12.137 & 1.290 & 10.488 & 0.653 & 0.057 \\
\hline $16482585+4245290$ & 1356678949313407360 & 10.827 & 0.735 & 10.543 & 1.157 & 9.042 & 0.610 & 0.015 \\
\hline $16483067+5232124$ & 1413923647824211456 & 12.955 & 0.952 & 12.705 & 1.160 & 11.191 & 0.626 & 0.037 \\
\hline $16485963+2050021$ & 4564767112786411648 & 12.375 & 0.914 & 12.174 & 1.182 & 10.558 & 0.615 & 0.048 \\
\hline $16491646+1922215$ & 4562883030895304960 & 12.715 & 0.639 & 12.444 & 0.979 & 11.104 & 0.517 & 0.067 \\
\hline $16492419+1810509$ & 4559694756410004480 & 12.656 & 0.893 & 16.473 & 0.000 & 10.708 & 0.608 & 0.080 \\
\hline $16501693+3227048$ & 1314079917484186880 & 12.849 & 0.950 & 12.551 & 1.232 & 10.972 & 0.649 & 0.032 \\
\hline $16501966+2135346$ & 4565674033785572096 & 12.256 & 0.910 & 11.953 & 1.178 & 10.420 & 0.615 & 0.043 \\
\hline $16505552+5951110$ & 1438058374730240256 & 11.854 & 0.996 & 11.534 & 1.296 & 9.896 & 0.646 & 0.040 \\
\hline $16510088+3648458$ & 1351373148578890624 & 12.509 & 0.651 & 12.459 & 0.831 & 11.302 & 0.546 & 0.015 \\
\hline $16511460+5352298$ & 1426065314211359488 & 12.916 & 0.773 & 12.636 & 1.100 & 11.223 & 0.627 & 0.031 \\
\hline $16514772+3844288$ & 1352397519754683136 & 12.117 & 0.808 & 11.841 & 1.077 & 10.431 & 0.587 & 0.013 \\
\hline $16523005+0311543$ & 4386055756985792640 & 12.001 & 1.076 & 11.625 & 1.357 & 9.866 & 0.669 & 0.075 \\
\hline $16534583+2531166$ & 1306113268546500352 & 12.477 & 1.110 & 11.722 & 1.551 & 9.814 & 0.729 & 0.049 \\
\hline $16534773+3049290$ & 1312862856894855040 & 13.040 & 0.823 & 12.793 & 1.079 & 11.384 & 0.569 & 0.029 \\
\hline $16545744+2603567$ & 1306294683667797248 & 11.368 & 0.847 & 11.170 & 1.135 & 9.688 & 0.572 & 0.046 \\
\hline $16553977-8810318$ & 5765193738828393600 & 12.870 & 0.818 & 12.573 & 1.171 & 11.073 & 0.605 & 0.098 \\
\hline $16563138+3921373$ & 1352143528272068864 & 12.901 & 0.724 & 12.654 & 0.996 & 11.338 & 0.542 & 0.015 \\
\hline $16563406+1516544$ & 4545782356611145728 & 11.376 & 1.125 & 10.961 & 1.424 & 9.149 & 0.736 & 0.081 \\
\hline $16574714+4326284$ & 1358072923898101120 & 11.721 & 0.994 & 13.532 & 0.000 & 9.724 & 0.691 & 0.015 \\
\hline $16581141+4516190$ & 1359217446784103296 & 13.016 & 0.776 & 12.648 & 1.268 & 11.086 & 0.658 & 0.013 \\
\hline $16583722+0153432$ & 4384878175734959744 & 12.064 & 0.737 & 11.766 & 1.089 & 10.301 & 0.561 & 0.090 \\
\hline $16590140+2521558$ & 4572761867992849920 & 12.408 & 0.822 & 12.038 & 1.106 & 10.590 & 0.581 & 0.037 \\
\hline 16591231-5353249 & 5935760874615991808 & 11.124 & 0.970 & 14.250 & 0.000 & 9.293 & 0.524 & 0.252 \\
\hline 17001311-7651059 & 5777695868934489600 & 12.286 & 0.186 & 12.004 & 0.661 & 10.855 & 0.514 & 0.073 \\
\hline $17024781+2559130$ & 4573174150493770368 & 12.797 & 1.000 & 12.548 & 1.250 & 10.946 & 0.642 & 0.032 \\
\hline $17034238+4012376$ & 1353472361090529536 & 12.994 & 0.797 & 12.693 & 1.254 & 11.029 & 0.630 & 0.028 \\
\hline $17034776+2709455$ & 4573403428730280064 & 12.770 & 0.766 & 12.528 & 1.059 & 11.119 & 0.566 & 0.062 \\
\hline
\end{tabular}


Table 2 (continued)

\begin{tabular}{|c|c|c|c|c|c|c|c|c|}
\hline $\begin{array}{l}\text { Star Name } \\
\text { (2MASS) }\end{array}$ & $\begin{array}{c}\text { Star Name } \\
\text { (Gaia EDR3) }\end{array}$ & $V$ & $(B-V)$ & G & $\left(G_{\mathrm{BP}}-G_{\mathrm{RP}}\right)$ & $J$ & $(J-K)$ & $E(B-V)$ \\
\hline $17035954+4513186$ & 1358789148349761152 & 12.805 & 0.593 & 12.577 & 1.006 & 11.236 & 0.503 & 0.024 \\
\hline $17041564+1932115$ & 4560834812533156480 & 13.040 & 0.988 & 12.689 & 1.296 & 11.015 & 0.662 & 0.072 \\
\hline $17041979+6325224$ & 1631229304871201024 & 12.666 & 1.074 & 12.392 & 1.226 & 10.815 & 0.657 & 0.021 \\
\hline $17042385+6726017$ & 1636646151984790784 & 12.675 & 1.066 & 12.271 & 1.513 & 10.422 & 0.724 & 0.032 \\
\hline $17045983+1948039$ & 4561595434057474304 & 11.961 & 1.120 & 11.579 & 1.390 & 9.813 & 0.709 & 0.061 \\
\hline $17054252-6742414$ & 5814397399577566848 & 12.423 & 0.974 & 12.169 & 1.261 & 10.505 & 0.705 & 0.059 \\
\hline $17060632+6430248$ & 1632107986461148928 & 12.675 & 0.639 & 12.551 & 1.002 & 11.222 & 0.542 & 0.021 \\
\hline $17095788+4825288$ & 1414140487836898048 & 13.049 & 0.969 & 12.701 & 1.290 & 11.058 & 0.665 & 0.011 \\
\hline $17104782+4615401$ & 1364943737701366144 & 11.833 & 0.868 & 11.530 & 1.125 & 9.987 & 0.631 & 0.031 \\
\hline $17115737+4056337$ & 1341931337289579648 & 12.449 & 0.879 & 12.223 & 1.129 & 10.701 & 0.576 & 0.028 \\
\hline 17125716-2214215 & 4115322081195173248 & 13.287 & 1.093 & 12.865 & 1.479 & 10.966 & 0.630 & 0.300 \\
\hline 17130596-3442468 & 5978819326162668288 & 12.308 & 1.016 & 11.901 & 1.409 & 10.119 & 0.521 & 0.300 \\
\hline $17130931+4245360$ & 1354380420256070272 & 13.007 & 0.821 & 12.666 & 1.137 & 11.198 & 0.590 & 0.016 \\
\hline $17133146+5029289$ & 1414644171538142464 & 12.952 & 0.779 & 12.612 & 1.124 & 11.176 & 0.598 & 0.018 \\
\hline $17134640-2252270$ & 4114531910303984896 & 11.467 & 1.015 & 11.152 & 1.279 & 9.569 & 0.594 & 0.300 \\
\hline $17134904+4643336$ & 1364979746707350016 & 12.998 & 0.883 & 12.709 & 1.147 & 11.192 & 0.575 & 0.036 \\
\hline 17151412-2251434 & 4114489441664092160 & 11.068 & 1.020 & 10.739 & 1.331 & 9.054 & 0.553 & 0.300 \\
\hline $17154790-5232570$ & 5925618184739644160 & 12.707 & 1.052 & 12.301 & 1.436 & 10.446 & 0.745 & 0.236 \\
\hline 17160251-2205199 & 4114972844488807680 & 11.804 & 0.980 & 11.488 & 1.294 & 9.835 & 0.681 & 0.300 \\
\hline $17162512+4249078$ & 1360187452261689472 & 11.890 & 0.612 & 11.694 & 0.971 & 10.415 & 0.527 & 0.015 \\
\hline $17163270+4302287$ & 1360381309905667840 & 10.753 & 0.740 & 10.554 & 1.052 & 9.212 & 0.559 & 0.020 \\
\hline 17163966-8711235 & 5765345368353739392 & 12.179 & 0.760 & 11.917 & 1.097 & 10.466 & 0.551 & 0.129 \\
\hline $17174451+3653087$ & 1339989290517076096 & 13.048 & 0.676 & 12.842 & 0.998 & 11.503 & 0.526 & 0.033 \\
\hline $17185527+3839047$ & 1340720740627444096 & 12.884 & 0.971 & 12.645 & 1.282 & 10.915 & 0.617 & 0.046 \\
\hline $17192865-6853306$ & 5811209090730279680 & 11.646 & 0.836 & 11.441 & 1.092 & 10.042 & 0.582 & 0.078 \\
\hline $17194406+4515228$ & 1361644549983238272 & 12.911 & 0.790 & 12.633 & 1.132 & 11.144 & 0.594 & 0.027 \\
\hline $17212704+5033288$ & 1414790574087583360 & 12.628 & 0.965 & 12.215 & 1.326 & 10.527 & 0.657 & 0.027 \\
\hline $17215395-6411338$ & 5814091284361742976 & 12.867 & 0.875 & 12.615 & 1.156 & 11.124 & 0.610 & 0.066 \\
\hline $17215498+1423078$ & 4543224965582361984 & 12.747 & 0.959 & 12.384 & 1.287 & 10.741 & 0.639 & 0.120 \\
\hline $17224220+0353126$ & 4389167576754184192 & 12.925 & 0.922 & 12.587 & 1.233 & 10.953 & 0.641 & 0.086 \\
\hline $17234737-5834124$ & 5916061474500671232 & 11.608 & 0.976 & 11.240 & 1.299 & 9.566 & 0.631 & 0.091 \\
\hline $17240759+3635398$ & 1337102866336482688 & 12.958 & 0.799 & 12.677 & 1.110 & 11.212 & 0.566 & 0.044 \\
\hline 17251201-7832462 & 5776783519098670464 & 12.870 & 1.384 & 12.257 & 1.821 & 10.098 & 0.790 & 0.250 \\
\hline $17252775+5318307$ & 1416272239020984576 & 12.727 & 0.561 & 12.481 & 1.056 & 11.056 & 0.539 & 0.028 \\
\hline $17254679-8513010$ & 5767118193414597632 & 12.928 & 0.781 & 12.633 & 1.110 & 11.143 & 0.622 & 0.102 \\
\hline $17255611+1556131$ & 4543997853536251264 & 12.811 & 0.817 & 12.523 & 1.098 & 11.052 & 0.620 & 0.076 \\
\hline $17265179+3527349$ & 1335862475484040064 & 12.135 & 0.887 & 11.821 & 1.197 & 10.271 & 0.635 & 0.029 \\
\hline $17272663+3847099$ & 1343578233909298816 & 10.174 & 0.535 & 10.402 & 0.762 & 8.982 & 0.526 & 0.036 \\
\hline $17283154-6945443$ & 5810934625139045248 & 10.403 & 0.746 & 10.249 & 0.938 & 9.239 & 0.521 & 0.066 \\
\hline $17285162-6941036$ & 5810941016050593536 & 11.634 & 0.899 & 11.336 & 1.194 & 9.788 & 0.628 & 0.068 \\
\hline $17285482-5720440$ & 5919234390177666304 & 11.222 & 0.765 & 11.048 & 0.961 & 9.888 & 0.535 & 0.080 \\
\hline $17291858+7510248$ & 1655949551783195520 & 12.654 & 1.122 & 12.441 & 1.374 & 10.742 & 0.694 & 0.029 \\
\hline
\end{tabular}


Table 2 (continued)

\begin{tabular}{|c|c|c|c|c|c|c|c|c|}
\hline $\begin{array}{l}\text { Star Name } \\
\text { (2MASS) }\end{array}$ & $\begin{array}{c}\text { Star Name } \\
\text { (Gaia EDR3) }\end{array}$ & $V$ & $(B-V)$ & $G$ & $\left(G_{\mathrm{BP}}-G_{\mathrm{RP}}\right)$ & $J$ & $(J-K)$ & $E(B-V)$ \\
\hline $17292254+0305472$ & 4388901086920961024 & 12.049 & 0.970 & 11.630 & 1.348 & 9.903 & 0.660 & 0.136 \\
\hline $17293008+5534560$ & 1421238419509501568 & 12.676 & 1.042 & 12.482 & 1.067 & 11.098 & 0.631 & 0.036 \\
\hline $17303697+0421414$ & 4389421362079616512 & 12.773 & 1.205 & 12.393 & 1.498 & 10.510 & 0.764 & 0.151 \\
\hline $17314553+2843156$ & 4598739479466205568 & 12.193 & 0.747 & 11.974 & 1.040 & 10.622 & 0.566 & 0.044 \\
\hline 17315874-5342306 & 5921618165752908928 & 11.806 & 0.933 & 11.496 & 1.266 & 9.862 & 0.636 & 0.165 \\
\hline $17330791+2548590$ & 4594030404311114240 & 12.447 & 0.985 & 12.139 & 1.321 & 10.426 & 0.658 & 0.062 \\
\hline $17331311+1713463$ & 4550007577936164864 & 12.891 & 0.888 & 12.470 & 1.271 & 10.860 & 0.635 & 0.044 \\
\hline $17331323+3152308$ & 4601590066440260096 & 12.691 & 1.019 & 12.383 & 1.288 & 10.750 & 0.711 & 0.043 \\
\hline $17332654+2633432$ & 4594840366419966208 & 12.379 & 0.694 & 12.172 & 1.015 & 10.812 & 0.543 & 0.054 \\
\hline $17333170+5456025$ & 1420926742322768640 & 12.238 & 1.101 & 11.863 & 1.307 & 10.296 & 0.685 & 0.048 \\
\hline $17333194-5022586$ & 5946394938738915584 & 12.735 & 0.932 & 12.399 & 1.272 & 10.746 & 0.608 & 0.175 \\
\hline $17334423+1911331$ & 4553866722012938496 & 12.349 & 0.739 & 12.076 & 1.060 & 10.713 & 0.541 & 0.064 \\
\hline $17334679-6744028$ & 5813027335062608384 & 12.762 & 1.061 & 12.379 & 1.339 & 10.617 & 0.725 & 0.067 \\
\hline $17335350+1815568$ & 4550692088345948672 & 12.789 & 0.774 & 12.478 & 1.136 & 11.003 & 0.634 & 0.049 \\
\hline $17335522+1719221$ & 4550032381370693376 & 12.102 & 0.991 & 11.654 & 1.414 & 9.851 & 0.709 & 0.046 \\
\hline $17355093+1411487$ & 4542596697766095232 & 12.615 & 0.788 & 12.418 & 1.069 & 10.989 & 0.570 & 0.080 \\
\hline 17370392-7408233 & 5803200381170398336 & 12.929 & 0.928 & 12.544 & 1.141 & 11.203 & 0.587 & 0.066 \\
\hline $17371472+1603347$ & 4549079418323779712 & 11.526 & 0.656 & 11.352 & 0.904 & 10.175 & 0.586 & 0.065 \\
\hline $17373165+0109351$ & 4375387436181302912 & 11.849 & 1.041 & 11.435 & 1.426 & 9.595 & 0.712 & 0.222 \\
\hline $17374593-4903259$ & 5946896105559051392 & 13.026 & 0.937 & 12.787 & 1.178 & 11.339 & 0.664 & 0.168 \\
\hline $17381613+1806130$ & 4550553412442483456 & 12.617 & 0.994 & 12.287 & 1.295 & 10.666 & 0.702 & 0.052 \\
\hline $17382795+8319481$ & 1711261033974731648 & 12.111 & 0.617 & 11.837 & 1.077 & 10.425 & 0.546 & 0.079 \\
\hline 17390078-7342041 & 5803233817494625536 & 12.870 & 1.330 & 13.442 & 0.929 & 11.486 & 0.534 & 0.052 \\
\hline $17390590+1504553$ & 4548685209048009600 & 11.551 & 1.037 & 11.224 & 1.303 & 9.580 & 0.691 & 0.058 \\
\hline 17393096-5334586 & 5921761544627396352 & 12.555 & 0.965 & 12.295 & 1.282 & 10.621 & 0.677 & 0.173 \\
\hline $17412217-6605479$ & 5812858972344007680 & 11.582 & 1.056 & 11.262 & 1.331 & 9.591 & 0.698 & 0.058 \\
\hline $17412978+2128403$ & 4556052314917665920 & 12.303 & 0.864 & 12.127 & 1.118 & 10.721 & 0.603 & 0.086 \\
\hline $17415044+2448137$ & 4581633552441283328 & 12.952 & 0.845 & 12.689 & 1.144 & 11.198 & 0.620 & 0.057 \\
\hline 17421570-5343061 & 5921703652781168768 & 11.398 & 1.010 & 11.034 & 1.355 & 9.282 & 0.705 & 0.163 \\
\hline $17422649+3217551$ & 4601113221991774976 & 12.968 & 1.058 & 12.674 & 1.281 & 11.009 & 0.652 & 0.042 \\
\hline $17425789+0417381$ & 4473307880166709248 & 12.740 & 0.876 & 12.414 & 1.241 & 10.799 & 0.653 & 0.215 \\
\hline $17431049+5633516$ & 1421354113045323904 & 13.010 & 1.109 & 12.603 & 1.547 & 10.666 & 0.740 & 0.034 \\
\hline 17435113-5359333 & 5921684136450078592 & 12.023 & 0.835 & 11.732 & 1.172 & 10.184 & 0.584 & 0.125 \\
\hline $17442011+0827068$ & 4488216467565808512 & 13.042 & 0.797 & 12.793 & 1.083 & 11.375 & 0.484 & 0.132 \\
\hline $17442313+1055585$ & 4489909058337283584 & 12.273 & 0.891 & 11.976 & 1.220 & 10.389 & 0.636 & 0.115 \\
\hline $17451045+1600587$ & 4501194587422903936 & 12.978 & 0.944 & 12.692 & 1.214 & 11.114 & 0.620 & 0.100 \\
\hline $17452465+0613284$ & 4474168965270090368 & 12.698 & 1.089 & 12.275 & 1.431 & 10.434 & 0.704 & 0.165 \\
\hline $17452911+0549214$ & 4474076782390335616 & 11.607 & 0.816 & 11.291 & 1.181 & 9.725 & 0.603 & 0.199 \\
\hline $17460256+1657355$ & 4549397894441550848 & 12.797 & 0.818 & 12.594 & 1.092 & 11.178 & 0.614 & 0.067 \\
\hline $17465957+1519406$ & 4501077351995658496 & 12.123 & 0.884 & 11.834 & 1.195 & 10.272 & 0.602 & 0.079 \\
\hline $17470290-5058143$ & 5945699978662588416 & 12.344 & 0.865 & 12.128 & 1.141 & 10.587 & 0.614 & 0.154 \\
\hline $17471725-6723320$ & 5812356121877182080 & 12.206 & 1.054 & 11.846 & 1.339 & 10.164 & 0.729 & 0.062 \\
\hline
\end{tabular}


Table 2 (continued)

\begin{tabular}{|c|c|c|c|c|c|c|c|c|}
\hline $\begin{array}{c}\text { Star Name } \\
\text { (2MASS) }\end{array}$ & $\begin{array}{c}\text { Star Name } \\
\text { (Gaia EDR3) }\end{array}$ & $V$ & $(B-V)$ & $G$ & $\left(G_{\mathrm{BP}}-G_{\mathrm{RP}}\right)$ & $J$ & $(J-K)$ & $E(B-V)$ \\
\hline $17471835-0035363$ & 4371256532336413056 & 10.968 & 0.911 & 10.717 & 1.173 & 9.264 & 0.562 & 0.300 \\
\hline $17472373+5750230$ & 1422587593292960640 & 12.299 & 1.018 & 12.693 & 1.304 & 11.024 & 0.701 & 0.031 \\
\hline $17473698+5229473$ & 1368840406910596992 & 12.405 & 0.773 & 12.208 & 1.066 & 10.802 & 0.584 & 0.030 \\
\hline 17485598-7415094 & 5803007691756413056 & 11.901 & 0.964 & 11.560 & 1.282 & 9.931 & 0.659 & 0.060 \\
\hline $17493192-5314272$ & 5921036111776622592 & 13.039 & 0.742 & 12.721 & 1.084 & 11.261 & 0.531 & 0.128 \\
\hline $17505960+1845110$ & 4551354475384137600 & 12.929 & 0.828 & 12.647 & 1.150 & 11.164 & 0.620 & 0.072 \\
\hline $17512033+4313226$ & 1346347658884884608 & 10.767 & 0.907 & 10.457 & 1.216 & 8.879 & 0.618 & 0.029 \\
\hline $17513368+0735474$ & 4475867611955393280 & 12.875 & 0.818 & 12.713 & 1.107 & 11.290 & 0.629 & 0.144 \\
\hline $17531820-5828173$ & 5918003040226875648 & 12.722 & 0.909 & 12.415 & 1.223 & 10.796 & 0.649 & 0.085 \\
\hline $17553087+1344549$ & 4499700449901916672 & 12.567 & 0.915 & 12.228 & 1.270 & 10.575 & 0.660 & 0.090 \\
\hline $17563856-6259025$ & 5911002106095737472 & 12.248 & 1.037 & 11.866 & 1.304 & 10.249 & 0.694 & 0.073 \\
\hline $17570985+6104567$ & 1435424254107968896 & 12.557 & 0.925 & 12.340 & 1.139 & 10.893 & 0.650 & 0.024 \\
\hline $17580876-5017025$ & 5947067839841987840 & 12.773 & 1.049 & 12.484 & 1.313 & 10.768 & 0.706 & 0.129 \\
\hline $17582442-5200036$ & 5945364387093681024 & 11.499 & 1.090 & 11.082 & 1.437 & 9.242 & 0.736 & 0.142 \\
\hline $17583367+0851134$ & 4476514330950969216 & 12.660 & 0.917 & 12.306 & 1.275 & 10.605 & 0.623 & 0.140 \\
\hline $17590214+0226114$ & 4468887736043966848 & 12.605 & 0.994 & 12.135 & 1.357 & 10.330 & 0.685 & 0.211 \\
\hline $17593067+0547081$ & 4474462912835838336 & 11.394 & 0.878 & 11.164 & 1.158 & 9.534 & 0.606 & 0.169 \\
\hline $18025682-7550189$ & 6414540257944747008 & 11.744 & 0.819 & 11.510 & 1.149 & 9.829 & 0.698 & 0.150 \\
\hline $18033495+4211510$ & 2113756098755468288 & 11.247 & 0.720 & 11.028 & 1.117 & 9.577 & 0.590 & 0.021 \\
\hline $18042621-7447453$ & 6417661629719563264 & 12.338 & 0.884 & 12.080 & 1.187 & 10.509 & 0.603 & 0.091 \\
\hline $18042842-7051331$ & 6431507302910109952 & 12.969 & 0.841 & 12.670 & 1.159 & 11.124 & 0.594 & 0.077 \\
\hline $18045298-5543550$ & 6652206220339855104 & 13.006 & 0.933 & 12.668 & 1.277 & 10.993 & 0.673 & 0.101 \\
\hline $18045953+0826177$ & 4476206639494994944 & 12.376 & 0.898 & 11.931 & 1.312 & 10.186 & 0.594 & 0.189 \\
\hline $18052892+0754210$ & 4475421897432191616 & 11.720 & 0.946 & 11.580 & 1.340 & 9.585 & 0.569 & 0.191 \\
\hline $18062441-6457049$ & 6629637992566177664 & 12.364 & 0.733 & 15.608 & 2.404 & 11.050 & 0.585 & 0.061 \\
\hline $18063457+0547198$ & 4471868065390279680 & 12.477 & 0.806 & 12.129 & 1.171 & 10.729 & 0.627 & 0.224 \\
\hline $18072175+5952412$ & 2158636960809774336 & 13.000 & 0.981 & 12.612 & 1.336 & 10.904 & 0.675 & 0.026 \\
\hline $18073369+5849547$ & 2152484604840257280 & 12.633 & 1.043 & 12.367 & 1.418 & 10.462 & 0.690 & 0.036 \\
\hline $18082002-5104378$ & 6702907209758894848 & 11.930 & 0.616 & 11.750 & 0.906 & 10.527 & 0.439 & 0.102 \\
\hline $18083624-6750220$ & 6435985682492232320 & 12.056 & 0.848 & 11.882 & 1.076 & 10.468 & 0.584 & 0.087 \\
\hline $18085682-2106299$ & 4094063947258804608 & 12.915 & 0.703 & 12.668 & 1.012 & 11.313 & 0.432 & 0.300 \\
\hline 18095421-6630506 & 6437097494904121600 & 12.748 & 0.613 & 12.615 & 0.823 & 11.673 & 0.372 & 0.059 \\
\hline
\end{tabular}


Table 2 (continued)

\begin{tabular}{|c|c|c|c|c|c|c|c|c|}
\hline $\begin{array}{c}\text { Star Name } \\
\text { (2MASS) }\end{array}$ & $\begin{array}{c}\text { Star Name } \\
\text { (Gaia EDR3) }\end{array}$ & $V$ & $(B-V)$ & $G$ & $\left(G_{\mathrm{BP}}-G_{\mathrm{RP}}\right)$ & $J$ & $(J-K)$ & $E(B-V)$ \\
\hline $18100815-6731119$ & 6436045915114732672 & 12.644 & 0.776 & 13.999 & 1.479 & 12.089 & 0.660 & 0.094 \\
\hline $18102490-7119083$ & 6431254307857238784 & 12.246 & 0.753 & 12.017 & 1.043 & 10.653 & 0.549 & 0.098 \\
\hline 18123519-6033510 & 6635102251817906304 & 11.185 & 0.960 & 11.187 & 1.192 & 9.411 & 0.646 & 0.090 \\
\hline $18124977+5854462$ & 2152380494834185984 & 12.708 & 0.720 & 12.360 & 1.103 & 10.905 & 0.606 & 0.025 \\
\hline $18135644+4824196$ & 2122140905709563904 & 12.068 & 0.806 & 11.781 & 1.182 & 10.256 & 0.583 & 0.037 \\
\hline $18141754+1612114$ & 4498809506188122624 & 10.582 & 0.654 & 10.394 & 0.967 & 8.922 & 0.601 & 0.183 \\
\hline $18145902+5852559$ & 2152342188021376384 & 12.863 & 0.810 & 12.517 & 1.168 & 10.999 & 0.573 & 0.028 \\
\hline $18150365-3751206$ & 6727766450449365248 & 12.867 & 1.007 & 12.541 & 1.309 & 10.803 & 0.613 & 0.125 \\
\hline $18162072-4725281$ & 6707603223921252608 & 11.761 & 0.757 & 11.486 & 1.081 & 10.062 & 0.537 & 0.082 \\
\hline $18165208+6146498$ & 2159095113561329024 & 12.585 & 0.893 & 12.333 & 1.194 & 10.773 & 0.630 & 0.037 \\
\hline $18171654+1311582$ & 4485396250300800768 & 12.904 & 0.854 & 12.724 & 1.077 & 11.247 & 0.606 & 0.132 \\
\hline $18190641-6824118$ & 6432812045255424128 & 11.915 & 1.031 & 11.501 & 1.266 & 9.848 & 0.665 & 0.124 \\
\hline 18191919-2029254 & 4091364130814956032 & 11.126 & 1.031 & 10.903 & 1.227 & 9.366 & 0.540 & 0.300 \\
\hline $18192007-5552473$ & 6649365494611393920 & 12.407 & 1.233 & 11.947 & 1.498 & 10.012 & 0.754 & 0.106 \\
\hline 18193127-3713134 & 6728130968571404800 & 11.629 & 1.050 & 11.317 & 1.334 & 9.575 & 0.672 & 0.099 \\
\hline 18194826-5310564 & 6653631805885843200 & 12.379 & 0.826 & 12.118 & 1.124 & 10.649 & 0.583 & 0.094 \\
\hline 18203066-2016012 & 4091455699522188416 & 10.755 & 0.787 & 10.608 & 0.981 & 9.337 & 0.443 & 0.300 \\
\hline $18204921-3419480$ & 4044557100560972800 & 12.920 & 0.520 & 12.891 & 1.286 & 11.234 & 0.707 & 0.134 \\
\hline $18211549+5653298$ & 2150956936513301632 & 12.069 & 0.801 & 11.900 & 1.097 & 10.463 & 0.585 & 0.049 \\
\hline $18220774-7608090$ & 6413638383531665664 & 11.927 & 0.807 & 11.726 & 1.057 & 10.320 & 0.587 & 0.145 \\
\hline $18223327-5258169$ & 6653722004497397120 & 12.255 & 1.129 & 11.961 & 1.427 & 10.145 & 0.713 & 0.074 \\
\hline 18231998-3926193 & 6726604713316398336 & 12.046 & 1.101 & 11.731 & 1.391 & 9.941 & 0.695 & 0.095 \\
\hline $18232072-7312133$ & 6418085663251356416 & 12.860 & -0.420 & 12.899 & 1.120 & 11.470 & 0.601 & 0.095 \\
\hline $18273263-5638304$ & 6648380469631584128 & 12.014 & 0.801 & 11.777 & 1.070 & 10.397 & 0.566 & 0.084 \\
\hline $18274709+1732530$ & 4522680968780032128 & 13.030 & 0.945 & 12.575 & 1.356 & 10.794 & 0.629 & 0.242 \\
\hline $18285509-3410250$ & 6734946570607247360 & 10.692 & 0.804 & 10.633 & 1.065 & 9.116 & 0.529 & 0.125 \\
\hline 18293868-2010483 & 4092688806190705152 & 11.978 & 0.935 & 11.680 & 1.252 & 10.117 & 0.503 & 0.300 \\
\hline 18301354-4555101 & 6708532208165979392 & 12.059 & 0.857 & 11.807 & 1.112 & 10.393 & 0.649 & 0.049 \\
\hline $18302241-3955233$ & 6723716880376076928 & 12.872 & 0.810 & 12.592 & 1.158 & 11.084 & 0.599 & 0.098 \\
\hline $18305087-6953306$ & 6431994249123338240 & 12.978 & 0.972 & 12.547 & 1.247 & 11.009 & 0.640 & 0.087 \\
\hline $18311217+4103160$ & 2110452925308798080 & 13.037 & 0.919 & 12.644 & 1.233 & 11.038 & 0.641 & 0.071 \\
\hline 18315993-6920161 & 6432068225640386432 & 11.913 & 0.994 & 11.630 & 1.257 & 10.028 & 0.662 & 0.078 \\
\hline $18321423-3829407$ & 6726896461847358848 & 13.871 & 0.800 & 13.624 & 1.083 & 12.195 & 0.550 & 0.086 \\
\hline $18324025-6202554$ & 6631161743881174400 & 12.923 & 0.970 & 12.610 & 1.258 & 10.979 & 0.618 & 0.135 \\
\hline $18334251+4115292$ & 2110471101610527616 & 11.958 & 0.735 & 11.781 & 0.966 & 10.424 & 0.536 & 0.055 \\
\hline $18340800-5627514$ & 6649667246135478144 & 11.677 & 0.821 & 11.478 & 1.044 & 10.168 & 0.606 & 0.102 \\
\hline 18352484-3639135 & 6733341416736455936 & 11.959 & 0.746 & 11.710 & 1.054 & 10.297 & 0.545 & 0.098 \\
\hline $18352710-4928306$ & 6703851728906337152 & 12.982 & 0.834 & 12.699 & 1.101 & 11.292 & 0.631 & 0.058 \\
\hline $18354018-3948416$ & 6723580609650147712 & 10.432 & 0.787 & 10.209 & 1.067 & 8.831 & 0.555 & 0.078 \\
\hline $18360814+1919587$ & 4524742759245230592 & 12.589 & 0.939 & 12.116 & 1.379 & 10.320 & 0.685 & 0.234 \\
\hline 18361214-7333443 & 6418205892271978624 & 11.798 & 1.006 & 11.425 & 1.339 & 9.692 & 0.659 & 0.119 \\
\hline 18371329-3141091 & 4046640705089371136 & 12.470 & 0.819 & 12.181 & 1.184 & 10.646 & 0.606 & 0.111 \\
\hline
\end{tabular}


Table 2 (continued)

\begin{tabular}{|c|c|c|c|c|c|c|c|c|}
\hline $\begin{array}{l}\text { Star Name } \\
\text { (2MASS) }\end{array}$ & $\begin{array}{c}\text { Star Name } \\
\text { (Gaia EDR3) }\end{array}$ & $V$ & $(B-V)$ & $G$ & $\left(G_{\mathrm{BP}}-G_{\mathrm{RP}}\right)$ & $J$ & $(J-K)$ & $E(B-V)$ \\
\hline $18373068-5538076$ & 6649914292651257984 & 12.092 & 0.942 & 11.785 & 1.252 & 10.164 & 0.645 & 0.089 \\
\hline $18395804+4820336$ & 2119887903305583104 & 12.040 & 1.079 & 11.688 & 1.381 & 9.957 & 0.696 & 0.051 \\
\hline $18404278-5822181$ & 6636472453764175744 & 12.098 & 0.821 & 11.841 & 1.105 & 10.302 & 0.635 & 0.086 \\
\hline $18405632+5805144$ & 2154314260908589184 & 11.743 & 0.881 & 11.469 & 1.164 & 10.017 & 0.626 & 0.040 \\
\hline 18411282-6105584 & 6632762873329420544 & 11.863 & 1.056 & 11.514 & 1.373 & 9.766 & 0.720 & 0.093 \\
\hline 18430593-7820300 & 6364899129015765248 & 12.099 & 0.958 & 11.702 & 1.301 & 10.031 & 0.662 & 0.101 \\
\hline 18462350-4939426 & 6656086710412617344 & 12.549 & 0.903 & 12.305 & 1.188 & 10.739 & 0.625 & 0.050 \\
\hline $18482959-4911163$ & 6656206965197014272 & 13.021 & 0.721 & 12.874 & 0.908 & 11.809 & 0.499 & 0.050 \\
\hline 18490576-5304063 & 6651390073476420736 & 13.017 & 0.966 & 12.757 & 1.237 & 11.207 & 0.647 & 0.060 \\
\hline $18491122+4005588$ & 2098297480667292160 & 12.942 & 0.967 & 12.992 & 1.241 & 11.427 & 0.649 & 0.082 \\
\hline $18512136+5319111$ & 2146237458947371520 & 12.316 & 0.702 & 12.152 & 1.015 & 10.818 & 0.554 & 0.045 \\
\hline $18515139+7849432$ & 2293311669034242432 & 13.016 & 0.713 & 12.772 & 1.041 & 11.419 & 0.525 & 0.067 \\
\hline $18523585+4032042$ & 2103587162389240064 & 13.243 & 1.122 & 12.888 & 1.419 & 11.123 & 0.731 & 0.095 \\
\hline $18523981+4129288$ & 2104473093882735104 & 13.693 & 0.996 & 13.425 & 1.173 & 11.907 & 0.626 & 0.086 \\
\hline $19024429+5351114$ & 2134680487931467648 & 11.703 & 0.684 & 11.880 & 0.933 & 10.364 & 0.591 & 0.045 \\
\hline $19025533+4219090$ & 2104015387809994496 & 11.973 & 0.599 & 11.726 & 1.119 & 10.250 & 0.605 & 0.066 \\
\hline 19032297-4757304 & 6662155460541591424 & 11.820 & 0.854 & 11.613 & 1.061 & 10.294 & 0.569 & 0.060 \\
\hline $19035178-5108429$ & 6657682239223060736 & 11.617 & 1.085 & 11.262 & 1.365 & 9.548 & 0.691 & 0.054 \\
\hline $19044854+5029122$ & 2133638842399952256 & 13.615 & 1.016 & 13.430 & 1.050 & 12.050 & 0.525 & 0.050 \\
\hline $19052503-7826440$ & 6364286563599950592 & 11.221 & 0.961 & 10.958 & 1.281 & 9.274 & 0.731 & 0.146 \\
\hline $19055032-5206090$ & 6656823172744784384 & 12.981 & 0.995 & 12.644 & 1.304 & 10.982 & 0.666 & 0.064 \\
\hline $19060062-5118110$ & 6657644374791106304 & 13.724 & 0.878 & 13.405 & 1.210 & 11.817 & 0.640 & 0.062 \\
\hline 19060226-6310122 & 6438853483690577024 & 11.209 & 0.956 & 10.823 & 1.294 & 9.106 & 0.701 & 0.047 \\
\hline 19120066-6302562 & 6438895574370061568 & 13.603 & 0.937 & 13.335 & 1.198 & 11.773 & 0.633 & 0.039 \\
\hline $19131047+3313392$ & 2043394428389668992 & 12.323 & 0.940 & 12.080 & 1.217 & 10.476 & 0.602 & 0.111 \\
\hline $19155183-4922541$ & 6658254874322598016 & 12.777 & 0.758 & 12.518 & 1.058 & 11.140 & 0.593 & 0.060 \\
\hline $19155497+4038463$ & 2101342715562410240 & 12.126 & 0.991 & 11.811 & 1.221 & 10.244 & 0.609 & 0.088 \\
\hline $19160765+4246319$ & 2102705938181969408 & 12.128 & 0.622 & 11.818 & 1.124 & 10.329 & 0.580 & 0.090 \\
\hline $19184233+8121549$ & 2295494715010792576 & 11.721 & 0.739 & 11.483 & 1.087 & 9.983 & 0.628 & 0.052 \\
\hline 19214950-7947382 & 6361002631604746752 & 11.345 & 1.103 & 10.964 & 1.434 & 9.012 & 0.740 & 0.300 \\
\hline $19232375+4720104$ & 2129167334770344704 & 13.023 & 0.834 & 12.744 & 1.196 & 11.169 & 0.615 & 0.075 \\
\hline $19233833+4018284$ & 2101121026531480960 & 13.472 & 1.195 & 13.195 & 1.257 & 11.554 & 0.671 & 0.101 \\
\hline $19242686+8215294$ & 2295685828172114688 & 12.039 & 0.532 & 12.300 & 0.726 & 11.180 & 0.516 & 0.057 \\
\hline 19253278-2828581 & 6764789893132461568 & 14.610 & 0.851 & 14.371 & 1.115 & 12.901 & 0.583 & 0.104 \\
\hline $19275678+3601156$ & 2049957756892384256 & 11.463 & 0.814 & 11.347 & 1.060 & 9.822 & 0.612 & 0.098 \\
\hline $19280042+4614069$ & 2126804793521418112 & 13.783 & 1.045 & 13.503 & 1.185 & 11.947 & 0.627 & 0.083 \\
\hline 19281201-2900223 & 6764699423940862080 & 12.027 & 1.013 & 11.707 & 1.306 & 10.060 & 0.694 & 0.106 \\
\hline 19281988-6339344 & 6441410874720278272 & 12.760 & 0.799 & 12.494 & 1.116 & 11.034 & 0.620 & 0.051 \\
\hline $19333143+8023242$ & 2295227331824267136 & 12.280 & 0.796 & 12.100 & 1.122 & 10.587 & 0.653 & 0.067 \\
\hline $19352572+5114261$ & 2136022446171534848 & 12.157 & 0.944 & 11.869 & 1.376 & 10.112 & 0.695 & 0.077 \\
\hline $19375420+5750304$ & 2238305511775896064 & 12.886 & 0.988 & 12.511 & 1.292 & 10.898 & 0.669 & 0.057 \\
\hline $19383369+4309290$ & 2077979845822635648 & 12.443 & 0.743 & 12.225 & 1.005 & 10.843 & 0.562 & 0.132 \\
\hline
\end{tabular}


Table 2 (continued)

\begin{tabular}{|c|c|c|c|c|c|c|c|c|}
\hline $\begin{array}{l}\text { Star Name } \\
\text { (2MASS) }\end{array}$ & $\begin{array}{c}\text { Star Name } \\
\text { (Gaia EDR3) }\end{array}$ & $V$ & $(B-V)$ & $G$ & $\left(G_{\mathrm{BP}}-G_{\mathrm{RP}}\right)$ & $J$ & $(J-K)$ & $E(B-V)$ \\
\hline 19411203-4509560 & 6684605636852993408 & 12.898 & 1.068 & 12.584 & 1.340 & 10.896 & 0.694 & 0.056 \\
\hline $19423665+0502288$ & 4290628386915732736 & 11.365 & 1.055 & 11.039 & 1.357 & 9.308 & 0.672 & 0.193 \\
\hline $19433935+7749001$ & 2291495585062080640 & 12.895 & 0.950 & 12.527 & 1.282 & 10.879 & 0.670 & 0.076 \\
\hline $19440238-4515172$ & 6684633743118584064 & 13.890 & 0.802 & 13.640 & 1.093 & 12.177 & 0.525 & 0.048 \\
\hline $19460015-4844202$ & 6671059481799424000 & 11.790 & 0.955 & 11.536 & 1.190 & 9.976 & 0.601 & 0.032 \\
\hline 19481329-4903448 & 6670987390771270912 & 12.666 & 0.932 & 12.385 & 1.200 & 10.829 & 0.640 & 0.033 \\
\hline $19491264+0358174$ & 4242240117122573440 & 12.781 & 0.897 & 12.610 & 1.116 & 11.184 & 0.637 & 0.171 \\
\hline $19493291+1245526$ & 4305164750954492032 & 12.192 & 1.048 & 11.867 & 1.350 & 10.154 & 0.685 & 0.238 \\
\hline 19501371-7714505 & 6365989359218055680 & 12.666 & 1.002 & 12.444 & 1.248 & 10.915 & 0.674 & 0.146 \\
\hline 19512662-5013221 & 6669955297247174784 & 11.133 & 0.903 & 10.860 & 1.188 & 9.359 & 0.687 & 0.038 \\
\hline $19523317+4918292$ & 2086984326351707008 & 12.210 & 0.919 & 12.146 & 1.175 & 10.541 & 0.624 & 0.152 \\
\hline 19533677-4832517 & 6670382182636447104 & 13.625 & 0.825 & 13.384 & 1.095 & 11.980 & 0.600 & 0.048 \\
\hline $19545002+0803021$ & 4298283770977735168 & 12.389 & 0.993 & 12.002 & 1.337 & 10.291 & 0.682 & 0.117 \\
\hline $19550782+0003520$ & 4237149584437046784 & 12.626 & 0.679 & 12.393 & 0.990 & 11.180 & 0.518 & 0.173 \\
\hline $19552158-4613569$ & 6671915382881993472 & 11.943 & 0.895 & 11.622 & 1.230 & 10.069 & 0.694 & 0.041 \\
\hline $19580641-5217166$ & & 13.675 & 0.724 & & & 14.830 & 0.687 & 0.039 \\
\hline $19583119+1403473$ & 1807178962362590336 & 11.509 & 1.084 & 10.968 & 1.514 & 9.039 & 0.735 & 0.203 \\
\hline 20000909-8240204 & 6347485957207236480 & 11.415 & 0.986 & 11.087 & 1.294 & 9.415 & 0.637 & 0.209 \\
\hline $20015470+1103254$ & 4300772996581672704 & 12.355 & 0.999 & 12.097 & 1.235 & 10.512 & 0.692 & 0.132 \\
\hline $20021812+0356003$ & 4247387510856965504 & 12.534 & 1.133 & 12.122 & 1.427 & 10.211 & 0.711 & 0.112 \\
\hline 20032253-1142028 & 4190620966764303488 & 11.872 & 0.783 & 11.577 & 1.161 & 10.041 & 0.566 & 0.134 \\
\hline 20035532-5028100 & 6667107184173771648 & 11.927 & 0.848 & 11.611 & 1.195 & 10.108 & 0.673 & 0.046 \\
\hline 20042821-0634042 & 4219932228775714816 & 12.538 & 1.045 & 12.074 & 1.368 & 10.396 & 0.737 & 0.146 \\
\hline 20052878-5431260 & 6473118900280458240 & 12.071 & 0.780 & 11.814 & 1.084 & 10.372 & 0.568 & 0.037 \\
\hline 20065053-0824044 & 4192486700559707136 & 12.899 & 0.821 & 12.541 & 1.222 & 10.981 & 0.664 & 0.102 \\
\hline 20065112-0135379 & 4235780417581602304 & 12.427 & 1.019 & 12.075 & 1.310 & 10.362 & 0.650 & 0.174 \\
\hline $20071356+0151191$ & 4243611826586899200 & 12.542 & 0.787 & 15.769 & 0.000 & 11.223 & 0.539 & 0.106 \\
\hline $20082836+1011584$ & 4299755432926365184 & 11.592 & 1.166 & 11.260 & 1.414 & 9.455 & 0.702 & 0.174 \\
\hline 20083729-1136333 & 6880822690942721024 & 10.765 & 0.699 & 10.485 & 1.110 & 9.003 & 0.598 & 0.110 \\
\hline 20111053-0042015 & 4236237642618470272 & 12.817 & 1.113 & 12.372 & 1.453 & 10.515 & 0.735 & 0.122 \\
\hline $20115949+0328555$ & 4244395262980430592 & 12.808 & 0.823 & 12.506 & 1.098 & 11.152 & 0.577 & 0.088 \\
\hline 20120663-1720171 & 6873767610163223552 & 10.435 & 0.732 & 10.370 & 0.903 & 9.161 & 0.515 & 0.110 \\
\hline 20121138-5616178 & 6471798631629401088 & 11.154 & 0.823 & 10.876 & 1.137 & 9.436 & 0.635 & 0.048 \\
\hline 20121853-1451042 & 6877707847518537088 & 12.951 & 1.038 & 12.541 & 1.430 & 10.692 & 0.723 & 0.151 \\
\hline $20124384+0008034$ & 4236447546259730816 & 12.981 & 1.155 & 12.583 & 1.469 & 10.710 & 0.696 & 0.108 \\
\hline $20125289+0124185$ & 4242787188566429184 & 12.927 & 1.065 & 12.519 & 1.425 & 10.707 & 0.683 & 0.144 \\
\hline 20125447-6143094 & 6442969093151242880 & 12.071 & 0.895 & 11.780 & 1.185 & 10.274 & 0.645 & 0.033 \\
\hline 20145497-0643013 & 4217089677680862080 & 12.842 & 0.928 & 12.437 & 1.299 & 10.752 & 0.650 & 0.068 \\
\hline 20152495-2220596 & 6852768686118280576 & 12.192 & 1.023 & 11.775 & 1.374 & 10.061 & 0.706 & 0.096 \\
\hline 20153131-5719468 & 6468635748993114112 & 11.899 & 0.907 & 11.580 & 1.191 & 10.068 & 0.677 & 0.038 \\
\hline 20160180-0523231 & 4217535808820767104 & 12.291 & 0.891 & 12.019 & 1.162 & 10.489 & 0.589 & 0.110 \\
\hline 20160744-0552554 & 4217300027997089280 & 12.994 & 0.804 & 12.743 & 1.093 & 11.299 & 0.544 & 0.071 \\
\hline
\end{tabular}


Table 2 (continued)

\begin{tabular}{|c|c|c|c|c|c|c|c|c|}
\hline $\begin{array}{l}\text { Star Name } \\
\text { (2MASS) }\end{array}$ & $\begin{array}{c}\text { Star Name } \\
\text { (Gaia EDR3) }\end{array}$ & $V$ & $(B-V)$ & G & $\left(G_{\mathrm{BP}}-G_{\mathrm{RP}}\right)$ & $J$ & $(J-K)$ & $E(B-V)$ \\
\hline 20165001-1040388 & 6881177219022208512 & 11.171 & 1.022 & 10.800 & 1.302 & 9.198 & 0.636 & 0.099 \\
\hline $20165024+1403416$ & 1803616579035764992 & 13.002 & 0.996 & 12.639 & 1.331 & 10.910 & 0.653 & 0.143 \\
\hline 20174990-4403599 & 6673167103853359232 & 12.516 & 0.715 & 12.313 & 0.978 & 10.959 & 0.518 & 0.035 \\
\hline 20180356-1301215 & 6879502009977573504 & 10.888 & 0.957 & 10.565 & 1.272 & 8.916 & 0.645 & 0.094 \\
\hline $20185485-1428523$ & 6876208079299850752 & 12.659 & 0.736 & 12.340 & 1.104 & 10.875 & 0.577 & 0.059 \\
\hline 20192202-6130149 & 6430979984003489024 & 11.799 & 1.020 & 11.465 & 1.307 & 9.832 & 0.695 & 0.037 \\
\hline 20194097-2922268 & 6797560699757276544 & 11.654 & 0.869 & 11.332 & 1.197 & 9.808 & 0.657 & 0.128 \\
\hline 20200915-4813436 & 6668894268525757696 & 12.416 & 0.982 & 12.083 & 1.295 & 10.485 & 0.708 & 0.034 \\
\hline $20203472-2757112$ & 6846116468972086016 & 12.992 & 1.078 & 12.441 & 1.301 & 10.945 & 0.727 & 0.057 \\
\hline 20204659-1002234 & 6905253392633848960 & 12.499 & 1.079 & 12.102 & 1.395 & 10.354 & 0.741 & 0.063 \\
\hline 20205529-1340439 & 6876402624138104576 & 11.976 & 1.002 & 11.575 & 1.371 & 9.835 & 0.671 & 0.050 \\
\hline 20210974-6637116 & 6425821762641148800 & 12.868 & 0.840 & 12.615 & 1.116 & 11.158 & 0.542 & 0.031 \\
\hline $20214042-5350224$ & 6472730429078350976 & 11.984 & 0.882 & 11.866 & 1.170 & 10.180 & 0.584 & 0.044 \\
\hline 20214109-5550310 & 6469232749446813824 & 11.839 & 0.741 & 11.633 & 1.024 & 10.292 & 0.547 & 0.053 \\
\hline 20214764-1610513 & $\ldots$ & 13.937 & 0.620 & $\ldots$ & $\ldots$ & 13.705 & 0.515 & 0.064 \\
\hline 20214838-2917466 & 6797535960745546624 & 13.569 & 0.846 & 13.397 & 1.052 & 11.993 & 0.520 & 0.059 \\
\hline 20231318-0728503 & 4216093593161355776 & 11.747 & 0.811 & 11.491 & 1.128 & 10.034 & 0.606 & 0.028 \\
\hline 20231543-2104080 & 6859284155805054848 & 13.048 & 0.843 & 12.708 & 1.193 & 11.135 & 0.579 & 0.050 \\
\hline 20232260-0807452 & 6906102627927525760 & 11.837 & 1.101 & 11.454 & 1.398 & 9.728 & 0.736 & 0.044 \\
\hline 20233743-1659533 & 6873254241311424896 & 11.658 & 0.935 & 11.366 & 1.234 & 9.764 & 0.643 & 0.074 \\
\hline 20235225-1628512 & 6873331791241472768 & 12.722 & 0.970 & 12.440 & 1.250 & 10.812 & 0.629 & 0.060 \\
\hline 20241045-6720324 & 6425543307024121088 & 12.656 & 1.028 & 12.333 & 1.288 & 10.711 & 0.675 & 0.033 \\
\hline 20242459-2529550 & 6848220487550880640 & 12.251 & 0.994 & 11.938 & 1.292 & 10.298 & 0.703 & 0.044 \\
\hline 20244286-2618599 & 6847828584672684416 & 12.397 & 0.683 & 12.250 & 0.906 & 11.027 & 0.448 & 0.054 \\
\hline 20244510-1605268 & 6874849529603646080 & 12.819 & 1.022 & 12.439 & 1.352 & 10.723 & 0.668 & 0.061 \\
\hline 20255659-4915524 & 6668166013871841536 & 12.063 & 0.870 & 11.862 & 1.201 & 10.225 & 0.647 & 0.030 \\
\hline 20263268-1025283 & 6904467555355890304 & 11.903 & 0.888 & 11.649 & 1.162 & 10.084 & 0.596 & 0.051 \\
\hline 20271323-1658595 & 6862731949391812992 & 10.030 & 0.438 & 9.845 & 0.678 & 9.235 & 0.495 & 0.052 \\
\hline 20273786-1444546 & 6875659938390010240 & 12.911 & 0.848 & 12.624 & 1.167 & 11.081 & 0.587 & 0.044 \\
\hline 20273791-2627414 & 6847786940670450944 & 10.615 & 0.830 & 10.422 & 1.038 & 9.119 & 0.553 & 0.047 \\
\hline $20274485-4223567$ & 6679325159242871808 & 11.517 & 0.761 & 11.326 & 1.008 & 10.006 & 0.538 & 0.044 \\
\hline 20275301-5141137 & 6475211236547281152 & 12.172 & 1.030 & 11.835 & 1.305 & 10.223 & 0.723 & 0.034 \\
\hline 20275840-1556595 & 6874956658968640512 & 12.507 & 1.142 & 12.026 & 1.481 & 10.228 & 0.736 & 0.059 \\
\hline $20281482-5351378$ & 6473996241838555392 & 12.768 & 0.742 & 12.549 & 1.009 & 11.193 & 0.523 & 0.039 \\
\hline 20284544-2638089 & 6847769863878031616 & 10.294 & 0.651 & 12.303 & 0.000 & 9.221 & 0.489 & 0.048 \\
\hline 20290062-2157354 & 6855992046192920960 & 12.552 & 0.874 & 12.328 & 1.099 & 10.916 & 0.581 & 0.064 \\
\hline 20290527-5059527 & 6475303698603197824 & 12.632 & 0.738 & 12.502 & 1.018 & 11.052 & 0.586 & 0.030 \\
\hline 20292008-4513468 & 6675597235006194432 & 11.359 & 0.915 & 11.188 & 1.181 & 9.778 & 0.698 & 0.031 \\
\hline 20293622-3652218 & 6694857444613732352 & 12.394 & 0.838 & 12.137 & 1.067 & 10.834 & 0.631 & 0.041 \\
\hline 20301912-2844399 & 6797804306004518400 & 12.969 & 0.872 & 12.743 & 1.119 & 11.280 & 0.569 & 0.048 \\
\hline 20313318-3054125 & 6796525780141266304 & 12.268 & 0.796 & 12.102 & 0.993 & 10.836 & 0.471 & 0.083 \\
\hline 20314518-5623277 & 6469004910021548672 & 12.997 & 0.801 & 12.801 & 1.057 & 11.466 & 0.582 & 0.050 \\
\hline
\end{tabular}


Table 2 (continued)

\begin{tabular}{|c|c|c|c|c|c|c|c|c|}
\hline $\begin{array}{c}\text { Star Name } \\
\text { (2MASS) }\end{array}$ & $\begin{array}{c}\text { Star Name } \\
\text { (Gaia EDR3) }\end{array}$ & $V$ & $(B-V)$ & $G$ & $\left(G_{\mathrm{BP}}-G_{\mathrm{RP}}\right)$ & $J$ & $(J-K)$ & $E(B-V)$ \\
\hline 20331212-4200270 & 6679615950005226240 & 12.197 & 0.722 & 11.989 & 0.992 & 10.706 & 0.537 & 0.038 \\
\hline $20331607-5047126$ & 6475629909958997376 & 12.365 & 0.765 & 12.205 & 0.988 & 10.949 & 0.525 & 0.031 \\
\hline $20351407-4625056$ & 6674758921813508736 & 12.855 & 0.946 & 12.468 & 1.241 & 10.890 & 0.710 & 0.032 \\
\hline $20354257-0714557$ & 6907591641550947072 & 10.884 & 0.942 & 10.580 & 1.235 & 9.038 & 0.731 & 0.044 \\
\hline 20373334-3645452 & 6683227639544432512 & 13.242 & 1.133 & 12.775 & 1.491 & 10.945 & 0.704 & 0.037 \\
\hline 20375779-2518252 & 6800352497219577728 & 14.468 & 0.790 & 14.233 & 1.058 & 12.821 & 0.531 & 0.040 \\
\hline $20381947-2750475$ & 6798666258699212672 & 12.703 & 0.675 & 12.480 & 0.973 & 11.199 & 0.495 & 0.049 \\
\hline $20385050-2657542$ & 6799133551141107712 & 11.866 & 0.442 & 11.649 & 0.793 & 10.398 & 0.555 & 0.066 \\
\hline 20404939-5843259 & 6455696764877344896 & 13.370 & 0.942 & 13.079 & 1.235 & 11.495 & 0.654 & 0.068 \\
\hline $20410627-3251350$ & 6792327226270824832 & 12.756 & 0.799 & 12.543 & 1.071 & 11.135 & 0.515 & 0.055 \\
\hline 20414841-5237274 & 6471501763492187520 & 12.601 & 1.092 & 12.204 & 1.355 & 10.514 & 0.670 & 0.027 \\
\hline $20430947-3600325$ & 6779175490951538560 & 12.959 & 0.780 & 12.725 & 1.075 & 11.320 & 0.580 & 0.040 \\
\hline 20440973-2940093 & 6795239665071836160 & 12.920 & 0.941 & 12.633 & 1.202 & 11.051 & 0.598 & 0.058 \\
\hline 20442721-7405038 & 6369772538209241216 & 12.600 & 0.651 & 12.313 & 0.968 & 11.110 & 0.515 & 0.051 \\
\hline 20443065-2936534 & 6795263648167892992 & 11.820 & 1.075 & 11.469 & 1.127 & 10.073 & 0.571 & 0.060 \\
\hline 20445501-3732403 & 6778221802053181824 & 12.992 & 0.911 & 12.737 & 1.111 & 11.356 & 0.618 & 0.032 \\
\hline 20451053-2934207 & 6795077693263710080 & 10.946 & 0.768 & 10.722 & 1.018 & 9.431 & 0.542 & 0.063 \\
\hline $20460102+1506250$ & 1762690140608564480 & 11.609 & 1.390 & 11.336 & 1.523 & 9.424 & 0.737 & 0.098 \\
\hline 20461039-3940468 & 6678691295087392640 & 12.875 & 0.787 & 12.631 & 1.086 & 11.169 & 0.504 & 0.036 \\
\hline 20461191-3833114 & 6681901765961577344 & 12.370 & 0.750 & 12.160 & 1.024 & 10.845 & 0.562 & 0.039 \\
\hline 20465470-3948150 & 6678675249089470464 & 11.574 & 0.847 & 11.315 & 1.126 & 9.866 & 0.606 & 0.037 \\
\hline 20474129-4949312 & 6480909150618081536 & 11.979 & 1.133 & 11.599 & 1.451 & 9.832 & 0.709 & 0.027 \\
\hline $20480642-3520259$ & 6779625977182979328 & 12.567 & 1.035 & 12.256 & 1.265 & 10.656 & 0.647 & 0.058 \\
\hline $20485047-7341446$ & 6370159673678547712 & 12.971 & 0.958 & 12.431 & 1.426 & 10.631 & 0.704 & 0.048 \\
\hline $20485505-4125356$ & 6678289698465087232 & 12.752 & 0.765 & 12.461 & 1.115 & 11.005 & 0.594 & 0.035 \\
\hline 20490816-2214501 & 6807184278357672576 & 12.923 & 0.913 & 12.503 & 1.311 & 10.841 & 0.723 & 0.074 \\
\hline 20492765-5124440 & 6477616903566840064 & 11.525 & 1.136 & 11.162 & 1.385 & 9.448 & 0.714 & 0.028 \\
\hline $20504877+1007551$ & 1751382561168000256 & 12.529 & 1.029 & 12.406 & 1.363 & 10.443 & 0.724 & 0.065 \\
\hline $20512785-4843325$ & 6481344866460154880 & 12.666 & 0.992 & 12.457 & 1.227 & 10.849 & 0.671 & 0.036 \\
\hline 20523629-5241033 & 6477303675894157824 & 13.050 & 0.874 & 12.662 & 1.205 & 11.155 & 0.633 & 0.028 \\
\hline $20530472-3836380$ & 6774883405575766400 & 12.448 & 0.907 & 12.169 & 1.223 & 10.606 & 0.638 & 0.043 \\
\hline 20531334-4520139 & 6484187516335125760 & 11.404 & 0.927 & 11.159 & 1.187 & 9.584 & 0.635 & 0.043 \\
\hline $20531555+1147415$ & 1757847139781935616 & 12.960 & 0.956 & 12.648 & 1.257 & 11.025 & 0.628 & 0.094 \\
\hline 20541462-4811494 & 6481473440600905472 & 13.035 & 0.726 & 12.805 & 1.019 & 11.469 & 0.542 & 0.027 \\
\hline 20545308-4710289 & 6481731516596154368 & 11.687 & 1.016 & 11.327 & 1.324 & 9.673 & 0.681 & 0.027 \\
\hline 20555702-3912091 & 6774608463246378880 & 11.632 & 0.800 & 11.408 & 1.074 & 10.053 & 0.563 & 0.036 \\
\hline 20565365-5609461 & 6457897089506392064 & 11.968 & 0.848 & 11.708 & 1.139 & 10.246 & 0.606 & 0.045 \\
\hline 20571292-4958553 & 6478163463924318208 & 12.925 & 0.824 & 12.642 & 1.131 & 11.150 & 0.577 & 0.035 \\
\hline $20574855-4154444$ & 6677344182887180800 & 12.847 & 0.885 & 12.898 & 1.124 & 11.129 & 0.597 & 0.036 \\
\hline $20575772-5637258$ & 6457695677015968384 & 11.949 & 0.957 & 11.672 & 1.208 & 10.114 & 0.619 & 0.052 \\
\hline $20580267+1427040$ & 1761667216837966848 & 12.518 & 0.821 & 12.209 & 1.176 & 10.694 & 0.598 & 0.082 \\
\hline 20585673-4013142 & 6773745346616608000 & 11.702 & 0.864 & 11.512 & 1.081 & 10.059 & 0.566 & 0.029 \\
\hline
\end{tabular}


Table 2 (continued)

\begin{tabular}{|c|c|c|c|c|c|c|c|c|}
\hline $\begin{array}{l}\text { Star Name } \\
\text { (2MASS) }\end{array}$ & $\begin{array}{c}\text { Star Name } \\
\text { (Gaia EDR3) }\end{array}$ & $V$ & $(B-V)$ & $G$ & $\left(G_{\mathrm{BP}}-G_{\mathrm{RP}}\right)$ & $J$ & $(J-K)$ & $E(B-V)$ \\
\hline $20590249+1847021$ & 1765354016765575680 & 11.314 & 0.997 & 10.948 & 1.358 & 9.139 & 0.731 & 0.114 \\
\hline 20592599-4724182 & 6483013375355411200 & 13.052 & 0.960 & 12.685 & 1.292 & 11.028 & 0.652 & 0.029 \\
\hline 20595162-4937337 & 6478288460356702464 & 12.872 & 0.541 & 12.719 & 0.752 & 11.911 & 0.498 & 0.027 \\
\hline 21011036-4537322 & 6483420263376508032 & 12.784 & 0.825 & 12.593 & 1.064 & 11.211 & 0.584 & 0.031 \\
\hline 21011096-4148546 & 6581257453716372352 & 12.738 & 0.960 & 12.492 & 1.267 & 10.814 & 0.668 & 0.032 \\
\hline 21014399-4913588 & 6478323339786839168 & 10.823 & 0.812 & 10.577 & 1.082 & 9.254 & 0.594 & 0.026 \\
\hline $21032352-4211400$ & 6581180590980731520 & 10.761 & 0.693 & 10.591 & 0.959 & 9.360 & 0.540 & 0.035 \\
\hline 21032466-7254213 & 6370267975574142592 & 13.618 & 0.866 & 13.331 & 1.176 & 11.783 & 0.580 & 0.053 \\
\hline 21032961-7321324 & 6370238112664426624 & 12.563 & 1.024 & 12.241 & 1.301 & 10.599 & 0.675 & 0.050 \\
\hline 21042794-4710017 & 6480078194704569088 & 12.929 & 0.909 & 12.649 & 1.169 & 11.173 & 0.662 & 0.030 \\
\hline 21051884-6831537 & 6376679571472808064 & 11.839 & 0.942 & 11.438 & 1.306 & 9.818 & 0.695 & 0.035 \\
\hline 21054066-4520565 & 6483621714522704128 & 11.772 & 0.767 & 11.491 & 1.085 & 10.094 & 0.565 & 0.029 \\
\hline $21080234+1835409$ & 1788340995265967488 & 12.376 & 0.822 & 11.816 & 1.306 & 10.115 & 0.639 & 0.091 \\
\hline $21091442-4721520$ & 6480023214826621696 & 12.492 & 0.895 & 12.304 & 1.212 & 10.706 & 0.659 & 0.027 \\
\hline 21092218-4250491 & 6580163542725955840 & 11.722 & 1.043 & 11.405 & 1.291 & 9.808 & 0.703 & 0.029 \\
\hline 21094841-5600060 & 6463024322681098368 & 13.545 & 0.765 & 13.347 & 1.027 & 12.019 & 0.546 & 0.028 \\
\hline $21095801+1725439$ & 1788003032879354752 & 10.747 & 0.799 & 10.473 & 1.136 & 8.979 & 0.557 & 0.091 \\
\hline $21102133+3016061$ & 1852687405024593024 & 11.413 & 0.981 & 11.087 & 1.261 & 9.467 & 0.659 & 0.144 \\
\hline $21105535+2140380$ & 1790165875330692352 & 12.559 & 0.829 & 15.432 & 1.751 & 10.638 & 0.621 & 0.154 \\
\hline 21110533-4239222 & 6580263048527430400 & 12.235 & 1.009 & 11.883 & 1.315 & 10.220 & 0.683 & 0.034 \\
\hline $21111175-4126536$ & 6581456534040000128 & 11.877 & 0.782 & 11.625 & 1.073 & 10.228 & 0.587 & 0.030 \\
\hline 21114008-5138220 & 6476892256683323008 & 13.899 & 0.778 & 13.693 & 1.026 & 12.360 & 0.555 & 0.022 \\
\hline $21115127-5257071$ & 6464688708407259008 & 10.822 & 0.786 & 10.523 & 1.111 & 9.099 & 0.597 & 0.032 \\
\hline $21120163+2520001$ & 1841468911788506112 & 11.870 & 0.908 & 11.647 & 1.195 & 10.091 & 0.641 & 0.110 \\
\hline $21125173+2110327$ & 1790077841384173312 & 12.102 & 0.887 & 11.767 & 1.206 & 10.175 & 0.603 & 0.118 \\
\hline 21134390-6802355 & 6399985335331112064 & 12.681 & 0.803 & 12.455 & 1.085 & 11.043 & 0.606 & 0.033 \\
\hline $21145602+2112242$ & 1791382721170477824 & 11.459 & 0.925 & 11.117 & 1.255 & 9.460 & 0.622 & 0.094 \\
\hline $21150824+2631245$ & 1847680160351457024 & 11.017 & 0.934 & 10.703 & 1.313 & 8.885 & 0.661 & 0.128 \\
\hline $21151790-4333404$ & 6579952677010742272 & 12.929 & 0.815 & 12.657 & 1.108 & 11.182 & 0.541 & 0.030 \\
\hline 21154971-6848520 & 6375872461218303488 & 12.815 & 0.800 & 12.552 & 1.099 & 11.120 & 0.590 & 0.042 \\
\hline $21171659-4115323$ & 6580773015762728704 & 11.609 & 0.862 & 11.285 & 1.209 & 9.761 & 0.668 & 0.026 \\
\hline $21190273+3318462$ & 1854629726683249024 & 12.168 & 1.012 & 11.927 & 1.148 & 10.421 & 0.599 & 0.159 \\
\hline 21192932-7715553 & 6356252535213950592 & 11.776 & 0.724 & 11.535 & 1.043 & 10.154 & 0.547 & 0.089 \\
\hline 21203573-5321426 & 6463751271665659264 & 12.264 & 0.800 & 12.060 & 1.065 & 10.656 & 0.546 & 0.022 \\
\hline $21211669+2032551$ & 1790500809763786624 & 12.503 & 0.942 & 12.133 & 1.244 & 10.520 & 0.627 & 0.088 \\
\hline $21214670+1916532$ & 1785668323017152384 & 12.826 & 0.927 & 12.418 & 1.270 & 10.807 & 0.685 & 0.071 \\
\hline $21232828-5328287$ & 6463713682111820160 & 12.078 & 0.822 & 11.842 & 1.090 & 10.433 & 0.603 & 0.019 \\
\hline $21240060-5241520$ & 6465286018099447680 & 11.684 & 0.831 & 11.420 & 1.106 & 10.003 & 0.601 & 0.017 \\
\hline 21243758-6400120 & 6403279953204895360 & 10.700 & 0.800 & 10.409 & 1.148 & 8.895 & 0.680 & 0.029 \\
\hline $21254398-6753045$ & 6399850542078013568 & 10.918 & 1.016 & 10.617 & 1.269 & 9.047 & 0.653 & 0.023 \\
\hline $21262879+1749436$ & 1784515446419862656 & 12.884 & 0.911 & 12.570 & 1.221 & 10.867 & 0.690 & 0.061 \\
\hline $21263180+2031469$ & 1790358392944627712 & 12.031 & 0.915 & 11.789 & 1.287 & 10.131 & 0.662 & 0.079 \\
\hline
\end{tabular}


Table 2 (continued)

\begin{tabular}{|c|c|c|c|c|c|c|c|c|}
\hline $\begin{array}{l}\text { Star Name } \\
\text { (2MASS) }\end{array}$ & $\begin{array}{c}\text { Star Name } \\
\text { (Gaia EDR3) }\end{array}$ & $V$ & $(B-V)$ & $G$ & $\left(G_{\mathrm{BP}}-G_{\mathrm{RP}}\right)$ & $J$ & $(J-K)$ & $E(B-V)$ \\
\hline $21281884-6406460$ & 6403090356168392192 & 12.689 & 0.923 & 12.421 & 1.221 & 10.867 & 0.663 & 0.031 \\
\hline 21292831-5558258 & 6460174865281654144 & 12.430 & 0.962 & 12.094 & 1.250 & 10.551 & 0.694 & 0.037 \\
\hline 21313828-6858199 & 6396651616076109184 & 13.751 & 0.801 & 13.502 & 1.098 & 12.067 & 0.565 & 0.025 \\
\hline 21322880-6607539 & 6400620298999247488 & 13.115 & 0.974 & 12.606 & 1.450 & 10.827 & 0.731 & 0.027 \\
\hline $21323054+1600097$ & 1772048878641647488 & 11.703 & 0.797 & 11.409 & 1.094 & 9.975 & 0.588 & 0.090 \\
\hline 21340283-6224209 & 6403844758583266688 & 12.342 & 0.789 & 12.096 & 1.072 & 10.694 & 0.563 & 0.032 \\
\hline $21343861+1538156$ & 1771832962045862656 & 12.895 & 0.905 & 12.563 & 1.193 & 11.022 & 0.598 & 0.092 \\
\hline 21351702-5533118 & 6460110479427930624 & 11.911 & 0.789 & 11.663 & 1.083 & 10.260 & 0.588 & 0.035 \\
\hline 21373999-2446495 & 6814831136913490048 & 12.156 & 0.952 & 11.901 & 1.246 & 10.264 & 0.660 & 0.041 \\
\hline $21391225+3849406$ & 1953863502940549504 & 12.616 & 0.763 & 12.998 & 1.162 & 11.425 & 0.597 & 0.235 \\
\hline $21393394-5845548$ & 6458363870847393792 & 12.537 & 1.099 & 12.210 & 1.347 & 10.532 & 0.701 & 0.035 \\
\hline $21414000-2854239$ & 6809748884932883712 & 12.057 & 0.926 & 11.782 & 1.194 & 10.271 & 0.675 & 0.033 \\
\hline 21430664-6338509 & 6402785069892389760 & 11.653 & 0.782 & 11.377 & 1.074 & 9.991 & 0.555 & 0.034 \\
\hline $21440820+3813139$ & 1952857308060989312 & 12.870 & 0.979 & 12.483 & 1.314 & 10.761 & 0.681 & 0.234 \\
\hline $21445442-6319381$ & 6402818531980755584 & 12.822 & 0.885 & 12.480 & 1.312 & 10.712 & 0.692 & 0.027 \\
\hline $21453847+2351116$ & 1794596868534480128 & 10.517 & 0.713 & 10.262 & 1.040 & 8.887 & 0.558 & 0.070 \\
\hline $21461136-5420472$ & 6461349457233919872 & 11.726 & 1.104 & 11.302 & 1.451 & 9.501 & 0.701 & 0.016 \\
\hline $21480606+4643071$ & 1974610531448247936 & 12.154 & 1.213 & 11.618 & 1.463 & 9.757 & 0.782 & 0.300 \\
\hline $21482057+2155222$ & 1793330952694172416 & 12.790 & 0.846 & 12.479 & 1.181 & 11.021 & 0.593 & 0.101 \\
\hline $21494865+1048431$ & 1765600930139450752 & 12.082 & 0.821 & 11.862 & 1.102 & 10.419 & 0.583 & 0.079 \\
\hline $21502426-6105576$ & 6409890217109702784 & 13.796 & 0.957 & 13.463 & 1.264 & 11.867 & 0.681 & 0.033 \\
\hline $21510307+3619543$ & 1949409175177462272 & 12.052 & 1.212 & 11.625 & 1.520 & 9.690 & 0.728 & 0.210 \\
\hline $21513710-7925459$ & 6355014691279286400 & 13.023 & 0.864 & 12.684 & 1.145 & 11.215 & 0.600 & 0.113 \\
\hline $21515415+0537172$ & 2697022209558112768 & 11.214 & 1.015 & 10.869 & 1.293 & 9.233 & 0.689 & 0.043 \\
\hline $21525027-6604240$ & 6398934786330198784 & 11.634 & 0.850 & 11.478 & 1.143 & 10.000 & 0.675 & 0.031 \\
\hline $21555532+3501402$ & 1948312515407711104 & 12.850 & 0.969 & 12.543 & 1.275 & 10.868 & 0.664 & 0.149 \\
\hline 21573551-0308043 & 2670534149811033088 & 11.800 & 0.680 & 11.452 & 1.074 & 10.033 & 0.571 & 0.084 \\
\hline $21573761+3544196$ & 1948748437403259776 & 12.982 & 0.944 & 12.651 & 1.342 & 10.921 & 0.667 & 0.171 \\
\hline $21584417+0052490$ & 2681491607815613952 & 12.214 & 0.721 & 11.952 & 1.024 & 10.592 & 0.513 & 0.040 \\
\hline $21584491+0129524$ & 2681597607608087040 & 11.663 & 1.163 & 11.413 & 1.355 & 9.711 & 0.712 & 0.051 \\
\hline 22013669-6918312 & 6396019706127003520 & 12.991 & 0.943 & 12.723 & 1.200 & 11.180 & 0.679 & 0.034 \\
\hline 22032935-5635128 & 6412646138709077888 & 12.703 & 0.872 & 12.478 & 1.141 & 10.976 & 0.577 & 0.019 \\
\hline $22040352+0016519$ & 2680470706974136192 & 12.150 & 1.028 & 11.702 & 1.374 & 9.954 & 0.699 & 0.049 \\
\hline 22043661-6044348 & 6409307304149253248 & 12.322 & 0.704 & 12.100 & 1.027 & 10.753 & 0.522 & 0.034 \\
\hline $22044480+4148205$ & 1959828147361822848 & 13.032 & 0.813 & 12.779 & 1.099 & 11.357 & 0.583 & 0.224 \\
\hline $22050237+3107332$ & 1898564081014685184 & 12.470 & 0.739 & 12.705 & 0.983 & 11.151 & 0.661 & 0.080 \\
\hline 22082896-6607320 & 6399063394830844544 & 12.961 & 0.727 & 12.763 & 1.018 & 11.422 & 0.529 & 0.029 \\
\hline 22104990-3947023 & 6573266443723504768 & 12.897 & 0.738 & 12.708 & 1.008 & 11.374 & 0.566 & 0.015 \\
\hline 22112456-3753100 & 6574080735161542784 & 10.855 & 0.952 & 10.585 & 1.195 & 9.046 & 0.624 & 0.015 \\
\hline 22115350-1209181 & 2612971211403496832 & 11.810 & 0.812 & 11.559 & 1.126 & 10.093 & 0.583 & 0.033 \\
\hline $22120815-4215210$ & 6569918602615687808 & 13.081 & 0.755 & 12.831 & 1.048 & 11.458 & 0.537 & 0.011 \\
\hline 22125424-0235414 & 2675834650555144576 & 12.218 & 0.705 & 11.993 & 0.982 & 10.749 & 0.566 & 0.068 \\
\hline
\end{tabular}


Table 2 (continued)

\begin{tabular}{|c|c|c|c|c|c|c|c|c|}
\hline $\begin{array}{l}\text { Star Name } \\
\text { (2MASS) }\end{array}$ & $\begin{array}{c}\text { Star Name } \\
\text { (Gaia EDR3) }\end{array}$ & $V$ & $(B-V)$ & $G$ & $\left(G_{\mathrm{BP}}-G_{\mathrm{RP}}\right)$ & $J$ & $(J-K)$ & $E(B-V)$ \\
\hline 22125459-0408594 & 2627229884351014144 & 11.671 & 0.952 & 11.352 & 1.247 & 9.763 & 0.656 & 0.080 \\
\hline 22140658-0016065 & 2678634350759886336 & 11.804 & 1.077 & 11.394 & 1.466 & 9.553 & 0.723 & 0.070 \\
\hline $22165631-1154207$ & 2612857755547023232 & 11.083 & 0.741 & 10.936 & 1.004 & 9.640 & 0.578 & 0.041 \\
\hline 22211976-4111021 & 6569481855982624640 & 11.522 & 0.857 & 11.246 & 1.165 & 9.750 & 0.632 & 0.011 \\
\hline $22213981+3725402$ & 1906965002686762496 & 12.888 & 0.851 & 12.763 & 1.135 & 11.088 & 0.655 & 0.105 \\
\hline $22221878-7406283$ & 6357952517629300352 & 12.497 & 0.828 & 12.046 & 1.267 & 10.413 & 0.624 & 0.038 \\
\hline $22224538+3555471$ & 1905936405260048384 & 12.611 & 0.966 & 12.319 & 1.275 & 10.659 & 0.660 & 0.118 \\
\hline $22224859+2804264$ & 1881899328730042368 & 11.703 & 0.806 & 11.493 & 1.102 & 10.039 & 0.565 & 0.069 \\
\hline $22225487+2954084$ & 1894651090929156096 & 12.834 & 0.823 & 12.545 & 1.116 & 11.087 & 0.575 & 0.065 \\
\hline $22234372-8627533$ & 6342106630928779392 & 12.969 & 1.148 & 12.543 & 1.558 & 10.608 & 0.740 & 0.140 \\
\hline $22235096-6512086$ & 6404249993041212928 & 11.550 & 0.742 & 11.471 & 0.965 & 10.145 & 0.520 & 0.025 \\
\hline $22241668+2548128$ & 1879125024671706624 & 13.047 & 0.759 & 12.896 & 1.003 & 11.541 & 0.610 & 0.050 \\
\hline $22241984+2430382$ & 1878702846566493440 & 12.076 & 0.825 & 11.684 & 1.120 & 10.155 & 0.642 & 0.058 \\
\hline $22244368+3236401$ & 1901779602770732032 & 13.028 & 0.987 & 12.656 & 1.189 & 11.127 & 0.672 & 0.071 \\
\hline $22251594+2911332$ & 1894379923873649536 & 11.472 & 0.735 & 11.221 & 1.026 & 9.849 & 0.563 & 0.059 \\
\hline $22280812+3546524$ & 1905786459361887488 & 12.448 & 1.081 & 11.936 & 1.394 & 10.206 & 0.741 & 0.102 \\
\hline $22284549-4124119$ & 6593293356532115712 & 12.926 & 0.793 & 12.480 & 1.247 & 10.906 & 0.645 & 0.012 \\
\hline $22290341+3954145$ & 1909092729485051776 & 12.040 & 0.883 & 11.764 & 1.147 & 10.415 & 0.656 & 0.122 \\
\hline $22293323+4332048$ & 1981952760850101376 & 11.527 & 0.816 & 11.224 & 1.172 & 9.671 & 0.615 & 0.126 \\
\hline 22294083-3305402 & 6600574876582217344 & 12.021 & 0.852 & 11.773 & 1.163 & 10.263 & 0.615 & 0.011 \\
\hline 22303946-1809055 & 2594309161890648320 & 11.503 & 1.122 & 11.025 & 1.484 & 9.212 & 0.706 & 0.037 \\
\hline $22310829+3023018$ & 1900531824573844608 & 12.603 & 0.721 & 12.391 & 1.080 & 11.024 & 0.589 & 0.074 \\
\hline $22311433-6656572$ & 6386075551166390144 & 11.560 & 0.878 & 11.306 & 1.157 & 9.813 & 0.629 & 0.021 \\
\hline $22315231+2320202$ & 1875411630307299456 & 12.493 & 0.710 & 12.365 & 0.979 & 11.051 & 0.653 & 0.042 \\
\hline $22342447+2739353$ & 1881261994239589888 & 12.000 & 0.961 & 11.591 & 1.220 & 10.050 & 0.686 & 0.040 \\
\hline $22345447-6605172$ & 6392134513070641408 & 11.359 & 0.776 & 11.135 & 1.087 & 9.722 & 0.592 & 0.021 \\
\hline 22354139-4305549 & 6520905878946665344 & 12.088 & 0.830 & 11.849 & 1.115 & 10.382 & 0.606 & 0.013 \\
\hline $22355721-2434108$ & 6623904314300239488 & 12.344 & 1.088 & 14.476 & 0.000 & 10.262 & 0.738 & 0.022 \\
\hline $22360602+3930413$ & 1908318669000212480 & 12.223 & 0.902 & 11.928 & 1.181 & 10.440 & 0.663 & 0.159 \\
\hline 22364074-7026524 & 6384994623861077376 & 12.884 & 0.625 & 12.937 & 0.797 & 11.922 & 0.409 & 0.022 \\
\hline 22373316-4341181 & 6520826714109323392 & 10.977 & 0.770 & 10.736 & 1.064 & 9.384 & 0.594 & 0.010 \\
\hline $22401067-3738259$ & 6595625729931613568 & 11.411 & 0.900 & 11.155 & 1.173 & 9.673 & 0.637 & 0.014 \\
\hline $22412632-3627304$ & 6596002145160945664 & 11.821 & 0.812 & 11.573 & 1.107 & 10.118 & 0.553 & 0.011 \\
\hline $22413573+2931038$ & 1887539965117822464 & 12.098 & 0.942 & 11.734 & 1.291 & 10.108 & 0.669 & 0.061 \\
\hline $22432022-7547340$ & 6357547759911189120 & 11.766 & 0.665 & 11.727 & 0.838 & 10.572 & 0.463 & 0.061 \\
\hline $22451263+3532066$ & 1903656636619168768 & 12.652 & 1.010 & 12.268 & 1.310 & 10.585 & 0.677 & 0.077 \\
\hline $22453503+1301210$ & 2731609959149919360 & 12.444 & 0.845 & 12.171 & 1.164 & 10.627 & 0.564 & 0.046 \\
\hline $22454796+2826260$ & 1887186850086385152 & 12.945 & 0.858 & 12.638 & 1.165 & 11.074 & 0.633 & 0.047 \\
\hline $22471965-7201440$ & 6382433144021702656 & 11.658 & 0.724 & 11.472 & 0.985 & 10.145 & 0.637 & 0.025 \\
\hline $22472643+3532411$ & 1903562838830927744 & 12.502 & 0.917 & 12.207 & 1.166 & 10.690 & 0.621 & 0.090 \\
\hline $22473447-5511534$ & 6505905757201226368 & 12.023 & 0.674 & 11.723 & 1.001 & 10.329 & 0.626 & 0.018 \\
\hline $22474545+2517113$ & 1876790894628571264 & 12.742 & 0.927 & 12.488 & 1.198 & 10.996 & 0.711 & 0.099 \\
\hline
\end{tabular}


Table 2 (continued)

\begin{tabular}{|c|c|c|c|c|c|c|c|c|}
\hline $\begin{array}{l}\text { Star Name } \\
\text { (2MASS) }\end{array}$ & $\begin{array}{c}\text { Star Name } \\
\text { (Gaia EDR3) }\end{array}$ & $V$ & $(B-V)$ & $G$ & $\left(G_{\mathrm{BP}}-G_{\mathrm{RP}}\right)$ & $J$ & $(J-K)$ & $E(B-V)$ \\
\hline 22481449-5703067 & 6504026829267642240 & 11.927 & 0.659 & 11.767 & 0.889 & 10.606 & 0.528 & 0.011 \\
\hline 22490353-5536242 & 6505879059684460032 & 11.009 & 0.880 & 12.480 & 0.000 & 9.278 & 0.635 & 0.012 \\
\hline 22492811-5717202 & 6503988105842414464 & 12.964 & 0.773 & 12.644 & 1.037 & 11.382 & 0.578 & 0.012 \\
\hline $22511876-3814381$ & 6547694891340666752 & 11.217 & 0.834 & 11.032 & 1.071 & 9.681 & 0.593 & 0.013 \\
\hline 22512739-5049405 & 6513929237145370880 & 11.082 & 0.825 & 10.874 & 1.059 & 9.508 & 0.561 & 0.008 \\
\hline 22531950-6647163 & 6391157150312476032 & 11.863 & 1.136 & 11.472 & 1.413 & 9.738 & 0.703 & 0.022 \\
\hline $22534476-5456485$ & 6506123701021637888 & 12.857 & 0.691 & 12.645 & 0.963 & 11.409 & 0.547 & 0.017 \\
\hline $22534538+7919586$ & 2285728337337892480 & 12.867 & 0.944 & 12.552 & 1.228 & 11.063 & 0.588 & 0.201 \\
\hline $22544750-3509160$ & 6554282379035795840 & 12.453 & 1.151 & 12.019 & 1.478 & 10.206 & 0.746 & 0.011 \\
\hline $22575548-5622538$ & 6493611254400172416 & 12.658 & 0.942 & 12.352 & 1.227 & 10.814 & 0.710 & 0.017 \\
\hline 22591090-4829425 & 6515325616912384256 & 12.176 & 0.855 & 11.962 & 1.101 & 10.531 & 0.587 & 0.009 \\
\hline 22594336-3819324 & 6546814427341069568 & 12.806 & 1.000 & 12.599 & 1.267 & 10.872 & 0.630 & 0.009 \\
\hline $23003637+2820257$ & 1885059776122105728 & 12.994 & 0.796 & 12.897 & 1.060 & 11.434 & 0.597 & 0.057 \\
\hline $23022817-4059099$ & 6543252452048113152 & 11.912 & 0.894 & 11.711 & 1.142 & 10.227 & 0.615 & 0.009 \\
\hline 23035329-4139482 & 6543169129682052608 & 13.707 & 0.803 & 13.493 & 1.055 & 12.104 & 0.539 & 0.008 \\
\hline $23043022+0155166$ & 2652540916900514304 & 12.079 & 1.086 & 11.894 & 1.276 & 10.278 & 0.664 & 0.056 \\
\hline $23044868-4311029$ & 6541868716664047744 & 12.876 & 1.126 & 12.632 & 1.473 & 10.674 & 0.767 & 0.008 \\
\hline $23055841-8636001$ & 6341894558326196480 & 12.740 & 1.122 & 12.125 & 1.504 & 10.169 & 0.778 & 0.126 \\
\hline $23064708+2802027$ & 1884897937457948672 & 11.285 & 0.965 & 10.949 & 1.311 & 9.302 & 0.668 & 0.099 \\
\hline 23083044-7441319 & 6378503729982312576 & 13.184 & 0.856 & 12.893 & 1.149 & 11.384 & 0.579 & 0.024 \\
\hline $23085453-5226130$ & 6500818969734685440 & 11.895 & 0.981 & 11.516 & 1.265 & 10.002 & 0.716 & 0.009 \\
\hline 23093209-7132507 & 6381003327932886144 & 12.573 & 0.857 & 12.266 & 1.198 & 10.718 & 0.605 & 0.030 \\
\hline 23100319-7702165 & 6354182945092770176 & 11.943 & 0.993 & 11.653 & 1.296 & 9.983 & 0.670 & 0.078 \\
\hline 23123243-0240516 & 2638139066923296128 & 12.915 & 1.116 & 12.532 & 1.386 & 10.800 & 0.691 & 0.040 \\
\hline $23124700+2701045$ & 2845306436489791232 & 11.553 & 0.905 & 11.348 & 1.231 & 9.636 & 0.607 & 0.082 \\
\hline 23130418-4332060 & 6541663554666433920 & 12.123 & 0.863 & 11.919 & 1.078 & 10.546 & 0.592 & 0.008 \\
\hline $23131220+5425552$ & 1996424051866554112 & 11.954 & 1.396 & 11.427 & 1.482 & 9.598 & 0.839 & 0.300 \\
\hline 23133742-5336585 & 6500438435631474560 & 12.001 & 1.033 & 11.702 & 1.327 & 9.968 & 0.746 & 0.009 \\
\hline $23164530-4047253$ & 6548299386513340288 & 11.567 & 0.919 & 11.355 & 1.191 & 9.773 & 0.654 & 0.015 \\
\hline 23235454-4730233 & 6526777614634204672 & 10.815 & 0.755 & 10.475 & 1.118 & 9.047 & 0.658 & 0.005 \\
\hline $23273196+5438201$ & 1995911404570328192 & 11.766 & 0.474 & 11.606 & 0.757 & 10.787 & 0.531 & 0.300 \\
\hline $23293844+3337097$ & 2872688983306031616 & 11.435 & 0.995 & 11.113 & 1.238 & 9.525 & 0.661 & 0.106 \\
\hline $23321307+1950398$ & 2824757388800259840 & 10.824 & 0.972 & 10.582 & 1.250 & 8.886 & 0.656 & 0.061 \\
\hline $23341995+4703450$ & 1938499679067388288 & 12.880 & 0.906 & 12.661 & 1.175 & 11.076 & 0.673 & 0.142 \\
\hline $23362842-5537423$ & 6496310731541441152 & 11.408 & 0.722 & 11.141 & 1.054 & 9.758 & 0.569 & 0.010 \\
\hline $23371202+2100145$ & 2826450808506230016 & 12.189 & 1.228 & 11.843 & 1.606 & 9.714 & 0.745 & 0.043 \\
\hline $23400099+4959092$ & 1943744250753474944 & 12.640 & 0.837 & 12.369 & 1.206 & 10.766 & 0.605 & 0.175 \\
\hline $23421250-6346584$ & 6485737793369175680 & 12.006 & 0.901 & 11.716 & 1.182 & 10.204 & 0.640 & 0.019 \\
\hline $23430472-8200221$ & 6350719930141819520 & 12.038 & 0.976 & 11.751 & 1.267 & 10.122 & 0.656 & 0.165 \\
\hline $23433753+5008599$ & 1943761190104941824 & 12.606 & 0.551 & 12.473 & 0.893 & 11.104 & 0.644 & 0.153 \\
\hline $23450930+5538498$ & 1994885560221975552 & 12.466 & 1.224 & 11.872 & 1.508 & 9.922 & 0.835 & 0.300 \\
\hline $23465497+1201211$ & 2763718305727188480 & 12.728 & 0.930 & 12.612 & 1.351 & 10.689 & 0.669 & 0.053 \\
\hline
\end{tabular}


Table 2 (continued)

\begin{tabular}{|c|c|c|c|c|c|c|c|c|}
\hline $\begin{array}{c}\text { Star Name } \\
\text { (2MASS) }\end{array}$ & $\begin{array}{c}\text { Star Name } \\
\text { (Gaia EDR3) }\end{array}$ & $V$ & $(B-V)$ & $G$ & $\left(G_{\mathrm{BP}}-G_{\mathrm{RP}}\right)$ & $J$ & $(J-K)$ & $E(B-V)$ \\
\hline $23472384+4835469$ & 1940559103007605120 & 11.378 & 0.990 & 11.025 & 1.297 & 9.378 & 0.718 & 0.123 \\
\hline $23505349+1142348$ & 2766637307235175296 & 12.560 & 0.672 & 12.213 & 1.074 & 10.793 & 0.543 & 0.053 \\
\hline $23550005+1255039$ & 2766874664308017408 & 13.025 & 1.008 & 12.590 & 1.384 & 10.854 & 0.709 & 0.048 \\
\hline $23555398+5702462$ & 1998062118035485056 & 12.298 & 0.885 & 12.062 & 1.192 & 10.369 & 0.620 & 0.300 \\
\hline $23560245+1109200$ & 2765621221052354816 & 12.378 & 0.886 & 11.982 & 1.292 & 10.352 & 0.657 & 0.075 \\
\hline $23562635+0651168$ & 2746230337064331776 & 13.016 & 0.960 & 12.696 & 1.216 & 11.148 & 0.656 & 0.060 \\
\hline $23563722+4615436$ & 1927134439687586560 & 12.410 & 1.113 & 12.131 & 1.869 & 9.931 & 0.779 & 0.106 \\
\hline $23564530-4429484$ & 6532542491534291968 & 12.930 & 0.811 & 12.616 & 1.138 & 11.142 & 0.588 & 0.010 \\
\hline
\end{tabular}


Table 3. Stellar Atmospheric Parameters and Abundances

\begin{tabular}{|c|c|c|c|c|c|c|c|c|}
\hline $\begin{array}{c}\text { Star Name } \\
(2 \mathrm{MASS})\end{array}$ & $\begin{array}{c}\text { Star Name } \\
\text { (Gaia EDR3) }\end{array}$ & $\begin{array}{l}T_{\text {eff }} \\
(\mathrm{K})\end{array}$ & $\begin{array}{l}\log g \\
(\operatorname{cgs})\end{array}$ & {$[\mathrm{Fe} / \mathrm{H}]$} & {$[\mathrm{C} / \mathrm{Fe}]$} & {$[\mathrm{C} / \mathrm{Fe}]_{c}$} & $A(\mathrm{C})_{c}$ & {$[\mathrm{Mg} / \mathrm{Fe}]$} \\
\hline 00003305-7953389 & 4634573766005607552 & 5280 & 2.06 & -1.93 & 0.13 & 0.17 & 6.67 & 0.38 \\
\hline 00020162-4430117 & 4994519032163925632 & 5036 & 2.00 & -1.81 & 0.30 & 0.37 & 6.99 & $\ldots$ \\
\hline 00040305-6106367 & 4905632480654004608 & 4648 & 4.13 & -1.27 & 0.15 & 0.15 & 7.31 & -0.19 \\
\hline $00043646+4124062$ & 384060304935385984 & 6068 & 3.51 & -1.29 & 0.95 & 0.95 & 8.09 & 0.14 \\
\hline $00045403+3524010$ & 2876804519751163008 & 5455 & 3.52 & -2.59 & 0.50 & 0.50 & 6.34 & 0.06 \\
\hline $00052731+0025510$ & 2546113345293028608 & 5397 & 4.41 & -0.25 & 0.36 & 0.36 & 8.54 & 0.37 \\
\hline 00060902-6238019 & 4904480471642380928 & 4810 & 1.41 & -2.33 & 0.26 & 0.73 & 6.82 & 0.01 \\
\hline $00071189+4724466$ & 393031258963415936 & 5868 & 3.98 & -0.53 & 0.46 & 0.46 & 8.36 & 0.30 \\
\hline $00111339+0152512$ & 2546752127188959232 & 5003 & 1.83 & -2.39 & 0.44 & 0.62 & 6.65 & 0.26 \\
\hline $00140089+3148167$ & 2861747777456341120 & 5090 & 2.13 & -0.83 & -0.24 & -0.19 & 7.42 & $\ldots$ \\
\hline 00144636-2246093 & 2361100512255883904 & 4973 & 2.92 & -1.14 & 0.22 & 0.24 & 7.54 & 0.59 \\
\hline 00150914-3736048 & 2308246644711975680 & 4737 & 3.77 & -1.68 & 0.77 & 0.77 & 7.52 & -0.06 \\
\hline 00152923-2436237 & 2336022438732700672 & 4768 & 4.66 & -1.02 & -0.31 & -0.31 & 7.10 & 0.42 \\
\hline 00152952-6121258 & 4904881106191486592 & 4875 & 1.52 & -2.33 & -0.07 & 0.44 & 6.54 & 0.21 \\
\hline 00154806-6253207 & 4901504815220315648 & 4860 & 1.37 & -2.50 & 0.25 & 0.76 & 6.69 & -0.10 \\
\hline 00162809-0505519 & 2443891577459590016 & 5430 & 3.08 & -2.75 & 0.94 & 0.95 & 6.63 & 0.22 \\
\hline $00163655+3538314$ & 2876439211309388672 & 4996 & 1.94 & -2.06 & 0.29 & 0.40 & 6.77 & 0.43 \\
\hline 00163809-4912369 & 4977077135617904640 & 4648 & 1.13 & -2.22 & -0.44 & 0.24 & 6.45 & 0.30 \\
\hline $00165353+3642326$ & 2876647328245159552 & 5221 & 1.40 & -3.01 & 0.61 & 1.07 & 6.49 & 0.42 \\
\hline $00170767+4614488$ & 392109932642310272 & 5265 & 3.57 & -1.03 & 0.16 & 0.16 & 7.55 & 0.11 \\
\hline 00175076-6819295 & 4706413931618489600 & 5069 & 1.84 & -2.07 & -0.18 & 0.01 & 6.36 & 0.21 \\
\hline $00182947-7829325$ & 4635466706886248448 & 5159 & 4.54 & -0.86 & 0.35 & 0.35 & 7.92 & 0.20 \\
\hline $00192284+4431554$ & 385734487485292928 & 4974 & 4.51 & -0.66 & 0.26 & 0.26 & 8.04 & 0.10 \\
\hline 00202312-3950260 & 4997141779713479680 & 4693 & 4.14 & -0.08 & -0.72 & -0.72 & 7.63 & -0.07 \\
\hline $00213396+0008219$ & 2545302077511395968 & 4967 & 4.73 & -1.03 & -0.10 & -0.10 & 7.30 & 0.39 \\
\hline 00223511-4231148 & 4992150679821873664 & 5058 & 2.05 & -2.48 & 0.43 & 0.46 & 6.41 & 0.19 \\
\hline 00234358-1117357 & 2424691974134738816 & 5057 & 4.46 & -0.85 & 0.25 & 0.25 & 7.82 & 0.29 \\
\hline 00235345-6649211 & 4707128682896207872 & 5072 & 2.25 & -1.61 & 0.28 & 0.30 & 7.12 & 0.11 \\
\hline $00241677+2941440$ & 2858965188404148480 & 5320 & 2.50 & -0.22 & -0.62 & -0.59 & 7.62 & 0.17 \\
\hline $00275509+3458068$ & 365999795656991616 & 4989 & 1.50 & -2.13 & -0.19 & 0.28 & 6.58 & 0.34 \\
\hline 00281899-6820268 & 4703742084003326080 & 4641 & 1.13 & -2.44 & -0.41 & 0.30 & 6.28 & 0.01 \\
\hline $00293797+2103054$ & 2796582811359131392 & 5207 & 2.09 & -2.11 & 0.42 & 0.44 & 6.75 & 0.43 \\
\hline $00301775+2957334$ & 2858881625520606848 & 4822 & 1.47 & -1.99 & -0.15 & 0.30 & 6.74 & 0.40 \\
\hline $00303445+2816193$ & 2857835710788936832 & 4465 & 4.35 & -1.67 & 0.42 & 0.42 & 7.18 & -0.09 \\
\hline $00311900+4957158$ & 391597358363860608 & 5308 & 4.32 & -0.26 & -0.23 & -0.23 & 7.95 & -0.05 \\
\hline $00325147+4107490$ & 381063654713703040 & 5216 & 2.11 & -2.81 & 0.45 & 0.46 & 6.08 & 0.41 \\
\hline $00331311+2033190$ & 2796309861892160384 & 5222 & 4.59 & -0.60 & 0.02 & 0.02 & 7.85 & 0.16 \\
\hline 00341417-3943068 & 4994368227272214784 & 4999 & 1.52 & -2.48 & -0.05 & 0.41 & 6.36 & -0.04 \\
\hline 00342795-2413107 & 2348080164279166208 & 4443 & 4.52 & -1.99 & 0.59 & 0.59 & 7.04 & 0.16 \\
\hline 00351869-2854190 & 2319002587146050304 & 5057 & 4.85 & -1.00 & 0.16 & 0.16 & 7.59 & 0.49 \\
\hline $00354136+1618228$ & 2780880960680375808 & 4843 & 4.83 & -0.88 & 0.04 & 0.04 & 7.60 & 0.37 \\
\hline
\end{tabular}


Table 3 (continued)

\begin{tabular}{|c|c|c|c|c|c|c|c|c|}
\hline $\begin{array}{l}\text { Star Name } \\
\text { (2MASS) }\end{array}$ & $\begin{array}{c}\text { Star Name } \\
\text { (Gaia EDR3) }\end{array}$ & $\begin{array}{l}T_{\text {eff }} \\
(\mathrm{K})\end{array}$ & $\begin{array}{l}\log g \\
(\operatorname{cgs})\end{array}$ & {$[\mathrm{Fe} / \mathrm{H}]$} & {$[\mathrm{C} / \mathrm{Fe}]$} & {$[\mathrm{C} / \mathrm{Fe}]_{c}$} & $A(\mathrm{C})_{c}$ & {$[\mathrm{Mg} / \mathrm{Fe}]$} \\
\hline $00370433+4341208$ & 387703815888550656 & 4608 & 0.75 & -2.83 & -0.01 & 0.72 & 6.32 & 0.28 \\
\hline 00370966-4224102 & 4992914501101243264 & 5326 & 4.20 & -0.73 & 0.29 & 0.29 & 8.00 & 0.23 \\
\hline 00374809-2751193 & 2343095974991737600 & 4539 & 4.39 & -0.46 & -1.19 & -1.19 & 6.78 & 0.05 \\
\hline $00400685-4325183$ & 4980747129334018944 & 4562 & 1.38 & -3.07 & -0.75 & -0.20 & 5.16 & $\cdots$ \\
\hline $00410089+3633397$ & 365840847508048384 & 5192 & 1.73 & -2.47 & -0.19 & 0.08 & 6.04 & 0.54 \\
\hline $00410467+1649478$ & 2782313903504860032 & 4709 & 3.30 & -0.97 & -0.39 & -0.38 & 7.09 & 0.32 \\
\hline $00425182+1925361$ & 2795241995584048000 & 5154 & 2.61 & -1.56 & 0.05 & 0.07 & 6.94 & 0.33 \\
\hline 00433651-2719379 & 2343181981712123520 & 4791 & 4.52 & -1.20 & 0.13 & 0.13 & 7.36 & 0.32 \\
\hline 00435264-2132285 & 2350389894612060544 & 5153 & 2.09 & -1.87 & 0.32 & 0.35 & 6.91 & 0.13 \\
\hline 00452879-5846450 & 4906911251332870144 & 4921 & 1.73 & -2.33 & 0.29 & 0.58 & 6.68 & -0.06 \\
\hline 00463619-3739335 & 5000753194373767424 & 5031 & 2.74 & -3.98 & -0.15 & -0.15 & 4.30 & 0.37 \\
\hline 00482546-7441092 & 4685477782827620992 & 5401 & 3.14 & -0.53 & -0.25 & -0.24 & 7.66 & 0.03 \\
\hline $00482715-8224023$ & 4629945985987660416 & 4870 & 1.38 & -2.02 & -0.10 & 0.41 & 6.81 & 0.44 \\
\hline 00504527-6351504 & 4709709305405862656 & 4920 & 2.10 & -3.25 & -0.27 & -0.27 & 4.91 & 0.25 \\
\hline 00510748-7809118 & 4635149768362820096 & 4936 & 4.66 & -0.17 & -0.20 & -0.20 & 8.06 & 0.00 \\
\hline 00521010-6006097 & 4903551070783622528 & 4869 & 4.52 & -0.05 & -0.15 & -0.15 & 8.24 & -0.02 \\
\hline $00522310-5804087$ & 4907332914042242304 & 5472 & 3.62 & -0.74 & 0.32 & 0.32 & 8.02 & -0.05 \\
\hline $00523111+3758286$ & 367656523458156544 & 5289 & 2.66 & -2.23 & 0.74 & 0.75 & 6.95 & 0.25 \\
\hline $00582707+0633561$ & 2553447805108682496 & 4906 & 1.49 & -2.57 & 0.33 & 0.76 & 6.62 & 0.37 \\
\hline $00594615+1223173$ & 2584343227151274752 & 4843 & 4.28 & -1.22 & 0.34 & 0.34 & 7.55 & 0.05 \\
\hline $01021265+0428241$ & 2551971405806340096 & 4774 & 1.41 & -2.76 & -0.51 & 0.04 & 5.70 & 0.29 \\
\hline $01024809+4300486$ & 376344555103575808 & 4641 & 2.15 & -0.63 & -0.49 & -0.43 & 7.37 & $\ldots$ \\
\hline $01031767+0908145$ & 2581189278047060224 & 5122 & 1.99 & -2.79 & -0.02 & 0.01 & 5.66 & 0.54 \\
\hline 01033338-7410471 & 4684860888081427840 & 5364 & 4.38 & -0.32 & 0.28 & 0.28 & 8.39 & 0.01 \\
\hline $01040440+0504477$ & 2552119633717667584 & 5348 & 4.36 & -0.69 & 0.55 & 0.55 & 8.29 & 0.43 \\
\hline $01042513+4011391$ & 371347102956265728 & 4906 & 4.45 & -1.01 & 0.18 & 0.18 & 7.60 & 0.17 \\
\hline $01042908+0755213$ & 2578034366869468800 & 5094 & 4.77 & -0.73 & 0.16 & 0.16 & 7.86 & 0.25 \\
\hline 01051545-0041328 & 2533223147709485312 & 4811 & 4.58 & -0.54 & -0.24 & -0.24 & 7.65 & 0.10 \\
\hline 01065190-5244105 & 4927175937828177280 & 5003 & 2.38 & -3.27 & 0.46 & 0.47 & 5.63 & 0.44 \\
\hline 01132198-6139522 & 4710458244623085184 & 4841 & 2.07 & -0.97 & -0.47 & -0.38 & 7.09 & 0.49 \\
\hline 01200289-0158201 & 2533312006288169600 & 5153 & 2.24 & -1.57 & 0.48 & 0.50 & 7.36 & 0.20 \\
\hline 01250922-5614027 & 4910744423745801472 & 4774 & 1.37 & -2.64 & 0.04 & 0.57 & 6.36 & 0.43 \\
\hline 01253364-4148345 & 4984027698370924672 & 5738 & 2.95 & -1.75 & 1.34 & 1.36 & 8.03 & 0.15 \\
\hline 01253802-2911025 & $\ldots$ & 4054 & 3.91 & -1.06 & -0.45 & -0.45 & 6.92 & $\ldots$ \\
\hline 01291742-7139220 & 4687775246726470144 & 4835 & 1.35 & -2.53 & 0.10 & 0.63 & 6.52 & -0.06 \\
\hline 01311599-4016510 & 5008222486100643200 & 4895 & 1.83 & -2.41 & 0.45 & 0.63 & 6.65 & 0.50 \\
\hline 01315199-6547540 & 4710799574264331904 & 5174 & 4.54 & -0.96 & 0.40 & 0.40 & 7.87 & 0.18 \\
\hline 01323787-1530302 & 2451901038631956224 & 5255 & 4.26 & -1.12 & 0.38 & 0.38 & 7.69 & 0.34 \\
\hline $01363655+5451319$ & 409152813849385216 & 4897 & 4.73 & -0.05 & -0.48 & -0.48 & 7.91 & -0.08 \\
\hline 01372246-4611110 & 4931138955692103424 & 5016 & 2.15 & -1.43 & -0.24 & -0.21 & 6.79 & 0.03 \\
\hline 01373378-6921368 & 4691261969896942848 & 4805 & 4.65 & -0.93 & 0.13 & 0.13 & 7.63 & 0.05 \\
\hline 01382048-7637319 & 4636801853303548416 & 5420 & 2.82 & -2.10 & 0.47 & 0.48 & 6.81 & 0.28 \\
\hline
\end{tabular}


Table 3 (continued)

\begin{tabular}{|c|c|c|c|c|c|c|c|c|}
\hline $\begin{array}{c}\text { Star Name } \\
\text { (2MASS) }\end{array}$ & $\begin{array}{c}\text { Star Name } \\
\text { (Gaia EDR3) }\end{array}$ & $\begin{array}{l}T_{\text {eff }} \\
(\mathrm{K})\end{array}$ & $\begin{array}{l}\log g \\
(\mathrm{cgs})\end{array}$ & {$[\mathrm{Fe} / \mathrm{H}]$} & {$[\mathrm{C} / \mathrm{Fe}]$} & {$[\mathrm{C} / \mathrm{Fe}]_{c}$} & $A(\mathrm{C})_{c}$ & {$[\mathrm{Mg} / \mathrm{Fe}]$} \\
\hline 01390079-7811248 & 4633446629147784320 & 4888 & 1.58 & -2.07 & 0.02 & 0.42 & 6.77 & 0.18 \\
\hline 01394297-1615477 & 2452308064092390784 & 4852 & 1.46 & -2.22 & -0.11 & 0.42 & 6.63 & 0.12 \\
\hline 01430726-6445174 & 4710973567683306624 & 4965 & 2.15 & -2.66 & 0.16 & 0.17 & 5.94 & 0.35 \\
\hline 01481644-5717024 & 4718885485854328064 & 5282 & 1.84 & -2.48 & 0.91 & 1.06 & 7.01 & 0.14 \\
\hline 01481836-6022102 & 4717520854484700288 & 5394 & 4.03 & -0.76 & -0.11 & -0.11 & 7.56 & 0.06 \\
\hline 01512909-5049125 & 4940475493039087872 & 4796 & 1.53 & -2.14 & -0.13 & 0.32 & 6.61 & 0.32 \\
\hline 01532990-7617103 & 4636731828156259712 & 5034 & 1.58 & -1.88 & -0.05 & 0.33 & 6.89 & 0.18 \\
\hline $01535484+4743406$ & 357061938652034816 & 5468 & 4.13 & -0.13 & -0.42 & -0.42 & 7.87 & -0.02 \\
\hline 01541382-4927292 & 4940875062436326784 & 5075 & 2.07 & -2.13 & 0.50 & 0.53 & 6.83 & 0.07 \\
\hline 01542953-4953166 & 4940665261873824640 & 4722 & 1.28 & -2.30 & -0.32 & 0.32 & 6.45 & 0.16 \\
\hline 01555066-6400155 & 4699467590815290368 & 4980 & 1.67 & -2.67 & 0.18 & 0.48 & 6.25 & 0.06 \\
\hline $01555808+5040276$ & 359446465839056512 & 5217 & 4.02 & -0.72 & 0.13 & 0.13 & 7.84 & $\cdots$ \\
\hline 01570453-6511318 & 4699066135928244224 & 5333 & 3.25 & -1.03 & -0.08 & -0.07 & 7.33 & 0.08 \\
\hline 01585657-1624249 & 5141897776909177856 & 5294 & 2.97 & -1.55 & 0.65 & 0.67 & 7.55 & 0.07 \\
\hline $01592159+8341476$ & 572808964949945088 & 5102 & 4.19 & -0.05 & -0.43 & -0.43 & 7.96 & -0.04 \\
\hline 02002105-2520170 & 5121763691780187008 & 4617 & 2.27 & -0.62 & -0.62 & -0.59 & 7.22 & 0.08 \\
\hline $02002992+5657571$ & 505258239019253760 & 5619 & 4.09 & -0.25 & -0.13 & -0.13 & 8.04 & -0.06 \\
\hline 02013041-0949339 & 2462500536881830528 & 5033 & 1.80 & -2.17 & 0.57 & 0.76 & 7.03 & -0.11 \\
\hline 02020691-8507254 & 4616783629211669888 & 4793 & 4.84 & -0.50 & -0.10 & -0.10 & 7.84 & 0.31 \\
\hline $02023240+5535052$ & 504560598882408960 & 5429 & 4.64 & -0.08 & -0.28 & -0.28 & 8.07 & -0.07 \\
\hline 02062328-0718389 & 2487426224965026688 & 5034 & 4.09 & -1.14 & 0.29 & 0.29 & 7.58 & 0.25 \\
\hline 02091623-2825011 & 5117222021562768256 & 4706 & 1.08 & -2.38 & 0.09 & 0.72 & 6.77 & -0.08 \\
\hline 02121057-2136569 & 5124244804192255104 & 5162 & 2.17 & -2.61 & 0.82 & 0.83 & 6.66 & 0.13 \\
\hline 02131127-3617490 & 4965179285932359552 & 4887 & 4.49 & -0.56 & 0.14 & 0.14 & 8.01 & 0.30 \\
\hline 02143996-2731561 & 5116644773662434176 & 4605 & 3.93 & -0.31 & -0.86 & -0.86 & 7.26 & -0.06 \\
\hline $02151298+4941500$ & 355587661060325120 & 5271 & 4.19 & -1.26 & 0.39 & 0.39 & 7.57 & -0.03 \\
\hline $02165682+4443112$ & 352161380966711936 & 4745 & 4.75 & -0.71 & -0.13 & -0.13 & 7.59 & 0.40 \\
\hline 02165716-7547064 & 4637170571951777280 & 4673 & 0.74 & -2.73 & 0.14 & 0.82 & 6.51 & 0.11 \\
\hline 02184236-5351323 & 4743265369493805696 & 4884 & 4.24 & -0.67 & -0.23 & -0.23 & 7.53 & 0.23 \\
\hline 02184254-6111160 & 4701711045508666112 & 5136 & 2.19 & -1.77 & 0.40 & 0.42 & 7.08 & 0.20 \\
\hline 02194944-2701309 & 5117093275622914688 & 5974 & 4.33 & -0.28 & 0.01 & 0.01 & 8.17 & 0.03 \\
\hline 02200131-5909599 & 4738094228868954496 & 4976 & 4.90 & -0.97 & -0.40 & -0.40 & 7.06 & $\cdots$ \\
\hline 02200416-3505332 & 4966846038184021888 & 5096 & 1.63 & -1.82 & 0.06 & 0.38 & 6.99 & 0.23 \\
\hline 02202248-3221349 & 4970735118186763520 & 4697 & 4.33 & -1.77 & 0.63 & 0.63 & 7.29 & -0.03 \\
\hline 02205873-6708044 & 4696223279895236992 & 4814 & 1.31 & -2.72 & 0.18 & 0.74 & 6.46 & 0.26 \\
\hline 02215591-1414291 & 5146733910084621440 & 5254 & 4.37 & -0.36 & 0.14 & 0.14 & 8.21 & 0.02 \\
\hline $02230945+3819551$ & 331739842266523264 & 5191 & 4.24 & -1.04 & 0.28 & 0.28 & 7.67 & -0.05 \\
\hline $02234760-4630467$ & 4940121690813101056 & 5123 & 2.56 & -1.27 & 0.13 & 0.15 & 7.31 & 0.22 \\
\hline $02242892+7959144$ & 562642266262613504 & 5395 & 4.44 & -0.70 & 0.78 & 0.78 & 8.50 & 0.40 \\
\hline 02243601-3101131 & 5066948639089274496 & 5509 & 4.53 & -0.02 & -0.22 & -0.22 & 8.18 & 0.05 \\
\hline 02255030-7822010 & 4632830898340209920 & 5275 & 2.40 & -2.33 & 0.70 & 0.71 & 6.81 & 0.12 \\
\hline 02260082-2250455 & 5120483443633370496 & 4781 & 2.57 & -1.04 & -0.54 & -0.52 & 6.88 & $\ldots$ \\
\hline
\end{tabular}


Table 3 (continued)

\begin{tabular}{|c|c|c|c|c|c|c|c|c|}
\hline $\begin{array}{l}\text { Star Name } \\
\text { (2MASS) }\end{array}$ & $\begin{array}{c}\text { Star Name } \\
\text { (Gaia EDR3) }\end{array}$ & $\begin{array}{l}T_{\text {eff }} \\
(\mathrm{K})\end{array}$ & $\begin{array}{l}\log g \\
(\operatorname{cgs})\end{array}$ & {$[\mathrm{Fe} / \mathrm{H}]$} & {$[\mathrm{C} / \mathrm{Fe}]$} & {$[\mathrm{C} / \mathrm{Fe}]_{c}$} & $A(\mathrm{C})_{c}$ & {$[\mathrm{Mg} / \mathrm{Fe}]$} \\
\hline 02263468-7052018 & 4645240501967006720 & 5003 & 1.69 & -2.31 & 0.25 & 0.58 & 6.71 & 0.45 \\
\hline $02263472-1610120$ & 5145471979973495040 & 4558 & 4.57 & -0.88 & -0.39 & -0.39 & 7.16 & 0.33 \\
\hline 02263559-6118229 & 4701661876723001088 & 4720 & 1.35 & -2.25 & -0.26 & 0.32 & 6.50 & 0.20 \\
\hline 02265573-3957537 & 4951576334231196160 & 4939 & 1.56 & -2.01 & -0.05 & 0.36 & 6.78 & 0.33 \\
\hline 02272940-1844371 & 5131351909915040640 & 5170 & 1.97 & -0.67 & -0.35 & -0.19 & 7.57 & 0.29 \\
\hline $02281118+3107248$ & 132459372685231872 & 5071 & 4.55 & -0.73 & 0.27 & 0.27 & 7.98 & 0.18 \\
\hline $02284342+8235565$ & 569513114421444480 & 6129 & 3.50 & -1.19 & 1.00 & 1.00 & 8.24 & 0.50 \\
\hline 02295644-7231067 & 4643772207267807104 & 5490 & 4.46 & -0.47 & 0.07 & 0.07 & 8.03 & 0.12 \\
\hline 02302280-1713235 & 5133294712601873024 & 5014 & 3.89 & -0.19 & -0.21 & -0.21 & 8.04 & -0.09 \\
\hline $02302823+3146355$ & 134052560743210624 & 5198 & 4.30 & -0.67 & -0.03 & -0.03 & 7.73 & 0.12 \\
\hline 02314914-4251147 & 4950048081786940160 & 5105 & 4.35 & 0.06 & 0.22 & 0.22 & 8.70 & -0.09 \\
\hline 02322533-2955268 & 5067349028825362176 & 5265 & 4.26 & -0.26 & -0.42 & -0.42 & 7.75 & 0.13 \\
\hline 02332987-2602004 & 5070849431466425600 & 5122 & 4.45 & -0.47 & 0.46 & 0.46 & 8.42 & -0.05 \\
\hline 02335901-5218323 & 4744814203419011456 & 5036 & 4.00 & -0.83 & 0.72 & 0.72 & 8.32 & 0.15 \\
\hline 02343390-3438203 & 5062336050373022976 & 5980 & 3.46 & -1.15 & 1.02 & 1.02 & 8.31 & 0.11 \\
\hline 02345434-3349391 & 5062448200558971136 & 4718 & 1.09 & -2.38 & -0.09 & 0.58 & 6.63 & 0.32 \\
\hline 02361077-1202559 & 5171442680844060160 & 5152 & 1.59 & -2.29 & 0.00 & 0.45 & 6.59 & 0.00 \\
\hline 02371057-4036121 & 4951834822543543040 & 5862 & 3.75 & -1.01 & 0.85 & 0.85 & 8.27 & -0.13 \\
\hline $02372192+4302214$ & 340142202749502464 & 5248 & 4.38 & -0.22 & -0.42 & -0.42 & 7.79 & 0.01 \\
\hline 02384449-3325102 & 5062531071950987392 & 5121 & 1.92 & -1.86 & 0.11 & 0.23 & 6.80 & 0.37 \\
\hline 02394381-3631306 & 4953863662014601216 & 5874 & 4.37 & -0.56 & 0.28 & 0.28 & 8.15 & 0.07 \\
\hline $02401392+2556291$ & 126323578110142848 & 5376 & 3.81 & -0.51 & 0.29 & 0.29 & 8.20 & $\ldots$ \\
\hline $02404390+4457499$ & 340915713477131008 & 4844 & 4.77 & -0.74 & -0.09 & -0.09 & 7.60 & 0.35 \\
\hline 02425864-3709379 & 4953584523499875712 & 5204 & 2.39 & -2.04 & 0.40 & 0.41 & 6.80 & 0.21 \\
\hline 02431746-8608453 & 4613478261037323776 & 4627 & 0.64 & -2.43 & -0.44 & 0.31 & 6.31 & 0.25 \\
\hline 02435125-2942551 & 5065856377366623872 & 4675 & 4.33 & -0.34 & -0.73 & -0.73 & 7.36 & -0.12 \\
\hline $02451977+1332222$ & 31933391083889152 & 5180 & 2.34 & -2.36 & 0.41 & 0.42 & 6.49 & 0.32 \\
\hline 02462323-3137296 & 5064607984993521792 & 5324 & 4.71 & -0.49 & 0.21 & 0.21 & 8.15 & 0.05 \\
\hline 02471497-6303000 & 4721297363392975488 & 4737 & 2.15 & -0.68 & -0.52 & -0.46 & 7.29 & 0.23 \\
\hline 02494852-2229202 & 5077735500993403648 & 5300 & 4.30 & -0.37 & 0.17 & 0.17 & 8.23 & 0.29 \\
\hline $02501156+3457476$ & 140083622534201088 & 4686 & 4.82 & -0.78 & -0.17 & -0.17 & 7.48 & 0.17 \\
\hline $02512543+8333571$ & 569663953674510464 & 4752 & 4.64 & -2.47 & 1.08 & 1.08 & 7.04 & 0.08 \\
\hline 02523341-4416060 & 4755176207159038080 & 4915 & 1.20 & -2.27 & 0.32 & 0.82 & 6.98 & $\ldots$ \\
\hline $02525369+2107466$ & 109283900954164992 & 4665 & 4.92 & -1.12 & -0.01 & -0.01 & 7.30 & 0.31 \\
\hline 02525416-3228483 & 5052264558220148352 & 4718 & 4.44 & -0.41 & -0.37 & -0.37 & 7.64 & 0.05 \\
\hline 02532757-3454050 & 5049605732946898432 & 5099 & 2.14 & -1.94 & 0.34 & 0.36 & 6.85 & 0.20 \\
\hline 02533614-6234511 & 4721372684235614848 & 5240 & 2.85 & -1.86 & 0.51 & 0.52 & 7.09 & 0.43 \\
\hline 02564805-1942473 & 5128076774013414912 & 5237 & 4.45 & -0.09 & -0.15 & -0.15 & 8.19 & 0.00 \\
\hline $02571027+3318455$ & 136508702211948672 & 4984 & 4.74 & -0.77 & 0.24 & 0.24 & 7.90 & 0.03 \\
\hline $02580877+0829424$ & 8791969854015488 & 5492 & 4.71 & -0.53 & 0.08 & 0.08 & 7.98 & 0.09 \\
\hline 02590016-7209504 & 4645469170322185984 & 5100 & 4.13 & -1.51 & 0.62 & 0.62 & 7.54 & 0.12 \\
\hline $03042561+3112028$ & 135287316592202880 & 5031 & 4.00 & -1.35 & 0.64 & 0.64 & 7.72 & -0.10 \\
\hline
\end{tabular}


Table 3 (continued)

\begin{tabular}{|c|c|c|c|c|c|c|c|c|}
\hline $\begin{array}{c}\text { Star Name } \\
\text { (2MASS) }\end{array}$ & $\begin{array}{c}\text { Star Name } \\
\text { (Gaia EDR3) }\end{array}$ & $\begin{array}{l}T_{\text {eff }} \\
(\mathrm{K})\end{array}$ & $\begin{array}{l}\log g \\
(\operatorname{cgs})\end{array}$ & {$[\mathrm{Fe} / \mathrm{H}]$} & {$[\mathrm{C} / \mathrm{Fe}]$} & {$[\mathrm{C} / \mathrm{Fe}]_{c}$} & $A(\mathrm{C})_{c}$ & {$[\mathrm{Mg} / \mathrm{Fe}]$} \\
\hline 03042843-3406046 & 5050991770433282688 & 4938 & 2.33 & -3.38 & 0.21 & 0.22 & 5.27 & 0.54 \\
\hline 03044130-3635405 & 5047482782152551808 & 5319 & 2.86 & -0.38 & -0.14 & -0.11 & 7.94 & 0.04 \\
\hline $03053573+2834227$ & 115967213665888000 & 5074 & 1.84 & -2.66 & 0.62 & 0.75 & 6.52 & 0.41 \\
\hline 03060595-5251430 & 4746980344406420736 & 5261 & 2.57 & -2.16 & 0.48 & 0.49 & 6.76 & 0.17 \\
\hline $03080797+3836489$ & 142648478221664256 & 6068 & 3.82 & -0.45 & 0.30 & 0.30 & 8.28 & 0.40 \\
\hline 03091996-5853578 & 4724087928200048768 & 5062 & 4.25 & -0.50 & -0.29 & -0.29 & 7.64 & 0.23 \\
\hline 03095592-0459281 & 5182694120770155264 & 4853 & 4.25 & -0.46 & -0.21 & -0.21 & 7.76 & 0.12 \\
\hline $03104602+4514503$ & 433115020506112512 & 4680 & 5.00 & -1.03 & -0.04 & -0.04 & 7.36 & 0.40 \\
\hline 03121034-5703094 & 4727345712434297984 & 4778 & 0.86 & -2.27 & -0.41 & 0.32 & 6.49 & 0.45 \\
\hline 03123270-2849566 & 5059444369153859200 & 5035 & 1.63 & -2.14 & -0.06 & 0.32 & 6.61 & 0.28 \\
\hline 03123346-5234570 & 4735321737284031232 & 5121 & 4.64 & -0.89 & 0.18 & 0.18 & 7.72 & 0.29 \\
\hline 03131491-8107109 & 4619392843320436608 & 5070 & 1.88 & -2.36 & 0.32 & 0.49 & 6.56 & 0.12 \\
\hline 03134048-8045218 & 4619419089865071744 & 5511 & 3.93 & -0.80 & 0.29 & 0.29 & 7.92 & -0.03 \\
\hline $03135196+4230102$ & 240424332130561024 & 5538 & 4.05 & -0.62 & 0.20 & 0.20 & 8.01 & 0.08 \\
\hline $03152783+3353586$ & 137535813574478592 & 5377 & 4.50 & -0.40 & 0.56 & 0.56 & 8.58 & 0.39 \\
\hline $03155572+3357169$ & 125528253246150656 & 4925 & 4.07 & -0.87 & 0.17 & 0.17 & 7.73 & $\ldots$ \\
\hline 03155933-7432577 & 4639776204054863232 & 4700 & 3.09 & -1.45 & 0.62 & 0.63 & 7.61 & -0.03 \\
\hline $03163710+2332211$ & 110907432953980800 & 4978 & 1.32 & -2.61 & -0.21 & 0.38 & 6.20 & 0.50 \\
\hline 03170396-3740469 & 4854289415101796608 & 5010 & 2.78 & -3.35 & 0.22 & 0.22 & 5.30 & $\ldots$ \\
\hline 03171573-3747479 & 4854284497363037696 & 4812 & 4.72 & -0.68 & 0.09 & 0.09 & 7.84 & 0.40 \\
\hline 03173348-3705188 & 4854694001021568896 & 4920 & 4.22 & 0.10 & -0.09 & -0.09 & 8.45 & -0.11 \\
\hline $03180842+1814447$ & 56110625281842816 & 5057 & 2.18 & -1.63 & -0.09 & -0.07 & 6.73 & 0.49 \\
\hline 03190720-5245069 & 4735169768457507968 & 5366 & 4.72 & -0.43 & 0.09 & 0.09 & 8.08 & 0.03 \\
\hline 03214149-5553303 & 4733473496892938368 & 5022 & 4.54 & -0.18 & -0.18 & -0.18 & 8.07 & -0.05 \\
\hline 03220165-0020329 & 3262842880464520576 & 5231 & 3.53 & -1.04 & 0.26 & 0.26 & 7.65 & 0.09 \\
\hline 03222245-3731294 & 4854443793406263040 & 5291 & 4.73 & -0.79 & 0.07 & 0.07 & 7.72 & 0.40 \\
\hline $03223653+0859382$ & 11176917949244800 & 4695 & 4.55 & -1.09 & 0.26 & 0.26 & 7.60 & 0.04 \\
\hline 03242169-3515217 & 4860920810247328256 & 5320 & 2.13 & -2.47 & 0.07 & 0.08 & 6.04 & $\ldots$ \\
\hline 03242519-1550054 & 5106733402188456320 & 4774 & 2.55 & -0.86 & -0.42 & -0.40 & 7.18 & $\ldots$ \\
\hline $03252266+8009505$ & 568038153934104320 & 4922 & 1.10 & -2.57 & -0.02 & 0.65 & 6.52 & 0.49 \\
\hline 03260086-4126000 & 4849648891917127424 & 4896 & 2.21 & -0.91 & -0.55 & -0.53 & 7.00 & 0.46 \\
\hline 03260534-2006507 & 5101544016542831232 & 4706 & 2.26 & -0.70 & -0.69 & -0.66 & 7.07 & 0.44 \\
\hline $03263031+0616326$ & 9265348264761984 & 5002 & 2.37 & -1.27 & -0.02 & 0.00 & 7.16 & 0.25 \\
\hline 03265306-0053348 & 3264007336293442048 & 4606 & 4.25 & -1.20 & 0.08 & 0.08 & 7.31 & -0.20 \\
\hline $03265389+0202281$ & 3268028903151246720 & 5212 & 2.75 & -3.41 & 0.75 & 0.76 & 5.79 & $\ldots$ \\
\hline 03275664-4544078 & 4846530329047408384 & 4881 & 4.45 & -0.31 & -0.19 & -0.19 & 7.93 & -0.02 \\
\hline 03283529-4000252 & 4853194778260569856 & 5337 & 4.48 & -0.42 & -0.03 & -0.03 & 7.98 & 0.11 \\
\hline $03283748+1856359$ & 57481853722629888 & 4907 & 1.45 & -2.26 & -0.04 & 0.47 & 6.63 & 0.35 \\
\hline 03292423-6057094 & 4722654951015751680 & 4984 & 1.79 & -2.66 & 0.25 & 0.46 & 6.23 & 0.25 \\
\hline 03304035-0321071 & 3249179288121898752 & 5558 & 4.12 & -0.17 & -0.55 & -0.55 & 7.72 & -0.07 \\
\hline 03305484-0304088 & 3249204710032811648 & 5105 & 2.79 & -0.21 & -0.53 & -0.50 & 7.72 & 0.21 \\
\hline $03312821+0749469$ & 11354660875621376 & 4777 & 4.84 & -1.35 & 0.12 & 0.12 & 7.20 & 0.33 \\
\hline
\end{tabular}


Table 3 (continued)

\begin{tabular}{|c|c|c|c|c|c|c|c|c|}
\hline $\begin{array}{c}\text { Star Name } \\
\text { (2MASS) }\end{array}$ & $\begin{array}{c}\text { Star Name } \\
\text { (Gaia EDR3) }\end{array}$ & $\begin{array}{l}T_{\text {eff }} \\
(\mathrm{K})\end{array}$ & $\begin{array}{l}\log g \\
(\operatorname{cgs})\end{array}$ & {$[\mathrm{Fe} / \mathrm{H}]$} & {$[\mathrm{C} / \mathrm{Fe}]$} & {$[\mathrm{C} / \mathrm{Fe}]_{c}$} & $A(\mathrm{C})_{c}$ & {$[\mathrm{Mg} / \mathrm{Fe}]$} \\
\hline 03343133-0555030 & 3247331455752180992 & 4537 & 4.33 & -1.36 & 0.14 & 0.14 & 7.21 & -0.09 \\
\hline $03351518+7823252$ & 555315734952674816 & 4771 & 0.96 & -2.41 & -0.32 & 0.43 & 6.45 & 0.40 \\
\hline 03354665-1356010 & 5113319751716905344 & 4927 & 3.92 & -1.31 & 0.70 & 0.70 & 7.81 & -0.09 \\
\hline $03362369+2036490$ & 58156438465188096 & 5257 & 2.39 & -1.64 & 0.15 & 0.17 & 6.96 & 0.32 \\
\hline $03362874-6005593$ & 4728790397697259392 & 5011 & 3.59 & -1.01 & -0.39 & -0.39 & 7.03 & 0.45 \\
\hline 03365068-2327557 & 5086423493982030848 & 4899 & 4.04 & -1.90 & 0.91 & 0.91 & 7.44 & 0.30 \\
\hline $03365778+2917172$ & 120001145732321536 & 4810 & 2.20 & -1.04 & -0.16 & -0.14 & 7.25 & $\ldots$ \\
\hline 03370393-4829266 & 4833641097832497408 & 5113 & 3.27 & -1.46 & 0.86 & 0.87 & 7.85 & 0.36 \\
\hline $03370580+1802236$ & 56464972968303104 & 6440 & 4.14 & -0.39 & 0.17 & 0.17 & 8.22 & 0.01 \\
\hline 03385093-0255535 & 3250559347013995136 & 4842 & 1.57 & -2.28 & -0.03 & 0.44 & 6.58 & 0.23 \\
\hline $03391370+0310345$ & 3271273935626311808 & 4925 & 1.77 & -2.31 & 0.05 & 0.32 & 6.44 & 0.42 \\
\hline 03401638-5917516 & 4728932754388052224 & 5326 & 4.35 & -0.47 & 0.30 & 0.30 & 8.26 & 0.26 \\
\hline 03410147-1812508 & 5107448320968631040 & 4736 & 4.86 & -1.06 & 0.08 & 0.08 & 7.46 & 0.37 \\
\hline $03421349+2729013$ & 71184787964000000 & 5205 & 4.80 & -0.36 & 0.06 & 0.06 & 8.13 & 0.23 \\
\hline 03425047-1013432 & 5116038431655398400 & 5097 & 1.62 & -2.35 & -0.20 & 0.19 & 6.28 & 0.27 \\
\hline $03431186+7807495$ & 554502989702092416 & 5317 & 3.96 & -1.26 & 0.34 & 0.34 & 7.51 & 0.06 \\
\hline 03435581-3212071 & 4863399349974669440 & 5214 & 2.24 & -2.17 & 0.54 & 0.55 & 6.81 & 0.24 \\
\hline 03440716-2841235 & 5080387454320334592 & 5576 & 4.05 & -0.29 & 0.10 & 0.10 & 8.24 & 0.03 \\
\hline 03440987-4057281 & 4854966508106088064 & 5174 & 4.24 & -0.53 & -0.38 & -0.38 & 7.53 & 0.12 \\
\hline 03445544-7517390 & 4628961755579625600 & 5301 & 4.47 & -0.32 & 0.19 & 0.19 & 8.30 & -0.02 \\
\hline 03453731-8211290 & 4616039813892309376 & 5090 & 2.20 & -2.31 & 0.46 & 0.47 & 6.59 & 0.15 \\
\hline $03462539+2125305$ & 63534390354375040 & 5014 & 4.34 & -0.23 & -0.27 & -0.27 & 7.93 & -0.07 \\
\hline 03463330-0023036 & 3251527020325320960 & 4457 & 4.06 & -0.26 & -1.78 & -1.78 & 6.40 & -0.09 \\
\hline 03464056-1703266 & 5108001001657825280 & 5099 & 1.56 & -2.19 & -0.09 & 0.34 & 6.58 & 0.21 \\
\hline 03470283-7933197 & 4625542858531568768 & 5108 & 2.22 & -1.71 & 0.24 & 0.26 & 6.99 & 0.13 \\
\hline $03470366+7232599$ & 543671700657298816 & 5661 & 3.19 & -0.25 & 0.18 & 0.20 & 8.38 & 0.59 \\
\hline 03475502-2219560 & 5087674875655599744 & 5220 & 4.20 & -0.74 & 0.39 & 0.39 & 8.09 & -0.06 \\
\hline 03481276-2657207 & 5081024380790201984 & 5210 & 1.80 & -2.22 & 0.29 & 0.52 & 6.73 & 0.26 \\
\hline 03494330-1035255 & 3194196834787169152 & 4738 & 4.60 & -0.60 & -0.21 & -0.21 & 7.62 & 0.08 \\
\hline 03494712-2012428 & 5094777381468797568 & 4769 & 1.23 & -2.37 & -0.11 & 0.52 & 6.58 & 0.09 \\
\hline $03505656+1915396$ & 50799709243487232 & 4765 & 4.77 & -1.03 & 0.15 & 0.15 & 7.54 & 0.06 \\
\hline 03511385-1222556 & 5114516604484590848 & 4909 & 3.97 & -0.30 & -0.57 & -0.57 & 7.56 & -0.05 \\
\hline 03514691-4821339 & 4830105381971162368 & 5007 & 4.50 & -0.68 & 0.22 & 0.22 & 7.97 & 0.03 \\
\hline $03515804+7834544$ & 554892216818025088 & 5056 & 4.38 & -0.47 & 0.37 & 0.37 & 8.34 & 0.27 \\
\hline 03522200-7822390 & 4626118933904711936 & 5022 & 1.85 & -1.99 & 0.31 & 0.48 & 6.92 & 0.11 \\
\hline 03525823-2712362 & 5080850520514122368 & 5075 & 4.20 & -0.44 & 0.47 & 0.47 & 8.46 & -0.05 \\
\hline $03532071+0332467$ & 3272181586771744640 & 5277 & 2.02 & -1.61 & 0.17 & 0.23 & 7.04 & 0.40 \\
\hline 03535378-8214319 & 4616030261884997504 & 5112 & 2.18 & -2.04 & 0.42 & 0.43 & 6.83 & 0.20 \\
\hline 03542750-4930350 & 4829790406249501824 & 4980 & 3.72 & -0.24 & -0.08 & -0.08 & 8.12 & -0.10 \\
\hline $03550575+0448406$ & 3273182073633848576 & 5221 & 2.47 & -1.29 & 0.21 & 0.23 & 7.37 & 0.23 \\
\hline 03551870-3540553 & 4858448073019453568 & 5100 & 2.02 & -2.20 & 0.57 & 0.63 & 6.85 & 0.13 \\
\hline 03552492-3137538 & 4886536888554252288 & 4963 & 1.97 & -1.39 & -0.12 & -0.02 & 7.02 & $\ldots$ \\
\hline
\end{tabular}


Table 3 (continued)

\begin{tabular}{|c|c|c|c|c|c|c|c|c|}
\hline $\begin{array}{l}\text { Star Name } \\
(2 \mathrm{MASS})\end{array}$ & $\begin{array}{c}\text { Star Name } \\
\text { (Gaia EDR3) }\end{array}$ & $\begin{array}{l}T_{\text {eff }} \\
(\mathrm{K})\end{array}$ & $\begin{array}{l}\log g \\
(\operatorname{cgs})\end{array}$ & {$[\mathrm{Fe} / \mathrm{H}]$} & {$[\mathrm{C} / \mathrm{Fe}]$} & {$[\mathrm{C} / \mathrm{Fe}]_{c}$} & $A(\mathrm{C})_{c}$ & {$[\mathrm{Mg} / \mathrm{Fe}]$} \\
\hline $03570714+1150353$ & 3304873572159327104 & 5369 & 3.71 & -0.88 & 0.22 & 0.22 & 7.76 & -0.04 \\
\hline 03575259-3507496 & 4858859741341036032 & 5146 & 3.95 & -0.93 & -0.30 & -0.30 & 7.21 & 0.21 \\
\hline 03580690-4125514 & 4842342190474113920 & 5469 & 3.64 & -0.41 & 0.04 & 0.04 & 8.06 & -0.04 \\
\hline $03582857+0851479$ & 3301417188637954304 & 4989 & 4.32 & -1.30 & 0.33 & 0.33 & 7.47 & 0.19 \\
\hline 03582979-0646599 & 3196842083667299968 & 4868 & 4.58 & -0.44 & -0.36 & -0.36 & 7.63 & 0.06 \\
\hline 03583609-3024329 & 4887268613542620160 & 5339 & 2.40 & -1.09 & 0.20 & 0.22 & 7.56 & 0.37 \\
\hline 03585372-2512589 & 5083157811305565184 & 5248 & 4.69 & -0.90 & 0.41 & 0.41 & 7.94 & 0.19 \\
\hline 03590972-7052417 & 4665955133531907840 & 5372 & 2.29 & -2.22 & 0.44 & 0.45 & 6.66 & 0.45 \\
\hline 04021386-2714330 & 4890018148526236416 & 5091 & 1.73 & -2.02 & 0.53 & 0.75 & 7.16 & $\ldots$ \\
\hline 04030414-2123136 & 5090671530172203648 & 5391 & 4.32 & -0.41 & 0.05 & 0.05 & 8.07 & 0.41 \\
\hline 04032141-5057006 & 4828667358199528064 & 4952 & 4.76 & -0.55 & -0.07 & -0.07 & 7.81 & 0.21 \\
\hline $04034276+0516322$ & 3272889569179614464 & 5155 & 4.09 & -0.51 & 0.20 & 0.20 & 8.11 & $\ldots$ \\
\hline 04051129-3610404 & 4857845781166112384 & 4821 & 4.45 & -0.35 & -0.42 & -0.42 & 7.66 & -0.06 \\
\hline 04062658-1417389 & 5110102993011576192 & 5198 & 2.55 & -1.41 & 0.11 & 0.13 & 7.15 & 0.30 \\
\hline 04064080-2822221 & 4889098510129080064 & 5491 & 3.92 & -1.15 & 0.33 & 0.33 & 7.61 & 0.03 \\
\hline 04065230-1132234 & 3190316692611552000 & 5562 & 4.65 & -0.44 & 0.31 & 0.31 & 8.30 & 0.02 \\
\hline 04065870-5405560 & 4779874227616110592 & 5294 & 2.38 & -2.40 & 0.67 & 0.68 & 6.71 & 0.26 \\
\hline 04071847-3844270 & 4844183765074837760 & 4931 & 4.58 & -0.80 & 0.36 & 0.36 & 7.98 & 0.10 \\
\hline 04073220-1508180 & 5097968885766682240 & 4678 & 4.38 & -1.45 & 0.63 & 0.63 & 7.61 & -0.06 \\
\hline 04081065-4723248 & 4831180463825371776 & 4397 & 4.38 & -1.88 & 0.04 & 0.04 & 6.60 & $\ldots$ \\
\hline 04082208-8054055 & 4622225700669724160 & 5017 & 1.69 & -2.12 & 0.12 & 0.44 & 6.76 & 0.16 \\
\hline 04082291-3306502 & 4882442375971513216 & 4906 & 4.74 & -0.82 & 0.26 & 0.26 & 7.87 & 0.31 \\
\hline 04095634-2018250 & 5091033814957326464 & 5344 & 3.39 & -1.56 & 0.50 & 0.50 & 7.37 & 0.29 \\
\hline 04123785-0354082 & 3203733925629461376 & 5054 & 3.25 & -0.49 & -0.62 & -0.60 & 7.34 & 0.13 \\
\hline 04133760-2855548 & 4885822480873873408 & 5183 & 1.68 & -2.22 & 0.00 & 0.35 & 6.56 & 0.10 \\
\hline $04144546-4431586$ & 4837831066064068480 & 4667 & 4.76 & -1.58 & 0.57 & 0.57 & 7.42 & 0.09 \\
\hline 04152012-4554089 & 4837327760319848576 & 5084 & 4.71 & -0.33 & -0.03 & -0.03 & 8.06 & 0.07 \\
\hline 04161576-0521188 & 3202575628785925888 & 6479 & 3.24 & -1.31 & 1.79 & 1.81 & 8.93 & 0.16 \\
\hline 04162853-6636540 & 4668867224438266368 & 5086 & 2.27 & -1.71 & 0.08 & 0.10 & 6.82 & 0.37 \\
\hline 04163240-0602269 & 3202394930922308096 & 5280 & 3.95 & -0.88 & 0.56 & 0.56 & 8.11 & 0.52 \\
\hline 04174265-5558407 & 4778645523371812480 & 5016 & 4.48 & -0.69 & 0.28 & 0.28 & 8.02 & 0.07 \\
\hline 04211598-4852031 & 4782730209069106432 & 5072 & 1.55 & -2.57 & 0.01 & 0.42 & 6.28 & $\ldots$ \\
\hline 04212925-5219577 & 4780985799511960576 & 4716 & 1.17 & -2.16 & -0.13 & 0.48 & 6.75 & 0.31 \\
\hline 04213031-2030287 & 5091573542023760384 & 5170 & 4.24 & -0.26 & -0.26 & -0.26 & 7.91 & 0.05 \\
\hline 04215801-2739310 & 4892329974803162752 & 4996 & 4.06 & -0.27 & 0.00 & 0.00 & 8.16 & -0.08 \\
\hline 04225741-8308401 & 4615741777521531136 & 5123 & 3.67 & -1.12 & 0.34 & 0.34 & 7.65 & -0.01 \\
\hline $04242725-8157493$ & 4621912305496245248 & 5375 & 2.85 & -2.12 & 0.42 & 0.43 & 6.75 & $\ldots$ \\
\hline 04251520-6213209 & 4675852078212908416 & 4984 & 1.53 & -2.32 & 0.19 & 0.63 & 6.74 & -0.04 \\
\hline 04262201-3615167 & 4869018507227798400 & 5032 & 1.87 & -2.16 & 0.45 & 0.59 & 6.87 & 0.01 \\
\hline 04282895-3353452 & 4871041608622321664 & 5247 & 4.54 & -0.47 & 0.44 & 0.44 & 8.40 & 0.09 \\
\hline 04291257-4921466 & 4787721991859465728 & 5607 & 2.98 & -0.28 & -0.01 & 0.02 & 8.18 & 0.22 \\
\hline 04293837-5448372 & 4776030816001572736 & 4934 & 4.66 & -0.62 & 0.21 & 0.21 & 8.02 & 0.01 \\
\hline
\end{tabular}


Table 3 (continued)

\begin{tabular}{|c|c|c|c|c|c|c|c|c|}
\hline $\begin{array}{c}\text { Star Name } \\
(2 \mathrm{MASS})\end{array}$ & $\begin{array}{c}\text { Star Name } \\
\text { (Gaia EDR3) }\end{array}$ & $\begin{array}{l}T_{\text {eff }} \\
(\mathrm{K})\end{array}$ & $\begin{array}{l}\log g \\
(\operatorname{cgs})\end{array}$ & {$[\mathrm{Fe} / \mathrm{H}]$} & {$[\mathrm{C} / \mathrm{Fe}]$} & {$[\mathrm{C} / \mathrm{Fe}]_{c}$} & $A(\mathrm{C})_{c}$ & {$[\mathrm{Mg} / \mathrm{Fe}]$} \\
\hline $04311275-2258353$ & 4898098833139598464 & 4845 & 1.14 & -2.22 & -0.09 & 0.54 & 6.75 & 0.29 \\
\hline 04313335-7337168 & 4652567991051879168 & 5288 & 2.55 & -1.69 & 0.34 & 0.36 & 7.10 & 0.13 \\
\hline 04325046-3205240 & 4871721965802188160 & 5552 & 4.53 & -0.14 & 0.05 & 0.05 & 8.34 & -0.06 \\
\hline 04330582-1605256 & 3172371116657963008 & 5564 & 3.57 & -1.38 & 0.61 & 0.61 & 7.66 & 0.03 \\
\hline $04332408+7049217$ & 500082940861162240 & 5439 & 4.39 & -0.48 & 0.39 & 0.39 & 8.35 & $\ldots$ \\
\hline 04332826-0431033 & 3201331222141772928 & 4895 & 1.58 & -1.84 & -0.08 & 0.28 & 6.87 & 0.31 \\
\hline $04334315+8752515$ & 575291322904958208 & 4778 & 4.82 & -1.12 & 0.28 & 0.28 & 7.59 & -0.01 \\
\hline 04354147-4750031 & 4788319503413123200 & 5504 & 3.79 & -0.78 & 0.30 & 0.30 & 7.94 & 0.27 \\
\hline 04354314-5355093 & 4777636927611794432 & 4883 & 1.19 & -2.24 & -0.13 & 0.49 & 6.69 & 0.18 \\
\hline $04363802+7949143$ & 556538739776801664 & 5135 & 3.89 & -0.23 & -0.40 & -0.40 & 7.80 & 0.40 \\
\hline 04391892-3404504 & 4868176796716806272 & 6152 & 4.07 & -0.99 & 0.76 & 0.76 & 8.21 & 0.06 \\
\hline 04392332-2434534 & 4894484605277323648 & 5393 & 2.79 & -1.95 & 0.46 & 0.47 & 6.95 & 0.28 \\
\hline 04392855-3957548 & 4816386358778340608 & 4835 & 4.59 & -0.78 & 0.22 & 0.22 & 7.87 & 0.02 \\
\hline 04400253-1922270 & 2978208560171576448 & 7596 & 2.12 & -3.00 & 2.95 & 2.98 & 8.41 & $\cdots$ \\
\hline 04404386-2741005 & 4879633570439853440 & 5052 & 1.68 & -2.33 & 0.63 & 0.91 & 7.01 & -0.02 \\
\hline 04404847-4214219 & 4814898930000598272 & 4971 & 1.38 & -2.59 & -0.15 & 0.41 & 6.25 & 0.17 \\
\hline 04404877-3530531 & 4867833886528092288 & 4693 & 1.39 & -2.50 & -0.48 & 0.09 & 6.02 & $\ldots$ \\
\hline 04414545-1703252 & 2980627661847718016 & 5284 & 4.44 & -0.24 & -0.23 & -0.23 & 7.96 & -0.01 \\
\hline 04452944-4656234 & 4786751256234998272 & 5147 & 3.33 & -0.24 & -0.68 & -0.68 & 7.51 & 0.02 \\
\hline $04455221+8238074$ & 569892445935443200 & 4610 & 4.78 & -1.19 & 0.10 & 0.10 & 7.35 & 0.25 \\
\hline 04460040-5244239 & 4777958977143271936 & 4976 & 4.58 & -1.02 & 0.43 & 0.43 & 7.84 & 0.41 \\
\hline 04460379-4440454 & 4790350855440186752 & 4762 & 1.57 & -2.12 & -0.10 & 0.32 & 6.63 & 0.22 \\
\hline 04480413-5133186 & 4784117101252301056 & 4832 & 4.75 & -0.85 & 0.36 & 0.36 & 7.95 & 0.17 \\
\hline 04481274-3428106 & 4873248568978164608 & 4787 & 4.76 & -0.68 & 0.10 & 0.10 & 7.85 & 0.38 \\
\hline 04482945-5127292 & 4784118686097335808 & 4842 & 2.70 & -0.11 & -0.88 & -0.85 & 7.47 & 0.16 \\
\hline 04504247-4831497 & 4786320148893885568 & 5113 & 4.45 & -0.19 & -0.08 & -0.08 & 8.16 & 0.01 \\
\hline $04505386+1803498$ & 3406732187621193600 & 7843 & 4.53 & 0.23 & 1.70 & 1.70 & 10.37 & $\cdots$ \\
\hline 04514248-3210438 & 4874664052759778304 & 4924 & 1.66 & -1.89 & 0.21 & 0.49 & 7.03 & 0.23 \\
\hline 04520803-2837181 & 4879979504285453184 & 5043 & 3.99 & -1.06 & 0.64 & 0.64 & 8.01 & -0.04 \\
\hline 04522717-3451392 & 4873001625538322688 & 4671 & 4.72 & -0.48 & -0.15 & -0.15 & 7.80 & 0.24 \\
\hline 04525913-4008568 & 4816836269489019904 & 4806 & 1.30 & -2.34 & -0.13 & 0.48 & 6.57 & 0.20 \\
\hline 04542625-4241431 & 4811990171989821824 & 4937 & 0.82 & -2.63 & 0.50 & 1.07 & 6.87 & $\ldots$ \\
\hline 04562421-3124177 & 4874779806424378496 & 4601 & 4.33 & -0.40 & -0.64 & -0.64 & 7.39 & 0.02 \\
\hline 04563790-5602511 & 4776246457719437440 & 4799 & 1.23 & -2.21 & -0.08 & 0.51 & 6.73 & 0.23 \\
\hline 04582722-4611154 & 4810231400062085504 & 5301 & 2.82 & -0.92 & 0.20 & 0.22 & 7.73 & $\cdots$ \\
\hline 04583735-0449416 & 3212422747548345216 & 4927 & 4.43 & -0.18 & -0.76 & -0.76 & 7.49 & 0.03 \\
\hline $05000638+0109473$ & 3228880619846899712 & 4772 & 4.61 & -0.97 & 0.22 & 0.22 & 7.69 & -0.05 \\
\hline $05011881+0803047$ & 3290174403068832640 & 5500 & 4.39 & -0.47 & 0.12 & 0.12 & 8.08 & 0.45 \\
\hline 05015088-4139079 & 4813429157832439168 & 4772 & 2.49 & -2.15 & 0.36 & 0.37 & 6.65 & $\cdots$ \\
\hline $05015244+6745243$ & 483790308838071424 & 5581 & 4.48 & -0.76 & 0.33 & 0.33 & 7.99 & 0.15 \\
\hline 05021896-5152056 & 4783532062283301120 & 5157 & 2.47 & -1.66 & 0.29 & 0.31 & 7.08 & 0.11 \\
\hline 05022161-4603414 & 4810297405118590592 & 4532 & 4.68 & -1.08 & -0.05 & -0.05 & 7.30 & 0.36 \\
\hline
\end{tabular}


Table 3 (continued)

\begin{tabular}{|c|c|c|c|c|c|c|c|c|}
\hline $\begin{array}{c}\text { Star Name } \\
(2 \mathrm{MASS})\end{array}$ & $\begin{array}{c}\text { Star Name } \\
\text { (Gaia EDR3) }\end{array}$ & $\begin{array}{l}T_{\text {eff }} \\
(\mathrm{K})\end{array}$ & $\begin{array}{l}\log g \\
(\operatorname{cgs})\end{array}$ & {$[\mathrm{Fe} / \mathrm{H}]$} & {$[\mathrm{C} / \mathrm{Fe}]$} & {$[\mathrm{C} / \mathrm{Fe}]_{c}$} & $A(\mathrm{C})_{c}$ & {$[\mathrm{Mg} / \mathrm{Fe}]$} \\
\hline $05024868+0053487$ & 3228646389509963008 & 5336 & 4.40 & -0.87 & 0.19 & 0.19 & 7.76 & -0.10 \\
\hline 05025851-3909387 & 4814331680783356800 & 5996 & 4.00 & -0.15 & 0.41 & 0.41 & 8.69 & -0.02 \\
\hline 05042173-6614399 & 4662031938654411008 & 4921 & 4.80 & -0.77 & 0.10 & 0.10 & 7.76 & 0.34 \\
\hline $05043110+0151252$ & 3229000397895064960 & 5441 & 4.01 & -0.48 & 0.07 & 0.07 & 8.02 & $\cdots$ \\
\hline 05065541-1056146 & 3181964218172190464 & 5060 & 3.67 & -1.85 & 0.62 & 0.62 & 7.20 & $\ldots$ \\
\hline 05070417-0543063 & 3211326195154437760 & 4889 & 4.16 & -0.12 & -0.43 & -0.43 & 7.89 & -0.14 \\
\hline $05071155+3428162$ & 184903053675527552 & 6158 & 1.40 & -0.69 & 0.23 & 0.27 & 8.02 & $\ldots$ \\
\hline $05071933+0950093$ & 3290839985560775552 & 5404 & 4.80 & -0.50 & -0.02 & -0.02 & 7.91 & -0.07 \\
\hline $05071949+2233222$ & 3415364831369329024 & 5757 & 4.44 & -1.34 & 0.65 & 0.65 & 7.75 & -0.11 \\
\hline $05085713+0345040$ & 3238657198066863616 & 5041 & 2.27 & -0.82 & -0.06 & -0.04 & 7.57 & $\ldots$ \\
\hline $05090948+0758512$ & 3242001298259483776 & 5073 & 1.75 & -1.63 & -0.06 & 0.18 & 6.98 & 0.30 \\
\hline 05104812-0827001 & 3206923024747054080 & 5298 & 3.98 & 0.06 & -0.43 & -0.43 & 8.06 & -0.08 \\
\hline 05115864-0512205 & 3211775654892063360 & 5356 & 3.89 & -0.06 & -0.53 & -0.53 & 7.85 & -0.03 \\
\hline $05120490+0354513$ & 3235687077858885248 & 5232 & 1.93 & -1.74 & -0.04 & 0.06 & 6.75 & 0.40 \\
\hline 05124167-4059465 & 4818944995352554496 & 4845 & 4.82 & -1.38 & 0.08 & 0.08 & 7.13 & 0.44 \\
\hline 05130026-2540113 & 2956447060393795200 & 4783 & 4.55 & -0.91 & 0.28 & 0.28 & 7.80 & 0.05 \\
\hline 05134078-0629405 & 3208349473581491968 & 4619 & 0.88 & -2.43 & 0.07 & 0.73 & 6.73 & 0.34 \\
\hline $05144882+7604501$ & 503757371280728448 & 5667 & 3.52 & -1.53 & 0.62 & 0.62 & 7.53 & 0.05 \\
\hline $05145383+3147370$ & 180592968094361472 & 5547 & 3.34 & -0.29 & -0.15 & -0.15 & 7.99 & 0.41 \\
\hline 05160558-1418474 & 2985144932353707264 & $\cdots$ & $\cdots$ & $\cdots$ & $\cdots$ & $\cdots$ & $\cdots$ & $\cdots$ \\
\hline 05161882-0759103 & 3207022255670687488 & 5102 & 4.56 & -0.40 & -0.24 & -0.24 & 7.79 & 0.17 \\
\hline 05163919-0740502 & 3207049193707252992 & 5284 & 4.57 & -0.10 & -0.35 & -0.35 & 7.98 & -0.02 \\
\hline 05165318-1711429 & 2982252980551829760 & 4565 & 0.85 & -2.24 & -0.26 & 0.45 & 6.64 & 0.33 \\
\hline $05170982+0306456$ & 3235326060087791232 & 4827 & 1.71 & -1.93 & 0.21 & 0.47 & 6.97 & 0.26 \\
\hline $05171185+7649087$ & 551880864924665088 & 5077 & 4.57 & -0.65 & 0.46 & 0.46 & 8.23 & 0.14 \\
\hline 05172978-4253358 & 4800721449115828352 & 4682 & 1.41 & -2.96 & -0.39 & 0.14 & 5.61 & 0.27 \\
\hline 05175177-1441274 & 2985070199923468416 & 4510 & 4.61 & -0.68 & -0.42 & -0.42 & 7.34 & -0.08 \\
\hline $05180022-2418191$ & 2958140484397025792 & 4979 & 1.32 & -2.53 & -0.25 & 0.34 & 6.24 & 0.31 \\
\hline $05181193+1750335$ & 3395305135071832448 & 5784 & 3.69 & -1.72 & 1.08 & 1.08 & 7.79 & -0.10 \\
\hline 05181299-0356513 & 3213469349475577472 & 5219 & 3.46 & -0.86 & 0.27 & 0.27 & 7.84 & 0.01 \\
\hline 05183018-4217511 & 4806766701483968640 & 5736 & 4.04 & -1.04 & 0.59 & 0.59 & 7.99 & -0.13 \\
\hline 05183839-0208303 & 3214278143356960256 & 4816 & 2.45 & -1.78 & 0.87 & 0.89 & 7.54 & -0.07 \\
\hline 05191853-0625013 & 3207648530621533056 & 5392 & 4.73 & -0.63 & 0.23 & 0.23 & 8.02 & 0.25 \\
\hline $05202360-2522274$ & 2957773415014463872 & 4619 & 4.64 & -1.22 & -0.49 & -0.49 & 6.72 & 0.21 \\
\hline 05204433-0757598 & 3207154098284136064 & 5462 & 3.82 & -0.16 & -0.49 & -0.49 & 7.78 & 0.04 \\
\hline 05211432-1032144 & 3014044878233010304 & 5166 & 4.70 & -1.38 & 0.66 & 0.66 & 7.70 & 0.12 \\
\hline $05221674+1850154$ & 3401393307050179968 & 5077 & 4.31 & -1.33 & 0.46 & 0.46 & 7.56 & -0.11 \\
\hline 05224909-0008545 & 3221372600399707520 & 5271 & 3.05 & -0.21 & -0.53 & -0.52 & 7.71 & 0.11 \\
\hline $05232882+6904581$ & 485426657021670784 & 7450 & 4.30 & -0.97 & 2.33 & 2.36 & 9.82 & 0.34 \\
\hline $05234159+0001547$ & 3221399469715891712 & 5242 & 4.19 & -0.20 & -0.55 & -0.55 & 7.68 & -0.04 \\
\hline $05234178+0140465$ & 3222180191690519296 & 5500 & 3.58 & -0.38 & 0.22 & 0.22 & 8.27 & $\cdots$ \\
\hline $05240221+0037377$ & 3221857420604615040 & 5970 & 3.77 & -1.74 & 1.45 & 1.45 & 8.14 & 0.44 \\
\hline
\end{tabular}


Table 3 (continued)

\begin{tabular}{|c|c|c|c|c|c|c|c|c|}
\hline $\begin{array}{c}\text { Star Name } \\
\text { (2MASS) }\end{array}$ & $\begin{array}{c}\text { Star Name } \\
\text { (Gaia EDR3) }\end{array}$ & $\begin{array}{l}T_{\text {eff }} \\
(\mathrm{K})\end{array}$ & $\begin{array}{l}\log g \\
(\operatorname{cgs})\end{array}$ & {$[\mathrm{Fe} / \mathrm{H}]$} & {$[\mathrm{C} / \mathrm{Fe}]$} & {$[\mathrm{C} / \mathrm{Fe}]_{c}$} & $A(\mathrm{C})_{c}$ & {$[\mathrm{Mg} / \mathrm{Fe}]$} \\
\hline 05240835-0847159 & 3014887683958579840 & 5258 & 3.58 & -0.26 & -0.41 & -0.41 & 7.77 & 0.08 \\
\hline $05244855+3052198$ & 3447044445721668096 & 7217 & 3.95 & -2.17 & 1.55 & 1.55 & 7.81 & $\cdots$ \\
\hline $05250902-4636237$ & 4798948177378743680 & 5121 & 4.65 & -0.01 & -0.38 & -0.38 & 8.03 & 0.16 \\
\hline 05251415-6236322 & 4757456177662951296 & 4512 & 4.75 & -0.87 & -0.11 & -0.11 & 7.45 & 0.33 \\
\hline 05254247-2935153 & 2906535455902590464 & 5742 & 3.47 & -1.84 & 1.26 & 1.26 & 7.86 & 0.07 \\
\hline 05255980-1225415 & 2985857656406840832 & 5099 & 2.93 & -0.25 & -0.65 & -0.62 & 7.56 & 0.12 \\
\hline 05261364-0409463 & 3210609210495286016 & 5107 & 4.05 & -1.43 & 0.73 & 0.73 & 7.73 & -0.07 \\
\hline $05261736+0054216$ & 3221894563481860352 & 4896 & 3.92 & -1.49 & 0.77 & 0.77 & 7.72 & -0.08 \\
\hline $05263189+5101299$ & 261986133108337024 & 6069 & 4.08 & -0.47 & 0.33 & 0.33 & 8.29 & 0.49 \\
\hline $05270704+0208344$ & 3222306291931030912 & 4854 & 3.71 & -1.60 & 0.62 & 0.62 & 7.45 & -0.11 \\
\hline 05271660-1148112 & 3009915249997569024 & 4455 & 4.59 & -1.05 & -0.25 & -0.25 & 7.13 & 0.26 \\
\hline 05272500-3754396 & 4821159308691479424 & 4630 & 4.67 & -0.75 & -0.50 & -0.50 & 7.18 & 0.22 \\
\hline 05284287-0020406 & 3220938052789255552 & 5227 & 3.94 & -0.70 & 0.30 & 0.30 & 8.03 & -0.05 \\
\hline $05284445+0105281$ & 3221936173125954432 & 4837 & 3.96 & -0.42 & 0.12 & 0.12 & 8.13 & 0.54 \\
\hline 05291238-3538496 & 4822053555242274688 & 5429 & 2.68 & -2.48 & 0.88 & 0.90 & 6.86 & 0.32 \\
\hline 05293906-0037377 & 3220726675974365184 & 5018 & 4.47 & -0.41 & -0.40 & -0.40 & 7.63 & 0.12 \\
\hline $05310644+1002437$ & 3338225775622947584 & 5620 & 3.78 & -0.43 & 0.24 & 0.24 & 8.23 & 0.43 \\
\hline 05310938-0734569 & 3016409408052028416 & 4828 & 1.46 & -2.18 & -0.05 & 0.45 & 6.70 & 0.19 \\
\hline $05311096+0119175$ & 3221955960038003968 & 5342 & 3.83 & -0.08 & -0.58 & -0.58 & 7.77 & -0.06 \\
\hline 05313358-5942455 & 4759644931651720064 & 4815 & 1.44 & -1.94 & -0.09 & 0.36 & 6.85 & 0.37 \\
\hline 05314665-7729338 & 4623987049577991808 & 6026 & 3.44 & -1.13 & 0.66 & 0.66 & 7.95 & -0.14 \\
\hline 05315984-0724275 & 3016418655117200896 & 5170 & 3.92 & -0.08 & -0.40 & -0.40 & 7.96 & -0.04 \\
\hline $05322358+0426172$ & 3236515250632113536 & 4888 & 4.15 & -1.47 & 0.57 & 0.57 & 7.53 & -0.10 \\
\hline $05323333+5054220$ & 215399069525282304 & 4884 & 4.74 & -0.45 & -0.15 & -0.15 & 7.83 & 0.38 \\
\hline 05323416-3555512 & 4821789049679265536 & 5138 & 2.86 & -0.15 & -0.85 & -0.82 & 7.45 & 0.08 \\
\hline $05330330+3425560$ & 3449578029750593408 & 6383 & 1.64 & -0.80 & 0.63 & 0.65 & 8.28 & ... \\
\hline 05331754-0208154 & 3216941882074480000 & 5459 & 3.97 & -0.22 & -0.54 & -0.54 & 7.67 & 0.00 \\
\hline 05332244-5030257 & 4793470921547465856 & 5084 & 1.77 & -2.34 & 0.56 & 0.78 & 6.87 & 0.17 \\
\hline 05333204-2332171 & 2963722876730308224 & 5283 & 4.49 & -0.54 & 0.00 & 0.00 & 7.89 & 0.06 \\
\hline 05341283-0120379 & 3217572555072525056 & 5289 & 4.22 & -0.28 & -0.41 & -0.41 & 7.75 & 0.06 \\
\hline $05343131+0352284$ & 3224421859677408384 & 5252 & 2.34 & -2.50 & 0.43 & 0.44 & 6.37 & $\ldots$ \\
\hline 05344241-0015449 & 3220804015449200384 & 5681 & 4.16 & -0.07 & -0.50 & -0.50 & 7.86 & -0.11 \\
\hline 05355964-3116059 & 2902351985957543552 & 4940 & 2.76 & -0.31 & -0.76 & -0.73 & 7.39 & 0.20 \\
\hline $05363390+0511260$ & 3332736468116552192 & 5263 & 3.71 & -0.88 & 0.16 & 0.16 & 7.71 & -0.07 \\
\hline 05365678-4350228 & 4802513236454812160 & 5365 & 3.61 & -0.14 & -0.44 & -0.44 & 7.86 & -0.01 \\
\hline $05365967+0411503$ & 3224519505758364160 & 5485 & 4.27 & -0.07 & -0.42 & -0.42 & 7.94 & -0.08 \\
\hline 05370169-5105089 & 4793302318311314176 & 4989 & 4.22 & -0.43 & -0.48 & -0.48 & 7.53 & 0.10 \\
\hline $05374341+5648268$ & 268483082894179072 & 4909 & 3.10 & 0.08 & -0.38 & -0.37 & 8.14 & -0.15 \\
\hline $05382360+7011135$ & 485911644728122624 & 5734 & 4.25 & -0.70 & 0.41 & 0.41 & 8.15 & 0.33 \\
\hline 05384334-5147228 & 4793246488033004160 & 5264 & 2.43 & -2.67 & 0.82 & 0.83 & 6.59 & 0.17 \\
\hline 05391044-2345290 & 2963434353708776320 & 6224 & 4.01 & -0.16 & 0.52 & 0.52 & 8.80 & 0.00 \\
\hline 05404660-1040257 & 3010342513344338688 & 5134 & 4.23 & -0.15 & -0.28 & -0.28 & 8.01 & -0.04 \\
\hline
\end{tabular}


Table 3 (continued)

\begin{tabular}{|c|c|c|c|c|c|c|c|c|}
\hline $\begin{array}{c}\text { Star Name } \\
(2 \mathrm{MASS})\end{array}$ & $\begin{array}{c}\text { Star Name } \\
\text { (Gaia EDR3) }\end{array}$ & $\begin{array}{l}T_{\text {eff }} \\
(\mathrm{K})\end{array}$ & $\begin{array}{l}\log g \\
(\operatorname{cgs})\end{array}$ & {$[\mathrm{Fe} / \mathrm{H}]$} & {$[\mathrm{C} / \mathrm{Fe}]$} & {$[\mathrm{C} / \mathrm{Fe}]_{c}$} & $A(\mathrm{C})_{c}$ & {$[\mathrm{Mg} / \mathrm{Fe}]$} \\
\hline $05415000+1525054$ & 3347851759405013248 & 5011 & 4.44 & -0.02 & -0.57 & -0.57 & 7.84 & -0.10 \\
\hline 05423307-5913398 & 4759918400809516416 & 5114 & 2.10 & -1.63 & 0.43 & 0.46 & 7.26 & 0.09 \\
\hline $05431543+7808008$ & 552468274714043392 & 4719 & 4.94 & -0.97 & -0.16 & -0.16 & 7.30 & 0.39 \\
\hline $05434309+5012096$ & 214379311147693312 & 5149 & 4.68 & -0.51 & 0.13 & 0.13 & 8.05 & 0.08 \\
\hline $05434572-2151551$ & 2964356642107544192 & 4741 & 4.79 & -1.24 & 0.17 & 0.17 & 7.36 & 0.42 \\
\hline 05434805-2538412 & 2914965720791328896 & 5286 & 2.64 & -1.55 & 0.25 & 0.27 & 7.15 & 0.18 \\
\hline 05440311-6050523 & 4758589400489342080 & 5217 & 3.80 & 0.00 & -0.47 & -0.47 & 7.97 & -0.12 \\
\hline 05440932-3820149 & 4808589176366959360 & 5200 & 2.53 & -1.36 & 0.26 & 0.28 & 7.35 & 0.33 \\
\hline 05450050-2105418 & 2965923995930380160 & 4635 & 4.72 & -0.88 & -0.05 & -0.05 & 7.50 & 0.28 \\
\hline 05455248-2556383 & 2914192523598690048 & 5299 & 2.32 & -2.09 & 0.34 & 0.35 & 6.69 & 0.21 \\
\hline $05463023+4210063$ & 192794656646602880 & 4919 & 4.75 & -0.77 & 0.12 & 0.12 & 7.78 & 0.14 \\
\hline 05463758-4103031 & 4804867874965676032 & 4765 & 4.73 & -1.17 & 0.46 & 0.46 & 7.72 & 0.15 \\
\hline 05464200-1802448 & 2967658131925635968 & $\ldots$ & $\ldots$ & $\ldots$ & $\ldots$ & $\ldots$ & $\ldots$ & $\ldots$ \\
\hline 05472677-5432402 & 4767980226222674944 & 4886 & 1.37 & -1.88 & -0.01 & 0.43 & 6.98 & 0.19 \\
\hline 05483017-5835567 & 4765250138851402112 & 4953 & 3.31 & -1.68 & 0.52 & 0.53 & 7.28 & 0.30 \\
\hline 05483336-2434285 & 2915518908282121472 & 5061 & 4.27 & -1.51 & 0.31 & 0.31 & 7.23 & 0.35 \\
\hline $05483820+3148424$ & 3445140602679798400 & 7458 & 3.84 & -2.78 & 2.04 & 2.04 & 7.69 & $\ldots$ \\
\hline 05505657-2008418 & 2966088402979538944 & 5137 & 2.20 & -1.88 & 0.35 & 0.36 & 6.91 & 0.08 \\
\hline 05511133-0653111 & 3018944989240573056 & 5504 & 2.68 & -0.27 & -0.03 & 0.00 & 8.16 & 0.03 \\
\hline 05521578-3953184 & 4805034691496015104 & 4921 & 1.49 & -2.26 & 0.29 & 0.71 & 6.88 & -0.01 \\
\hline 05530383-2007355 & 2966268035692065280 & 5477 & 3.66 & -0.73 & 0.03 & 0.03 & 7.72 & 0.02 \\
\hline 05531036-4113596 & 4804147870945271168 & 4577 & 2.01 & -0.53 & -0.71 & -0.51 & 7.39 & 0.14 \\
\hline $05542509+1632212$ & 3349664785359713280 & 5340 & 4.57 & -0.63 & 0.20 & 0.20 & 8.00 & -0.11 \\
\hline 05544693-8301197 & 4620849386990258560 & 4914 & 1.49 & -2.08 & -0.08 & 0.39 & 6.75 & 0.28 \\
\hline $05545543+5131242$ & 214916074682985856 & 4819 & 2.26 & -1.89 & -0.01 & 0.00 & 6.55 & 0.13 \\
\hline 05583688-2438574 & 2914576528036091136 & 5057 & 3.98 & -0.96 & -0.27 & -0.27 & 7.20 & 0.18 \\
\hline 05583844-3434436 & 2889188186073231104 & 5069 & 3.70 & -1.00 & 0.41 & 0.41 & 7.84 & 0.00 \\
\hline $05584204+5040146$ & 211594935389482240 & 5189 & 4.90 & -0.61 & -0.05 & -0.05 & 7.77 & 0.20 \\
\hline 05592533-7744016 & 4647752959052853632 & 5287 & 1.97 & -2.40 & -0.08 & -0.02 & 6.01 & 0.30 \\
\hline 05594737-2708140 & 2911019229961694208 & 4637 & 0.73 & -2.34 & -0.17 & 0.54 & 6.63 & 0.45 \\
\hline 06000654-3034592 & 2891276807192892800 & 5263 & 4.32 & -0.25 & -0.25 & -0.25 & 7.93 & 0.02 \\
\hline $06000928+6830331$ & 1105490157831460352 & 5811 & 3.94 & -1.77 & 1.27 & 1.27 & 7.92 & -0.04 \\
\hline 06010912-2251504 & 2916542798424334208 & 4807 & 4.26 & -0.23 & -0.06 & -0.06 & 8.14 & 0.15 \\
\hline 06013941-3304567 & 2889974641827298048 & 4768 & 3.82 & -1.49 & 0.78 & 0.78 & 7.72 & -0.09 \\
\hline 06023445-3332108 & 2889528175684527104 & 5632 & 3.20 & -0.18 & -0.27 & -0.25 & 8.01 & 0.26 \\
\hline 06032005-7531388 & 5261685578730080000 & 5558 & 3.47 & -0.53 & -0.30 & -0.30 & 7.61 & 0.06 \\
\hline 06032024-2059364 & 2917926946123381248 & 5226 & 2.60 & -1.63 & 0.29 & 0.31 & 7.11 & 0.34 \\
\hline 06035878-3445341 & 2886205760783047808 & 5151 & 4.75 & -0.85 & 0.24 & 0.24 & 7.83 & 0.29 \\
\hline 06040505-3922438 & 2882964602365617408 & 5209 & 4.58 & -0.23 & -0.09 & -0.09 & 8.11 & 0.05 \\
\hline 06043058-3232288 & 2889972447101620480 & 5122 & 2.68 & -1.49 & 0.74 & 0.76 & 7.70 & $\ldots$ \\
\hline 06050821-3625551 & 2885764135065967488 & 5598 & 4.25 & -0.20 & -0.20 & -0.20 & 8.03 & 0.01 \\
\hline $06050851+6114505$ & 1005911306711125632 & 4621 & 4.82 & -1.39 & -0.04 & -0.04 & 7.00 & 0.43 \\
\hline
\end{tabular}


Table 3 (continued)

\begin{tabular}{|c|c|c|c|c|c|c|c|c|}
\hline $\begin{array}{l}\text { Star Name } \\
(2 \mathrm{MASS})\end{array}$ & $\begin{array}{c}\text { Star Name } \\
\text { (Gaia EDR3) }\end{array}$ & $\begin{array}{l}T_{\text {eff }} \\
(\mathrm{K})\end{array}$ & $\begin{array}{l}\log g \\
(\operatorname{cgs})\end{array}$ & {$[\mathrm{Fe} / \mathrm{H}]$} & {$[\mathrm{C} / \mathrm{Fe}]$} & {$[\mathrm{C} / \mathrm{Fe}]_{c}$} & $A(\mathrm{C})_{c}$ & {$[\mathrm{Mg} / \mathrm{Fe}]$} \\
\hline $06050978+6654472$ & 1105001321832556800 & 5391 & 4.49 & -0.22 & -0.19 & -0.19 & 8.02 & -0.02 \\
\hline $06052881-4438188$ & 5567973273001201152 & 5522 & 3.22 & -1.54 & 0.24 & 0.25 & 7.15 & 0.29 \\
\hline 06055284-3537284 & 2885916589224697088 & 5043 & 1.73 & -2.33 & 0.59 & 0.84 & 6.93 & 0.09 \\
\hline 06055999-6907423 & 5279730523050938624 & 5109 & 1.73 & -2.03 & 0.02 & 0.30 & 6.70 & 0.10 \\
\hline 06063114-1605358 & 2991490626275600640 & 5072 & 4.22 & -1.34 & 0.65 & 0.65 & 7.73 & -0.07 \\
\hline 06063142-5032308 & 5549982410912756992 & 5087 & 4.80 & -1.10 & 0.07 & 0.07 & 7.40 & 0.21 \\
\hline 06074714-3724498 & 2884863016567274240 & 5692 & 3.70 & -1.22 & 0.65 & 0.65 & 7.85 & 0.00 \\
\hline 06085789-3536279 & 2885882774945629824 & 5621 & 4.31 & -0.38 & 0.23 & 0.23 & 8.28 & 0.07 \\
\hline 06090353-7122260 & 5278332292157596160 & 5033 & 1.68 & -2.73 & 0.42 & 0.70 & 6.40 & 0.14 \\
\hline 06120579-3152561 & 2895882146005313152 & 4958 & 4.59 & -0.40 & -0.17 & -0.17 & 7.86 & 0.07 \\
\hline 06134289-6937017 & 5279560687166096896 & 5038 & 1.86 & -1.65 & 0.68 & 0.78 & 7.57 & 0.18 \\
\hline 06135706-3555284 & 2885428298685730816 & 5172 & 4.50 & -0.97 & 0.36 & 0.36 & 7.82 & 0.19 \\
\hline 06155680-7459448 & 5261728773219036800 & 5118 & 2.12 & -2.39 & 0.49 & 0.50 & 6.54 & 0.24 \\
\hline $06171171-7328560$ & 5265153816361777920 & 4806 & 2.28 & -1.28 & -0.94 & -0.92 & 6.23 & 0.15 \\
\hline 06181310-0348135 & 3116844057174980864 & 4307 & 4.59 & -1.47 & -0.64 & -0.64 & 6.32 & 0.07 \\
\hline 06183086-2506582 & 2912205186393737472 & 4948 & 4.44 & -0.39 & -0.05 & -0.05 & 7.99 & -0.02 \\
\hline 06192867-8743048 & 5189368469885240832 & 5081 & 1.79 & -1.87 & 0.52 & 0.70 & 7.26 & 0.26 \\
\hline 06194462-2030436 & 2938430256702303360 & 4871 & 1.35 & -2.74 & 0.17 & 0.70 & 6.39 & -0.14 \\
\hline 06200929-5719169 & 5495322423720756352 & 5056 & 3.72 & -1.29 & 0.84 & 0.84 & 7.98 & 0.35 \\
\hline 06220380-2155289 & 2937664962250734592 & 5126 & 3.45 & -0.88 & -0.47 & -0.47 & 7.08 & 0.39 \\
\hline 06234993-5946248 & 5482612241102802304 & 5426 & 4.54 & -0.28 & 0.20 & 0.20 & 8.36 & 0.00 \\
\hline $06244840+7752154$ & 1140661709439350528 & 4623 & 4.62 & -1.08 & -0.11 & -0.11 & 7.24 & 0.14 \\
\hline 06250234-4659051 & 5553993768631973120 & 5311 & 3.27 & -0.46 & -0.95 & -0.93 & 7.04 & $\ldots$ \\
\hline 06290787-6709523 & 5283390938997970176 & 4766 & 1.16 & -2.73 & -0.14 & 0.53 & 6.23 & 0.21 \\
\hline $06325088+6530326$ & 1103660291306745856 & 4632 & 3.81 & -1.92 & 1.03 & 1.03 & 7.54 & -0.07 \\
\hline $06325555-4214034$ & 5569242345641089408 & 4838 & 4.52 & -1.11 & 0.38 & 0.38 & 7.70 & 0.10 \\
\hline 06332771-3519240 & 5581669477032806912 & 5412 & 2.71 & -1.93 & 0.54 & 0.55 & 7.05 & 0.20 \\
\hline $06345221-4425441$ & 5556592090703647744 & 4705 & 4.32 & -1.30 & 0.98 & 0.98 & 8.11 & $\ldots$ \\
\hline 06363111-2255559 & 2924798167948814208 & 4984 & 4.68 & -0.64 & 0.48 & 0.48 & 8.28 & -0.03 \\
\hline 06382886-8309319 & 5206227036859558272 & 5183 & 1.57 & -2.50 & 0.06 & 0.47 & 6.40 & 0.29 \\
\hline 06393860-3406395 & 5583340111934906112 & 4787 & 4.47 & -0.84 & -0.04 & -0.04 & 7.55 & 0.47 \\
\hline 06421665-5008385 & 5503130777344639488 & 5120 & 4.36 & -0.28 & -0.29 & -0.29 & 7.87 & 0.08 \\
\hline $06425079+7014409$ & 1112486380380023040 & 6222 & 4.49 & -1.72 & 1.47 & 1.47 & 8.18 & -0.11 \\
\hline 06451421-3217294 & 5583930313459131008 & 5075 & 2.11 & -2.03 & 0.48 & 0.50 & 6.90 & 0.25 \\
\hline 06451920-0857165 & 3098535676741247744 & 5304 & 4.13 & -1.17 & 0.43 & 0.43 & 7.69 & -0.01 \\
\hline 06460710-4155202 & 5563430395897276032 & 5140 & 3.71 & -0.70 & -0.26 & -0.26 & 7.48 & 0.23 \\
\hline 06464646-4440364 & 5556009212103193472 & 5264 & 4.63 & -0.10 & -0.07 & -0.07 & 8.26 & -0.03 \\
\hline $06484021+1625173$ & 3358091992230790656 & 4618 & 2.59 & -1.96 & 0.34 & 0.35 & 6.82 & 0.15 \\
\hline 06484979-2428341 & 2922058189267952384 & 5124 & 3.41 & -0.63 & -0.03 & -0.03 & 7.77 & 0.08 \\
\hline $06510773+0943416$ & 3158574272957723776 & 4743 & 4.72 & -0.96 & 0.07 & 0.07 & 7.54 & 0.20 \\
\hline $06523842+1513163$ & 3354530639711957376 & 5732 & 4.11 & -0.53 & 0.39 & 0.39 & 8.29 & 0.43 \\
\hline $06525096+2440261$ & 3381304160163213056 & 4699 & 1.47 & -1.93 & -0.19 & 0.25 & 6.76 & 0.43 \\
\hline
\end{tabular}


Table 3 (continued)

\begin{tabular}{|c|c|c|c|c|c|c|c|c|}
\hline $\begin{array}{l}\text { Star Name } \\
(2 \mathrm{MASS})\end{array}$ & $\begin{array}{c}\text { Star Name } \\
\text { (Gaia EDR3) }\end{array}$ & $\begin{array}{l}T_{\text {eff }} \\
(\mathrm{K})\end{array}$ & $\begin{array}{l}\log g \\
(\operatorname{cgs})\end{array}$ & {$[\mathrm{Fe} / \mathrm{H}]$} & {$[\mathrm{C} / \mathrm{Fe}]$} & {$[\mathrm{C} / \mathrm{Fe}]_{c}$} & $A(\mathrm{C})_{c}$ & {$[\mathrm{Mg} / \mathrm{Fe}]$} \\
\hline $06525451+1446083$ & 3354323111187785216 & 5344 & 3.75 & -0.16 & -0.49 & -0.49 & 7.78 & -0.03 \\
\hline $06533075+1226225$ & 3351515542604966912 & 7500 & 4.53 & 0.07 & 1.02 & 1.02 & 9.52 & $\ldots$ \\
\hline $06533083+6836050$ & 1103319172123035136 & 4933 & 1.71 & -2.21 & 0.19 & 0.50 & 6.71 & 0.44 \\
\hline 06554143-4313578 & 5562195201959849984 & 4784 & 1.06 & -2.28 & -0.44 & 0.28 & 6.43 & 0.23 \\
\hline $06560363+2846500$ & 887636359876055424 & 5253 & 4.65 & -0.08 & -0.30 & -0.30 & 8.05 & -0.06 \\
\hline 06570150-6751413 & 5280814817608368000 & 5093 & 2.08 & -1.43 & 0.07 & 0.11 & 7.11 & -0.02 \\
\hline 06571276-5526234 & 5485259315346971136 & 5202 & 4.67 & -0.55 & 0.08 & 0.08 & 7.96 & 0.21 \\
\hline 06580901-8334199 & 5194228654877524096 & 5039 & 1.48 & -1.97 & -0.03 & 0.40 & 6.86 & 0.34 \\
\hline $06585640+0850479$ & 3157301205994883840 & 5497 & 2.96 & -0.33 & -0.34 & -0.31 & 7.79 & 0.54 \\
\hline $06593152-5853177$ & 5480647173305937280 & 4829 & 1.23 & -2.09 & -0.20 & 0.40 & 6.74 & 0.34 \\
\hline $06593924+2056439$ & 3366133201801121536 & 5291 & 2.44 & -2.57 & 0.89 & 0.91 & 6.77 & 0.43 \\
\hline 07001955-3637535 & 5578346443656712960 & 5489 & 4.22 & -0.56 & 0.50 & 0.50 & 8.38 & -0.08 \\
\hline $07003916+2701430$ & 884148846432586240 & 5358 & 3.66 & -0.61 & -0.04 & -0.04 & 7.78 & -0.03 \\
\hline $07004613+2933178$ & 887873162895952768 & 5062 & 4.58 & -0.29 & -0.16 & -0.16 & 7.98 & -0.02 \\
\hline $07012363+1813452$ & 3364377384810729088 & 5313 & 2.47 & -2.30 & 0.28 & 0.29 & 6.42 & 0.44 \\
\hline 07013933-5842103 & 5480743208774977024 & 5156 & 1.66 & -2.14 & -0.01 & 0.34 & 6.63 & 0.15 \\
\hline $07023862+0659243$ & 3153768440772179584 & 5867 & 4.27 & -0.30 & -0.49 & -0.49 & 7.64 & -0.05 \\
\hline $07030318+4006286$ & 947804380629842816 & 5298 & 2.60 & -1.59 & 0.01 & 0.03 & 6.87 & 0.42 \\
\hline $07033238+2225136$ & 3368024709696717824 & 4836 & 1.62 & -2.01 & -0.06 & 0.30 & 6.73 & 0.32 \\
\hline $07035093+1955164$ & 3365212055873231104 & 5787 & 3.86 & -1.06 & 0.54 & 0.54 & 7.91 & -0.13 \\
\hline $07041471+1901123$ & 3364898931280232448 & 4654 & 4.82 & -1.22 & 0.13 & 0.13 & 7.34 & 0.36 \\
\hline 07041738-1908113 & 2932674107172462592 & 5435 & 2.08 & -0.64 & -0.36 & -0.27 & 7.52 & 0.18 \\
\hline $07041880+0246488$ & 3115642187883221632 & 7079 & 4.50 & -1.48 & 2.19 & 2.20 & 9.16 & 0.00 \\
\hline $07041957+2340564$ & 3368538353422137344 & 6411 & 3.40 & -0.93 & 0.88 & 0.89 & 8.39 & 0.30 \\
\hline $07054097+1733350$ & 3361464812868710144 & 5010 & 2.15 & -1.43 & -0.24 & -0.21 & 6.79 & 0.27 \\
\hline $07070364+2657035$ & 883254423785454080 & 5165 & 2.56 & -2.62 & 0.65 & 0.66 & 6.47 & 0.31 \\
\hline $07073503+2356476$ & 3368586663216905984 & 4783 & 4.79 & -0.51 & -0.19 & -0.19 & 7.73 & 0.30 \\
\hline 07081426-3557115 & 5566430245872289408 & 5980 & 3.42 & -1.37 & 0.90 & 0.91 & 7.97 & 0.07 \\
\hline 07083867-4621128 & 5510018805377253632 & 4704 & 2.34 & -0.52 & -0.70 & -0.67 & 7.25 & 0.19 \\
\hline $07085997-5424564$ & 5491233099459990528 & 5367 & 1.94 & -2.06 & -0.05 & 0.04 & 6.41 & 0.33 \\
\hline $07090577+2142312$ & 3367308652748446464 & 4907 & 3.92 & -1.58 & 0.60 & 0.60 & 7.44 & -0.02 \\
\hline $07093534+2210136$ & 3367373077257509120 & 5914 & 3.97 & -0.35 & 0.24 & 0.24 & 8.32 & 0.57 \\
\hline 07093868-0419118 & 3107527762016884096 & 6302 & 3.59 & -3.50 & 2.59 & 2.59 & 7.52 & 0.08 \\
\hline 07101117-2219008 & 2928210850185413120 & 6828 & 3.88 & -2.58 & 3.29 & 3.30 & 9.15 & 0.24 \\
\hline $07102845+1245411$ & 3161108024483929600 & 5088 & 1.77 & -2.65 & 0.33 & 0.53 & 6.31 & 0.36 \\
\hline $07103372-0244311$ & 3108335765621350656 & 5890 & 3.97 & -0.14 & -0.41 & -0.41 & 7.88 & -0.09 \\
\hline $07104970+0826444$ & 3154409902728232320 & 4856 & 1.66 & -1.68 & -0.53 & -0.18 & 6.57 & 0.54 \\
\hline 07110190-6301262 & 5285981079149837184 & 5330 & 4.04 & -1.33 & 0.38 & 0.38 & 7.48 & 0.30 \\
\hline $07113988+8541053$ & 1150345383223043712 & 5407 & 3.86 & -1.57 & 0.88 & 0.88 & 7.75 & 0.08 \\
\hline $07121704+1402591$ & 3167279926849037568 & 4687 & 0.88 & -2.22 & -0.38 & 0.34 & 6.55 & 0.21 \\
\hline $07122036+4221574$ & 949584631690298368 & 5030 & 4.79 & -0.05 & -0.35 & -0.35 & 8.03 & -0.02 \\
\hline $07122182-8422429$ & 5193956796331371264 & 5431 & 4.74 & -0.39 & 0.11 & 0.11 & 8.15 & 0.03 \\
\hline
\end{tabular}


Table 3 (continued)

\begin{tabular}{|c|c|c|c|c|c|c|c|c|}
\hline $\begin{array}{c}\text { Star Name } \\
\text { (2MASS) }\end{array}$ & $\begin{array}{c}\text { Star Name } \\
\text { (Gaia EDR3) }\end{array}$ & $\begin{array}{l}T_{\text {eff }} \\
(\mathrm{K})\end{array}$ & $\begin{array}{l}\log g \\
(\mathrm{cgs})\end{array}$ & {$[\mathrm{Fe} / \mathrm{H}]$} & {$[\mathrm{C} / \mathrm{Fe}]$} & {$[\mathrm{C} / \mathrm{Fe}]_{c}$} & $A(\mathrm{C})_{c}$ & {$[\mathrm{Mg} / \mathrm{Fe}]$} \\
\hline $07122917+2231366$ & 3367505087372386944 & 4909 & 4.11 & -0.29 & -0.19 & -0.19 & 7.95 & 0.49 \\
\hline 07122989-0700106 & 3052191643892060672 & 6739 & 0.95 & -1.26 & 2.88 & 2.92 & 10.09 & $\ldots$ \\
\hline 07123398-4814049 & 5508686643961355648 & 4773 & 1.56 & -2.91 & -0.16 & 0.21 & 5.73 & 0.35 \\
\hline $07123461+3153463$ & 890055835212749696 & 4766 & 4.83 & -0.96 & 0.07 & 0.07 & 7.54 & 0.01 \\
\hline 07130783-7052481 & 5267185649427578496 & 5074 & 4.67 & -0.36 & -0.12 & -0.12 & 7.95 & 0.03 \\
\hline $07150401+2714015$ & 883489998448405504 & 5504 & 4.49 & -0.40 & -0.04 & -0.04 & 7.99 & 0.12 \\
\hline $07151852-5252051$ & 5491893184393739776 & 5019 & 1.53 & -2.92 & 0.40 & 0.78 & 6.29 & 0.20 \\
\hline 07152806-4111161 & 5561098267315218048 & 4799 & 3.94 & -1.25 & 0.42 & 0.42 & 7.60 & -0.14 \\
\hline $07160059+5240188$ & 986603229693072128 & 5704 & 3.75 & -1.54 & 0.67 & 0.67 & 7.56 & 0.11 \\
\hline $07170438+2402438$ & 869715076257527808 & 6150 & 3.24 & -0.30 & 0.01 & 0.03 & 8.15 & 0.24 \\
\hline $07174600+1259027$ & 3166159975473025280 & 5135 & 3.83 & -1.30 & 0.36 & 0.36 & 7.50 & 0.04 \\
\hline 07174929-0924096 & 3048017378051885056 & 5710 & 4.27 & -0.04 & 0.25 & 0.25 & 8.64 & -0.09 \\
\hline $07175677-2704124$ & 5616006675420673664 & 5350 & 4.41 & -1.07 & 0.38 & 0.38 & 7.74 & -0.04 \\
\hline $07181152+4406479$ & 973981901357263104 & 5077 & 3.93 & -1.24 & 0.55 & 0.55 & 7.74 & -0.08 \\
\hline 07194881-5748087 & 5485933071160480768 & 4984 & 4.25 & -0.30 & -0.10 & -0.10 & 8.04 & -0.04 \\
\hline 07203081-6240451 & 5291814602512817024 & 4907 & 1.40 & -2.05 & -0.24 & 0.27 & 6.65 & 0.24 \\
\hline 07204786-7623393 & 5214066868299703040 & 5411 & 4.72 & -0.73 & 0.23 & 0.23 & 7.93 & 0.18 \\
\hline $07213106+2755248$ & 872994571549210240 & 5632 & 3.47 & -1.89 & 1.34 & 1.34 & 7.88 & 0.38 \\
\hline $07220367+0253575$ & 3136142616543571456 & 4737 & 1.09 & -2.14 & -0.41 & 0.26 & 6.54 & 0.46 \\
\hline $07224055+3618087$ & 897278351003895424 & 5068 & 4.66 & -0.73 & 0.33 & 0.33 & 8.04 & 0.11 \\
\hline $07224582-2959375$ & 5605629995767584000 & 5638 & 4.30 & -0.82 & 0.52 & 0.52 & 8.13 & -0.06 \\
\hline $07233679+1412112$ & 3166723543901552000 & 4773 & 2.44 & -0.21 & -0.89 & -0.86 & 7.36 & -0.11 \\
\hline 07234039-2959296 & 5605624738733670912 & 5685 & 3.54 & -0.14 & 0.20 & 0.20 & 8.48 & 0.06 \\
\hline $07234262+1516310$ & 3167138884419576064 & 5223 & 3.57 & -0.14 & -0.52 & -0.52 & 7.77 & -0.03 \\
\hline $07242428+5822232$ & 989627947524580864 & 4716 & 4.71 & -1.33 & 0.29 & 0.29 & 7.39 & 0.33 \\
\hline $07254156+4948144$ & 977051149410569344 & 5219 & 4.19 & -0.09 & -0.59 & -0.59 & 7.75 & -0.07 \\
\hline 07254769-3453506 & 5590014289255060480 & 5651 & 3.68 & -0.39 & -0.09 & -0.09 & 7.95 & -0.20 \\
\hline 07260648-4637077 & 5510340717467331456 & 4831 & 4.06 & -0.11 & -0.68 & -0.68 & 7.65 & -0.07 \\
\hline $07270798+4352580$ & 973215713552094848 & 4980 & 4.61 & -0.90 & 0.38 & 0.38 & 7.91 & 0.06 \\
\hline $07273288+3819555$ & 899749713837935872 & 4795 & 4.38 & -1.41 & 0.34 & 0.34 & 7.36 & 0.34 \\
\hline $07280531+1637140$ & 3169561074176213376 & 5309 & 4.75 & -0.83 & 0.18 & 0.18 & 7.78 & 0.30 \\
\hline 07281913-8333342 & 5194416392190823040 & 5269 & 2.42 & -1.77 & 0.49 & 0.51 & 7.17 & 0.06 \\
\hline 07282428-3705347 & 5586496638018390784 & 5356 & 3.12 & -0.21 & -0.03 & -0.02 & 8.20 & 0.17 \\
\hline $07284694+8149439$ & 1142689281896442880 & 5012 & 1.69 & -2.69 & 0.55 & 0.80 & 6.53 & 0.42 \\
\hline 07295359-6910360 & 5267963068571164928 & 5163 & 4.71 & -0.30 & -0.13 & -0.13 & 8.01 & 0.22 \\
\hline $07304303+4111480$ & 900640730573598976 & 4633 & 4.35 & -1.74 & 0.66 & 0.66 & 7.36 & -0.06 \\
\hline 07310602-2742595 & 5611975414810555264 & 5391 & 4.60 & -0.13 & 0.20 & 0.20 & 8.50 & 0.02 \\
\hline 07311836-6659351 & 5269135942537559680 & 4643 & 4.78 & -0.75 & -0.19 & -0.19 & 7.49 & 0.30 \\
\hline $07314455+5553592$ & 988736827710024704 & 4816 & 4.71 & -0.53 & -0.10 & -0.10 & 7.81 & 0.32 \\
\hline 07314605-3324217 & 5591704921522389888 & 6884 & 4.17 & -1.63 & 0.35 & 0.35 & 7.15 & $\cdots$ \\
\hline $07321280+1523588$ & 3168444240942341248 & 5114 & 4.41 & -1.05 & 0.37 & 0.37 & 7.75 & -0.02 \\
\hline 07321479-1300309 & 3033439198205025024 & 5234 & 3.11 & -3.39 & -0.17 & -0.17 & 4.87 & $\ldots$ \\
\hline
\end{tabular}


Table 3 (continued)

\begin{tabular}{|c|c|c|c|c|c|c|c|c|}
\hline $\begin{array}{c}\text { Star Name } \\
\text { (2MASS) }\end{array}$ & $\begin{array}{c}\text { Star Name } \\
\text { (Gaia EDR3) }\end{array}$ & $\begin{array}{l}T_{\text {eff }} \\
(\mathrm{K})\end{array}$ & $\begin{array}{l}\log g \\
(\operatorname{cgs})\end{array}$ & {$[\mathrm{Fe} / \mathrm{H}]$} & {$[\mathrm{C} / \mathrm{Fe}]$} & {$[\mathrm{C} / \mathrm{Fe}]_{c}$} & $A(\mathrm{C})_{c}$ & {$[\mathrm{Mg} / \mathrm{Fe}]$} \\
\hline $07325519+3655267$ & 898637038134266752 & 4770 & 1.23 & -2.28 & -0.30 & 0.35 & 6.50 & 0.32 \\
\hline $07331792+0531033$ & 3141404295078246400 & 4877 & 4.72 & -0.87 & 0.19 & 0.19 & 7.75 & 0.07 \\
\hline $07333190+5310203$ & 983909666723291392 & 6890 & 4.16 & -3.87 & 2.10 & 2.10 & 6.67 & $\cdots$ \\
\hline $07334024+4425362$ & 973487288627865728 & 5119 & 4.76 & -0.65 & 0.07 & 0.07 & 7.85 & -0.02 \\
\hline $07345045+0736494$ & 3142272222070528384 & 4516 & 4.16 & -0.68 & -1.03 & -1.03 & 6.73 & -0.20 \\
\hline 07350484-0004500 & 3086417752932494848 & 4832 & 4.52 & -0.80 & 0.35 & 0.35 & 7.97 & 0.11 \\
\hline $07351435+2626331$ & 872041539782121984 & 4754 & 3.72 & -1.85 & 0.77 & 0.77 & 7.35 & 0.22 \\
\hline 07351699-4815064 & 5506540186806281728 & 5212 & 4.08 & -1.20 & 0.38 & 0.38 & 7.61 & -0.01 \\
\hline $07353628+1938556$ & 672591122640513280 & 5387 & 4.61 & -0.54 & 0.17 & 0.17 & 8.06 & 0.06 \\
\hline $07354551+0455028$ & 3138308624388237568 & 5491 & 3.54 & -1.10 & 0.17 & 0.17 & 7.50 & -0.08 \\
\hline 07354757-5036104 & 5493916801186715648 & 5506 & 4.07 & -0.37 & -0.26 & -0.26 & 7.80 & -0.08 \\
\hline $07363720+1126504$ & 3162146822454143360 & 4853 & 3.64 & -1.36 & 0.61 & 0.61 & 7.68 & -0.13 \\
\hline $07363729+4034177$ & 924375132168707200 & 4769 & 4.66 & -0.90 & 0.15 & 0.15 & 7.68 & -0.03 \\
\hline $07364486+4508331$ & 926998601271917184 & 5684 & 3.43 & -0.80 & 0.28 & 0.28 & 7.91 & 0.42 \\
\hline $07365679+5950385$ & 1086214756921278976 & 4516 & 4.49 & -0.48 & -1.03 & -1.03 & 6.93 & -0.15 \\
\hline 07374300-5630568 & 5487737374038584704 & 5116 & 1.85 & -2.96 & 0.82 & 0.93 & 6.41 & 0.44 \\
\hline 07380041-2129196 & 5619082589253324544 & 5200 & 2.40 & -0.44 & -0.88 & -0.85 & 7.14 & 0.24 \\
\hline 07384500-0331557 & 3057721981216813568 & 5329 & 2.03 & -1.82 & 0.18 & 0.23 & 6.84 & 0.19 \\
\hline 07393047-1530275 & 3029020260970100736 & 5120 & 2.86 & -1.06 & 0.36 & 0.38 & 7.75 & -0.06 \\
\hline $07395820+1311537$ & 3164103029736387200 & 5051 & 2.10 & -1.73 & -0.07 & -0.04 & 6.65 & 0.36 \\
\hline 07400327-0740587 & 3042138568557247360 & 5175 & 1.57 & -2.39 & 0.04 & 0.47 & 6.51 & 0.31 \\
\hline $07403555+5352561$ & 985231550281510144 & 4686 & 3.88 & -0.51 & -0.40 & -0.40 & 7.53 & 0.52 \\
\hline $07404482+1243437$ & 3163865500863822592 & 4976 & 4.26 & -0.27 & -0.16 & -0.16 & 8.00 & 0.51 \\
\hline 07410359-6714551 & 5274884459907753344 & 4756 & 1.03 & -1.64 & -0.43 & 0.13 & 6.92 & -0.19 \\
\hline 07410851-5943543 & 5292748779377543168 & 5023 & 4.48 & -0.52 & -0.32 & -0.32 & 7.59 & 0.18 \\
\hline 07412077-2900201 & 5599777230966529024 & 5400 & 1.97 & -2.29 & 0.23 & 0.31 & 6.45 & 0.22 \\
\hline 07414196-0741080 & 3042148704679838336 & 5317 & 4.69 & -0.26 & -0.07 & -0.07 & 8.11 & 0.15 \\
\hline $07435834+1341353$ & 3164056506649463040 & 5531 & 3.64 & -1.36 & 0.63 & 0.63 & 7.69 & -0.07 \\
\hline $07440179+0312226$ & 3136905750633082624 & 4991 & 3.72 & -1.57 & 0.55 & 0.55 & 7.41 & 0.22 \\
\hline $07441635+5322545$ & 984534979601381120 & 4941 & 4.69 & -0.57 & 0.19 & 0.19 & 8.05 & 0.00 \\
\hline $07443402+0742591$ & 3145150125036773760 & 6196 & 3.56 & -0.58 & 0.23 & 0.23 & 8.08 & 0.17 \\
\hline 07443970-4425135 & 5532006972050819584 & 5202 & 2.18 & -2.65 & 0.80 & 0.81 & 6.59 & 0.04 \\
\hline 07445367-4653249 & 5530553525058225152 & 5350 & 3.98 & -0.14 & -0.54 & -0.54 & 7.76 & -0.02 \\
\hline $07450351+0956148$ & 3148979071201226880 & 4702 & 1.06 & -2.64 & -0.28 & 0.45 & 6.25 & 0.34 \\
\hline 07450424-7231255 & 5263155252116820608 & 7552 & 4.62 & -0.81 & 0.90 & 0.90 & 8.52 & .. \\
\hline $07451488+1000107$ & 3149076244837702784 & 5644 & 4.14 & -0.24 & -0.36 & -0.36 & 7.83 & -0.01 \\
\hline $07452477+2524147$ & 868026810512553856 & 5054 & 4.72 & -0.62 & 0.14 & 0.14 & 7.95 & 0.05 \\
\hline $07455398+0103107$ & 3086833647505226752 & 5802 & 3.65 & -0.99 & 0.47 & 0.47 & 7.91 & 0.12 \\
\hline $07461952+3903325$ & 920303430156012160 & 4781 & 0.97 & -2.67 & -0.11 & 0.61 & 6.37 & 0.47 \\
\hline 07464924-4313456 & 5532490211705643904 & 5601 & 3.38 & -0.13 & 0.11 & 0.11 & 8.41 & -0.06 \\
\hline 07480156-4924040 & 5518045893097566976 & 4955 & 1.59 & -2.73 & 0.15 & 0.51 & 6.21 & 0.19 \\
\hline $07485521+5623334$ & 1082018707015827584 & 5353 & 3.67 & -0.78 & 0.20 & 0.20 & 7.85 & -0.12 \\
\hline
\end{tabular}


Table 3 (continued)

\begin{tabular}{|c|c|c|c|c|c|c|c|c|}
\hline $\begin{array}{c}\text { Star Name } \\
\text { (2MASS) }\end{array}$ & $\begin{array}{c}\text { Star Name } \\
\text { (Gaia EDR3) }\end{array}$ & $\begin{array}{l}T_{\text {eff }} \\
(\mathrm{K})\end{array}$ & $\begin{array}{l}\log g \\
(\operatorname{cgs})\end{array}$ & {$[\mathrm{Fe} / \mathrm{H}]$} & {$[\mathrm{C} / \mathrm{Fe}]$} & {$[\mathrm{C} / \mathrm{Fe}]_{c}$} & $A(\mathrm{C})_{c}$ & {$[\mathrm{Mg} / \mathrm{Fe}]$} \\
\hline $07494258+6938239$ & 1098142705736502528 & 4957 & 3.76 & -0.73 & -0.38 & -0.38 & 7.32 & 0.30 \\
\hline $07503801+1119098$ & 3150778696857500928 & 5164 & 4.77 & -0.54 & 0.06 & 0.06 & 7.95 & 0.00 \\
\hline $07504673+0558247$ & 3143849888115383040 & 5027 & 2.00 & -1.96 & 0.24 & 0.30 & 6.77 & 0.35 \\
\hline $07533988+4933361$ & 934072756004889216 & 5378 & 3.01 & -1.94 & 0.47 & 0.48 & 6.97 & 0.10 \\
\hline $07545950+6639532$ & 1095319709633456000 & 5186 & 4.67 & -1.05 & 0.26 & 0.26 & 7.64 & 0.29 \\
\hline $07551255+1944081$ & 670305998176686720 & 4615 & 1.08 & -2.83 & -0.12 & 0.60 & 6.20 & 0.44 \\
\hline $07552395+3520063$ & 906645575890268416 & 4701 & 4.67 & -0.93 & 0.09 & 0.09 & 7.59 & 0.02 \\
\hline $07555507+8148305$ & 1148451100783017984 & 4638 & 4.72 & -0.89 & -0.42 & -0.42 & 7.13 & 0.45 \\
\hline $07575895+4035155$ & 922132399029177088 & 4984 & 4.57 & -0.16 & -0.77 & -0.77 & 7.51 & 0.07 \\
\hline $07580568+3504110$ & 906456700407472512 & 5122 & 4.72 & -0.23 & -0.04 & -0.04 & 8.16 & 0.09 \\
\hline $07584532+4211214$ & 922545677962459904 & 5289 & 3.43 & -1.46 & 0.46 & 0.46 & 7.42 & 0.18 \\
\hline $07590580+4748300$ & 933111228791082240 & 5079 & 4.05 & -0.29 & 0.09 & 0.09 & 8.23 & 0.45 \\
\hline $08005431+3135329$ & 878032675562889728 & 4469 & 4.91 & -1.19 & 0.74 & 0.74 & 7.97 & $\ldots$ \\
\hline $08005634+1633254$ & 667243544758539136 & 5337 & 4.74 & -0.17 & -0.19 & -0.19 & 8.07 & -0.03 \\
\hline $08011676+6358383$ & 1094412028423846144 & 4892 & 4.62 & -1.01 & 0.10 & 0.10 & 7.52 & 0.39 \\
\hline $08021251-2821568$ & 5597762543400727680 & 8401 & 4.07 & -0.13 & 0.00 & 0.00 & 0.00 & ... \\
\hline 08022771-4804247 & 5517895294366243968 & 5286 & 3.93 & -0.04 & -0.54 & -0.54 & 7.85 & -0.03 \\
\hline $08024574-4803573$ & 5517708957205335168 & 5200 & 2.74 & -0.18 & -0.30 & -0.27 & 7.98 & 0.03 \\
\hline 08042788-6440110 & 5275614368128678528 & 5288 & 2.29 & -2.21 & 0.63 & 0.64 & 6.86 & 0.05 \\
\hline $08045600+3310059$ & 905235555306335104 & 4959 & 4.88 & -0.65 & 0.14 & 0.14 & 7.92 & 0.09 \\
\hline $08050775+2604105$ & 681971846610387712 & 5980 & 3.51 & -0.36 & 0.07 & 0.07 & 8.14 & 0.34 \\
\hline 08052894-6502132 & 5275590007074529152 & 5149 & 1.59 & -1.98 & -0.04 & 0.36 & 6.81 & 0.33 \\
\hline 08053267-6119242 & 5289953163686735232 & 5443 & 4.67 & -0.40 & 0.24 & 0.24 & 8.27 & -0.03 \\
\hline 08074491-5257227 & 5512588600272046848 & 5403 & 4.48 & -0.09 & 0.02 & 0.02 & 8.35 & -0.06 \\
\hline $08080715+5915114$ & 1083546379639153664 & 4949 & 4.79 & -0.13 & -0.40 & -0.40 & 7.90 & -0.04 \\
\hline 08085268-6128294 & 5289751265864064128 & 5309 & 2.59 & -1.91 & 0.39 & 0.40 & 6.93 & 0.22 \\
\hline $08090031+2850085$ & 876519232166668800 & 4979 & 1.60 & -2.67 & 0.34 & 0.68 & 6.45 & 0.46 \\
\hline $08092156+2521288$ & 681134774663802496 & 5135 & 4.65 & -0.34 & -0.21 & -0.21 & 7.88 & 0.08 \\
\hline 08100860-7523572 & 5213699150380190720 & 4943 & 1.21 & -2.40 & -0.27 & 0.41 & 6.44 & 0.21 \\
\hline $08102898+4020333$ & 909546843477733504 & 5188 & 3.64 & -1.17 & 0.10 & 0.10 & 7.36 & 0.02 \\
\hline $08111719+4456352$ & 929054103899434752 & 5057 & 4.84 & -0.41 & 0.09 & 0.09 & 8.11 & 0.14 \\
\hline 08125962-7004235 & 5270057397705227904 & 4973 & 4.21 & -0.01 & -0.39 & -0.39 & 8.02 & -0.11 \\
\hline $08130864+3925564$ & 909255575975553920 & 4904 & 1.33 & -2.48 & -0.43 & 0.17 & 6.13 & 0.43 \\
\hline $08135674+5113349$ & 935284722760688384 & 5834 & 3.66 & -0.91 & 0.22 & 0.22 & 7.74 & -0.08 \\
\hline $08140229+3940332$ & 909264917528317952 & 4907 & 4.78 & -0.56 & 0.01 & 0.01 & 7.88 & 0.15 \\
\hline $08152203+3827587$ & 908032124475412224 & 5500 & 4.18 & -0.21 & -0.23 & -0.23 & 7.99 & -0.02 \\
\hline $08153796+6531162$ & 1092014470303207552 & 4823 & 4.69 & -0.89 & 0.19 & 0.19 & 7.73 & 0.00 \\
\hline 08162824-4611151 & 5519745360123251200 & 5467 & 2.39 & -0.18 & -0.01 & 0.03 & 8.28 & 0.56 \\
\hline 08173327-2728418 & 5693425380770623616 & 5168 & 3.39 & -1.68 & 0.84 & 0.84 & 7.60 & 0.25 \\
\hline $08182058+5923087$ & 1083715257753356800 & 5153 & 3.56 & -1.16 & 0.30 & 0.30 & 7.57 & 0.09 \\
\hline 08202927-2458369 & 5696053561463700992 & 5187 & 4.58 & -1.26 & 0.50 & 0.50 & 7.67 & 0.12 \\
\hline 08204593-7457097 & 5219742375524510720 & 5647 & 3.78 & -1.23 & 0.53 & 0.53 & 7.72 & -0.01 \\
\hline
\end{tabular}


Table 3 (continued)

\begin{tabular}{|c|c|c|c|c|c|c|c|c|}
\hline $\begin{array}{l}\text { Star Name } \\
\text { (2MASS) }\end{array}$ & $\begin{array}{c}\text { Star Name } \\
\text { (Gaia EDR3) }\end{array}$ & $\begin{array}{l}T_{\text {eff }} \\
(\mathrm{K})\end{array}$ & $\begin{array}{l}\log g \\
(\operatorname{cgs})\end{array}$ & {$[\mathrm{Fe} / \mathrm{H}]$} & {$[\mathrm{C} / \mathrm{Fe}]$} & {$[\mathrm{C} / \mathrm{Fe}]_{c}$} & $A(\mathrm{C})_{c}$ & {$[\mathrm{Mg} / \mathrm{Fe}]$} \\
\hline $08225420+6259266$ & 1090362836337748864 & 4674 & 0.80 & -2.50 & -0.19 & 0.54 & 6.46 & 0.46 \\
\hline 08242044-1026147 & 5751499802540091776 & 4605 & 4.34 & -0.18 & -0.62 & -0.62 & 7.64 & 0.07 \\
\hline 08244155-6859542 & 5270209581283002240 & 5499 & 3.60 & -0.30 & -0.28 & -0.28 & 7.86 & 0.00 \\
\hline $08255445+7726501$ & 1137625614237615232 & 5276 & 4.72 & -0.52 & 0.09 & 0.09 & 8.00 & 0.06 \\
\hline 08282505-2119200 & 5706111756390126080 & 5277 & 4.23 & -1.23 & 0.67 & 0.67 & 7.86 & 0.10 \\
\hline 08284828-2858468 & 5644860540592289536 & 6079 & 4.38 & -1.71 & 1.15 & 1.15 & 7.87 & -0.11 \\
\hline 08295142-2428117 & 5695713503130206848 & 5063 & 2.40 & -0.33 & -0.80 & -0.77 & 7.32 & 0.17 \\
\hline 08295611-1354544 & 5722897519371974272 & 5161 & 4.64 & -0.25 & -0.09 & -0.09 & 8.08 & 0.04 \\
\hline 08302532-6949046 & 5221888519143226240 & 4937 & 4.54 & -0.46 & 0.02 & 0.02 & 7.98 & 0.26 \\
\hline 08312114-2126535 & 5703185367534835584 & 5188 & 2.31 & -0.52 & -0.90 & -0.87 & 7.03 & 0.26 \\
\hline $08315869+6949533$ & 1121420775645432320 & 5526 & 4.42 & -0.23 & -0.15 & -0.15 & 8.05 & 0.04 \\
\hline 08322899-2137273 & 5703121870736546304 & 4887 & 2.22 & -1.74 & 0.37 & 0.39 & 7.08 & 0.14 \\
\hline 08331960-1350331 & 5722870096004958208 & 5693 & 3.33 & -1.99 & 1.19 & 1.19 & 7.63 & -0.14 \\
\hline $08343393+7518493$ & 1125113279289236096 & 5623 & 3.68 & -0.37 & -0.07 & -0.07 & 7.99 & 0.49 \\
\hline $08350584+4839306$ & 1026860572337001088 & 5012 & 1.50 & -2.28 & -0.25 & 0.25 & 6.39 & 0.47 \\
\hline 08355055-2033433 & 5703594458875614848 & 4506 & 4.02 & -1.42 & -0.07 & -0.07 & 6.95 & 0.21 \\
\hline 08355851-0839549 & 5753641990492978816 & 5098 & 1.68 & -2.05 & -0.25 & 0.08 & 6.46 & 0.24 \\
\hline 08360617-0450149 & 3065446698936865152 & 4897 & 4.64 & -0.78 & 0.02 & 0.02 & 7.67 & 0.55 \\
\hline $08364858+7742308$ & 1137832945195154560 & 5088 & 1.54 & -2.58 & 0.83 & 1.14 & 7.00 & 0.56 \\
\hline $08370406+7830159$ & 1138150772775117312 & 4988 & 1.70 & -2.55 & 0.50 & 0.77 & 6.64 & 0.31 \\
\hline 08400533-4428341 & 5522379549464634752 & 5299 & 2.71 & -0.50 & -0.84 & -0.81 & 7.12 & 0.20 \\
\hline $08401347+4216411$ & 913518485634602112 & 4901 & 4.73 & -1.32 & 0.43 & 0.43 & 7.53 & 0.16 \\
\hline 08413166-0629117 & 5754432023955507712 & 5578 & 3.56 & -1.11 & 0.21 & 0.21 & 7.53 & 0.04 \\
\hline 08422928-0849161 & 5750516216373911680 & 4969 & 4.69 & -1.74 & 0.10 & 0.10 & 6.79 & 0.50 \\
\hline $08430958+6038165$ & 1041278571391227392 & 4700 & 4.46 & -1.79 & 0.60 & 0.60 & 7.24 & -0.02 \\
\hline $08433739+6717034$ & 1093203940022382720 & 4531 & 4.83 & -0.59 & -0.48 & -0.48 & 7.36 & 0.16 \\
\hline 08434698-1812314 & 5705872333433002880 & 5203 & 4.36 & -0.58 & 0.44 & 0.44 & 8.29 & 0.28 \\
\hline $08441851+0346257$ & 581348807340055424 & 4581 & 4.46 & -0.55 & -0.93 & -0.93 & 6.96 & -0.05 \\
\hline 08443695-7241450 & $\ldots$ & 4821 & 4.43 & -0.70 & -0.05 & -0.05 & 7.69 & 0.35 \\
\hline 08444676-2845157 & 5642803457417047552 & 5316 & 3.39 & -0.21 & -0.80 & -0.80 & 7.42 & -0.01 \\
\hline 08445228-7438443 & 5216771215930182912 & 5489 & 4.06 & -1.00 & 0.46 & 0.46 & 7.89 & -0.06 \\
\hline 08454426-2141380 & 5702387126388268160 & 5002 & 1.96 & -1.68 & 0.30 & 0.38 & 7.13 & 0.32 \\
\hline 08460663-0426024 & 5761503056249343360 & 5559 & 3.30 & -0.14 & -0.35 & -0.33 & 7.97 & -0.07 \\
\hline $08472137+5830138$ & 1040480914362286592 & 5034 & 1.21 & -2.23 & -0.19 & 0.45 & 6.65 & 0.23 \\
\hline $08473213+0548147$ & 582973163970364416 & 5177 & 2.26 & -2.02 & 0.51 & 0.52 & 6.93 & 0.51 \\
\hline 08490347-6323398 & 5297691668265349120 & 6269 & 3.14 & -0.87 & 1.10 & 1.12 & 8.68 & -0.03 \\
\hline 08490462-6853235 & 5223489373715006976 & 5298 & 4.60 & -0.30 & 0.08 & 0.08 & 8.21 & 0.01 \\
\hline 08494240-3836003 & 5621994959343177856 & 5041 & 2.37 & -0.30 & -0.52 & -0.49 & 7.64 & 0.12 \\
\hline $08501165+4242063$ & 913730107265419136 & 4833 & 4.81 & -0.30 & -0.45 & -0.45 & 7.69 & 0.14 \\
\hline 08511780-2736072 & 5648942030830217344 & 5497 & 3.63 & -0.75 & 0.16 & 0.16 & 7.84 & 0.02 \\
\hline $08514213+1250467$ & 608133907024045952 & 5237 & 4.10 & -0.23 & 0.26 & 0.26 & 8.46 & 0.46 \\
\hline $08515469+5402598$ & 1029765967389921408 & 5598 & 4.60 & -0.32 & -0.08 & -0.08 & 8.03 & -0.06 \\
\hline
\end{tabular}


Table 3 (continued)

\begin{tabular}{|c|c|c|c|c|c|c|c|c|}
\hline $\begin{array}{l}\text { Star Name } \\
\text { (2MASS) }\end{array}$ & $\begin{array}{c}\text { Star Name } \\
\text { (Gaia EDR3) }\end{array}$ & $\begin{array}{l}T_{\text {eff }} \\
(\mathrm{K})\end{array}$ & $\begin{array}{l}\log g \\
(\operatorname{cgs})\end{array}$ & {$[\mathrm{Fe} / \mathrm{H}]$} & {$[\mathrm{C} / \mathrm{Fe}]$} & {$[\mathrm{C} / \mathrm{Fe}]_{c}$} & $A(\mathrm{C})_{c}$ & {$[\mathrm{Mg} / \mathrm{Fe}]$} \\
\hline 08523133-0804579 & 5750792365590211328 & 5060 & 4.72 & -1.30 & 0.55 & 0.55 & 7.68 & 0.20 \\
\hline $08524186+6924318$ & 1118241228535948672 & 5388 & 4.57 & -0.01 & -0.50 & -0.50 & 7.92 & -0.02 \\
\hline $08525722+6423125$ & 1044196095432573952 & 5274 & 4.01 & -0.68 & 0.09 & 0.09 & 7.84 & 0.22 \\
\hline $08533398+1644112$ & 611494731817426560 & 4883 & 4.18 & -1.34 & 0.64 & 0.64 & 7.73 & -0.02 \\
\hline 08543649-4744205 & 5328756032854574592 & 6680 & 1.12 & -0.31 & 1.83 & 1.83 & 9.95 & $\ldots$ \\
\hline 08544986-1159089 & 5736694942894396672 & 5382 & 3.16 & -1.43 & 0.35 & 0.36 & 7.35 & -0.09 \\
\hline 08554225-6955045 & 5222449162695490432 & 5190 & 1.46 & -2.28 & 0.05 & 0.54 & 6.70 & 0.20 \\
\hline 08565578-7603590 & 5215675105917524608 & 4614 & 3.18 & -0.43 & -0.59 & -0.57 & 7.43 & 0.19 \\
\hline $08571123+0040261$ & 576743880843785088 & 5069 & 4.14 & -0.34 & 0.17 & 0.17 & 8.26 & 0.47 \\
\hline 08571600-6556117 & 5296305287178801280 & 5297 & 2.27 & -2.45 & 0.56 & 0.57 & 6.54 & 0.00 \\
\hline 09002061-2220490 & 5654811563195280512 & 5599 & 3.80 & -1.06 & 0.85 & 0.85 & 8.22 & -0.11 \\
\hline 09002174-3342353 & 5627698985144472448 & 5822 & 3.55 & -0.74 & 0.25 & 0.25 & 7.94 & -0.14 \\
\hline 09012132-7055369 & 5222157448519411584 & 5339 & 4.69 & -0.76 & 0.25 & 0.25 & 7.92 & 0.13 \\
\hline 09023125-0810363 & 5755853112670730112 & 4985 & 4.71 & -0.20 & -0.12 & -0.12 & 8.11 & 0.14 \\
\hline 09030506-2047415 & 5656156368995636608 & 4995 & 1.74 & -2.07 & 0.42 & 0.66 & 7.03 & 0.13 \\
\hline 09030861-0427115 & 5759379998080221824 & 5639 & 3.51 & -0.98 & 0.09 & 0.09 & 7.54 & 0.01 \\
\hline 09040262-0523492 & 5759068836288541696 & 4842 & 1.93 & -1.50 & 0.11 & 0.21 & 7.14 & 0.33 \\
\hline $09043992+0733498$ & 584226980887837824 & 5064 & 1.63 & -2.55 & -0.47 & -0.11 & 5.77 & 0.52 \\
\hline 09050894-2050426 & 5656143484093610368 & 4900 & 2.54 & -0.45 & -0.72 & -0.69 & 7.29 & 0.33 \\
\hline $09065688+5836407$ & 1036942720302654080 & 5298 & 4.32 & -1.09 & 0.22 & 0.22 & 7.56 & -0.07 \\
\hline 09073395-0340147 & 5759902820154063744 & 4949 & 4.22 & 0.03 & -0.42 & -0.42 & 8.04 & -0.06 \\
\hline 09090697-0324211 & 5759936900718820864 & 5042 & 1.61 & -2.32 & 0.62 & 0.93 & 7.04 & 0.22 \\
\hline 09093944-2006527 & 5679552155368264704 & 5409 & 4.67 & -0.49 & 0.24 & 0.24 & 8.19 & 0.03 \\
\hline $09102690+6033156$ & 1039441978952648064 & 4671 & 2.10 & -1.05 & -0.18 & -0.12 & 7.26 & $\ldots$ \\
\hline $09103058+6419220$ & 1043675820274039040 & 4848 & 1.55 & -2.25 & 0.00 & 0.45 & 6.63 & 0.46 \\
\hline 09104309-1444185 & 5731383034718059520 & 4887 & 2.25 & -3.41 & 0.08 & 0.09 & 5.11 & -0.02 \\
\hline 09110582-2014595 & 5679493090978018304 & 5243 & 4.32 & -0.14 & 0.12 & 0.12 & 8.41 & -0.02 \\
\hline $09110758+0217287$ & 3843821216808928512 & 5281 & 4.60 & -0.44 & 0.07 & 0.07 & 8.07 & 0.14 \\
\hline $09114163+1017524$ & 591768294919763072 & 4925 & 1.92 & -1.59 & 0.06 & 0.18 & 7.03 & 0.28 \\
\hline 09122631-0037340 & 3842047846288227712 & 5085 & 4.22 & -0.02 & 0.04 & 0.04 & 8.46 & -0.06 \\
\hline $09123863+6218061$ & 1040178033268005504 & 5497 & 4.81 & -0.63 & 0.12 & 0.12 & 7.92 & 0.34 \\
\hline 09131573-1716172 & 5682721055254568576 & 4949 & 1.56 & -2.52 & 0.01 & 0.44 & 6.35 & 0.22 \\
\hline 09152673-0018170 & 3842238950857112064 & 4841 & 2.65 & -0.38 & -0.95 & -0.92 & 7.13 & 0.15 \\
\hline $09155397+1212314$ & 593696288558989312 & 5776 & 3.50 & -1.25 & 0.72 & 0.72 & 7.90 & 0.36 \\
\hline $09161100+0140496$ & 3845027557158758144 & 4507 & 4.22 & -1.25 & -0.87 & -0.87 & 6.30 & 0.50 \\
\hline 09164357-0512382 & 5758526021141799552 & 4796 & 1.14 & -2.20 & -0.41 & 0.27 & 6.50 & 0.20 \\
\hline 09171078-6147067 & 5298830659236242688 & 5057 & 1.83 & -2.37 & 0.63 & 0.80 & 6.86 & -0.02 \\
\hline 09172903-1021252 & 5742681753643854464 & 4593 & 4.73 & -0.54 & -0.28 & -0.28 & 7.61 & 0.14 \\
\hline 09180855-0852126 & 5743138252832504576 & 5099 & 2.20 & -1.99 & 0.25 & 0.26 & 6.69 & 0.21 \\
\hline $09184848+0713360$ & 586821454667385344 & 5106 & 1.87 & -2.07 & -0.13 & 0.02 & 6.38 & 0.39 \\
\hline $09185431+1211488$ & 593613206711676800 & 4746 & 4.73 & -0.65 & -0.13 & -0.13 & 7.65 & 0.33 \\
\hline 09201726-0554052 & 5746372328846303360 & 4736 & 4.58 & -1.00 & 0.46 & 0.46 & 7.89 & 0.03 \\
\hline
\end{tabular}


Table 3 (continued)

\begin{tabular}{|c|c|c|c|c|c|c|c|c|}
\hline $\begin{array}{l}\text { Star Name } \\
\text { (2MASS) }\end{array}$ & $\begin{array}{c}\text { Star Name } \\
\text { (Gaia EDR3) }\end{array}$ & $\begin{array}{l}T_{\text {eff }} \\
(\mathrm{K})\end{array}$ & $\begin{array}{l}\log g \\
(\operatorname{cgs})\end{array}$ & {$[\mathrm{Fe} / \mathrm{H}]$} & {$[\mathrm{C} / \mathrm{Fe}]$} & {$[\mathrm{C} / \mathrm{Fe}]_{c}$} & $A(\mathrm{C})_{c}$ & {$[\mathrm{Mg} / \mathrm{Fe}]$} \\
\hline $09205040+1820344$ & 632540488180370176 & 5319 & 3.95 & -1.09 & 0.24 & 0.24 & 7.58 & -0.05 \\
\hline $09214717+6219538$ & 1040038326571016704 & 4929 & 1.60 & -1.90 & 0.14 & 0.46 & 6.99 & 0.47 \\
\hline $09220002+0957599$ & 592248296169483264 & 4635 & 3.78 & -1.70 & 0.63 & 0.63 & 7.36 & -0.04 \\
\hline $09221426+5824580$ & 1038229698662764160 & 5305 & 2.61 & -1.96 & 0.36 & 0.37 & 6.84 & 0.22 \\
\hline 09221563-7701423 & 5203340376458000128 & 5110 & 2.11 & -1.81 & 0.90 & 0.93 & 7.55 & 0.11 \\
\hline 09222115-4317486 & 5424356193758211456 & 5713 & 3.73 & -0.04 & -0.44 & -0.44 & 7.95 & 0.04 \\
\hline $09230164+5819465$ & 1026218874158310272 & 4471 & 4.23 & -1.24 & -0.35 & -0.35 & 6.84 & -0.08 \\
\hline $09270301+4204494$ & 814375831397158144 & 4950 & 1.92 & -1.92 & 0.13 & 0.25 & 6.76 & 0.42 \\
\hline $09270844+1544438$ & 630694854833847168 & 4983 & 1.71 & -2.32 & 0.30 & 0.61 & 6.73 & 0.50 \\
\hline 09282183-3930462 & 5429709754532474880 & 5544 & 2.47 & -0.55 & 0.36 & 0.39 & 8.27 & $\ldots$ \\
\hline $09285510+1543302$ & 619055111209080832 & 5414 & 4.40 & -0.22 & 0.06 & 0.06 & 8.27 & 0.01 \\
\hline $09290292+3549540$ & 798505274404072832 & 5512 & 3.73 & -0.15 & -0.51 & -0.51 & 7.78 & -0.09 \\
\hline 09291542-1847544 & 5678392273680123520 & 5542 & 3.55 & -1.01 & 0.63 & 0.63 & 8.05 & -0.06 \\
\hline $09301030+0243320$ & 3844656021013252992 & 4844 & 1.26 & -2.49 & 0.15 & 0.72 & 6.66 & 0.46 \\
\hline 09304755-3932097 & 5426696130598924416 & 5167 & 4.53 & -0.47 & 0.19 & 0.19 & 8.16 & 0.41 \\
\hline $09304964+8016095$ & 1144494542550685824 & 4752 & 4.53 & -0.97 & 0.21 & 0.21 & 7.66 & -0.04 \\
\hline $09313142+7905121$ & 1132002131953311232 & 5111 & 4.76 & -0.75 & 0.20 & 0.20 & 7.87 & 0.44 \\
\hline $09315651+2335316$ & 644413044801144064 & 4989 & 4.77 & -0.86 & 0.20 & 0.20 & 7.77 & 0.13 \\
\hline $09325648+1823093$ & 633101995024420480 & 5120 & 1.93 & -2.03 & -0.30 & -0.19 & 6.21 & 0.46 \\
\hline 09341289-8700397 & 5189077198087438208 & 5200 & 4.07 & -1.19 & 0.51 & 0.51 & 7.75 & 0.02 \\
\hline $09343649+1036027$ & 589530578303563648 & 5261 & 2.65 & -2.25 & 0.55 & 0.56 & 6.75 & 0.42 \\
\hline $09344298+0353574$ & 3851012229012923264 & 4653 & 0.86 & -2.29 & -0.48 & 0.27 & 6.41 & 0.53 \\
\hline $09345116+1214576$ & 614001725703023488 & 4718 & 4.15 & -0.68 & -0.17 & -0.17 & 7.58 & 0.50 \\
\hline $09372216+0925058$ & 588581463545772928 & 5285 & 3.33 & -0.79 & 0.06 & 0.06 & 7.70 & 0.37 \\
\hline $09375748+2559307$ & 645906319030740864 & 4835 & 4.54 & -0.93 & 0.17 & 0.17 & 7.67 & 0.18 \\
\hline $09382460+4205266$ & 813812915803635712 & 4771 & 2.47 & -0.69 & 0.23 & 0.26 & 8.00 & -0.13 \\
\hline $09383941+1227080$ & 614063912534411136 & 5129 & 2.51 & -1.37 & 0.24 & 0.26 & 7.33 & 0.34 \\
\hline $09384661+7833575$ & 1131785214629423488 & 5189 & 2.33 & -2.19 & 0.45 & 0.46 & 6.70 & 0.41 \\
\hline $09391251+3445386$ & 797731523158972416 & 4819 & 2.46 & -0.33 & -0.72 & -0.69 & 7.42 & 0.07 \\
\hline $09403979+2921557$ & 696431047286005632 & 5386 & 4.50 & -0.35 & -0.17 & -0.17 & 7.92 & 0.05 \\
\hline $09455700+2135550$ & 640001361769756032 & 4830 & 4.55 & -0.97 & 0.19 & 0.19 & 7.65 & 0.15 \\
\hline $09472261+3354159$ & 794484841418902912 & 5261 & 4.25 & -0.19 & -0.48 & -0.48 & 7.77 & -0.03 \\
\hline 09473545-2638039 & 5658256784099611008 & 6163 & 4.16 & -0.46 & 0.08 & 0.08 & 8.06 & -0.04 \\
\hline 09474496-7045530 & 5242578991517893120 & 5064 & 1.84 & -2.18 & 0.51 & 0.68 & 6.94 & -0.20 \\
\hline $09485248+1837465$ & 627201117981349888 & 5121 & 2.13 & -1.99 & 0.00 & 0.02 & 6.46 & 0.33 \\
\hline 09485477-3811139 & 5432401118477539456 & 6795 & 3.47 & -0.34 & 1.13 & 1.13 & 9.22 & 0.26 \\
\hline $09491888+3201060$ & 793274382192849664 & 4688 & 0.87 & -2.29 & -0.26 & 0.47 & 6.61 & 0.41 \\
\hline 09501353-7925417 & 5202088268933375232 & 4884 & 1.11 & -2.39 & -0.25 & 0.45 & 6.49 & 0.23 \\
\hline $09502173+3716070$ & 799953365577763712 & 5092 & 3.07 & -0.44 & -0.55 & -0.54 & 7.45 & 0.02 \\
\hline 09502215-6930058 & 5243501550494934400 & 5400 & 4.08 & 0.18 & -0.59 & -0.59 & 8.02 & -0.06 \\
\hline 09513068-3848520 & 5432133353036744448 & 5047 & 4.59 & -1.09 & 0.57 & 0.57 & 7.91 & -0.12 \\
\hline $09513210+3330203$ & 793674978087183616 & 4870 & 4.68 & -0.98 & 0.27 & 0.27 & 7.72 & 0.24 \\
\hline
\end{tabular}


Table 3 (continued)

\begin{tabular}{|c|c|c|c|c|c|c|c|c|}
\hline $\begin{array}{l}\text { Star Name } \\
\text { (2MASS) }\end{array}$ & $\begin{array}{c}\text { Star Name } \\
\text { (Gaia EDR3) }\end{array}$ & $\begin{array}{l}T_{\text {eff }} \\
(\mathrm{K})\end{array}$ & $\begin{array}{l}\log g \\
(\operatorname{cgs})\end{array}$ & {$[\mathrm{Fe} / \mathrm{H}]$} & {$[\mathrm{C} / \mathrm{Fe}]$} & {$[\mathrm{C} / \mathrm{Fe}]_{c}$} & $A(\mathrm{C})_{c}$ & {$[\mathrm{Mg} / \mathrm{Fe}]$} \\
\hline 09532759-2717101 & 5657259969431486336 & 4752 & 4.12 & -0.62 & -0.20 & -0.20 & 7.61 & 0.21 \\
\hline $09535089+3329284$ & 794978139884594560 & 5138 & 1.54 & -2.10 & 0.03 & 0.46 & 6.80 & 0.35 \\
\hline $09541478+2735210$ & 647086477259746176 & 5134 & 4.09 & -1.31 & 0.55 & 0.55 & 7.67 & -0.11 \\
\hline $09545564+6756128$ & 1069803755602231168 & 4799 & 1.16 & -1.91 & -0.33 & 0.26 & 6.78 & 0.50 \\
\hline $09553934+4319179$ & 808168980883073792 & 5705 & 3.91 & -1.58 & 0.92 & 0.92 & 7.78 & -0.02 \\
\hline 09555639-2403072 & 5660496214405409152 & 4991 & 2.01 & -1.99 & 0.80 & 0.86 & 7.30 & 0.25 \\
\hline 09560894-5924282 & 5257538328273646336 & 5382 & 3.04 & -0.48 & 0.00 & 0.01 & 7.96 & 0.11 \\
\hline $09574469+1400136$ & 615228711960558464 & 4846 & 4.63 & -0.89 & 0.15 & 0.15 & 7.69 & 0.16 \\
\hline 09580008-4318227 & 5418253457547492096 & 5252 & 3.20 & -1.54 & 0.76 & 0.77 & 7.66 & 0.07 \\
\hline $09580109+2933087$ & 743582087319504640 & 4937 & 4.61 & -0.57 & 0.03 & 0.03 & 7.89 & 0.04 \\
\hline $09593411+3708378$ & 796772718958610304 & 4526 & 4.29 & -1.55 & 0.40 & 0.40 & 7.29 & -0.15 \\
\hline $09594843+7246373$ & 1125921282896321792 & 5390 & 2.14 & -1.64 & 0.00 & 0.02 & 6.81 & 0.43 \\
\hline $10003325-2537597$ & 5658983939239396352 & 5185 & 4.54 & -0.78 & 0.24 & 0.24 & 7.89 & 0.15 \\
\hline $10020621-1554291$ & 5673966327061493760 & 4695 & 4.66 & -1.61 & 0.65 & 0.65 & 7.48 & $\ldots$ \\
\hline $10030615+7054115$ & 1071492949060164992 & 5078 & 1.53 & -2.06 & -0.10 & 0.35 & 6.72 & 0.34 \\
\hline 10033379-2329108 & 5665883610926876672 & 4981 & 4.52 & -0.72 & 0.28 & 0.28 & 7.99 & 0.08 \\
\hline $10045623-4228179$ & 5418445670219076224 & 5373 & 1.90 & -1.80 & 0.27 & 0.40 & 7.03 & 0.04 \\
\hline $10052247+3945408$ & 803621576887555840 & 4927 & 1.57 & -2.25 & 0.08 & 0.51 & 6.69 & 0.40 \\
\hline 10053264-1827501 & 5672581221584085504 & 4992 & 4.61 & -1.43 & 0.79 & 0.79 & 7.78 & 0.16 \\
\hline $10063882+2403476$ & 630450488374469120 & 4816 & 1.28 & -2.87 & -0.41 & 0.21 & 5.76 & 0.42 \\
\hline 10075999-2736413 & 5465659970825328896 & $\ldots$ & $\ldots$ & $\ldots$ & $\ldots$ & $\ldots$ & $\ldots$ & $\ldots$ \\
\hline $10085157-3236041$ & 5459310600414841856 & 4889 & 3.53 & -0.29 & -0.35 & -0.35 & 7.79 & -0.13 \\
\hline $10100365+2227088$ & 629233049829242368 & 4670 & 4.75 & -0.73 & -0.05 & -0.05 & 7.65 & 0.33 \\
\hline 10111521-6620282 & 5245373292941453952 & 5533 & 4.19 & 0.00 & -0.48 & -0.48 & 7.95 & -0.09 \\
\hline 10115056-4309343 & 5415268253418659200 & 5533 & 3.72 & 0.08 & -0.36 & -0.36 & 8.15 & -0.08 \\
\hline $10115917+5502205$ & 852773418843119616 & 4808 & 4.75 & -0.80 & 0.14 & 0.14 & 7.77 & 0.06 \\
\hline 10121964-3221347 & 5459373341297312384 & 4640 & 0.88 & -2.87 & -0.31 & 0.43 & 5.99 & 0.10 \\
\hline $10124027-2249482$ & 5666123373181152000 & 4693 & 4.56 & -0.80 & -0.40 & -0.40 & 7.23 & 0.25 \\
\hline $10124666+4624575$ & 810408381126766592 & 5069 & 2.15 & -2.16 & 0.28 & 0.29 & 6.56 & 0.31 \\
\hline $10143521+2324515$ & 725459455633859840 & 4830 & 1.98 & -1.49 & -0.17 & -0.08 & 6.86 & $\ldots$ \\
\hline $10151784+5551061$ & 853205526912187264 & 4815 & 4.78 & -0.45 & -0.14 & -0.14 & 7.84 & 0.15 \\
\hline 10152469-1057352 & 3767574556484311424 & 4624 & 0.84 & -2.42 & -0.11 & 0.59 & 6.60 & 0.12 \\
\hline $10152705+6456284$ & 1053650967717923072 & 4944 & 4.80 & -0.19 & -0.26 & -0.26 & 7.98 & -0.01 \\
\hline $10160573+6533551$ & 1065697491989952896 & 5462 & 2.95 & -0.92 & 0.45 & 0.47 & 7.98 & 0.56 \\
\hline $10171294+4654224$ & 810350480671638784 & 5152 & 4.79 & -0.53 & 0.19 & 0.19 & 8.09 & 0.00 \\
\hline $10193367+7257464$ & 1077961238527864704 & 5033 & 4.57 & -0.70 & 0.28 & 0.28 & 8.01 & -0.05 \\
\hline $10203763+7221398$ & 1077708213414112128 & 5360 & 3.90 & -0.54 & 0.17 & 0.17 & 8.07 & -0.05 \\
\hline $10211155+4557314$ & 809444964126986240 & 5168 & 4.38 & -0.43 & 0.31 & 0.31 & 8.31 & 0.27 \\
\hline $10213675+7058455$ & 1076692848785487104 & 5346 & 4.23 & -0.56 & 0.44 & 0.44 & 8.31 & 0.04 \\
\hline $10221281-4123416$ & 5416543996142159488 & 5119 & 1.71 & -2.30 & 0.08 & 0.41 & 6.53 & 0.17 \\
\hline $10244576-7355423$ & 5229049672736749184 & 5171 & 4.70 & -0.08 & -0.02 & -0.02 & 8.34 & -0.04 \\
\hline $10250250+7723238$ & 1128041244394048000 & 5254 & 4.61 & -0.43 & -0.07 & -0.07 & 7.93 & 0.10 \\
\hline
\end{tabular}


Table 3 (continued)

\begin{tabular}{|c|c|c|c|c|c|c|c|c|}
\hline $\begin{array}{l}\text { Star Name } \\
(2 \mathrm{MASS})\end{array}$ & $\begin{array}{c}\text { Star Name } \\
\text { (Gaia EDR3) }\end{array}$ & $\begin{array}{l}T_{\text {eff }} \\
(\mathrm{K})\end{array}$ & $\begin{array}{l}\log g \\
(\operatorname{cgs})\end{array}$ & {$[\mathrm{Fe} / \mathrm{H}]$} & {$[\mathrm{C} / \mathrm{Fe}]$} & {$[\mathrm{C} / \mathrm{Fe}]_{c}$} & $A(\mathrm{C})_{c}$ & {$[\mathrm{Mg} / \mathrm{Fe}]$} \\
\hline $10263327-2231158$ & 5475397761196528896 & 5025 & 2.12 & -1.48 & 0.07 & 0.10 & 7.06 & 0.30 \\
\hline $10280894+4658364$ & 833602269677094272 & 4569 & 0.61 & -2.91 & -0.12 & 0.61 & 6.13 & 0.39 \\
\hline 10281330-2946530 & 5461386169127203200 & 4675 & 2.30 & -0.04 & -0.92 & -0.89 & 7.50 & -0.17 \\
\hline 10295444-0801026 & 3774466329726812672 & 5158 & 1.61 & -2.69 & 0.18 & 0.53 & 6.27 & -0.14 \\
\hline $10340832-7354406$ & 5229004382300835840 & 5196 & 4.57 & -0.02 & 0.18 & 0.18 & 8.60 & -0.10 \\
\hline $10350987-1416284$ & 3750801231724757760 & 5253 & 2.21 & -2.48 & 0.76 & 0.77 & 6.72 & 0.10 \\
\hline $10352666+4804049$ & 834056058741414400 & 5404 & 3.20 & -1.51 & 0.57 & 0.58 & 7.50 & 0.58 \\
\hline 10363889-1917043 & 3554709655102839552 & 5700 & 3.75 & -1.46 & 0.73 & 0.73 & 7.70 & 0.02 \\
\hline $10371075-1056303$ & 3760861312146553216 & 4941 & 3.94 & -0.85 & -0.26 & -0.26 & 7.32 & 0.15 \\
\hline $10375744+4851237$ & 834186007272278016 & 4842 & 4.72 & -0.80 & 0.05 & 0.05 & 7.68 & 0.16 \\
\hline $10382409-5248250$ & 5354554767533822336 & 4911 & 1.41 & -2.35 & 0.25 & 0.72 & 6.80 & 0.14 \\
\hline $10383538+4953297$ & 835062146240700160 & 4931 & 1.60 & -2.73 & 0.12 & 0.47 & 6.17 & 0.37 \\
\hline $10385482-0518037$ & 3776786466765131520 & 4875 & 1.47 & -2.25 & 0.05 & 0.53 & 6.71 & 0.16 \\
\hline $10391495+7348283$ & 1078279478424242432 & 5280 & 4.69 & -1.03 & 0.31 & 0.31 & 7.70 & 0.36 \\
\hline $10391566+7340387$ & 1078266108189870592 & 5347 & 4.54 & -0.54 & 0.06 & 0.06 & 7.94 & 0.16 \\
\hline $10394895+3459288$ & 750368410525129728 & 4728 & 1.33 & -2.55 & -0.35 & 0.25 & 6.13 & 0.49 \\
\hline $10420484+0246381$ & 3857349504797962496 & 4955 & 1.65 & -2.12 & 0.05 & 0.40 & 6.70 & 0.29 \\
\hline $10425611-6355510$ & 5239913019452422784 & 5573 & 2.83 & -0.31 & 0.34 & 0.37 & 8.49 & 0.40 \\
\hline $10430163-0230226$ & 3802519784793984000 & 5035 & 4.68 & -0.33 & -0.05 & -0.05 & 8.06 & 0.04 \\
\hline $10430365-0643033$ & 3776307354572694272 & 5363 & 3.39 & -1.19 & 0.56 & 0.56 & 7.80 & 0.33 \\
\hline $10432543+7515589$ & 1126646406518426752 & 4622 & 4.57 & -0.50 & -0.60 & -0.60 & 7.33 & 0.04 \\
\hline $10440416+7616213$ & 1128438924005715456 & 5257 & 4.79 & -0.65 & 0.17 & 0.17 & 7.95 & 0.03 \\
\hline $10440597-0853008$ & 3762337131628855680 & 4953 & 4.76 & -0.61 & -0.38 & -0.38 & 7.44 & 0.25 \\
\hline $10453280+0345108$ & 3857542022411847040 & 4812 & 1.33 & -2.31 & -0.31 & 0.30 & 6.42 & 0.48 \\
\hline 10493965-1719329 & 3556866862556915072 & 5085 & 2.03 & -2.11 & 0.47 & 0.52 & 6.85 & 0.04 \\
\hline $10502726-2300446$ & 3549234430794364160 & 5365 & 3.37 & -1.37 & 0.11 & 0.11 & 7.17 & 0.36 \\
\hline $10511274-0817026$ & 3763102327298064128 & 4975 & 4.73 & -0.54 & 0.21 & 0.21 & 8.10 & 0.26 \\
\hline $10521859+0528265$ & 3864195618243548032 & 4767 & 4.78 & -0.74 & -0.12 & -0.12 & 7.57 & 0.41 \\
\hline $10530608-2253102$ & 3549276036141218176 & 4796 & 1.21 & -1.91 & -0.33 & 0.24 & 6.76 & 0.28 \\
\hline $10531802-0055302$ & 3803159838000339200 & 4801 & 1.62 & -1.76 & -0.18 & 0.16 & 6.84 & 0.18 \\
\hline $10540939-5233263$ & 5359711992455305344 & 4912 & 4.19 & -0.25 & -0.34 & -0.34 & 7.84 & -0.02 \\
\hline $10541225+0549178$ & 3864303022490267136 & 5193 & 2.61 & -2.94 & 0.45 & 0.46 & 5.95 & 0.52 \\
\hline $10543311+0528128$ & 3864140775805950208 & 5159 & 2.45 & -3.01 & 0.60 & 0.61 & 6.03 & 0.56 \\
\hline $10554570-1654038$ & 3556496876894238464 & 4958 & 1.81 & -1.63 & 0.24 & 0.41 & 7.20 & 0.18 \\
\hline $10563216-0555480$ & 3764649748179457280 & 5053 & 4.72 & -1.45 & 0.58 & 0.58 & 7.57 & 0.25 \\
\hline $10570875-5207081$ & 5359563558390131968 & 4594 & 4.07 & -0.11 & -0.61 & -0.61 & 7.72 & -0.04 \\
\hline $10582963+0011130$ & 3804842056430901120 & 5263 & 2.09 & -3.21 & 0.72 & 0.73 & 5.94 & 0.28 \\
\hline $10590836-6924479$ & 5231719699295439488 & 5527 & 2.58 & -2.45 & 2.16 & 2.18 & 8.16 & $\ldots$ \\
\hline $11000776+4630029$ & 783425712959288576 & 4953 & 1.51 & -1.83 & -0.01 & 0.38 & 6.98 & 0.41 \\
\hline 11004069-1102492 & 3758563126686411776 & 4891 & 1.47 & -2.97 & 0.40 & 0.83 & 6.29 & 0.05 \\
\hline $11010971+1310060$ & 3967986487953405312 & 5084 & 2.17 & -1.85 & 0.35 & 0.36 & 6.94 & 0.46 \\
\hline 11023256-0636095 & 3787611983373749120 & 4762 & 4.88 & -1.34 & 0.65 & 0.65 & 7.74 & 0.22 \\
\hline
\end{tabular}


Table 3 (continued)

\begin{tabular}{|c|c|c|c|c|c|c|c|c|}
\hline $\begin{array}{l}\text { Star Name } \\
\text { (2MASS) }\end{array}$ & $\begin{array}{c}\text { Star Name } \\
\text { (Gaia EDR3) }\end{array}$ & $\begin{array}{l}T_{\text {eff }} \\
(\mathrm{K})\end{array}$ & $\begin{array}{l}\log g \\
(\operatorname{cgs})\end{array}$ & {$[\mathrm{Fe} / \mathrm{H}]$} & {$[\mathrm{C} / \mathrm{Fe}]$} & {$[\mathrm{C} / \mathrm{Fe}]_{c}$} & $A(\mathrm{C})_{c}$ & {$[\mathrm{Mg} / \mathrm{Fe}]$} \\
\hline $11023598+0102539$ & 3805298933576500480 & 5159 & 2.45 & -2.46 & 0.54 & 0.55 & 6.52 & 0.08 \\
\hline $11051574-4752557$ & 5362354531210538624 & 5713 & 3.54 & -1.36 & 0.78 & 0.78 & 7.84 & -0.13 \\
\hline 11051929-1329347 & 3564247918473953792 & 4774 & 4.44 & -0.92 & 0.22 & 0.22 & 7.73 & 0.04 \\
\hline $11064962-5323068$ & 5347385401964090240 & 7311 & 3.53 & -2.57 & 3.08 & 3.09 & 8.95 & $\ldots$ \\
\hline $11065789-4643237$ & 5386525267120422912 & 4997 & 4.10 & -1.39 & 0.79 & 0.79 & 7.83 & -0.10 \\
\hline $11065939-4920517$ & 5362109546275295872 & 6300 & 3.13 & -1.17 & 1.45 & 1.47 & 8.73 & -0.01 \\
\hline $11090121+0754418$ & 3818459160048340352 & 4863 & 1.46 & -3.04 & -0.02 & 0.43 & 5.82 & 0.55 \\
\hline 11092807-0242021 & 3791025112280181248 & 5656 & 4.19 & -0.18 & 0.27 & 0.27 & 8.52 & -0.02 \\
\hline $11093391+2327348$ & 3995259156621469440 & 4923 & 4.81 & -0.79 & 0.16 & 0.16 & 7.81 & 0.14 \\
\hline $11112337+6943386$ & 1074121743923049344 & 4497 & 4.51 & -0.51 & -1.16 & -1.16 & 6.76 & -0.05 \\
\hline $11132240-0910000$ & 3783099076553128064 & 5261 & 4.56 & -0.44 & 0.24 & 0.24 & 8.22 & 0.00 \\
\hline $11133490+1015249$ & 3963264914441096320 & 5074 & 1.67 & -2.47 & -0.26 & 0.08 & 6.05 & 0.36 \\
\hline $11144345-1133225$ & 3566319673258222208 & 4850 & 1.34 & -2.49 & -0.06 & 0.52 & 6.46 & 0.19 \\
\hline $11172319-5030507$ & 5349769001420934784 & 4998 & 1.55 & -1.82 & 0.04 & 0.39 & 7.01 & 0.30 \\
\hline $11213850+3100109$ & 4023379441379492096 & 4658 & 2.59 & -0.53 & -0.30 & -0.27 & 7.62 & 0.21 \\
\hline $11215118-4555200$ & 5376213596097250176 & 5248 & 4.78 & -0.53 & 0.17 & 0.17 & 8.08 & 0.18 \\
\hline $11232110+6118098$ & 862722319742958592 & 5091 & 2.18 & -2.16 & 0.40 & 0.41 & 6.67 & 0.26 \\
\hline $11235327+0025363$ & 3797935886458318336 & 4663 & 1.02 & -2.07 & -0.27 & 0.39 & 6.75 & 0.37 \\
\hline $11242364-0642128$ & 3785352182036872320 & 4939 & 1.50 & -1.98 & 0.06 & 0.47 & 6.92 & 0.06 \\
\hline 11245109-0118132 & 3796858154608999424 & 4852 & 1.83 & -3.26 & -0.64 & -0.57 & 4.60 & .. \\
\hline $11245186+8132096$ & 1133615145575572992 & 5113 & 3.27 & -1.38 & 0.21 & 0.22 & 7.27 & 0.28 \\
\hline $11251752+4810412$ & 789033325338645888 & 5411 & 4.79 & -0.71 & 0.13 & 0.13 & 7.85 & 0.35 \\
\hline 11252946-0035203 & 3797041055791986048 & 5186 & 4.14 & -1.28 & 0.54 & 0.54 & 7.69 & 0.09 \\
\hline $11255206+5922341$ & 859132616140394880 & 5056 & 3.42 & -0.36 & -0.55 & -0.55 & 7.52 & 0.04 \\
\hline 11260849-0718091 & 3591618782978505472 & 5290 & 3.72 & -1.91 & 0.65 & 0.65 & 7.17 & 0.21 \\
\hline $11264720+2321085$ & 3992675231281619584 & 4750 & 4.30 & -1.50 & 0.61 & 0.61 & 7.54 & -0.03 \\
\hline $11284462+1159546$ & 3917369061419260928 & 4872 & 4.79 & -0.72 & 0.01 & 0.01 & 7.72 & 0.08 \\
\hline $11293922+6718355$ & 1057741219692489856 & 4944 & 4.94 & -0.93 & -0.07 & -0.07 & 7.43 & 0.41 \\
\hline $11320178+4540156$ & 784645621097082624 & 5092 & 1.69 & -2.14 & -0.02 & 0.31 & 6.61 & 0.12 \\
\hline $11322424+7217439$ & 1075031310621259776 & 4829 & 4.74 & -0.54 & -0.12 & -0.12 & 7.77 & 0.39 \\
\hline $11332470+1251440$ & 3917497017084939776 & 4785 & 1.44 & -1.91 & -0.13 & 0.32 & 6.84 & 0.39 \\
\hline $11343570+1932184$ & 3977488952772409856 & 4824 & 4.00 & -1.55 & 0.63 & 0.63 & 7.52 & -0.02 \\
\hline $11344124+5314269$ & 841082728317714048 & 5222 & 1.99 & -2.42 & 0.42 & 0.49 & 6.50 & 0.39 \\
\hline 11351928-3430096 & 3477077051780069632 & 4687 & 4.50 & -0.62 & -0.56 & -0.56 & 7.25 & 0.09 \\
\hline $11364104+3220057$ & 4024512758694334336 & 4684 & 4.10 & -0.70 & -0.34 & -0.34 & 7.39 & 0.54 \\
\hline $11372004+1845309$ & 3974320228980706816 & 4933 & 1.60 & -2.58 & -0.20 & 0.18 & 6.04 & 0.53 \\
\hline $11373800-5202251$ & 5345681227653768064 & 5161 & 1.75 & -2.49 & 0.72 & 0.93 & 6.87 & -0.20 \\
\hline $11381456+1534117$ & 3972403604119095808 & 4983 & 4.06 & -1.54 & 0.49 & 0.49 & 7.38 & -0.05 \\
\hline $11403298+6340440$ & 864134573709331584 & 4949 & 4.75 & -0.91 & 0.09 & 0.09 & 7.61 & 0.13 \\
\hline $11415054+5035361$ & 790901636102569472 & 5270 & 4.69 & -0.41 & 0.34 & 0.34 & 8.36 & 0.58 \\
\hline $11423344+0800282$ & 3910233647567741056 & 5203 & 2.60 & -2.73 & 0.36 & 0.37 & 6.08 & 0.17 \\
\hline $11444533-4644384$ & 5372404612941931392 & 5379 & 4.36 & -0.57 & 0.30 & 0.30 & 8.17 & 0.09 \\
\hline
\end{tabular}


Table 3 (continued)

\begin{tabular}{|c|c|c|c|c|c|c|c|c|}
\hline $\begin{array}{c}\text { Star Name } \\
\text { (2MASS) }\end{array}$ & $\begin{array}{c}\text { Star Name } \\
\text { (Gaia EDR3) }\end{array}$ & $\begin{array}{l}T_{\text {eff }} \\
(\mathrm{K})\end{array}$ & $\begin{array}{l}\log g \\
(\operatorname{cgs})\end{array}$ & {$[\mathrm{Fe} / \mathrm{H}]$} & {$[\mathrm{C} / \mathrm{Fe}]$} & {$[\mathrm{C} / \mathrm{Fe}]_{c}$} & $A(\mathrm{C})_{c}$ & {$[\mathrm{Mg} / \mathrm{Fe}]$} \\
\hline $11465844+4835156$ & 787263141676539648 & 5050 & 3.82 & -1.17 & 0.53 & 0.53 & 7.78 & -0.03 \\
\hline $11471027+0341265$ & 3895958344506982784 & 5095 & 2.05 & -2.62 & 0.48 & 0.51 & 6.33 & 0.23 \\
\hline $11483814+1952557$ & 3976143459777843968 & 4878 & 1.40 & -2.07 & -0.25 & 0.26 & 6.62 & 0.39 \\
\hline $11492027+3132066$ & 4021576517188258688 & 4927 & 4.81 & -0.75 & 0.01 & 0.01 & 7.70 & 0.13 \\
\hline $11505337-8036338$ & 5199459645869304704 & 5836 & 3.81 & -1.30 & 0.71 & 0.71 & 7.84 & 0.10 \\
\hline $11515346+8151152$ & 1133680021057772544 & 5208 & 2.02 & -2.09 & 0.05 & 0.10 & 6.43 & 0.16 \\
\hline $11515556-4738558$ & 5371554866553160960 & 4801 & 1.16 & -2.64 & 0.40 & 0.95 & 6.74 & -0.14 \\
\hline $11524486-4709547$ & 5377584485241041408 & 5183 & 2.60 & -1.34 & 0.29 & 0.31 & 7.40 & 0.21 \\
\hline $11541040-5208317$ & 5368942804889403520 & 5200 & 4.71 & -1.21 & 0.38 & 0.38 & 7.59 & 0.15 \\
\hline $11543955+7023508$ & 1061694681253422336 & 5079 & 3.75 & -1.28 & 0.19 & 0.19 & 7.34 & 0.24 \\
\hline $11545004+4749009$ & 786606630149557248 & 5440 & 4.33 & -0.70 & 0.21 & 0.21 & 7.95 & 0.01 \\
\hline $11564526+3145522$ & 4026626810316850048 & 4612 & 3.95 & -2.32 & 0.99 & 0.99 & 7.10 & 0.17 \\
\hline $11593802+2922115$ & 4008146360612154112 & 4669 & 0.78 & -2.39 & -0.30 & 0.44 & 6.48 & 0.48 \\
\hline 11595151-3905280 & 3459312659023799552 & 4890 & 4.49 & -0.94 & 0.41 & 0.41 & 7.89 & 0.07 \\
\hline $12001449+1641370$ & 3925430066494830336 & 4761 & 4.80 & -0.56 & -0.17 & -0.17 & 7.71 & 0.23 \\
\hline $12004306+2949116$ & 4008186046109996672 & 5007 & 1.47 & -2.17 & -0.14 & 0.35 & 6.61 & 0.46 \\
\hline 12005811-3929233 & 3459242084121356288 & 5126 & 4.63 & -0.76 & 0.93 & 0.93 & 8.61 & -0.03 \\
\hline $12022430+6255274$ & 1582997852929561600 & 4653 & 3.82 & -1.64 & 0.77 & 0.77 & 7.55 & 0.19 \\
\hline $12034594+3035029$ & 4014343925404883328 & 4915 & 4.68 & -0.97 & 0.26 & 0.26 & 7.72 & -0.03 \\
\hline $12052554-4408587$ & 6147464275054037120 & 5701 & 3.50 & -1.10 & 1.03 & 1.03 & 8.36 & 0.27 \\
\hline $12055188+1735548$ & 3925930962760630656 & 5424 & 4.60 & -0.68 & 0.02 & 0.02 & 7.77 & 0.33 \\
\hline $12070423-5345229$ & 6076898344653529344 & 5123 & 2.01 & -2.70 & 0.66 & 0.70 & 6.43 & 0.02 \\
\hline $12070444+7600223$ & 1692730414755815552 & 5151 & 4.76 & -0.75 & 0.31 & 0.31 & 7.99 & 0.14 \\
\hline $12074090+3519591$ & 4029760766348866944 & 5033 & 2.34 & -1.45 & 0.50 & 0.52 & 7.50 & -0.10 \\
\hline $12083074+3954084$ & 1536035581002935424 & 4798 & 1.40 & -2.63 & -0.09 & 0.44 & 6.24 & 0.47 \\
\hline $12094665+6450371$ & 1585030811273958400 & 4681 & 1.42 & -1.89 & -0.28 & 0.19 & 6.73 & 0.38 \\
\hline $12100140+4520564$ & 1539687570219614080 & 5080 & 1.89 & -2.53 & -0.20 & -0.09 & 5.81 & 0.33 \\
\hline $12104009+7032545$ & 1684079324125218944 & 5769 & 4.08 & -0.88 & 0.41 & 0.41 & 7.96 & 0.43 \\
\hline $12111339+2220415$ & 4001468785978496768 & 4848 & 4.81 & -0.93 & 0.09 & 0.09 & 7.59 & 0.23 \\
\hline 12132563-3800203 & 3461061226110222080 & 5632 & 4.72 & -0.67 & 0.85 & 0.85 & 8.60 & 0.57 \\
\hline $12143659-1517191$ & 3569366385619378432 & 4786 & 4.20 & -0.98 & 0.48 & 0.48 & 7.93 & -0.14 \\
\hline $12155841-3547529$ & 3461905891557813248 & 5249 & 4.63 & 0.00 & -0.07 & -0.07 & 8.37 & -0.05 \\
\hline $12161019+4524243$ & 1538985944361745536 & 4886 & 4.85 & -0.82 & 0.02 & 0.02 & 7.63 & 0.15 \\
\hline $12181234-3721065$ & 6151711177372668928 & 4967 & 1.90 & -1.73 & 0.35 & 0.47 & 7.17 & 0.35 \\
\hline $12214951+4244520$ & 1538031641283723008 & 4722 & 1.18 & -2.74 & -0.06 & 0.59 & 6.28 & 0.12 \\
\hline $12221869-3853368$ & 6150224495918526848 & 5195 & 2.30 & -2.88 & 0.60 & 0.61 & 6.16 & 0.28 \\
\hline $12240228-7202466$ & 5842561802238077952 & 6218 & 3.75 & -0.64 & 0.60 & 0.60 & 8.38 & -0.08 \\
\hline $12245683+7007242$ & 1683832243246834304 & 4998 & 3.39 & -0.30 & -0.67 & -0.67 & 7.47 & 0.05 \\
\hline $12261008+3926273$ & 1532300402563759360 & 5344 & 4.24 & -1.07 & 0.17 & 0.17 & 7.54 & 0.03 \\
\hline 12280652-3319179 & 3468446817511036160 & 5154 & 2.03 & -1.95 & 0.15 & 0.20 & 6.68 & 0.19 \\
\hline $12284069+1942295$ & 3948963287525544576 & 4982 & 1.91 & -2.69 & 0.07 & 0.15 & 5.89 & 0.32 \\
\hline $12293447-3233073$ & 3468579201286803200 & 5109 & 2.21 & -1.50 & 0.14 & 0.16 & 7.09 & 0.48 \\
\hline
\end{tabular}


Table 3 (continued)

\begin{tabular}{|c|c|c|c|c|c|c|c|c|}
\hline $\begin{array}{c}\text { Star Name } \\
(2 \mathrm{MASS})\end{array}$ & $\begin{array}{c}\text { Star Name } \\
\text { (Gaia EDR3) }\end{array}$ & $\begin{array}{l}T_{\text {eff }} \\
(\mathrm{K})\end{array}$ & $\begin{array}{l}\log g \\
(\operatorname{cgs})\end{array}$ & {$[\mathrm{Fe} / \mathrm{H}]$} & {$[\mathrm{C} / \mathrm{Fe}]$} & {$[\mathrm{C} / \mathrm{Fe}]_{c}$} & $A(\mathrm{C})_{c}$ & {$[\mathrm{Mg} / \mathrm{Fe}]$} \\
\hline $12334194+1952177$ & 3949121587136124160 & 4619 & 0.98 & -3.10 & -0.03 & 0.72 & 6.06 & 0.47 \\
\hline $12335935-3246361$ & 6160173770478603520 & 5429 & 3.46 & -1.76 & 0.63 & 0.63 & 7.31 & 0.30 \\
\hline $12335980-2944042$ & 3471719754389184384 & 5113 & 1.63 & -2.16 & 0.05 & 0.43 & 6.70 & 0.22 \\
\hline 12340964-3453438 & 6158168604865990016 & 4861 & 0.98 & -2.44 & 0.12 & 0.77 & 6.76 & -0.02 \\
\hline $12342913+4826025$ & 1543862488889757568 & 4967 & 2.02 & -1.81 & -0.09 & -0.04 & 6.57 & 0.19 \\
\hline $12353682+4854057$ & 1544253571429564800 & 4721 & 4.68 & -0.51 & -0.47 & -0.47 & 7.46 & 0.10 \\
\hline $12353793-4301524$ & 6145744883683553408 & 5029 & 2.60 & -0.13 & -0.51 & -0.48 & 7.82 & 0.16 \\
\hline $12360829-7040522$ & 5843251466600455808 & 6510 & 2.92 & -1.06 & 1.51 & 1.53 & 8.91 & 0.01 \\
\hline $12363406+4334497$ & 1540600409684268032 & 5188 & 4.38 & -0.01 & -0.37 & -0.37 & 8.05 & -0.04 \\
\hline $12382743-3400315$ & 6158315870705750912 & 5047 & 1.58 & -2.62 & 0.50 & 0.85 & 6.66 & 0.35 \\
\hline $12383506+2039384$ & 3949279882451077888 & 4475 & 4.55 & -1.32 & -0.47 & -0.47 & 6.65 & 0.23 \\
\hline $12392838+5038459$ & 1568524878294905088 & 4858 & 4.28 & -0.56 & 0.18 & 0.18 & 8.05 & 0.59 \\
\hline 12395834-3131208 & 6160731124090635520 & 5268 & 3.86 & 0.02 & -0.05 & -0.05 & 8.40 & -0.12 \\
\hline $12401578-7012252$ & 5843372485918189440 & 5705 & 3.33 & -0.29 & 0.53 & 0.55 & 8.69 & -0.08 \\
\hline $12413756-5412247$ & 6074478212169867904 & 5054 & 4.16 & -1.00 & 0.67 & 0.67 & 8.10 & 0.07 \\
\hline $12432213-5955445$ & 6056631317380990208 & 5403 & 3.49 & -0.37 & 0.06 & 0.06 & 8.12 & 0.01 \\
\hline 12433114-8753309 & 5765813554148627328 & 4632 & 2.53 & -0.17 & -1.03 & -1.00 & 7.26 & 0.02 \\
\hline $12433200-2408376$ & 3501624164266183552 & 4984 & 1.92 & -1.85 & 0.15 & 0.27 & 6.84 & 0.29 \\
\hline $12435927+7222303$ & 1689741705929430016 & 5268 & 2.87 & -1.64 & 0.35 & 0.37 & 7.16 & 0.45 \\
\hline 12450496-1907283 & 3521841434322113152 & 5192 & 1.43 & -2.29 & -0.01 & 0.52 & 6.66 & 0.44 \\
\hline $12493821+6415038$ & 1676938094886271104 & 5049 & 4.82 & -0.68 & 0.11 & 0.11 & 7.86 & 0.23 \\
\hline $12503487+4033500$ & 1527854729160394496 & 5439 & 4.31 & -0.30 & 0.23 & 0.23 & 8.36 & 0.44 \\
\hline $12505600+5649548$ & 1576792896497757440 & 4688 & 3.72 & -0.58 & -0.30 & -0.30 & 7.54 & $\cdots$ \\
\hline $12510042-1942157$ & 3509552399017285632 & 4793 & 4.71 & -1.03 & 0.38 & 0.38 & 7.78 & 0.16 \\
\hline $12511944+6940558$ & 1688859141689049984 & 5519 & 3.06 & -2.88 & 0.73 & 0.73 & 6.27 & 0.46 \\
\hline $12534156+4512260$ & 1530523729214664192 & 5127 & 4.66 & -0.52 & 0.11 & 0.11 & 8.02 & 0.59 \\
\hline $12540492-1445540$ & 3525348811990850560 & 5387 & 4.66 & -0.49 & 0.00 & 0.00 & 7.94 & 0.12 \\
\hline $12541483-6940177$ & 5844853661205358208 & 5663 & 3.94 & -0.04 & 0.40 & 0.40 & 8.80 & -0.09 \\
\hline $12550382+4640597$ & 1530993770436582784 & 4783 & 1.25 & -2.39 & -0.03 & 0.57 & 6.61 & 0.51 \\
\hline $12552381-8428164$ & 5770402155472131712 & 5049 & 1.62 & -2.55 & 0.23 & 0.59 & 6.47 & -0.05 \\
\hline $12553388+6801121$ & 1679621251151114624 & 4750 & 3.84 & -1.51 & 0.65 & 0.65 & 7.57 & 0.00 \\
\hline $12572833-5125217$ & 6081346139752486784 & 4921 & 1.63 & -2.33 & 0.30 & 0.66 & 6.76 & -0.03 \\
\hline $12592188+5338040$ & 1558199669539397376 & 5315 & 4.48 & -0.01 & -0.60 & -0.60 & 7.82 & -0.06 \\
\hline $12595962-0829171$ & 3627268897025456000 & 5338 & 3.59 & -1.20 & 0.37 & 0.37 & 7.60 & 0.03 \\
\hline $13003028+6135085$ & 1579614380772959104 & 5192 & 2.63 & -2.63 & 0.72 & 0.73 & 6.53 & 0.44 \\
\hline $13022750+5836042$ & 1578633165428231552 & 5040 & 3.79 & -0.64 & -0.34 & -0.34 & 7.45 & 0.16 \\
\hline $13031323+4812574$ & 1555059567409267328 & 4757 & 4.87 & -0.94 & 0.05 & 0.05 & 7.55 & 0.16 \\
\hline $13044809-8623048$ & 5769157650045097984 & 5154 & 1.97 & -2.52 & 0.62 & 0.68 & 6.60 & 0.44 \\
\hline $13070832-5007271$ & 6081477913650691456 & 5586 & 4.01 & -0.12 & -0.45 & -0.45 & 7.86 & 0.02 \\
\hline $13073032+4948349$ & 1556370498804511360 & 4888 & 1.34 & -2.57 & -0.11 & 0.46 & 6.32 & 0.38 \\
\hline $13091593+4640598$ & 1553980847721855616 & 4864 & 4.70 & -0.97 & 0.24 & 0.24 & 7.70 & 0.28 \\
\hline $13091772+4524279$ & 1529793344255574016 & 4908 & 4.23 & -1.19 & 0.59 & 0.59 & 7.83 & -0.06 \\
\hline
\end{tabular}


Table 3 (continued)

\begin{tabular}{|c|c|c|c|c|c|c|c|c|}
\hline $\begin{array}{c}\text { Star Name } \\
\text { (2MASS) }\end{array}$ & $\begin{array}{c}\text { Star Name } \\
\text { (Gaia EDR3) }\end{array}$ & $\begin{array}{l}T_{\text {eff }} \\
(\mathrm{K})\end{array}$ & $\begin{array}{l}\log g \\
(\operatorname{cgs})\end{array}$ & {$[\mathrm{Fe} / \mathrm{H}]$} & {$[\mathrm{C} / \mathrm{Fe}]$} & {$[\mathrm{C} / \mathrm{Fe}]_{c}$} & $A(\mathrm{C})_{c}$ & {$[\mathrm{Mg} / \mathrm{Fe}]$} \\
\hline $13123590+7259464$ & 1688176585486569600 & 4817 & 4.88 & -1.11 & 0.10 & 0.10 & 7.42 & 0.38 \\
\hline 13133130-6138303 & 5863379783621954944 & 5769 & 0.93 & -0.56 & 0.58 & 0.58 & 8.45 & 0.40 \\
\hline $13134248+3405127$ & 1473046861950780032 & 5041 & 1.77 & -2.38 & 0.27 & 0.52 & 6.56 & 0.44 \\
\hline $13140302-0929340$ & 3624508096342874240 & 5324 & 4.37 & -0.38 & -0.10 & -0.10 & 7.95 & 0.08 \\
\hline $13145513+3825569$ & 1522993032339838848 & 5167 & 2.40 & -2.75 & 0.70 & 0.71 & 6.39 & 0.27 \\
\hline $13151765+5708386$ & 1566249645139278720 & 5372 & 4.33 & -0.77 & 0.46 & 0.46 & 8.13 & -0.02 \\
\hline $13175280+3411307$ & 1473007485690704128 & 5090 & 2.54 & -1.58 & 0.22 & 0.24 & 7.08 & 0.43 \\
\hline $13222653+7020284$ & 1685430349037732864 & 4725 & 4.38 & -1.55 & 0.66 & 0.66 & 7.54 & 0.00 \\
\hline $13222932+3936562$ & 1476579283573419264 & 5152 & 4.16 & -0.49 & 0.10 & 0.10 & 8.05 & 0.04 \\
\hline $13230288-1704404$ & 3604328999956748032 & 5193 & 2.24 & -2.57 & 0.65 & 0.66 & 6.53 & 0.29 \\
\hline 13231009-0531428 & 3635041726974665600 & 5508 & 3.60 & -0.86 & 0.14 & 0.14 & 7.71 & -0.10 \\
\hline $13231283+4320557$ & 1549603412755666944 & 5019 & 1.70 & -2.25 & -0.20 & 0.12 & 6.30 & 0.49 \\
\hline 13240005-1748099 & 3604036456848334720 & 5125 & 1.98 & -2.02 & 0.42 & 0.49 & 6.90 & 0.09 \\
\hline $13241831+4610307$ & 1551088548024093952 & 4641 & 0.48 & -2.59 & -0.43 & 0.32 & 6.16 & 0.49 \\
\hline $13242995-4551146$ & 6087082811610225536 & 5531 & 4.09 & -0.23 & -0.23 & -0.23 & 7.97 & -0.04 \\
\hline $13244275-1607008$ & 3604537731071348992 & 4929 & 1.71 & -2.27 & 0.02 & 0.34 & 6.51 & 0.26 \\
\hline $13253853-1412517$ & 3608192335923918208 & 5070 & 2.08 & -2.07 & 0.40 & 0.43 & 6.79 & 0.29 \\
\hline $13282335+7427122$ & 1712581998412032768 & 4980 & 3.55 & -1.48 & 0.19 & 0.19 & 7.15 & 0.29 \\
\hline $13294999+0115237$ & 3711389512304903168 & 4853 & 1.43 & -2.58 & 0.01 & 0.52 & 6.37 & 0.48 \\
\hline $13303726-4125545$ & 6160938171579414016 & 5132 & 4.06 & -1.20 & 0.24 & 0.24 & 7.47 & 0.08 \\
\hline 13351401-0110524 & 3638534188221336960 & 4703 & 1.17 & -2.49 & -0.34 & 0.34 & 6.28 & 0.14 \\
\hline $13360938-4408536$ & 6111481627465700352 & 5192 & 1.72 & -2.29 & 0.49 & 0.75 & 6.90 & 0.30 \\
\hline $13373017-7717500$ & 5789490093947866624 & 5162 & 2.01 & -2.73 & 0.55 & 0.59 & 6.29 & 0.10 \\
\hline $13373672+5902017$ & 1662055552169232896 & 5318 & 4.79 & -0.43 & 0.09 & 0.09 & 8.09 & 0.13 \\
\hline $13375051+4742311$ & 1552172322891498240 & 5017 & 1.96 & -1.74 & -0.06 & 0.03 & 6.73 & 0.32 \\
\hline $13394721+6824054$ & 1672939136736222464 & 4889 & 4.65 & -0.99 & 0.31 & 0.31 & 7.75 & 0.49 \\
\hline $13401321+7237543$ & 1687511071713939712 & 5070 & 2.06 & -2.54 & 0.44 & 0.47 & 6.36 & 0.28 \\
\hline $13404811+0717285$ & 3724481569055443712 & 5134 & 4.73 & -0.52 & -0.04 & -0.04 & 7.87 & 0.33 \\
\hline 13425404-0717005 & 3620124270467822848 & 4597 & 0.72 & -2.77 & -0.57 & 0.18 & 5.85 & 0.37 \\
\hline $13433867+4844266$ & 1558284370590734336 & 6030 & 3.34 & -3.15 & 1.29 & 1.29 & 6.57 & 0.03 \\
\hline $13434635-0806060$ & 3618345883425109632 & 4914 & 3.32 & -1.14 & 0.20 & 0.20 & 7.49 & 0.06 \\
\hline $13443166+1523410$ & 3742083100949072640 & 4519 & 4.30 & -0.51 & -0.68 & -0.68 & 7.23 & -0.02 \\
\hline $13443667-4143163$ & 6112289699794699264 & 5134 & 1.89 & -2.19 & 0.64 & 0.77 & 7.01 & 0.05 \\
\hline $13452111-0730545$ & 3619873547457548160 & 4710 & 1.06 & -2.38 & -0.29 & 0.44 & 6.49 & 0.21 \\
\hline $13454247-7355568$ & 5791382177358811264 & 5196 & 4.56 & -0.30 & 0.29 & 0.29 & 8.42 & -0.02 \\
\hline $13455046+0513062$ & 3714276142644562432 & 4869 & 1.39 & -2.66 & 0.27 & 0.76 & 6.54 & 0.42 \\
\hline $13455076+1426436$ & 3741132710585731840 & 4853 & 4.73 & -0.84 & 0.47 & 0.47 & 8.06 & 0.03 \\
\hline $13460278-6854556$ & 5850193237592455552 & 6724 & 2.61 & -1.65 & 2.73 & 2.76 & 9.54 & $\ldots$ \\
\hline $13461713-4155246$ & 6112273791235652352 & 5202 & 1.70 & -2.30 & 0.19 & 0.53 & 6.67 & 0.29 \\
\hline $13465223-4334387$ & 6108883889504179072 & 4845 & 1.42 & -2.36 & -0.08 & 0.46 & 6.53 & 0.18 \\
\hline $13481581-7052139$ & 5840796639405915648 & 4947 & 4.20 & -0.50 & -0.21 & -0.21 & 7.71 & 0.28 \\
\hline $13510697+5853470$ & 1659341789968532736 & 4936 & 4.72 & -0.91 & 0.11 & 0.11 & 7.63 & 0.40 \\
\hline
\end{tabular}


Table 3 (continued)

\begin{tabular}{|c|c|c|c|c|c|c|c|c|}
\hline $\begin{array}{l}\text { Star Name } \\
\text { (2MASS) }\end{array}$ & $\begin{array}{c}\text { Star Name } \\
\text { (Gaia EDR3) }\end{array}$ & $\begin{array}{l}T_{\text {eff }} \\
(\mathrm{K})\end{array}$ & $\begin{array}{l}\log g \\
(\operatorname{cgs})\end{array}$ & {$[\mathrm{Fe} / \mathrm{H}]$} & {$[\mathrm{C} / \mathrm{Fe}]$} & {$[\mathrm{C} / \mathrm{Fe}]_{c}$} & $A(\mathrm{C})_{c}$ & {$[\mathrm{Mg} / \mathrm{Fe}]$} \\
\hline $13512378+0741110$ & 3721736844435125760 & 5084 & 4.47 & -0.30 & -0.16 & -0.16 & 7.96 & -0.03 \\
\hline $13521659-3554258$ & 6115884072018761728 & 5327 & 2.78 & -1.72 & 0.59 & 0.61 & 7.31 & 0.42 \\
\hline $13533557+7552137$ & 1712349073745277056 & 5014 & 4.76 & -0.50 & -0.23 & -0.23 & 7.70 & 0.13 \\
\hline $13550687+1707259$ & 1243948839616012160 & 4612 & 4.79 & -1.26 & -0.01 & -0.01 & 7.17 & 0.24 \\
\hline $13580646+1002348$ & 3723801761632006784 & 4918 & 4.29 & -0.54 & 0.09 & 0.09 & 7.98 & 0.59 \\
\hline $13584157-3151091$ & 6171032134996732416 & 5541 & 4.65 & -0.34 & 0.87 & 0.87 & 8.97 & 0.54 \\
\hline $13592706+1141130$ & 3727361838547995648 & 5051 & 2.18 & -1.99 & 0.15 & 0.16 & 6.60 & 0.56 \\
\hline $14002121+5538479$ & 1657538212943126400 & 4861 & 4.73 & -0.78 & -0.13 & -0.13 & 7.52 & 0.17 \\
\hline $14004596+1215235$ & 3727546045400955136 & 5084 & 1.96 & -2.83 & 0.97 & 1.02 & 6.62 & 0.19 \\
\hline $14012934+1126361$ & 3724429582771428352 & 4756 & 1.22 & -2.21 & -0.31 & 0.33 & 6.55 & 0.36 \\
\hline $14020262-6530100$ & 5851263646573358848 & 6026 & 3.28 & -1.16 & 1.11 & 1.12 & 8.39 & 0.15 \\
\hline $14043744+1255144$ & 1229600350311933952 & 4603 & 0.58 & -2.43 & -0.39 & 0.35 & 6.36 & 0.54 \\
\hline $14053819+7503388$ & 1712061237921283456 & 4707 & 4.73 & -0.29 & -0.54 & -0.54 & 7.61 & 0.08 \\
\hline $14063229+4115356$ & 1498298211635183744 & 5224 & 4.68 & -1.34 & 0.44 & 0.44 & 7.53 & 0.10 \\
\hline $14070810+6936102$ & 1674781746425953792 & 5020 & 2.02 & -2.17 & 0.10 & 0.14 & 6.40 & 0.21 \\
\hline $14071697+1212488$ & 1226501033191248640 & 4787 & 4.96 & -0.85 & -0.22 & -0.22 & 7.35 & 0.36 \\
\hline $14095514-2844258$ & 6269381255374610176 & 5101 & 1.59 & -2.65 & 0.10 & 0.47 & 6.25 & 0.16 \\
\hline $14103285-2816339$ & 6270148611411493888 & 4657 & 1.10 & -1.97 & -0.45 & 0.18 & 6.64 & 0.40 \\
\hline $14164140+6136563$ & 1666637942972460672 & 4840 & 4.70 & -0.19 & -0.53 & -0.53 & 7.72 & -0.07 \\
\hline $14173352-2745144$ & 6269861295279466752 & 5048 & 3.55 & -0.37 & 0.55 & 0.55 & 8.60 & 0.22 \\
\hline $14192484-2307370$ & 6275972209107871872 & 5069 & 4.34 & -0.41 & 0.23 & 0.23 & 8.26 & 0.60 \\
\hline $14193074+4035121$ & 1491839749053036160 & 5317 & 3.99 & -0.79 & 0.34 & 0.34 & 7.99 & $\ldots$ \\
\hline $14200302+5936293$ & 1660185630782829952 & 4640 & 4.79 & -1.16 & -0.08 & -0.08 & 7.19 & 0.29 \\
\hline 14203031-2729455 & 6269707565513913088 & 5181 & 3.40 & -1.07 & 0.41 & 0.41 & 7.77 & 0.26 \\
\hline $14211081-5015172$ & 6091186773125289728 & 5677 & 4.37 & -0.68 & 0.12 & 0.12 & 7.88 & 0.33 \\
\hline $14223584+4045568$ & 1491104691170130944 & 5128 & 2.40 & -3.04 & 0.59 & 0.60 & 5.99 & 0.09 \\
\hline $14243397+5624475$ & 1610452860137779584 & 5287 & 2.90 & -1.67 & 0.18 & 0.20 & 6.96 & 0.27 \\
\hline $14255416+2648176$ & 1256475262057930112 & 4738 & 4.39 & -0.27 & -0.47 & -0.47 & 7.70 & -0.07 \\
\hline $14261661+8102449$ & 1721820606208706176 & 4670 & 0.90 & -2.54 & -0.14 & 0.57 & 6.46 & 0.41 \\
\hline $14280573-1353174$ & 6300021547768039808 & 4947 & 1.75 & -2.17 & 0.16 & 0.45 & 6.71 & 0.10 \\
\hline $14294135+6525010$ & 1669746193625166976 & 5872 & 3.72 & -1.79 & 1.21 & 1.21 & 7.85 & -0.01 \\
\hline $14295841+4502025$ & 1494491496221024512 & 5142 & 1.67 & -2.00 & 0.07 & 0.40 & 6.83 & 0.40 \\
\hline $14324019+3238042$ & 1287120579569866496 & 4784 & 0.95 & -2.75 & -0.09 & 0.64 & 6.32 & 0.54 \\
\hline 14341379-6707026 & 5848443708440147072 & 4863 & 4.09 & -1.15 & 0.40 & 0.40 & 7.68 & -0.19 \\
\hline $14364248-0715099$ & 6331121238455373696 & 5241 & 4.66 & -0.89 & 0.08 & 0.08 & 7.62 & 0.39 \\
\hline $14385266+3937037$ & 1487940434144428416 & 4724 & 1.30 & -1.98 & -0.17 & 0.36 & 6.81 & 0.42 \\
\hline $14402612+0655539$ & 1171807334801223808 & 5293 & 2.13 & -2.58 & 0.25 & 0.26 & 6.11 & 0.02 \\
\hline $14410823+4223420$ & 1490063071404892544 & 5395 & 3.47 & -1.26 & 0.46 & 0.46 & 7.62 & 0.40 \\
\hline $14411830+1044242$ & 1177885611173494016 & 4864 & 1.40 & -2.62 & 0.03 & 0.55 & 6.36 & 0.19 \\
\hline $14421990+5654506$ & 1607745454489656960 & 5306 & 4.42 & -0.21 & 0.28 & 0.28 & 8.50 & 0.47 \\
\hline 14431485-0206178 & 3648915222959836800 & 4755 & 4.50 & -0.38 & -0.48 & -0.48 & 7.58 & -0.03 \\
\hline $14442119+4758464$ & 1591067649802389632 & 4867 & 1.61 & -2.33 & -0.08 & 0.33 & 6.42 & 0.39 \\
\hline
\end{tabular}


Table 3 (continued)

\begin{tabular}{|c|c|c|c|c|c|c|c|c|}
\hline $\begin{array}{c}\text { Star Name } \\
\text { (2MASS) }\end{array}$ & $\begin{array}{c}\text { Star Name } \\
\text { (Gaia EDR3) }\end{array}$ & $\begin{array}{l}T_{\text {eff }} \\
(\mathrm{K})\end{array}$ & $\begin{array}{l}\log g \\
(\operatorname{cgs})\end{array}$ & {$[\mathrm{Fe} / \mathrm{H}]$} & {$[\mathrm{C} / \mathrm{Fe}]$} & {$[\mathrm{C} / \mathrm{Fe}]_{c}$} & $A(\mathrm{C})_{c}$ & {$[\mathrm{Mg} / \mathrm{Fe}]$} \\
\hline $14443681+3939231$ & 1487238292890443776 & 4677 & 4.62 & -1.08 & -0.04 & -0.04 & 7.31 & 0.24 \\
\hline $14444228+4247368$ & 1490052389822427648 & 4794 & 4.69 & -0.98 & 0.16 & 0.16 & 7.61 & 0.35 \\
\hline 14453096-0214079 & 3648730612380753664 & 5730 & 4.19 & -0.93 & 0.37 & 0.37 & 7.87 & 0.44 \\
\hline $14455563+5838081$ & 1616958709094540672 & 4661 & 4.39 & -0.32 & -0.42 & -0.42 & 7.69 & -0.05 \\
\hline $14460403-7303141$ & 5796756182184708096 & 4711 & 1.22 & -2.35 & -0.26 & 0.41 & 6.49 & 0.06 \\
\hline $14473151+0306259$ & 1157525159792413440 & 5253 & 3.87 & -0.34 & -0.34 & -0.34 & 7.75 & 0.12 \\
\hline $14504173+6652564$ & 1669581885356163456 & 4747 & 1.16 & -2.78 & 0.10 & 0.74 & 6.39 & 0.55 \\
\hline $14512223+0339397$ & 1157672906667474432 & 4663 & 4.75 & -0.94 & 0.17 & 0.17 & 7.65 & 0.13 \\
\hline $14520851+7234433$ & 1698671492693440512 & 5125 & 4.69 & -0.36 & -0.04 & -0.04 & 8.03 & -0.05 \\
\hline $14535190-4724035$ & 5905262548019849856 & 4704 & 4.17 & -0.37 & -0.67 & -0.67 & 7.39 & -0.13 \\
\hline $14550996+1452303$ & 1185448189587672960 & 5182 & 4.24 & -0.34 & 0.32 & 0.32 & 8.41 & 0.45 \\
\hline $14561630+4534315$ & 1586671424357440000 & 5380 & 3.96 & -0.89 & 0.18 & 0.18 & 7.72 & 0.33 \\
\hline $14561931+0829491$ & 1161734987952738176 & 4857 & 1.42 & -2.49 & 0.06 & 0.57 & 6.51 & 0.46 \\
\hline $14572828+3910442$ & 1296184266229998976 & 4643 & 4.51 & -0.96 & 0.27 & 0.27 & 7.73 & 0.06 \\
\hline $14572980+5047347$ & 1593299585391391232 & 4824 & 4.73 & -0.91 & 0.13 & 0.13 & 7.65 & 0.10 \\
\hline $14584270+6055051$ & 1619069599620600576 & 4944 & 4.27 & -1.04 & 0.62 & 0.62 & 8.01 & 0.44 \\
\hline $14584982+4500407$ & 1586583807024523520 & 5428 & 4.52 & -0.72 & 0.49 & 0.49 & 8.20 & 0.39 \\
\hline $14590313+0544031$ & 1159807818946952064 & 4913 & 4.44 & -1.03 & 0.25 & 0.25 & 7.65 & 0.55 \\
\hline $14591786+4755555$ & 1587515844991289600 & 5342 & 4.53 & -0.18 & -0.37 & -0.37 & 7.88 & -0.08 \\
\hline $15002374+1131178$ & 1180473277429900288 & 5203 & 4.79 & -0.50 & -0.03 & -0.03 & 7.90 & 0.19 \\
\hline $15010054+5654074$ & 1612825747965425152 & 5048 & 4.78 & -1.00 & 0.39 & 0.39 & 7.82 & 0.33 \\
\hline $15010753+1708495$ & 1187645868519130240 & 5101 & 1.57 & -2.19 & -0.13 & 0.30 & 6.54 & 0.46 \\
\hline $15012803+3616594$ & 1294826884765960192 & 4769 & 1.91 & -1.57 & -0.15 & -0.02 & 6.83 & $\ldots$ \\
\hline 15015996-2613494 & 6225828809526332416 & 5048 & 2.05 & -1.37 & 0.22 & 0.27 & 7.33 & 0.27 \\
\hline $15021465+3507093$ & 1291694234403435648 & 5065 & 4.61 & -0.51 & 0.15 & 0.15 & 8.06 & 0.06 \\
\hline $15023312+0549057$ & 1159849256791308160 & 5162 & 2.35 & -0.44 & -0.14 & -0.11 & 7.88 & $\ldots$ \\
\hline $15023572+0940189$ & 1167946644533913728 & 4814 & 1.66 & -1.62 & -0.09 & 0.21 & 7.01 & 0.55 \\
\hline $15023742-2442191$ & 6227668258118329088 & 5431 & 3.47 & -0.68 & 0.47 & 0.47 & 8.22 & 0.48 \\
\hline $15025030+1345255$ & 1181911644797448832 & 4376 & 3.97 & -1.45 & 0.01 & 0.01 & 6.99 & -0.09 \\
\hline $15025884+4539160$ & 1586250517562417024 & 4993 & 1.53 & -2.30 & -0.12 & 0.36 & 6.50 & 0.41 \\
\hline $15031286+0602157$ & 1159876916380773120 & 4963 & 1.91 & -1.95 & 0.05 & 0.17 & 6.64 & 0.34 \\
\hline $15040464+5927327$ & 1614255147440846720 & 5263 & 4.76 & -0.28 & 0.03 & 0.03 & 8.17 & 0.07 \\
\hline $15043325+0441214$ & 1156620193003216256 & 4885 & 1.92 & -1.83 & 0.06 & 0.18 & 6.78 & 0.55 \\
\hline $15050841+0701547$ & 1160377499113263360 & 4830 & 1.74 & -1.72 & -0.18 & 0.09 & 6.80 & 0.45 \\
\hline $15060136-2508317$ & 6227460583562691968 & 5360 & 4.76 & -0.95 & 0.48 & 0.48 & 7.95 & 0.50 \\
\hline $15060189+0444205$ & 1156532506951177472 & 4839 & 1.27 & -2.02 & -0.17 & 0.38 & 6.79 & 0.40 \\
\hline $15065236-2547072$ & 6226609772026895104 & 5052 & 4.40 & -0.35 & 0.22 & 0.22 & 8.30 & 0.59 \\
\hline $15071495+5704053$ & 1612981535018964224 & 4709 & 4.59 & -0.86 & -0.04 & -0.04 & 7.53 & -0.07 \\
\hline $15085620-7020101$ & 5799023787486633984 & 5114 & 4.18 & -0.18 & -0.15 & -0.15 & 8.10 & -0.07 \\
\hline $15092359+3623180$ & 1292060685309150336 & 5025 & 4.34 & -1.17 & 0.37 & 0.37 & 7.63 & 0.20 \\
\hline 15094309-2025300 & 6255898665838008960 & 5367 & 4.67 & -0.31 & 0.39 & 0.39 & 8.51 & 0.49 \\
\hline $15095381+7303555$ & 1697243020930244608 & 4656 & 4.64 & -0.65 & -0.56 & -0.56 & 7.22 & 0.38 \\
\hline
\end{tabular}


Table 3 (continued)

\begin{tabular}{|c|c|c|c|c|c|c|c|c|}
\hline $\begin{array}{c}\text { Star Name } \\
\text { (2MASS) }\end{array}$ & $\begin{array}{c}\text { Star Name } \\
\text { (Gaia EDR3) }\end{array}$ & $\begin{array}{l}T_{\text {eff }} \\
(\mathrm{K})\end{array}$ & $\begin{array}{l}\log g \\
(\operatorname{cgs})\end{array}$ & {$[\mathrm{Fe} / \mathrm{H}]$} & {$[\mathrm{C} / \mathrm{Fe}]$} & {$[\mathrm{C} / \mathrm{Fe}]_{c}$} & $A(\mathrm{C})_{c}$ & {$[\mathrm{Mg} / \mathrm{Fe}]$} \\
\hline $15102643+0809151$ & 1166563287107603328 & 5145 & 2.15 & -2.05 & 0.40 & 0.42 & 6.80 & 0.50 \\
\hline $15114226+2557063$ & 1265182569515722880 & 5019 & 1.95 & -2.78 & 0.51 & 0.57 & 6.23 & 0.36 \\
\hline $15122018-6551473$ & 5824755211457838848 & 5076 & 3.28 & -1.35 & 0.32 & 0.33 & 7.42 & 0.00 \\
\hline $15140783+1824326$ & 1211721397891599872 & 5038 & 2.01 & -2.12 & 0.32 & 0.38 & 6.69 & 0.41 \\
\hline $15142395+2613341$ & 1265208133161061888 & 5038 & 1.39 & -2.32 & -0.14 & 0.43 & 6.54 & 0.40 \\
\hline 15174310-6858306 & 5799218602888406272 & 4601 & 2.02 & -0.79 & -0.77 & -0.59 & 7.05 & 0.27 \\
\hline $15185830+2620276$ & 1270426797601163008 & 5104 & 2.13 & -1.96 & 0.42 & 0.44 & 6.90 & 0.14 \\
\hline $15193496+4256501$ & 1394164216985937152 & 5052 & 4.29 & -1.23 & 0.66 & 0.66 & 7.86 & -0.09 \\
\hline $15193617+2304315$ & 1215718152722386816 & 4914 & 1.65 & -2.21 & 0.00 & 0.39 & 6.62 & 0.34 \\
\hline 15200891-6701472 & 5823675417983108096 & 5107 & 4.11 & -1.31 & 0.56 & 0.56 & 7.68 & 0.00 \\
\hline $15210142+7816076$ & 1708318332837616256 & 4877 & 1.73 & -3.54 & 0.49 & 0.63 & 5.52 & 0.20 \\
\hline $15243997-8015248$ & 5772460033220024576 & 5171 & 2.86 & -1.42 & 0.81 & 0.83 & 7.84 & 0.24 \\
\hline $15244942+6935080$ & 1695610791623488128 & 5057 & 4.77 & -0.11 & -0.29 & -0.29 & 8.03 & 0.04 \\
\hline $15252134+6351159$ & 1640849688017226496 & 4655 & 4.78 & -0.75 & -0.45 & -0.45 & 7.23 & 0.40 \\
\hline $15255933+5026270$ & 1594780288252588032 & 4744 & 4.90 & -1.05 & 0.00 & 0.00 & 7.38 & 0.20 \\
\hline $15264322-7017087$ & 5796025453632860544 & 4897 & 1.49 & -2.37 & 0.15 & 0.61 & 6.68 & 0.26 \\
\hline $15272716+2937502$ & 1273518040182639872 & 5262 & 3.78 & -0.13 & -0.48 & -0.48 & 7.82 & -0.04 \\
\hline $15282654+6737516$ & 1645668263365914368 & 4999 & 4.74 & -1.36 & 0.04 & 0.04 & 7.11 & 0.40 \\
\hline $15304031+2345045$ & 1220893455176256512 & 4918 & 1.48 & -2.13 & -0.30 & 0.19 & 6.49 & 0.49 \\
\hline $15312758+2528504$ & 1222449092327405056 & 5306 & 4.75 & -0.41 & -0.02 & -0.02 & 8.00 & 0.18 \\
\hline $15330912+8158562$ & 1721465807550041856 & 5024 & 4.71 & -0.66 & 0.26 & 0.26 & 8.03 & -0.01 \\
\hline $15345353+5610250$ & 1601294344891081472 & 6097 & 4.35 & -1.14 & 0.42 & 0.42 & 7.71 & 0.51 \\
\hline $15351593+0251226$ & 4427077397649058944 & 4851 & 1.33 & -2.48 & -0.03 & 0.54 & 6.48 & 0.52 \\
\hline $15352535+2843009$ & 1272505179518285696 & 4643 & 4.52 & -0.47 & -0.62 & -0.62 & 7.34 & 0.02 \\
\hline $15371627+3318345$ & 1370624708124173312 & 4871 & 1.36 & -1.93 & -0.10 & 0.39 & 6.89 & 0.48 \\
\hline $15381851+4804330$ & 1401181854246440704 & 4772 & 4.90 & -0.99 & -0.03 & -0.03 & 7.41 & 0.23 \\
\hline $15391788+5403318$ & 1597864040410848896 & 5270 & 4.76 & -0.32 & -0.09 & -0.09 & 8.01 & 0.05 \\
\hline $15392367+4037228$ & 1389938076542244992 & 4959 & 1.23 & -2.61 & -0.15 & 0.49 & 6.31 & 0.49 \\
\hline $15392783+5557319$ & 1601593274615155712 & 5012 & 4.39 & 0.04 & -0.41 & -0.41 & 8.05 & -0.08 \\
\hline $15393011+7252178$ & 1696583898069432704 & 5052 & 4.51 & -0.74 & -0.07 & -0.07 & 7.63 & 0.11 \\
\hline $15394006+4426174$ & 1397344691808122624 & 4834 & 1.28 & -1.99 & -0.33 & 0.23 & 6.67 & 0.35 \\
\hline $15414028+8245352$ & 1723003508921638656 & 5334 & 4.63 & -0.40 & 0.16 & 0.16 & 8.19 & -0.06 \\
\hline $15420239+0515389$ & 4427879525740941056 & 5216 & 2.54 & -1.54 & 0.23 & 0.25 & 7.14 & 0.30 \\
\hline $15431257+6424109$ & 1641066429247008128 & 5227 & 2.60 & -2.32 & 0.72 & 0.73 & 6.84 & 0.20 \\
\hline $15433525+3739578$ & 1376344024013884800 & 5182 & 4.06 & -0.29 & 0.15 & 0.15 & 8.29 & 0.48 \\
\hline $15441676+0459116$ & 4429308311037974272 & 4731 & 0.84 & -2.49 & -0.27 & 0.46 & 6.41 & 0.49 \\
\hline $15452566-4444040$ & 5988917073480792704 & 5040 & 1.83 & -1.92 & 0.49 & 0.64 & 7.15 & -0.03 \\
\hline $15460715+0559221$ & 4429522058673688192 & 5050 & 2.04 & -2.50 & 0.44 & 0.48 & 6.41 & 0.25 \\
\hline $15470901+0505149$ & 4426320800506688384 & 5048 & 4.35 & -1.34 & 0.39 & 0.39 & 7.48 & 0.25 \\
\hline $15471083-4404210$ & 5989331898604674176 & 5138 & 2.94 & -0.51 & -0.24 & -0.21 & 7.71 & $\ldots$ \\
\hline $15475041+6321134$ & 1640197196585984896 & 4728 & 4.32 & -0.83 & -0.09 & -0.09 & 7.50 & 0.36 \\
\hline 15482592-3959257 & 6008057268799998848 & 5835 & 3.28 & -0.34 & 0.57 & 0.59 & 8.68 & 0.38 \\
\hline
\end{tabular}


Table 3 (continued)

\begin{tabular}{|c|c|c|c|c|c|c|c|c|}
\hline $\begin{array}{l}\text { Star Name } \\
\text { (2MASS) }\end{array}$ & $\begin{array}{c}\text { Star Name } \\
\text { (Gaia EDR3) }\end{array}$ & $\begin{array}{l}T_{\text {eff }} \\
(\mathrm{K})\end{array}$ & $\begin{array}{l}\log g \\
(\operatorname{cgs})\end{array}$ & {$[\mathrm{Fe} / \mathrm{H}]$} & {$[\mathrm{C} / \mathrm{Fe}]$} & {$[\mathrm{C} / \mathrm{Fe}]_{c}$} & $A(\mathrm{C})_{c}$ & {$[\mathrm{Mg} / \mathrm{Fe}]$} \\
\hline $15482651+1917200$ & 1203885900077833984 & 4615 & 0.91 & -2.52 & -0.19 & 0.53 & 6.45 & 0.42 \\
\hline $15484895+0949113$ & 4455582481414836096 & 4733 & 1.01 & -2.29 & -0.20 & 0.51 & 6.65 & 0.56 \\
\hline $15494232+5707537$ & 1598759730070071808 & 4794 & 4.74 & -1.02 & -0.14 & -0.14 & 7.28 & 0.36 \\
\hline $15495840-4306370$ & 5989480401391030528 & 4967 & 2.81 & -0.76 & -0.33 & -0.30 & 7.38 & $\ldots$ \\
\hline $15501637+3536243$ & 1372799645203241856 & 5280 & 2.88 & -1.61 & 0.43 & 0.45 & 7.27 & 0.55 \\
\hline $15511496+0734493$ & 4430292717546018304 & 5154 & 2.60 & -1.64 & 0.16 & 0.18 & 6.96 & 0.21 \\
\hline $15512062+0140120$ & 4423150667969419264 & 5227 & 3.86 & -1.26 & 0.43 & 0.43 & 7.60 & -0.04 \\
\hline $15514567-3935382$ & 6008406329380234368 & 5793 & 2.92 & -0.47 & 0.38 & 0.41 & 8.36 & 0.40 \\
\hline $15521212-3934227$ & 6008407257093139456 & 6122 & 3.93 & -0.05 & 0.29 & 0.29 & 8.67 & 0.38 \\
\hline $15525776+0136148$ & 4411131043072307456 & 4791 & 1.31 & -2.58 & 0.03 & 0.61 & 6.46 & 0.43 \\
\hline $15533843+0145539$ & 4411228560308157184 & 4808 & 1.48 & -2.66 & 0.05 & 0.51 & 6.28 & 0.51 \\
\hline $15541062-3255166$ & 6015313976817734784 & 5144 & 2.32 & -1.45 & 0.49 & 0.51 & 7.49 & 0.45 \\
\hline $15542259-3341566$ & 6012240773099364992 & 5637 & 3.58 & -0.51 & 0.86 & 0.86 & 8.78 & 0.33 \\
\hline $15542887-3332103$ & 6012255341628535680 & 5125 & 1.80 & -2.67 & 0.85 & 1.00 & 6.76 & 0.06 \\
\hline $15545039-4652323$ & 5987712279288235648 & 5351 & 4.61 & -0.20 & 0.30 & 0.30 & 8.53 & 0.00 \\
\hline $15555135+1256054$ & 1191328721374511744 & 4862 & 1.66 & -2.03 & -0.01 & 0.33 & 6.73 & 0.49 \\
\hline $15563950+0754021$ & 4454167994065860352 & 4735 & 1.34 & -2.22 & -0.10 & 0.47 & 6.69 & 0.41 \\
\hline $15570603+0757417$ & 4454127621373692032 & 4492 & 4.23 & -0.62 & -0.64 & -0.64 & 7.18 & -0.13 \\
\hline $15583081+1505320$ & 1192938543835309952 & 4844 & 4.81 & -0.86 & -0.07 & -0.07 & 7.50 & 0.42 \\
\hline $15583757-3734113$ & 6010214613689309056 & 6615 & 3.67 & -1.15 & 1.29 & 1.29 & 8.57 & 0.07 \\
\hline $15584850-3603371$ & 6010918747795620480 & 5581 & 4.70 & -0.40 & 0.61 & 0.61 & 8.63 & 0.56 \\
\hline $15591458+0507554$ & 4425763554271082112 & 4615 & 1.05 & -1.80 & -0.60 & 0.03 & 6.66 & 0.40 \\
\hline $15592133-3416261$ & 6011940778224281856 & 6037 & 3.96 & -0.52 & 0.89 & 0.89 & 8.80 & 0.37 \\
\hline $15592229-3853560$ & 5997979351379581312 & 5285 & 3.23 & -0.75 & 0.79 & 0.81 & 8.49 & 0.58 \\
\hline $15592785+0314441$ & 4424940775976241664 & 5481 & 3.11 & -1.72 & 0.29 & 0.30 & 7.02 & 0.38 \\
\hline 15593606-3220592 & 6036353337976099840 & 5297 & 3.34 & -0.92 & 0.20 & 0.20 & 7.71 & -0.03 \\
\hline $15593767+1613419$ & 1199172465527378176 & 5043 & 2.16 & -1.25 & -0.31 & -0.28 & 6.91 & 0.26 \\
\hline $15595245-3207381$ & 6036461468074424448 & 5625 & 4.60 & -1.30 & 0.97 & 0.97 & 8.10 & 0.46 \\
\hline $16001273+2435560$ & 1219123340233877376 & 5182 & 3.77 & -0.75 & 0.24 & 0.24 & 7.92 & $\ldots$ \\
\hline $16004212+1105313$ & 4457460855293872128 & 5199 & 4.40 & -0.66 & 0.46 & 0.46 & 8.23 & 0.43 \\
\hline $16005880-3307564$ & 6036077979033846528 & 5995 & 3.89 & -0.44 & 0.96 & 0.96 & 8.96 & 0.28 \\
\hline $16020475+0622249$ & 4450709720758088192 & 5221 & 4.81 & -0.46 & 0.18 & 0.18 & 8.15 & 0.07 \\
\hline $16025295+3733291$ & 1379240098985809408 & 4808 & 1.47 & -2.37 & 0.04 & 0.54 & 6.59 & 0.45 \\
\hline $16025382-8226516$ & 5768895377865607808 & 5033 & 1.98 & -3.01 & 0.07 & 0.09 & 5.51 & 0.02 \\
\hline $16025490+0102272$ & 4411633352385123840 & 4725 & 4.12 & -0.49 & -0.20 & -0.20 & 7.74 & 0.56 \\
\hline $16030891+1940229$ & 1203268485640442880 & 5126 & 2.23 & -2.12 & 0.51 & 0.52 & 6.83 & 0.32 \\
\hline $16032024+1641034$ & 1199294713178394496 & 5058 & 4.75 & -0.83 & 0.30 & 0.30 & 7.90 & 0.20 \\
\hline $16033629+3116414$ & 1322162667977621632 & 5325 & 4.49 & -0.01 & -0.21 & -0.21 & 8.22 & 0.02 \\
\hline $16034976+0832565$ & 4451626679096707456 & 5204 & 4.38 & -0.29 & 0.36 & 0.36 & 8.50 & 0.18 \\
\hline $16040396+1841499$ & 1200065646925933056 & 4890 & 4.95 & -0.90 & 0.00 & 0.00 & 7.53 & 0.40 \\
\hline $16040780+4241470$ & 1383653195061929728 & 5567 & 4.63 & -0.34 & 0.23 & 0.23 & 8.32 & -0.02 \\
\hline $16042932-2253301$ & 6242404084762732800 & 5344 & 4.53 & -0.46 & 0.37 & 0.37 & 8.34 & -0.01 \\
\hline
\end{tabular}


Table 3 (continued)

\begin{tabular}{|c|c|c|c|c|c|c|c|c|}
\hline $\begin{array}{l}\text { Star Name } \\
\text { (2MASS) }\end{array}$ & $\begin{array}{c}\text { Star Name } \\
\text { (Gaia EDR3) }\end{array}$ & $\begin{array}{l}T_{\text {eff }} \\
(\mathrm{K})\end{array}$ & $\begin{array}{l}\log g \\
(\operatorname{cgs})\end{array}$ & {$[\mathrm{Fe} / \mathrm{H}]$} & {$[\mathrm{C} / \mathrm{Fe}]$} & {$[\mathrm{C} / \mathrm{Fe}]_{c}$} & $A(\mathrm{C})_{c}$ & {$[\mathrm{Mg} / \mathrm{Fe}]$} \\
\hline 16045324-2005064 & 6244194055324808576 & 5594 & 3.17 & -1.13 & 0.89 & 0.90 & 8.21 & 0.30 \\
\hline $16051879+1359570$ & 1191908675099304704 & 5642 & 3.93 & -0.70 & 0.54 & 0.54 & 8.27 & 0.28 \\
\hline $16052710+0024235$ & 4409850528641208192 & 6151 & 3.76 & -0.67 & 0.70 & 0.70 & 8.47 & 0.40 \\
\hline $16052946-2543348$ & 6043663818991262208 & 4834 & 3.67 & -0.45 & -0.33 & -0.33 & 7.64 & 0.38 \\
\hline $16053749+1843052$ & 1200145322861774080 & 4724 & 4.07 & -1.56 & 0.77 & 0.77 & 7.64 & -0.11 \\
\hline $16055339+1944171$ & 1203234576873824384 & 4802 & 4.19 & -0.44 & 0.17 & 0.17 & 8.16 & 0.54 \\
\hline $16060454-3508190$ & 6034898993336955008 & 5388 & 4.40 & 0.02 & 0.53 & 0.53 & 8.98 & 0.42 \\
\hline $16061119-3620355$ & 5998751552140270976 & 5485 & 3.53 & -0.93 & 1.06 & 1.06 & 8.57 & 0.53 \\
\hline $16061643-3237103$ & 6036155219728437760 & 5921 & 3.68 & -1.11 & 0.94 & 0.94 & 8.26 & 0.38 \\
\hline $16064542-3216179$ & 6036585644175848576 & 6068 & 3.78 & -0.63 & 0.88 & 0.88 & 8.68 & 0.39 \\
\hline $16065604-3707315$ & 5998563982328033920 & 6568 & 3.67 & -0.72 & 0.53 & 0.53 & 8.25 & 0.09 \\
\hline $16073239-2250378$ & 6242439784523836416 & 6191 & 4.11 & -0.55 & 0.42 & 0.42 & 8.30 & 0.28 \\
\hline $16073532-3208471$ & 6036547126907295616 & 5160 & 4.61 & -1.20 & 0.58 & 0.58 & 7.80 & 0.56 \\
\hline $16074138-3314575$ & 6035358520474835456 & 5775 & 3.86 & -0.73 & 0.49 & 0.49 & 8.20 & 0.46 \\
\hline $16074907+1420135$ & 4458558752013944960 & 4717 & 3.85 & -1.47 & 0.53 & 0.53 & 7.50 & -0.01 \\
\hline 16080889-2943209 & 6041589418507890688 & 5743 & 3.63 & -0.54 & 0.79 & 0.79 & 8.68 & 0.39 \\
\hline 16081391-2017182 & 6244117639267067008 & 5901 & 4.02 & -0.77 & 0.72 & 0.72 & 8.39 & 0.48 \\
\hline $16083267-3438595$ & 6034940156304474112 & 6002 & 3.79 & -0.77 & 0.76 & 0.76 & 8.41 & 0.36 \\
\hline $16084292-2820180$ & 6041824610913270656 & 5785 & 4.47 & -0.69 & 0.62 & 0.62 & 8.36 & 0.56 \\
\hline $16091164+3008425$ & 1318809707269021184 & 4691 & 1.16 & -2.28 & -0.06 & 0.56 & 6.70 & 0.53 \\
\hline $16101125-3446225$ & 6034969392126840320 & 5591 & 3.94 & -0.64 & 0.98 & 0.98 & 8.77 & 0.49 \\
\hline $16102112+0023264$ & 4408313235288945024 & 5082 & 1.59 & -2.00 & 0.09 & 0.46 & 6.89 & 0.47 \\
\hline $16102340+3952510$ & 1379957603338177280 & 4481 & 4.59 & -0.90 & -0.62 & -0.62 & 6.92 & 0.38 \\
\hline $16102544+1810108$ & 1199821898940802944 & 4828 & 1.44 & -2.26 & 0.05 & 0.56 & 6.73 & 0.49 \\
\hline $16103301+0031597$ & 4408318943304571776 & 5298 & 2.14 & -1.60 & -0.05 & -0.02 & 6.81 & 0.55 \\
\hline $16103970-2443561$ & 6049772323329029632 & 5094 & 2.74 & -0.77 & 0.52 & 0.55 & 8.21 & 0.56 \\
\hline $16105877-2811435$ & 6041842237458491520 & 5365 & 2.41 & -1.46 & 0.60 & 0.62 & 7.59 & 0.49 \\
\hline 16111495-3204491 & 6035900167382119808 & 5580 & 3.70 & -0.79 & 0.86 & 0.86 & 8.50 & 0.49 \\
\hline 16113577-1909453 & 6245765566678852608 & 5947 & 3.59 & -0.39 & 0.53 & 0.53 & 8.57 & 0.03 \\
\hline $16114668-2427053$ & 6049806927886876928 & 5835 & 3.71 & -0.52 & 0.41 & 0.41 & 8.31 & 0.40 \\
\hline 16121101-2818128 & 6042164669240420864 & 6127 & 4.38 & -0.08 & 0.13 & 0.13 & 8.48 & 0.31 \\
\hline $16123013+4456046$ & 1385790890480677376 & 5148 & 2.39 & -1.78 & 0.32 & 0.34 & 7.00 & 0.21 \\
\hline 16132830-2013405 & 6245397122905040000 & 6208 & 4.17 & -0.74 & 0.92 & 0.92 & 8.61 & 0.39 \\
\hline $16134375-2517381$ & 6049518065566679040 & 6110 & 3.77 & -0.28 & 0.54 & 0.54 & 8.69 & 0.31 \\
\hline 16135700-2757099 & 6042198238705334144 & 5984 & 4.00 & -0.93 & 1.17 & 1.17 & 8.66 & 0.39 \\
\hline 16141120-2033379 & 6244625270041451648 & 6254 & 3.65 & -0.93 & 0.89 & 0.89 & 8.39 & 0.25 \\
\hline 16150157-2339354 & 6050327233103808768 & 5423 & 3.81 & -0.68 & 0.77 & 0.77 & 8.51 & 0.31 \\
\hline 16150863-0734143 & 4348947961102169344 & 5165 & 2.38 & -1.49 & 0.21 & 0.23 & 7.16 & 0.36 \\
\hline $16154084+3326445$ & 1322837974275568384 & 5178 & 2.42 & -2.15 & 0.49 & 0.50 & 6.78 & 0.43 \\
\hline 16154634-1423377 & 4329332948541070976 & 5735 & 3.61 & -0.98 & 0.89 & 0.89 & 8.34 & 0.05 \\
\hline $16154808-2355376$ & 6050304972294952064 & 6068 & 3.50 & -0.90 & 0.83 & 0.83 & 8.36 & 0.29 \\
\hline $16154986-0751166$ & 4345832009572003840 & 4731 & 1.15 & -2.30 & -0.36 & 0.32 & 6.45 & 0.19 \\
\hline
\end{tabular}


Table 3 (continued)

\begin{tabular}{|c|c|c|c|c|c|c|c|c|}
\hline $\begin{array}{c}\text { Star Name } \\
\text { (2MASS) }\end{array}$ & $\begin{array}{c}\text { Star Name } \\
\text { (Gaia EDR3) }\end{array}$ & $\begin{array}{l}T_{\text {eff }} \\
(\mathrm{K})\end{array}$ & $\begin{array}{l}\log g \\
(\operatorname{cgs})\end{array}$ & {$[\mathrm{Fe} / \mathrm{H}]$} & {$[\mathrm{C} / \mathrm{Fe}]$} & {$[\mathrm{C} / \mathrm{Fe}]_{c}$} & $A(\mathrm{C})_{c}$ & {$[\mathrm{Mg} / \mathrm{Fe}]$} \\
\hline $16163380+4615010$ & 1386708295494907904 & 5272 & 4.51 & -0.85 & 0.48 & 0.48 & 8.07 & 0.04 \\
\hline 16163935-3233268 & 6035641953958688896 & 5115 & 1.37 & -2.63 & -0.07 & 0.47 & 6.27 & 0.13 \\
\hline $16164213-3256588$ & 6035579999042793856 & 5524 & 3.33 & -1.47 & 0.53 & 0.54 & 7.51 & 0.39 \\
\hline 16165205-2154235 & 6242759089572065920 & 5164 & 3.73 & -0.49 & 0.76 & 0.76 & 8.70 & 0.54 \\
\hline $16171415+1832404$ & 1200608324633174784 & 4890 & 1.46 & -2.30 & 0.15 & 0.63 & 6.76 & 0.48 \\
\hline 16173056-1932253 & 6245550268557026432 & 6249 & 3.00 & -1.09 & 1.32 & 1.34 & 8.68 & -0.04 \\
\hline $16173244+0248331$ & 4436119304542479616 & 4974 & 1.70 & -2.22 & 0.35 & 0.65 & 6.86 & 0.28 \\
\hline $16173704+3125396$ & 1319323522796448512 & 4879 & 1.14 & -3.03 & 0.49 & 1.11 & 6.50 & 0.31 \\
\hline $16175168-3344503$ & 6023449568284510592 & 6012 & 4.24 & -0.29 & 0.57 & 0.57 & 8.71 & 0.39 \\
\hline $16175506-2501165$ & 6048881207813795456 & 6253 & 3.70 & -1.07 & 1.44 & 1.44 & 8.80 & 0.34 \\
\hline 16180650-1903385 & 6245955610391354880 & 5943 & 3.76 & -0.28 & 0.22 & 0.22 & 8.36 & -0.01 \\
\hline $16194310-0224311$ & 4358730552275110272 & 5308 & 2.72 & -1.99 & 0.61 & 0.62 & 7.06 & 0.14 \\
\hline $16195744+3711488$ & 1330482668168550016 & 4983 & 4.68 & -0.22 & -0.17 & -0.17 & 8.04 & -0.03 \\
\hline $16201273+8036085$ & 1710329438388205824 & 5185 & 2.48 & -2.20 & 0.45 & 0.46 & 6.69 & 0.38 \\
\hline $16201770+4208508$ & 1382053238141320192 & 4628 & 4.92 & -1.14 & -0.12 & -0.12 & 7.17 & 0.35 \\
\hline $16202566+0328284$ & 4436260965440303232 & 4860 & 4.23 & -0.35 & -0.05 & -0.05 & 8.03 & 0.54 \\
\hline $16203416+3132541$ & 1319232263331506304 & 4743 & 4.03 & -0.50 & -0.23 & -0.23 & 7.71 & 0.54 \\
\hline 16203444-2056578 & 6244454330343783936 & 5554 & 3.79 & -0.86 & 1.04 & 1.04 & 8.61 & 0.57 \\
\hline 16204714-2606162 & 6048548399391137152 & 5920 & 3.02 & -0.56 & 1.07 & 1.10 & 8.97 & 0.32 \\
\hline $16204887+7014507$ & 1652927273492300160 & 4981 & 4.16 & -0.22 & -0.68 & -0.68 & 7.53 & -0.06 \\
\hline $16211341+3512582$ & 1329412882010974976 & 4914 & 4.78 & -0.76 & 0.18 & 0.18 & 7.85 & 0.01 \\
\hline $16213044-7002350$ & 5807311386428745984 & 5949 & 3.52 & -0.90 & 0.35 & 0.35 & 7.88 & -0.12 \\
\hline 16213929-7135317 & 5806920991081963136 & 4816 & 1.16 & -2.17 & -0.20 & 0.44 & 6.69 & 0.18 \\
\hline $16220240+0700378$ & 4439734017734002944 & 4982 & 1.39 & -2.40 & -0.14 & 0.43 & 6.46 & 0.39 \\
\hline $16221768+1134274$ & 4459979806371200512 & 4968 & 1.34 & -2.17 & -0.14 & 0.42 & 6.68 & 0.53 \\
\hline $16224822+5100127$ & 1423692705557972992 & 4793 & 4.34 & -0.69 & -0.14 & -0.14 & 7.60 & 0.51 \\
\hline $16225698-3234118$ & 6025040458531718016 & 5486 & 4.18 & -0.05 & 0.17 & 0.17 & 8.55 & -0.12 \\
\hline 16231806-3110431 & 6037281562002354048 & 5407 & 3.51 & -0.53 & 0.57 & 0.57 & 8.47 & 0.30 \\
\hline $16232673+0535156$ & 4437155113207686400 & 5240 & 4.73 & -0.50 & 0.00 & 0.00 & 7.93 & 0.23 \\
\hline $16234350+2958278$ & 1318193396641660544 & 4808 & 4.00 & -1.34 & 0.62 & 0.62 & 7.71 & 0.05 \\
\hline $16241901+0630059$ & 4439457593640443392 & 4621 & 0.55 & -2.64 & -0.12 & 0.59 & 6.39 & 0.43 \\
\hline $16243746+8055162$ & 1710372289277885440 & 5199 & 4.76 & -0.05 & -0.02 & -0.02 & 8.36 & 0.03 \\
\hline $16244834+1804359$ & 4467003074331962880 & 4704 & 2.78 & -1.77 & 0.48 & 0.50 & 7.15 & 0.32 \\
\hline $16251518+0520267$ & 4437084641390808960 & 4605 & 0.95 & -2.47 & -0.33 & 0.42 & 6.38 & 0.53 \\
\hline 16253573-0834395 & 4350830702966299648 & 5147 & 1.83 & -2.55 & 0.67 & 0.82 & 6.70 & 0.21 \\
\hline 16254695-3354120 & 6023951392267153408 & 6065 & 3.83 & -0.64 & 0.83 & 0.83 & 8.61 & 0.38 \\
\hline 16254871-1359117 & 4328986293140183680 & 5069 & 1.62 & -2.53 & 0.07 & 0.45 & 6.36 & 0.17 \\
\hline $16260791-2025280$ & 6052684487252718080 & 5605 & 3.72 & -1.08 & 1.37 & 1.37 & 8.71 & 0.53 \\
\hline $16260857+3126550$ & 1324425389891741696 & 4505 & 4.25 & -1.44 & 0.01 & 0.01 & 7.01 & -0.19 \\
\hline 16263668-0628581 & 4352076965036319872 & 5301 & 4.31 & -0.23 & 0.07 & 0.07 & 8.28 & 0.02 \\
\hline $16270418+0322290$ & 4433540296934619520 & 4763 & 1.00 & -2.49 & 0.02 & 0.69 & 6.63 & 0.55 \\
\hline $16272230+3655279$ & 1331122622592397568 & 4853 & 1.15 & -2.59 & -0.44 & 0.25 & 6.09 & 0.24 \\
\hline
\end{tabular}


Table 3 (continued)

\begin{tabular}{|c|c|c|c|c|c|c|c|c|}
\hline $\begin{array}{c}\text { Star Name } \\
(2 \mathrm{MASS})\end{array}$ & $\begin{array}{c}\text { Star Name } \\
(\text { Gaia EDR3) }\end{array}$ & $\begin{array}{l}T_{\text {eff }} \\
(\mathrm{K})\end{array}$ & $\begin{array}{l}\log g \\
(\mathrm{cgs})\end{array}$ & {$[\mathrm{Fe} / \mathrm{H}]$} & {$[\mathrm{C} / \mathrm{Fe}]$} & {$[\mathrm{C} / \mathrm{Fe}]_{c}$} & $A(\mathrm{C})_{c}$ & {$[\mathrm{Mg} / \mathrm{Fe}]$} \\
\hline $16272250-0848362$ & 4350762533245253632 & 4830 & 1.27 & -2.54 & 0.03 & 0.62 & 6.52 & 0.25 \\
\hline $16272730-1210551$ & 4331507503365927680 & 5173 & 4.14 & -0.39 & -0.55 & -0.55 & 7.49 & 0.10 \\
\hline $16272732+1123305$ & 4459078580497051136 & 5242 & 4.73 & -0.74 & 0.15 & 0.15 & 7.83 & 0.42 \\
\hline $16274702-3314031$ & 6024841279438699008 & 5108 & 2.69 & -0.95 & 0.66 & 0.68 & 8.16 & 0.40 \\
\hline $16283300+1722329$ & 4467079971426476160 & 4698 & 1.05 & -2.57 & -0.33 & 0.40 & 6.26 & 0.40 \\
\hline $16290198+1430507$ & 4463821083383973760 & 4674 & 1.23 & -3.55 & -0.20 & 0.44 & 5.32 & $\cdots$ \\
\hline $16293666+0213503$ & 4432101100636150400 & 4916 & 4.29 & -0.89 & 0.36 & 0.36 & 7.90 & 0.09 \\
\hline $16300807+3459161$ & 1326275047622823552 & 5254 & 4.60 & -0.81 & 0.37 & 0.37 & 7.99 & 0.43 \\
\hline $16302614-3342496$ & 6024015541893607808 & 6448 & 3.36 & -1.15 & 1.12 & 1.13 & 8.41 & 0.14 \\
\hline $16303876+1329513$ & 4460685211799513600 & 4920 & 1.84 & -1.86 & 0.01 & 0.19 & 6.76 & 0.24 \\
\hline $16305318+4405187$ & 1405543067118031488 & 4748 & 4.73 & -1.37 & 0.10 & 0.10 & 7.15 & 0.41 \\
\hline $16312561+1408014$ & 4460744722866525824 & 5232 & 4.48 & -0.46 & 0.28 & 0.28 & 8.25 & 0.00 \\
\hline $16320549+0401083$ & 4433503532014896512 & 5511 & 3.47 & -2.27 & 0.71 & 0.71 & 6.86 & 0.32 \\
\hline $16322157+1615449$ & 4465786018039320320 & 4791 & 1.53 & -1.66 & -0.35 & 0.06 & 6.83 & 0.35 \\
\hline $16323169+1555079$ & 4465571613272013440 & 5068 & 1.53 & -2.39 & -0.10 & 0.39 & 6.44 & 0.23 \\
\hline $16325211-8327479$ & 5768596864756350976 & 5082 & 3.52 & -1.37 & 0.34 & 0.34 & 7.40 & -0.10 \\
\hline $16325439-7525259$ & 5781162663455262080 & 4779 & 2.31 & -0.34 & -0.63 & -0.60 & 7.49 & 0.23 \\
\hline $16333188+5906052$ & 1623752935039977088 & 5433 & 3.17 & -2.85 & 0.18 & 0.18 & 5.75 & 0.13 \\
\hline $16341169+4636439$ & 1407185565691196672 & 4876 & 4.03 & -0.30 & -0.09 & -0.09 & 8.04 & 0.52 \\
\hline $16342046+1157567$ & 4459450868260931456 & 5211 & 2.81 & -2.18 & 0.42 & 0.43 & 6.68 & 0.41 \\
\hline $16343097+0620345$ & 4438931438666007296 & 4822 & 1.17 & -2.33 & -0.40 & 0.29 & 6.39 & 0.37 \\
\hline $16344239+2231419$ & 1298095453661818880 & 4786 & 4.75 & -1.04 & 0.28 & 0.28 & 7.67 & 0.01 \\
\hline $16350481+1137192$ & 4459250349123575808 & 4597 & 0.60 & -2.67 & -0.05 & 0.67 & 6.43 & 0.36 \\
\hline $16352407-3359508$ & 6024133674994546944 & 5795 & 4.36 & -0.83 & 0.98 & 0.98 & 8.58 & 0.45 \\
\hline $16353942+1442022$ & 4462370655749089792 & 4929 & 4.77 & -0.61 & -0.18 & -0.18 & 7.64 & 0.26 \\
\hline $16354720+1246210$ & 4459657718184334720 & 4909 & 4.89 & -0.42 & -0.16 & -0.16 & 7.85 & 0.24 \\
\hline $16360774+5058387$ & 1411871031413851648 & 5539 & 4.74 & -0.50 & 0.12 & 0.12 & 8.05 & 0.11 \\
\hline $16373418+3852001$ & 1331616986212008192 & 5206 & 2.43 & -2.93 & 0.57 & 0.58 & 6.08 & 0.31 \\
\hline $16374852+0734375$ & 4439132546216071296 & 6244 & 3.78 & -0.89 & 0.68 & 0.68 & 8.22 & 0.36 \\
\hline $16382363-6644037$ & 5815570715927353216 & 5149 & 4.06 & -0.51 & -0.49 & -0.49 & 7.43 & 0.14 \\
\hline $16383378+0540276$ & 4435778760872577024 & 4906 & 4.64 & -0.06 & -0.29 & -0.29 & 8.08 & -0.02 \\
\hline $16384693+1812116$ & 4562485587504234880 & 5095 & 1.53 & -2.31 & -0.26 & 0.22 & 6.34 & 0.58 \\
\hline $16385168+1723396$ & 4466157103214240256 & 5010 & 1.81 & -2.56 & 0.56 & 0.73 & 6.60 & -0.17 \\
\hline $16385848+3000087$ & 1311872265637358336 & 4949 & 4.74 & -0.67 & 0.20 & 0.20 & 7.96 & -0.06 \\
\hline $16391895+0338467$ & 4434135338883534336 & 5310 & 3.57 & -1.90 & 0.77 & 0.77 & 7.29 & 0.05 \\
\hline $16393846+0740507$ & 4445130901837683584 & 4686 & 4.23 & -1.35 & 0.48 & 0.48 & 7.56 & -0.10 \\
\hline $16394169+4228154$ & 1356989076015732864 & 4838 & 1.75 & -1.91 & -0.03 & 0.22 & 6.74 & 0.20 \\
\hline $16394396+4249163$ & 1357571404861995136 & 4634 & 1.29 & -1.82 & -0.36 & 0.16 & 6.77 & 0.10 \\
\hline $16402468+1550407$ & 4462850115836027648 & 4885 & 1.22 & -2.90 & -0.38 & 0.28 & 5.81 & 0.52 \\
\hline $16413086+2631045$ & 1301433231662419840 & 4925 & 1.88 & -2.23 & -0.15 & 0.00 & 6.20 & 0.21 \\
\hline $16414836+1216122$ & 4460825777486994048 & 5070 & 2.04 & -2.64 & 0.45 & 0.49 & 6.28 & 0.28 \\
\hline $16420651+2604225$ & 1301214463209079040 & 4647 & 4.78 & -1.26 & 0.26 & 0.26 & 7.44 & 0.12 \\
\hline
\end{tabular}


Table 3 (continued)

\begin{tabular}{|c|c|c|c|c|c|c|c|c|}
\hline $\begin{array}{c}\text { Star Name } \\
\text { (2MASS) }\end{array}$ & $\begin{array}{c}\text { Star Name } \\
\text { (Gaia EDR3) }\end{array}$ & $\begin{array}{l}T_{\text {eff }} \\
(\mathrm{K})\end{array}$ & $\begin{array}{l}\log g \\
(\mathrm{cgs})\end{array}$ & {$[\mathrm{Fe} / \mathrm{H}]$} & {$[\mathrm{C} / \mathrm{Fe}]$} & {$[\mathrm{C} / \mathrm{Fe}]_{c}$} & $A(\mathrm{C})_{c}$ & {$[\mathrm{Mg} / \mathrm{Fe}]$} \\
\hline $16421928+2344032$ & 1299750291677336960 & 4570 & 0.81 & -2.94 & 0.27 & 0.95 & 6.44 & 0.18 \\
\hline $16423938-8306366$ & 5768700008393198464 & 5090 & 2.01 & -2.28 & 0.42 & 0.48 & 6.62 & 0.11 \\
\hline $16430853+0426005$ & 4434657430811775232 & 4789 & 4.68 & -0.99 & 0.23 & 0.23 & 7.67 & 0.12 \\
\hline $16432853+1024103$ & 4446900634521443584 & 4603 & 1.12 & -2.50 & -0.30 & 0.42 & 6.35 & 0.44 \\
\hline $16435657+3232386$ & 1314234398867571072 & 4808 & 1.39 & -2.09 & -0.11 & 0.41 & 6.75 & 0.35 \\
\hline $16440315+1154212$ & 4448745095340450944 & 5265 & 4.34 & -0.50 & 0.28 & 0.28 & 8.21 & 0.42 \\
\hline 16443996-7951474 & 5775442729091999616 & 4898 & 1.28 & -2.44 & -0.21 & 0.42 & 6.42 & 0.24 \\
\hline $16461012+3554551$ & 1327313154102801664 & 5019 & 1.74 & -1.88 & -0.26 & 0.02 & 6.57 & 0.32 \\
\hline $16462075+1719330$ & 4559215674287937536 & 5178 & 4.78 & -0.43 & 0.08 & 0.08 & 8.08 & -0.06 \\
\hline $16462090+0333477$ & 4433981853933019264 & 4849 & 1.22 & -2.47 & -0.20 & 0.46 & 6.41 & 0.45 \\
\hline $16464088+0311203$ & 4385915672332609280 & 4920 & 4.64 & -0.28 & -0.21 & -0.21 & 7.94 & 0.13 \\
\hline $16471020+5015587$ & 1412373954904373760 & 5058 & 2.11 & -2.25 & 0.53 & 0.55 & 6.74 & 0.39 \\
\hline $16472419+1507161$ & 4461858837385161088 & 5318 & 4.77 & -1.11 & 0.18 & 0.18 & 7.50 & 0.55 \\
\hline $16472795+2336019$ & 1299862235704972928 & 5136 & 2.36 & -1.54 & 0.35 & 0.37 & 7.25 & 0.16 \\
\hline $16474061+1340279$ & 4461395668115705344 & 4787 & 0.91 & -2.05 & -0.21 & 0.43 & 6.81 & 0.51 \\
\hline $16482585+4245290$ & 1356678949313407360 & 5118 & 3.04 & -0.57 & 0.12 & 0.13 & 7.99 & 0.29 \\
\hline $16483067+5232124$ & 1413923647824211456 & 5150 & 3.15 & -0.48 & -0.37 & -0.35 & 7.60 & 0.01 \\
\hline $16485963+2050021$ & 4564767112786411648 & 5039 & 4.66 & -0.95 & 0.24 & 0.24 & 7.72 & 0.40 \\
\hline $16491646+1922215$ & 4562883030895304960 & 5572 & 3.32 & -1.69 & 0.49 & 0.50 & 7.24 & 0.08 \\
\hline $16492419+1810509$ & 4559694756410004480 & 5147 & 4.71 & -0.63 & 0.20 & 0.20 & 8.00 & 0.03 \\
\hline $16501693+3227048$ & 1314079917484186880 & 4733 & 0.93 & -2.31 & -0.46 & 0.29 & 6.41 & 0.53 \\
\hline $16501966+2135346$ & 4565674033785572096 & 4932 & 1.55 & -2.03 & -0.28 & 0.15 & 6.56 & 0.17 \\
\hline $16505552+5951110$ & 1438058374730240256 & 4714 & 0.90 & -2.69 & 0.11 & 0.79 & 6.52 & 0.34 \\
\hline $16510088+3648458$ & 1351373148578890624 & 5651 & 4.13 & -1.02 & 0.55 & 0.55 & 7.96 & 0.11 \\
\hline $16511460+5352298$ & 1426065314211359488 & 5097 & 2.40 & -1.94 & 0.36 & 0.37 & 6.86 & -0.14 \\
\hline $16514772+3844288$ & 1352397519754683136 & 5141 & 2.56 & -2.31 & 0.28 & 0.29 & 6.41 & 0.03 \\
\hline $16523005+0311543$ & 4386055756985792640 & 4763 & 1.34 & -2.00 & 0.02 & 0.50 & 6.93 & 0.52 \\
\hline $16534583+2531166$ & 1306113268546500352 & 4614 & 4.50 & -0.60 & -0.70 & -0.70 & 7.14 & 0.02 \\
\hline $16534773+3049290$ & 1312862856894855040 & 5092 & 2.18 & -1.92 & 0.30 & 0.31 & 6.82 & 0.37 \\
\hline $16545744+2603567$ & 1306294683667797248 & 5193 & 4.49 & -0.47 & 0.33 & 0.33 & 8.30 & 0.42 \\
\hline $16553977-8810318$ & 5765193738828393600 & 5063 & 1.45 & -2.15 & -0.57 & -0.04 & 6.24 & $\ldots$ \\
\hline $16563138+3921373$ & 1352143528272068864 & 5145 & 1.94 & -2.07 & -0.13 & -0.03 & 6.33 & 0.12 \\
\hline $16563406+1516544$ & 4545782356611145728 & 4657 & 1.09 & -2.27 & -0.17 & 0.50 & 6.66 & 0.46 \\
\hline $16574714+4326284$ & 1358072923898101120 & 4902 & 4.32 & -0.72 & 0.22 & 0.22 & 7.93 & 0.59 \\
\hline $16581141+4516190$ & 1359217446784103296 & 4960 & 4.76 & -0.21 & -0.32 & -0.32 & 7.90 & -0.05 \\
\hline $16583722+0153432$ & 4384878175734959744 & 5285 & 2.63 & -2.62 & 0.79 & 0.80 & 6.61 & 0.45 \\
\hline $16590140+2521558$ & 4572761867992849920 & 5028 & 1.34 & -2.76 & -0.13 & 0.43 & 6.10 & 0.45 \\
\hline $16591231-5353249$ & 5935760874615991808 & 5071 & 4.49 & 0.01 & 0.52 & 0.52 & 8.96 & -0.09 \\
\hline 17001311-7651059 & 5777695868934489600 & 6137 & 3.44 & -1.26 & 0.84 & 0.84 & 8.01 & -0.07 \\
\hline $17024781+2559130$ & 4573174150493770368 & 4754 & 1.26 & -2.50 & -0.15 & 0.49 & 6.41 & 0.34 \\
\hline $17034238+4012376$ & 1353472361090529536 & 5018 & 4.72 & -0.43 & 0.11 & 0.11 & 8.11 & 0.04 \\
\hline $17034776+2709455$ & 4573403428730280064 & 5423 & 4.32 & -0.53 & 0.10 & 0.10 & 8.00 & -0.01 \\
\hline
\end{tabular}


Table 3 (continued)

\begin{tabular}{|c|c|c|c|c|c|c|c|c|}
\hline $\begin{array}{c}\text { Star Name } \\
\text { (2MASS) }\end{array}$ & $\begin{array}{c}\text { Star Name } \\
\text { (Gaia EDR3) }\end{array}$ & $\begin{array}{l}T_{\text {eff }} \\
(\mathrm{K})\end{array}$ & $\begin{array}{l}\log g \\
(\operatorname{cgs})\end{array}$ & {$[\mathrm{Fe} / \mathrm{H}]$} & {$[\mathrm{C} / \mathrm{Fe}]$} & {$[\mathrm{C} / \mathrm{Fe}]_{c}$} & $A(\mathrm{C})_{c}$ & {$[\mathrm{Mg} / \mathrm{Fe}]$} \\
\hline $17035954+4513186$ & 1358789148349761152 & 5270 & 2.68 & -1.89 & 0.34 & 0.35 & 6.89 & 0.44 \\
\hline $17041564+1932115$ & 4560834812533156480 & 4798 & 1.59 & -2.96 & 0.06 & 0.36 & 5.82 & 0.04 \\
\hline $17041979+6325224$ & 1631229304871201024 & 4769 & 0.91 & -2.15 & -0.36 & 0.36 & 6.64 & 0.44 \\
\hline $17042385+6726017$ & 1636646151984790784 & 4651 & 4.51 & -0.41 & -0.77 & -0.77 & 7.25 & -0.12 \\
\hline $17045983+1948039$ & 4561595434057474304 & 4704 & 1.14 & -2.36 & -0.06 & 0.59 & 6.66 & 0.51 \\
\hline $17054252-6742414$ & 5814397399577566848 & 4839 & 2.45 & -0.31 & -0.83 & -0.80 & 7.32 & 0.11 \\
\hline $17060632+6430248$ & 1632107986461148928 & 5188 & 1.75 & -2.24 & -0.20 & 0.07 & 6.26 & 0.39 \\
\hline $17095788+4825288$ & 1414140487836898048 & 5025 & 4.39 & -0.20 & -0.37 & -0.37 & 7.86 & -0.03 \\
\hline $17104782+4615401$ & 1364943737701366144 & 5461 & 3.47 & -0.57 & 0.52 & 0.52 & 8.38 & 0.12 \\
\hline $17115737+4056337$ & 1341931337289579648 & 5295 & 4.16 & -0.73 & 0.31 & 0.31 & 8.01 & -0.03 \\
\hline $17125716-2214215$ & 4115322081195173248 & 6334 & 3.08 & -1.37 & 1.54 & 1.56 & 8.62 & 0.18 \\
\hline $17130596-3442468$ & 5978819326162668288 & 6827 & 3.01 & -1.44 & 1.85 & 1.87 & 8.87 & 0.12 \\
\hline $17130931+4245360$ & 1354380420256070272 & 5159 & 4.50 & -0.32 & -0.40 & -0.40 & 7.71 & 0.10 \\
\hline $17133146+5029289$ & 1414644171538142464 & 5201 & 4.78 & -0.58 & -0.10 & -0.10 & 7.75 & 0.17 \\
\hline $17134640-2252270$ & 4114531910303984896 & 5755 & 3.03 & -0.95 & 0.97 & 0.99 & 8.47 & 0.22 \\
\hline $17134904+4643336$ & 1364979746707350016 & 4971 & 1.79 & -2.71 & 0.15 & 0.35 & 6.07 & 0.32 \\
\hline $17151412-2251434$ & 4114489441664092160 & 5937 & 3.22 & -0.80 & 0.97 & 0.98 & 8.61 & 0.30 \\
\hline $17154790-5232570$ & 5925618184739644160 & 4928 & 1.72 & -3.19 & 0.38 & 0.54 & 5.78 & 0.07 \\
\hline 17160251-2205199 & 4114972844488807680 & 5451 & 3.95 & -0.78 & 0.65 & 0.65 & 8.30 & -0.01 \\
\hline $17162512+4249078$ & 1360187452261689472 & 5322 & 3.21 & -1.34 & 0.45 & 0.46 & 7.55 & 0.35 \\
\hline $17163270+4302287$ & 1360381309905667840 & 5455 & 4.35 & -0.22 & -0.15 & -0.15 & 8.06 & 0.01 \\
\hline 17163966-8711235 & 5765345368353739392 & 5328 & 2.53 & -2.37 & 0.66 & 0.67 & 6.73 & 0.14 \\
\hline $17174451+3653087$ & 1339989290517076096 & 5252 & 2.56 & -2.60 & 0.63 & 0.64 & 6.47 & 0.28 \\
\hline $17185527+3839047$ & 1340720740627444096 & 4963 & 4.60 & -0.83 & 0.19 & 0.19 & 7.80 & 0.02 \\
\hline 17192865-6853306 & 5811209090730279680 & 5135 & 2.62 & -0.51 & -0.19 & -0.16 & 7.76 & 0.14 \\
\hline $17194406+4515228$ & 1361644549983238272 & 4950 & 1.95 & -2.47 & 0.15 & 0.23 & 6.18 & -0.04 \\
\hline $17212704+5033288$ & 1414790574087583360 & 4731 & 0.97 & -2.18 & -0.03 & 0.60 & 6.85 & 0.47 \\
\hline 17215395-6411338 & 5814091284361742976 & 5022 & 1.30 & -2.92 & 0.34 & 0.88 & 6.39 & 0.30 \\
\hline $17215498+1423078$ & 4543224965582361984 & 4885 & 1.48 & -2.65 & -0.36 & 0.13 & 5.91 & 0.18 \\
\hline $17224220+0353126$ & 4389167576754184192 & 4912 & 1.95 & -1.86 & -0.06 & 0.04 & 6.61 & 0.42 \\
\hline $17234737-5834124$ & 5916061474500671232 & 4827 & 1.43 & -1.97 & 0.00 & 0.45 & 6.91 & -0.01 \\
\hline $17240759+3635398$ & 1337102866336482688 & 5045 & 1.99 & -2.61 & 0.74 & 0.79 & 6.62 & 0.45 \\
\hline 17251201-7832462 & 5776783519098670464 & $\ldots$ & $\ldots$ & $\ldots$ & $\cdots$ & $\cdots$ & $\cdots$ & $\ldots$ \\
\hline $17252775+5318307$ & 1416272239020984576 & 5126 & 2.45 & -2.37 & 0.42 & 0.43 & 6.49 & 0.23 \\
\hline $17254679-8513010$ & 5767118193414597632 & 5642 & 3.87 & -1.10 & 0.57 & 0.57 & 7.91 & -0.05 \\
\hline $17255611+1556131$ & 4543997853536251264 & 5448 & 3.89 & 0.09 & -0.52 & -0.52 & 8.00 & -0.05 \\
\hline $17265179+3527349$ & 1335862475484040064 & 4891 & 1.45 & -2.41 & -0.08 & 0.44 & 6.47 & 0.47 \\
\hline $17272663+3847099$ & 1343578233909298816 & 6867 & 4.60 & -1.11 & 1.21 & 1.21 & 8.53 & 0.23 \\
\hline $17283154-6945443$ & 5810934625139045248 & 5540 & 3.24 & -0.98 & 0.85 & 0.86 & 8.31 & -0.12 \\
\hline $17285162-6941036$ & 5810941016050593536 & 4914 & 1.47 & -2.37 & 0.40 & 0.82 & 6.89 & 0.06 \\
\hline $17285482-5720440$ & 5919234390177666304 & 5559 & 4.61 & -0.39 & 0.25 & 0.25 & 8.29 & 0.00 \\
\hline $17291858+7510248$ & 1655949551783195520 & 4640 & 0.76 & -2.50 & -0.57 & 0.19 & 6.12 & 0.51 \\
\hline
\end{tabular}


Table 3 (continued)

\begin{tabular}{|c|c|c|c|c|c|c|c|c|}
\hline $\begin{array}{l}\text { Star Name } \\
\text { (2MASS) }\end{array}$ & $\begin{array}{c}\text { Star Name } \\
\text { (Gaia EDR3) }\end{array}$ & $\begin{array}{l}T_{\text {eff }} \\
(\mathrm{K})\end{array}$ & $\begin{array}{l}\log g \\
(\operatorname{cgs})\end{array}$ & {$[\mathrm{Fe} / \mathrm{H}]$} & {$[\mathrm{C} / \mathrm{Fe}]$} & {$[\mathrm{C} / \mathrm{Fe}]_{c}$} & $A(\mathrm{C})_{c}$ & {$[\mathrm{Mg} / \mathrm{Fe}]$} \\
\hline $17292254+0305472$ & 4388901086920961024 & 4852 & 1.48 & -2.39 & 0.15 & 0.61 & 6.65 & 0.48 \\
\hline $17293008+5534560$ & 1421238419509501568 & 4983 & 1.68 & -2.88 & 0.51 & 0.76 & 6.31 & 0.31 \\
\hline $17303697+0421414$ & 4389421362079616512 & 4613 & 0.65 & -2.60 & -0.32 & 0.43 & 6.27 & 0.46 \\
\hline $17314553+2843156$ & 4598739479466205568 & 5293 & 2.87 & -2.15 & 0.37 & 0.38 & 6.67 & 0.16 \\
\hline $17315874-5342306$ & 5921618165752908928 & 5066 & 1.86 & -2.34 & 0.52 & 0.68 & 6.77 & 0.12 \\
\hline $17330791+2548590$ & 4594030404311114240 & 4894 & 4.68 & -0.45 & -0.22 & -0.22 & 7.76 & 0.09 \\
\hline $17331311+1713463$ & 4550007577936164864 & 4763 & 4.75 & -1.15 & 0.53 & 0.53 & 7.81 & 0.21 \\
\hline $17331323+3152308$ & 4601590066440260096 & 4778 & 1.65 & -1.98 & -0.38 & -0.03 & 6.42 & 0.02 \\
\hline $17332654+2633432$ & 4594840366419966208 & 5443 & 3.00 & -3.02 & 0.56 & 0.56 & 5.97 & 0.57 \\
\hline $17333170+5456025$ & 1420926742322768640 & 4887 & 4.54 & -0.91 & 0.17 & 0.17 & 7.68 & -0.06 \\
\hline $17333194-5022586$ & 5946394938738915584 & 5170 & 2.08 & -1.24 & 0.15 & 0.20 & 7.39 & 0.25 \\
\hline $17334423+1911331$ & 4553866722012938496 & 5530 & 4.61 & -0.33 & 0.15 & 0.15 & 8.25 & -0.03 \\
\hline $17334679-6744028$ & 5813027335062608384 & 4757 & 2.43 & -0.51 & -0.65 & -0.62 & 7.29 & 0.18 \\
\hline $17335350+1815568$ & 4550692088345948672 & 4921 & 1.65 & -2.31 & 0.12 & 0.51 & 6.63 & 0.20 \\
\hline $17335522+1719221$ & 4550032381370693376 & 4784 & 4.47 & -0.29 & -0.56 & -0.56 & 7.58 & -0.06 \\
\hline $17355093+1411487$ & 4542596697766095232 & 5358 & 4.18 & -0.34 & -0.48 & -0.48 & 7.62 & -0.12 \\
\hline $17370392-7408233$ & 5803200381170398336 & 5175 & 4.23 & -0.06 & -0.60 & -0.60 & 7.77 & -0.03 \\
\hline $17371472+1603347$ & 4549079418323779712 & 6600 & 3.75 & -0.69 & 1.16 & 1.16 & 8.90 & 0.43 \\
\hline $17373165+0109351$ & 4375387436181302912 & 5025 & 1.79 & -2.65 & 0.63 & 0.80 & 6.58 & 0.42 \\
\hline $17374593-4903259$ & 5946896105559051392 & 5310 & 4.24 & -0.09 & -0.52 & -0.52 & 7.82 & -0.01 \\
\hline $17381613+1806130$ & 4550553412442483456 & 4785 & 3.38 & -1.91 & 0.83 & 0.83 & 7.35 & 0.01 \\
\hline $17382795+8319481$ & 1711261033974731648 & 5292 & 4.94 & -0.72 & 0.17 & 0.17 & 7.89 & 0.39 \\
\hline $17390078-7342041$ & 5803233817494625536 & 5903 & 3.11 & -1.03 & 0.54 & 0.55 & 7.95 & 0.05 \\
\hline $17390590+1504553$ & 4548685209048009600 & 4698 & 1.14 & -2.20 & -0.13 & 0.50 & 6.73 & 0.53 \\
\hline $17393096-5334586$ & 5921761544627396352 & 5088 & 2.04 & -2.32 & 0.37 & 0.42 & 6.52 & 0.14 \\
\hline $17412217-6605479$ & 5812858972344007680 & 4679 & 1.03 & -2.51 & -0.24 & 0.48 & 6.40 & -0.10 \\
\hline $17412978+2128403$ & 4556052314917665920 & 5352 & 3.61 & -0.24 & -0.33 & -0.33 & 7.86 & 0.05 \\
\hline $17415044+2448137$ & 4581633552441283328 & 4979 & 1.92 & -2.67 & 0.33 & 0.42 & 6.18 & 0.36 \\
\hline $17421570-5343061$ & 5921703652781168768 & 4775 & 1.17 & -2.60 & -0.13 & 0.53 & 6.37 & 0.01 \\
\hline $17422649+3217551$ & 4601113221991774976 & 4874 & 4.70 & -0.72 & 0.24 & 0.24 & 7.95 & 0.00 \\
\hline $17425789+0417381$ & 4473307880166709248 & 5223 & 1.72 & -2.75 & 0.14 & 0.39 & 6.08 & 0.25 \\
\hline $17431049+5633516$ & 1421354113045323904 & 4560 & 4.07 & -0.45 & -0.91 & -0.91 & 7.07 & 0.20 \\
\hline $17435113-5359333$ & 5921684136450078592 & 5124 & 1.37 & -2.45 & 0.11 & 0.64 & 6.62 & 0.14 \\
\hline $17442011+0827068$ & 4488216467565808512 & 5273 & 2.36 & -1.77 & 0.35 & 0.37 & 7.04 & 0.28 \\
\hline $17442313+1055585$ & 4489909058337283584 & 5258 & 4.51 & -0.23 & -0.23 & -0.23 & 7.97 & 0.04 \\
\hline $17451045+1600587$ & 4501194587422903936 & 5225 & 4.62 & -0.21 & -0.25 & -0.25 & 7.97 & -0.04 \\
\hline $17452465+0613284$ & 4474168965270090368 & 4715 & 1.18 & -2.75 & -0.57 & 0.14 & 5.81 & 0.38 \\
\hline $17452911+0549214$ & 4474076782390335616 & 5146 & 1.66 & -2.68 & -0.42 & -0.10 & 5.65 & 0.54 \\
\hline $17460256+1657355$ & 4549397894441550848 & 5226 & 3.62 & -0.68 & 0.15 & 0.15 & 7.91 & 0.19 \\
\hline $17465957+1519406$ & 4501077351995658496 & 5131 & 2.34 & -3.54 & 0.71 & 0.72 & 5.62 & 0.17 \\
\hline $17470290-5058143$ & 5945699978662588416 & 5286 & 4.57 & -1.07 & 0.57 & 0.57 & 7.93 & 0.19 \\
\hline $17471725-6723320$ & 5812356121877182080 & 4713 & 1.09 & -2.33 & -0.30 & 0.42 & 6.52 & 0.26 \\
\hline
\end{tabular}


Table 3 (continued)

\begin{tabular}{|c|c|c|c|c|c|c|c|c|}
\hline $\begin{array}{l}\text { Star Name } \\
(2 \mathrm{MASS})\end{array}$ & $\begin{array}{c}\text { Star Name } \\
\text { (Gaia EDR3) }\end{array}$ & $\begin{array}{l}T_{\text {eff }} \\
(\mathrm{K})\end{array}$ & $\begin{array}{l}\log g \\
(\operatorname{cgs})\end{array}$ & {$[\mathrm{Fe} / \mathrm{H}]$} & {$[\mathrm{C} / \mathrm{Fe}]$} & {$[\mathrm{C} / \mathrm{Fe}]_{c}$} & $A(\mathrm{C})_{c}$ & {$[\mathrm{Mg} / \mathrm{Fe}]$} \\
\hline $17471835-0035363$ & 4371256532336413056 & 5829 & 4.28 & -0.51 & 0.20 & 0.20 & 8.12 & 0.01 \\
\hline $17472373+5750230$ & 1422587593292960640 & 4684 & 0.81 & -2.19 & -0.29 & 0.41 & 6.65 & 0.53 \\
\hline $17473698+5229473$ & 1368840406910596992 & 5194 & 2.31 & -2.39 & 0.57 & 0.58 & 6.62 & 0.18 \\
\hline 17485598-7415094 & 5803007691756413056 & 4816 & 1.64 & -2.85 & -0.21 & 0.09 & 5.67 & 0.09 \\
\hline $17493006+1616540$ & 4501261550253801344 & 5032 & 2.26 & -1.74 & 0.16 & 0.18 & 6.88 & 0.32 \\
\hline $17493192-5314272$ & 5921036111776622592 & 5429 & 2.96 & -1.75 & 0.36 & 0.38 & 7.06 & 0.22 \\
\hline $17505960+1845110$ & 4551354475384137600 & 4976 & 1.61 & -1.98 & -0.09 & 0.27 & 6.72 & 0.21 \\
\hline $17512033+4313226$ & 1346347658884884608 & 5040 & 4.61 & -0.81 & 0.32 & 0.32 & 7.94 & 0.51 \\
\hline $17513368+0735474$ & 4475867611955393280 & 5424 & 4.01 & -1.56 & 0.31 & 0.31 & 7.18 & 0.10 \\
\hline $17514375-8447409$ & 5767473713627782016 & 4793 & 1.25 & -2.80 & 0.24 & 0.81 & 6.44 & -0.15 \\
\hline $17515635+0549550$ & 4473933497983061632 & 5401 & 4.30 & -0.20 & -0.20 & -0.20 & 8.03 & 0.03 \\
\hline $17530760+0355502$ & 4472708298435677312 & 5628 & 4.25 & -0.63 & 0.35 & 0.35 & 8.15 & 0.03 \\
\hline $17531820-5828173$ & 5918003040226875648 & 5032 & 1.93 & -1.62 & -0.09 & 0.02 & 6.83 & 0.22 \\
\hline $17553087+1344549$ & 4499700449901916672 & 4877 & 0.91 & -2.31 & -0.26 & 0.47 & 6.59 & 0.50 \\
\hline $17563856-6259025$ & 5911002106095737472 & 4604 & 4.74 & -2.37 & 0.83 & 0.83 & 6.89 & 0.12 \\
\hline $17570985+6104567$ & 1435424254107968896 & 5018 & 2.56 & -0.83 & 0.15 & 0.17 & 7.77 & -0.04 \\
\hline $17580876-5017025$ & 5947067839841987840 & 4861 & 1.60 & -1.87 & -0.31 & 0.07 & 6.64 & 0.32 \\
\hline $17582442-5200036$ & 5945364387093681024 & 4666 & 1.14 & -2.80 & -0.14 & 0.55 & 6.18 & 0.15 \\
\hline $17583367+0851134$ & 4476514330950969216 & 4943 & 1.80 & -2.93 & 0.45 & 0.59 & 6.09 & 0.24 \\
\hline $17590214+0226114$ & 4468887736043966848 & 5035 & 4.30 & -1.18 & 0.49 & 0.49 & 7.75 & -0.08 \\
\hline $17593067+0547081$ & 4474462912835838336 & 5378 & 4.38 & -0.73 & 0.85 & 0.85 & 8.55 & 0.37 \\
\hline $17595240+0845349$ & 4476464986070993664 & 4743 & 1.01 & -2.20 & -0.39 & 0.31 & 6.54 & 0.42 \\
\hline $17595579+0402394$ & 4469713473577956352 & 4919 & 1.36 & -2.22 & -0.18 & 0.41 & 6.62 & 0.53 \\
\hline $18004880+4241389$ & 2113828593508701696 & 5907 & 4.63 & -0.32 & 0.13 & 0.13 & 8.24 & 0.07 \\
\hline $18012521+1607266$ & 4501806912321934592 & 5021 & 3.43 & -1.10 & 0.27 & 0.27 & 7.60 & 0.04 \\
\hline 18013011-6117020 & 6635006422507626880 & 5090 & 3.03 & -0.10 & -0.79 & -0.78 & 7.55 & 0.18 \\
\hline $18015056-5628090$ & 6651913342230612608 & 5458 & 4.48 & -0.45 & 0.27 & 0.27 & 8.25 & -0.01 \\
\hline $18025682-7550189$ & 6414540257944747008 & 5997 & 3.36 & -1.22 & 1.01 & 1.02 & 8.23 & -0.08 \\
\hline $18033495+4211510$ & 2113756098755468288 & 5009 & 1.65 & -2.49 & 0.00 & 0.36 & 6.30 & 0.35 \\
\hline $18042621-7447453$ & 6417661629719563264 & 5053 & 1.41 & -2.21 & 0.18 & 0.65 & 6.87 & 0.16 \\
\hline $18042842-7051331$ & 6431507302910109952 & 4992 & 1.65 & -2.40 & 0.35 & 0.68 & 6.71 & 0.17 \\
\hline $18045298-5543550$ & 6652206220339855104 & 4935 & 1.58 & -2.37 & 0.06 & 0.48 & 6.54 & 0.19 \\
\hline $18045953+0826177$ & 4476206639494994944 & 5221 & 2.44 & -1.64 & 0.38 & 0.40 & 7.19 & 0.07 \\
\hline $18052892+0754210$ & 4475421897432191616 & 5569 & 0.99 & -1.66 & 0.05 & 0.47 & 7.24 & $\ldots$ \\
\hline $18062441-6457049$ & 6629637992566177664 & 5653 & 4.29 & -0.87 & 0.37 & 0.37 & 7.93 & 0.04 \\
\hline $18063457+0547198$ & 4471868065390279680 & 5433 & 3.04 & -1.23 & 0.14 & 0.15 & 7.35 & 0.26 \\
\hline $18072175+5952412$ & 2158636960809774336 & 4723 & 4.79 & -0.81 & 0.02 & 0.02 & 7.64 & 0.10 \\
\hline $18073369+5849547$ & 2152484604840257280 & 4747 & 4.67 & -0.97 & 0.02 & 0.02 & 7.48 & 0.00 \\
\hline $18082002-5104378$ & 6702907209758894848 & 5689 & 2.73 & -3.81 & 1.40 & 1.40 & 6.02 & $\ldots$ \\
\hline $18083624-6750220$ & 6435985682492232320 & 5281 & 4.25 & -0.61 & 0.31 & 0.31 & 8.13 & 0.31 \\
\hline $18085682-2106299$ & 4094063947258804608 & 6448 & 3.46 & -1.05 & 0.83 & 0.83 & 8.21 & 0.15 \\
\hline $18095421-6630506$ & 6437097494904121600 & 5988 & 4.11 & -0.54 & 0.34 & 0.34 & 8.22 & 0.12 \\
\hline
\end{tabular}


Table 3 (continued)

\begin{tabular}{|c|c|c|c|c|c|c|c|c|}
\hline $\begin{array}{l}\text { Star Name } \\
(2 \mathrm{MASS})\end{array}$ & $\begin{array}{c}\text { Star Name } \\
\text { (Gaia EDR3) }\end{array}$ & $\begin{array}{l}T_{\text {eff }} \\
(\mathrm{K})\end{array}$ & $\begin{array}{l}\log g \\
(\operatorname{cgs})\end{array}$ & {$[\mathrm{Fe} / \mathrm{H}]$} & {$[\mathrm{C} / \mathrm{Fe}]$} & {$[\mathrm{C} / \mathrm{Fe}]_{c}$} & $A(\mathrm{C})_{c}$ & {$[\mathrm{Mg} / \mathrm{Fe}]$} \\
\hline 18100815-6731119 & 6436045915114732672 & 4837 & 4.83 & -1.36 & 0.78 & 0.78 & 7.85 & -0.03 \\
\hline $18102490-7119083$ & 6431254307857238784 & 5440 & 3.00 & -1.85 & 0.42 & 0.43 & 7.01 & 0.20 \\
\hline $18123519-6033510$ & 6635102251817906304 & 5202 & 4.75 & -0.73 & 0.20 & 0.20 & 7.90 & 0.33 \\
\hline $18124977+5854462$ & 2152380494834185984 & 5007 & 1.01 & -2.16 & -0.04 & 0.58 & 6.85 & 0.54 \\
\hline $18135644+4824196$ & 2122140905709563904 & 5230 & 4.76 & -0.48 & 0.12 & 0.12 & 8.08 & 0.08 \\
\hline $18141754+1612114$ & 4498809506188122624 & 6923 & 4.34 & -1.73 & 2.47 & 2.48 & 9.17 & -0.03 \\
\hline $18145902+5852559$ & 2152342188021376384 & 4972 & 4.94 & -0.82 & 0.13 & 0.13 & 7.74 & 0.29 \\
\hline $18150365-3751206$ & 6727766450449365248 & 5034 & 1.51 & -2.74 & 0.55 & 0.92 & 6.61 & 0.14 \\
\hline $18162072-4725281$ & 6707603223921252608 & 5196 & 1.95 & -2.17 & 0.18 & 0.27 & 6.52 & -0.10 \\
\hline $18165208+6146498$ & 2159095113561329024 & 4840 & 1.22 & -2.64 & -0.05 & 0.58 & 6.36 & 0.41 \\
\hline $18171654+1311582$ & 4485396250300800768 & 5520 & 4.35 & -1.07 & 0.43 & 0.43 & 7.78 & 0.01 \\
\hline $18190641-6824118$ & 6432812045255424128 & 5352 & 2.01 & -0.37 & -0.67 & -0.48 & 7.59 & $\ldots$ \\
\hline 18191919-2029254 & 4091364130814956032 & 5772 & 2.10 & -0.49 & 0.50 & 0.54 & 8.48 & 0.56 \\
\hline $18192007-5552473$ & 6649365494611393920 & 4555 & 3.24 & -1.58 & 0.71 & 0.72 & 7.57 & -0.01 \\
\hline $18193127-3713134$ & 6728130968571404800 & 5054 & 1.88 & -1.69 & 0.23 & 0.38 & 7.12 & 0.42 \\
\hline $18194826-5310564$ & 6653631805885843200 & 5193 & 2.01 & -2.32 & 0.62 & 0.68 & 6.79 & 0.14 \\
\hline 18203066-2016012 & 4091455699522188416 & 5881 & 4.20 & -0.76 & 0.69 & 0.69 & 8.36 & 0.34 \\
\hline 18204921-3419480 & 4044557100560972800 & 5144 & 2.15 & -1.75 & 0.29 & 0.31 & 6.99 & 0.31 \\
\hline $18211549+5653298$ & 2150956936513301632 & 5079 & 1.79 & -2.10 & -0.02 & 0.21 & 6.54 & 0.36 \\
\hline $18220774-7608090$ & 6413638383531665664 & 5512 & 4.33 & -0.21 & -0.18 & -0.18 & 8.04 & -0.09 \\
\hline $18223327-5258169$ & 6653722004497397120 & 4704 & 4.62 & -0.73 & -0.15 & -0.15 & 7.55 & 0.12 \\
\hline 18231998-3926193 & 6726604713316398336 & 4674 & 1.05 & -2.57 & -0.36 & 0.37 & 6.23 & 0.05 \\
\hline $18232072-7312133$ & 6418085663251356416 & 5477 & 4.54 & -0.52 & 0.02 & 0.02 & 7.93 & 0.00 \\
\hline $18273263-5638304$ & 6648380469631584128 & 5330 & 2.56 & -1.54 & 0.89 & 0.91 & 7.80 & 0.32 \\
\hline $18274709+1732530$ & 4522680968780032128 & 5144 & 1.49 & -2.40 & -0.09 & 0.42 & 6.45 & 0.49 \\
\hline $18285509-3410250$ & 6734946570607247360 & 5568 & 4.46 & -0.17 & 0.39 & 0.39 & 8.65 & -0.06 \\
\hline $18293868-2010483$ & 4092688806190705152 & 6617 & 2.93 & -1.35 & 1.58 & 1.60 & 8.68 & 0.15 \\
\hline $18301354-4555101$ & 6708532208165979392 & 5124 & 1.97 & -3.50 & 2.86 & 2.91 & 7.84 & 0.32 \\
\hline $18302241-3955233$ & 6723716880376076928 & 5106 & 1.47 & -2.47 & -0.12 & 0.38 & 6.35 & 0.26 \\
\hline $18305087-6953306$ & 6431994249123338240 & 5064 & 3.40 & -1.28 & 0.47 & 0.47 & 7.62 & 0.26 \\
\hline $18311217+4103160$ & 2110452925308798080 & 4866 & 1.47 & -2.53 & 0.21 & 0.67 & 6.57 & 0.36 \\
\hline $18315993-6920161$ & 6432068225640386432 & 4720 & 1.48 & -2.30 & -0.02 & 0.48 & 6.61 & 0.24 \\
\hline $18321423-3829407$ & 6726896461847358848 & 5397 & 2.61 & -1.13 & 0.12 & 0.14 & 7.45 & 0.34 \\
\hline $18324025-6202554$ & 6631161743881174400 & 5075 & 1.93 & -2.35 & 0.53 & 0.64 & 6.71 & 0.29 \\
\hline $18334251+4115292$ & 2110471101610527616 & 5537 & 4.14 & -0.44 & -0.40 & -0.40 & 7.59 & 0.05 \\
\hline $18340800-5627514$ & 6649667246135478144 & 5300 & 3.80 & -0.27 & 0.11 & 0.11 & 8.28 & -0.03 \\
\hline $18352484-3639135$ & 6733341416736455936 & 5390 & 2.42 & -1.93 & 0.75 & 0.76 & 7.26 & 0.03 \\
\hline $18352710-4928306$ & 6703851728906337152 & 5183 & 2.48 & -1.50 & 0.28 & 0.30 & 7.24 & 0.18 \\
\hline $18354018-3948416$ & 6723580609650147712 & 5244 & 1.58 & -2.11 & 0.73 & 1.00 & 7.32 & 0.15 \\
\hline $18360814+1919587$ & 4524742759245230592 & 5021 & 1.99 & -2.56 & 0.03 & 0.08 & 5.96 & 0.13 \\
\hline $18361214-7333443$ & 6418205892271978624 & 4865 & 1.47 & -2.70 & 0.53 & 0.93 & 6.65 & -0.04 \\
\hline 18371329-3141091 & 4046640705089371136 & 5081 & 1.17 & -2.74 & -0.15 & 0.52 & 6.21 & 0.30 \\
\hline
\end{tabular}


Table 3 (continued)

\begin{tabular}{|c|c|c|c|c|c|c|c|c|}
\hline $\begin{array}{c}\text { Star Name } \\
\text { (2MASS) }\end{array}$ & $\begin{array}{c}\text { Star Name } \\
\text { (Gaia EDR3) }\end{array}$ & $\begin{array}{l}T_{\text {eff }} \\
(\mathrm{K})\end{array}$ & $\begin{array}{l}\log g \\
(\operatorname{cgs})\end{array}$ & {$[\mathrm{Fe} / \mathrm{H}]$} & {$[\mathrm{C} / \mathrm{Fe}]$} & {$[\mathrm{C} / \mathrm{Fe}]_{c}$} & $A(\mathrm{C})_{c}$ & {$[\mathrm{Mg} / \mathrm{Fe}]$} \\
\hline $18373068-5538076$ & 6649914292651257984 & 4908 & 1.49 & -2.09 & 0.30 & 0.68 & 7.02 & 0.01 \\
\hline $18395804+4820336$ & 2119887903305583104 & 4731 & 1.24 & -2.44 & -0.20 & 0.44 & 6.43 & 0.37 \\
\hline $18404278-5822181$ & 6636472453764175744 & 5615 & 3.45 & -1.04 & 0.68 & 0.68 & 8.07 & -0.07 \\
\hline $18405632+5805144$ & 2154314260908589184 & 4974 & 1.77 & -2.05 & 0.27 & 0.50 & 6.88 & 0.45 \\
\hline $18411282-6105584$ & 6632762873329420544 & 4766 & 1.18 & -2.29 & -0.34 & 0.34 & 6.48 & 0.27 \\
\hline 18430593-7820300 & 6364899129015765248 & 4947 & 4.47 & -0.42 & -0.21 & -0.21 & 7.80 & 0.11 \\
\hline $18462350-4939426$ & 6656086710412617344 & 5211 & 4.65 & -0.15 & -0.17 & -0.17 & 8.11 & -0.01 \\
\hline 18482959-4911163 & 6656206965197014272 & 5571 & 3.87 & -0.54 & 0.24 & 0.24 & 8.13 & -0.06 \\
\hline $18490576-5304063$ & 6651390073476420736 & 5048 & 4.00 & -0.12 & -0.34 & -0.34 & 7.97 & -0.06 \\
\hline $18491122+4005588$ & 2098297480667292160 & 4969 & 4.69 & -0.28 & -0.39 & -0.39 & 7.76 & 0.01 \\
\hline $18512136+5319111$ & 2146237458947371520 & 5408 & 3.04 & -1.64 & 0.20 & 0.21 & 7.01 & 0.06 \\
\hline $18515139+7849432$ & 2293311669034242432 & 5371 & 2.33 & -1.66 & -0.02 & 0.00 & 6.77 & 0.06 \\
\hline $18523585+4032042$ & 2103587162389240064 & 4800 & 4.15 & -0.38 & -0.14 & -0.14 & 7.91 & 0.47 \\
\hline $18523981+4129288$ & 2104473093882735104 & 4939 & 1.97 & -1.77 & 0.13 & 0.21 & 6.87 & 0.59 \\
\hline $19024429+5351114$ & 2134680487931467648 & 5668 & 4.30 & -0.46 & 0.08 & 0.08 & 8.05 & -0.01 \\
\hline $19025533+4219090$ & 2104015387809994496 & 5113 & 2.08 & -2.51 & 0.63 & 0.66 & 6.59 & 0.39 \\
\hline 19032297-4757304 & 6662155460541591424 & 5306 & 4.42 & -0.31 & 0.50 & 0.50 & 8.62 & 0.00 \\
\hline $19035178-5108429$ & 6657682239223060736 & 4640 & 0.94 & -2.30 & -0.62 & 0.15 & 6.28 & 0.39 \\
\hline $19044854+5029122$ & 2133638842399952256 & 5272 & 3.01 & -1.35 & 0.44 & 0.46 & 7.54 & 0.37 \\
\hline $19052503-7826440$ & 6364286563599950592 & 4957 & 2.30 & -0.26 & -0.84 & -0.81 & 7.36 & 0.06 \\
\hline $19055032-5206090$ & 6656823172744784384 & 4802 & 1.28 & -2.59 & -0.08 & 0.52 & 6.36 & 0.27 \\
\hline $19060062-5118110$ & 6657644374791106304 & 4746 & 0.65 & -3.27 & 0.83 & 1.55 & 6.72 & $\cdots$ \\
\hline 19060226-6310122 & 6438853483690577024 & 4587 & 2.10 & -0.46 & -0.54 & -0.44 & 7.53 & 0.08 \\
\hline 19120066-6302562 & 6438895574370061568 & 4879 & 1.80 & -1.68 & 0.07 & 0.27 & 7.02 & 0.27 \\
\hline $19131047+3313392$ & 2043394428389668992 & 4977 & 1.48 & -2.52 & -0.15 & 0.34 & 6.25 & 0.26 \\
\hline $19155183-4922541$ & 6658254874322598016 & 5218 & 2.45 & -1.99 & 0.41 & 0.42 & 6.86 & -0.04 \\
\hline $19155497+4038463$ & 2101342715562410240 & 4877 & 1.45 & -2.88 & 0.26 & 0.71 & 6.27 & 0.51 \\
\hline $19160765+4246319$ & 2102705938181969408 & 5141 & 2.57 & -2.16 & 0.34 & 0.35 & 6.62 & 0.06 \\
\hline $19184233+8121549$ & 2295494715010792576 & 5641 & 3.49 & -1.42 & 0.82 & 0.82 & 7.83 & 0.54 \\
\hline $19214950-7947382$ & 6361002631604746752 & 6159 & 2.87 & -0.58 & 0.97 & 1.00 & 8.85 & 0.09 \\
\hline $19232375+4720104$ & 2129167334770344704 & 4917 & 1.56 & -2.82 & 0.26 & 0.63 & 6.24 & 0.40 \\
\hline $19233833+4018284$ & 2101121026531480960 & 4884 & 1.83 & -1.76 & -0.15 & 0.05 & 6.72 & 0.49 \\
\hline $19242686+8215294$ & 2295685828172114688 & 6268 & 3.90 & -0.61 & 0.56 & 0.56 & 8.39 & 0.39 \\
\hline 19253278-2828581 & 6764789893132461568 & 5394 & 3.78 & -0.82 & 0.61 & 0.61 & 8.22 & 0.39 \\
\hline $19275678+3601156$ & 2049957756892384256 & 5635 & 3.72 & -0.51 & 0.54 & 0.54 & 8.46 & 0.41 \\
\hline $19280042+4614069$ & 2126804793521418112 & 4893 & 1.61 & -2.02 & 0.21 & 0.54 & 6.95 & 0.41 \\
\hline 19281201-2900223 & 6764699423940862080 & 4834 & 1.22 & -2.47 & -0.17 & 0.48 & 6.44 & 0.18 \\
\hline 19281988-6339344 & 6441410874720278272 & 5139 & 2.15 & -2.13 & 0.42 & 0.43 & 6.73 & 0.31 \\
\hline $19333143+8023242$ & 2295227331824267136 & 5462 & 3.62 & -0.20 & -0.53 & -0.53 & 7.71 & 0.04 \\
\hline $19352572+5114261$ & 2136022446171534848 & 4936 & 4.45 & -0.05 & -0.57 & -0.57 & 7.81 & -0.09 \\
\hline $19375420+5750304$ & 2238305511775896064 & 4898 & 4.83 & -0.56 & -0.07 & -0.07 & 7.81 & 0.08 \\
\hline $19383369+4309290$ & 2077979845822635648 & 5514 & 4.23 & -0.08 & -0.53 & -0.53 & 7.82 & -0.09 \\
\hline
\end{tabular}


Table 3 (continued)

\begin{tabular}{|c|c|c|c|c|c|c|c|c|}
\hline $\begin{array}{c}\text { Star Name } \\
(2 \mathrm{MASS})\end{array}$ & $\begin{array}{c}\text { Star Name } \\
\text { (Gaia EDR3) }\end{array}$ & $\begin{array}{l}T_{\text {eff }} \\
(\mathrm{K})\end{array}$ & $\begin{array}{l}\log g \\
(\operatorname{cgs})\end{array}$ & {$[\mathrm{Fe} / \mathrm{H}]$} & {$[\mathrm{C} / \mathrm{Fe}]$} & {$[\mathrm{C} / \mathrm{Fe}]_{c}$} & $A(\mathrm{C})_{c}$ & {$[\mathrm{Mg} / \mathrm{Fe}]$} \\
\hline 19411203-4509560 & 6684605636852993408 & 4674 & 1.35 & -2.88 & 0.02 & 0.56 & 6.11 & 0.20 \\
\hline $19423665+0502288$ & 4290628386915732736 & 4862 & 1.04 & -2.47 & -0.23 & 0.49 & 6.45 & 0.45 \\
\hline $19433935+7749001$ & 2291495585062080640 & 4793 & 1.19 & -2.71 & 0.00 & 0.64 & 6.36 & 0.28 \\
\hline $19440238-4515172$ & 6684633743118584064 & 5211 & 2.38 & -2.38 & 0.57 & 0.58 & 6.63 & 0.23 \\
\hline $19460015-4844202$ & 6671059481799424000 & 4856 & 1.93 & -1.61 & -0.05 & 0.05 & 6.87 & 0.12 \\
\hline $19481329-4903448$ & 6670987390771270912 & 4856 & 1.59 & -2.50 & 0.10 & 0.49 & 6.42 & 0.37 \\
\hline $19491264+0358174$ & 4242240117122573440 & 5217 & 4.76 & -0.96 & 0.09 & 0.09 & 7.56 & 0.42 \\
\hline 19493291+1245526 & 4305164750954492032 & 4982 & 1.81 & -1.95 & 0.07 & 0.28 & 6.75 & 0.30 \\
\hline 19501371-7714505 & 6365989359218055680 & 5204 & 3.50 & -1.30 & 0.31 & 0.31 & 7.44 & -0.07 \\
\hline $19512662-5013221$ & 6669955297247174784 & 5015 & 1.92 & -1.82 & 0.11 & 0.23 & 6.84 & 0.06 \\
\hline $19523317+4918292$ & 2086984326351707008 & 5254 & 4.44 & -0.03 & -0.41 & -0.41 & 7.98 & -0.09 \\
\hline $19533677-4832517$ & 6670382182636447104 & 5101 & 2.00 & -1.88 & 0.47 & 0.53 & 7.08 & 0.13 \\
\hline $19545002+0803021$ & 4298283770977735168 & 4879 & 1.45 & -2.44 & -0.25 & 0.27 & 6.26 & 0.14 \\
\hline $19550782+0003520$ & 4237149584437046784 & 5929 & 4.00 & -0.35 & -0.06 & -0.06 & 8.02 & 0.06 \\
\hline 19552158-4613569 & 6671915382881993472 & 4893 & 1.49 & -2.45 & 0.14 & 0.59 & 6.57 & -0.16 \\
\hline $19580641-5217166$ & & 4709 & 1.12 & 0.00 & 0.00 & 0.00 & 0.00 & $\ldots$ \\
\hline $19583119+1403473$ & 1807178962362590336 & 4760 & 1.15 & -2.51 & -0.09 & 0.56 & 6.48 & 0.43 \\
\hline 20000909-8240204 & 6347485957207236480 & 5123 & 2.05 & -2.05 & 0.57 & 0.61 & 6.99 & 0.00 \\
\hline $20015470+1103254$ & 4300772996581672704 & 5057 & 4.26 & -1.26 & 0.52 & 0.52 & 7.69 & -0.03 \\
\hline $20021812+0356003$ & 4247387510856965504 & 4657 & 3.32 & -1.63 & 0.67 & 0.68 & 7.48 & 0.15 \\
\hline 20032253-1142028 & 4190620966764303488 & 5323 & 2.19 & -3.45 & 0.87 & 0.88 & 5.86 & $\ldots$ \\
\hline $20035532-5028100$ & 6667107184173771648 & 4854 & 1.61 & -2.58 & -0.35 & 0.03 & 5.88 & 0.25 \\
\hline 20042821-0634042 & 4219932228775714816 & 4698 & 4.28 & -1.80 & 1.18 & 1.18 & 7.81 & 0.12 \\
\hline $20052878-5431260$ & 6473118900280458240 & 5198 & 2.05 & -2.70 & 0.36 & 0.39 & 6.12 & 0.12 \\
\hline 20065053-0824044 & 4192486700559707136 & 5174 & 2.16 & -2.62 & 0.76 & 0.77 & 6.57 & 0.18 \\
\hline 20065112-0135379 & 4235780417581602304 & 5031 & 1.12 & -2.25 & 0.67 & 1.07 & 7.26 & $\ldots$ \\
\hline $20071356+0151191$ & 4243611826586899200 & 5666 & 4.03 & -1.10 & 0.69 & 0.69 & 8.02 & 0.21 \\
\hline $20082836+1011584$ & 4299755432926365184 & 4772 & 0.89 & -2.52 & 0.13 & 0.79 & 6.70 & 0.38 \\
\hline 20083729-1136333 & 6880822690942721024 & 5407 & 4.25 & -0.23 & -0.01 & -0.01 & 8.20 & -0.08 \\
\hline 20111053-0042015 & 4236237642618470272 & 4735 & 1.06 & -2.19 & -0.28 & 0.41 & 6.65 & 0.27 \\
\hline $20115949+0328555$ & 4244395262980430592 & 5346 & 4.37 & -0.24 & -0.42 & -0.42 & 7.77 & -0.04 \\
\hline 20120663-1720171 & 6873767610163223552 & 5538 & 3.99 & -0.81 & 0.60 & 0.60 & 8.22 & 0.09 \\
\hline 20121138-5616178 & 6471798631629401088 & 5003 & 0.99 & -2.28 & 0.43 & 0.94 & 7.09 & $\ldots$ \\
\hline 20121853-1451042 & 6877707847518537088 & 4889 & 1.19 & -2.43 & -0.15 & 0.50 & 6.49 & 0.26 \\
\hline $20124384+0008034$ & 4236447546259730816 & 4748 & 4.74 & -0.38 & -0.31 & -0.31 & 7.74 & 0.21 \\
\hline $20125289+0124185$ & 4242787188566429184 & 4821 & 1.09 & -2.25 & -0.25 & 0.44 & 6.63 & 0.54 \\
\hline 20125447-6143094 & 6442969093151242880 & 5188 & 4.59 & -0.60 & 0.00 & 0.00 & 7.83 & 0.33 \\
\hline 20145497-0643013 & 4217089677680862080 & 4928 & 1.87 & -1.91 & 0.17 & 0.32 & 6.84 & 0.24 \\
\hline 20152495-2220596 & 6852768686118280576 & 4914 & 4.28 & -0.91 & 0.57 & 0.57 & 8.09 & 0.47 \\
\hline 20153131-5719468 & 6468635748993114112 & 4918 & 1.44 & -2.56 & 0.04 & 0.53 & 6.41 & 0.02 \\
\hline 20160180-0523231 & 4217535808820767104 & 5185 & 1.60 & -2.13 & 0.78 & 1.03 & 7.33 & $\cdots$ \\
\hline 20160744-0552554 & 4217300027997089280 & 5523 & 4.58 & -0.22 & -0.21 & -0.21 & 8.00 & 0.00 \\
\hline
\end{tabular}


Table 3 (continued)

\begin{tabular}{|c|c|c|c|c|c|c|c|c|}
\hline $\begin{array}{c}\text { Star Name } \\
\text { (2MASS) }\end{array}$ & $\begin{array}{c}\text { Star Name } \\
\text { (Gaia EDR3) }\end{array}$ & $\begin{array}{l}T_{\text {eff }} \\
(\mathrm{K})\end{array}$ & $\begin{array}{l}\log g \\
(\operatorname{cgs})\end{array}$ & {$[\mathrm{Fe} / \mathrm{H}]$} & {$[\mathrm{C} / \mathrm{Fe}]$} & {$[\mathrm{C} / \mathrm{Fe}]_{c}$} & $A(\mathrm{C})_{c}$ & {$[\mathrm{Mg} / \mathrm{Fe}]$} \\
\hline 20165001-1040388 & 6881177219022208512 & 5022 & 4.39 & -0.03 & -0.23 & -0.23 & 8.17 & -0.08 \\
\hline $20165024+1403416$ & 1803616579035764992 & 4810 & 0.93 & -2.70 & 0.02 & 0.72 & 6.45 & 0.38 \\
\hline 20174990-4403599 & 6673167103853359232 & 5479 & 4.73 & -0.73 & 0.22 & 0.22 & 7.92 & 0.34 \\
\hline 20180356-1301215 & 6879502009977573504 & 4806 & 1.19 & -2.11 & 0.17 & 0.68 & 7.00 & 0.16 \\
\hline $20185485-1428523$ & 6876208079299850752 & 5207 & 1.76 & -1.87 & 0.20 & 0.44 & 7.00 & 0.35 \\
\hline 20192202-6130149 & 6430979984003489024 & 4652 & 1.50 & -2.91 & 0.07 & 0.48 & 5.99 & 0.35 \\
\hline 20194097-2922268 & 6797560699757276544 & 6051 & 3.47 & -0.23 & 0.24 & 0.24 & 8.44 & 0.13 \\
\hline 20200915-4813436 & 6668894268525757696 & 4911 & 3.98 & -0.14 & -0.44 & -0.44 & 7.85 & -0.04 \\
\hline $20203472-2757112$ & 6846116468972086016 & 4916 & 3.55 & -1.02 & 0.30 & 0.30 & 7.71 & -0.02 \\
\hline 20204659-1002234 & 6905253392633848960 & 4626 & 0.80 & -2.72 & -0.21 & 0.53 & 6.24 & -0.10 \\
\hline 20205529-1340439 & 6876402624138104576 & 4635 & 3.79 & -1.03 & 0.51 & 0.51 & 7.92 & 0.41 \\
\hline 20210974-6637116 & 6425821762641148800 & 5072 & 2.00 & -2.15 & 0.42 & 0.48 & 6.76 & 0.20 \\
\hline $20214042-5350224$ & 6472730429078350976 & 5255 & 4.32 & -0.31 & -0.21 & -0.21 & 7.91 & -0.01 \\
\hline 20214109-5550310 & 6469232749446813824 & 5224 & 2.36 & -2.03 & 0.22 & 0.23 & 6.64 & 0.49 \\
\hline 20214764-1610513 & & $\ldots$ & $\ldots$ & $\ldots$ & $\ldots$ & $\ldots$ & $\ldots$ & $\ldots$ \\
\hline 20214838-2917466 & 6797535960745546624 & 5599 & 4.61 & -0.46 & 0.78 & 0.78 & 8.75 & 0.42 \\
\hline 20231318-0728503 & 4216093593161355776 & 4920 & 1.41 & -2.49 & 0.35 & 0.81 & 6.75 & -0.04 \\
\hline 20231543-2104080 & 6859284155805054848 & 4965 & 1.72 & -3.18 & 0.35 & 0.53 & 5.79 & 0.20 \\
\hline 20232260-0807452 & 6906102627927525760 & 4685 & 1.14 & -2.13 & -0.25 & 0.39 & 6.69 & 0.17 \\
\hline 20233743-1659533 & 6873254241311424896 & 4848 & 1.47 & -2.61 & 0.17 & 0.63 & 6.45 & -0.15 \\
\hline 20235225-1628512 & 6873331791241472768 & 4855 & 0.95 & -2.69 & -0.05 & 0.66 & 6.40 & 0.16 \\
\hline 20241045-6720324 & 6425543307024121088 & 4703 & 1.22 & -3.93 & 0.86 & 1.52 & 6.03 & 0.32 \\
\hline 20242459-2529550 & 6848220487550880640 & 4824 & 1.50 & -2.59 & -0.06 & 0.41 & 6.26 & 0.30 \\
\hline 20244286-2618599 & 6847828584672684416 & 5790 & 4.28 & -0.81 & 0.72 & 0.72 & 8.34 & 0.44 \\
\hline 20244510-1605268 & 6874849529603646080 & 4758 & 1.17 & -2.40 & -0.23 & 0.46 & 6.49 & 0.12 \\
\hline 20255659-4915524 & 6668166013871841536 & 5086 & 4.51 & -0.68 & 0.30 & 0.30 & 8.05 & 0.05 \\
\hline 20263268-1025283 & 6904467555355890304 & 5177 & 2.66 & -0.73 & -0.23 & -0.20 & 7.50 & $\cdots$ \\
\hline 20271323-1658595 & 6862731949391812992 & 6484 & 3.86 & -0.05 & 0.44 & 0.44 & 8.82 & -0.02 \\
\hline 20273786-1444546 & 6875659938390010240 & 5158 & 4.51 & -0.91 & 0.14 & 0.14 & 7.66 & 0.36 \\
\hline 20273791-2627414 & 6847786940670450944 & 5417 & 4.07 & -0.74 & 0.54 & 0.54 & 8.23 & 0.29 \\
\hline $20274485-4223567$ & 6679325159242871808 & 5513 & 4.46 & -0.41 & 0.20 & 0.20 & 8.22 & 0.04 \\
\hline 20275301-5141137 & 6475211236547281152 & 4653 & 0.92 & -2.66 & -0.27 & 0.48 & 6.25 & 0.14 \\
\hline 20275840-1556595 & 6874956658968640512 & 4667 & 4.74 & -0.75 & -0.19 & -0.19 & 7.49 & 0.29 \\
\hline 20281482-5351378 & 6473996241838555392 & 5266 & 2.01 & -2.41 & 0.28 & 0.33 & 6.35 & -0.09 \\
\hline 20284544-2638089 & 6847769863878031616 & 5893 & 4.23 & -0.88 & 0.48 & 0.48 & 8.03 & 0.50 \\
\hline 20290062-2157354 & 6855992046192920960 & 5371 & 4.02 & -0.69 & 0.47 & 0.47 & 8.21 & 0.38 \\
\hline $20290527-5059527$ & 6475303698603197824 & 5663 & 3.86 & -1.46 & 0.73 & 0.73 & 7.70 & 0.03 \\
\hline 20292008-4513468 & 6675597235006194432 & 5019 & 3.00 & -1.10 & 0.09 & 0.10 & 7.43 & 0.08 \\
\hline 20293622-3652218 & 6694857444613732352 & 5253 & 3.86 & -0.69 & 0.06 & 0.06 & 7.80 & 0.34 \\
\hline 20301912-2844399 & 6797804306004518400 & 5448 & 4.71 & -0.32 & 0.26 & 0.26 & 8.38 & 0.54 \\
\hline 20313318-3054125 & 6796525780141266304 & 5621 & 4.64 & -0.72 & 0.85 & 0.85 & 8.55 & 0.59 \\
\hline 20314518-5623277 & 6469004910021548672 & 5210 & 2.15 & -2.15 & 0.69 & 0.70 & 6.98 & 0.14 \\
\hline
\end{tabular}


Table 3 (continued)

\begin{tabular}{|c|c|c|c|c|c|c|c|c|}
\hline $\begin{array}{l}\text { Star Name } \\
(2 \mathrm{MASS})\end{array}$ & $\begin{array}{c}\text { Star Name } \\
\text { (Gaia EDR3) }\end{array}$ & $\begin{array}{l}T_{\text {eff }} \\
(\mathrm{K})\end{array}$ & $\begin{array}{l}\log g \\
(\operatorname{cgs})\end{array}$ & {$[\mathrm{Fe} / \mathrm{H}]$} & {$[\mathrm{C} / \mathrm{Fe}]$} & {$[\mathrm{C} / \mathrm{Fe}]_{c}$} & $A(\mathrm{C})_{c}$ & {$[\mathrm{Mg} / \mathrm{Fe}]$} \\
\hline $20331212-4200270$ & 6679615950005226240 & 5249 & 2.52 & -1.97 & 0.23 & 0.24 & 6.70 & 0.36 \\
\hline $20331607-5047126$ & 6475629909958997376 & 5523 & 4.53 & -0.58 & 0.09 & 0.09 & 7.94 & 0.11 \\
\hline $20351407-4625056$ & 6674758921813508736 & 4751 & 2.43 & -0.51 & -0.71 & -0.68 & 7.24 & 0.30 \\
\hline 20354257-0714557 & 6907591641550947072 & 4826 & 1.18 & -2.35 & 0.06 & 0.66 & 6.74 & 0.03 \\
\hline 20373334-3645452 & 6683227639544432512 & 4702 & 3.99 & -0.38 & -0.46 & -0.46 & 7.59 & 0.31 \\
\hline 20375779-2518252 & 6800352497219577728 & 5620 & 4.65 & -0.48 & 0.90 & 0.90 & 8.85 & 0.52 \\
\hline $20381947-2750475$ & 6798666258699212672 & 5573 & 3.85 & -1.58 & 1.10 & 1.10 & 7.96 & 0.37 \\
\hline $20385050-2657542$ & 6799133551141107712 & 5536 & 2.92 & -2.52 & 1.34 & 1.36 & 7.27 & 0.01 \\
\hline 20404939-5843259 & 6455696764877344896 & 4830 & 1.24 & -2.33 & -0.43 & 0.23 & 6.33 & 0.15 \\
\hline $20410627-3251350$ & 6792327226270824832 & 5283 & 4.66 & -0.90 & 0.46 & 0.46 & 7.99 & 0.20 \\
\hline $20414841-5237274$ & 6471501763492187520 & 4697 & 1.00 & -2.31 & -0.28 & 0.46 & 6.58 & 0.19 \\
\hline $20430947-3600325$ & 6779175490951538560 & 5088 & 2.00 & -2.19 & 0.47 & 0.53 & 6.77 & 0.17 \\
\hline $20440973-2940093$ & 6795239665071836160 & 4965 & 1.76 & -2.23 & 0.11 & 0.42 & 6.62 & 0.08 \\
\hline 20442721-7405038 & 6369772538209241216 & 5624 & 4.24 & -0.16 & -0.47 & -0.47 & 7.80 & -0.05 \\
\hline 20443065-2936534 & 6795263648167892992 & 5208 & 4.50 & -0.86 & 0.94 & 0.94 & 8.52 & 0.38 \\
\hline 20445501-3732403 & 6778221802053181824 & 5187 & 3.53 & -0.14 & -0.48 & -0.48 & 7.82 & 0.04 \\
\hline 20451053-2934207 & 6795077693263710080 & 5311 & 1.99 & -1.88 & 0.39 & 0.46 & 7.01 & 0.17 \\
\hline $20460102+1506250$ & 1762690140608564480 & 4488 & 0.70 & -2.12 & -0.63 & 0.10 & 6.42 & 0.45 \\
\hline 20461039-3940468 & 6678691295087392640 & 5116 & 1.65 & -2.51 & 0.80 & 1.06 & 6.97 & 0.37 \\
\hline 20461191-3833114 & 6681901765961577344 & 5290 & 2.60 & -1.51 & 0.45 & 0.47 & 7.39 & 0.23 \\
\hline $20465470-3948150$ & 6678675249089470464 & 5033 & 1.85 & -1.62 & 0.38 & 0.51 & 7.33 & -0.01 \\
\hline 20474129-4949312 & 6480909150618081536 & 4616 & 1.68 & -3.32 & -0.49 & -0.29 & 4.83 & $\ldots$ \\
\hline $20480642-3520259$ & 6779625977182979328 & 4906 & 4.09 & -0.23 & -0.31 & -0.31 & 7.89 & -0.01 \\
\hline $20485047-7341446$ & 6370159673678547712 & 4688 & 1.09 & -2.38 & -0.30 & 0.42 & 6.48 & 0.15 \\
\hline $20485505-4125356$ & 6678289698465087232 & 5043 & 1.38 & -2.64 & 0.04 & 0.57 & 6.36 & 0.11 \\
\hline $20490816-2214501$ & 6807184278357672576 & 4770 & 1.19 & -2.79 & -0.08 & 0.57 & 6.21 & 0.01 \\
\hline $20492765-5124440$ & 6477616903566840064 & 4583 & 0.80 & -2.76 & -0.06 & 0.66 & 6.33 & 0.16 \\
\hline $20504877+1007551$ & 1751382561168000256 & 4882 & 4.75 & -0.79 & 0.18 & 0.18 & 7.81 & 0.27 \\
\hline $20512785-4843325$ & 6481344866460154880 & 5031 & 4.69 & -0.28 & 0.03 & 0.03 & 8.18 & 0.07 \\
\hline $20523629-5241033$ & 6477303675894157824 & 4889 & 1.39 & -2.32 & -0.06 & 0.49 & 6.60 & 0.27 \\
\hline $20530472-3836380$ & 6774883405575766400 & 4916 & 1.53 & -2.26 & -0.59 & -0.10 & 6.07 & 0.23 \\
\hline 20531334-4520139 & 6484187516335125760 & 5194 & 4.20 & 0.04 & 0.00 & 0.00 & 8.48 & -0.08 \\
\hline $20531555+1147415$ & 1757847139781935616 & 4863 & 1.33 & -2.34 & 0.17 & 0.69 & 6.78 & 0.43 \\
\hline $20541462-4811494$ & 6481473440600905472 & 5138 & 1.50 & -2.43 & 0.04 & 0.51 & 6.50 & 0.17 \\
\hline 20545308-4710289 & 6481731516596154368 & 4645 & 1.07 & -2.49 & -0.13 & 0.57 & 6.50 & 0.05 \\
\hline 20555702-3912091 & 6774608463246378880 & 5033 & 1.56 & -1.85 & 0.27 & 0.57 & 7.15 & 0.10 \\
\hline $20565365-5609461$ & 6457897089506392064 & 4934 & 1.68 & -2.23 & 0.32 & 0.64 & 6.84 & 0.11 \\
\hline $20571292-4958553$ & 6478163463924318208 & 5007 & 1.46 & -2.13 & -0.02 & 0.46 & 6.75 & 0.25 \\
\hline $20574855-4154444$ & 6677344182887180800 & 5225 & 4.29 & 0.00 & -0.24 & -0.24 & 8.19 & 0.01 \\
\hline $20575772-5637258$ & 6457695677015968384 & 4996 & 2.17 & -1.55 & 0.48 & 0.50 & 7.38 & 0.05 \\
\hline $20580267+1427040$ & 1761667216837966848 & 5026 & 1.63 & -2.06 & -0.35 & 0.03 & 6.40 & -0.20 \\
\hline 20585673-4013142 & 6773745346616608000 & 5264 & 4.56 & -0.34 & -0.89 & -0.89 & 7.20 & $\ldots$ \\
\hline
\end{tabular}


Table 3 (continued)

\begin{tabular}{|c|c|c|c|c|c|c|c|c|}
\hline $\begin{array}{l}\text { Star Name } \\
\text { (2MASS) }\end{array}$ & $\begin{array}{c}\text { Star Name } \\
\text { (Gaia EDR3) }\end{array}$ & $\begin{array}{l}T_{\text {eff }} \\
(\mathrm{K})\end{array}$ & $\begin{array}{l}\log g \\
(\operatorname{cgs})\end{array}$ & {$[\mathrm{Fe} / \mathrm{H}]$} & {$[\mathrm{C} / \mathrm{Fe}]$} & {$[\mathrm{C} / \mathrm{Fe}]_{c}$} & $A(\mathrm{C})_{c}$ & {$[\mathrm{Mg} / \mathrm{Fe}]$} \\
\hline $20590249+1847021$ & 1765354016765575680 & 4906 & 3.17 & -1.38 & 0.69 & 0.70 & 7.75 & 0.11 \\
\hline 20592599-4724182 & 6483013375355411200 & 4940 & 4.68 & -0.28 & -0.10 & -0.10 & 8.05 & 0.00 \\
\hline 20595162-4937337 & 6478288460356702464 & 6092 & 4.10 & -0.10 & -0.15 & -0.15 & 8.18 & -0.06 \\
\hline $21011036-4537322$ & 6483420263376508032 & 5153 & 2.14 & -2.31 & 0.42 & 0.43 & 6.55 & 0.47 \\
\hline 21011096-4148546 & 6581257453716372352 & 5048 & 4.07 & -1.41 & 0.54 & 0.54 & 7.56 & 0.18 \\
\hline 21014399-4913588 & 6478323339786839168 & 5326 & 4.62 & -0.39 & 0.15 & 0.15 & 8.19 & -0.05 \\
\hline $21032352-4211400$ & 6581180590980731520 & 5485 & 4.37 & -0.37 & 0.24 & 0.24 & 8.31 & 0.09 \\
\hline 21032466-7254213 & 6370267975574142592 & 4987 & 1.71 & -2.30 & 0.05 & 0.38 & 6.52 & 0.11 \\
\hline 21032961-7321324 & 6370238112664426624 & 4731 & 0.97 & -2.40 & -0.10 & 0.60 & 6.63 & 0.12 \\
\hline $21042794-4710017$ & 6480078194704569088 & 4863 & 1.29 & -2.05 & -0.16 & 0.38 & 6.76 & 0.35 \\
\hline $21051884-6831537$ & 6376679571472808064 & 4668 & 1.06 & -2.84 & -0.13 & 0.59 & 6.18 & 0.17 \\
\hline 21054066-4520565 & 6483621714522704128 & 5092 & 1.98 & -2.55 & 0.50 & 0.56 & 6.44 & 0.10 \\
\hline $21080234+1835409$ & 1788340995265967488 & 5078 & 4.62 & -1.04 & 0.52 & 0.52 & 7.91 & -0.06 \\
\hline $21091442-4721520$ & 6480023214826621696 & 4810 & 2.23 & -0.81 & -0.65 & -0.63 & 6.99 & 0.44 \\
\hline 21092218-4250491 & 6580163542725955840 & 4713 & 0.92 & -2.11 & -0.44 & 0.26 & 6.58 & 0.44 \\
\hline 21094841-5600060 & 6463024322681098368 & 5175 & 2.06 & -2.36 & -0.27 & -0.25 & 5.82 & 0.39 \\
\hline $21095801+1725439$ & 1788003032879354752 & 5132 & 2.21 & -2.50 & 0.48 & 0.49 & 6.42 & 0.40 \\
\hline $21102133+3016061$ & 1852687405024593024 & 5044 & 4.70 & -0.79 & 0.35 & 0.35 & 7.99 & 0.43 \\
\hline $21105535+2140380$ & 1790165875330692352 & 5147 & 4.72 & -0.98 & 0.12 & 0.12 & 7.57 & 0.38 \\
\hline 21110533-4239222 & 6580263048527430400 & 4778 & 1.22 & -2.13 & -0.29 & 0.33 & 6.62 & 0.00 \\
\hline $21111175-4126536$ & 6581456534040000128 & 5120 & 1.92 & -1.96 & 0.56 & 0.66 & 7.14 & 0.00 \\
\hline $21114008-5138220$ & 6476892256683323008 & 5307 & 2.35 & -1.93 & 0.65 & 0.66 & 7.16 & $\ldots$ \\
\hline $21115127-5257071$ & 6464688708407259008 & 5164 & 2.56 & -1.32 & 0.26 & 0.28 & 7.39 & 0.13 \\
\hline $21120163+2520001$ & 1841468911788506112 & 5194 & 3.95 & -0.98 & 0.27 & 0.27 & 7.72 & $\ldots$ \\
\hline $21125173+2110327$ & 1790077841384173312 & 4981 & 1.03 & -2.53 & -0.15 & 0.56 & 6.46 & -0.10 \\
\hline $21134390-6802355$ & 6399985335331112064 & 5140 & 1.87 & -2.73 & 0.73 & 0.83 & 6.54 & 0.05 \\
\hline $21145602+2112242$ & 1791382721170477824 & 4896 & 1.58 & -2.29 & -0.04 & 0.41 & 6.55 & 0.46 \\
\hline $21150824+2631245$ & 1847680160351457024 & 5423 & 2.28 & 0.00 & 0.00 & 0.00 & 0.00 & $\ldots$ \\
\hline 21151790-4333404 & 6579952677010742272 & 5018 & 1.57 & -2.54 & 0.38 & 0.76 & 6.65 & 0.11 \\
\hline 21154971-6848520 & 6375872461218303488 & 5041 & 1.38 & -2.36 & 0.10 & 0.62 & 6.69 & 0.26 \\
\hline $21171659-4115323$ & 6580773015762728704 & 4982 & 3.20 & -0.87 & -0.38 & -0.37 & 7.18 & 0.40 \\
\hline $21190273+3318462$ & 1854629726683249024 & 5194 & 1.81 & -2.11 & -0.10 & 0.11 & 6.43 & 0.21 \\
\hline $21192932-7715553$ & 6356252535213950592 & 5395 & 2.14 & -2.25 & 0.82 & 0.83 & 7.01 & 0.20 \\
\hline $21203573-5321426$ & 6463751271665659264 & 5089 & 2.18 & -1.93 & 0.43 & 0.44 & 6.94 & 0.37 \\
\hline $21211669+2032551$ & 1790500809763786624 & 5164 & 4.51 & -0.07 & -0.21 & -0.21 & 8.15 & -0.05 \\
\hline $21214670+1916532$ & 1785668323017152384 & 5070 & 3.77 & -0.98 & 0.09 & 0.09 & 7.54 & 0.10 \\
\hline $21232828-5328287$ & 6463713682111820160 & 5110 & 2.65 & -1.39 & 0.25 & 0.27 & 7.31 & 0.31 \\
\hline $21240060-5241520$ & 6465286018099447680 & 5089 & 2.54 & -1.32 & 0.17 & 0.19 & 7.31 & 0.26 \\
\hline 21243758-6400120 & 6403279953204895360 & 5152 & 3.59 & -0.34 & 0.06 & 0.06 & 8.14 & 0.03 \\
\hline 21254398-6753045 & 6399850542078013568 & 4870 & 4.69 & -1.00 & 0.31 & 0.31 & 7.74 & 0.14 \\
\hline $21262879+1749436$ & 1784515446419862656 & 5304 & 3.72 & -0.12 & -0.55 & -0.55 & 7.76 & 0.00 \\
\hline $21263180+2031469$ & 1790358392944627712 & 4745 & 1.08 & -3.04 & 0.04 & 0.76 & 6.15 & 0.45 \\
\hline
\end{tabular}


Table 3 (continued)

\begin{tabular}{|c|c|c|c|c|c|c|c|c|}
\hline $\begin{array}{c}\text { Star Name } \\
\text { (2MASS) }\end{array}$ & $\begin{array}{c}\text { Star Name } \\
\text { (Gaia EDR3) }\end{array}$ & $\begin{array}{l}T_{\text {eff }} \\
(\mathrm{K})\end{array}$ & $\begin{array}{l}\log g \\
(\operatorname{cgs})\end{array}$ & {$[\mathrm{Fe} / \mathrm{H}]$} & {$[\mathrm{C} / \mathrm{Fe}]$} & {$[\mathrm{C} / \mathrm{Fe}]_{c}$} & $A(\mathrm{C})_{c}$ & {$[\mathrm{Mg} / \mathrm{Fe}]$} \\
\hline 21281884-6406460 & 6403090356168392192 & 4858 & 1.60 & -2.66 & -0.05 & 0.32 & 6.10 & -0.04 \\
\hline $21292831-5558258$ & 6460174865281654144 & 4908 & 4.05 & -0.65 & -0.37 & -0.37 & 7.42 & 0.13 \\
\hline 21313828-6858199 & 6396651616076109184 & 5017 & 1.53 & -2.79 & 0.48 & 0.86 & 6.51 & 0.25 \\
\hline 21322880-6607539 & 6400620298999247488 & 4499 & 4.58 & -1.47 & -0.12 & -0.12 & 6.85 & 0.36 \\
\hline $21323054+1600097$ & 1772048878641647488 & 5228 & 2.54 & -1.99 & 0.41 & 0.42 & 6.86 & 0.40 \\
\hline 21340283-6224209 & 6403844758583266688 & 4888 & 3.03 & -3.54 & 0.11 & 0.11 & 5.00 & $\ldots$ \\
\hline $21343861+1538156$ & 1771832962045862656 & 5026 & 4.80 & -0.74 & 0.10 & 0.10 & 7.79 & 0.15 \\
\hline $21351702-5533118$ & 6460110479427930624 & 5112 & 1.79 & -1.66 & 0.00 & 0.21 & 6.98 & 0.28 \\
\hline 21373999-2446495 & 6814831136913490048 & 4931 & 4.67 & -0.68 & 0.22 & 0.22 & 7.98 & 0.06 \\
\hline $21391225+3849406$ & 1953863502940549504 & 5333 & 4.81 & -0.43 & -0.05 & -0.05 & 7.95 & 0.18 \\
\hline 21393394-5845548 & 6458363870847393792 & 5014 & 1.99 & -2.43 & 0.23 & 0.29 & 6.29 & $\ldots$ \\
\hline $21414000-2854239$ & 6809748884932883712 & 4870 & 1.67 & -1.94 & -0.05 & 0.27 & 6.76 & 0.27 \\
\hline 21430664-6338509 & 6402785069892389760 & 5348 & 4.43 & -0.11 & -0.03 & -0.03 & 8.29 & -0.03 \\
\hline $21440820+3813139$ & 1952857308060989312 & 5023 & 1.86 & -1.53 & -0.35 & -0.16 & 6.75 & 0.04 \\
\hline $21445442-6319381$ & 6402818531980755584 & 4847 & 3.64 & -1.40 & 0.79 & 0.79 & 7.83 & 0.04 \\
\hline $21453847+2351116$ & 1794596868534480128 & 5189 & 1.98 & -2.51 & 0.47 & 0.54 & 6.46 & 0.33 \\
\hline $21461136-5420472$ & 6461349457233919872 & 4637 & 4.66 & -0.65 & -0.43 & -0.43 & 7.35 & 0.21 \\
\hline $21480606+4643071$ & 1974610531448247936 & 4772 & 2.89 & -1.46 & 0.39 & 0.41 & 7.38 & 0.04 \\
\hline $21482057+2155222$ & 1793330952694172416 & 5485 & 4.08 & -0.91 & 0.39 & 0.39 & 7.91 & 0.01 \\
\hline $21494865+1048431$ & 1765600930139450752 & 5155 & 2.12 & -2.13 & 0.39 & 0.40 & 6.70 & 0.46 \\
\hline 21502426-6105576 & 6409890217109702784 & 4668 & 1.24 & -2.45 & -0.26 & 0.40 & 6.39 & 0.33 \\
\hline $21510307+3619543$ & 1949409175177462272 & 4807 & 1.23 & -2.27 & -0.15 & 0.48 & 6.65 & 0.46 \\
\hline 21513710-7925459 & 6355014691279286400 & 5465 & 3.42 & -1.45 & 0.58 & 0.58 & 7.56 & 0.05 \\
\hline $21515415+0537172$ & 2697022209558112768 & 4757 & 1.49 & -2.01 & -0.31 & 0.16 & 6.58 & 0.49 \\
\hline $21525027-6604240$ & 6398934786330198784 & 5109 & 2.70 & -0.36 & -0.52 & -0.49 & 7.58 & 0.15 \\
\hline $21555532+3501402$ & 1948312515407711104 & 5035 & 1.92 & -1.95 & 0.07 & 0.19 & 6.66 & 0.26 \\
\hline 21573551-0308043 & 2670534149811033088 & 5301 & 2.54 & -1.90 & 0.68 & 0.69 & 7.23 & 0.15 \\
\hline $21573761+3544196$ & 1948748437403259776 & 4901 & 4.84 & -0.72 & 0.02 & 0.02 & 7.72 & 0.11 \\
\hline $21584417+0052490$ & 2681491607815613952 & 5277 & 2.65 & -2.05 & 0.43 & 0.44 & 6.82 & 0.11 \\
\hline $21584491+0129524$ & 2681597607608087040 & 4616 & 0.49 & -2.65 & -0.05 & 0.66 & 6.45 & 0.20 \\
\hline 22013669-6918312 & 6396019706127003520 & 4795 & 1.77 & -2.02 & 0.12 & 0.38 & 6.78 & 0.35 \\
\hline 22032935-5635128 & 6412646138709077888 & 4907 & 1.47 & -2.20 & -0.16 & 0.33 & 6.56 & 0.40 \\
\hline $22040352+0016519$ & 2680470706974136192 & 4725 & 1.07 & -2.36 & -0.30 & 0.43 & 6.50 & 0.35 \\
\hline 22043661-6044348 & 6409307304149253248 & 5224 & 2.47 & -2.03 & 0.28 & 0.29 & 6.69 & 0.18 \\
\hline $22044480+4148205$ & 1959828147361822848 & 5459 & 4.70 & -0.65 & 0.31 & 0.31 & 8.09 & 0.06 \\
\hline $22050237+3107332$ & 1898564081014685184 & 5464 & 4.19 & -0.87 & 0.27 & 0.27 & 7.83 & 0.16 \\
\hline 22082896-6607320 & 6399063394830844544 & 5166 & 1.56 & -2.34 & -0.03 & 0.45 & 6.54 & 0.34 \\
\hline 22104990-3947023 & 6573266443723504768 & 5161 & 2.20 & -2.55 & 0.54 & 0.55 & 6.43 & -0.07 \\
\hline $22112456-3753100$ & 6574080735161542784 & 4820 & 0.94 & -2.20 & -0.47 & 0.26 & 6.49 & 0.25 \\
\hline 22115350-1209181 & 2612971211403496832 & 5030 & 1.46 & -2.27 & -0.15 & 0.36 & 6.53 & 0.07 \\
\hline $22120815-4215210$ & 6569918602615687808 & 5158 & 2.19 & -1.91 & 0.34 & 0.35 & 6.87 & 0.23 \\
\hline 22125424-0235414 & 2675834650555144576 & 5406 & 2.90 & -2.21 & 0.56 & 0.57 & 6.79 & 0.24 \\
\hline
\end{tabular}


Table 3 (continued)

\begin{tabular}{|c|c|c|c|c|c|c|c|c|}
\hline $\begin{array}{c}\text { Star Name } \\
\text { (2MASS) }\end{array}$ & $\begin{array}{c}\text { Star Name } \\
\text { (Gaia EDR3) }\end{array}$ & $\begin{array}{l}T_{\text {eff }} \\
(\mathrm{K})\end{array}$ & $\begin{array}{l}\log g \\
(\operatorname{cgs})\end{array}$ & {$[\mathrm{Fe} / \mathrm{H}]$} & {$[\mathrm{C} / \mathrm{Fe}]$} & {$[\mathrm{C} / \mathrm{Fe}]_{c}$} & $A(\mathrm{C})_{c}$ & {$[\mathrm{Mg} / \mathrm{Fe}]$} \\
\hline 22125459-0408594 & 2627229884351014144 & 4881 & 1.49 & -2.46 & 0.16 & 0.61 & 6.57 & 0.25 \\
\hline 22140658-0016065 & 2678634350759886336 & 4666 & 4.28 & -0.62 & 0.06 & 0.06 & 7.87 & -0.10 \\
\hline 22165631-1154207 & 2612857755547023232 & 5448 & 4.26 & -0.35 & 0.06 & 0.06 & 8.14 & -0.09 \\
\hline $22211976-4111021$ & 6569481855982624640 & 4885 & 1.42 & -2.49 & 0.06 & 0.57 & 6.51 & -0.13 \\
\hline $22213981+3725402$ & 1906965002686762496 & 5447 & 3.65 & -1.04 & 0.18 & 0.18 & 7.57 & -0.05 \\
\hline $22221878-7406283$ & 6357952517629300352 & 4810 & 1.32 & -2.18 & -0.20 & 0.39 & 6.65 & 0.27 \\
\hline $22224538+3555471$ & 1905936405260048384 & 4879 & 1.23 & -2.22 & -0.35 & 0.29 & 6.50 & 0.35 \\
\hline $22224859+2804264$ & 1881899328730042368 & 5144 & 2.10 & -2.16 & 0.43 & 0.45 & 6.73 & 0.46 \\
\hline $22225487+2954084$ & 1894651090929156096 & 5248 & 4.56 & -0.98 & 0.06 & 0.06 & 7.51 & 0.18 \\
\hline $22234372-8627533$ & 6342106630928779392 & 4631 & 4.36 & -0.59 & -0.57 & -0.57 & 7.28 & 0.00 \\
\hline 22235096-6512086 & 6404249993041212928 & 5470 & 4.65 & -0.84 & 0.24 & 0.24 & 7.83 & 0.35 \\
\hline $22241668+2548128$ & 1879125024671706624 & 5651 & 3.83 & -1.36 & 0.63 & 0.63 & 7.70 & -0.01 \\
\hline $22241984+2430382$ & 1878702846566493440 & 5415 & 2.57 & -0.32 & -0.37 & -0.34 & 7.78 & 0.35 \\
\hline $22244368+3236401$ & 1901779602770732032 & 4898 & 3.75 & -1.50 & 0.42 & 0.42 & 7.34 & -0.02 \\
\hline $22251594+2911332$ & 1894379923873649536 & 5205 & 1.57 & -2.76 & 0.00 & 0.39 & 6.06 & $\ldots$ \\
\hline $22280812+3546524$ & 1905786459361887488 & 4750 & 4.38 & -1.32 & 0.53 & 0.53 & 7.64 & -0.06 \\
\hline $22284549-4124119$ & 6593293356532115712 & 4992 & 4.59 & -0.64 & 0.08 & 0.08 & 7.86 & 0.12 \\
\hline $22290341+3954145$ & 1909092729485051776 & 5531 & 3.03 & -1.08 & 0.51 & 0.53 & 7.88 & 0.44 \\
\hline $22293323+4332048$ & 1981952760850101376 & 5109 & 2.20 & -2.74 & 0.47 & 0.48 & 6.17 & 0.25 \\
\hline 22294083-3305402 & 6600574876582217344 & 4909 & 1.41 & -2.70 & -0.07 & 0.46 & 6.19 & -0.15 \\
\hline 22303946-1809055 & 2594309161890648320 & 4716 & 4.70 & -0.37 & -0.22 & -0.22 & 7.84 & 0.18 \\
\hline $22310829+3023018$ & 1900531824573844608 & 5396 & 4.23 & -0.12 & -0.61 & -0.61 & 7.71 & -0.07 \\
\hline $22311433-6656572$ & 6386075551166390144 & 4764 & 1.24 & -2.09 & -0.11 & 0.46 & 6.80 & 0.17 \\
\hline $22315231+2320202$ & 1875411630307299456 & 6294 & 4.18 & -0.76 & 0.54 & 0.54 & 8.21 & -0.13 \\
\hline $22342447+2739353$ & 1881261994239589888 & 5093 & 3.06 & -0.82 & 0.14 & 0.15 & 7.76 & 0.38 \\
\hline $22345447-6605172$ & 6392134513070641408 & 4951 & 1.48 & -2.80 & 0.10 & 0.55 & 6.18 & -0.17 \\
\hline 22354139-4305549 & 6520905878946665344 & 4950 & 1.43 & -2.85 & -0.16 & 0.36 & 5.94 & 0.14 \\
\hline $22355721-2434108$ & 6623904314300239488 & 4748 & 4.64 & -0.95 & 0.31 & 0.31 & 7.80 & 0.06 \\
\hline $22360602+3930413$ & 1908318669000212480 & 5279 & 3.71 & -0.25 & -0.36 & -0.36 & 7.82 & 0.02 \\
\hline $22364074-7026524$ & 6384994623861077376 & 5827 & 4.12 & -1.24 & 0.61 & 0.61 & 7.80 & 0.27 \\
\hline 22373316-4341181 & 6520826714109323392 & 5176 & 2.37 & -2.14 & 0.18 & 0.19 & 6.48 & 0.14 \\
\hline 22401067-3738259 & 6595625729931613568 & 4863 & 1.44 & -2.58 & 0.04 & 0.53 & 6.38 & 0.21 \\
\hline $22412632-3627304$ & 6596002145160945664 & 5005 & 1.65 & -2.31 & -0.03 & 0.35 & 6.47 & 0.39 \\
\hline $22413573+2931038$ & 1887539965117822464 & 5193 & 4.32 & -0.44 & 0.31 & 0.31 & 8.30 & 0.57 \\
\hline $22432022-7547340$ & 6357547759911189120 & 5877 & 3.78 & -0.85 & 0.77 & 0.77 & 8.35 & 0.09 \\
\hline $22451263+3532066$ & 1903656636619168768 & 4749 & 1.24 & -2.19 & -0.29 & 0.32 & 6.56 & 0.33 \\
\hline $22453503+1301210$ & 2731609959149919360 & 4990 & 1.31 & -2.88 & 0.10 & 0.67 & 6.21 & 0.49 \\
\hline $22454796+2826260$ & 1887186850086385152 & 5128 & 4.13 & -1.25 & 0.44 & 0.44 & 7.63 & -0.12 \\
\hline 22471965-7201440 & 6382433144021702656 & 5595 & 3.90 & -0.43 & -0.23 & -0.23 & 7.77 & 0.02 \\
\hline $22472643+3532411$ & 1903562838830927744 & 5230 & 4.65 & -0.33 & -0.09 & -0.09 & 8.01 & 0.04 \\
\hline $22473447-5511534$ & 6505905757201226368 & 5627 & 3.81 & -1.40 & 0.62 & 0.62 & 7.65 & 0.01 \\
\hline $22474545+2517113$ & 1876790894628571264 & 5056 & 4.33 & -1.04 & 0.32 & 0.32 & 7.72 & -0.09 \\
\hline
\end{tabular}


Table 3 (continued)

\begin{tabular}{|c|c|c|c|c|c|c|c|c|}
\hline $\begin{array}{c}\text { Star Name } \\
\text { (2MASS) }\end{array}$ & $\begin{array}{c}\text { Star Name } \\
\text { (Gaia EDR3) }\end{array}$ & $\begin{array}{l}T_{\text {eff }} \\
(\mathrm{K})\end{array}$ & $\begin{array}{l}\log g \\
(\operatorname{cgs})\end{array}$ & {$[\mathrm{Fe} / \mathrm{H}]$} & {$[\mathrm{C} / \mathrm{Fe}]$} & {$[\mathrm{C} / \mathrm{Fe}]_{c}$} & $A(\mathrm{C})_{c}$ & {$[\mathrm{Mg} / \mathrm{Fe}]$} \\
\hline $22481449-5703067$ & 6504026829267642240 & 5512 & 3.00 & -1.93 & 0.27 & 0.28 & 6.77 & 0.18 \\
\hline $22490353-5536242$ & 6505879059684460032 & 5125 & 4.57 & -0.49 & 0.08 & 0.08 & 8.02 & 0.15 \\
\hline 22492811-5717202 & 6503988105842414464 & 5418 & 4.07 & 0.09 & -0.43 & -0.43 & 8.10 & 0.00 \\
\hline $22511876-3814381$ & 6547694891340666752 & 5112 & 2.22 & -2.06 & 0.47 & 0.48 & 6.85 & 0.16 \\
\hline 22512739-5049405 & 6513929237145370880 & 5055 & 1.65 & -1.88 & -0.13 & 0.20 & 6.75 & 0.20 \\
\hline 22531950-6647163 & 6391157150312476032 & 4673 & 0.93 & -2.48 & 0.42 & 0.98 & 6.93 & -0.04 \\
\hline $22534476-5456485$ & 6506123701021637888 & 5295 & 3.07 & -1.45 & 0.15 & 0.16 & 7.15 & 0.30 \\
\hline $22534538+7919586$ & 2285728337337892480 & 4938 & 4.86 & -0.62 & 0.01 & 0.01 & 7.82 & 0.10 \\
\hline $22544750-3509160$ & 6554282379035795840 & 4577 & 4.57 & -0.83 & -0.56 & -0.56 & 7.04 & 0.33 \\
\hline $22575548-5622538$ & 6493611254400172416 & 4833 & 1.68 & -2.30 & 0.10 & 0.46 & 6.59 & 0.13 \\
\hline $22591090-4829425$ & 6515325616912384256 & 4976 & 1.57 & -2.05 & -0.08 & 0.32 & 6.70 & 0.08 \\
\hline 22594336-3819324 & 6546814427341069568 & 4900 & 4.29 & -1.05 & 0.24 & 0.24 & 7.62 & 0.29 \\
\hline $23003637+2820257$ & 1885059776122105728 & 5261 & 4.55 & -0.86 & 0.11 & 0.11 & 7.68 & 0.39 \\
\hline 23022817-4059099 & 6543252452048113152 & 4912 & 1.78 & -1.80 & 0.10 & 0.32 & 6.95 & 0.29 \\
\hline 23035329-4139482 & 6543169129682052608 & 5107 & 1.86 & -2.02 & 0.03 & 0.19 & 6.59 & 0.03 \\
\hline $23043022+0155166$ & 2652540916900514304 & 4891 & 1.68 & -2.36 & 0.07 & 0.42 & 6.49 & 0.09 \\
\hline 23044868-4311029 & 6541868716664047744 & 4594 & 4.22 & -0.32 & -0.80 & -0.80 & 7.32 & $\ldots$ \\
\hline $23055841-8636001$ & 6341894558326196480 & 4633 & 3.82 & -1.14 & 0.19 & 0.19 & 7.49 & -0.15 \\
\hline $23064708+2802027$ & 1884897937457948672 & 4798 & 4.54 & -1.50 & 0.03 & 0.03 & 6.96 & 0.44 \\
\hline 23083044-7441319 & 6378503729982312576 & 5006 & 1.91 & -1.77 & 0.22 & 0.34 & 7.00 & 0.18 \\
\hline $23085453-5226130$ & 6500818969734685440 & 4993 & 3.88 & 0.05 & -0.05 & -0.05 & 8.42 & -0.15 \\
\hline 23093209-7132507 & 6381003327932886144 & 4888 & 1.40 & -2.38 & -0.37 & 0.19 & 6.24 & 0.23 \\
\hline 23100319-7702165 & 6354182945092770176 & 4778 & 1.28 & -2.75 & 0.09 & 0.67 & 6.35 & 0.05 \\
\hline 23123243-0240516 & 2638139066923296128 & 4824 & 4.38 & -0.24 & -0.43 & -0.43 & 7.76 & -0.11 \\
\hline $23124700+2701045$ & 2845306436489791232 & 5153 & 4.41 & -0.30 & 0.37 & 0.37 & 8.51 & 0.44 \\
\hline 23130418-4332060 & 6541663554666433920 & 5010 & 1.76 & -1.75 & 0.19 & 0.39 & 7.07 & 0.45 \\
\hline $23131220+5425552$ & 1996424051866554112 & 4751 & 3.17 & -1.41 & 0.45 & 0.46 & 7.48 & 0.02 \\
\hline $23133742-5336585$ & 6500438435631474560 & 4509 & 2.48 & -1.58 & 0.62 & 0.64 & 7.49 & 0.08 \\
\hline $23164530-4047253$ & 6548299386513340288 & 4963 & 4.58 & -0.84 & 0.12 & 0.12 & 7.71 & $\ldots$ \\
\hline $23235454-4730233$ & 6526777614634204672 & 4870 & 2.45 & -1.62 & 0.99 & 1.01 & 7.82 & $\ldots$ \\
\hline $23273196+5438201$ & 1995911404570328192 & 6154 & 4.22 & -0.80 & 0.64 & 0.64 & 8.27 & 0.45 \\
\hline $23293844+3337097$ & 2872688983306031616 & 4953 & 1.52 & -2.96 & 0.30 & 0.71 & 6.17 & 0.39 \\
\hline $23321307+1950398$ & 2824757388800259840 & 5028 & 4.40 & -0.35 & 0.32 & 0.32 & 8.40 & 0.39 \\
\hline $23341995+4703450$ & 1938499679067388288 & 5357 & 3.71 & -1.30 & 0.38 & 0.38 & 7.52 & 0.01 \\
\hline $23362842-5537423$ & 6496310731541441152 & 5178 & 1.43 & -1.97 & 0.35 & 0.70 & 7.16 & $\ldots$ \\
\hline $23371202+2100145$ & 2826450808506230016 & 4447 & 4.40 & -0.54 & -1.08 & -1.08 & 6.81 & 0.02 \\
\hline $23400099+4959092$ & 1943744250753474944 & 5232 & 2.76 & -2.79 & 0.55 & 0.56 & 6.19 & 0.14 \\
\hline $23421250-6346584$ & 6485737793369175680 & 5008 & 4.19 & -1.06 & 0.62 & 0.62 & 7.99 & 0.09 \\
\hline $23430472-8200221$ & 6350719930141819520 & 4977 & 4.48 & -0.62 & 0.37 & 0.37 & 8.18 & 0.05 \\
\hline $23433753+5008599$ & 1943761190104941824 & 7429 & 4.52 & -0.07 & 1.20 & 1.20 & 9.56 & 0.29 \\
\hline $23450930+5538498$ & 1994885560221975552 & 4600 & 2.23 & -1.89 & 0.61 & 0.62 & 7.16 & 0.00 \\
\hline $23465497+1201211$ & 2763718305727188480 & 5029 & 4.67 & -0.83 & 0.37 & 0.37 & 7.97 & 0.26 \\
\hline
\end{tabular}


Table 3 (continued)

\begin{tabular}{|c|c|c|c|c|c|c|c|c|}
\hline $\begin{array}{c}\text { Star Name } \\
\text { (2MASS) }\end{array}$ & $\begin{array}{c}\text { Star Name } \\
\text { (Gaia EDR3) }\end{array}$ & $\begin{array}{l}T_{\text {eff }} \\
(\mathrm{K})\end{array}$ & $\begin{array}{l}\log g \\
(\operatorname{cgs})\end{array}$ & {$[\mathrm{Fe} / \mathrm{H}]$} & {$[\mathrm{C} / \mathrm{Fe}]$} & {$[\mathrm{C} / \mathrm{Fe}]_{c}$} & $A(\mathrm{C})_{c}$ & {$[\mathrm{Mg} / \mathrm{Fe}]$} \\
\hline $23472384+4835469$ & 1940559103007605120 & 4795 & 1.16 & -2.02 & -0.17 & 0.42 & 6.82 & 0.43 \\
\hline $23505349+1142348$ & 2766637307235175296 & 5235 & 1.87 & -2.05 & -0.05 & 0.11 & 6.48 & 0.38 \\
\hline $23550005+1255039$ & 2766874664308017408 & 4644 & 4.77 & -1.28 & 0.11 & 0.11 & 7.26 & 0.12 \\
\hline $23555398+5702462$ & 1998062118035485056 & 5458 & 3.87 & -0.27 & -0.23 & -0.23 & 7.93 & -0.03 \\
\hline $23560245+1109200$ & 2765621221052354816 & 5080 & 4.38 & -0.40 & 0.32 & 0.32 & 8.36 & 0.44 \\
\hline $23562635+0651168$ & 2746230337064331776 & 4958 & 4.14 & -1.40 & 0.58 & 0.58 & 7.61 & -0.10 \\
\hline $23563722+4615436$ & 1927134439687586560 & 4161 & 3.23 & -1.72 & 0.64 & 0.65 & 7.35 & $\ldots$ \\
\hline $23564530-4429484$ & 6532542491534291968 & 5127 & 4.70 & -0.31 & -0.07 & -0.07 & 8.05 & 0.10 \\
\hline
\end{tabular}


Table 4. Phase-space Information from Gaia EDR3

\begin{tabular}{|c|c|c|c|c|c|c|c|c|}
\hline $\begin{array}{c}\text { Star Name } \\
\text { (2MASS) }\end{array}$ & $\begin{array}{c}\text { Star Name } \\
\text { (Gaia EDR3) }\end{array}$ & $\begin{array}{c}V_{\mathrm{los}} \\
\left(\mathrm{km} \mathrm{s}^{-1}\right)\end{array}$ & $\begin{array}{l}d_{\text {helio }} \\
(\mathrm{kpc})\end{array}$ & $\begin{array}{c}\sigma_{d} \\
(\mathrm{kpc})\end{array}$ & 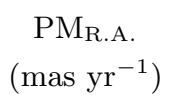 & $\begin{array}{c}\mathrm{PM}_{\text {Decl. }} \\
\left(\text { mas yr }^{-1}\right)\end{array}$ & $\begin{array}{l}Z_{\mathrm{Gal}} \\
(\mathrm{kpc})\end{array}$ & $\begin{array}{c}V_{\mathrm{TAV}} \\
\left(\mathrm{km} \mathrm{s}^{-1}\right)\end{array}$ \\
\hline 00003305-7953389 & 4634573766005607552 & 269.3 & 2.51 & 0.06 & -4.538 & -4.538 & -1.48 & 274.3 \\
\hline $00020162-4430117$ & 4994519032163925632 & 58.5 & 3.13 & 0.19 & 14.656 & 14.656 & -2.92 & 202.6 \\
\hline 00040305-6106367 & 4905632480654004608 & -6.0 & 0.12 & 0.00 & 53.448 & 53.448 & -0.07 & 15.7 \\
\hline $00043646+4124062$ & 384060304935385984 & -13.7 & 1.01 & 0.01 & -7.058 & -7.058 & -0.33 & 28.8 \\
\hline $00045403+3524010$ & 2876804519751163008 & -91.5 & 1.06 & 0.02 & 9.525 & 9.525 & -0.45 & 154.2 \\
\hline $00052731+0025510$ & 2546113345293028608 & -2.9 & 0.19 & 0.01 & -9.668 & -9.668 & -0.14 & 41.4 \\
\hline 00060902-6238019 & 4904480471642380928 & 65.5 & 5.74 & 0.41 & -4.387 & -4.387 & -4.60 & 198.3 \\
\hline $00071189+4724466$ & 393031258963415936 & 13.4 & 0.15 & 0.01 & 74.884 & 74.884 & -0.01 & 74.5 \\
\hline $00111339+0152512$ & 2546752127188959232 & 176.0 & 3.36 & 0.19 & 4.350 & 4.350 & -2.87 & 288.9 \\
\hline $00140089+3148167$ & 2861747777456341120 & $\ldots$ & 0.80 & 0.01 & -14.106 & -14.106 & -0.38 & 45.5 \\
\hline $00144636-2246093$ & 2361100512255883904 & 10.7 & 0.77 & 0.01 & 22.865 & 22.865 & -0.73 & 101.1 \\
\hline 00150914-3736048 & 2308246644711975680 & $\ldots$ & 0.26 & 0.01 & 34.186 & 34.186 & -0.22 & 43.3 \\
\hline 00152923-2436237 & 2336022438732700672 & -17.0 & 0.11 & 0.00 & 190.327 & 190.327 & -0.08 & 93.4 \\
\hline $00152952-6121258$ & 4904881106191486592 & 68.5 & 6.67 & 0.63 & 4.618 & 4.618 & -5.46 & 109.1 \\
\hline 00154806-6253207 & 4901504815220315648 & 207.8 & 2.26 & 0.06 & 25.055 & 25.055 & -1.80 & 259.1 \\
\hline 00162809-0505519 & 2443891577459590016 & -173.5 & 2.28 & 0.09 & 29.212 & 29.212 & -2.06 & 391.8 \\
\hline $00163655+3538314$ & 2876439211309388672 & -9.7 & 1.88 & 0.09 & 13.297 & 13.297 & -0.82 & 188.4 \\
\hline 00163809-4912369 & 4977077135617904640 & 241.5 & 6.12 & 0.58 & 3.195 & 3.195 & -5.61 & 274.5 \\
\hline $00165353+3642326$ & 2876647328245159552 & -87.6 & 3.90 & 0.24 & 5.221 & 5.221 & -1.66 & 148.5 \\
\hline $00170767+4614488$ & 392109932642310272 & 24.3 & 0.68 & 0.01 & 23.695 & 23.695 & -0.16 & 59.4 \\
\hline 00175076-6819295 & 4706413931618489600 & 200.6 & 5.11 & 0.27 & 0.792 & 0.792 & -3.80 & 401.9 \\
\hline $00182947-7829325$ & 4635466706886248448 & 62.1 & 0.22 & 0.01 & -9.678 & -9.678 & -0.11 & 62.5 \\
\hline $00192284+4431554$ & 385734487485292928 & 10.2 & 0.09 & 0.00 & 15.571 & 15.571 & 0.00 & 21.6 \\
\hline 00202312-3950260 & 4997141779713479680 & 12.8 & 0.08 & 0.00 & -28.842 & -28.842 & -0.06 & 41.8 \\
\hline $00213396+0008219$ & 2545302077511395968 & 71.2 & $\ldots$ & $\ldots$ & $\ldots$ & $\ldots$ & 0.03 & 71.2 \\
\hline $00223511-4231148$ & 4992150679821873664 & -52.3 & 3.22 & 0.14 & 0.676 & 0.676 & -3.06 & 293.1 \\
\hline 00234358-1117357 & 2424691974134738816 & 20.0 & 0.24 & 0.01 & 6.954 & 6.954 & -0.21 & 93.3 \\
\hline 00235345-6649211 & 4707128682896207872 & 2.4 & 1.00 & 0.01 & 27.544 & 27.544 & -0.74 & 51.9 \\
\hline $00241677+2941440$ & 2858965188404148480 & $\ldots$ & 0.74 & 0.01 & 2.487 & 2.487 & -0.38 & 22.1 \\
\hline $00275509+3458068$ & 365999795656991616 & -222.1 & 3.19 & 0.19 & 8.430 & 8.430 & -1.46 & 253.2 \\
\hline 00281899-6820268 & 4703742084003326080 & 299.3 & 6.23 & 0.39 & 1.197 & 1.197 & -4.65 & 327.5 \\
\hline $00293797+2103054$ & 2796582811359131392 & $\ldots$ & 2.77 & 0.15 & 43.194 & 43.194 & -1.81 & 564.7 \\
\hline $00301775+2957334$ & 2858881625520606848 & -404.2 & 3.80 & 0.17 & 6.526 & 6.526 & -2.03 & 495.0 \\
\hline $00303445+2816193$ & 2857835710788936832 & 22.8 & 0.11 & 0.00 & 66.853 & 66.853 & -0.04 & 40.0 \\
\hline $00311900+4957158$ & 391597358363860608 & 2.1 & 0.25 & 0.01 & 7.126 & 7.126 & -0.03 & 11.7 \\
\hline $00325147+4107490$ & 381063654713703040 & -394.7 & 2.64 & 0.10 & -5.534 & -5.534 & -0.95 & 398.7 \\
\hline $00331311+2033190$ & 2796309861892160384 & 9.8 & $\ldots$ & $\ldots$ & $\ldots$ & $\ldots$ & 0.03 & 9.8 \\
\hline 00341417-3943068 & 4994368227272214784 & 250.1 & 3.24 & 0.16 & 13.041 & 13.041 & -3.13 & 294.0 \\
\hline 00342795-2413107 & 2348080164279166208 & 3.1 & 0.13 & 0.00 & -51.758 & -51.758 & -0.10 & 33.5 \\
\hline 00351869-2854190 & 2319002587146050304 & 37.2 & 0.20 & 0.00 & 112.358 & 112.358 & -0.17 & 99.4 \\
\hline $00354136+1618228$ & 2780880960680375808 & -41.0 & 0.18 & 0.00 & 44.539 & 44.539 & -0.10 & 59.7 \\
\hline
\end{tabular}


Table 4 (continued)

\begin{tabular}{|c|c|c|c|c|c|c|c|c|}
\hline $\begin{array}{l}\text { Star Name } \\
\text { (2MASS) }\end{array}$ & $\begin{array}{c}\text { Star Name } \\
\text { (Gaia EDR3) }\end{array}$ & $\begin{array}{c}V_{\mathrm{los}} \\
\left(\mathrm{km} \mathrm{s}^{-1}\right)\end{array}$ & $\begin{array}{l}d_{\text {helio }} \\
(\mathrm{kpc})\end{array}$ & $\begin{array}{c}\sigma_{d} \\
(\mathrm{kpc})\end{array}$ & $\begin{array}{c}\mathrm{PM}_{\mathrm{R} . \mathrm{A}} \\
\left(\mathrm{mas} \mathrm{yr}^{-1}\right)\end{array}$ & $\begin{array}{c}\mathrm{PM}_{\text {Decl. }} \\
\left(\mathrm{mas} \mathrm{yr}^{-1}\right)\end{array}$ & $\begin{array}{l}Z_{\mathrm{Gal}} \\
(\mathrm{kpc})\end{array}$ & $\begin{array}{c}V_{\mathrm{TAV}} \\
\left(\mathrm{km} \mathrm{s}^{-1}\right)\end{array}$ \\
\hline $00370433+4341208$ & 387703815888550656 & -33.6 & 6.63 & 0.91 & 2.657 & 2.657 & -2.15 & 83.1 \\
\hline 00370966-4224102 & 4992914501101243264 & -14.3 & 0.14 & 0.00 & -43.446 & -43.446 & -0.11 & 97.9 \\
\hline 00374809-2751193 & 2343095974991737600 & 7.8 & 0.12 & 0.00 & 104.044 & 104.044 & -0.09 & 56.0 \\
\hline $00400685-4325183$ & 4980747129334018944 & 47.1 & 7.70 & 0.79 & 3.057 & 3.057 & -7.36 & 320.7 \\
\hline $00410089+3633397$ & 365840847508048384 & -153.9 & 3.18 & 0.11 & 5.212 & 5.212 & -1.38 & 248.6 \\
\hline $00410467+1649478$ & 2782313903504860032 & -57.9 & 0.54 & 0.00 & 8.886 & 8.886 & -0.37 & 141.9 \\
\hline $00425182+1925361$ & 2795241995584048000 & 4.7 & 1.68 & 0.04 & 35.604 & 35.604 & -1.13 & 291.1 \\
\hline 00433651-2719379 & 2343181981712123520 & 21.8 & 0.13 & 0.00 & 151.268 & 151.268 & -0.10 & 83.4 \\
\hline 00435264-2132285 & 2350389894612060544 & 67.4 & 2.70 & 0.12 & 19.345 & 19.345 & -2.66 & 269.3 \\
\hline 00452879-5846450 & 4906911251332870144 & 187.9 & 2.33 & 0.06 & -16.287 & -16.287 & -1.96 & 332.4 \\
\hline 00463619-3739335 & 5000753194373767424 & 48.3 & 4.06 & 0.26 & 15.259 & 15.259 & -3.97 & 278.9 \\
\hline 00482546-7441092 & 4685477782827620992 & 15.6 & 1.27 & 0.02 & 4.258 & 4.258 & -0.83 & 17.4 \\
\hline 00482715-8224023 & 4629945985987660416 & 139.1 & 3.47 & 0.10 & 6.495 & 6.495 & -1.95 & 145.8 \\
\hline 00504527-6351504 & 4709709305405862656 & -33.1 & 7.69 & 0.57 & 2.040 & 2.040 & -6.13 & 238.8 \\
\hline 00510748-7809118 & 4635149768362820096 & -0.5 & 0.18 & 0.00 & 36.727 & 36.727 & -0.09 & 8.5 \\
\hline 00521010-6006097 & 4903551070783622528 & 6.0 & 0.11 & 0.00 & -2.542 & -2.542 & -0.06 & 6.9 \\
\hline 00522310-5804087 & 4907332914042242304 & 3.4 & 0.52 & 0.00 & 18.114 & 18.114 & -0.42 & 26.0 \\
\hline $00523111+3758286$ & 367656523458156544 & -236.2 & 1.55 & 0.03 & 1.710 & 1.710 & -0.63 & 295.8 \\
\hline $00582707+0633561$ & 2553447805108682496 & 4.6 & 3.62 & 0.23 & 5.477 & 5.477 & -2.99 & 98.6 \\
\hline $00594615+1223173$ & 2584343227151274752 & -9.4 & 0.16 & 0.00 & -14.409 & -14.409 & -0.10 & 32.4 \\
\hline $01021265+0428241$ & 2551971405806340096 & -172.8 & 5.42 & 0.79 & 9.874 & 9.874 & -4.59 & 452.2 \\
\hline $01024809+4300486$ & 376344555103575808 & -21.9 & 0.93 & 0.02 & -0.255 & -0.255 & -0.29 & 23.3 \\
\hline $01031767+0908145$ & 2581189278047060224 & -20.3 & 3.09 & 0.21 & 8.386 & 8.386 & -2.46 & 145.9 \\
\hline 01033338-7410471 & 4684860888081427840 & 5.0 & 0.17 & 0.00 & 10.983 & 10.983 & -0.09 & 12.4 \\
\hline $01040440+0504477$ & 2552119633717667584 & -20.5 & 0.12 & 0.00 & -16.251 & -16.251 & -0.07 & 34.3 \\
\hline $01042513+4011391$ & 371347102956265728 & 17.9 & 0.22 & 0.00 & -8.887 & -8.887 & -0.06 & 33.3 \\
\hline $01042908+0755213$ & 2578034366869468800 & 3.6 & 0.19 & 0.00 & -10.960 & -10.960 & -0.13 & 39.7 \\
\hline 01051545-0041328 & 2533223147709485312 & 5.2 & 0.12 & 0.00 & 29.517 & 29.517 & -0.08 & 37.4 \\
\hline 01065190-5244105 & 4927175937828177280 & $\cdots$ & 8.55 & 0.82 & 9.022 & 9.022 & -7.68 & 238.3 \\
\hline 01132198-6139522 & 4710458244623085184 & $\ldots$ & 0.66 & 0.00 & 4.926 & 4.926 & -0.51 & 11.3 \\
\hline 01200289-0158201 & 2533312006288169600 & 83.1 & 0.74 & 0.01 & 30.210 & 30.210 & -0.64 & 162.9 \\
\hline 01250922-5614027 & 4910744423745801472 & $\ldots$ & 8.34 & 0.78 & 2.353 & 2.353 & -7.22 & 269.7 \\
\hline 01253364-4148345 & 4984027698370924672 & $\ldots$ & 0.43 & 0.00 & 0.256 & 0.256 & -0.39 & 44.0 \\
\hline 01253802-2911025 & $\ldots$ & 32.2 & $\ldots$ & $\ldots$ & $\ldots$ & $\ldots$ & $\ldots$ & $\ldots$ \\
\hline 01291742-7139220 & 4687775246726470144 & 208.4 & 7.12 & 0.51 & 1.975 & 1.975 & -5.03 & 338.1 \\
\hline 01311599-4016510 & 5008222486100643200 & -58.2 & 2.24 & 0.08 & 8.840 & 8.840 & -2.13 & 295.6 \\
\hline 01315199-6547540 & 4710799574264331904 & -33.9 & 0.35 & 0.03 & 99.318 & 99.318 & -0.25 & 121.0 \\
\hline 01323787-1530302 & 2451901038631956224 & 63.5 & 0.24 & 0.00 & 1.760 & 1.760 & -0.20 & 182.7 \\
\hline $01363655+5451319$ & 409152813849385216 & 0.7 & 0.18 & 0.00 & 16.368 & 16.368 & 0.00 & 14.2 \\
\hline 01372246-4611110 & 4931138955692103424 & 100.5 & 1.97 & 0.04 & 8.330 & 8.330 & -1.81 & 182.4 \\
\hline 01373378-6921368 & 4691261969896942848 & 53.7 & 0.13 & 0.00 & -84.055 & -84.055 & -0.07 & 57.4 \\
\hline 01382048-7637319 & 4636801853303548416 & 134.8 & 1.16 & 0.01 & 4.982 & 4.982 & -0.72 & 201.7 \\
\hline
\end{tabular}


Table 4 (continued)

\begin{tabular}{|c|c|c|c|c|c|c|c|c|}
\hline $\begin{array}{c}\text { Star Name } \\
\text { (2MASS) }\end{array}$ & $\begin{array}{c}\text { Star Name } \\
\text { (Gaia EDR3) }\end{array}$ & $\begin{array}{c}V_{\mathrm{los}} \\
\left(\mathrm{km} \mathrm{s}^{-1}\right)\end{array}$ & $\begin{array}{l}d_{\text {helio }} \\
(\mathrm{kpc})\end{array}$ & $\begin{array}{c}\sigma_{d} \\
(\mathrm{kpc})\end{array}$ & $\begin{array}{c}\mathrm{PM}_{\mathrm{R} . \mathrm{A}} \\
\left(\mathrm{mas} \mathrm{yr}^{-1}\right)\end{array}$ & $\begin{array}{c}\mathrm{PM}_{\text {Decl. }} \\
\left(\text { mas yr }^{-1}\right)\end{array}$ & $\begin{array}{l}Z_{\mathrm{Gal}} \\
(\mathrm{kpc})\end{array}$ & $\begin{array}{c}V_{\mathrm{TAV}} \\
\left(\mathrm{km} \mathrm{s}^{-1}\right)\end{array}$ \\
\hline 01390079-7811248 & 4633446629147784320 & 330.2 & 4.88 & 0.25 & 10.324 & 10.324 & -3.02 & 362.4 \\
\hline 01394297-1615477 & 2452308064092390784 & -220.1 & 3.98 & 0.24 & 7.862 & 7.862 & -3.80 & 406.1 \\
\hline 01430726-6445174 & 4710973567683306624 & 162.6 & 5.17 & 0.29 & 11.803 & 11.803 & -4.02 & 234.6 \\
\hline 01481644-5717024 & 4718885485854328064 & -85.4 & 1.77 & 0.03 & -5.596 & -5.596 & -1.48 & 343.2 \\
\hline 01481836-6022102 & 4717520854484700288 & $\ldots$ & 0.29 & 0.00 & -20.484 & -20.484 & -0.21 & 20.3 \\
\hline 01512909-5049125 & 4940475493039087872 & $\ldots$ & 10.56 & 1.25 & 9.844 & 9.844 & -9.44 & 316.4 \\
\hline 01532990-7617103 & 4636731828156259712 & 181.1 & 3.55 & 0.14 & 13.921 & 13.921 & -2.27 & 191.1 \\
\hline $01535484+4743406$ & 357061938652034816 & $\ldots$ & 0.30 & 0.00 & 9.471 & 9.471 & -0.05 & 21.9 \\
\hline 01541382-4927292 & 4940875062436326784 & 9.4 & 2.83 & 0.08 & 8.951 & 8.951 & -2.53 & 193.8 \\
\hline 01542953-4953166 & 4940665261873824640 & -34.8 & 5.21 & 0.30 & 4.610 & 4.610 & -4.66 & 125.4 \\
\hline 01555066-6400155 & 4699467590815290368 & 240.0 & 4.40 & 0.23 & 11.492 & 11.492 & -3.42 & 352.4 \\
\hline $01555808+5040276$ & 359446465839056512 & -15.6 & 0.16 & 0.00 & 26.903 & 26.903 & 0.00 & 25.0 \\
\hline 01570453-6511318 & 4699066135928244224 & -4.0 & 1.19 & 0.02 & 9.354 & 9.354 & -0.89 & 61.7 \\
\hline 01585657-1624249 & 5141897776909177856 & -7.5 & 0.83 & 0.02 & 17.531 & 17.531 & -0.76 & 99.5 \\
\hline $01592159+8341476$ & 572808964949945088 & -2.6 & 0.21 & 0.00 & 0.883 & 0.883 & 0.10 & 34.4 \\
\hline 02002105-2520170 & 5121763691780187008 & 46.9 & 0.69 & 0.01 & 30.965 & 30.965 & -0.64 & 118.2 \\
\hline $02002992+5657571$ & 505258239019253760 & 22.6 & 0.88 & 0.06 & 4.403 & 4.403 & -0.05 & 38.8 \\
\hline 02013041-0949339 & 2462500536881830528 & 213.0 & 2.03 & 0.09 & 22.264 & 22.264 & -1.83 & 321.7 \\
\hline 02020691-8507254 & 4616783629211669888 & 7.8 & 0.16 & 0.00 & 9.187 & 9.187 & -0.06 & 13.1 \\
\hline $02023240+5535052$ & 504560598882408960 & -4.2 & 0.26 & 0.01 & 20.266 & 20.266 & 0.00 & 21.0 \\
\hline 02062328-0718389 & 2487426224965026688 & $\ldots$ & 0.26 & 0.00 & -22.845 & -22.845 & -0.20 & 98.4 \\
\hline 02091623-2825011 & 5117222021562768256 & 64.6 & 4.03 & 0.24 & 17.853 & 17.853 & -3.82 & 352.9 \\
\hline 02121057-2136569 & 5124244804192255104 & -6.7 & 3.00 & 0.13 & 7.289 & 7.289 & -2.80 & 128.6 \\
\hline 02131127-3617490 & 4965179285932359552 & 9.7 & 0.17 & 0.00 & 26.291 & 26.291 & -0.14 & 28.3 \\
\hline 02143996-2731561 & 5116644773662434176 & -0.5 & 0.16 & 0.00 & -23.539 & -23.539 & -0.13 & 17.5 \\
\hline $02151298+4941500$ & 355587661060325120 & $\ldots$ & 0.32 & 0.00 & 17.132 & 17.132 & -0.04 & 20.0 \\
\hline $02165682+4443112$ & 352161380966711936 & 15.5 & 0.12 & 0.00 & -8.012 & -8.012 & -0.01 & 23.5 \\
\hline 02165716-7547064 & 4637170571951777280 & -6.1 & 4.55 & 0.23 & -3.316 & -3.316 & -2.91 & 37.8 \\
\hline 02184236-5351323 & 4743265369493805696 & -45.1 & 0.21 & 0.00 & -3.544 & -3.544 & -0.15 & 48.2 \\
\hline 02184254-6111160 & 4701711045508666112 & 159.4 & 2.05 & 0.05 & 33.257 & 33.257 & -1.61 & 228.7 \\
\hline 02194944-2701309 & 5117093275622914688 & 44.0 & 0.49 & 0.00 & 14.825 & 14.825 & -0.44 & 53.7 \\
\hline 02200131-5909599 & 4738094228868954496 & $\ldots$ & 0.09 & 0.00 & 19.189 & 19.189 & -0.05 & 14.2 \\
\hline 02200416-3505332 & 4966846038184021888 & -17.0 & 3.74 & 0.17 & 6.072 & 6.072 & -3.48 & 206.6 \\
\hline 02202248-3221349 & 4970735118186763520 & 43.5 & 0.17 & 0.00 & 70.075 & 70.075 & -0.14 & 74.5 \\
\hline 02205873-6708044 & 4696223279895236992 & -70.4 & 4.61 & 0.25 & 10.768 & 10.768 & -3.38 & 252.0 \\
\hline 02215591-1414291 & 5146733910084621440 & $\ldots$ & $\ldots$ & $\ldots$ & $\ldots$ & $\ldots$ & 0.03 & 0.0 \\
\hline $02230945+3819551$ & 331739842266523264 & -5.1 & 0.17 & 0.01 & 16.461 & 16.461 & -0.04 & 23.3 \\
\hline 02234760-4630467 & 4940121690813101056 & 138.2 & 1.98 & 0.04 & 12.889 & 12.889 & -1.75 & 199.1 \\
\hline $02242892+7959144$ & 562642266262613504 & 25.1 & 0.14 & 0.00 & 239.113 & 239.113 & 0.07 & 39.5 \\
\hline 02243601-3101131 & 5066948639089274496 & 23.2 & 0.26 & 0.02 & 7.840 & 7.840 & -0.22 & 32.3 \\
\hline 02255030-7822010 & 4632830898340209920 & 66.1 & 1.62 & 0.03 & 0.690 & 0.690 & -0.97 & 101.5 \\
\hline 02260082-2250455 & 5120483443633370496 & 101.1 & 0.65 & 0.01 & 27.400 & 27.400 & -0.58 & 136.6 \\
\hline
\end{tabular}


Table 4 (continued)

\begin{tabular}{|c|c|c|c|c|c|c|c|c|}
\hline $\begin{array}{c}\text { Star Name } \\
\text { (2MASS) }\end{array}$ & $\begin{array}{c}\text { Star Name } \\
\text { (Gaia EDR3) }\end{array}$ & $\begin{array}{c}V_{\mathrm{los}} \\
\left(\mathrm{km} \mathrm{s}^{-1}\right)\end{array}$ & $\begin{array}{l}d_{\text {helio }} \\
(\mathrm{kpc})\end{array}$ & $\begin{array}{c}\sigma_{d} \\
(\mathrm{kpc})\end{array}$ & 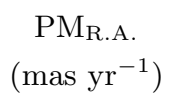 & 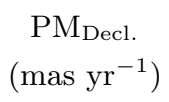 & $\begin{array}{l}Z_{\mathrm{Gal}} \\
(\mathrm{kpc})\end{array}$ & $\begin{array}{c}V_{\mathrm{TAV}} \\
\left(\mathrm{km} \mathrm{s}^{-1}\right)\end{array}$ \\
\hline 02263468-7052018 & 4645240501967006720 & $\ldots$ & 6.24 & 0.42 & 4.656 & 4.656 & -4.33 & 334.5 \\
\hline 02263472-1610120 & 5145471979973495040 & 2.9 & 0.13 & 0.00 & 75.713 & 75.713 & -0.09 & 62.1 \\
\hline 02263559-6118229 & 4701661876723001088 & 223.7 & 7.99 & 0.86 & 1.870 & 1.870 & -6.30 & 232.4 \\
\hline 02265573-3957537 & 4951576334231196160 & 20.2 & 3.43 & 0.14 & 6.183 & 6.183 & -3.12 & 212.9 \\
\hline 02272940-1844371 & 5131351909915040640 & 5.2 & 0.56 & 0.01 & 9.690 & 9.690 & -0.49 & 29.8 \\
\hline $02281118+3107248$ & 132459372685231872 & 24.0 & $\ldots$ & $\ldots$ & $\ldots$ & $\ldots$ & 0.03 & 24.0 \\
\hline $02284342+8235565$ & 569513114421444480 & $\ldots$ & 0.56 & 0.00 & -4.274 & -4.274 & 0.22 & 1.7 \\
\hline 02295644-7231067 & 4643772207267807104 & -28.5 & 0.26 & 0.00 & 17.933 & 17.933 & -0.15 & 63.3 \\
\hline 02302280-1713235 & 5133294712601873024 & $\ldots$ & 0.17 & 0.00 & -12.634 & -12.634 & -0.13 & 13.1 \\
\hline $02302823+3146355$ & 134052560743210624 & $\ldots$ & 0.20 & 0.00 & 16.616 & 16.616 & -0.06 & 45.8 \\
\hline 02314914-4251147 & 4950048081786940160 & 16.5 & 0.12 & 0.00 & 29.764 & 29.764 & -0.08 & 22.9 \\
\hline 02322533-2955268 & 5067349028825362176 & 26.8 & 0.22 & 0.00 & 19.293 & 19.293 & -0.18 & 34.1 \\
\hline 02332987-2602004 & 5070849431466425600 & $\ldots$ & 0.11 & 0.00 & 80.899 & 80.899 & -0.08 & 55.9 \\
\hline 02335901-5218323 & 4744814203419011456 & 55.1 & 0.11 & 0.00 & 98.925 & 98.925 & -0.07 & 63.2 \\
\hline 02343390-3438203 & 5062336050373022976 & 21.8 & 0.60 & 0.01 & -9.000 & -9.000 & -0.52 & 35.8 \\
\hline 02345434-3349391 & 5062448200558971136 & 113.0 & 6.72 & 0.52 & 2.169 & 2.169 & -6.16 & 306.9 \\
\hline 02361077-1202559 & 5171442680844060160 & 150.9 & 2.93 & 0.16 & 19.595 & 19.595 & -2.54 & 369.3 \\
\hline 02371057-4036121 & 4951834822543543040 & $\ldots$ & 0.21 & 0.00 & 6.449 & 6.449 & -0.17 & 24.8 \\
\hline $02372192+4302214$ & 340142202749502464 & $\ldots$ & 0.35 & 0.00 & -6.867 & -6.867 & -0.07 & 8.6 \\
\hline 02384449-3325102 & 5062531071950987392 & 50.5 & 2.70 & 0.11 & 9.318 & 9.318 & -2.44 & 160.9 \\
\hline 02394381-3631306 & 4953863662014601216 & 23.7 & 0.37 & 0.00 & 16.298 & 16.298 & -0.31 & 35.0 \\
\hline $02401392+2556291$ & 126323578110142848 & 11.1 & 0.22 & 0.00 & 5.150 & 5.150 & -0.09 & 19.2 \\
\hline $02404390+4457499$ & 340915713477131008 & -21.2 & 0.22 & 0.01 & -9.164 & -9.164 & -0.03 & 22.9 \\
\hline 02425864-3709379 & 4953584523499875712 & 152.3 & 1.72 & 0.03 & 9.897 & 9.897 & -1.52 & 176.5 \\
\hline 02431746-8608453 & 4613478261037323776 & 0.0 & 6.66 & 0.52 & 1.146 & 1.146 & -3.36 & 115.2 \\
\hline 02435125-2942551 & 5065856377366623872 & 5.8 & 0.12 & 0.00 & -4.724 & -4.724 & -0.08 & 10.3 \\
\hline $02451977+1332222$ & 31933391083889152 & -23.8 & 2.55 & 0.10 & 19.962 & 19.962 & -1.64 & 295.5 \\
\hline 02462323-3137296 & 5064607984993521792 & 12.8 & 0.20 & 0.00 & -9.950 & -9.950 & -0.16 & 15.8 \\
\hline 02471497-6303000 & 4721297363392975488 & 56.1 & 0.59 & 0.00 & 5.156 & 5.156 & -0.42 & 59.1 \\
\hline 02494852-2229202 & 5077735500993403648 & 124.8 & 0.12 & 0.00 & 66.542 & 66.542 & -0.08 & 135.3 \\
\hline $02501156+3457476$ & 140083622534201088 & $\ldots$ & 0.18 & 0.01 & 20.274 & 20.274 & -0.04 & 14.0 \\
\hline $02512543+8333571$ & 569663953674510464 & $\ldots$ & 0.20 & 0.00 & 63.748 & 63.748 & 0.10 & 72.0 \\
\hline 02523341-4416060 & 4755176207159038080 & 71.4 & 9.43 & 1.16 & -0.018 & -0.018 & -8.17 & 185.0 \\
\hline $02525369+2107466$ & 109283900954164992 & 36.7 & 0.14 & 0.00 & 121.112 & 121.112 & -0.05 & 89.9 \\
\hline 02525416-3228483 & 5052264558220148352 & 30.8 & $\ldots$ & $\ldots$ & $\ldots$ & $\ldots$ & 0.03 & 30.8 \\
\hline 02532757-3454050 & 5049605732946898432 & 37.9 & 2.93 & 0.10 & 6.447 & 6.447 & -2.59 & 86.8 \\
\hline 02533614-6234511 & 4721372684235614848 & 125.7 & 1.77 & 0.04 & 19.840 & 19.840 & -1.32 & 156.6 \\
\hline 02564805-1942473 & 5128076774013414912 & 19.2 & 0.17 & 0.00 & 7.097 & 7.097 & -0.12 & 20.6 \\
\hline $02571027+3318455$ & 136508702211948672 & 22.9 & 0.15 & 0.00 & 23.347 & 23.347 & -0.03 & 27.3 \\
\hline $02580877+0829424$ & 8791969854015488 & 47.1 & 0.22 & 0.00 & 19.669 & 19.669 & -0.12 & 53.4 \\
\hline 02590016-7209504 & 4645469170322185984 & $\ldots$ & 0.16 & 0.00 & 78.043 & 78.043 & -0.08 & 60.0 \\
\hline $03042561+3112028$ & 135287316592202880 & $\ldots$ & 0.18 & 0.00 & 17.415 & 17.415 & -0.05 & 30.8 \\
\hline
\end{tabular}


Table 4 (continued)

\begin{tabular}{|c|c|c|c|c|c|c|c|c|}
\hline $\begin{array}{c}\text { Star Name } \\
\text { (2MASS) }\end{array}$ & $\begin{array}{c}\text { Star Name } \\
\text { (Gaia EDR3) }\end{array}$ & $\begin{array}{c}V_{\mathrm{los}} \\
\left(\mathrm{km} \mathrm{s}^{-1}\right)\end{array}$ & $\begin{array}{l}d_{\text {helio }} \\
(\mathrm{kpc})\end{array}$ & $\begin{array}{c}\sigma_{d} \\
(\mathrm{kpc})\end{array}$ & $\begin{array}{c}\mathrm{PM}_{\mathrm{R} . \mathrm{A}} \\
\left(\mathrm{mas} \mathrm{yr}^{-1}\right)\end{array}$ & $\begin{array}{c}\mathrm{PM}_{\text {Decl. }} \\
\left(\text { mas yr }^{-1}\right)\end{array}$ & $\begin{array}{l}Z_{\mathrm{Gal}} \\
(\mathrm{kpc})\end{array}$ & $\begin{array}{c}V_{\mathrm{TAV}} \\
\left(\mathrm{km} \mathrm{s}^{-1}\right)\end{array}$ \\
\hline 03042843-3406046 & 5050991770433282688 & 122.5 & 2.86 & 0.10 & 4.617 & 4.617 & -2.47 & 307.8 \\
\hline 03044130-3635405 & 5047482782152551808 & 27.9 & 0.23 & 0.00 & 20.603 & 20.603 & -0.17 & 36.8 \\
\hline $03053573+2834227$ & 115967213665888000 & -36.5 & 2.08 & 0.08 & 30.401 & 30.401 & -0.88 & 343.9 \\
\hline 03060595-5251430 & 4746980344406420736 & 92.9 & 1.86 & 0.04 & 16.988 & 16.988 & -1.49 & 169.8 \\
\hline $03080797+3836489$ & 142648478221664256 & $\ldots$ & 0.33 & 0.00 & 16.552 & 16.552 & -0.07 & 35.3 \\
\hline 03091996-5853578 & 4724087928200048768 & $\ldots$ & 0.23 & 0.00 & 36.367 & 36.367 & -0.15 & 37.3 \\
\hline 03095592-0459281 & 5182694120770155264 & 25.2 & 0.09 & 0.00 & -7.807 & -7.807 & -0.04 & 43.0 \\
\hline $03104602+4514503$ & 433115020506112512 & 19.9 & 0.11 & 0.00 & 210.154 & 210.154 & 0.00 & 98.4 \\
\hline 03121034-5703094 & 4727345712434297984 & $\ldots$ & 11.20 & 1.40 & 6.794 & 6.794 & -8.69 & 196.5 \\
\hline 03123270-2849566 & 5059444369153859200 & -8.0 & 4.69 & 0.27 & 8.056 & 8.056 & -3.99 & 283.9 \\
\hline 03123346-5234570 & 4735321737284031232 & 33.8 & 0.23 & 0.01 & -5.571 & -5.571 & -0.16 & 42.8 \\
\hline 03131491-8107109 & 4619392843320436608 & 175.8 & 3.40 & 0.12 & 12.803 & 12.803 & -1.89 & 283.0 \\
\hline 03134048-8045218 & 4619419089865071744 & $\ldots$ & 0.33 & 0.00 & -4.002 & -4.002 & -0.16 & 2.2 \\
\hline $03135196+4230102$ & 240424332130561024 & $\ldots$ & 0.29 & 0.00 & 41.528 & 41.528 & -0.04 & 49.3 \\
\hline $03152783+3353586$ & 137535813574478592 & 36.6 & 0.13 & 0.00 & 21.657 & 21.657 & -0.02 & 56.3 \\
\hline $03155572+3357169$ & 125528253246150656 & -8.3 & 0.13 & 0.00 & -15.817 & -15.817 & -0.02 & 23.5 \\
\hline 03155933-7432577 & 4639776204054863232 & 6.5 & 0.44 & 0.00 & 34.730 & 34.730 & -0.26 & 46.5 \\
\hline $03163710+2332211$ & 110907432953980800 & -186.6 & 1.92 & 0.06 & 25.393 & 25.393 & -0.89 & 327.1 \\
\hline 03170396-3740469 & 4854289415101796608 & 147.9 & 4.14 & 0.18 & 2.982 & 2.982 & -3.48 & 165.9 \\
\hline 03171573-3747479 & 4854284497363037696 & 12.1 & 0.12 & 0.00 & -19.602 & -19.602 & -0.08 & 61.6 \\
\hline 03173348-3705188 & 4854694001021568896 & 41.1 & $\ldots$ & $\ldots$ & $\ldots$ & & 0.03 & 41.1 \\
\hline $03180842+1814447$ & 56110625281842816 & 119.5 & 1.02 & 0.02 & 30.257 & 30.257 & -0.52 & 189.6 \\
\hline 03190720-5245069 & 4735169768457507968 & 8.8 & 0.21 & 0.01 & 22.589 & 22.589 & -0.14 & 17.7 \\
\hline 03214149-5553303 & 4733473496892938368 & 16.7 & 0.18 & 0.00 & 39.385 & 39.385 & -0.11 & 47.8 \\
\hline 03220165-0020329 & 3262842880464520576 & -68.2 & 0.50 & 0.00 & -3.261 & -3.261 & -0.33 & 69.2 \\
\hline 03222245-3731294 & 4854443793406263040 & 25.4 & 0.20 & 0.00 & 46.146 & 46.146 & -0.14 & 43.8 \\
\hline $03223653+0859382$ & 11176917949244800 & -16.2 & 0.16 & 0.01 & 0.780 & 0.780 & -0.07 & 18.1 \\
\hline 03242169-3515217 & 4860920810247328256 & 137.2 & 1.66 & 0.03 & 15.340 & 15.340 & -1.36 & 171.5 \\
\hline 03242519-1550054 & 5106733402188456320 & 19.1 & 0.12 & 0.00 & -0.432 & -0.432 & -0.07 & 19.3 \\
\hline $03252266+8009505$ & 568038153934104320 & -55.4 & 2.30 & 0.07 & 3.106 & 3.106 & 0.79 & 61.5 \\
\hline 03260086-4126000 & 4849648891917127424 & 55.6 & 0.75 & 0.01 & 20.915 & 20.915 & -0.59 & 104.4 \\
\hline 03260534-2006507 & 5101544016542831232 & -20.4 & 0.48 & 0.00 & 16.759 & 16.759 & -0.37 & 51.5 \\
\hline $03263031+0616326$ & 9265348264761984 & 67.3 & 1.97 & 0.06 & 12.244 & 12.244 & -1.23 & 242.6 \\
\hline 03265306-0053348 & 3264007336293442048 & 14.4 & 0.12 & 0.00 & 31.705 & 31.705 & -0.06 & 25.4 \\
\hline $03265389+0202281$ & 3268028903151246720 & $\ldots$ & 1.74 & 0.05 & 22.686 & 22.686 & -1.15 & 220.3 \\
\hline 03275664-4544078 & 4846530329047408384 & 9.9 & 0.15 & 0.00 & -32.451 & -32.451 & -0.09 & 30.3 \\
\hline 03283529-4000252 & 4853194778260569856 & -5.2 & 0.22 & 0.00 & -18.959 & -18.959 & -0.15 & 43.1 \\
\hline $03283748+1856359$ & 57481853722629888 & -20.0 & 2.67 & 0.09 & 8.639 & 8.639 & -1.32 & 171.9 \\
\hline 03292423-6057094 & 4722654951015751680 & 148.3 & 4.28 & 0.19 & 12.577 & 12.577 & -3.11 & 204.6 \\
\hline 03304035-0321071 & 3249179288121898752 & 21.7 & 0.33 & 0.00 & -4.870 & -4.870 & -0.21 & 24.3 \\
\hline 03305484-0304088 & 3249204710032811648 & $\ldots$ & 0.18 & 0.00 & 30.999 & 30.999 & -0.10 & 43.2 \\
\hline $03312821+0749469$ & 11354660875621376 & $\ldots$ & 0.10 & 0.00 & 170.741 & 170.741 & -0.04 & 83.3 \\
\hline
\end{tabular}


Table 4 (continued)

\begin{tabular}{|c|c|c|c|c|c|c|c|c|}
\hline $\begin{array}{c}\text { Star Name } \\
\text { (2MASS) }\end{array}$ & $\begin{array}{c}\text { Star Name } \\
\text { (Gaia EDR3) }\end{array}$ & $\begin{array}{c}V_{\mathrm{los}} \\
\left(\mathrm{km} \mathrm{s}^{-1}\right)\end{array}$ & $\begin{array}{l}d_{\text {helio }} \\
(\mathrm{kpc})\end{array}$ & $\begin{array}{c}\sigma_{d} \\
(\mathrm{kpc})\end{array}$ & 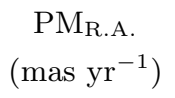 & $\begin{array}{c}\mathrm{PM}_{\text {Decl. }} \\
\left(\mathrm{mas}^{-1}{ }^{-1}\right)\end{array}$ & $\begin{array}{l}Z_{\text {Gal }} \\
(\mathrm{kpc})\end{array}$ & $\begin{array}{c}V_{\mathrm{TAV}} \\
\left(\mathrm{km} \mathrm{s}^{-1}\right)\end{array}$ \\
\hline 03343133-0555030 & 3247331455752180992 & 4.4 & 0.11 & 0.00 & -4.909 & -4.909 & -0.05 & 15.6 \\
\hline $03351518+7823252$ & 555315734952674816 & -319.8 & 4.21 & 0.17 & 6.994 & 6.994 & 1.34 & 331.3 \\
\hline 03354665-1356010 & 5113319751716905344 & 12.6 & 0.13 & 0.00 & -2.858 & -2.858 & -0.08 & 12.9 \\
\hline $03362369+2036490$ & 58156438465188096 & -91.4 & 1.69 & 0.08 & 18.292 & 18.292 & -0.76 & 280.2 \\
\hline $03362874-6005593$ & 4728790397697259392 & 76.8 & 0.43 & 0.00 & 55.488 & 55.488 & -0.29 & 110.7 \\
\hline 03365068-2327557 & 5086423493982030848 & -3.0 & 0.16 & 0.00 & 6.202 & 6.202 & -0.10 & 24.7 \\
\hline $03365778+2917172$ & 120001145732321536 & 7.8 & 0.18 & 0.00 & 15.551 & 15.551 & -0.04 & 33.9 \\
\hline 03370393-4829266 & 4833641097832497408 & -29.0 & 0.47 & 0.00 & 20.033 & 20.033 & -0.35 & 43.4 \\
\hline $03370580+1802236$ & 56464972968303104 & $\ldots$ & 0.85 & 0.01 & -5.037 & -5.037 & -0.39 & 44.2 \\
\hline 03385093-0255535 & 3250559347013995136 & -66.5 & 4.02 & 0.25 & 2.541 & 2.541 & -2.73 & 230.6 \\
\hline $03391370+0310345$ & 3271273935626311808 & 198.8 & 2.89 & 0.11 & 3.347 & 3.347 & -1.81 & 308.6 \\
\hline 03401638-5917516 & 4728932754388052224 & -9.8 & 0.14 & 0.00 & 90.244 & 90.244 & -0.07 & 77.7 \\
\hline 03410147-1812508 & 5107448320968631040 & 28.7 & 0.15 & 0.00 & -39.942 & -39.942 & -0.09 & 103.6 \\
\hline $03421349+2729013$ & 71184787964000000 & $\ldots$ & 0.17 & 0.00 & 34.641 & 34.641 & -0.04 & 32.7 \\
\hline 03425047-1013432 & 5116038431655398400 & $\ldots$ & 4.02 & 0.23 & 3.622 & 3.622 & -2.88 & 185.7 \\
\hline $03431186+7807495$ & 554502989702092416 & $\ldots$ & 0.38 & 0.00 & 10.011 & 10.011 & 0.14 & 19.9 \\
\hline 03435581-3212071 & 4863399349974669440 & 97.6 & 1.89 & 0.03 & 32.521 & 32.521 & -1.47 & 284.0 \\
\hline 03440716-2841235 & 5080387454320334592 & -31.8 & 0.24 & 0.00 & -24.650 & -24.650 & -0.16 & 71.1 \\
\hline 03440987-4057281 & 4854966508106088064 & -12.1 & 0.18 & 0.00 & 5.906 & 5.906 & -0.12 & 14.1 \\
\hline 03445544-7517390 & 4628961755579625600 & 2.1 & 0.28 & 0.02 & 22.659 & 22.659 & -0.14 & 35.4 \\
\hline 03453731-8211290 & 4616039813892309376 & 237.1 & 2.51 & 0.07 & 22.271 & 22.271 & -1.33 & 257.3 \\
\hline $03462539+2125305$ & 63534390354375040 & 8.1 & 0.13 & 0.00 & 20.311 & 20.311 & -0.03 & 28.4 \\
\hline 03463330-0023036 & 3251527020325320960 & 13.1 & 0.05 & 0.00 & -69.236 & -69.236 & -0.01 & 21.5 \\
\hline 03464056-1703266 & 5108001001657825280 & 58.3 & 3.62 & 0.20 & 13.360 & 13.360 & -2.68 & 473.2 \\
\hline 03470283-7933197 & 4625542858531568768 & 147.3 & 2.47 & 0.05 & 11.866 & 11.866 & -1.37 & 149.7 \\
\hline $03470366+7232599$ & 543671700657298816 & -20.8 & 0.65 & 0.03 & 8.940 & 8.940 & 0.18 & 24.8 \\
\hline $03475502-2219560$ & 50876748756555999744 & 27.3 & 0.21 & 0.00 & 30.762 & 30.762 & -0.14 & 43.6 \\
\hline 03481276-2657207 & 5081024380790201984 & -62.5 & 2.24 & 0.09 & 5.715 & 5.715 & -1.71 & 325.9 \\
\hline 03494330-1035255 & 3194196834787169152 & -24.9 & 0.09 & 0.00 & -49.489 & -49.489 & -0.04 & 47.3 \\
\hline 03494712-2012428 & 5094777381468797568 & 102.2 & 4.05 & 0.28 & 6.973 & 6.973 & -3.02 & 201.3 \\
\hline $03505656+1915396$ & 50799709243487232 & 14.8 & 0.11 & 0.00 & 22.875 & 22.875 & -0.03 & 26.9 \\
\hline 03511385-1222556 & 5114516604484590848 & 26.0 & 0.15 & 0.00 & -5.112 & -5.112 & -0.08 & 26.2 \\
\hline 03514691-4821339 & 4830105381971162368 & -2.3 & 0.17 & 0.00 & -17.221 & -17.221 & -0.10 & 9.9 \\
\hline $03515804+7834544$ & 554892216818025088 & -16.7 & 0.12 & 0.00 & 3.419 & 3.419 & 0.06 & 23.7 \\
\hline 03522200-7822390 & 4626118933904711936 & 293.1 & 3.23 & 0.12 & 10.741 & 10.741 & -1.83 & 367.2 \\
\hline 03525823-2712362 & 5080850520514122368 & 18.4 & 0.15 & 0.00 & 44.705 & 44.705 & -0.09 & 47.7 \\
\hline $03532071+0332467$ & 3272181586771744640 & 58.5 & 1.86 & 0.05 & 11.931 & 11.931 & -1.08 & 135.8 \\
\hline 03535378-8214319 & 4616030261884997504 & 41.5 & 2.86 & 0.11 & 14.260 & 14.260 & -1.51 & 92.9 \\
\hline 03542750-4930350 & 4829790406249501824 & $\ldots$ & 0.10 & 0.00 & 22.877 & 22.877 & -0.05 & 20.5 \\
\hline $03550575+0448406$ & 3273182073633848576 & 130.7 & 1.40 & 0.03 & 23.053 & 23.053 & -0.78 & 270.8 \\
\hline $03551870-3540553$ & 4858448073019453568 & -71.9 & 2.78 & 0.08 & 21.927 & 21.927 & -2.11 & 270.4 \\
\hline 03552492-3137538 & 4886536888554252288 & 191.5 & 0.55 & 0.00 & 46.702 & 46.702 & -0.39 & 221.1 \\
\hline
\end{tabular}


Table 4 (continued)

\begin{tabular}{|c|c|c|c|c|c|c|c|c|}
\hline $\begin{array}{c}\text { Star Name } \\
\text { (2MASS) }\end{array}$ & $\begin{array}{c}\text { Star Name } \\
\text { (Gaia EDR3) }\end{array}$ & $\begin{array}{c}V_{\mathrm{los}} \\
\left(\mathrm{km} \mathrm{s}^{-1}\right)\end{array}$ & $\begin{array}{l}d_{\text {helio }} \\
(\mathrm{kpc})\end{array}$ & $\begin{array}{c}\sigma_{d} \\
(\mathrm{kpc})\end{array}$ & $\begin{array}{c}\mathrm{PM}_{\mathrm{R} . \mathrm{A}} \\
\left(\mathrm{mas} \mathrm{yr}^{-1}\right)\end{array}$ & $\begin{array}{c}\mathrm{PM}_{\text {Decl. }} \\
\left(\text { mas yr }^{-1}\right)\end{array}$ & $\begin{array}{c}Z_{\mathrm{Gal}} \\
(\mathrm{kpc})\end{array}$ & $\begin{array}{c}V_{\mathrm{TAV}} \\
\left(\mathrm{km} \mathrm{s}^{-1}\right)\end{array}$ \\
\hline $03570714+1150353$ & 3304873572159327104 & 86.9 & 0.61 & 0.01 & 11.143 & 11.143 & -0.29 & 92.5 \\
\hline 03575259-3507496 & 4858859741341036032 & 73.6 & 0.34 & 0.00 & 11.523 & 11.523 & -0.23 & 75.3 \\
\hline 03580690-4125514 & 4842342190474113920 & $\ldots$ & 0.16 & 0.00 & 27.947 & 27.947 & -0.09 & 15.8 \\
\hline $03582857+0851479$ & 3301417188637954304 & $\ldots$ & 0.22 & 0.00 & 15.624 & 15.624 & -0.09 & 17.1 \\
\hline 03582979-0646599 & 3196842083667299968 & $\ldots$ & 0.16 & 0.00 & -0.695 & -0.695 & -0.08 & 16.1 \\
\hline 03583609-3024329 & 4887268613542620160 & 187.2 & 0.86 & 0.01 & 63.216 & 63.216 & -0.63 & 294.7 \\
\hline 03585372-2512589 & 5083157811305565184 & 45.1 & 0.17 & 0.00 & -11.421 & -11.421 & -0.10 & 78.9 \\
\hline 03590972-7052417 & 4665955133531907840 & 173.6 & 2.24 & 0.05 & 1.149 & 1.149 & -1.39 & 175.2 \\
\hline 04021386-2714330 & 4890018148526236416 & 104.3 & 1.68 & 0.04 & 7.713 & 7.713 & -1.22 & 318.5 \\
\hline 04030414-2123136 & 5090671530172203648 & 175.9 & 0.14 & 0.00 & 229.019 & 229.019 & -0.07 & 223.8 \\
\hline 04032141-5057006 & 4828667358199528064 & 38.8 & 0.14 & 0.00 & 24.806 & 24.806 & -0.08 & 66.6 \\
\hline $04034276+0516322$ & 3272889569179614464 & -16.2 & 0.14 & 0.00 & -13.406 & -13.406 & -0.05 & 22.3 \\
\hline 04051129-3610404 & 4857845781166112384 & 29.1 & 0.14 & 0.00 & 32.299 & 32.299 & -0.08 & 42.2 \\
\hline 04062658-1417389 & 5110102993011576192 & -53.2 & 1.45 & 0.04 & 7.586 & 7.586 & -0.96 & 272.1 \\
\hline 04064080-2822221 & 4889098510129080064 & 5.8 & 0.41 & 0.01 & -5.041 & -5.041 & -0.27 & 22.0 \\
\hline 04065230-1132234 & 3190316692611552000 & 53.6 & 0.23 & 0.00 & 43.919 & 43.919 & -0.13 & 71.7 \\
\hline 04065870-5405560 & 4779874227616110592 & 95.5 & 2.14 & 0.05 & 5.793 & 5.793 & -1.50 & 151.8 \\
\hline $04071847-3844270$ & 4844183765074837760 & 11.3 & 0.16 & 0.00 & -29.704 & -29.704 & -0.09 & 47.3 \\
\hline 04073220-1508180 & 5097968885766682240 & 11.8 & 0.18 & 0.00 & 0.633 & 0.633 & -0.10 & 12.7 \\
\hline 04081065-4723248 & 4831180463825371776 & 13.2 & $\ldots$ & $\ldots$ & $\ldots$ & $\cdots$ & 0.03 & 13.2 \\
\hline 04082208-8054055 & 4622225700669724160 & $\ldots$ & 4.33 & 0.21 & 7.381 & 7.381 & -2.32 & 117.8 \\
\hline 04082291-3306502 & 4882442375971513216 & 9.9 & 0.17 & 0.00 & -30.636 & -30.636 & -0.10 & 25.1 \\
\hline 04095634-2018250 & 5091033814957326464 & $\ldots$ & 0.64 & 0.00 & 10.401 & 10.401 & -0.43 & 30.2 \\
\hline 04123785-0354082 & 3203733925629461376 & 17.1 & 0.74 & 0.01 & 10.202 & 10.202 & -0.42 & 47.9 \\
\hline 04133760-2855548 & 4885822480873873408 & 181.0 & 3.08 & 0.10 & 6.087 & 6.087 & -2.18 & 237.2 \\
\hline 04144546-4431586 & 4837831066064068480 & $\ldots$ & 0.23 & 0.01 & -5.567 & -5.567 & -0.14 & 59.6 \\
\hline 04152012-4554089 & 4837327760319848576 & 9.0 & 0.17 & 0.00 & 3.381 & 3.381 & -0.10 & 28.2 \\
\hline 04161576-0521188 & 3202575628785925888 & -16.4 & 0.14 & 0.00 & 20.966 & 20.966 & -0.06 & 21.7 \\
\hline 04162853-6636540 & 4668867224438266368 & 28.5 & 3.44 & 0.12 & 7.803 & 7.803 & -2.18 & 75.9 \\
\hline 04163240-0602269 & 3202394930922308096 & 90.2 & 0.24 & 0.02 & 107.285 & 107.285 & -0.12 & 155.1 \\
\hline 04174265-5558407 & 4778645523371812480 & 11.7 & 0.23 & 0.00 & 29.465 & 29.465 & -0.13 & 40.3 \\
\hline 04211598-4852031 & 4782730209069106432 & -4.5 & 3.11 & 0.10 & 16.139 & 16.139 & -2.15 & 208.5 \\
\hline 04212925-5219577 & 4780985799511960576 & 100.1 & 6.77 & 0.50 & 2.069 & 2.069 & -4.66 & 219.4 \\
\hline 04213031-2030287 & 5091573542023760384 & 22.5 & 0.18 & 0.00 & -14.980 & -14.980 & -0.10 & 30.5 \\
\hline 04215801-2739310 & 4892329974803162752 & $\ldots$ & 0.10 & 0.00 & 13.945 & 13.945 & -0.04 & 32.8 \\
\hline 04225741-8308401 & 4615741777521531136 & 29.0 & 0.26 & 0.01 & 2.949 & 2.949 & -0.11 & 38.4 \\
\hline 04242725-8157493 & 4621912305496245248 & 206.1 & 1.17 & 0.02 & 6.986 & 6.986 & -0.59 & 206.9 \\
\hline 04251520-6213209 & 4675852078212908416 & 281.6 & 5.61 & 0.36 & 6.295 & 6.295 & -3.62 & 307.9 \\
\hline 04262201-3615167 & 4869018507227798400 & -16.4 & 1.87 & 0.05 & 20.637 & 20.637 & -1.27 & 278.6 \\
\hline 04282895-3353452 & 4871041608622321664 & 16.4 & 0.13 & 0.00 & -12.051 & -12.051 & -0.06 & 18.5 \\
\hline 04291257-4921466 & 4787721991859465728 & $\ldots$ & 0.72 & 0.01 & 5.411 & 5.411 & -0.47 & 12.9 \\
\hline 04293837-5448372 & 4776030816001572736 & 45.2 & 0.08 & 0.00 & 88.079 & 88.079 & -0.03 & 61.9 \\
\hline
\end{tabular}


Table 4 (continued)

\begin{tabular}{|c|c|c|c|c|c|c|c|c|}
\hline $\begin{array}{c}\text { Star Name } \\
\text { (2MASS) }\end{array}$ & $\begin{array}{c}\text { Star Name } \\
\text { (Gaia EDR3) }\end{array}$ & $\begin{array}{c}V_{\mathrm{los}} \\
\left(\mathrm{km} \mathrm{s}^{-1}\right)\end{array}$ & $\begin{array}{l}d_{\text {helio }} \\
(\mathrm{kpc})\end{array}$ & $\begin{array}{c}\sigma_{d} \\
(\mathrm{kpc})\end{array}$ & 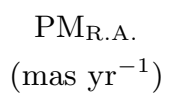 & 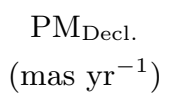 & $\begin{array}{l}Z_{\mathrm{Gal}} \\
(\mathrm{kpc})\end{array}$ & $\begin{array}{c}V_{\mathrm{TAV}} \\
\left(\mathrm{km} \mathrm{s}^{-1}\right)\end{array}$ \\
\hline $04311275-2258353$ & 4898098833139598464 & $\ldots$ & 7.85 & 0.66 & 2.604 & 2.604 & -5.07 & 116.2 \\
\hline 04313335-7337168 & 4652567991051879168 & 135.6 & 1.43 & 0.02 & 22.810 & 22.810 & -0.81 & 289.8 \\
\hline 04325046-3205240 & 4871721965802188160 & -7.8 & 0.11 & 0.00 & -2.312 & -2.312 & -0.05 & 11.0 \\
\hline 04330582-1605256 & 3172371116657963008 & $\ldots$ & $\ldots$ & $\ldots$ & $\ldots$ & $\ldots$ & 0.03 & 0.0 \\
\hline $04332408+7049217$ & 500082940861162240 & 9.3 & 0.18 & 0.00 & 4.847 & 4.847 & 0.07 & 18.0 \\
\hline 04332826-0431033 & 3201331222141772928 & -159.3 & 3.76 & 0.34 & 1.850 & 1.850 & -2.00 & 262.1 \\
\hline $04334315+8752515$ & 575291322904958208 & -31.6 & 0.18 & 0.01 & -17.597 & -17.597 & 0.11 & 66.8 \\
\hline $04354147-4750031$ & 4788319503413123200 & -24.0 & 0.31 & 0.00 & 29.006 & 29.006 & -0.18 & 104.8 \\
\hline 04354314-5355093 & 4777636927611794432 & 121.4 & 4.86 & 0.25 & 3.034 & 3.034 & -3.19 & 288.4 \\
\hline $04363802+7949143$ & 556538739776801664 & $\ldots$ & 0.27 & 0.00 & -12.261 & -12.261 & 0.12 & 16.6 \\
\hline 04391892-3404504 & 4868176796716806272 & $\ldots$ & 0.55 & 0.00 & 8.944 & 8.944 & -0.34 & 79.4 \\
\hline 04392332-2434534 & 4894484605277323648 & 215.7 & 0.33 & 0.00 & 8.493 & 8.493 & -0.18 & 252.9 \\
\hline 04392855-3957548 & 4816386358778340608 & 14.3 & 0.17 & 0.01 & -11.547 & -11.547 & -0.09 & 16.6 \\
\hline 04400253-1922270 & 2978208560171576448 & $\ldots$ & 0.73 & 0.02 & 11.086 & 11.086 & -0.42 & 40.2 \\
\hline 04404386-2741005 & 4879633570439853440 & 90.5 & 1.87 & 0.05 & 4.293 & 4.293 & -1.17 & 98.8 \\
\hline 04404847-4214219 & 4814898930000598272 & 289.9 & 4.79 & 0.21 & 1.374 & 1.374 & -3.14 & 297.2 \\
\hline 04404877-3530531 & 4867833886528092288 & 287.9 & 3.51 & 0.16 & 9.361 & 9.361 & -2.28 & 323.2 \\
\hline 04414545-1703252 & 2980627661847718016 & 2.1 & 0.20 & 0.00 & -7.033 & -7.033 & -0.09 & 21.7 \\
\hline 04452944-4656234 & 4786751256234998272 & 19.4 & 0.92 & 0.01 & 3.694 & 3.694 & -0.57 & 49.3 \\
\hline $04455221+8238074$ & 569892445935443200 & -74.6 & 0.14 & 0.00 & 41.570 & 41.570 & 0.08 & 76.4 \\
\hline 04460040-5244239 & 4777958977143271936 & 18.1 & 0.15 & 0.00 & -6.810 & -6.810 & -0.07 & 19.6 \\
\hline 04460379-4440454 & 4790350855440186752 & 195.6 & 4.07 & 0.20 & 11.452 & 11.452 & -2.62 & 257.4 \\
\hline 04480413-5133186 & 4784117101252301056 & 27.9 & 0.12 & 0.00 & 3.070 & 3.070 & -0.05 & 37.1 \\
\hline 04481274-3428106 & 4873248568978164608 & 10.4 & 0.12 & 0.00 & -24.481 & -24.481 & -0.05 & 22.7 \\
\hline 04482945-5127292 & 4784118686097335808 & 6.3 & 0.82 & 0.01 & -7.365 & -7.365 & -0.50 & 49.4 \\
\hline 04504247-4831497 & 4786320148893885568 & 19.5 & 0.16 & 0.00 & 1.990 & 1.990 & -0.08 & 45.4 \\
\hline $04505386+1803498$ & 3406732187621193600 & $\ldots$ & 0.97 & 0.02 & 5.936 & 5.936 & -0.25 & 52.5 \\
\hline 04514248-3210438 & 4874664052759778304 & 118.8 & 3.89 & 0.15 & 9.143 & 9.143 & -2.38 & 257.5 \\
\hline 04520803-2837181 & 4879979504285453184 & $\ldots$ & 0.17 & 0.00 & 47.758 & 47.758 & -0.08 & 34.7 \\
\hline 04522717-3451392 & 4873001625538322688 & 19.3 & 0.09 & 0.00 & 14.682 & 14.682 & -0.03 & 50.5 \\
\hline 04525913-4008568 & 4816836269489019904 & 116.9 & 5.77 & 0.34 & 2.151 & 2.151 & -3.61 & 213.8 \\
\hline $04542625-4241431$ & 4811990171989821824 & -20.0 & 5.48 & 0.29 & -1.754 & -1.754 & -3.42 & 153.2 \\
\hline 04562421-3124177 & 4874779806424378496 & 39.4 & 0.13 & 0.00 & 91.251 & 91.251 & -0.05 & 105.1 \\
\hline 04563790-5602511 & 4776246457719437440 & 112.5 & 6.78 & 0.59 & 5.697 & 5.697 & -4.16 & 190.8 \\
\hline $04582722-4611154$ & 4810231400062085504 & 30.3 & 0.46 & 0.00 & -2.133 & -2.133 & -0.26 & 31.2 \\
\hline 04583735-0449416 & 3212422747548345216 & $\ldots$ & 0.17 & 0.00 & 13.169 & 13.169 & -0.05 & 15.1 \\
\hline $05000638+0109473$ & 3228880619846899712 & 25.2 & 0.16 & 0.00 & 7.232 & 7.232 & -0.04 & 35.7 \\
\hline $05011881+0803047$ & 3290174403068832640 & 31.0 & 0.09 & 0.00 & 6.945 & 6.945 & -0.01 & 31.1 \\
\hline 05015088-4139079 & 4813429157832439168 & $\ldots$ & 0.10 & 0.00 & 31.560 & 31.560 & -0.04 & 12.1 \\
\hline $05015244+6745243$ & 483790308838071424 & $\ldots$ & 0.32 & 0.00 & -11.716 & -11.716 & 0.11 & 11.6 \\
\hline 05021896-5152056 & 4783532062283301120 & 247.7 & 2.94 & 0.10 & 12.096 & 12.096 & -1.77 & 276.7 \\
\hline 05022161-4603414 & 4810297405118590592 & 78.8 & $\ldots$ & $\ldots$ & $\ldots$ & $\ldots$ & 0.03 & 78.8 \\
\hline
\end{tabular}


Table 4 (continued)

\begin{tabular}{|c|c|c|c|c|c|c|c|c|}
\hline $\begin{array}{l}\text { Star Name } \\
\text { (2MASS) }\end{array}$ & $\begin{array}{c}\text { Star Name } \\
\text { (Gaia EDR3) }\end{array}$ & $\begin{array}{c}V_{\mathrm{los}} \\
\left(\mathrm{km} \mathrm{s}^{-1}\right)\end{array}$ & $\begin{array}{l}d_{\text {helio }} \\
(\mathrm{kpc})\end{array}$ & $\begin{array}{c}\sigma_{d} \\
(\mathrm{kpc})\end{array}$ & 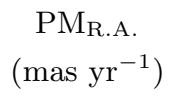 & $\begin{array}{c}\mathrm{PM}_{\text {Decl. }} \\
\left(\mathrm{mas}^{-1} \mathrm{yr}^{-1}\right)\end{array}$ & $\begin{array}{l}Z_{\text {Gal }} \\
(\mathrm{kpc})\end{array}$ & $\begin{array}{c}V_{\mathrm{TAV}} \\
\left(\mathrm{km} \mathrm{s}^{-1}\right)\end{array}$ \\
\hline $05024868+0053487$ & 3228646389509963008 & 33.5 & 0.30 & 0.00 & 6.712 & 6.712 & -0.10 & 35.0 \\
\hline 05025851-3909387 & 48143316807833568000 & 76.6 & 0.36 & 0.00 & 21.490 & 21.490 & -0.19 & 81.7 \\
\hline 05042173-6614399 & 4662031938654411008 & 27.6 & 0.08 & 0.00 & -83.523 & -83.523 & -0.02 & 72.5 \\
\hline $05043110+0151252$ & 3229000397895064960 & $\ldots$ & 0.31 & 0.00 & -6.371 & -6.371 & -0.10 & 19.5 \\
\hline 05065541-1056146 & 3181964218172190464 & -20.7 & 0.06 & 0.00 & -55.515 & -55.515 & -0.01 & 37.3 \\
\hline $05070417-0543063$ & 3211326195154437760 & 11.4 & 0.13 & 0.00 & -4.919 & -4.919 & -0.03 & 14.5 \\
\hline $05071155+3428162$ & 184903053675527552 & -5.4 & 4.48 & 0.40 & 1.349 & 1.349 & -0.26 & 26.4 \\
\hline $05071933+0950093$ & 3290839985560775552 & $\ldots$ & 0.41 & 0.03 & 2.439 & 2.439 & -0.10 & 20.8 \\
\hline $05071949+2233222$ & 3415364831369329024 & $\ldots$ & 0.34 & 0.03 & 1.365 & 1.365 & -0.04 & 9.6 \\
\hline $05085713+0345040$ & 3238657198066863616 & 28.8 & 0.33 & 0.01 & 2.745 & 2.745 & -0.09 & 30.0 \\
\hline $05090948+0758512$ & 3242001298259483776 & 347.2 & 3.02 & 0.15 & 10.447 & 10.447 & -0.93 & 420.9 \\
\hline $05104812-0827001$ & 3206923024747054080 & 11.4 & 0.27 & 0.00 & 1.309 & 1.309 & -0.10 & 11.9 \\
\hline 05115864-0512205 & 3211775654892063360 & 5.6 & 0.33 & 0.00 & 1.101 & 1.101 & -0.11 & 5.9 \\
\hline $05120490+0354513$ & 3235687077858885248 & -173.9 & 2.14 & 0.10 & 15.144 & 15.144 & -0.71 & 360.1 \\
\hline 05124167-4059465 & 4818944995352554496 & 97.8 & 0.12 & 0.00 & 32.779 & 32.779 & -0.05 & 175.8 \\
\hline 05130026-2540113 & 2956447060393795200 & 12.3 & 0.16 & 0.00 & -37.367 & -37.367 & -0.06 & 34.4 \\
\hline 05134078-0629405 & 3208349473581491968 & 331.2 & 3.78 & 0.47 & 8.440 & 8.440 & -1.55 & 374.2 \\
\hline $05144882+7604501$ & 503757371280728448 & -7.7 & 0.82 & 0.01 & -10.662 & -10.662 & 0.32 & 22.8 \\
\hline $05145383+3147370$ & 180592968094361472 & -6.3 & 0.53 & 0.01 & -5.895 & -5.895 & -0.01 & 30.0 \\
\hline 05160558-1418474 & 2985144932353707264 & 101.4 & 0.16 & 0.00 & 32.774 & 32.774 & -0.05 & 111.0 \\
\hline 05161882-0759103 & 3207022255670687488 & 8.0 & 0.22 & 0.00 & 0.519 & 0.519 & -0.07 & 8.4 \\
\hline 05163919-0740502 & 3207049193707252992 & 7.0 & 0.24 & 0.01 & 3.754 & 3.754 & -0.08 & 14.2 \\
\hline 05165318-1711429 & 2982252980551829760 & 177.1 & 4.57 & 0.39 & 4.684 & 4.684 & -2.15 & 202.0 \\
\hline $05170982+0306456$ & 3235326060087791232 & 86.3 & 3.55 & 0.21 & 2.123 & 2.123 & -1.15 & 150.4 \\
\hline $05171185+7649087$ & 551880864924665088 & -15.1 & 0.16 & 0.00 & 92.861 & 92.861 & 0.08 & 42.9 \\
\hline $05172978-4253358$ & 4800721449115828352 & $\ldots$ & 4.27 & 0.24 & 8.119 & 8.119 & -2.41 & 168.6 \\
\hline 05175177-1441274 & 2985070199923468416 & 18.2 & 0.08 & 0.00 & -81.537 & -81.537 & -0.01 & 36.9 \\
\hline $05180022-2418191$ & 2958140484397025792 & 186.0 & 4.64 & 0.26 & 9.156 & 9.156 & -2.34 & 263.3 \\
\hline $05181193+1750335$ & 3395305135071832448 & 10.8 & 0.64 & 0.01 & 7.126 & 7.126 & -0.10 & 23.9 \\
\hline 05181299-0356513 & 3213469349475577472 & 31.4 & 0.33 & 0.00 & 1.152 & 1.152 & -0.10 & 31.5 \\
\hline 05183018-4217511 & 48067667014839686640 & 5.2 & 0.77 & 0.01 & 7.157 & 7.157 & -0.41 & 47.5 \\
\hline 05183839-0208303 & 3214278143356960256 & $\ldots$ & 0.39 & 0.00 & 1.470 & 1.470 & -0.12 & 2.8 \\
\hline 05191853-0625013 & 3207648530621533056 & 142.5 & 0.20 & 0.00 & 91.397 & 91.397 & -0.06 & 167.6 \\
\hline 05202360-2522274 & 2957773415014463872 & 416.1 & 0.10 & 0.00 & 141.588 & 141.588 & -0.03 & 429.2 \\
\hline 05204433-0757598 & 3207154098284136064 & 8.0 & 0.35 & 0.03 & 3.304 & 3.304 & -0.12 & 12.2 \\
\hline 05211432-1032144 & 3014044878233010304 & -49.7 & 0.17 & 0.00 & 2.270 & 2.270 & -0.05 & 57.7 \\
\hline $05221674+1850154$ & 3401393307050179968 & 11.6 & 0.30 & 0.01 & 0.391 & 0.391 & -0.03 & 13.7 \\
\hline 05224909-0008545 & 3221372600399707520 & $\ldots$ & 0.34 & 0.00 & 1.455 & 1.455 & -0.09 & 2.7 \\
\hline $05232882+6904581$ & 485426657021670784 & $\ldots$ & 0.83 & 0.01 & -4.104 & -4.104 & 0.28 & 5.7 \\
\hline $05234159+0001547$ & 3221399469715891712 & 19.6 & 0.34 & 0.00 & 1.415 & 1.415 & -0.09 & 19.8 \\
\hline $05234178+0140465$ & 3222180191690519296 & 24.8 & 0.35 & 0.00 & 1.425 & 1.425 & -0.09 & 24.9 \\
\hline $05240221+0037377$ & 3221857420604615040 & $\ldots$ & 0.61 & 0.01 & 4.713 & 4.713 & -0.18 & 28.0 \\
\hline
\end{tabular}


Table 4 (continued)

\begin{tabular}{|c|c|c|c|c|c|c|c|c|}
\hline $\begin{array}{c}\text { Star Name } \\
\text { (2MASS) }\end{array}$ & $\begin{array}{c}\text { Star Name } \\
\text { (Gaia EDR3) }\end{array}$ & $\begin{array}{c}V_{\mathrm{los}} \\
\left(\mathrm{km} \mathrm{s}^{-1}\right)\end{array}$ & $\begin{array}{l}d_{\text {helio }} \\
(\mathrm{kpc})\end{array}$ & $\begin{array}{c}\sigma_{d} \\
(\mathrm{kpc})\end{array}$ & 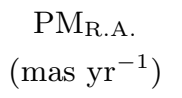 & $\begin{array}{c}\mathrm{PM}_{\text {Decl. }} \\
\left(\mathrm{mas}^{-1}{ }^{-1}\right)\end{array}$ & $\begin{array}{l}Z_{\text {Gal }} \\
(\mathrm{kpc})\end{array}$ & $\begin{array}{c}V_{\mathrm{TAV}} \\
\left(\mathrm{km} \mathrm{s}^{-1}\right)\end{array}$ \\
\hline 05240835-0847159 & 3014887683958579840 & 45.4 & 0.18 & 0.00 & 9.030 & 9.030 & -0.05 & 46.4 \\
\hline $05244855+3052198$ & 3447044445721668096 & $\ldots$ & 1.35 & 0.02 & 0.403 & 0.403 & -0.04 & 9.9 \\
\hline $05250902-4636237$ & 4798948177378743680 & 43.9 & 0.18 & 0.00 & 42.407 & 42.407 & -0.07 & 50.3 \\
\hline $05251415-6236322$ & 4757456177662951296 & 24.5 & 0.11 & 0.00 & 66.624 & 66.624 & -0.03 & 58.1 \\
\hline 05254247-2935153 & 2906535455902590464 & $\ldots$ & 0.69 & 0.01 & -4.702 & -4.702 & -0.33 & 13.5 \\
\hline 05255980-1225415 & 2985857656406840832 & 7.1 & 0.28 & 0.00 & -0.086 & -0.086 & -0.09 & 7.3 \\
\hline 05261364-0409463 & 3210609210495286016 & $\ldots$ & 0.32 & 0.00 & -1.147 & -1.147 & -0.09 & 8.6 \\
\hline $05261736+0054216$ & 3221894563481860352 & 14.6 & 0.32 & 0.00 & 1.373 & 1.373 & -0.08 & 14.8 \\
\hline $05263189+5101299$ & 261986133108337024 & 27.3 & 0.50 & 0.01 & 3.646 & 3.646 & 0.10 & 48.5 \\
\hline $05270704+0208344$ & 3222306291931030912 & $\ldots$ & 0.31 & 0.01 & 2.733 & 2.733 & -0.07 & 4.5 \\
\hline 05271660-1148112 & 3009915249997569024 & 68.1 & 0.10 & 0.00 & -75.260 & -75.260 & -0.02 & 83.3 \\
\hline 05272500-3754396 & 4821159308691479424 & -38.8 & 0.08 & 0.00 & 5.204 & 5.204 & -0.02 & 55.0 \\
\hline 05284287-0020406 & 3220938052789255552 & $\ldots$ & 0.41 & 0.01 & -0.233 & -0.233 & -0.11 & 0.5 \\
\hline $05284445+0105281$ & 3221936173125954432 & 16.7 & 0.13 & 0.00 & -3.106 & -3.106 & -0.01 & 17.3 \\
\hline 05291238-3538496 & 4822053555242274688 & 85.7 & 1.43 & 0.03 & 16.487 & 16.487 & -0.72 & 186.3 \\
\hline 05293906-0037377 & 3220726675974365184 & 19.2 & 0.34 & 0.00 & 1.454 & 1.454 & -0.08 & 19.4 \\
\hline $05310644+1002437$ & 3338225775622947584 & 29.5 & 0.35 & 0.04 & -1.073 & -1.073 & -0.05 & 29.6 \\
\hline 05310938-0734569 & 3016409408052028416 & 76.5 & 2.14 & 0.08 & 7.267 & 7.267 & -0.75 & 352.9 \\
\hline $05311096+0119175$ & 3221955960038003968 & 24.4 & 0.35 & 0.00 & 1.745 & 1.745 & -0.08 & 24.6 \\
\hline 05313358-5942455 & 4759644931651720064 & 217.5 & 4.20 & 0.24 & 3.859 & 3.859 & -2.27 & 245.3 \\
\hline 05314665-7729338 & 4623987049577991808 & $\ldots$ & 0.77 & 0.01 & -7.724 & -7.724 & -0.37 & 26.4 \\
\hline 05315984-0724275 & 3016418655117200896 & 31.6 & 0.36 & 0.00 & 2.853 & 2.853 & -0.10 & 31.9 \\
\hline $05322358+0426172$ & 3236515250632113536 & 54.1 & 0.19 & 0.00 & 11.655 & 11.655 & -0.03 & 55.5 \\
\hline $05323333+5054220$ & 215399069525282304 & $\ldots$ & 0.13 & 0.00 & -16.380 & -16.380 & 0.05 & 7.8 \\
\hline 05323416-3555512 & 4821789049679265536 & $\ldots$ & 0.38 & 0.00 & -0.635 & -0.635 & -0.17 & 7.4 \\
\hline $05330330+3425560$ & 3449578029750593408 & -22.6 & 2.53 & 0.13 & -0.066 & -0.066 & 0.05 & 32.7 \\
\hline 05331754-0208154 & 3216941882074480000 & 26.8 & 0.41 & 0.02 & -0.649 & -0.649 & -0.10 & 26.8 \\
\hline 05332244-5030257 & 4793470921547465856 & 287.3 & 2.93 & 0.08 & -0.437 & -0.437 & -1.56 & 325.2 \\
\hline 05333204-2332171 & 2963722876730308224 & 60.3 & 0.25 & 0.02 & -14.510 & -14.510 & -0.09 & 72.2 \\
\hline 05341283-0120379 & 3217572555072525056 & 33.5 & 0.41 & 0.00 & 1.195 & 1.195 & -0.10 & 33.6 \\
\hline $05343131+0352284$ & 3224421859677408384 & 152.0 & 2.95 & 0.15 & -2.794 & -2.794 & -0.75 & 363.8 \\
\hline 05344241-0015449 & 3220804015449200384 & $\ldots$ & 0.39 & 0.01 & -1.034 & -1.034 & -0.09 & 3.3 \\
\hline 05355964-3116059 & 2902351985957543552 & 40.6 & 0.62 & 0.14 & 0.547 & 0.547 & -0.27 & 46.5 \\
\hline $05363390+0511260$ & 3332736468116552192 & $\ldots$ & 0.37 & 0.01 & -1.820 & -1.820 & -0.07 & 3.3 \\
\hline $05365678-4350228$ & 4802513236454812160 & $\ldots$ & 0.71 & 0.00 & 0.318 & 0.318 & -0.34 & 68.5 \\
\hline $05365967+0411503$ & 3224519505758364160 & 30.4 & 0.40 & 0.01 & -0.612 & -0.612 & -0.08 & 30.4 \\
\hline 05370169-5105089 & 4793302318311314176 & 2.2 & 0.25 & 0.00 & -2.475 & -2.475 & -0.11 & 17.8 \\
\hline $05374341+5648268$ & 268483082894179072 & -23.8 & 0.26 & 0.00 & -3.257 & -3.257 & 0.08 & 34.8 \\
\hline $05382360+7011135$ & 485911644728122624 & -26.8 & 0.26 & 0.00 & 4.291 & 4.291 & 0.11 & 27.6 \\
\hline 05384334-5147228 & 4793246488033004160 & $\ldots$ & 3.24 & 0.11 & 13.252 & 13.252 & -1.69 & 135.7 \\
\hline 05391044-2345290 & 2963434353708776320 & 44.0 & 0.43 & 0.00 & 1.582 & 1.582 & -0.16 & 61.0 \\
\hline 05404660-1040257 & 3010342513344338688 & 66.6 & 0.14 & 0.00 & 12.838 & 12.838 & -0.02 & 68.3 \\
\hline
\end{tabular}


Table 4 (continued)

\begin{tabular}{|c|c|c|c|c|c|c|c|c|}
\hline $\begin{array}{l}\text { Star Name } \\
\text { (2MASS) }\end{array}$ & $\begin{array}{c}\text { Star Name } \\
\text { (Gaia EDR3) }\end{array}$ & $\begin{array}{c}V_{\mathrm{los}} \\
\left(\mathrm{km} \mathrm{s}^{-1}\right)\end{array}$ & $\begin{array}{l}d_{\text {helio }} \\
(\mathrm{kpc})\end{array}$ & $\begin{array}{c}\sigma_{d} \\
(\mathrm{kpc})\end{array}$ & $\begin{array}{c}\mathrm{PM}_{\mathrm{R} . \mathrm{A}} \\
\left({\left.\mathrm{mas} \mathrm{yr}^{-1}\right)}\right.\end{array}$ & $\begin{array}{c}\mathrm{PM}_{\text {Decl. }} \\
\left(\mathrm{mas} \mathrm{yr}^{-1}\right)\end{array}$ & $\begin{array}{l}Z_{\mathrm{Gal}} \\
(\mathrm{kpc})\end{array}$ & $\begin{array}{c}V_{\mathrm{TAV}} \\
\left(\mathrm{km} \mathrm{s}^{-1}\right)\end{array}$ \\
\hline $05415000+1525054$ & 3347851759405013248 & 24.4 & 0.21 & 0.01 & 10.654 & 10.654 & 0.00 & 32.9 \\
\hline 05423307-5913398 & 4759918400809516416 & 222.9 & 1.49 & 0.03 & 19.782 & 19.782 & -0.76 & 235.0 \\
\hline $05431543+7808008$ & 552468274714043392 & -45.4 & 0.15 & 0.00 & 16.266 & 16.266 & 0.08 & 57.9 \\
\hline $05434309+5012096$ & 214379311147693312 & $\ldots$ & 0.27 & 0.00 & 3.577 & 3.577 & 0.07 & 30.2 \\
\hline $05434572-2151551$ & 2964356642107544192 & 191.1 & 0.10 & 0.00 & 100.852 & 100.852 & -0.02 & 197.0 \\
\hline 05434805-2538412 & 2914965720791328896 & -14.9 & 1.64 & 0.03 & 16.642 & 16.642 & -0.68 & 372.9 \\
\hline 05440311-6050523 & 4758589400489342080 & 25.8 & 0.85 & 0.11 & 3.792 & 3.792 & -0.42 & 27.3 \\
\hline 05440932-3820149 & 4808589176366959360 & 107.5 & 1.95 & 0.03 & 11.620 & 11.620 & -0.92 & 152.3 \\
\hline 05450050-2105418 & 2965923995930380160 & 39.2 & 0.10 & 0.00 & 14.388 & 14.388 & -0.01 & 40.1 \\
\hline $05455248-2556383$ & 2914192523598690048 & 294.7 & 2.52 & 0.07 & 27.407 & 27.407 & -1.05 & 423.8 \\
\hline $05463023+4210063$ & 192794656646602880 & 41.5 & 0.12 & 0.00 & 3.161 & 3.161 & 0.04 & 50.5 \\
\hline 05463758-4103031 & 4804867874965676032 & 14.3 & 0.17 & 0.00 & 31.083 & 31.083 & -0.06 & 78.5 \\
\hline 05464200-1802448 & 2967658131925635968 & 16.3 & 0.09 & 0.00 & -29.518 & -29.518 & -0.01 & 20.6 \\
\hline 05472677-5432402 & 4767980226222674944 & 192.8 & 3.82 & 0.13 & 2.635 & 2.635 & -1.94 & 235.3 \\
\hline $05483017-5835567$ & 4765250138851402112 & 113.9 & 0.64 & 0.00 & -7.970 & -7.970 & -0.30 & 118.3 \\
\hline 05483336-2434285 & 2915518908282121472 & -43.2 & 0.19 & 0.00 & 29.060 & 29.060 & -0.05 & 60.8 \\
\hline $05483820+3148424$ & 3445140602679798400 & $\ldots$ & 2.23 & 0.08 & -0.069 & -0.069 & 0.10 & 39.7 \\
\hline 05505657-2008418 & 2966088402979538944 & 245.1 & 2.34 & 0.07 & 13.501 & 13.501 & -0.85 & 284.0 \\
\hline 05511133-0653111 & 3018944989240573056 & 31.0 & 0.35 & 0.00 & 2.574 & 2.574 & -0.08 & 31.5 \\
\hline 05521578-3953184 & 4805034691496015104 & 103.8 & 2.36 & 0.07 & 4.519 & 4.519 & -1.08 & 137.4 \\
\hline 05530383-2007355 & 2966268035692065280 & $\ldots$ & 0.85 & 0.01 & 1.914 & 1.914 & -0.29 & 8.9 \\
\hline 05531036-4113596 & 4804147870945271168 & -15.0 & 0.94 & 0.01 & -4.486 & -4.486 & -0.42 & 21.9 \\
\hline $05542509+1632212$ & 3349664785359713280 & $\ldots$ & 0.27 & 0.02 & 8.105 & 8.105 & 0.00 & 23.5 \\
\hline 05544693-8301197 & 4620849386990258560 & 168.4 & 4.56 & 0.24 & 5.693 & 5.693 & -2.16 & 176.5 \\
\hline $05545543+5131242$ & 214916074682985856 & -9.2 & 2.87 & 0.10 & 0.297 & 0.297 & 0.66 & 9.6 \\
\hline 05583688-2438574 & 2914576528036091136 & 12.0 & 0.22 & 0.01 & 3.727 & 3.727 & -0.06 & 17.8 \\
\hline 05583844-3434436 & 2889188186073231104 & $\ldots$ & 0.81 & 0.01 & 2.500 & 2.500 & -0.32 & 24.8 \\
\hline $05584204+5040146$ & 211594935389482240 & 55.4 & 0.19 & 0.00 & -6.961 & -6.961 & 0.07 & 73.6 \\
\hline 05592533-7744016 & 4647752959052853632 & $\cdots$ & 3.55 & 0.17 & 7.200 & 7.200 & -1.71 & 204.2 \\
\hline 05594737-2708140 & 2911019229961694208 & 15.1 & 6.26 & 0.39 & 2.083 & 2.083 & -2.39 & 206.4 \\
\hline 06000654-3034592 & 2891276807192892800 & 15.4 & 0.23 & 0.00 & -3.437 & -3.437 & -0.07 & 19.7 \\
\hline $06000928+6830331$ & 1105490157831460352 & $\ldots$ & 0.85 & 0.01 & -0.343 & -0.343 & 0.32 & 9.5 \\
\hline 06010912-2251504 & 2916542798424334208 & 72.4 & 0.12 & 0.00 & 25.477 & 25.477 & -0.02 & 76.8 \\
\hline 06013941-3304567 & 2889974641827298048 & -13.8 & 0.15 & 0.00 & -44.143 & -44.143 & -0.04 & 30.5 \\
\hline 06023445-3332108 & 2889528175684527104 & $\ldots$ & 0.50 & 0.00 & -2.054 & -2.054 & -0.18 & 5.2 \\
\hline 06032005-7531388 & 5261685578730080000 & 36.6 & 0.53 & 0.00 & -3.028 & -3.028 & -0.23 & 50.0 \\
\hline 06032024-2059364 & 2917926946123381248 & $\cdots$ & 2.81 & 0.10 & 8.565 & 8.565 & -0.92 & 244.8 \\
\hline 06035878-3445341 & 2886205760783047808 & $\ldots$ & 0.15 & 0.00 & 62.687 & 62.687 & -0.04 & 61.1 \\
\hline 06040505-3922438 & 2882964602365617408 & -2.4 & 0.15 & 0.00 & -13.304 & -13.304 & -0.04 & 18.4 \\
\hline 06043058-3232288 & 2889972447101620480 & 26.0 & 0.59 & 0.01 & -2.492 & -2.492 & -0.21 & 27.3 \\
\hline 06050821-3625551 & 2885764135065967488 & $\ldots$ & 0.21 & 0.02 & -15.244 & -15.244 & -0.06 & 19.3 \\
\hline $06050851+6114505$ & 1005911306711125632 & 27.4 & 0.12 & 0.00 & 168.401 & 168.401 & 0.06 & 85.7 \\
\hline
\end{tabular}


Table 4 (continued)

\begin{tabular}{|c|c|c|c|c|c|c|c|c|}
\hline $\begin{array}{l}\text { Star Name } \\
\text { (2MASS) }\end{array}$ & $\begin{array}{c}\text { Star Name } \\
\text { (Gaia EDR3) }\end{array}$ & $\begin{array}{c}V_{\mathrm{los}} \\
\left(\mathrm{km} \mathrm{s}^{-1}\right)\end{array}$ & $\begin{array}{l}d_{\text {helio }} \\
(\mathrm{kpc})\end{array}$ & $\begin{array}{c}\sigma_{d} \\
(\mathrm{kpc})\end{array}$ & $\begin{array}{c}\mathrm{PM}_{\mathrm{R} . \mathrm{A}} \\
\left(\mathrm{mas} \mathrm{yr}^{-1}\right)\end{array}$ & $\begin{array}{c}\mathrm{PM}_{\text {Decl. }} \\
\left(\mathrm{mas} \mathrm{yr}^{-1}\right)\end{array}$ & $\begin{array}{l}Z_{\text {Gal }} \\
(\mathrm{kpc})\end{array}$ & $\begin{array}{c}V_{\mathrm{TAV}} \\
\left(\mathrm{km} \mathrm{s}^{-1}\right)\end{array}$ \\
\hline $06050978+6654472$ & 1105001321832556800 & -19.2 & 0.26 & 0.00 & 1.992 & 1.992 & 0.12 & 23.5 \\
\hline 06052881-4438188 & 5567973273001201152 & $\ldots$ & 0.67 & 0.00 & 39.707 & 39.707 & -0.28 & 94.1 \\
\hline 06055284-3537284 & 2885916589224697088 & 181.2 & 0.98 & 0.01 & 3.061 & 3.061 & -0.38 & 214.7 \\
\hline 06055999-6907423 & 5279730523050938624 & 193.5 & 2.84 & 0.09 & 7.405 & 7.405 & -1.36 & 228.0 \\
\hline 06063114-1605358 & 2991490626275600640 & 17.8 & 0.29 & 0.00 & -5.445 & -5.445 & -0.06 & 20.9 \\
\hline 06063142-5032308 & 5549982410912756992 & $\ldots$ & 0.39 & 0.00 & -5.561 & -5.561 & -0.15 & 14.6 \\
\hline 06074714-3724498 & 2884863016567274240 & -2.0 & 0.60 & 0.00 & -3.097 & -3.097 & -0.22 & 14.6 \\
\hline 06085789-3536279 & 2885882774945629824 & 6.8 & 0.17 & 0.00 & -1.292 & -1.292 & -0.04 & 68.4 \\
\hline 06090353-7122260 & 5278332292157596160 & 190.5 & 2.98 & 0.11 & 19.498 & 19.498 & -1.42 & 256.5 \\
\hline 06120579-3152561 & 2895882146005313152 & 1.1 & 0.15 & 0.00 & 4.804 & 4.804 & -0.03 & 17.6 \\
\hline 06134289-6937017 & 5279560687166096896 & 212.2 & 1.92 & 0.05 & 18.928 & 18.928 & -0.89 & 223.8 \\
\hline 06135706-3555284 & 2885428298685730816 & 3.1 & 0.22 & 0.01 & 34.066 & 34.066 & -0.06 & 75.4 \\
\hline 06155680-7459448 & 5261728773219036800 & 136.6 & 2.15 & 0.05 & -2.711 & -2.711 & -1.00 & 195.8 \\
\hline 06171171-7328560 & 5265153816361777920 & $\ldots$ & 0.21 & 0.00 & -8.263 & -8.263 & -0.08 & 118.8 \\
\hline 06181310-0348135 & 3116844057174980864 & $\ldots$ & 0.13 & 0.00 & 23.051 & 23.051 & 0.00 & 26.5 \\
\hline 06183086-2506582 & 2912205186393737472 & 17.7 & 0.12 & 0.00 & 27.244 & 27.244 & -0.01 & 23.1 \\
\hline 06192867-8743048 & 5189368469885240832 & 200.9 & 1.61 & 0.03 & 3.383 & 3.383 & -0.71 & 327.4 \\
\hline 06194462-2030436 & 2938430256702303360 & -93.9 & 3.88 & 0.19 & -5.437 & -5.437 & -1.04 & 142.8 \\
\hline 06200929-5719169 & 5495322423720756352 & 39.5 & 0.10 & 0.00 & 210.836 & 210.836 & -0.02 & 84.9 \\
\hline 06220380-2155289 & 2937664962250734592 & 59.1 & 0.29 & 0.00 & -1.694 & -1.694 & -0.06 & 59.9 \\
\hline 06234993-5946248 & 5482612241102802304 & $\ldots$ & $\ldots$ & $\ldots$ & $\ldots$ & $\ldots$ & 0.03 & 0.0 \\
\hline $06244840+7752154$ & 1140661709439350528 & -12.0 & 0.14 & 0.00 & -67.044 & -67.044 & 0.09 & 24.6 \\
\hline 06250234-4659051 & 5553993768631973120 & -4.9 & 0.74 & 0.01 & 3.564 & 3.564 & -0.28 & 27.7 \\
\hline 06290787-6709523 & 5283390938997970176 & $\ldots$ & 4.67 & 0.27 & 5.013 & 5.013 & -2.09 & 112.0 \\
\hline $06325088+6530326$ & 1103660291306745856 & -35.5 & 0.52 & 0.00 & -0.116 & -0.116 & 0.23 & 51.4 \\
\hline $06325555-4214034$ & 5569242345641089408 & 22.8 & 0.16 & 0.00 & 8.484 & 8.484 & -0.03 & 24.9 \\
\hline 06332771-3519240 & 5581669477032806912 & 186.7 & 0.69 & 0.01 & 47.709 & 47.709 & -0.20 & 358.2 \\
\hline 06345221-4425441 & 5556592090703647744 & 29.7 & 0.10 & 0.00 & -1.477 & -1.477 & -0.01 & 30.0 \\
\hline 06363111-2255559 & 2924798167948814208 & $\cdots$ & 0.27 & 0.00 & -5.327 & -5.327 & -0.04 & 14.0 \\
\hline 06382886-8309319 & 5206227036859558272 & 123.9 & 3.05 & 0.10 & 2.605 & 2.605 & -1.38 & 141.6 \\
\hline 06393860-3406395 & 5583340111934906112 & 6.0 & 0.17 & 0.00 & 29.810 & 29.810 & -0.02 & 46.5 \\
\hline 06421665-5008385 & 5503130777344639488 & 27.8 & 0.19 & 0.00 & 8.378 & 8.378 & -0.05 & 39.5 \\
\hline $06425079+7014409$ & 1112486380380023040 & $\ldots$ & 1.82 & 0.08 & -1.467 & -1.467 & 0.79 & 12.9 \\
\hline 06451421-3217294 & 5583930313459131008 & 105.2 & 2.23 & 0.05 & 1.053 & 1.053 & -0.56 & 188.9 \\
\hline 06451920-0857165 & 3098535676741247744 & 5.7 & 0.22 & 0.01 & -11.052 & -11.052 & 0.00 & 12.9 \\
\hline 06460710-4155202 & 5563430395897276032 & 38.7 & 0.47 & 0.00 & -20.460 & -20.460 & -0.13 & 53.6 \\
\hline 06464646-4440364 & 5556009212103193472 & 32.6 & 0.19 & 0.01 & -4.807 & -4.807 & -0.04 & 32.9 \\
\hline $06484021+1625173$ & 3358091992230790656 & $\ldots$ & 1.35 & 0.03 & 0.195 & 0.195 & 0.19 & 12.2 \\
\hline 06484979-2428341 & 2922058189267952384 & 101.5 & 1.42 & 0.03 & 2.385 & 2.385 & -0.26 & 105.4 \\
\hline $06510773+0943416$ & 3158574272957723776 & -47.5 & 0.14 & 0.00 & 30.653 & 30.653 & 0.04 & 54.7 \\
\hline $06523842+1513163$ & 3354530639711957376 & $\ldots$ & 0.31 & 0.00 & -9.853 & -9.853 & 0.06 & 18.8 \\
\hline $06525096+2440261$ & 3381304160163213056 & -98.0 & 6.36 & 0.74 & 2.166 & 2.166 & 1.27 & 318.0 \\
\hline
\end{tabular}


Table 4 (continued)

\begin{tabular}{|c|c|c|c|c|c|c|c|c|}
\hline $\begin{array}{l}\text { Star Name } \\
\text { (2MASS) }\end{array}$ & $\begin{array}{c}\text { Star Name } \\
\text { (Gaia EDR3) }\end{array}$ & $\begin{array}{c}V_{\mathrm{los}} \\
\left(\mathrm{km} \mathrm{s}^{-1}\right)\end{array}$ & $\begin{array}{l}d_{\text {helio }} \\
(\mathrm{kpc})\end{array}$ & $\begin{array}{c}\sigma_{d} \\
(\mathrm{kpc})\end{array}$ & $\begin{array}{c}\mathrm{PM}_{\mathrm{R} . \mathrm{A}} \\
\left({\left.\mathrm{mas} \mathrm{yr}^{-1}\right)}\right.\end{array}$ & $\begin{array}{c}\mathrm{PM}_{\text {Decl. }} \\
\left(\mathrm{mas} \mathrm{yr}^{-1}\right)\end{array}$ & $\begin{array}{l}Z_{\mathrm{Gal}} \\
(\mathrm{kpc})\end{array}$ & $\begin{array}{c}V_{\mathrm{TAV}} \\
\left(\mathrm{km} \mathrm{s}^{-1}\right)\end{array}$ \\
\hline $06525451+1446083$ & 3354323111187785216 & -26.3 & 0.95 & 0.01 & 3.742 & 3.742 & 0.14 & 39.8 \\
\hline $06533075+1226225$ & 3351515542604966912 & $\cdots$ & 2.75 & 0.13 & 0.052 & 0.052 & 0.32 & 1.5 \\
\hline $06533083+6836050$ & 1103319172123035136 & -199.6 & 3.12 & 0.11 & 5.701 & 5.701 & 1.36 & 243.6 \\
\hline 06554143-4313578 & 5562195201959849984 & 123.0 & 4.69 & 0.19 & 1.482 & 1.482 & -1.38 & 201.8 \\
\hline $06560363+2846500$ & 887636359876055424 & 38.5 & 0.23 & 0.00 & -3.176 & -3.176 & 0.08 & 44.2 \\
\hline 06570150-6751413 & 5280814817608368000 & 127.5 & 1.25 & 0.02 & -9.128 & -9.128 & -0.49 & 133.0 \\
\hline 06571276-5526234 & 5485259315346971136 & 35.1 & 0.26 & 0.00 & -15.893 & -15.893 & -0.07 & 45.9 \\
\hline 06580901-8334199 & 5194228654877524096 & 198.7 & 3.34 & 0.13 & 5.771 & 5.771 & -1.48 & 209.5 \\
\hline $06585640+0850479$ & 3157301205994883840 & 75.5 & 3.47 & 0.17 & 1.262 & 1.262 & 0.37 & 79.1 \\
\hline 06593152-5853177 & 5480647173305937280 & 165.3 & 4.26 & 0.16 & 2.651 & 2.651 & -1.57 & 181.3 \\
\hline $06593924+2056439$ & 3366133201801121536 & 88.5 & 1.72 & 0.04 & 7.248 & 7.248 & 0.36 & 222.8 \\
\hline 07001955-3637535 & 5578346443656712960 & 29.2 & 0.15 & 0.00 & -2.484 & -2.484 & -0.01 & 29.8 \\
\hline $07003916+2701430$ & 884148846432586240 & -42.4 & 0.80 & 0.01 & 0.778 & 0.778 & 0.22 & 47.8 \\
\hline $07004613+2933178$ & 887873162895952768 & 5.1 & 0.26 & 0.02 & -5.985 & -5.985 & 0.09 & 8.2 \\
\hline $07012363+1813452$ & 3364377384810729088 & 162.5 & 1.78 & 0.05 & -1.782 & -1.782 & 0.34 & 195.7 \\
\hline 07013933-5842103 & 5480743208774977024 & 469.0 & 3.63 & 0.13 & 1.891 & 1.891 & -1.32 & 526.4 \\
\hline $07023862+0659243$ & 3153768440772179584 & $\ldots$ & 0.72 & 0.01 & 3.649 & 3.649 & 0.10 & 14.0 \\
\hline $07030318+4006286$ & 947804380629842816 & 21.4 & 1.55 & 0.04 & -0.683 & -0.683 & 0.53 & 53.4 \\
\hline $07033238+2225136$ & 3368024709696717824 & 223.8 & 4.14 & 0.24 & 0.741 & 0.741 & 0.92 & 238.4 \\
\hline $07035093+1955164$ & 3365212055873231104 & $\ldots$ & 3.56 & 4.19 & -6.860 & -6.860 & 0.74 & 114.9 \\
\hline $07041471+1901123$ & 3364898931280232448 & 70.7 & 0.14 & 0.00 & 47.252 & 47.252 & 0.05 & 82.3 \\
\hline 07041738-1908113 & 2932674107172462592 & 51.9 & 2.05 & 0.07 & -3.733 & -3.733 & -0.18 & 75.1 \\
\hline $07041880+0246488$ & 3115642187883221632 & $\ldots$ & 2.09 & 0.08 & -4.119 & -4.119 & 0.18 & 47.4 \\
\hline $07041957+2340564$ & 3368538353422137344 & 40.7 & 0.58 & 0.06 & -4.305 & -4.305 & 0.16 & 42.5 \\
\hline $07054097+1733350$ & 3361464812868710144 & -119.9 & 2.37 & 0.07 & 18.373 & 18.373 & 0.48 & 355.9 \\
\hline $07070364+2657035$ & 883254423785454080 & 95.2 & 2.58 & 0.11 & 17.608 & 17.608 & 0.70 & 306.1 \\
\hline $07073503+2356476$ & 3368586663216905984 & 33.9 & 0.14 & 0.00 & -19.877 & -19.877 & 0.06 & 42.3 \\
\hline 07081426-3557115 & 5566430245872289408 & 11.2 & 1.48 & 0.03 & -1.781 & -1.781 & -0.29 & 39.6 \\
\hline 07083867-4621128 & 5510018805377253632 & 24.2 & 0.92 & 0.04 & 8.756 & 8.756 & -0.24 & 36.7 \\
\hline 07085997-5424564 & 5491233099459990528 & 145.3 & 2.84 & 0.09 & 2.822 & 2.822 & -0.91 & 172.4 \\
\hline $07090577+2142312$ & 3367308652748446464 & $\cdots$ & 0.29 & 0.00 & -2.868 & -2.868 & 0.09 & 7.1 \\
\hline $07093534+2210136$ & 3367373077257509120 & 10.6 & 0.29 & 0.00 & -6.868 & -6.868 & 0.09 & 20.1 \\
\hline 07093868-0419118 & 3107527762016884096 & 85.4 & 2.77 & 0.33 & -0.848 & -0.848 & 0.13 & 86.8 \\
\hline 07101117-2219008 & 2928210850185413120 & $\ldots$ & 1.99 & 0.05 & -3.363 & -3.363 & -0.19 & 43.2 \\
\hline $07102845+1245411$ & 3161108024483929600 & $\cdots$ & 5.36 & 0.49 & -8.364 & -8.364 & 0.95 & 316.7 \\
\hline 07103372-0244311 & 3108335765621350656 & $\ldots$ & 0.89 & 0.01 & -7.763 & -7.763 & 0.07 & 32.9 \\
\hline $07104970+0826444$ & 3154409902728232320 & 86.8 & 7.07 & 0.77 & -9.655 & -9.655 & 1.02 & 443.1 \\
\hline 07110190-6301262 & 5285981079149837184 & 95.5 & 0.13 & 0.00 & 51.459 & 51.459 & -0.02 & 135.3 \\
\hline $07113988+8541053$ & 1150345383223043712 & 4.5 & 0.74 & 0.01 & 1.777 & 1.777 & 0.37 & 41.7 \\
\hline $07121704+1402591$ & 3167279926849037568 & 186.1 & 5.55 & 0.46 & 3.506 & 3.506 & 1.08 & 263.8 \\
\hline $07122036+4221574$ & 949584631690298368 & 21.9 & 0.19 & 0.00 & -15.315 & -15.315 & 0.10 & 44.4 \\
\hline $07122182-8422429$ & 5193956796331371264 & 1.4 & 0.22 & 0.00 & -17.776 & -17.776 & -0.07 & 30.5 \\
\hline
\end{tabular}


Table 4 (continued)

\begin{tabular}{|c|c|c|c|c|c|c|c|c|}
\hline $\begin{array}{l}\text { Star Name } \\
\text { (2MASS) }\end{array}$ & $\begin{array}{c}\text { Star Name } \\
\text { (Gaia EDR3) }\end{array}$ & $\begin{array}{c}V_{\mathrm{los}} \\
\left(\mathrm{km} \mathrm{s}^{-1}\right)\end{array}$ & $\begin{array}{l}d_{\text {helio }} \\
(\mathrm{kpc})\end{array}$ & $\begin{array}{c}\sigma_{d} \\
(\mathrm{kpc})\end{array}$ & 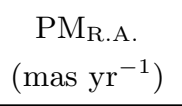 & $\begin{array}{c}\mathrm{PM}_{\text {Decl. }} \\
\left(\mathrm{mas} \mathrm{yr}^{-1}\right)\end{array}$ & $\begin{array}{l}Z_{\mathrm{Gal}} \\
(\mathrm{kpc})\end{array}$ & $\begin{array}{c}V_{\mathrm{TAV}} \\
\left(\mathrm{km} \mathrm{s}^{-1}\right)\end{array}$ \\
\hline $07122917+2231366$ & 3367505087372386944 & -6.6 & 0.07 & 0.00 & -51.921 & -51.921 & 0.04 & 19.7 \\
\hline 07122989-0700106 & 3052191643892060672 & $\cdots$ & 3.95 & 0.28 & -0.807 & -0.807 & 0.13 & 27.6 \\
\hline 07123398-4814049 & 5508686643961355648 & 352.6 & 3.46 & 0.15 & 6.957 & 6.957 & -0.96 & 368.7 \\
\hline $07123461+3153463$ & 890055835212749696 & $\cdots$ & 0.23 & 0.00 & 23.621 & 23.621 & 0.10 & 21.7 \\
\hline 07130783-7052481 & 5267185649427578496 & -0.5 & 0.18 & 0.00 & -22.414 & -22.414 & -0.05 & 39.6 \\
\hline $07150401+2714015$ & 883489998448405504 & $\ldots$ & 0.25 & 0.00 & 12.475 & 12.475 & 0.10 & 39.1 \\
\hline $07151852-5252051$ & 5491893184393739776 & 230.3 & 3.18 & 0.09 & 9.504 & 9.504 & -0.95 & 283.6 \\
\hline 07152806-4111161 & 5561098267315218048 & 9.2 & 0.16 & 0.01 & -17.602 & -17.602 & -0.01 & 18.6 \\
\hline $07160059+5240188$ & 986603229693072128 & $\ldots$ & 1.15 & 0.02 & -2.867 & -2.867 & 0.51 & 29.1 \\
\hline $07170438+2402438$ & 869715076257527808 & 22.9 & 0.93 & 0.02 & -3.041 & -3.041 & 0.28 & 26.2 \\
\hline $07174600+1259027$ & 3166159975473025280 & $\ldots$ & 0.86 & 0.01 & -11.119 & -11.119 & 0.20 & 47.6 \\
\hline 07174929-0924096 & 3048017378051885056 & 30.3 & 0.20 & 0.01 & -6.129 & -6.129 & 0.03 & 30.9 \\
\hline $07175677-2704124$ & 5616006675420673664 & $\ldots$ & 0.27 & 0.00 & -12.715 & -12.715 & -0.01 & 18.5 \\
\hline $07181152+4406479$ & 973981901357263104 & $\ldots$ & 0.23 & 0.00 & 9.699 & 9.699 & 0.11 & 23.4 \\
\hline 07194881-5748087 & 5485933071160480768 & $\ldots$ & 0.15 & 0.00 & -11.550 & -11.550 & -0.03 & 4.9 \\
\hline 07203081-6240451 & 5291814602512817024 & 205.8 & 3.36 & 0.15 & 5.240 & 5.240 & -1.16 & 227.1 \\
\hline 07204786-7623393 & 5214066868299703040 & -16.1 & 0.23 & 0.00 & -54.501 & -54.501 & -0.07 & 128.1 \\
\hline $07213106+2755248$ & 872994571549210240 & $\ldots$ & 0.39 & 0.00 & -25.934 & -25.934 & 0.15 & 42.2 \\
\hline $07220367+0253575$ & 3136142616543571456 & $\ldots$ & 5.64 & 0.69 & 4.212 & 4.212 & 0.82 & 234.8 \\
\hline $07224055+3618087$ & 897278351003895424 & $\ldots$ & 0.26 & 0.01 & -15.579 & -15.579 & 0.12 & 18.9 \\
\hline 07224582-2959375 & 5605629995767584000 & $\ldots$ & 0.32 & 0.01 & -8.563 & -8.563 & -0.01 & 13.9 \\
\hline $07233679+1412112$ & 3166723543901552000 & 5.8 & 0.76 & 0.01 & 5.331 & 5.331 & 0.20 & 19.8 \\
\hline 07234039-2959296 & 5605624738733670912 & $\ldots$ & 9.44 & 2.44 & -0.901 & -0.901 & -1.11 & 128.0 \\
\hline $07234262+1516310$ & 3167138884419576064 & $\ldots$ & 0.71 & 0.01 & -1.336 & -1.336 & 0.20 & 31.6 \\
\hline $07242428+5822232$ & 989627947524580864 & 1.6 & 0.05 & 0.00 & 1.892 & 1.892 & 0.05 & 1.6 \\
\hline $07254156+4948144$ & 977051149410569344 & $\ldots$ & 0.31 & 0.00 & -3.254 & -3.254 & 0.16 & 5.4 \\
\hline 07254769-3453506 & 5590014289255060480 & $\ldots$ & 0.40 & 0.00 & -5.124 & -5.124 & -0.04 & 22.0 \\
\hline 07260648-4637077 & 5510340717467331456 & 43.5 & 0.15 & 0.00 & -36.597 & -36.597 & -0.01 & 48.8 \\
\hline $07270798+4352580$ & 973215713552094848 & -4.6 & 0.18 & 0.00 & -0.914 & -0.914 & 0.10 & 5.0 \\
\hline $07273288+3819555$ & 899749713837935872 & -10.8 & 0.18 & 0.00 & 59.225 & 59.225 & 0.09 & 45.9 \\
\hline $07280531+1637140$ & 3169561074176213376 & 46.3 & 0.17 & 0.00 & -21.420 & -21.420 & 0.07 & 53.2 \\
\hline 07281913-8333342 & 5194416392190823040 & 179.5 & 0.81 & 0.01 & -21.358 & -21.358 & -0.33 & 336.0 \\
\hline 07282428-3705347 & 5586496638018390784 & 59.9 & 0.17 & 0.00 & 22.135 & 22.135 & 0.00 & 81.9 \\
\hline $07284694+8149439$ & 1142689281896442880 & -19.5 & 2.05 & 0.05 & 0.901 & 0.901 & 0.99 & 140.7 \\
\hline 07295359-6910360 & 5267963068571164928 & 6.9 & 0.15 & 0.00 & -6.256 & -6.256 & -0.03 & 9.3 \\
\hline $07304303+4111480$ & 900640730573598976 & 30.9 & 0.13 & 0.00 & -41.388 & -41.388 & 0.08 & 41.6 \\
\hline 07310602-2742595 & 5611975414810555264 & 33.1 & 0.11 & 0.00 & -14.729 & -14.729 & 0.02 & 54.1 \\
\hline 07311836-6659351 & 5269135942537559680 & -21.1 & 0.09 & 0.00 & -81.454 & -81.454 & -0.01 & 25.8 \\
\hline $07314455+5553592$ & 988736827710024704 & -39.2 & 0.24 & 0.02 & 5.851 & 5.851 & 0.14 & 40.5 \\
\hline 07314605-3324217 & 5591704921522389888 & $\ldots$ & 3.06 & 0.10 & -2.223 & -2.223 & -0.34 & 74.5 \\
\hline $07321280+1523588$ & 3168444240942341248 & 27.6 & 0.24 & 0.00 & -6.270 & -6.270 & 0.09 & 35.8 \\
\hline 07321479-1300309 & 3033439198205025024 & 306.4 & 4.40 & 0.31 & 7.346 & 7.346 & 0.25 & 400.0 \\
\hline
\end{tabular}


Table 4 (continued)

\begin{tabular}{|c|c|c|c|c|c|c|c|c|}
\hline $\begin{array}{l}\text { Star Name } \\
\text { (2MASS) }\end{array}$ & $\begin{array}{c}\text { Star Name } \\
\text { (Gaia EDR3) }\end{array}$ & $\begin{array}{c}V_{\mathrm{los}} \\
\left(\mathrm{km} \mathrm{s}^{-1}\right)\end{array}$ & $\begin{array}{l}d_{\text {helio }} \\
(\mathrm{kpc})\end{array}$ & $\begin{array}{c}\sigma_{d} \\
(\mathrm{kpc})\end{array}$ & 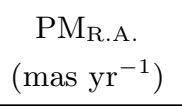 & $\begin{array}{c}\mathrm{PM}_{\text {Decl. }} \\
\left(\mathrm{mas} \mathrm{yr}^{-1}\right)\end{array}$ & $\begin{array}{l}Z_{\mathrm{Gal}} \\
(\mathrm{kpc})\end{array}$ & $\begin{array}{c}V_{\mathrm{TAV}} \\
\left(\mathrm{km} \mathrm{s}^{-1}\right)\end{array}$ \\
\hline $07325519+3655267$ & 898637038134266752 & $\cdots$ & 8.26 & 0.88 & -2.478 & -2.478 & 3.34 & 489.2 \\
\hline $07331792+0531033$ & 3141404295078246400 & 45.3 & 0.17 & 0.00 & 2.006 & 2.006 & 0.06 & 45.9 \\
\hline $07333190+5310203$ & 983909666723291392 & $\ldots$ & 1.29 & 0.04 & 0.861 & 0.861 & 0.62 & 7.3 \\
\hline $07334024+4425362$ & 973487288627865728 & 28.1 & 0.19 & 0.00 & -10.097 & -10.097 & 0.11 & 34.2 \\
\hline $07345045+0736494$ & 3142272222070528384 & 29.6 & 0.12 & 0.00 & -24.337 & -24.337 & 0.05 & 32.7 \\
\hline 07350484-0004500 & 3086417752932494848 & 36.4 & 0.10 & 0.00 & -34.819 & -34.819 & 0.04 & 40.1 \\
\hline $07351435+2626331$ & 872041539782121984 & 59.6 & 0.52 & 0.01 & -11.724 & -11.724 & 0.21 & 99.7 \\
\hline 07351699-4815064 & 5506540186806281728 & 18.9 & 0.30 & 0.01 & -9.939 & -9.939 & -0.04 & 22.8 \\
\hline $07353628+1938556$ & 672591122640513280 & $\ldots$ & 0.29 & 0.00 & -27.725 & -27.725 & 0.12 & 36.6 \\
\hline $07354551+0455028$ & 3138308624388237568 & $\ldots$ & 0.90 & 0.01 & -2.557 & -2.557 & 0.21 & 15.2 \\
\hline 07354757-5036104 & 5493916801186715648 & $\ldots$ & 0.26 & 0.01 & -8.396 & -8.396 & -0.04 & 12.9 \\
\hline $07363720+1126504$ & 3162146822454143360 & -11.6 & 0.47 & 0.01 & -17.589 & -17.589 & 0.15 & 40.4 \\
\hline $07363729+4034177$ & 924375132168707200 & 3.9 & 0.12 & 0.00 & -40.912 & -40.912 & 0.08 & 26.8 \\
\hline $07364486+4508331$ & 926998601271917184 & $\cdots$ & 0.51 & 0.00 & -5.058 & -5.058 & 0.25 & 41.4 \\
\hline $07365679+5950385$ & 1086214756921278976 & 5.4 & 0.10 & 0.00 & -18.690 & -18.690 & 0.07 & 30.4 \\
\hline $07374300-5630568$ & 5487737374038584704 & 173.6 & 1.46 & 0.02 & 18.477 & 18.477 & -0.39 & 188.7 \\
\hline 07380041-2129196 & 5619082589253324544 & $\ldots$ & 1.86 & 0.07 & -3.326 & -3.326 & 0.03 & 38.4 \\
\hline 07384500-0331557 & 3057721981216813568 & -46.3 & 1.98 & 0.07 & 11.815 & 11.815 & 0.33 & 125.5 \\
\hline 07393047-1530275 & 3029020260970100736 & $\ldots$ & 0.41 & 0.00 & 4.746 & 4.746 & 0.05 & 13.4 \\
\hline $07395820+1311537$ & 3164103029736387200 & 297.1 & 2.91 & 0.16 & -4.003 & -4.003 & 0.86 & 346.5 \\
\hline 07400327-0740587 & 3042138568557247360 & 166.4 & 1.77 & 0.05 & -6.393 & -6.393 & 0.25 & 178.7 \\
\hline $07403555+5352561$ & 985231550281510144 & 70.4 & 0.09 & 0.00 & -188.395 & -188.395 & 0.07 & 105.3 \\
\hline $07404482+1243437$ & 3163865500863822592 & -8.5 & 0.11 & 0.00 & -27.391 & -27.391 & 0.06 & 26.0 \\
\hline 07410359-6714551 & 5274884459907753344 & 146.2 & 3.84 & 0.16 & 1.126 & 1.126 & -1.31 & 249.2 \\
\hline 07410851-5943543 & 5292748779377543168 & $\ldots$ & 0.22 & 0.00 & -22.644 & -22.644 & -0.04 & 49.1 \\
\hline 07412077-2900201 & 5599777230966529024 & 129.7 & 2.38 & 0.06 & 11.590 & 11.590 & -0.10 & 175.0 \\
\hline 07414196-0741080 & 3042148704679838336 & 39.2 & 0.28 & 0.00 & -27.894 & -27.894 & 0.06 & 59.7 \\
\hline $07435834+1341353$ & 3164056506649463040 & $\ldots$ & 0.97 & 0.01 & -7.498 & -7.498 & 0.32 & 54.1 \\
\hline $07440179+0312226$ & 3136905750633082624 & 57.0 & 0.57 & 0.00 & 19.335 & 19.335 & 0.16 & 83.5 \\
\hline $07441635+5322545$ & 984534979601381120 & 22.6 & 0.12 & 0.00 & -31.675 & -31.675 & 0.08 & 45.1 \\
\hline $07443402+0742591$ & 3145150125036773760 & -0.2 & 1.10 & 0.02 & 4.613 & 4.613 & 0.32 & 36.9 \\
\hline 07443970-4425135 & 5532006972050819584 & 155.8 & 1.91 & 0.04 & -24.993 & -24.993 & -0.31 & 224.7 \\
\hline 07445367-4653249 & 5530553525058225152 & 20.4 & 0.37 & 0.02 & -3.264 & -3.264 & -0.05 & 25.1 \\
\hline $07450351+0956148$ & 3148979071201226880 & 20.7 & 3.96 & 0.25 & 7.369 & 7.369 & 1.14 & 256.7 \\
\hline 07450424-7231255 & 5263155252116820608 & $\cdots$ & 1.21 & 0.02 & -3.792 & -3.792 & -0.43 & 47.3 \\
\hline $07451488+1000107$ & 3149076244837702784 & 13.4 & 0.77 & 0.01 & -5.782 & -5.782 & 0.24 & 84.4 \\
\hline $07452477+2524147$ & 868026810512553856 & 66.5 & 0.23 & 0.01 & -21.954 & -21.954 & 0.11 & 70.2 \\
\hline $07455398+0103107$ & 3086833647505226752 & -17.0 & 0.80 & 0.01 & 0.872 & 0.872 & 0.20 & 17.7 \\
\hline $07461952+3903325$ & 920303430156012160 & -36.6 & 4.57 & 0.33 & 8.956 & 8.956 & 2.09 & 307.6 \\
\hline 07464924-4313456 & 5532490211705643904 & $\ldots$ & 0.18 & 0.00 & -22.130 & -22.130 & 0.00 & 20.1 \\
\hline 07480156-4924040 & 5518045893097566976 & 84.7 & 2.86 & 0.08 & 5.674 & 5.674 & -0.56 & 176.8 \\
\hline $07485521+5623334$ & 1082018707015827584 & 18.6 & 0.68 & 0.01 & -14.878 & -14.878 & 0.37 & 47.6 \\
\hline
\end{tabular}


Table 4 (continued)

\begin{tabular}{|c|c|c|c|c|c|c|c|c|}
\hline $\begin{array}{l}\text { Star Name } \\
\text { (2MASS) }\end{array}$ & $\begin{array}{c}\text { Star Name } \\
\text { (Gaia EDR3) }\end{array}$ & $\begin{array}{c}V_{\mathrm{los}} \\
\left(\mathrm{km} \mathrm{s}^{-1}\right)\end{array}$ & $\begin{array}{l}d_{\text {helio }} \\
(\mathrm{kpc})\end{array}$ & $\begin{array}{c}\sigma_{d} \\
(\mathrm{kpc})\end{array}$ & $\begin{array}{c}\mathrm{PM}_{\mathrm{R} . \mathrm{A}} \\
\left({\left.\mathrm{mas} \mathrm{yr}^{-1}\right)}\right.\end{array}$ & $\begin{array}{c}\mathrm{PM}_{\text {Decl. }} \\
\left(\mathrm{mas} \mathrm{yr}^{-1}\right)\end{array}$ & $\begin{array}{l}Z_{\mathrm{Gal}} \\
(\mathrm{kpc})\end{array}$ & $\begin{array}{c}V_{\mathrm{TAV}} \\
\left(\mathrm{km} \mathrm{s}^{-1}\right)\end{array}$ \\
\hline $07494258+6938239$ & 1098142705736502528 & -1.1 & 0.52 & 0.00 & -16.294 & -16.294 & 0.29 & 123.6 \\
\hline $07503801+1119098$ & 3150778696857500928 & 6.3 & 0.18 & 0.00 & -1.057 & -1.057 & 0.08 & 17.9 \\
\hline $07504673+0558247$ & 3143849888115383040 & 371.1 & 1.76 & 0.05 & -9.594 & -9.594 & 0.51 & 401.2 \\
\hline $07533988+4933361$ & 934072756004889216 & $\ldots$ & 0.97 & 0.01 & 17.279 & 17.279 & 0.51 & 271.0 \\
\hline $07545950+6639532$ & 1095319709633456000 & 48.9 & 0.22 & 0.01 & -13.826 & -13.826 & 0.14 & 49.2 \\
\hline $07551255+1944081$ & 670305998176686720 & -48.9 & 3.84 & 0.19 & 14.512 & 14.512 & 1.50 & 298.1 \\
\hline $07552395+3520063$ & 906645575890268416 & 19.6 & 0.13 & 0.00 & 34.856 & 34.856 & 0.09 & 47.9 \\
\hline $07555507+8148305$ & 1148451100783017984 & -82.1 & 0.16 & 0.00 & 24.326 & 24.326 & 0.11 & 83.6 \\
\hline $07575895+4035155$ & 922132399029177088 & -2.1 & 0.21 & 0.00 & 29.874 & 29.874 & 0.13 & 22.4 \\
\hline $07580568+3504110$ & 906456700407472512 & 15.5 & 0.35 & 0.01 & -5.773 & -5.773 & 0.19 & 20.0 \\
\hline $07584532+4211214$ & 922545677962459904 & -49.5 & 0.63 & 0.01 & 2.269 & 2.269 & 0.34 & 55.2 \\
\hline $07590580+4748300$ & 933111228791082240 & $\ldots$ & 0.16 & 0.00 & 49.371 & 49.371 & 0.11 & 36.3 \\
\hline $08005431+3135329$ & 878032675562889728 & $\ldots$ & 0.33 & 0.00 & -22.213 & -22.213 & 0.18 & 32.0 \\
\hline $08005634+1633254$ & 667243544758539136 & 23.3 & 0.20 & 0.01 & -12.290 & -12.290 & 0.10 & 26.2 \\
\hline $08011676+6358383$ & 1094412028423846144 & -97.6 & 0.15 & 0.00 & -25.176 & -25.176 & 0.10 & 123.7 \\
\hline 08021251-2821568 & 5597762543400727680 & $\ldots$ & 3.79 & 0.58 & -3.165 & -3.165 & 0.11 & 79.9 \\
\hline 08022771-4804247 & 5517895294366243968 & 26.5 & 0.35 & 0.00 & -8.273 & -8.273 & -0.03 & 31.6 \\
\hline 08024574-4803573 & 5517708957205335168 & 18.5 & 0.34 & 0.01 & -6.100 & -6.100 & -0.03 & 24.2 \\
\hline 08042788-6440110 & 5275614368128678528 & 97.1 & 0.88 & 0.01 & -3.929 & -3.929 & -0.23 & 135.2 \\
\hline $08045600+3310059$ & 905235555306335104 & 37.5 & 0.19 & 0.00 & -44.030 & -44.030 & 0.12 & 59.5 \\
\hline $08050775+2604105$ & 681971846610387712 & 49.3 & 1.41 & 0.03 & -2.544 & -2.544 & 0.67 & 52.1 \\
\hline 08052894-6502132 & 5275590007074529152 & 176.6 & 2.41 & 0.06 & 0.136 & 0.136 & -0.68 & 179.6 \\
\hline 08053267-6119242 & 5289953163686735232 & 28.0 & 0.21 & 0.00 & -16.357 & -16.357 & -0.03 & 30.1 \\
\hline 08074491-5257227 & 5512588600272046848 & 24.0 & 0.16 & 0.00 & -6.065 & -6.065 & -0.01 & 26.0 \\
\hline $08080715+5915114$ & 1083546379639153664 & -15.0 & 0.14 & 0.00 & -8.307 & -8.307 & 0.10 & 15.3 \\
\hline 08085268-6128294 & 5289751265864064128 & 172.8 & 1.35 & 0.02 & -20.164 & -20.164 & -0.32 & 257.0 \\
\hline $08090031+2850085$ & 876519232166668800 & -10.8 & 2.23 & 0.10 & 15.811 & 15.811 & 1.09 & 267.3 \\
\hline $08092156+2521288$ & 681134774663802496 & -18.4 & 0.22 & 0.00 & 18.719 & 18.719 & 0.13 & 35.4 \\
\hline 08100860-7523572 & 5213699150380190720 & 117.6 & 4.35 & 0.19 & 0.660 & 0.660 & -1.57 & 120.9 \\
\hline $08102898+4020333$ & 909546843477733504 & -43.5 & 0.98 & 0.01 & 1.062 & 1.062 & 0.54 & 45.6 \\
\hline $08111719+4456352$ & 929054103899434752 & 0.8 & 0.19 & 0.00 & -48.812 & -48.812 & 0.13 & 34.2 \\
\hline 08125962-7004235 & 5270057397705227904 & 19.9 & 0.17 & 0.00 & -5.517 & -5.517 & -0.03 & 20.2 \\
\hline $08130864+3925564$ & 909255575975553920 & 67.3 & 3.91 & 0.24 & -8.775 & -8.775 & 2.09 & 220.5 \\
\hline $08135674+5113349$ & 935284722760688384 & -35.7 & 0.72 & 0.01 & -0.456 & -0.456 & 0.42 & 35.7 \\
\hline $08140229+3940332$ & 909264917528317952 & $\cdots$ & 0.12 & 0.00 & -8.485 & -8.485 & 0.09 & 43.7 \\
\hline $08152203+3827587$ & 908032124475412224 & $\ldots$ & 0.25 & 0.00 & -22.591 & -22.591 & 0.16 & 36.6 \\
\hline $08153796+6531162$ & 1092014470303207552 & 20.2 & 0.13 & 0.00 & -5.352 & -5.352 & 0.10 & 26.0 \\
\hline 08162824-4611151 & 5519745360123251200 & 20.4 & 0.34 & 0.00 & -6.608 & -6.608 & -0.01 & 26.8 \\
\hline 08173327-2728418 & 5693425380770623616 & 32.0 & 0.94 & 0.01 & -14.050 & -14.050 & 0.10 & 64.2 \\
\hline $08182058+5923087$ & 1083715257753356800 & 10.2 & 0.51 & 0.00 & -8.735 & -8.735 & 0.31 & 43.0 \\
\hline 08202927-2458369 & 5696053561463700992 & 21.0 & 0.16 & 0.00 & -20.854 & -20.854 & 0.04 & 27.2 \\
\hline 08204593-7457097 & 5219742375524510720 & -9.1 & 0.93 & 0.01 & -2.510 & -2.510 & -0.30 & 14.8 \\
\hline
\end{tabular}


Table 4 (continued)

\begin{tabular}{|c|c|c|c|c|c|c|c|c|}
\hline $\begin{array}{c}\text { Star Name } \\
\text { (2MASS) }\end{array}$ & $\begin{array}{c}\text { Star Name } \\
\text { (Gaia EDR3) }\end{array}$ & $\begin{array}{c}V_{\mathrm{los}} \\
\left(\mathrm{km} \mathrm{s}^{-1}\right)\end{array}$ & $\begin{array}{l}d_{\text {helio }} \\
(\mathrm{kpc})\end{array}$ & $\begin{array}{c}\sigma_{d} \\
(\mathrm{kpc})\end{array}$ & $\begin{array}{c}\mathrm{PM}_{\mathrm{R} . \mathrm{A}} \\
\left(\operatorname{mas~yr}^{-1}\right)\end{array}$ & $\begin{array}{c}\mathrm{PM}_{\text {Decl. }} \\
\left(\text { mas yr }^{-1}\right)\end{array}$ & $\begin{array}{l}Z_{\mathrm{Gal}} \\
(\mathrm{kpc})\end{array}$ & $\begin{array}{c}V_{\mathrm{TAV}} \\
\left(\mathrm{km} \mathrm{s}^{-1}\right)\end{array}$ \\
\hline $08225420+6259266$ & 1090362836337748864 & 13.5 & 6.09 & 0.37 & 8.063 & 8.063 & 3.46 & 139.7 \\
\hline 08242044-1026147 & 5751499802540091776 & 32.2 & 0.21 & 0.00 & -20.188 & -20.188 & 0.08 & 37.8 \\
\hline 08244155-6859542 & 5270209581283002240 & $\ldots$ & 0.45 & 0.00 & -17.370 & -17.370 & -0.11 & 16.1 \\
\hline $08255445+7726501$ & 1137625614237615232 & -13.3 & 0.24 & 0.00 & -11.439 & -11.439 & 0.15 & 16.4 \\
\hline 08282505-2119200 & 5706111756390126080 & 6.0 & 0.33 & 0.03 & -0.040 & -0.040 & 0.08 & 60.2 \\
\hline 08284828-2858468 & 5644860540592289536 & $\ldots$ & 1.17 & 0.02 & -6.186 & -6.186 & 0.14 & 39.0 \\
\hline 08295142-2428117 & 5695713503130206848 & 26.1 & 0.92 & 0.02 & -8.666 & -8.666 & 0.16 & 43.3 \\
\hline 08295611-1354544 & 5722897519371974272 & -5.9 & 0.15 & 0.01 & 0.560 & 0.560 & 0.06 & 19.7 \\
\hline 08302532-6949046 & 5221888519143226240 & -11.5 & 0.13 & 0.00 & -83.753 & -83.753 & -0.01 & 63.5 \\
\hline 08312114-2126535 & 5703185367534835584 & 40.9 & 2.56 & 0.10 & -1.806 & -1.806 & 0.49 & 52.8 \\
\hline $08315869+6949533$ & 1121420775645432320 & -14.9 & 0.31 & 0.00 & 11.472 & 11.472 & 0.20 & 29.9 \\
\hline 08322899-2137273 & 5703121870736546304 & 11.8 & 1.00 & 0.01 & -5.252 & -5.252 & 0.21 & 34.4 \\
\hline 08331960-1350331 & 5722870096004958208 & $\ldots$ & 0.78 & 0.01 & -14.429 & -14.429 & 0.23 & 51.6 \\
\hline $08343393+7518493$ & 1125113279289236096 & -12.5 & 0.49 & 0.00 & 7.102 & 7.102 & 0.29 & 24.6 \\
\hline $08350584+4839306$ & 1026860572337001088 & 93.2 & 3.38 & 0.15 & -7.498 & -7.498 & 2.05 & 228.7 \\
\hline 08355055-2033433 & 5703594458875614848 & -32.4 & 0.05 & 0.00 & -177.252 & -177.252 & 0.04 & 62.6 \\
\hline 08355851-0839549 & 5753641990492978816 & 200.0 & 5.77 & 0.59 & -3.172 & -3.172 & 1.86 & 217.6 \\
\hline 08360617-0450149 & 3065446698936865152 & . & 0.13 & 0.00 & -45.393 & -45.393 & 0.07 & 47.0 \\
\hline $08364858+7742308$ & 1137832945195154560 & -10.4 & 2.81 & 0.08 & -0.338 & -0.338 & 1.51 & 465.2 \\
\hline $08370406+7830159$ & 1138150772775117312 & $\ldots$ & 2.73 & 0.08 & -4.247 & -4.247 & 1.46 & 126.9 \\
\hline 08400533-4428341 & 5522379549464634752 & 37.2 & 1.32 & 0.02 & -8.119 & -8.119 & -0.01 & 74.7 \\
\hline $08401347+4216411$ & 913518485634602112 & 32.2 & 0.10 & 0.00 & -124.428 & -124.428 & 0.09 & 55.6 \\
\hline 08413166-0629117 & 5754432023955507712 & $\ldots$ & 0.75 & 0.01 & -4.612 & -4.612 & 0.29 & 26.7 \\
\hline 08422928-0849161 & 5750516216373911680 & 103.6 & 0.11 & 0.00 & -169.313 & -169.313 & 0.06 & 139.2 \\
\hline $08430958+6038165$ & 1041278571391227392 & $\cdots$ & 0.21 & 0.00 & 3.206 & 3.206 & 0.15 & 12.6 \\
\hline $08433739+6717034$ & 1093203940022382720 & 9.8 & 0.10 & 0.00 & -83.104 & -83.104 & 0.08 & 29.7 \\
\hline 08434698-1812314 & 5705872333433002880 & 5.7 & 0.13 & 0.00 & -28.791 & -28.791 & 0.06 & 41.8 \\
\hline $08441851+0346257$ & 581348807340055424 & -18.6 & 0.10 & 0.00 & 85.815 & 85.815 & 0.07 & 69.3 \\
\hline 08443695-7241450 & & 59.2 & . & $\cdots$ & $\cdots$ & & $\cdots$ & .. \\
\hline 08444676-2845157 & 5642803457417047552 & .. & 0.96 & 0.01 & -9.639 & -9.639 & 0.17 & 46.5 \\
\hline 08445228-7438443 & 5216771215930182912 & 7.8 & 0.30 & 0.00 & -2.961 & -2.961 & -0.07 & 19.9 \\
\hline 08454426-2141380 & 5702387126388268160 & 276.6 & 0.82 & 0.01 & 21.625 & 21.625 & 0.21 & 339.3 \\
\hline 08460663-0426024 & 5761503056249343360 & $\cdots$ & 0.51 & 0.01 & -10.877 & -10.877 & 0.22 & 29.0 \\
\hline $08472137+5830138$ & 1040480914362286592 & -241.2 & 3.13 & 0.15 & 0.296 & 0.296 & 1.94 & 277.0 \\
\hline $08473213+0548147$ & 582973163970364416 & 213.8 & 1.25 & 0.04 & -10.678 & -10.678 & 0.62 & 359.4 \\
\hline 08490347-6323398 & 5297691668265349120 & -4.1 & 0.50 & 0.00 & -12.005 & -12.005 & -0.08 & 18.0 \\
\hline 08490462-6853235 & 5223489373715006976 & 37.6 & 0.17 & 0.00 & -1.905 & -1.905 & -0.02 & 40.3 \\
\hline 08494240-3836003 & 5621994959343177856 & $\ldots$ & 0.81 & 0.01 & -16.178 & -16.178 & 0.07 & 70.7 \\
\hline $08501165+4242063$ & 913730107265419136 & -1.4 & 0.13 & 0.00 & -37.648 & -37.648 & 0.11 & 19.0 \\
\hline 08511780-2736072 & 5648942030830217344 & 50.7 & 0.62 & 0.01 & -14.853 & -14.853 & 0.14 & 81.1 \\
\hline $08514213+1250467$ & 608133907024045952 & 30.7 & $\ldots$ & $\ldots$ & $\cdots$ & $\ldots$ & 0.03 & 30.7 \\
\hline $08515469+5402598$ & 1029765967389921408 & 31.1 & 0.32 & 0.00 & -21.917 & -21.917 & 0.23 & 46.6 \\
\hline
\end{tabular}


Table 4 (continued)

\begin{tabular}{|c|c|c|c|c|c|c|c|c|}
\hline $\begin{array}{l}\text { Star Name } \\
\text { (2MASS) }\end{array}$ & $\begin{array}{c}\text { Star Name } \\
\text { (Gaia EDR3) }\end{array}$ & $\begin{array}{c}V_{\mathrm{los}} \\
\left(\mathrm{km} \mathrm{s}^{-1}\right)\end{array}$ & $\begin{array}{l}d_{\text {helio }} \\
(\mathrm{kpc})\end{array}$ & $\begin{array}{c}\sigma_{d} \\
(\mathrm{kpc})\end{array}$ & $\begin{array}{c}\mathrm{PM}_{\mathrm{R} . \mathrm{A}} \\
\left(\mathrm{mas} \mathrm{yr}^{-1}\right)\end{array}$ & $\begin{array}{c}\mathrm{PM}_{\text {Decl. }} \\
\left(\mathrm{mas} \mathrm{yr}^{-1}\right)\end{array}$ & $\begin{array}{l}Z_{\mathrm{Gal}} \\
(\mathrm{kpc})\end{array}$ & $\begin{array}{c}V_{\mathrm{TAV}} \\
\left(\mathrm{km} \mathrm{s}^{-1}\right)\end{array}$ \\
\hline 08523133-0804579 & 5750792365590211328 & 21.3 & 0.14 & 0.00 & -33.560 & -33.560 & 0.08 & 30.7 \\
\hline $08524186+6924318$ & 1118241228535948672 & -7.9 & 0.23 & 0.00 & -4.064 & -4.064 & 0.16 & 17.4 \\
\hline $08525722+6423125$ & 1044196095432573952 & 20.5 & 0.56 & 0.01 & 6.630 & 6.630 & 0.37 & 80.2 \\
\hline $08533398+1644112$ & 611494731817426560 & $\ldots$ & 0.23 & 0.00 & -19.564 & -19.564 & 0.15 & 20.2 \\
\hline 08543649-4744205 & 5328756032854574592 & $\ldots$ & 5.31 & 0.37 & -3.141 & -3.141 & -0.14 & 105.0 \\
\hline 08544986-1159089 & 57366949428943966672 & $\ldots$ & 1.21 & 0.02 & -6.811 & -6.811 & 0.45 & 44.0 \\
\hline 08554225-6955045 & 5222449162695490432 & 204.0 & 2.98 & 0.08 & -4.211 & -4.211 & -0.78 & 209.2 \\
\hline 08565578-7603590 & 5215675105917524608 & $\cdots$ & 0.53 & 0.00 & 13.626 & 13.626 & -0.15 & 17.8 \\
\hline $08571123+0040261$ & 576743880843785088 & -25.0 & 0.09 & 0.00 & 72.471 & 72.471 & 0.07 & 48.0 \\
\hline 08571600-6556117 & 5296305287178801280 & -5.4 & 1.42 & 0.02 & 4.943 & 4.943 & -0.30 & 297.9 \\
\hline 09002061-2220490 & 5654811563195280512 & 1.5 & 0.28 & 0.01 & -22.666 & -22.666 & 0.10 & 27.9 \\
\hline 09002174-3342353 & 5627698985144472448 & 42.3 & 1.21 & 0.02 & -5.904 & -5.904 & 0.20 & 55.6 \\
\hline 09012132-7055369 & 5222157448519411584 & 10.2 & 0.19 & 0.00 & 17.163 & 17.163 & -0.03 & 29.8 \\
\hline 09023125-0810363 & 5755853112670730112 & 81.8 & 0.11 & 0.00 & -89.341 & -89.341 & 0.07 & 106.6 \\
\hline 09030506-2047415 & 5656156368995636608 & 196.6 & 1.08 & 0.02 & -16.814 & -16.814 & 0.34 & 213.1 \\
\hline 09030861-0427115 & 5759379998080221824 & 12.7 & 1.18 & 0.04 & -5.644 & -5.644 & 0.55 & 34.0 \\
\hline 09040262-0523492 & 5759068836288541696 & 301.6 & 2.89 & 0.22 & -6.489 & -6.489 & 1.30 & 320.5 \\
\hline $09043992+0733498$ & 584226980887837824 & 193.3 & 2.61 & 0.17 & -1.153 & -1.153 & 1.44 & 245.5 \\
\hline 09050894-2050426 & 5656143484093610368 & $\ldots$ & 0.50 & 0.00 & -9.391 & -9.391 & 0.17 & 27.6 \\
\hline $09065688+5836407$ & 1036942720302654080 & 1.9 & 0.27 & 0.00 & -16.136 & -16.136 & 0.20 & 22.1 \\
\hline 09073395-0340147 & 5759902820154063744 & $\ldots$ & 0.18 & 0.00 & -93.185 & -93.185 & 0.11 & 79.0 \\
\hline 09090697-0324211 & 5759936900718820864 & 52.3 & 3.08 & 0.14 & -2.041 & -2.041 & 1.49 & 149.6 \\
\hline 09093944-2006527 & 5679552155368264704 & 29.4 & 0.25 & 0.00 & -22.073 & -22.073 & 0.11 & 39.1 \\
\hline $09102690+6033156$ & 1039441978952648064 & -65.3 & 0.71 & 0.01 & -14.631 & -14.631 & 0.48 & 71.4 \\
\hline $09103058+6419220$ & 1043675820274039040 & -58.4 & 3.45 & 0.12 & 4.295 & 4.295 & 2.20 & 180.1 \\
\hline 09104309-1444185 & 5731383034718059520 & 199.7 & 5.04 & 0.48 & -3.637 & -3.637 & 1.92 & 216.6 \\
\hline 09110582-2014595 & 5679493090978018304 & 14.7 & 0.12 & 0.00 & -20.584 & -20.584 & 0.06 & 20.5 \\
\hline $09110758+0217287$ & 3843821216808928512 & 30.5 & 0.31 & 0.01 & -2.467 & -2.467 & 0.19 & 52.4 \\
\hline $09114163+1017524$ & 591768294919763072 & -140.8 & 3.10 & 0.14 & -0.048 & -0.048 & 1.84 & 281.2 \\
\hline 09122631-0037340 & 3842047846288227712 & $\ldots$ & 0.13 & 0.00 & 7.049 & 7.049 & 0.09 & 19.3 \\
\hline $09123863+6218061$ & 1040178033268005504 & -91.2 & 0.24 & 0.01 & -7.310 & -7.310 & 0.18 & 125.9 \\
\hline 09131573-1716172 & 56827210552545685576 & $\ldots$ & 2.75 & 0.16 & -1.754 & -1.754 & 1.01 & 44.7 \\
\hline 09152673-0018170 & 3842238950857112064 & $\ldots$ & 0.48 & 0.01 & 20.346 & 20.346 & 0.28 & 72.5 \\
\hline $09155397+1212314$ & 593696288558989312 & $\ldots$ & 0.75 & 0.01 & -3.461 & -3.461 & 0.48 & 41.2 \\
\hline $09161100+0140496$ & 3845027557158758144 & 5.2 & 0.06 & 0.00 & 81.129 & 81.129 & 0.06 & 23.8 \\
\hline 09164357-0512382 & 5758526021141799552 & 21.2 & 4.57 & 0.28 & 3.774 & 3.774 & 2.23 & 227.8 \\
\hline 09171078-6147067 & 5298830659236242688 & 269.2 & 1.24 & 0.02 & 0.769 & 0.769 & -0.16 & 269.2 \\
\hline 09172903-1021252 & 5742681753643854464 & -9.5 & 0.07 & 0.00 & -9.311 & -9.311 & 0.06 & 11.4 \\
\hline 09180855-0852126 & 5743138252832504576 & 234.2 & 2.75 & 0.15 & 11.681 & 11.681 & 1.28 & 327.4 \\
\hline $09184848+0713360$ & 586821454667385344 & 112.2 & 4.34 & 0.28 & -0.045 & -0.045 & 2.57 & 234.6 \\
\hline $09185431+1211488$ & 593613206711676800 & -30.0 & 0.15 & 0.00 & 18.174 & 18.174 & 0.12 & 43.0 \\
\hline 09201726-0554052 & 5746372328846303360 & -18.1 & 0.17 & 0.00 & 12.317 & 12.317 & 0.11 & 36.3 \\
\hline
\end{tabular}


Table 4 (continued)

\begin{tabular}{|c|c|c|c|c|c|c|c|c|}
\hline $\begin{array}{l}\text { Star Name } \\
\text { (2MASS) }\end{array}$ & $\begin{array}{c}\text { Star Name } \\
\text { (Gaia EDR3) }\end{array}$ & $\begin{array}{c}V_{\mathrm{los}} \\
\left(\mathrm{km} \mathrm{s}^{-1}\right)\end{array}$ & $\begin{array}{l}d_{\text {helio }} \\
(\mathrm{kpc})\end{array}$ & $\begin{array}{c}\sigma_{d} \\
(\mathrm{kpc})\end{array}$ & $\begin{array}{c}\mathrm{PM}_{\mathrm{R} . \mathrm{A}} \\
\left({\left.\mathrm{mas} \mathrm{yr}^{-1}\right)}\right.\end{array}$ & $\begin{array}{c}\mathrm{PM}_{\text {Decl. }} \\
\left(\mathrm{mas} \mathrm{yr}^{-1}\right)\end{array}$ & $\begin{array}{l}Z_{\mathrm{Gal}} \\
(\mathrm{kpc})\end{array}$ & $\begin{array}{c}V_{\mathrm{TAV}} \\
\left(\mathrm{km} \mathrm{s}^{-1}\right)\end{array}$ \\
\hline $09205040+1820344$ & 632540488180370176 & $\cdots$ & 0.88 & 0.02 & 0.485 & 0.485 & 0.60 & 59.2 \\
\hline $09214717+6219538$ & 1040038326571016704 & -237.5 & 2.84 & 0.13 & 3.527 & 3.527 & 1.89 & 307.8 \\
\hline $09220002+0957599$ & 592248296169483264 & $\ldots$ & 0.16 & 0.00 & -34.364 & -34.364 & 0.12 & 25.9 \\
\hline $09221426+5824580$ & 1038229698662764160 & 303.8 & 1.75 & 0.03 & -16.804 & -16.804 & 1.21 & 390.7 \\
\hline 09221563-7701423 & 5203340376458000128 & 16.8 & 1.00 & 0.01 & 6.305 & 6.305 & -0.30 & 21.0 \\
\hline 09222115-4317486 & 5424356193758211456 & -28.2 & 0.79 & 0.01 & -7.417 & -7.417 & 0.09 & 35.4 \\
\hline $09230164+5819465$ & 1026218874158310272 & 2.6 & 0.15 & 0.01 & 1.540 & 1.540 & 0.13 & 7.1 \\
\hline $09270301+4204494$ & 814375831397158144 & 32.1 & 3.65 & 0.18 & -2.996 & -2.996 & 2.65 & 251.9 \\
\hline $09270844+1544438$ & 630694854833847168 & 11.5 & 2.75 & 0.15 & 6.147 & 6.147 & 1.84 & 310.1 \\
\hline 09282183-3930462 & 5429709754532474880 & 60.6 & 0.70 & 0.01 & -18.313 & -18.313 & 0.13 & 80.3 \\
\hline $09285510+1543302$ & 619055111209080832 & 4.7 & 0.26 & 0.01 & -24.829 & -24.829 & 0.20 & 33.5 \\
\hline $09290292+3549540$ & 798505274404072832 & 24.7 & 0.77 & 0.01 & 0.407 & 0.407 & 0.58 & 33.5 \\
\hline 09291542-1847544 & 5678392273680123520 & 88.8 & 0.63 & 0.01 & -20.350 & -20.350 & 0.27 & 108.5 \\
\hline $09301030+0243320$ & 3844656021013252992 & 96.3 & 4.22 & 0.27 & 1.377 & 1.377 & 2.51 & 196.8 \\
\hline 09304755-3932097 & 5426696130598924416 & 98.5 & 0.09 & 0.00 & -95.259 & -95.259 & 0.04 & 105.5 \\
\hline $09304964+8016095$ & 1144494542550685824 & -1.6 & 0.08 & 0.00 & -22.045 & -22.045 & 0.07 & 11.3 \\
\hline $09313142+7905121$ & 1132002131953311232 & 38.5 & 0.15 & 0.00 & -61.481 & -61.481 & 0.11 & 45.4 \\
\hline $09315651+2335316$ & 644413044801144064 & -41.8 & 0.16 & 0.00 & 58.193 & 58.193 & 0.14 & 63.2 \\
\hline $09325648+1823093$ & 633101995024420480 & 154.2 & 1.90 & 0.06 & -7.231 & -7.231 & 1.34 & 190.3 \\
\hline 09341289-8700397 & 5189077198087438208 & $\ldots$ & 0.15 & 0.00 & -1.853 & -1.853 & -0.04 & 21.9 \\
\hline $09343649+1036027$ & 589530578303563648 & 124.3 & 1.74 & 0.04 & -4.731 & -4.731 & 1.17 & 182.0 \\
\hline $09344298+0353574$ & 3851012229012923264 & 41.7 & 6.51 & 0.67 & -0.482 & -0.482 & 4.01 & 154.7 \\
\hline $09345116+1214576$ & 614001725703023488 & -6.8 & $\ldots$ & $\ldots$ & $\ldots$ & $\ldots$ & 0.03 & 6.8 \\
\hline $09372216+0925058$ & 588581463545772928 & 24.4 & 0.34 & 0.00 & -0.455 & -0.455 & 0.25 & 34.0 \\
\hline $09375748+2559307$ & 645906319030740864 & -39.4 & 0.17 & 0.00 & -50.272 & -50.272 & 0.15 & 72.2 \\
\hline $09382460+4205266$ & 813812915803635712 & 53.6 & 1.78 & 0.05 & -4.143 & -4.143 & 1.35 & 82.8 \\
\hline $09383941+1227080$ & 614063912534411136 & $\cdots$ & 2.25 & 0.10 & -16.659 & -16.659 & 1.55 & 208.4 \\
\hline $09384661+7833575$ & 1131785214629423488 & -26.7 & 2.26 & 0.05 & -5.970 & -5.970 & 1.30 & 196.5 \\
\hline $09391251+3445386$ & 797731523158972416 & $\ldots$ & 0.55 & 0.01 & 1.077 & 1.077 & 0.43 & 24.1 \\
\hline $09403979+2921557$ & 696431047286005632 & 29.9 & 0.19 & 0.00 & -45.512 & -45.512 & 0.17 & 61.1 \\
\hline $09455700+2135550$ & 640001361769756032 & $\cdots$ & 0.22 & 0.01 & 73.681 & 73.681 & 0.19 & 73.2 \\
\hline $09472261+3354159$ & 794484841418902912 & 57.8 & 0.26 & 0.00 & -21.031 & -21.031 & 0.23 & 62.5 \\
\hline 09473545-2638039 & 5658256784099611008 & $\ldots$ & 0.57 & 0.02 & -13.656 & -13.656 & 0.22 & 40.3 \\
\hline 09474496-7045530 & 5242578991517893120 & 319.6 & 1.89 & 0.04 & -7.380 & -7.380 & -0.40 & 374.2 \\
\hline $09485248+1837465$ & 627201117981349888 & $\cdots$ & 3.34 & 0.23 & -2.521 & -2.521 & 2.48 & 185.3 \\
\hline 09485477-3811139 & 5432401118477539456 & 20.7 & 0.85 & 0.02 & -12.405 & -12.405 & 0.20 & 55.3 \\
\hline $09491888+3201060$ & 793274382192849664 & 194.7 & 8.06 & 0.95 & 1.350 & 1.350 & 6.23 & 285.4 \\
\hline 09501353-7925417 & 5202088268933375232 & 428.3 & 3.26 & 0.09 & 2.083 & 2.083 & -1.06 & 436.9 \\
\hline $09502173+3716070$ & 799953365577763712 & 22.0 & 1.10 & 0.08 & -3.136 & -3.136 & 0.88 & 26.1 \\
\hline 09502215-6930058 & 5243501550494934400 & $\ldots$ & 0.28 & 0.00 & -27.062 & -27.062 & -0.03 & 26.4 \\
\hline 09513068-3848520 & 5432133353036744448 & 30.4 & 0.37 & 0.00 & -16.205 & -16.205 & 0.10 & 41.3 \\
\hline $09513210+3330203$ & 793674978087183616 & -4.2 & 0.13 & 0.00 & -4.097 & -4.097 & 0.12 & 16.8 \\
\hline
\end{tabular}


Table 4 (continued)

\begin{tabular}{|c|c|c|c|c|c|c|c|c|}
\hline $\begin{array}{c}\text { Star Name } \\
\text { (2MASS) }\end{array}$ & $\begin{array}{c}\text { Star Name } \\
\text { (Gaia EDR3) }\end{array}$ & $\begin{array}{c}V_{\mathrm{los}} \\
\left(\mathrm{km} \mathrm{s}^{-1}\right)\end{array}$ & $\begin{array}{l}d_{\text {helio }} \\
(\mathrm{kpc})\end{array}$ & $\begin{array}{c}\sigma_{d} \\
(\mathrm{kpc})\end{array}$ & 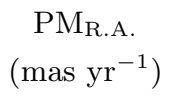 & $\begin{array}{c}\mathrm{PM}_{\text {Decl. }} \\
\left(\mathrm{mas}^{-1}{ }^{-1}\right)\end{array}$ & $\begin{array}{l}Z_{\text {Gal }} \\
(\mathrm{kpc})\end{array}$ & $\begin{array}{c}V_{\mathrm{TAV}} \\
\left(\mathrm{km} \mathrm{s}^{-1}\right)\end{array}$ \\
\hline 09532759-2717101 & 5657259969431486336 & 40.0 & 0.21 & 0.00 & -3.430 & -3.430 & 0.10 & 40.6 \\
\hline $09535089+3329284$ & 794978139884594560 & 99.4 & 4.21 & 0.32 & 0.165 & 0.165 & 3.32 & 241.0 \\
\hline $09541478+2735210$ & 647086477259746176 & $\ldots$ & 0.21 & 0.00 & -19.516 & -19.516 & 0.19 & 30.3 \\
\hline $09545564+6756128$ & 1069803755602231168 & -99.4 & 1.75 & 0.04 & 16.958 & 16.958 & 1.18 & 173.4 \\
\hline $09553934+4319179$ & 808168980883073792 & $\ldots$ & 0.74 & 0.01 & -0.826 & -0.826 & 0.60 & 20.7 \\
\hline 09555639-2403072 & 5660496214405409152 & $\ldots$ & 0.29 & 0.00 & -57.350 & -57.350 & 0.14 & 74.3 \\
\hline 09560894-5924282 & 5257538328273646336 & 16.3 & 0.15 & 0.00 & -18.431 & -18.431 & 0.02 & 18.2 \\
\hline $09574469+1400136$ & 615228711960558464 & -18.6 & 0.18 & 0.00 & 3.786 & 3.786 & 0.16 & 27.3 \\
\hline $09580008-4318227$ & 5418253457547492096 & 43.9 & 0.60 & 0.00 & -14.662 & -14.662 & 0.12 & 68.7 \\
\hline $09580109+2933087$ & 743582087319504640 & 15.5 & 0.21 & 0.00 & -46.308 & -46.308 & 0.19 & 48.3 \\
\hline $09593411+3708378$ & 796772718958610304 & -2.1 & 0.11 & 0.00 & -40.613 & -40.613 & 0.11 & 30.1 \\
\hline $09594843+7246373$ & 1125921282896321792 & -129.6 & 2.64 & 0.09 & -5.372 & -5.372 & 1.69 & 235.8 \\
\hline $10003325-2537597$ & 5658983939239396352 & 14.9 & 0.16 & 0.00 & -65.373 & -65.373 & 0.09 & 49.0 \\
\hline $10020621-1554291$ & 5673966327061493760 & $\ldots$ & 0.09 & 0.00 & -37.953 & -37.953 & 0.07 & 16.0 \\
\hline $10030615+7054115$ & 1071492949060164992 & -265.3 & 3.08 & 0.11 & 9.403 & 9.403 & 2.02 & 275.2 \\
\hline $10033379-2329108$ & 5665883610926876672 & 36.2 & 0.18 & 0.00 & -45.496 & -45.496 & 0.10 & 67.5 \\
\hline $10045623-4228179$ & 5418445670219076224 & 26.8 & 1.14 & 0.01 & -25.537 & -25.537 & 0.23 & 109.8 \\
\hline $10052247+3945408$ & 803621576887555840 & -188.2 & 3.71 & 0.17 & 2.800 & 2.800 & 3.01 & 292.2 \\
\hline 10053264-1827501 & 5672581221584085504 & -18.3 & 0.18 & 0.00 & -58.691 & -58.691 & 0.11 & 56.7 \\
\hline $10063882+2403476$ & 630450488374469120 & $\ldots$ & 4.99 & 0.37 & -6.915 & -6.915 & 4.00 & 196.3 \\
\hline 10075999-2736413 & 5465659970825328896 & $\ldots$ & 0.12 & 0.00 & -65.183 & -65.183 & 0.07 & 44.5 \\
\hline $10085157-3236041$ & 5459310600414841856 & 12.5 & 0.15 & 0.00 & -45.986 & -45.986 & 0.07 & 29.8 \\
\hline $10100365+2227088$ & 629233049829242368 & -16.9 & 0.14 & 0.00 & -1.856 & -1.856 & 0.14 & 16.9 \\
\hline 10111521-6620282 & 5245373292941453952 & 16.0 & 0.22 & 0.00 & -23.962 & -23.962 & -0.01 & 22.4 \\
\hline $10115056-4309343$ & 5415268253418659200 & 23.9 & 0.53 & 0.00 & -6.423 & -6.423 & 0.12 & 29.0 \\
\hline $10115917+5502205$ & 852773418843119616 & 1.0 & 0.15 & 0.00 & 6.192 & 6.192 & 0.14 & 13.5 \\
\hline 10121964-3221347 & 5459373341297312384 & 365.5 & 3.19 & 0.13 & -9.952 & -9.952 & 1.09 & 388.7 \\
\hline $10124027-2249482$ & 5666123373181152000 & -38.0 & 0.15 & 0.00 & -33.996 & -33.996 & 0.09 & 45.8 \\
\hline $10124666+4624575$ & 810408381126766592 & $\ldots$ & 3.03 & 0.13 & 6.681 & 6.681 & 2.46 & 287.5 \\
\hline $10143521+2324515$ & 725459455633859840 & $\ldots$ & 0.40 & 0.00 & 12.694 & 12.694 & 0.35 & 252.3 \\
\hline $10151784+5551061$ & 853205526912187264 & -15.3 & 0.16 & 0.00 & -16.823 & -16.823 & 0.14 & 16.8 \\
\hline $10152469-1057352$ & 3767574556484311424 & 126.2 & 6.68 & 0.82 & 0.685 & 0.685 & 3.98 & 145.1 \\
\hline $10152705+6456284$ & 1053650967717923072 & 0.3 & 0.13 & 0.00 & -37.775 & -37.775 & 0.11 & 23.3 \\
\hline $10160573+6533551$ & 1065697491989952896 & 20.0 & $\ldots$ & $\ldots$ & 8.128 & 8.128 & 0.03 & 20.0 \\
\hline $10171294+4654224$ & 810350480671638784 & -29.0 & 0.18 & 0.00 & 13.861 & 13.861 & 0.17 & 31.6 \\
\hline $10193367+7257464$ & 1077961238527864704 & 15.6 & 0.17 & 0.01 & -10.908 & -10.908 & 0.14 & 15.9 \\
\hline $10203763+7221398$ & 1077708213414112128 & 16.9 & 0.56 & 0.00 & -17.091 & -17.091 & 0.39 & 53.4 \\
\hline $10211155+4557314$ & 809444964126986240 & 13.7 & 0.14 & 0.00 & -63.114 & -63.114 & 0.14 & 37.5 \\
\hline $10213675+7058455$ & 1076692848785487104 & -37.3 & 0.16 & 0.00 & 93.688 & 93.688 & 0.13 & 53.2 \\
\hline $10221281-4123416$ & 5416543996142159488 & 118.1 & 3.97 & 0.19 & -9.056 & -9.056 & 0.94 & 351.7 \\
\hline $10244576-7355423$ & 5229049672736749184 & 19.1 & 0.15 & 0.00 & -33.353 & -33.353 & -0.01 & 21.1 \\
\hline $10250250+7723238$ & 1128041244394048000 & -18.6 & 0.25 & 0.01 & 10.640 & 10.640 & 0.17 & 18.8 \\
\hline
\end{tabular}


Table 4 (continued)

\begin{tabular}{|c|c|c|c|c|c|c|c|c|}
\hline $\begin{array}{c}\text { Star Name } \\
\text { (2MASS) }\end{array}$ & $\begin{array}{c}\text { Star Name } \\
\text { (Gaia EDR3) }\end{array}$ & $\begin{array}{c}V_{\mathrm{los}} \\
\left(\mathrm{km} \mathrm{s}^{-1}\right)\end{array}$ & $\begin{array}{l}d_{\text {helio }} \\
(\mathrm{kpc})\end{array}$ & $\begin{array}{c}\sigma_{d} \\
(\mathrm{kpc})\end{array}$ & 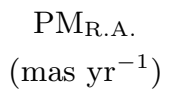 & $\begin{array}{c}\mathrm{PM}_{\text {Decl. }} \\
\left(\mathrm{mas}^{-1}{ }^{-1}\right)\end{array}$ & $\begin{array}{l}Z_{\text {Gal }} \\
(\mathrm{kpc})\end{array}$ & $\begin{array}{c}V_{\mathrm{TAV}} \\
\left(\mathrm{km} \mathrm{s}^{-1}\right)\end{array}$ \\
\hline $10263327-2231158$ & 5475397761196528896 & 244.6 & 3.96 & 0.25 & -13.606 & -13.606 & 1.96 & 364.6 \\
\hline $10280894+4658364$ & 833602269677094272 & 201.6 & 6.65 & 0.59 & -4.237 & -4.237 & 5.52 & 299.1 \\
\hline $10281330-2946530$ & 5461386169127203200 & 23.1 & 0.35 & 0.00 & -9.639 & -9.639 & 0.16 & 38.0 \\
\hline $10295444-0801026$ & 3774466329726812672 & 76.9 & 4.55 & 0.33 & -8.071 & -8.071 & 3.01 & 188.8 \\
\hline $10340832-7354406$ & 5229004382300835840 & 18.4 & 0.12 & 0.00 & -43.013 & -43.013 & 0.00 & 19.7 \\
\hline $10350987-1416284$ & 3750801231724757760 & 187.0 & 2.12 & 0.12 & -7.360 & -7.360 & 1.30 & 204.7 \\
\hline $10352666+4804049$ & 834056058741414400 & 187.8 & 0.79 & 0.01 & -55.523 & -55.523 & 0.69 & 499.1 \\
\hline 10363889-1917043 & 3554709655102839552 & -39.3 & 0.61 & 0.06 & 10.326 & 10.326 & 0.36 & 48.4 \\
\hline $10371075-1056303$ & 3760861312146553216 & $\ldots$ & 0.16 & 0.00 & 17.853 & 17.853 & 0.13 & 52.2 \\
\hline $10375744+4851237$ & 834186007272278016 & -58.6 & 0.23 & 0.02 & -31.223 & -31.223 & 0.22 & 65.5 \\
\hline $10382409-5248250$ & 5354554767533822336 & 171.4 & 1.53 & 0.03 & -53.742 & -53.742 & 0.16 & 359.7 \\
\hline $10383538+4953297$ & 835062146240700160 & -24.1 & 3.79 & 0.29 & -12.147 & -12.147 & 3.16 & 347.2 \\
\hline $10385482-0518037$ & 3776786466765131520 & 103.8 & 3.55 & 0.16 & -9.024 & -9.024 & 2.51 & 225.1 \\
\hline $10391495+7348283$ & 1078279478424242432 & -81.9 & 0.27 & 0.00 & -12.129 & -12.129 & 0.20 & 95.4 \\
\hline $10391566+7340387$ & 1078266108189870592 & 18.8 & 0.26 & 0.00 & -33.484 & -33.484 & 0.19 & 22.1 \\
\hline $10394895+3459288$ & 750368410525129728 & 58.9 & 4.33 & 0.45 & -6.616 & -6.616 & 3.81 & 271.7 \\
\hline $10420484+0246381$ & 3857349504797962496 & 162.8 & 3.22 & 0.14 & -14.480 & -14.480 & 2.51 & 287.7 \\
\hline $10425611-6355510$ & 5239913019452422784 & 17.3 & 0.15 & 0.00 & -17.242 & -17.242 & 0.01 & 20.0 \\
\hline 10430163-0230226 & 3802519784793984000 & $\ldots$ & 0.18 & 0.00 & -47.126 & -47.126 & 0.16 & 40.7 \\
\hline $10430365-0643033$ & 3776307354572694272 & 36.5 & 0.37 & 0.00 & -7.424 & -7.424 & 0.28 & 90.0 \\
\hline $10432543+7515589$ & 1126646406518426752 & -14.8 & 0.11 & 0.00 & 37.015 & 37.015 & 0.10 & 30.3 \\
\hline $10440416+7616213$ & 1128438924005715456 & -22.5 & 0.26 & 0.00 & 11.976 & 11.976 & 0.19 & 33.8 \\
\hline $10440597-0853008$ & 3762337131628855680 & $\ldots$ & 0.22 & 0.00 & 10.072 & 10.072 & 0.17 & 24.9 \\
\hline $10453280+0345108$ & 3857542022411847040 & 131.9 & 5.77 & 0.56 & -8.333 & -8.333 & 4.57 & 331.5 \\
\hline 10493965-1719329 & 3556866862556915072 & -19.7 & 2.60 & 0.10 & 10.412 & 10.412 & 1.58 & 329.6 \\
\hline $10502726-2300446$ & 3549234430794364160 & 71.5 & 0.94 & 0.01 & -2.033 & -2.033 & 0.52 & 92.0 \\
\hline $10511274-0817026$ & 3763102327298064128 & -7.7 & 0.08 & 0.00 & -45.229 & -45.229 & 0.08 & 18.4 \\
\hline $10521859+0528265$ & 3864195618243548032 & 18.3 & 0.14 & 0.00 & -23.355 & -23.355 & 0.14 & 38.1 \\
\hline $10530608-2253102$ & 3549276036141218176 & 127.4 & 5.45 & 0.44 & 4.828 & 4.828 & 2.94 & 227.0 \\
\hline $10531802-0055302$ & 3803159838000339200 & 213.2 & 3.53 & 0.39 & -1.828 & -1.828 & 2.73 & 256.5 \\
\hline $10540939-5233263$ & 5359711992455305344 & $\cdots$ & 0.16 & 0.00 & -3.128 & -3.128 & 0.04 & 7.5 \\
\hline $10541225+0549178$ & 3864303022490267136 & 67.8 & 2.08 & 0.08 & 10.787 & 10.787 & 1.73 & 331.8 \\
\hline $10543311+0528128$ & 3864140775805950208 & 82.6 & 4.06 & 0.22 & -8.674 & -8.674 & 3.34 & 201.6 \\
\hline $10554570-1654038$ & 3556496876894238464 & 257.2 & 1.88 & 0.05 & -5.156 & -5.156 & 1.17 & 362.7 \\
\hline $10563216-0555480$ & 3764649748179457280 & $\ldots$ & 0.21 & 0.00 & -28.513 & -28.513 & 0.18 & 30.5 \\
\hline $10570875-5207081$ & 5359563558390131968 & -11.4 & 0.09 & 0.00 & -88.328 & -88.328 & 0.04 & 36.0 \\
\hline $10582963+0011130$ & 3804842056430901120 & 172.0 & 2.59 & 0.12 & -15.700 & -15.700 & 2.06 & 348.8 \\
\hline $10590836-6924479$ & 5231719699295439488 & 0.7 & 0.33 & 0.00 & -15.665 & -15.665 & -0.02 & 19.6 \\
\hline $11000776+4630029$ & 783425712959288576 & -79.1 & 4.42 & 0.23 & -1.640 & -1.640 & 3.88 & 246.0 \\
\hline $11004069-1102492$ & 3758563126686411776 & 259.6 & 4.90 & 0.36 & -7.759 & -7.759 & 3.39 & 531.4 \\
\hline $11010971+1310060$ & 3967986487953405312 & 126.9 & 2.93 & 0.13 & -9.905 & -9.905 & 2.58 & 210.7 \\
\hline 11023256-0636095 & 3787611983373749120 & 11.5 & 0.11 & 0.00 & -87.308 & -87.308 & 0.11 & 48.1 \\
\hline
\end{tabular}


Table 4 (continued)

\begin{tabular}{|c|c|c|c|c|c|c|c|c|}
\hline $\begin{array}{l}\text { Star Name } \\
\text { (2MASS) }\end{array}$ & $\begin{array}{c}\text { Star Name } \\
\text { (Gaia EDR3) }\end{array}$ & $\begin{array}{c}V_{\mathrm{los}} \\
\left(\mathrm{km} \mathrm{s}^{-1}\right)\end{array}$ & $\begin{array}{l}d_{\text {helio }} \\
(\mathrm{kpc})\end{array}$ & $\begin{array}{c}\sigma_{d} \\
(\mathrm{kpc})\end{array}$ & 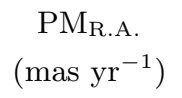 & $\begin{array}{c}\mathrm{PM}_{\text {Decl. }} \\
\left(\mathrm{mas} \mathrm{yr}^{-1}\right)\end{array}$ & $\begin{array}{l}Z_{\mathrm{Gal}} \\
(\mathrm{kpc})\end{array}$ & $\begin{array}{c}V_{\mathrm{TAV}} \\
\left(\mathrm{km} \mathrm{s}^{-1}\right)\end{array}$ \\
\hline $11023598+0102539$ & 3805298933576500480 & $\cdots$ & 3.13 & 0.16 & -10.293 & -10.293 & 2.53 & 344.1 \\
\hline $11051574-4752557$ & 5362354531210538624 & -11.9 & 0.55 & 0.00 & -9.000 & -9.000 & 0.13 & 22.7 \\
\hline 11051929-1329347 & 3564247918473953792 & 19.8 & 0.13 & 0.00 & -67.626 & -67.626 & 0.11 & 52.9 \\
\hline $11064962-5323068$ & 5347385401964090240 & $\cdots$ & 1.47 & 0.03 & -9.625 & -9.625 & 0.19 & 41.6 \\
\hline $11065789-4643237$ & 5386525267120422912 & 8.6 & 0.19 & 0.00 & -37.028 & -37.028 & 0.07 & 24.4 \\
\hline $11065939-4920517$ & 5362109546275295872 & $\cdots$ & 0.88 & 0.03 & -6.340 & -6.340 & 0.18 & 17.5 \\
\hline $11090121+0754418$ & 3818459160048340352 & -98.6 & 6.42 & 0.62 & 2.075 & 2.075 & 5.53 & 297.3 \\
\hline $11092807-0242021$ & 3791025112280181248 & 33.9 & 0.18 & 0.01 & -56.563 & -56.563 & 0.16 & 91.5 \\
\hline $11093391+2327348$ & 3995259156621469440 & 25.2 & 0.14 & 0.00 & -6.728 & -6.728 & 0.15 & 26.8 \\
\hline $11112337+6943386$ & 1074121743923049344 & 27.4 & 0.10 & 0.00 & -111.598 & -111.598 & 0.10 & 34.2 \\
\hline $11132240-0910000$ & 3783099076553128064 & -1.2 & 0.22 & 0.00 & -4.014 & -4.014 & 0.19 & 14.6 \\
\hline $11133490+1015249$ & 3963264914441096320 & 375.4 & 3.64 & 0.18 & -13.509 & -13.509 & 3.22 & 603.1 \\
\hline $11144345-1133225$ & 3566319673258222208 & 328.5 & 3.26 & 0.18 & -10.373 & -10.373 & 2.32 & 364.4 \\
\hline 11172319-5030507 & 5349769001420934784 & 300.3 & 2.88 & 0.09 & -4.224 & -4.224 & 0.51 & 305.8 \\
\hline $11213850+3100109$ & 4023379441379492096 & $\ldots$ & 0.33 & 0.00 & -21.924 & -21.924 & 0.33 & 45.7 \\
\hline $11215118-4555200$ & 5376213596097250176 & 25.4 & 0.18 & 0.00 & -81.924 & -81.924 & 0.07 & 91.1 \\
\hline $11232110+6118098$ & 862722319742958592 & -67.9 & 2.65 & 0.09 & -6.653 & -6.653 & 2.13 & 112.0 \\
\hline $11235327+0025363$ & 3797935886458318336 & 36.9 & 3.97 & 0.23 & 5.630 & 5.630 & 3.32 & 363.2 \\
\hline $11242364-0642128$ & 3785352182036872320 & 71.6 & 4.70 & 0.39 & 4.023 & 4.023 & 3.64 & 177.5 \\
\hline 11245109-0118132 & 3796858154608999424 & 139.6 & 5.49 & 0.58 & -1.147 & -1.147 & 4.51 & 262.8 \\
\hline $11245186+8132096$ & 1133615145575572992 & 50.8 & 1.06 & 0.01 & -3.797 & -3.797 & 0.63 & 89.2 \\
\hline $11251752+4810412$ & 789033325338645888 & 15.5 & 0.24 & 0.00 & -114.356 & -114.356 & 0.24 & 89.7 \\
\hline $11252946-0035203$ & 3797041055791986048 & 39.8 & 0.22 & 0.00 & -112.094 & -112.094 & 0.20 & 123.9 \\
\hline $11255206+5922341$ & 859132616140394880 & 57.4 & 0.84 & 0.01 & -22.077 & -22.077 & 0.71 & 73.0 \\
\hline 11260849-0718091 & 3591618782978505472 & $\cdots$ & 0.75 & 0.01 & -13.115 & -13.115 & 0.60 & 75.4 \\
\hline $11264720+2321085$ & 3992675231281619584 & $\cdots$ & 0.19 & 0.00 & -11.646 & -11.646 & 0.20 & 9.4 \\
\hline $11284462+1159546$ & 3917369061419260928 & 26.9 & 0.16 & 0.00 & -58.467 & -58.467 & 0.17 & 53.2 \\
\hline $11293922+6718355$ & 1057741219692489856 & -13.3 & 0.17 & 0.00 & 48.576 & 48.576 & 0.15 & 28.5 \\
\hline $11320178+4540156$ & 784645621097082624 & 51.6 & 3.51 & 0.18 & -9.471 & -9.471 & 3.23 & 206.7 \\
\hline $11322424+7217439$ & 1075031310621259776 & -17.3 & 0.14 & 0.00 & 12.530 & 12.530 & 0.12 & 28.7 \\
\hline $11332470+1251440$ & 3917497017084939776 & 221.2 & 6.29 & 0.52 & -16.401 & -16.401 & 5.81 & 674.7 \\
\hline $11343570+1932184$ & 3977488952772409856 & $\ldots$ & 0.20 & 0.00 & -16.705 & -16.705 & 0.21 & 18.4 \\
\hline $11344124+5314269$ & 841082728317714048 & -153.4 & 2.63 & 0.09 & -20.068 & -20.068 & 2.31 & 251.7 \\
\hline 11351928-3430096 & 3477077051780069632 & -18.7 & 0.19 & 0.00 & -20.516 & -20.516 & 0.11 & 47.2 \\
\hline $11364104+3220057$ & 4024512758694334336 & 19.2 & 0.08 & 0.00 & -43.519 & -43.519 & 0.11 & 52.8 \\
\hline $11372004+1845309$ & 3974320228980706816 & 178.7 & 5.29 & 0.46 & -13.067 & -13.067 & 5.03 & 380.1 \\
\hline $11373800-5202251$ & 5345681227653768064 & 300.8 & 1.18 & 0.02 & 2.068 & 2.068 & 0.21 & 331.5 \\
\hline $11381456+1534117$ & 3972403604119095808 & 6.7 & 0.31 & 0.00 & 11.753 & 11.753 & 0.32 & 18.7 \\
\hline $11403298+6340440$ & 864134573709331584 & -0.7 & 0.21 & 0.00 & -40.038 & -40.038 & 0.19 & 20.3 \\
\hline $11415054+5035361$ & 790901636102569472 & -7.3 & 0.10 & 0.00 & -45.556 & -45.556 & 0.12 & 15.7 \\
\hline $11423344+0800282$ & 3910233647567741056 & -216.2 & 2.83 & 0.12 & 0.589 & 0.589 & 2.59 & 220.2 \\
\hline 11444533-4644384 & 5372404612941931392 & 5.3 & 0.28 & 0.01 & 9.102 & 9.102 & 0.09 & 46.1 \\
\hline
\end{tabular}


Table 4 (continued)

\begin{tabular}{|c|c|c|c|c|c|c|c|c|}
\hline $\begin{array}{c}\text { Star Name } \\
\text { (2MASS) }\end{array}$ & $\begin{array}{c}\text { Star Name } \\
\text { (Gaia EDR3) }\end{array}$ & $\begin{array}{c}V_{\mathrm{los}} \\
\left(\mathrm{km} \mathrm{s}^{-1}\right)\end{array}$ & $\begin{array}{l}d_{\text {helio }} \\
(\mathrm{kpc})\end{array}$ & $\begin{array}{c}\sigma_{d} \\
(\mathrm{kpc})\end{array}$ & 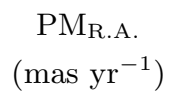 & $\begin{array}{c}\mathrm{PM}_{\text {Decl. }} \\
\left(\mathrm{mas} \mathrm{yr}^{-1}\right)\end{array}$ & $\begin{array}{l}Z_{\mathrm{Gal}} \\
(\mathrm{kpc})\end{array}$ & $\begin{array}{c}V_{\mathrm{TAV}} \\
\left(\mathrm{km} \mathrm{s}^{-1}\right)\end{array}$ \\
\hline $11465844+4835156$ & 787263141676539648 & -20.6 & 0.44 & 0.01 & -25.923 & -25.923 & 0.42 & 47.9 \\
\hline $11471027+0341265$ & 3895958344506982784 & 241.6 & 3.79 & 0.24 & -22.995 & -22.995 & 3.37 & 580.0 \\
\hline $11483814+1952557$ & 3976143459777843968 & 51.2 & 4.90 & 0.59 & -2.567 & -2.567 & 4.74 & 178.0 \\
\hline $11492027+3132066$ & 4021576517188258688 & $\cdots$ & 0.17 & 0.00 & 43.539 & 43.539 & 0.19 & 42.6 \\
\hline $11505337-8036338$ & 5199459645869304704 & -14.3 & 0.22 & 0.00 & 7.342 & 7.342 & -0.04 & 26.6 \\
\hline $11515346+8151152$ & 1133680021057772544 & -112.7 & 3.05 & 0.09 & -11.602 & -11.602 & 1.77 & 199.7 \\
\hline $11515556-4738558$ & 5371554866553160960 & 401.9 & 2.27 & 0.10 & 9.679 & 9.679 & 0.58 & 408.7 \\
\hline $11524486-4709547$ & 5377584485241041408 & 165.2 & 2.17 & 0.08 & -16.820 & -16.820 & 0.57 & 209.6 \\
\hline $11541040-5208317$ & 5368942804889403520 & 21.9 & 0.21 & 0.00 & -64.998 & -64.998 & 0.06 & 44.9 \\
\hline $11543955+7023508$ & 1061694681253422336 & -12.9 & 0.76 & 0.01 & -15.647 & -15.647 & 0.57 & 50.1 \\
\hline $11545004+4749009$ & 786606630149557248 & $\ldots$ & 0.34 & 0.00 & -23.789 & -23.789 & 0.34 & 66.1 \\
\hline $11564526+3145522$ & 4026626810316850048 & -71.0 & 0.22 & 0.00 & 7.749 & 7.749 & 0.24 & 76.7 \\
\hline $11593802+2922115$ & 4008146360612154112 & 14.5 & 6.40 & 0.62 & -5.114 & -5.114 & 6.30 & 332.5 \\
\hline $11595151-3905280$ & 3459312659023799552 & 22.4 & 0.18 & 0.00 & -53.545 & -53.545 & 0.10 & 45.0 \\
\hline $12001449+1641370$ & 3925430066494830336 & -23.9 & 0.19 & 0.01 & -38.839 & -38.839 & 0.20 & 41.5 \\
\hline $12004306+2949116$ & 4008186046109996672 & -97.7 & 3.84 & 0.23 & -10.467 & -10.467 & 3.79 & 346.3 \\
\hline $12005811-3929233$ & 3459242084121356288 & 16.1 & 0.07 & 0.00 & 63.913 & 63.913 & 0.05 & 22.8 \\
\hline $12022430+6255274$ & 1582997852929561600 & -32.5 & 0.46 & 0.00 & -26.675 & -26.675 & 0.39 & 51.7 \\
\hline $12034594+3035029$ & 4014343925404883328 & -1.4 & 0.14 & 0.00 & -35.593 & -35.593 & 0.16 & 29.5 \\
\hline $12052554-4408587$ & 6147464275054037120 & 89.3 & 0.23 & 0.00 & 81.846 & 81.846 & 0.10 & 111.6 \\
\hline $12055188+1735548$ & 3925930962760630656 & -57.7 & 0.24 & 0.00 & -76.045 & -76.045 & 0.26 & 108.4 \\
\hline $12070423-5345229$ & 6076898344653529344 & 193.5 & 2.51 & 0.07 & -19.384 & -19.384 & 0.40 & 239.5 \\
\hline $12070444+7600223$ & 1692730414755815552 & 13.2 & 0.15 & 0.00 & -2.544 & -2.544 & 0.12 & 17.0 \\
\hline $12074090+3519591$ & 4029760766348866944 & $\ldots$ & 0.76 & 0.01 & 10.270 & 10.270 & 0.77 & 46.1 \\
\hline $12083074+3954084$ & 1536035581002935424 & 88.6 & 5.68 & 0.50 & 3.950 & 3.950 & 5.50 & 232.2 \\
\hline $12094665+6450371$ & 1585030811273958400 & -167.4 & 6.57 & 0.50 & -3.115 & -3.115 & 5.18 & 237.1 \\
\hline $12100140+4520564$ & 1539687570219614080 & & 4.56 & 0.30 & -8.271 & -8.271 & 4.31 & 229.3 \\
\hline $12104009+7032545$ & 1684079324125218944 & 20.5 & 0.23 & 0.00 & -108.526 & -108.526 & 0.19 & 58.0 \\
\hline $12111339+2220415$ & 4001468785978496768 & -6.4 & 0.17 & 0.00 & -132.130 & -132.130 & 0.19 & 104.7 \\
\hline 12132563-3800203 & 3461061226110222080 & 39.5 & 0.12 & 0.00 & -101.804 & -101.804 & 0.07 & 88.1 \\
\hline 12143659-1517191 & 3569366385619378432 & -26.9 & 0.09 & 0.00 & -77.101 & -77.101 & 0.09 & 42.6 \\
\hline 12155841-3547529 & 3461905891557813248 & -9.2 & 0.34 & 0.01 & -24.300 & -24.300 & 0.18 & 33.4 \\
\hline $12161019+4524243$ & 1538985944361745536 & 12.5 & 0.13 & 0.00 & -24.820 & -24.820 & 0.15 & 26.9 \\
\hline 12181234-3721065 & 6151711177372668928 & $\ldots$ & 8.69 & 1.83 & -4.497 & -4.497 & 3.70 & 165.4 \\
\hline $12214951+4244520$ & 1538031641283723008 & 15.6 & 7.03 & 0.72 & -5.250 & -5.250 & 6.75 & 129.4 \\
\hline $12221869-3853368$ & 6150224495918526848 & 342.6 & 2.29 & 0.07 & -5.940 & -5.940 & 0.94 & 376.4 \\
\hline $12240228-7202466$ & 5842561802238077952 & $\ldots$ & 0.31 & 0.01 & 11.928 & 11.928 & -0.03 & 12.2 \\
\hline $12245683+7007242$ & 1683832243246834304 & -22.8 & 0.67 & 0.00 & 5.926 & 5.926 & 0.52 & 46.3 \\
\hline $12261008+3926273$ & 1532300402563759360 & & 0.55 & 0.02 & -21.132 & -21.132 & 0.56 & 43.7 \\
\hline $12280652-3319179$ & 3468446817511036160 & 232.0 & 3.91 & 0.28 & -0.223 & -0.223 & 1.94 & 265.0 \\
\hline $12284069+1942295$ & 3948963287525544576 & -30.8 & 4.44 & 0.31 & 5.104 & 5.104 & 4.41 & 322.6 \\
\hline $12293447-3233073$ & 3468579201286803200 & 243.4 & 4.20 & 0.30 & -13.894 & -13.894 & 2.13 & 339.7 \\
\hline
\end{tabular}


Table 4 (continued)

\begin{tabular}{|c|c|c|c|c|c|c|c|c|}
\hline $\begin{array}{c}\text { Star Name } \\
\text { (2MASS) }\end{array}$ & $\begin{array}{c}\text { Star Name } \\
\text { (Gaia EDR3) }\end{array}$ & $\begin{array}{c}V_{\mathrm{los}} \\
\left(\mathrm{km} \mathrm{s}^{-1}\right)\end{array}$ & $\begin{array}{l}d_{\text {helio }} \\
(\mathrm{kpc})\end{array}$ & $\begin{array}{c}\sigma_{d} \\
(\mathrm{kpc})\end{array}$ & 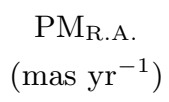 & 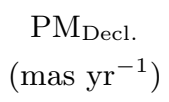 & $\begin{array}{l}Z_{\text {Gal }} \\
(\mathrm{kpc})\end{array}$ & $\begin{array}{c}V_{\mathrm{TAV}} \\
\left(\mathrm{km} \mathrm{s}^{-1}\right)\end{array}$ \\
\hline $12334194+1952177$ & 3949121587136124160 & 69.9 & 17.39 & 5.11 & 0.705 & 0.705 & 17.23 & 413.7 \\
\hline 12335935-3246361 & 6160173770478603520 & -11.6 & 0.64 & 0.01 & -55.534 & -55.534 & 0.35 & 147.7 \\
\hline 12335980-2944042 & 3471719754389184384 & 67.9 & 2.03 & 0.09 & -25.346 & -25.346 & 1.13 & 226.2 \\
\hline 12340964-3453438 & 6158168604865990016 & 85.6 & 4.10 & 0.35 & -19.584 & -19.584 & 1.94 & 437.6 \\
\hline $12342913+4826025$ & 1543862488889757568 & -177.7 & 4.03 & 0.20 & -2.067 & -2.067 & 3.77 & 268.9 \\
\hline $12353682+4854057$ & 1544253571429564800 & 16.6 & 0.14 & 0.00 & -84.074 & -84.074 & 0.15 & 39.6 \\
\hline $12353793-4301524$ & 6145744883683553408 & 60.1 & 0.31 & 0.00 & -53.060 & -53.060 & 0.13 & 83.3 \\
\hline 12360829-7040522 & 5843251466600455808 & -7.0 & 0.26 & 0.00 & -40.566 & -40.566 & -0.01 & 21.0 \\
\hline $12363406+4334497$ & 1540600409684268032 & -24.2 & 0.24 & 0.00 & -34.298 & -34.298 & 0.26 & 55.7 \\
\hline $12382743-3400315$ & 6158315870705750912 & -67.6 & 3.84 & 0.22 & 0.544 & 0.544 & 1.87 & 89.4 \\
\hline $12383506+2039384$ & 3949279882451077888 & -102.6 & $\ldots$ & $\ldots$ & $\ldots$ & $\ldots$ & 0.03 & 102.6 \\
\hline $12392838+5038459$ & 1568524878294905088 & 32.3 & 0.17 & 0.00 & -19.761 & -19.761 & 0.18 & 36.2 \\
\hline 12395834-3131208 & 6160731124090635520 & -16.1 & 0.12 & 0.00 & -51.828 & -51.828 & 0.09 & 31.2 \\
\hline $12401578-7012252$ & 5843372485918189440 & 59.4 & 0.21 & 0.00 & -25.660 & -25.660 & 0.00 & 60.6 \\
\hline $12413756-5412247$ & 6074478212169867904 & -25.7 & 0.16 & 0.00 & -25.894 & -25.894 & 0.05 & 60.0 \\
\hline $12432213-5955445$ & 6056631317380990208 & 18.4 & $\ldots$ & $\ldots$ & $\ldots$ & $\ldots$ & 0.03 & 18.4 \\
\hline $12433114-8753309$ & 5765813554148627328 & 3.6 & 0.87 & 0.02 & -0.675 & -0.675 & -0.34 & 4.0 \\
\hline $12433200-2408376$ & 3501624164266183552 & 197.9 & 2.07 & 0.10 & -11.797 & -11.797 & 1.32 & 305.6 \\
\hline $12435927+7222303$ & 1689741705929430016 & -75.0 & 0.58 & 0.00 & -59.320 & -59.320 & 0.43 & 141.9 \\
\hline $12450496-1907283$ & 3521841434322113152 & $\ldots$ & 3.52 & 0.19 & -6.236 & -6.236 & 2.46 & 236.9 \\
\hline $12493821+6415038$ & 1676938094886271104 & -4.0 & 0.15 & 0.00 & 35.080 & 35.080 & 0.14 & 21.7 \\
\hline $12503487+4033500$ & 1527854729160394496 & -9.1 & 0.20 & 0.00 & -1.243 & -1.243 & 0.22 & 31.1 \\
\hline $12505600+5649548$ & 1576792896497757440 & $\ldots$ & 0.13 & 0.00 & -90.373 & -90.373 & 0.13 & 48.3 \\
\hline $12510042-1942157$ & 3509552399017285632 & -25.8 & 0.14 & 0.00 & 34.753 & 34.753 & 0.12 & 49.9 \\
\hline $12511944+6940558$ & 1688859141689049984 & $\cdots$ & 1.20 & 0.02 & -63.881 & -63.881 & 0.91 & 251.3 \\
\hline $12534156+4512260$ & 1530523729214664192 & 19.8 & 0.14 & 0.00 & -28.915 & -28.915 & 0.16 & 45.1 \\
\hline $12540492-1445540$ & 3525348811990850560 & -38.7 & 0.08 & 0.00 & -56.364 & -56.364 & 0.09 & 46.7 \\
\hline $12541483-6940177$ & 5844853661205358208 & $\ldots$ & 0.96 & 0.08 & -18.522 & -18.522 & -0.09 & 36.1 \\
\hline $12550382+4640597$ & 1530993770436582784 & -16.9 & 4.64 & 0.35 & -14.226 & -14.226 & 4.39 & 312.7 \\
\hline $12552381-8428164$ & 5770402155472131712 & 185.6 & 4.79 & 0.24 & -8.690 & -8.690 & -1.74 & 205.2 \\
\hline $12553388+6801121$ & 1679621251151114624 & -81.4 & 0.25 & 0.00 & 28.530 & 28.530 & 0.22 & 93.0 \\
\hline $12572833-5125217$ & 6081346139752486784 & 41.3 & 2.49 & 0.12 & -10.486 & -10.486 & 0.52 & 90.2 \\
\hline $12592188+5338040$ & 1558199669539397376 & -10.8 & 0.27 & 0.00 & -1.367 & -1.367 & 0.27 & 24.3 \\
\hline $12595962-0829171$ & 3627268897025456000 & $\ldots$ & 0.47 & 0.00 & -28.012 & -28.012 & 0.41 & 63.5 \\
\hline $13003028+6135085$ & 1579614380772959104 & -129.7 & 0.93 & 0.01 & -69.293 & -69.293 & 0.79 & 325.8 \\
\hline $13022750+5836042$ & 1578633165428231552 & $\ldots$ & 0.63 & 0.00 & -13.387 & -13.387 & 0.57 & 26.8 \\
\hline $13031323+4812574$ & 1555059567409267328 & 24.7 & 0.15 & 0.00 & 46.783 & 46.783 & 0.17 & 51.6 \\
\hline $13044809-8623048$ & 5769157650045097984 & 6.5 & 3.14 & 0.10 & 1.228 & 1.228 & -1.23 & 150.2 \\
\hline $13070832-5007271$ & 6081477913650691456 & 8.1 & 0.41 & 0.00 & -25.849 & -25.849 & 0.11 & 33.1 \\
\hline $13073032+4948349$ & 1556370498804511360 & -65.5 & 4.07 & 0.17 & -10.343 & -10.343 & 3.77 & 174.5 \\
\hline $13091593+4640598$ & 1553980847721855616 & -0.9 & 0.12 & 0.00 & -136.326 & -136.326 & 0.14 & 61.1 \\
\hline $13091772+4524279$ & 1529793344255574016 & $\ldots$ & 0.14 & 0.00 & 1.640 & 1.640 & 0.16 & 30.0 \\
\hline
\end{tabular}


Table 4 (continued)

\begin{tabular}{|c|c|c|c|c|c|c|c|c|}
\hline $\begin{array}{c}\text { Star Name } \\
\text { (2MASS) }\end{array}$ & $\begin{array}{c}\text { Star Name } \\
\text { (Gaia EDR3) }\end{array}$ & $\begin{array}{c}V_{\mathrm{los}} \\
\left(\mathrm{km} \mathrm{s}^{-1}\right)\end{array}$ & $\begin{array}{l}d_{\text {helio }} \\
(\mathrm{kpc})\end{array}$ & $\begin{array}{c}\sigma_{d} \\
(\mathrm{kpc})\end{array}$ & $\begin{array}{c}\mathrm{PM}_{\mathrm{R} . \mathrm{A}} \\
\left(\operatorname{mas~yr}^{-1}\right)\end{array}$ & $\begin{array}{c}\mathrm{PM}_{\text {Decl. }} \\
\left(\text { mas yr }^{-1}\right)\end{array}$ & $\begin{array}{c}Z_{\mathrm{Gal}} \\
(\mathrm{kpc})\end{array}$ & $\begin{array}{c}V_{\mathrm{TAV}} \\
\left(\mathrm{km} \mathrm{s}^{-1}\right)\end{array}$ \\
\hline $13123590+7259464$ & 1688176585486569600 & -1.1 & 0.16 & 0.00 & -117.941 & -117.941 & 0.14 & 39.5 \\
\hline 13133130-6138303 & 5863379783621954944 & -27.5 & 2.00 & 0.06 & -4.561 & -4.561 & 0.06 & 34.3 \\
\hline $13134248+3405127$ & 1473046861950780032 & $\ldots$ & 5.26 & 0.33 & 3.928 & 3.928 & 5.23 & 333.9 \\
\hline $13140302-0929340$ & 3624508096342874240 & -7.1 & 0.27 & 0.00 & -41.343 & -41.343 & 0.24 & 52.1 \\
\hline $13145513+3825569$ & 1522993032339838848 & 156.6 & 4.11 & 0.21 & -10.615 & -10.615 & 4.04 & 229.2 \\
\hline $13151765+5708386$ & 1566249645139278720 & -8.5 & 0.29 & 0.01 & -1.676 & -1.676 & 0.28 & 22.4 \\
\hline $13175280+3411307$ & 1473007485690704128 & -135.9 & 2.69 & 0.10 & 3.018 & 3.018 & 2.68 & 383.7 \\
\hline $13222653+7020284$ & 1685430349037732864 & -41.8 & 0.19 & 0.00 & 26.324 & 26.324 & 0.16 & 42.8 \\
\hline $13222932+3936562$ & 1476579283573419264 & -0.5 & 0.43 & 0.12 & -5.065 & -5.065 & 0.45 & 17.1 \\
\hline $13230288-1704404$ & 3604328999956748032 & 15.1 & 2.68 & 0.13 & -14.892 & -14.892 & 1.92 & 217.2 \\
\hline 13231009-0531428 & 3635041726974665600 & $\ldots$ & 0.43 & 0.00 & -6.447 & -6.447 & 0.38 & 25.6 \\
\hline $13231283+4320557$ & 15496034127556666944 & 102.0 & 4.11 & 0.22 & -15.365 & -15.365 & 3.95 & 252.3 \\
\hline $13240005-1748099$ & 3604036456848334720 & 278.3 & 2.05 & 0.07 & 9.740 & 9.740 & 1.46 & 343.2 \\
\hline $13241831+4610307$ & 1551088548024093952 & -5.8 & 8.08 & 0.67 & -12.800 & -12.800 & 7.62 & 376.2 \\
\hline $13242995-4551146$ & 6087082811610225536 & 3.5 & 0.18 & 0.00 & -26.912 & -26.912 & 0.08 & 17.2 \\
\hline $13244275-1607008$ & 3604537731071348992 & 265.5 & 3.41 & 0.20 & -0.330 & -0.330 & 2.48 & 328.7 \\
\hline 13253853-1412517 & 3608192335923918208 & 168.2 & 4.74 & 0.40 & 2.178 & 2.178 & 3.54 & 309.7 \\
\hline $13282335+7427122$ & 1712581998412032768 & 2.4 & 0.63 & 0.00 & -3.652 & -3.652 & 0.45 & 6.9 \\
\hline $13294999+0115237$ & 3711389512304903168 & 34.2 & 3.63 & 0.21 & -7.893 & -7.893 & 3.24 & 272.5 \\
\hline $13303726-4125545$ & 6160938171579414016 & $\ldots$ & 0.28 & 0.00 & 23.031 & 23.031 & 0.12 & 27.7 \\
\hline 13351401-0110524 & 3638534188221336960 & 104.4 & 4.92 & 0.66 & 4.925 & 4.925 & 4.28 & 288.5 \\
\hline $13360938-4408536$ & 6111481627465700352 & 181.9 & 1.89 & 0.05 & -8.089 & -8.089 & 0.61 & 255.7 \\
\hline 13373017-7717500 & 5789490093947866624 & 299.9 & 2.48 & 0.06 & -27.405 & -27.405 & -0.60 & 314.1 \\
\hline $13373672+5902017$ & 1662055552169232896 & 21.7 & 0.17 & 0.00 & -20.338 & -20.338 & 0.16 & 56.7 \\
\hline $13375051+4742311$ & 1552172322891498240 & 93.0 & 2.85 & 0.12 & -12.538 & -12.538 & 2.66 & 170.8 \\
\hline $13394721+6824054$ & 1672939136736222464 & -85.1 & 0.17 & 0.00 & -2.554 & -2.554 & 0.15 & 86.9 \\
\hline $13401321+7237543$ & 1687511071713939712 & -117.7 & 4.10 & 0.23 & -1.218 & -1.218 & 2.87 & 149.3 \\
\hline $13404811+0717285$ & 3724481569055443712 & -34.1 & 0.20 & 0.00 & 44.519 & 44.519 & 0.21 & 81.6 \\
\hline 13425404-0717005 & 3620124270467822848 & 149.7 & 7.06 & 0.74 & 2.545 & 2.545 & 5.69 & 191.3 \\
\hline $13433867+4844266$ & 1558284370590734336 & .. & 0.88 & 0.01 & 0.434 & 0.434 & 0.83 & 183.4 \\
\hline $13434635-0806060$ & 3618345883425109632 & -30.2 & 0.79 & 0.01 & -9.786 & -9.786 & 0.65 & 48.4 \\
\hline $13443166+1523410$ & 3742083100949072640 & -2.4 & 0.12 & 0.00 & -94.515 & -94.515 & 0.14 & 53.0 \\
\hline $13443667-4143163$ & 6112289699794699264 & -22.1 & 0.87 & 0.01 & -39.979 & -39.979 & 0.32 & 142.5 \\
\hline 13452111-0730545 & 3619873547457548160 & 177.0 & 6.36 & 0.62 & -4.550 & -4.550 & 5.10 & 223.3 \\
\hline $13454247-7355568$ & 57913821773588811264 & -27.7 & 0.12 & 0.00 & 12.516 & 12.516 & 0.00 & 33.9 \\
\hline $13455046+0513062$ & 3714276142644562432 & 27.9 & 6.11 & 0.65 & -4.399 & -4.399 & 5.54 & 270.6 \\
\hline $13455076+1426436$ & 3741132710585731840 & -7.0 & 0.18 & 0.00 & -29.815 & -29.815 & 0.19 & 30.4 \\
\hline $13460278-6854556$ & 5850193237592455552 & .. & 1.18 & 0.02 & -8.218 & -8.218 & -0.11 & 28.8 \\
\hline $13461713-4155246$ & 6112273791235652352 & . & 2.02 & 0.11 & -23.549 & -23.549 & 0.71 & 350.3 \\
\hline $13465223-4334387$ & 6108883889504179072 & 213.7 & 5.91 & 0.54 & -12.875 & -12.875 & 1.87 & 354.0 \\
\hline 13481581-7052139 & 5840796639405915648 & -8.2 & 0.16 & 0.00 & -113.938 & -113.938 & 0.00 & 60.3 \\
\hline $13510697+5853470$ & 1659341789968532736 & 48.9 & 0.21 & 0.00 & -12.227 & -12.227 & 0.20 & 49.4 \\
\hline
\end{tabular}


Table 4 (continued)

\begin{tabular}{|c|c|c|c|c|c|c|c|c|}
\hline $\begin{array}{l}\text { Star Name } \\
\text { (2MASS) }\end{array}$ & $\begin{array}{c}\text { Star Name } \\
\text { (Gaia EDR3) }\end{array}$ & $\begin{array}{c}V_{\mathrm{los}} \\
\left(\mathrm{km} \mathrm{s}^{-1}\right)\end{array}$ & $\begin{array}{l}d_{\text {helio }} \\
(\mathrm{kpc})\end{array}$ & $\begin{array}{c}\sigma_{d} \\
(\mathrm{kpc})\end{array}$ & $\begin{array}{c}\mathrm{PM}_{\mathrm{R} . \mathrm{A}} \\
\left(\mathrm{mas} \mathrm{yr}^{-1}\right)\end{array}$ & $\begin{array}{c}\mathrm{PM}_{\text {Decl. }} \\
\left(\mathrm{mas} \mathrm{yr}^{-1}\right)\end{array}$ & $\begin{array}{l}Z_{\mathrm{Gal}} \\
(\mathrm{kpc})\end{array}$ & $\begin{array}{c}V_{\mathrm{TAV}} \\
\left(\mathrm{km} \mathrm{s}^{-1}\right)\end{array}$ \\
\hline $13512378+0741110$ & 3721736844435125760 & -39.2 & 0.15 & 0.00 & -64.142 & -64.142 & 0.16 & 62.5 \\
\hline $13521659-3554258$ & 6115884072018761728 & $\cdots$ & 4.20 & 0.38 & -16.688 & -16.688 & 1.82 & 283.5 \\
\hline $13533557+7552137$ & 1712349073745277056 & -33.0 & 0.22 & 0.00 & 39.657 & 39.657 & 0.17 & 43.2 \\
\hline $13550687+1707259$ & 1243948839616012160 & 45.0 & 0.07 & 0.00 & 137.007 & 137.007 & 0.10 & 72.2 \\
\hline $13580646+1002348$ & 3723801761632006784 & 46.9 & 0.08 & 0.00 & 61.416 & 61.416 & 0.10 & 56.0 \\
\hline $13584157-3151091$ & 6171032134996732416 & -9.0 & 0.13 & 0.00 & -106.555 & -106.555 & 0.09 & 81.5 \\
\hline $13592706+1141130$ & 3727361838547995648 & 12.6 & 2.24 & 0.12 & -14.679 & -14.679 & 2.10 & 276.3 \\
\hline $14002121+5538479$ & 1657538212943126400 & -30.6 & 0.25 & 0.02 & 30.009 & 30.009 & 0.24 & 86.3 \\
\hline $14004596+1215235$ & 3727546045400955136 & -9.9 & 2.91 & 0.13 & -12.503 & -12.503 & 2.72 & 405.2 \\
\hline $14012934+1126361$ & 3724429582771428352 & 28.3 & 4.71 & 0.36 & -2.166 & -2.166 & 4.37 & 133.4 \\
\hline $14020262-6530100$ & 58512636465733588848 & 12.0 & 0.66 & 0.01 & -5.396 & -5.396 & -0.02 & 21.4 \\
\hline $14043744+1255144$ & 1229600350311933952 & 113.2 & 7.75 & 1.32 & -5.369 & -5.369 & 7.19 & 246.1 \\
\hline $14053819+7503388$ & 1712061237921283456 & -6.5 & 0.12 & 0.00 & 19.022 & 19.022 & 0.11 & 7.3 \\
\hline $14063229+4115356$ & 1498298211635183744 & $\ldots$ & 0.22 & 0.00 & -81.394 & -81.394 & 0.23 & 63.5 \\
\hline $14070810+6936102$ & 1674781746425953792 & -162.4 & 3.98 & 0.16 & -6.147 & -6.147 & 2.89 & 199.1 \\
\hline $14071697+1212488$ & 1226501033191248640 & -36.8 & 0.15 & 0.00 & -128.472 & -128.472 & 0.16 & 102.3 \\
\hline $14095514-2844258$ & 6269381255374610176 & 290.5 & 2.40 & 0.13 & -19.832 & -19.832 & 1.26 & 359.3 \\
\hline 14103285-2816339 & 6270148611411493888 & 91.3 & 4.98 & 0.32 & -5.713 & -5.713 & 2.62 & 181.0 \\
\hline $14164140+6136563$ & 1666637942972460672 & -10.5 & 0.11 & 0.00 & -65.361 & -65.361 & 0.12 & 34.7 \\
\hline 14173352-2745144 & 6269861295279466752 & 5.4 & 0.97 & 0.01 & -7.807 & -7.807 & 0.53 & 38.7 \\
\hline 14192484-2307370 & 6275972209107871872 & 8.7 & 0.20 & 0.01 & -17.466 & -17.466 & 0.14 & 19.2 \\
\hline $14193074+4035121$ & 1491839749053036160 & -71.6 & 0.13 & 0.00 & -27.457 & -27.457 & 0.15 & 73.3 \\
\hline $14200302+5936293$ & 1660185630782829952 & -41.0 & 0.11 & 0.00 & -61.812 & -61.812 & 0.12 & 65.1 \\
\hline $14203031-2729455$ & 6269707565513913088 & -38.0 & 0.67 & 0.01 & 12.039 & 12.039 & 0.37 & 118.1 \\
\hline $14211081-5015172$ & 6091186773125289728 & 6.0 & 0.26 & 0.00 & -11.834 & -11.834 & 0.07 & 38.2 \\
\hline $14223584+4045568$ & 1491104691170130944 & -268.4 & 3.15 & 0.10 & -8.333 & -8.333 & 2.92 & 402.2 \\
\hline $14243397+5624475$ & 1610452860137779584 & -300.5 & 2.10 & 0.05 & -17.544 & -17.544 & 1.77 & 342.9 \\
\hline $14255416+2648176$ & 1256475262057930112 & 15.1 & 0.15 & 0.00 & 2.565 & 2.565 & 0.17 & 31.6 \\
\hline $14261661+8102449$ & 1721820606208706176 & -119.1 & 5.35 & 0.29 & -7.813 & -7.813 & 3.12 & 125.3 \\
\hline 14280573-1353174 & 6300021547768039808 & -11.0 & 3.46 & 0.17 & -10.934 & -10.934 & 2.37 & 182.8 \\
\hline $14294135+6525010$ & 1669746193625166976 & -78.1 & 0.65 & 0.01 & -4.787 & -4.787 & 0.51 & 80.7 \\
\hline $14295841+4502025$ & 1494491496221024512 & -76.5 & 4.26 & 0.21 & -7.376 & -7.376 & 3.84 & 244.2 \\
\hline $14324019+3238042$ & 1287120579569866496 & 68.2 & 8.08 & 1.23 & -3.410 & -3.410 & 7.48 & 213.8 \\
\hline $14341379-6707026$ & 5848443708440147072 & 11.6 & 0.11 & 0.00 & 14.967 & 14.967 & 0.01 & 15.1 \\
\hline 14364248-0715099 & 6331121238455373696 & 38.0 & 0.14 & 0.00 & 46.381 & 46.381 & 0.13 & 55.5 \\
\hline $14385266+3937037$ & 1487940434144428416 & -80.9 & 6.93 & 0.45 & -6.953 & -6.953 & 6.27 & 198.5 \\
\hline $14402612+0655539$ & 1171807334801223808 & -122.4 & 3.43 & 0.19 & -20.152 & -20.152 & 2.91 & 430.7 \\
\hline $14410823+4223420$ & 1490063071404892544 & -63.4 & 0.57 & 0.01 & 7.850 & 7.850 & 0.53 & 84.2 \\
\hline $14411830+1044242$ & 1177885611173494016 & -115.2 & 5.82 & 0.46 & -3.934 & -3.934 & 5.03 & 257.7 \\
\hline $14421990+5654506$ & 1607745454489656960 & -23.5 & 0.13 & 0.00 & 6.728 & 6.728 & 0.13 & 29.4 \\
\hline $14431485-0206178$ & 3648915222959836800 & 2.8 & 0.10 & 0.00 & -48.546 & -48.546 & 0.11 & 29.1 \\
\hline $14442119+4758464$ & 1591067649802389632 & -101.6 & 4.60 & 0.21 & -11.429 & -11.429 & 4.00 & 198.2 \\
\hline
\end{tabular}


Table 4 (continued)

\begin{tabular}{|c|c|c|c|c|c|c|c|c|}
\hline $\begin{array}{l}\text { Star Name } \\
\text { (2MASS) }\end{array}$ & $\begin{array}{c}\text { Star Name } \\
\text { (Gaia EDR3) }\end{array}$ & $\begin{array}{c}V_{\mathrm{los}} \\
\left(\mathrm{km} \mathrm{s}^{-1}\right)\end{array}$ & $\begin{array}{l}d_{\text {helio }} \\
(\mathrm{kpc})\end{array}$ & $\begin{array}{c}\sigma_{d} \\
(\mathrm{kpc})\end{array}$ & $\begin{array}{c}\mathrm{PM}_{\mathrm{R} . \mathrm{A}} \\
\left({\left.\mathrm{mas} \mathrm{yr}^{-1}\right)}\right.\end{array}$ & $\begin{array}{c}\mathrm{PM}_{\text {Decl. }} \\
\left(\mathrm{mas} \mathrm{yr}^{-1}\right)\end{array}$ & $\begin{array}{l}Z_{\mathrm{Gal}} \\
(\mathrm{kpc})\end{array}$ & $\begin{array}{c}V_{\mathrm{TAV}} \\
\left(\mathrm{km} \mathrm{s}^{-1}\right)\end{array}$ \\
\hline $14443681+3939231$ & 1487238292890443776 & -17.6 & 0.13 & 0.00 & -41.719 & -41.719 & 0.14 & 43.9 \\
\hline $14444228+4247368$ & 1490052389822427648 & 53.7 & 0.12 & 0.00 & -4.924 & -4.924 & 0.14 & 61.4 \\
\hline 14453096-0214079 & 3648730612380753664 & $\ldots$ & 0.55 & 0.02 & -6.476 & -6.476 & 0.44 & 68.0 \\
\hline $14455563+5838081$ & 1616958709094540672 & $\ldots$ & 0.14 & 0.00 & 19.115 & 19.115 & 0.14 & 7.0 \\
\hline 14460403-7303141 & 5796756182184708096 & 275.6 & 3.48 & 0.13 & -6.152 & -6.152 & -0.70 & 292.2 \\
\hline $14473151+0306259$ & 1157525159792413440 & $\cdots$ & 0.55 & 0.00 & -13.317 & -13.317 & 0.47 & 62.0 \\
\hline $14504173+6652564$ & 1669581885356163456 & -62.7 & 3.04 & 0.08 & -8.800 & -8.800 & 2.22 & 129.5 \\
\hline $14512223+0339397$ & 11576729066674744432 & $\cdots$ & 0.14 & 0.00 & -176.503 & -176.503 & 0.14 & 123.7 \\
\hline $14520851+7234433$ & 1698671492693440512 & -17.8 & 0.20 & 0.00 & -15.775 & -15.775 & 0.16 & 31.4 \\
\hline $14535190-4724035$ & 5905262548019849856 & $\ldots$ & 0.13 & 0.00 & -20.242 & -20.242 & 0.05 & 16.2 \\
\hline $14550996+1452303$ & 1185448189587672960 & -37.5 & 0.11 & 0.00 & -52.056 & -52.056 & 0.12 & 46.4 \\
\hline $14561630+4534315$ & 1586671424357440000 & -61.3 & 0.49 & 0.00 & -24.932 & -24.932 & 0.45 & 161.6 \\
\hline $14561931+0829491$ & 1161734987952738176 & -155.8 & 2.32 & 0.11 & -4.153 & -4.153 & 1.92 & 349.1 \\
\hline $14572828+3910442$ & 1296184266229998976 & -26.3 & 0.15 & 0.00 & -21.091 & -21.091 & 0.15 & 37.9 \\
\hline $14572980+5047347$ & 1593299585391391232 & $\ldots$ & 0.12 & 0.00 & -39.634 & -39.634 & 0.12 & 19.8 \\
\hline $14584270+6055051$ & 1619069599620600576 & 5.3 & 0.12 & 0.00 & 42.045 & 42.045 & 0.12 & 13.2 \\
\hline $14584982+4500407$ & 1586583807024523520 & 11.4 & 0.14 & 0.00 & 21.138 & 21.138 & 0.15 & 23.7 \\
\hline $14590313+0544031$ & 1159807818946952064 & 18.1 & 0.16 & 0.01 & -40.819 & -40.819 & 0.16 & 49.8 \\
\hline $14591786+4755555$ & 1587515844991289600 & 0.2 & 0.28 & 0.00 & -0.265 & -0.265 & 0.26 & 27.7 \\
\hline $15002374+1131178$ & 1180473277429900288 & -13.8 & 0.22 & 0.00 & -72.241 & -72.241 & 0.20 & 73.7 \\
\hline $15010054+5654074$ & 1612825747965425152 & -40.4 & $\ldots$ & $\ldots$ & $\ldots$ & $\ldots$ & 0.03 & 40.4 \\
\hline $15010753+1708495$ & 1187645868519130240 & -64.5 & 3.39 & 0.18 & -0.459 & -0.459 & 2.91 & 150.6 \\
\hline $15012803+3616594$ & 1294826884765960192 & -124.6 & 0.91 & 0.01 & -37.896 & -37.896 & 0.82 & 274.9 \\
\hline 15015996-2613494 & 6225828809526332416 & -77.1 & 1.35 & 0.03 & -55.922 & -55.922 & 0.66 & 331.0 \\
\hline $15021465+3507093$ & 1291694234403435648 & -39.3 & 0.21 & 0.00 & -7.918 & -7.918 & 0.21 & 55.3 \\
\hline $15023312+0549057$ & 1159849256791308160 & -11.0 & 0.41 & 0.00 & -5.138 & -5.138 & 0.35 & 47.8 \\
\hline $15023572+0940189$ & 1167946644533913728 & -44.9 & 4.42 & 0.31 & -9.672 & -9.672 & 3.62 & 213.0 \\
\hline $15023742-2442191$ & 6227668258118329088 & -54.4 & 0.54 & 0.01 & -5.267 & -5.267 & 0.29 & 55.7 \\
\hline $15025030+1345255$ & 1181911644797448832 & -76.9 & 0.09 & 0.00 & -142.077 & -142.077 & 0.10 & 101.2 \\
\hline $15025884+4539160$ & 1586250517562417024 & -89.7 & 5.87 & 0.34 & -8.430 & -8.430 & 5.00 & 187.3 \\
\hline $15031286+0602157$ & 1159876916380773120 & 100.6 & 1.61 & 0.05 & 13.809 & 13.809 & 1.30 & 209.4 \\
\hline $15040464+5927327$ & 1614255147440846720 & -22.6 & 0.26 & 0.00 & 4.828 & 4.828 & 0.22 & 28.7 \\
\hline $15043325+0441214$ & 1156620193003216256 & -29.6 & 3.74 & 0.20 & -12.647 & -12.647 & 2.94 & 324.1 \\
\hline $15050841+0701547$ & 1160377499113263360 & -2.6 & 4.18 & 0.29 & -3.422 & -3.422 & 3.34 & 255.3 \\
\hline 15060136-2508317 & 6227460583562691968 & 15.3 & 0.18 & 0.00 & -10.256 & -10.256 & 0.11 & 24.9 \\
\hline $15060189+0444205$ & 1156532506951177472 & 24.4 & 4.42 & 0.26 & -18.957 & -18.957 & 3.46 & 399.8 \\
\hline $15065236-2547072$ & 6226609772026895104 & -18.2 & $\ldots$ & $\cdots$ & $\ldots$ & $\ldots$ & 0.03 & 18.2 \\
\hline $15071495+5704053$ & 1612981535018964224 & -2.2 & 0.17 & 0.00 & -24.518 & -24.518 & 0.16 & 29.9 \\
\hline $15085620-7020101$ & 5799023787486633984 & 2.1 & 0.20 & 0.00 & -29.786 & -29.786 & -0.01 & 19.6 \\
\hline $15092359+3623180$ & 1292060685309150336 & -16.9 & 0.16 & 0.00 & -106.501 & -106.501 & 0.17 & 71.8 \\
\hline 15094309-2025300 & 6255898665838008960 & $\ldots$ & 0.27 & 0.00 & 10.236 & 10.236 & 0.17 & 12.5 \\
\hline $15095381+7303555$ & 1697243020930244608 & -48.4 & 0.17 & 0.00 & -51.675 & -51.675 & 0.14 & 51.8 \\
\hline
\end{tabular}


Table 4 (continued)

\begin{tabular}{|c|c|c|c|c|c|c|c|c|}
\hline $\begin{array}{l}\text { Star Name } \\
\text { (2MASS) }\end{array}$ & $\begin{array}{c}\text { Star Name } \\
\text { (Gaia EDR3) }\end{array}$ & $\begin{array}{c}V_{\mathrm{los}} \\
\left(\mathrm{km} \mathrm{s}^{-1}\right)\end{array}$ & $\begin{array}{l}d_{\text {helio }} \\
(\mathrm{kpc})\end{array}$ & $\begin{array}{c}\sigma_{d} \\
(\mathrm{kpc})\end{array}$ & $\begin{array}{c}\mathrm{PM}_{\mathrm{R} . \mathrm{A}} \\
\left(\mathrm{mas} \mathrm{yr}^{-1}\right)\end{array}$ & $\begin{array}{c}\mathrm{PM}_{\text {Decl. }} \\
\left(\mathrm{mas} \mathrm{yr}^{-1}\right)\end{array}$ & $\begin{array}{l}Z_{\mathrm{Gal}} \\
(\mathrm{kpc})\end{array}$ & $\begin{array}{c}V_{\mathrm{TAV}} \\
\left(\mathrm{km} \mathrm{s}^{-1}\right)\end{array}$ \\
\hline $15102643+0809151$ & 1166563287107603328 & 113.9 & 2.21 & 0.09 & 0.215 & 0.215 & 1.77 & 170.2 \\
\hline $15114226+2557063$ & 1265182569515722880 & -64.7 & 2.80 & 0.11 & -14.797 & -14.797 & 2.42 & 194.4 \\
\hline $15122018-6551473$ & 5824755211457838848 & -45.9 & 0.58 & 0.00 & -13.551 & -13.551 & -0.04 & 56.9 \\
\hline $15140783+1824326$ & 1211721397891599872 & -35.0 & 1.92 & 0.06 & 5.503 & 5.503 & 1.62 & 263.7 \\
\hline $15142395+2613341$ & 1265208133161061888 & -93.2 & 3.85 & 0.19 & -7.344 & -7.344 & 3.29 & 212.8 \\
\hline $15174310-6858306$ & 5799218602888406272 & -17.5 & 0.69 & 0.01 & -10.793 & -10.793 & -0.09 & 75.9 \\
\hline $15185830+2620276$ & 1270426797601163008 & 9.2 & 3.07 & 0.14 & -4.209 & -4.209 & 2.60 & 364.8 \\
\hline $15193496+4256501$ & 1394164216985937152 & -27.4 & 0.21 & 0.00 & -22.629 & -22.629 & 0.20 & 35.6 \\
\hline $15193617+2304315$ & 12157181527223868816 & 54.0 & 4.03 & 0.23 & -12.015 & -12.015 & 3.38 & 317.7 \\
\hline 15200891-6701472 & 5823675417983108096 & $\ldots$ & 0.19 & 0.00 & -16.960 & -16.960 & 0.00 & 19.3 \\
\hline $15210142+7816076$ & 1708318332837616256 & -152.5 & 5.39 & 0.30 & -10.865 & -10.865 & 3.21 & 252.7 \\
\hline $15243997-8015248$ & 5772460033220024576 & -53.9 & 0.48 & 0.00 & -50.670 & -50.670 & -0.13 & 91.1 \\
\hline $15244942+6935080$ & 1695610791623488128 & -7.9 & 0.25 & 0.00 & -7.182 & -7.182 & 0.20 & 45.9 \\
\hline $15252134+6351159$ & 1640849688017226496 & -15.2 & 0.15 & 0.00 & -166.651 & -166.651 & 0.13 & 57.5 \\
\hline $15255933+5026270$ & 15947802882525888032 & 9.4 & 0.11 & 0.00 & 51.652 & 51.652 & 0.11 & 50.3 \\
\hline $15264322-7017087$ & 5796025453632860544 & -53.6 & 5.30 & 0.29 & -5.540 & -5.540 & -1.01 & 72.3 \\
\hline $15272716+2937502$ & 1273518040182639872 & 29.3 & 0.60 & 0.00 & -7.566 & -7.566 & 0.52 & 38.2 \\
\hline $15282654+6737516$ & 1645668263365914368 & -31.3 & 0.18 & 0.00 & -120.215 & -120.215 & 0.15 & 108.0 \\
\hline $15304031+2345045$ & 1220893455176256512 & -32.7 & 3.62 & 0.30 & -8.340 & -8.340 & 2.96 & 138.9 \\
\hline $15312758+2528504$ & 1222449092327405056 & -17.4 & 0.18 & 0.00 & -56.957 & -56.957 & 0.17 & 59.3 \\
\hline $15330912+8158562$ & 1721465807550041856 & -16.0 & 0.16 & 0.00 & -33.533 & -33.533 & 0.11 & 21.5 \\
\hline $15345353+5610250$ & 1601294344891081472 & -92.2 & 0.29 & 0.00 & -31.603 & -31.603 & 0.25 & 103.3 \\
\hline $15351593+0251226$ & 4427077397649058944 & -69.6 & 4.68 & 0.34 & -10.210 & -10.210 & 3.27 & 280.4 \\
\hline $15352535+2843009$ & 1272505179518285696 & 5.3 & 0.11 & 0.00 & 3.852 & 3.852 & 0.12 & 24.7 \\
\hline $15371627+3318345$ & 1370624708124173312 & -19.8 & 3.72 & 0.18 & -5.305 & -5.305 & 3.03 & 93.1 \\
\hline $15381851+4804330$ & 1401181854246440704 & -63.7 & 0.16 & 0.01 & -32.067 & -32.067 & 0.15 & 66.4 \\
\hline $15391788+5403318$ & 1597864040410848896 & 12.7 & 0.20 & 0.01 & 31.283 & 31.283 & 0.17 & 36.1 \\
\hline $15392367+4037228$ & 1389938076542244992 & -285.3 & 1.73 & 0.04 & -35.192 & -35.192 & 1.41 & 378.7 \\
\hline $15392783+5557319$ & 1601593274615155712 & -26.1 & 0.22 & 0.02 & -17.235 & -17.235 & 0.19 & 27.9 \\
\hline $15393011+7252178$ & 1696583898069432704 & -44.8 & 0.23 & 0.02 & 4.303 & 4.303 & 0.17 & 48.7 \\
\hline $15394006+4426174$ & 1397344691808122624 & -80.2 & 4.38 & 0.20 & -10.900 & -10.900 & 3.49 & 180.7 \\
\hline $15414028+8245352$ & 1723003508921638656 & -15.7 & 0.27 & 0.00 & -20.880 & -20.880 & 0.17 & 16.8 \\
\hline $15420239+0515389$ & 4427879525740941056 & -1.2 & 1.77 & 0.04 & -17.168 & -17.168 & 1.25 & 238.1 \\
\hline $15431257+6424109$ & 1641066429247008128 & 73.2 & 3.10 & 0.11 & -17.218 & -17.218 & 2.17 & 162.5 \\
\hline $15433525+3739578$ & 1376344024013884800 & -6.3 & 0.16 & 0.00 & 18.650 & 18.650 & 0.15 & 36.3 \\
\hline $15441676+0459116$ & 4429308311037974272 & -91.7 & 6.39 & 0.61 & -3.586 & -3.586 & 4.41 & 262.7 \\
\hline $15452566-4444040$ & 5988917073480792704 & -6.6 & 1.11 & 0.02 & -34.347 & -34.347 & 0.18 & 148.9 \\
\hline $15460715+0559221$ & 44295220586736888192 & -3.7 & 2.66 & 0.10 & -15.826 & -15.826 & 1.86 & 273.7 \\
\hline $15470901+0505149$ & 4426320800506688384 & -6.4 & 0.22 & 0.00 & -24.205 & -24.205 & 0.18 & 50.6 \\
\hline $15471083-4404210$ & 5989331898604674176 & $\ldots$ & 0.27 & 0.01 & -14.486 & -14.486 & 0.06 & 29.4 \\
\hline $15475041+6321134$ & 1640197196585984896 & -87.6 & 0.09 & 0.00 & -30.458 & -30.458 & 0.09 & 88.8 \\
\hline $15482592-3959257$ & 6008057268799998848 & $\ldots$ & 0.63 & 0.01 & -7.404 & -7.404 & 0.15 & 17.1 \\
\hline
\end{tabular}


Table 4 (continued)

\begin{tabular}{|c|c|c|c|c|c|c|c|c|}
\hline $\begin{array}{c}\text { Star Name } \\
\text { (2MASS) }\end{array}$ & $\begin{array}{c}\text { Star Name } \\
\text { (Gaia EDR3) }\end{array}$ & $\begin{array}{c}V_{\mathrm{los}} \\
\left(\mathrm{km} \mathrm{s}^{-1}\right)\end{array}$ & $\begin{array}{l}d_{\text {helio }} \\
(\mathrm{kpc})\end{array}$ & $\begin{array}{c}\sigma_{d} \\
(\mathrm{kpc})\end{array}$ & 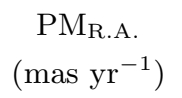 & 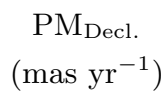 & $\begin{array}{l}Z_{\mathrm{Gal}} \\
(\mathrm{kpc})\end{array}$ & $\begin{array}{c}V_{\mathrm{TAV}} \\
\left(\mathrm{km} \mathrm{s}^{-1}\right)\end{array}$ \\
\hline $15482651+1917200$ & 1203885900077833984 & -245.2 & 6.97 & 0.78 & 4.213 & 4.213 & 5.27 & 319.5 \\
\hline $15484895+0949113$ & 4455582481414836096 & -66.5 & 7.26 & 0.71 & -8.147 & -8.147 & 5.14 & 334.3 \\
\hline $15494232+5707537$ & 1598759730070071808 & -38.4 & 0.14 & 0.00 & -132.733 & -132.733 & 0.13 & 73.2 \\
\hline $15495840-4306370$ & 5989480401391030528 & 6.3 & 0.17 & 0.00 & -17.293 & -17.293 & 0.05 & 19.1 \\
\hline $15501637+3536243$ & 1372799645203241856 & -100.2 & 0.74 & 0.01 & -28.525 & -28.525 & 0.60 & 157.8 \\
\hline $15511496+0734493$ & 4430292717546018304 & -2.3 & 2.27 & 0.08 & -21.610 & -21.610 & 1.58 & 237.1 \\
\hline $15512062+0140120$ & 4423150667969419264 & 31.7 & 0.19 & 0.00 & 4.097 & 4.097 & 0.15 & 42.7 \\
\hline $15514567-3935382$ & 6008406329380234368 & -16.9 & 0.75 & 0.01 & 6.927 & 6.927 & 0.17 & 26.8 \\
\hline $15521212-3934227$ & 6008407257093139456 & -1.9 & 0.61 & 0.01 & 3.418 & 3.418 & 0.14 & 18.7 \\
\hline $15525776+0136148$ & 4411131043072307456 & -16.4 & 3.96 & 0.21 & -7.435 & -7.435 & 2.55 & 157.8 \\
\hline $15533843+0145539$ & 4411228560308157184 & 123.0 & 3.09 & 0.14 & -6.501 & -6.501 & 1.99 & 352.8 \\
\hline $15541062-3255166$ & 6015313976817734784 & 121.1 & 4.91 & 0.45 & -4.846 & -4.846 & 1.37 & 429.0 \\
\hline $15542259-3341566$ & 6012240773099364992 & -27.7 & 0.36 & 0.00 & -15.817 & -15.817 & 0.12 & 43.1 \\
\hline 15542887-3332103 & 6012255341628535680 & -148.4 & 2.64 & 0.09 & -26.231 & -26.231 & 0.73 & 315.7 \\
\hline $15545039-4652323$ & 5987712279288235648 & -18.7 & 0.09 & 0.00 & 36.536 & 36.536 & 0.03 & 24.5 \\
\hline $15555135+1256054$ & 1191328721374511744 & 43.2 & 4.71 & 0.31 & -7.990 & -7.990 & 3.34 & 363.4 \\
\hline $15563950+0754021$ & 4454167994065860352 & -37.4 & 6.96 & 0.67 & -4.627 & -4.627 & 4.70 & 197.4 \\
\hline $15570603+0757417$ & 4454127621373692032 & 0.7 & 0.12 & 0.00 & -25.364 & -25.364 & 0.10 & 17.1 \\
\hline $15583081+1505320$ & 1192938543835309952 & -18.8 & 0.17 & 0.00 & 10.871 & 10.871 & 0.15 & 105.8 \\
\hline $15583757-3734113$ & 6010214613689309056 & $\ldots$ & 0.24 & 0.00 & -8.879 & -8.879 & 0.07 & 13.8 \\
\hline $15584850-3603371$ & 6010918747795620480 & -6.8 & 0.23 & 0.00 & -10.204 & -10.204 & 0.08 & 11.3 \\
\hline $15591458+0507554$ & 4425763554271082112 & -3.1 & 5.13 & 0.43 & -4.285 & -4.285 & 3.34 & 297.5 \\
\hline 15592133-3416261 & 6011940778224281856 & -37.6 & 0.37 & 0.00 & -9.480 & -9.480 & 0.12 & 40.6 \\
\hline $15592229-3853560$ & 5997979351379581312 & -47.4 & 0.79 & 0.01 & -11.796 & -11.796 & 0.17 & 65.1 \\
\hline $15592785+0314441$ & 4424940775976241664 & -15.6 & 0.53 & 0.00 & -56.624 & -56.624 & 0.36 & 222.6 \\
\hline $15593606-3220592$ & 6036353337976099840 & -2.9 & 0.79 & 0.01 & -0.405 & -0.405 & 0.24 & 8.3 \\
\hline $15593767+1613419$ & 1199172465527378176 & -25.0 & 4.32 & 0.25 & -17.575 & -17.575 & 3.09 & 359.1 \\
\hline $15595245-3207381$ & 6036461468074424448 & & 0.50 & 0.01 & -110.204 & -110.204 & 0.16 & 265.3 \\
\hline $16001273+2435560$ & 1219123340233877376 & 39.5 & 0.16 & 0.00 & 21.409 & 21.409 & 0.14 & 52.6 \\
\hline $16004212+1105313$ & 4457460855293872128 & -104.8 & 0.11 & 0.00 & -121.326 & -121.326 & 0.10 & 122.5 \\
\hline $16005880-3307564$ & 6036077979033846528 & 19.9 & 0.59 & 0.00 & 0.125 & 0.125 & 0.18 & 20.0 \\
\hline $16020475+0622249$ & 4450709720758088192 & 20.2 & 0.08 & 0.00 & 36.802 & 36.802 & 0.08 & 66.6 \\
\hline $16025295+3733291$ & 1379240098985809408 & -285.7 & 4.52 & 0.18 & -2.446 & -2.446 & 3.42 & 355.6 \\
\hline $16025382-8226516$ & 5768895377865607808 & 86.9 & 3.45 & 0.13 & -7.547 & -7.547 & -1.26 & 88.8 \\
\hline $16025490+0102272$ & 4411633352385123840 & -79.7 & 0.11 & 0.00 & -122.006 & -122.006 & 0.09 & 102.7 \\
\hline $16030891+1940229$ & 1203268485640442880 & -158.0 & 3.13 & 0.13 & -19.409 & -19.409 & 2.26 & 377.5 \\
\hline $16032024+1641034$ & 1199294713178394496 & -15.0 & 0.15 & 0.01 & 13.984 & 13.984 & 0.13 & 39.0 \\
\hline $16033629+3116414$ & 1322162667977621632 & -48.7 & 0.26 & 0.00 & -7.563 & -7.563 & 0.22 & 61.8 \\
\hline $16034976+0832565$ & 4451626679096707456 & -24.9 & 0.15 & 0.00 & -0.702 & -0.702 & 0.12 & 25.8 \\
\hline $16040396+1841499$ & 1200065646925933056 & 15.3 & 0.13 & 0.00 & -63.390 & -63.390 & 0.11 & 125.1 \\
\hline $16040780+4241470$ & 1383653195061929728 & 22.2 & 0.26 & 0.01 & -37.358 & -37.358 & 0.22 & 58.8 \\
\hline $16042932-2253301$ & 6242404084762732800 & -24.8 & 0.18 & 0.00 & -23.387 & -23.387 & 0.09 & 50.9 \\
\hline
\end{tabular}


Table 4 (continued)

\begin{tabular}{|c|c|c|c|c|c|c|c|c|}
\hline $\begin{array}{l}\text { Star Name } \\
\text { (2MASS) }\end{array}$ & $\begin{array}{c}\text { Star Name } \\
\text { (Gaia EDR3) }\end{array}$ & $\begin{array}{c}V_{\mathrm{los}} \\
\left(\mathrm{km} \mathrm{s}^{-1}\right)\end{array}$ & $\begin{array}{l}d_{\text {helio }} \\
(\mathrm{kpc})\end{array}$ & $\begin{array}{c}\sigma_{d} \\
(\mathrm{kpc})\end{array}$ & 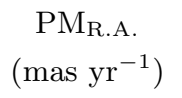 & $\begin{array}{c}\mathrm{PM}_{\text {Decl. }} \\
\left(\mathrm{mas} \mathrm{yr}^{-1}\right)\end{array}$ & $\begin{array}{l}Z_{\text {Gal }} \\
(\mathrm{kpc})\end{array}$ & $\begin{array}{c}V_{\mathrm{TAV}} \\
\left(\mathrm{km} \mathrm{s}^{-1}\right)\end{array}$ \\
\hline 16045324-2005064 & 6244194055324808576 & $\ldots$ & 0.37 & 0.00 & -30.699 & -30.699 & 0.17 & 58.5 \\
\hline $16051879+1359570$ & 1191908675099304704 & -9.4 & 0.32 & 0.01 & 7.060 & 7.060 & 0.24 & 24.4 \\
\hline $16052710+0024235$ & 4409850528641208192 & 0.1 & 0.14 & 0.00 & -27.148 & -27.148 & 0.11 & 19.7 \\
\hline $16052946-2543348$ & 6043663818991262208 & -3.0 & 0.10 & 0.00 & -18.640 & -18.640 & 0.06 & 17.6 \\
\hline $16053749+1843052$ & 1200145322861774080 & -26.4 & 0.11 & 0.00 & 33.220 & 33.220 & 0.10 & 31.4 \\
\hline $16055339+1944171$ & 1203234576873824384 & $\ldots$ & 0.10 & 0.00 & -18.528 & -18.528 & 0.10 & 9.7 \\
\hline $16060454-3508190$ & 6034898993336955008 & $\ldots$ & 0.25 & 0.00 & -7.601 & -7.601 & 0.08 & 42.7 \\
\hline $16061119-3620355$ & 5998751552140270976 & -27.0 & 0.85 & 0.01 & 6.182 & 6.182 & 0.20 & 33.6 \\
\hline $16061643-3237103$ & 6036155219728437760 & 25.9 & 0.46 & 0.00 & 20.837 & 20.837 & 0.14 & 54.6 \\
\hline $16064542-3216179$ & 6036585644175848576 & $\ldots$ & 0.90 & 0.04 & -2.701 & -2.701 & 0.25 & 21.4 \\
\hline $16065604-3707315$ & 5998563982328033920 & 14.8 & 0.30 & 0.00 & 12.598 & 12.598 & 0.08 & 21.2 \\
\hline $16073239-2250378$ & 6242439784523836416 & -24.3 & 0.23 & 0.00 & -8.200 & -8.200 & 0.11 & 34.0 \\
\hline $16073532-3208471$ & 6036547126907295616 & $\ldots$ & 0.63 & 0.01 & -2.706 & -2.706 & 0.18 & 12.3 \\
\hline $16074138-3314575$ & 6035358520474835456 & -13.2 & 0.23 & 0.00 & 4.261 & 4.261 & 0.08 & 14.2 \\
\hline $16074907+1420135$ & 4458558752013944960 & -16.7 & 0.52 & 0.00 & -0.786 & -0.786 & 0.38 & 39.0 \\
\hline 16080889-2943209 & 6041589418507890688 & $\ldots$ & 0.87 & 0.01 & 6.224 & 6.224 & 0.27 & 24.8 \\
\hline 16081391-2017182 & 6244117639267067008 & -34.6 & 0.33 & 0.00 & -11.290 & -11.290 & 0.15 & 39.9 \\
\hline $16083267-3438595$ & 6034940156304474112 & $\ldots$ & 0.79 & 0.01 & -0.076 & -0.076 & 0.20 & 19.9 \\
\hline $16084292-2820180$ & 6041824610913270656 & 38.4 & 0.20 & 0.00 & 13.808 & 13.808 & 0.08 & 46.6 \\
\hline $16091164+3008425$ & 1318809707269021184 & -264.4 & 4.80 & 0.33 & -0.854 & -0.854 & 3.53 & 306.1 \\
\hline $16101125-3446225$ & 6034969392126840320 & -15.4 & 0.31 & 0.00 & 2.922 & 2.922 & 0.09 & 30.0 \\
\hline $16102112+0023264$ & 4408313235288945024 & -43.2 & 2.97 & 0.11 & -8.139 & -8.139 & 1.74 & 219.1 \\
\hline $16102340+3952510$ & 1379957603338177280 & -65.8 & 0.11 & 0.00 & 84.748 & 84.748 & 0.10 & 120.9 \\
\hline $16102544+1810108$ & 1199821898940802944 & -34.9 & 3.54 & 0.21 & -7.497 & -7.497 & 2.47 & 135.3 \\
\hline $16103301+0031597$ & 4408318943304571776 & 11.4 & 1.88 & 0.05 & -9.468 & -9.468 & 1.12 & 130.5 \\
\hline $16103970-2443561$ & 6049772323329029632 & $\ldots$ & 1.84 & 0.05 & -23.071 & -23.071 & 0.63 & 335.7 \\
\hline $16105877-2811435$ & 6041842237458491520 & $\ldots$ & 3.16 & 0.19 & -9.119 & -9.119 & 0.94 & 208.6 \\
\hline $16111495-3204491$ & 6035900167382119808 & -9.1 & 0.30 & 0.00 & -3.163 & -3.163 & 0.10 & 33.8 \\
\hline $16113577-1909453$ & 6245765566678852608 & -36.5 & 0.19 & 0.00 & -16.128 & -16.128 & 0.10 & 56.8 \\
\hline $16114668-2427053$ & 6049806927886876928 & $\ldots$ & 0.68 & 0.01 & -8.758 & -8.758 & 0.25 & 50.5 \\
\hline 16121101-2818128 & 6042164669240420864 & -0.9 & 0.13 & 0.00 & -13.891 & -13.891 & 0.06 & 17.7 \\
\hline $16123013+4456046$ & 1385790890480677376 & -13.7 & 2.47 & 0.06 & -21.469 & -21.469 & 1.81 & 287.7 \\
\hline $16132830-2013405$ & 6245397122905040000 & -41.4 & 0.29 & 0.00 & -19.293 & -19.293 & 0.13 & 48.1 \\
\hline $16134375-2517381$ & 6049518065566679040 & 34.6 & 0.21 & 0.00 & 4.566 & 4.566 & 0.09 & 43.7 \\
\hline 16135700-2757099 & 6042198238705334144 & 7.9 & 0.27 & 0.00 & 2.697 & 2.697 & 0.10 & 18.2 \\
\hline 16141120-2033379 & 6244625270041451648 & -78.9 & 0.61 & 0.01 & -3.673 & -3.673 & 0.25 & 83.4 \\
\hline 16150157-2339354 & 6050327233103808768 & 34.8 & 0.41 & 0.02 & 9.618 & 9.618 & 0.16 & 42.5 \\
\hline $16150863-0734143$ & 4348947961102169344 & 69.0 & 1.45 & 0.03 & 3.717 & 3.717 & 0.75 & 266.4 \\
\hline $16154084+3326445$ & 1322837974275568384 & -150.8 & 2.45 & 0.06 & -17.553 & -17.553 & 1.79 & 253.4 \\
\hline $16154634-1423377$ & 4329332948541070976 & 73.3 & 0.19 & 0.00 & 31.508 & 31.508 & 0.11 & 94.3 \\
\hline $16154808-2355376$ & 6050304972294952064 & $\ldots$ & 0.37 & 0.00 & -12.707 & -12.707 & 0.15 & 33.1 \\
\hline 16154986-0751166 & 4345832009572003840 & -86.5 & 5.55 & 0.44 & -8.273 & -8.273 & 2.75 & 291.0 \\
\hline
\end{tabular}


Table 4 (continued)

\begin{tabular}{|c|c|c|c|c|c|c|c|c|}
\hline $\begin{array}{c}\text { Star Name } \\
\text { (2MASS) }\end{array}$ & $\begin{array}{c}\text { Star Name } \\
\text { (Gaia EDR3) }\end{array}$ & $\begin{array}{c}V_{\mathrm{los}} \\
\left(\mathrm{km} \mathrm{s}^{-1}\right)\end{array}$ & $\begin{array}{l}d_{\text {helio }} \\
(\mathrm{kpc})\end{array}$ & $\begin{array}{c}\sigma_{d} \\
(\mathrm{kpc})\end{array}$ & $\begin{array}{c}\mathrm{PM}_{\mathrm{R} . \mathrm{A}} \\
\left(\mathrm{mas} \mathrm{yr}^{-1}\right)\end{array}$ & $\begin{array}{c}\mathrm{PM}_{\text {Decl. }} \\
\left(\mathrm{mas} \mathrm{yr}^{-1}\right)\end{array}$ & $\begin{array}{l}Z_{\mathrm{Gal}} \\
(\mathrm{kpc})\end{array}$ & $\begin{array}{c}V_{\mathrm{TAV}} \\
\left(\mathrm{km} \mathrm{s}^{-1}\right)\end{array}$ \\
\hline $16163380+4615010$ & 1386708295494907904 & -13.5 & 0.25 & 0.00 & -27.954 & -27.954 & 0.20 & 42.9 \\
\hline $16163935-3233268$ & 60356419539586888896 & -156.2 & 2.91 & 0.14 & -3.452 & -3.452 & 0.68 & 163.9 \\
\hline $16164213-3256588$ & 6035579999042793856 & -143.9 & 0.53 & 0.01 & -95.709 & -95.709 & 0.14 & 366.2 \\
\hline $16165205-2154235$ & 6242759089572065920 & 76.9 & 0.17 & 0.00 & -23.252 & -23.252 & 0.08 & 80.4 \\
\hline $16171415+1832404$ & 1200608324633174784 & -174.7 & 1.69 & 0.04 & -28.344 & -28.344 & 1.16 & 340.9 \\
\hline 16173056-1932253 & 6245550268557026432 & -17.3 & 0.20 & 0.00 & -24.152 & -24.152 & 0.10 & 39.6 \\
\hline $16173244+0248331$ & 4436119304542479616 & -147.8 & 4.85 & 0.29 & -9.079 & -9.079 & 2.82 & 262.3 \\
\hline $16173704+3125396$ & 1319323522796448512 & -148.8 & 5.02 & 0.28 & -0.774 & -0.774 & 3.59 & 154.6 \\
\hline $16175168-3344503$ & 6023449568284510592 & 7.2 & 0.21 & 0.00 & -18.579 & -18.579 & 0.07 & 29.8 \\
\hline 16175506-2501165 & 6048881207813795456 & -47.9 & 0.34 & 0.00 & -8.452 & -8.452 & 0.13 & 51.4 \\
\hline 16180650-1903385 & 6245955610391354880 & -3.2 & 0.15 & 0.00 & 25.535 & 25.535 & 0.08 & 30.0 \\
\hline 16194310-0224311 & 4358730552275110272 & -226.0 & 1.15 & 0.02 & -15.097 & -15.097 & 0.63 & 276.1 \\
\hline $16195744+3711488$ & 1330482668168550016 & -5.5 & 0.19 & 0.00 & -19.824 & -19.824 & 0.16 & 15.5 \\
\hline $16201273+8036085$ & 1710329438388205824 & -104.9 & 2.05 & 0.04 & -8.357 & -8.357 & 1.13 & 107.6 \\
\hline $16201770+4208508$ & 1382053238141320192 & -22.9 & 0.12 & 0.00 & 34.852 & 34.852 & 0.11 & 29.2 \\
\hline $16202566+0328284$ & 4436260965440303232 & -15.8 & 0.10 & 0.00 & -35.910 & -35.910 & 0.08 & 29.6 \\
\hline $16203416+3132541$ & 1319232263331506304 & 6.6 & 0.09 & 0.00 & -38.354 & -38.354 & 0.09 & 16.7 \\
\hline $16203444-2056578$ & 6244454330343783936 & 46.7 & 0.30 & 0.00 & -7.170 & -7.170 & 0.13 & 47.8 \\
\hline $16204714-2606162$ & 6048548399391137152 & $\ldots$ & 1.15 & 0.03 & 1.219 & 1.219 & 0.36 & 11.7 \\
\hline $16204887+7014507$ & 1652927273492300160 & $\ldots$ & 0.17 & 0.00 & 30.019 & 30.019 & 0.13 & 33.2 \\
\hline $16211341+3512582$ & 1329412882010974976 & -13.4 & 0.12 & 0.00 & 14.326 & 14.326 & 0.11 & 15.6 \\
\hline $16213044-7002350$ & 5807311386428745984 & -57.6 & 1.05 & 0.01 & 3.205 & 3.205 & -0.23 & 59.1 \\
\hline 16213929-7135317 & 5806920991081963136 & $\ldots$ & 8.71 & 0.71 & -1.159 & -1.159 & -2.26 & 154.9 \\
\hline $16220240+0700378$ & 4439734017734002944 & -236.8 & 4.13 & 0.22 & -10.719 & -10.719 & 2.47 & 334.5 \\
\hline $16221768+1134274$ & 4459979806371200512 & -1.3 & 3.49 & 0.21 & -6.206 & -6.206 & 2.18 & 122.9 \\
\hline $16224822+5100127$ & 1423692705557972992 & -32.5 & 0.08 & 0.00 & -91.008 & -91.008 & 0.08 & 39.8 \\
\hline $16225698-3234118$ & 6025040458531718016 & -7.8 & 0.23 & 0.00 & -3.548 & -3.548 & 0.07 & 9.3 \\
\hline 16231806-3110431 & 6037281562002354048 & .. & 1.21 & 0.03 & 3.160 & 3.160 & 0.29 & 16.5 \\
\hline $16232673+0535156$ & 4437155113207686400 & -21.4 & . & $\cdots$ & $\cdots$ & & 0.03 & 21.4 \\
\hline $16234350+2958278$ & 1318193396641660544 & 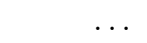 & 0.91 & 0.05 & -8.848 & -8.848 & 0.66 & 132.6 \\
\hline $16241901+0630059$ & 4439457593640443392 & -88.4 & 6.60 & 0.72 & -11.848 & -11.848 & 3.86 & 428.9 \\
\hline $16243746+8055162$ & 1710372289277885440 & 1.6 & 0.25 & 0.00 & -62.945 & -62.945 & 0.16 & 67.5 \\
\hline $16244834+1804359$ & 4467003074331962880 & -136.6 & 1.41 & 0.03 & -3.775 & -3.775 & 0.94 & 148.7 \\
\hline $16251518+0520267$ & 4437084641390808960 & -163.2 & 7.54 & 0.94 & -1.720 & -1.720 & 4.32 & 467.0 \\
\hline 16253573-0834395 & 4350830702966299648 & 33.8 & 2.14 & 0.08 & -6.353 & -6.353 & 1.00 & 162.9 \\
\hline $16254695-3354120$ & 6023951392267153408 & 8.5 & 0.29 & 0.00 & -5.305 & -5.305 & 0.08 & 10.7 \\
\hline 16254871-1359117 & 4328986293140183680 & 124.6 & 1.91 & 0.07 & -29.107 & -29.107 & 0.79 & 435.6 \\
\hline $16260791-2025280$ & 6052684487252718080 & $\ldots$ & 0.97 & 0.02 & 7.601 & 7.601 & 0.35 & 37.7 \\
\hline $16260857+3126550$ & 1324425389891741696 & -51.9 & 0.13 & 0.00 & -68.050 & -68.050 & 0.11 & 65.8 \\
\hline $16263668-0628581$ & 4352076965036319872 & -21.6 & 0.12 & 0.00 & -28.798 & -28.798 & 0.08 & 35.4 \\
\hline $16270418+0322290$ & 4433540296934619520 & -428.2 & 4.47 & 0.34 & -10.165 & -10.165 & 2.48 & 488.6 \\
\hline $16272230+3655279$ & 1331122622592397568 & -240.2 & 3.28 & 0.11 & -14.621 & -14.621 & 2.30 & 323.5 \\
\hline
\end{tabular}


Table 4 (continued)

\begin{tabular}{|c|c|c|c|c|c|c|c|c|}
\hline $\begin{array}{l}\text { Star Name } \\
\text { (2MASS) }\end{array}$ & $\begin{array}{c}\text { Star Name } \\
\text { (Gaia EDR3) }\end{array}$ & $\begin{array}{c}V_{\mathrm{los}} \\
\left(\mathrm{km} \mathrm{s}^{-1}\right)\end{array}$ & $\begin{array}{l}d_{\text {helio }} \\
(\mathrm{kpc})\end{array}$ & $\begin{array}{c}\sigma_{d} \\
(\mathrm{kpc})\end{array}$ & $\begin{array}{c}\mathrm{PM}_{\mathrm{R} . \mathrm{A}} \\
\left({\left.\mathrm{mas} \mathrm{yr}^{-1}\right)}\right.\end{array}$ & $\begin{array}{c}\mathrm{PM}_{\text {Decl. }} \\
\left(\mathrm{mas} \mathrm{yr}^{-1}\right)\end{array}$ & $\begin{array}{l}Z_{\mathrm{Gal}} \\
(\mathrm{kpc})\end{array}$ & $\begin{array}{c}V_{\mathrm{TAV}} \\
\left(\mathrm{km} \mathrm{s}^{-1}\right)\end{array}$ \\
\hline $16272250-0848362$ & 4350762533245253632 & 112.6 & 3.55 & 0.19 & 0.554 & 0.554 & 1.61 & 271.7 \\
\hline $16272730-1210551$ & 4331507503365927680 & 31.7 & 0.17 & 0.00 & 11.653 & 11.653 & 0.09 & 34.1 \\
\hline $16272732+1123305$ & 4459078580497051136 & -46.8 & 0.16 & 0.00 & -81.786 & -81.786 & 0.12 & 75.3 \\
\hline $16274702-3314031$ & 6024841279438699008 & -24.3 & 1.10 & 0.03 & -1.465 & -1.465 & 0.23 & 26.1 \\
\hline $16283300+1722329$ & 4467079971426476160 & 14.7 & 4.54 & 0.34 & -4.376 & -4.376 & 2.89 & 201.7 \\
\hline $16290198+1430507$ & 4463821083383973760 & $\cdots$ & 8.67 & 0.93 & -1.388 & -1.388 & 5.37 & 245.1 \\
\hline $16293666+0213503$ & 4432101100636150400 & 8.7 & 0.19 & 0.00 & -15.748 & -15.748 & 0.13 & 21.3 \\
\hline $16300807+3459161$ & 1326275047622823552 & 43.3 & 0.10 & 0.00 & -134.903 & -134.903 & 0.09 & 67.4 \\
\hline $16302614-3342496$ & 6024015541893607808 & -22.4 & 0.54 & 0.02 & -11.408 & -11.408 & 0.12 & 43.6 \\
\hline $16303876+1329513$ & 4460685211799513600 & -186.7 & 3.93 & 0.19 & -15.147 & -15.147 & 2.40 & 335.9 \\
\hline $16305318+4405187$ & 1405543067118031488 & -91.1 & 0.14 & 0.00 & -22.423 & -22.423 & 0.12 & 93.4 \\
\hline $16312561+1408014$ & 4460744722866525824 & -15.0 & 0.14 & 0.00 & -2.317 & -2.317 & 0.11 & 15.6 \\
\hline $16320549+0401083$ & 4433503532014896512 & 7.5 & 1.06 & 0.01 & -8.858 & -8.858 & 0.60 & 161.1 \\
\hline $16322157+1615449$ & 4465786018039320320 & -117.3 & 3.95 & 0.21 & 2.891 & 2.891 & 2.46 & 429.5 \\
\hline $16323169+1555079$ & 4465571613272013440 & -189.4 & 3.18 & 0.11 & -13.931 & -13.931 & 1.97 & 371.4 \\
\hline $16325211-8327479$ & 5768596864756350976 & -29.3 & 0.66 & 0.00 & 4.305 & 4.305 & -0.24 & 45.1 \\
\hline $16325439-7525259$ & 5781162663455262080 & -50.2 & 0.58 & 0.00 & 7.247 & 7.247 & -0.16 & 81.2 \\
\hline $16333188+5906052$ & 1623752935039977088 & -291.0 & 3.99 & 0.19 & 0.335 & 0.335 & 2.62 & 454.7 \\
\hline $16341169+4636439$ & 1407185565691196672 & -11.6 & 0.10 & 0.00 & -3.054 & -3.054 & 0.09 & 14.0 \\
\hline $16342046+1157567$ & 4459450868260931456 & -193.9 & 1.68 & 0.03 & -24.727 & -24.727 & 1.01 & 291.5 \\
\hline $16343097+0620345$ & 4438931438666007296 & -107.0 & 7.03 & 0.66 & -5.367 & -5.367 & 3.88 & 301.0 \\
\hline $16344239+2231419$ & 1298095453661818880 & 16.7 & 0.12 & 0.00 & 12.655 & 12.655 & 0.10 & 48.5 \\
\hline $16350481+1137192$ & 4459250349123575808 & -258.9 & 6.61 & 1.02 & -1.455 & -1.455 & 3.86 & 273.7 \\
\hline $16352407-3359508$ & 6024133674994546944 & $\ldots$ & 0.39 & 0.00 & -1.773 & -1.773 & 0.09 & 3.0 \\
\hline $16353942+1442022$ & 4462370655749089792 & -80.9 & 0.17 & 0.00 & -33.920 & -33.920 & 0.13 & 85.2 \\
\hline $16354720+1246210$ & 4459657718184334720 & -42.8 & 0.18 & 0.00 & -24.072 & -24.072 & 0.13 & 66.4 \\
\hline $16360774+5058387$ & 1411871031413851648 & 11.9 & 0.25 & 0.00 & 19.143 & 19.143 & 0.19 & 45.5 \\
\hline $16373418+3852001$ & 1331616986212008192 & -65.0 & 3.02 & 0.08 & -5.035 & -5.035 & 2.04 & 332.7 \\
\hline $16374852+0734375$ & 4439132546216071296 & -0.2 & 0.48 & 0.00 & -6.806 & -6.806 & 0.29 & 73.1 \\
\hline $16382363-6644037$ & 5815570715927353216 & 4.5 & 0.20 & 0.00 & 5.385 & 5.385 & -0.02 & 17.2 \\
\hline $16383378+0540276$ & 4435778760872577024 & 14.3 & 0.18 & 0.01 & -6.993 & -6.993 & 0.12 & 18.4 \\
\hline $16384693+1812116$ & 4562485587504234880 & -110.4 & 3.37 & 0.12 & -2.475 & -2.475 & 2.06 & 287.3 \\
\hline $16385168+1723396$ & 4466157103214240256 & 118.1 & 2.58 & 0.07 & -19.849 & -19.849 & 1.57 & 260.1 \\
\hline $16385848+3000087$ & 1311872265637358336 & -49.7 & 0.15 & 0.00 & -13.715 & -13.715 & 0.12 & 52.2 \\
\hline $16391895+0338467$ & 4434135338883534336 & $\cdots$ & 0.16 & 0.00 & -11.506 & -11.506 & 0.11 & 33.0 \\
\hline $16393846+0740507$ & 4445130901837683584 & -68.1 & 0.18 & 0.00 & -47.359 & -47.359 & 0.12 & 80.6 \\
\hline $16394169+4228154$ & 1356989076015732864 & -181.8 & 5.45 & 0.24 & -1.084 & -1.084 & 3.65 & 184.9 \\
\hline $16394396+4249163$ & 1357571404861995136 & $\ldots$ & 5.85 & 0.30 & -4.892 & -4.892 & 3.91 & 256.8 \\
\hline $16402468+1550407$ & 4462850115836027648 & -8.4 & 3.38 & 0.17 & -10.449 & -10.449 & 2.01 & 183.3 \\
\hline $16413086+2631045$ & 1301433231662419840 & -85.8 & 3.55 & 0.13 & -2.411 & -2.411 & 2.27 & 238.9 \\
\hline $16414836+1216122$ & 4460825777486994048 & 21.3 & 2.21 & 0.16 & -39.669 & -39.669 & 1.27 & 415.3 \\
\hline $16420651+2604225$ & 1301214463209079040 & 44.5 & 0.11 & 0.01 & 56.501 & 56.501 & 0.09 & 52.2 \\
\hline
\end{tabular}


Table 4 (continued)

\begin{tabular}{|c|c|c|c|c|c|c|c|c|}
\hline $\begin{array}{l}\text { Star Name } \\
\text { (2MASS) }\end{array}$ & $\begin{array}{c}\text { Star Name } \\
\text { (Gaia EDR3) }\end{array}$ & $\begin{array}{c}V_{\mathrm{los}} \\
\left(\mathrm{km} \mathrm{s}^{-1}\right)\end{array}$ & $\begin{array}{l}d_{\text {helio }} \\
(\mathrm{kpc})\end{array}$ & $\begin{array}{c}\sigma_{d} \\
(\mathrm{kpc})\end{array}$ & $\begin{array}{c}\mathrm{PM}_{\mathrm{R} . \mathrm{A}} \\
\left({\left.\mathrm{mas} \mathrm{yr}^{-1}\right)}\right.\end{array}$ & $\begin{array}{c}\mathrm{PM}_{\text {Decl. }} \\
\left(\mathrm{mas} \mathrm{yr}^{-1}\right)\end{array}$ & $\begin{array}{l}Z_{\mathrm{Gal}} \\
(\mathrm{kpc})\end{array}$ & $\begin{array}{c}V_{\mathrm{TAV}} \\
\left(\mathrm{km} \mathrm{s}^{-1}\right)\end{array}$ \\
\hline $16421928+2344032$ & 1299750291677336960 & $\ldots$ & 4.68 & 0.24 & -5.704 & -5.704 & 2.92 & 224.7 \\
\hline $16423938-8306366$ & 5768700008393198464 & $\ldots$ & 2.32 & 0.06 & -12.141 & -12.141 & -0.89 & 63.4 \\
\hline $16430853+0426005$ & 4434657430811775232 & $\ldots$ & 0.18 & 0.00 & 30.653 & 30.653 & 0.11 & 26.1 \\
\hline $16432853+1024103$ & 4446900634521443584 & 76.1 & 5.03 & 0.34 & -6.755 & -6.755 & 2.77 & 183.7 \\
\hline $16435657+3232386$ & 1314234398867571072 & -85.4 & 4.15 & 0.16 & -8.464 & -8.464 & 2.68 & 183.6 \\
\hline $16440315+1154212$ & 4448745095340450944 & 14.4 & 0.15 & 0.00 & -0.458 & -0.458 & 0.11 & 37.8 \\
\hline 16443996-7951474 & 5775442729091999616 & 299.9 & 3.99 & 0.16 & -16.199 & -16.199 & -1.44 & 352.1 \\
\hline $16461012+3554551$ & 1327313154102801664 & -99.9 & 4.51 & 0.25 & -2.786 & -2.786 & 2.92 & 193.6 \\
\hline $16462075+1719330$ & 4559215674287937536 & -39.1 & 0.18 & 0.00 & 9.798 & 9.798 & 0.13 & 40.7 \\
\hline $16462090+0333477$ & 4433981853933019264 & $\ldots$ & 3.75 & 0.25 & -18.988 & -18.988 & 1.86 & 338.5 \\
\hline $16464088+0311203$ & 4385915672332609280 & 17.6 & 0.13 & 0.00 & -40.919 & -40.919 & 0.09 & 42.8 \\
\hline $16471020+5015587$ & 1412373954904373760 & -114.0 & 2.31 & 0.06 & -10.385 & -10.385 & 1.51 & 136.4 \\
\hline $16472419+1507161$ & 4461858837385161088 & -29.0 & 0.15 & 0.00 & -129.084 & -129.084 & 0.11 & 91.8 \\
\hline $16472795+2336019$ & 1299862235704972928 & -236.6 & 2.35 & 0.05 & -6.087 & -6.087 & 1.44 & 293.4 \\
\hline $16474061+1340279$ & 4461395668115705344 & -168.6 & 5.46 & 0.46 & -10.780 & -10.780 & 3.04 & 502.5 \\
\hline $16482585+4245290$ & 1356678949313407360 & 16.5 & 1.10 & 0.01 & -5.920 & -5.920 & 0.73 & 28.7 \\
\hline $16483067+5232124$ & 1413923647824211456 & $\ldots$ & 1.01 & 0.01 & 3.562 & 3.562 & 0.67 & 14.0 \\
\hline $16485963+2050021$ & 4564767112786411648 & -108.2 & 0.15 & 0.00 & -43.513 & -43.513 & 0.11 & 111.9 \\
\hline $16491646+1922215$ & 4562883030895304960 & -39.7 & 1.48 & 0.05 & 2.845 & 2.845 & 0.88 & 434.0 \\
\hline $16492419+1810509$ & 4559694756410004480 & -38.9 & 0.16 & 0.00 & 49.132 & 49.132 & 0.12 & 84.3 \\
\hline $16501693+3227048$ & 1314079917484186880 & -240.6 & 5.36 & 0.27 & -7.607 & -7.607 & 3.36 & 292.5 \\
\hline $16501966+2135346$ & 4565674033785572096 & -447.4 & 3.55 & 0.17 & -16.750 & -16.750 & 2.10 & 518.9 \\
\hline $16505552+5951110$ & 1438058374730240256 & -225.9 & 6.78 & 0.56 & -12.560 & -12.560 & 4.22 & 372.9 \\
\hline $16510088+3648458$ & 1351373148578890624 & 34.5 & 0.31 & 0.00 & 9.025 & 9.025 & 0.22 & 36.3 \\
\hline $16511460+5352298$ & 1426065314211359488 & 23.9 & 3.19 & 0.12 & -12.507 & -12.507 & 2.04 & 249.0 \\
\hline $16514772+3844288$ & 1352397519754683136 & -155.8 & 3.00 & 0.09 & -10.699 & -10.699 & 1.92 & 211.9 \\
\hline $16523005+0311543$ & 4386055756985792640 & -110.2 & 5.39 & 0.49 & -6.969 & -6.969 & 2.54 & 309.7 \\
\hline $16534583+2531166$ & 1306113268546500352 & 34.3 & 0.09 & 0.00 & 20.450 & 20.450 & 0.08 & 36.5 \\
\hline $16534773+3049290$ & 1312862856894855040 & $\cdots$ & 3.23 & 0.13 & -11.851 & -11.851 & 1.99 & 240.3 \\
\hline $16545744+2603567$ & 1306294683667797248 & 0.2 & 0.11 & 0.00 & 78.702 & 78.702 & 0.09 & 67.2 \\
\hline $16553977-8810318$ & 5765193738828393600 & 84.4 & 5.72 & 0.36 & -4.622 & -4.622 & -2.50 & 120.2 \\
\hline $16563138+3921373$ & 1352143528272068864 & -286.0 & 3.94 & 0.15 & -5.390 & -5.390 & 2.47 & 312.2 \\
\hline $16563406+1516544$ & 4545782356611145728 & -284.1 & 3.86 & 0.20 & -4.422 & -4.422 & 2.08 & 295.2 \\
\hline $16574714+4326284$ & 1358072923898101120 & -22.8 & 0.09 & 0.00 & -53.204 & -53.204 & 0.08 & 30.0 \\
\hline $16581141+4516190$ & 1359217446784103296 & -6.2 & 0.23 & 0.00 & -19.060 & -19.060 & 0.17 & 32.4 \\
\hline $16583722+0153432$ & 4384878175734959744 & $\ldots$ & 1.68 & 0.06 & -18.636 & -18.636 & 0.75 & 197.1 \\
\hline $16590140+2521558$ & 4572761867992849920 & -85.5 & 3.60 & 0.13 & -11.653 & -11.653 & 2.09 & 236.1 \\
\hline $16591231-5353249$ & 5935760874615991808 & $\ldots$ & 0.10 & 0.00 & -22.899 & -22.899 & 0.01 & 40.1 \\
\hline 17001311-7651059 & 5777695868934489600 & $\ldots$ & 0.68 & 0.01 & 3.600 & 3.600 & -0.21 & 51.3 \\
\hline $17024781+2559130$ & 4573174150493770368 & -25.3 & 6.02 & 0.36 & -10.097 & -10.097 & 3.43 & 263.5 \\
\hline $17034238+4012376$ & 1353472361090529536 & -4.3 & 0.19 & 0.00 & 18.856 & 18.856 & 0.14 & 26.8 \\
\hline $17034776+2709455$ & 4573403428730280064 & -49.9 & 0.24 & 0.01 & -12.216 & -12.216 & 0.16 & 51.6 \\
\hline
\end{tabular}


Table 4 (continued)

\begin{tabular}{|c|c|c|c|c|c|c|c|c|}
\hline $\begin{array}{c}\text { Star Name } \\
\text { (2MASS) }\end{array}$ & $\begin{array}{c}\text { Star Name } \\
\text { (Gaia EDR3) }\end{array}$ & $\begin{array}{c}V_{\text {los }} \\
\left(\mathrm{km} \mathrm{s}^{-1}\right)\end{array}$ & $\begin{array}{l}d_{\text {helio }} \\
(\mathrm{kpc})\end{array}$ & $\begin{array}{c}\sigma_{d} \\
(\mathrm{kpc})\end{array}$ & 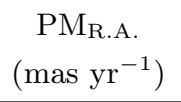 & $\begin{array}{c}\mathrm{PM}_{\text {Decl. }} \\
\left(\mathrm{mas} \mathrm{yr}^{-1}\right)\end{array}$ & $\begin{array}{l}Z_{\text {Gal }} \\
(\mathrm{kpc})\end{array}$ & $\begin{array}{c}V_{\mathrm{TAV}} \\
\left(\mathrm{km} \mathrm{s}^{-1}\right)\end{array}$ \\
\hline $17035954+4513186$ & 1358789148349761152 & -420.9 & 1.96 & 0.04 & -7.745 & -7.745 & 1.21 & 445.2 \\
\hline $17041564+1932115$ & 4560834812533156480 & 24.0 & 5.48 & 0.34 & -8.217 & -8.217 & 2.93 & 216.0 \\
\hline $17041979+6325224$ & 1631229304871201024 & -211.5 & 5.29 & 0.28 & -5.158 & -5.158 & 3.13 & 224.0 \\
\hline $17042385+6726017$ & 1636646151984790784 & -8.1 & 0.12 & 0.00 & -51.571 & -51.571 & 0.09 & 34.4 \\
\hline $17045983+1948039$ & 4561595434057474304 & -132.4 & 5.11 & 0.38 & -8.488 & -8.488 & 2.73 & 268.0 \\
\hline $17054252-6742414$ & 5814397399577566848 & -15.8 & 0.82 & 0.01 & 2.974 & 2.974 & -0.20 & 18.7 \\
\hline $17060632+6430248$ & 1632107986461148928 & -182.5 & 3.58 & 0.14 & -3.431 & -3.431 & 2.11 & 257.4 \\
\hline $17095788+4825288$ & 1414140487836898048 & -7.0 & 0.25 & 0.00 & -11.781 & -11.781 & 0.17 & 15.4 \\
\hline $17104782+4615401$ & 1364943737701366144 & $\ldots$ & 0.54 & 0.00 & -27.729 & -27.729 & 0.35 & 72.1 \\
\hline $17115737+4056337$ & 1341931337289579648 & -35.2 & 0.21 & 0.00 & -14.968 & -14.968 & 0.15 & 45.4 \\
\hline $17125716-2214215$ & 4115322081195173248 & -46.3 & 0.31 & 0.00 & -13.921 & -13.921 & 0.08 & 50.2 \\
\hline $17130596-3442468$ & 5978819326162668288 & -4.8 & 2.23 & 1.37 & -3.436 & -3.436 & 0.12 & 30.3 \\
\hline $17130931+4245360$ & 1354380420256070272 & $\ldots$ & 0.29 & 0.00 & -11.431 & -11.431 & 0.19 & 14.1 \\
\hline $17133146+5029289$ & 1414644171538142464 & -27.1 & 0.24 & 0.01 & -22.751 & -22.751 & 0.17 & 37.8 \\
\hline $17134640-2252270$ & 4114531910303984896 & -7.7 & 0.71 & 0.01 & -2.636 & -2.636 & 0.14 & 26.5 \\
\hline $17134904+4643336$ & 1364979746707350016 & -281.0 & 4.99 & 0.31 & -2.161 & -2.161 & 2.94 & 285.0 \\
\hline $17151412-2251434$ & 4114489441664092160 & -33.8 & 0.26 & 0.00 & 6.676 & 6.676 & 0.07 & 40.3 \\
\hline $17154790-5232570$ & 5925618184739644160 & -119.1 & 3.51 & 0.17 & -21.258 & -21.258 & -0.48 & 392.6 \\
\hline $17160251-2205199$ & 4114972844488807680 & -73.4 & 0.15 & 0.00 & -40.277 & -40.277 & 0.05 & 84.3 \\
\hline $17162512+4249078$ & 1360187452261689472 & -40.3 & 0.75 & 0.01 & -43.687 & -43.687 & 0.45 & 133.1 \\
\hline $17163270+4302287$ & 1360381309905667840 & $\ldots$ & 0.13 & 0.00 & -12.091 & -12.091 & 0.10 & 10.6 \\
\hline 17163966-8711235 & 5765345368353739392 & 47.9 & 1.58 & 0.03 & -25.556 & -25.556 & -0.67 & 244.0 \\
\hline $17174451+3653087$ & 1339989290517076096 & $\cdots$ & 2.31 & 0.07 & -8.774 & -8.774 & 1.31 & 83.5 \\
\hline $17185527+3839047$ & 1340720740627444096 & -25.3 & 0.17 & 0.00 & -12.911 & -12.911 & 0.12 & 26.6 \\
\hline $17192865-6853306$ & 5811209090730279680 & 24.7 & 0.40 & 0.00 & -21.319 & -21.319 & -0.09 & 53.6 \\
\hline $17194406+4515228$ & 1361644549983238272 & -69.5 & 3.92 & 0.16 & -4.442 & -4.442 & 2.25 & 124.0 \\
\hline $17212704+5033288$ & 1414790574087583360 & -137.6 & 7.86 & 0.66 & -6.514 & -6.514 & 4.49 & 304.4 \\
\hline $17215395-6411338$ & 5814091284361742976 & 98.3 & 4.69 & 0.26 & -3.071 & -3.071 & -1.21 & 133.8 \\
\hline $17215498+1423078$ & 4543224965582361984 & -106.9 & 3.72 & 0.16 & -7.078 & -7.078 & 1.67 & 279.5 \\
\hline $17224220+0353126$ & 4389167576754184192 & -179.5 & 3.14 & 0.14 & -10.172 & -10.172 & 1.17 & 325.8 \\
\hline $17234737-5834124$ & 5916061474500671232 & 117.6 & 2.55 & 0.15 & 17.354 & 17.354 & -0.52 & 212.0 \\
\hline $17240759+3635398$ & 1337102866336482688 & $\cdots$ & 3.52 & 0.14 & -8.225 & -8.225 & 1.92 & 256.6 \\
\hline $17251201-7832462$ & 5776783519098670464 & 1.6 & 0.08 & 0.00 & 27.731 & 27.731 & 0.00 & 10.8 \\
\hline $17252775+5318307$ & 1416272239020984576 & -225.4 & 2.84 & 0.09 & -6.598 & -6.598 & 1.62 & 300.1 \\
\hline $17254679-8513010$ & 5767118193414597632 & $\cdots$ & 0.81 & 0.01 & -8.340 & -8.340 & -0.32 & 99.5 \\
\hline $17255611+1556131$ & 4543997853536251264 & -21.3 & 0.72 & 0.01 & -2.459 & -2.459 & 0.34 & 47.2 \\
\hline $17265179+3527349$ & 1335862475484040064 & -30.2 & 4.22 & 0.18 & -4.771 & -4.771 & 2.25 & 268.5 \\
\hline $17272663+3847099$ & 1343578233909298816 & $\ldots$ & 0.40 & 0.01 & 15.982 & 15.982 & 0.24 & 96.5 \\
\hline $17283154-6945443$ & 5810934625139045248 & -8.5 & 0.48 & 0.00 & -2.646 & -2.646 & -0.13 & 30.2 \\
\hline $17285162-6941036$ & 5810941016050593536 & 225.5 & 2.30 & 0.10 & 2.581 & 2.581 & -0.71 & 291.5 \\
\hline $17285482-5720440$ & 5919234390177666304 & -2.9 & 0.15 & 0.00 & -35.900 & -35.900 & -0.01 & 53.9 \\
\hline $17291858+7510248$ & 1655949551783195520 & -173.9 & 7.86 & 0.57 & -3.397 & -3.397 & 4.12 & 199.2 \\
\hline
\end{tabular}


Table 4 (continued)

\begin{tabular}{|c|c|c|c|c|c|c|c|c|}
\hline $\begin{array}{l}\text { Star Name } \\
\text { (2MASS) }\end{array}$ & $\begin{array}{c}\text { Star Name } \\
\text { (Gaia EDR3) }\end{array}$ & $\begin{array}{c}V_{\mathrm{los}} \\
\left(\mathrm{km} \mathrm{s}^{-1}\right)\end{array}$ & $\begin{array}{l}d_{\text {helio }} \\
(\mathrm{kpc})\end{array}$ & $\begin{array}{c}\sigma_{d} \\
(\mathrm{kpc})\end{array}$ & 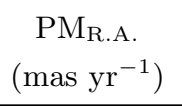 & $\begin{array}{c}\mathrm{PM}_{\text {Decl. }} \\
\left(\mathrm{mas} \mathrm{yr}^{-1}\right)\end{array}$ & $\begin{array}{l}Z_{\mathrm{Gal}} \\
(\mathrm{kpc})\end{array}$ & $\begin{array}{c}V_{\mathrm{TAV}} \\
\left(\mathrm{km} \mathrm{s}^{-1}\right)\end{array}$ \\
\hline $17292254+0305472$ & 4388901086920961024 & -43.9 & 2.96 & 0.16 & -12.370 & -12.370 & 1.02 & 373.8 \\
\hline $17293008+5534560$ & 1421238419509501568 & -282.0 & 3.19 & 0.11 & -4.169 & -4.169 & 1.79 & 319.8 \\
\hline $17303697+0421414$ & 4389421362079616512 & -11.6 & 6.33 & 0.51 & -4.477 & -4.477 & 2.19 & 137.0 \\
\hline $17314553+2843156$ & 4598739479466205568 & $\ldots$ & 1.79 & 0.03 & -14.515 & -14.515 & 0.89 & 231.0 \\
\hline $17315874-5342306$ & 5921618165752908928 & 135.3 & 1.79 & 0.06 & -14.685 & -14.685 & -0.31 & 222.1 \\
\hline $17330791+2548590$ & 4594030404311114240 & -26.6 & 0.17 & 0.01 & -64.286 & -64.286 & 0.10 & 61.6 \\
\hline $17331311+1713463$ & 4550007577936164864 & $\ldots$ & 0.15 & 0.00 & -18.220 & -18.220 & 0.09 & 25.3 \\
\hline $17331323+3152308$ & 4601590066440260096 & -165.3 & 4.35 & 0.19 & 0.838 & 0.838 & 2.17 & 235.8 \\
\hline $17332654+2633432$ & 4594840366419966208 & 42.1 & 1.72 & 0.03 & -2.762 & -2.762 & 0.83 & 47.1 \\
\hline $17333170+5456025$ & 1420926742322768640 & -13.0 & 0.12 & 0.00 & -1.866 & -1.866 & 0.09 & 17.1 \\
\hline $17333194-5022586$ & 5946394938738915584 & -150.3 & 2.37 & 0.08 & -4.026 & -4.026 & -0.36 & 382.1 \\
\hline $17334423+1911331$ & 4553866722012938496 & -2.1 & 0.21 & 0.00 & -0.653 & -0.653 & 0.11 & 2.2 \\
\hline $17334679-6744028$ & 5813027335062608384 & -11.3 & 0.60 & 0.00 & -5.799 & -5.799 & -0.16 & 39.5 \\
\hline $17335350+1815568$ & 4550692088345948672 & -279.7 & 3.57 & 0.15 & -5.097 & -5.097 & 1.53 & 458.1 \\
\hline $17335522+1719221$ & 4550032381370693376 & -2.8 & 0.10 & 0.00 & -0.847 & -0.847 & 0.07 & 4.4 \\
\hline $17355093+1411487$ & 4542596697766095232 & $\ldots$ & 0.25 & 0.00 & -2.973 & -2.973 & 0.12 & 8.9 \\
\hline $17370392-7408233$ & 5803200381170398336 & -3.4 & 0.21 & 0.00 & -2.489 & -2.489 & -0.05 & 26.4 \\
\hline $17371472+1603347$ & 4549079418323779712 & -22.1 & 1.06 & 0.11 & -4.736 & -4.736 & 0.45 & 95.1 \\
\hline $17373165+0109351$ & 4375387436181302912 & -161.1 & 1.43 & 0.04 & -15.205 & -15.205 & 0.44 & 242.2 \\
\hline $17374593-4903259$ & 5946896105559051392 & $\ldots$ & 0.32 & 0.00 & 14.056 & 14.056 & -0.03 & 15.0 \\
\hline $17381613+1806130$ & 4550553412442483456 & -30.3 & 0.92 & 0.02 & -6.533 & -6.533 & 0.40 & 56.7 \\
\hline $17382795+8319481$ & 1711261033974731648 & -40.8 & 0.17 & 0.00 & -45.355 & -45.355 & 0.11 & 154.6 \\
\hline $17390078-7342041$ & 5803233817494625536 & 173.5 & 3.46 & 0.45 & -6.853 & -6.853 & -1.22 & 275.6 \\
\hline $17390590+1504553$ & 4548685209048009600 & $\ldots$ & 3.71 & 0.26 & -4.858 & -4.858 & 1.45 & 101.0 \\
\hline 17393096-5334586 & 5921761544627396352 & 21.3 & 2.36 & 0.09 & 3.194 & 3.194 & -0.46 & 203.5 \\
\hline $17412217-6605479$ & 5812858972344007680 & 50.8 & 3.89 & 0.28 & -11.114 & -11.114 & -1.17 & 411.0 \\
\hline $17412978+2128403$ & 4556052314917665920 & -18.1 & 0.61 & 0.01 & -14.185 & -14.185 & 0.28 & 42.7 \\
\hline $17415044+2448137$ & 4581633552441283328 & 30.8 & 3.69 & 0.16 & -11.574 & -11.574 & 1.62 & 187.8 \\
\hline $17421570-5343061$ & 5921703652781168768 & 18.5 & 2.90 & 0.19 & 2.954 & 2.954 & -0.59 & 246.3 \\
\hline $17422649+3217551$ & 4601113221991774976 & 4.0 & 0.17 & 0.00 & 21.693 & 21.693 & 0.11 & 19.7 \\
\hline $17425789+0417381$ & 4473307880166709248 & 5.1 & 2.54 & 0.09 & -9.300 & -9.300 & 0.78 & 145.8 \\
\hline $17431049+5633516$ & 1421354113045323904 & -56.8 & 0.12 & 0.00 & -10.778 & -10.778 & 0.09 & 99.5 \\
\hline $17435113-5359333$ & 5921684136450078592 & 23.1 & 2.45 & 0.09 & 3.374 & 3.374 & -0.51 & 205.3 \\
\hline $17442011+0827068$ & 4488216467565808512 & -122.7 & 1.31 & 0.03 & -1.522 & -1.522 & 0.45 & 153.3 \\
\hline $17442313+1055585$ & 4489909058337283584 & -9.5 & 0.16 & 0.00 & -1.873 & -1.873 & 0.08 & 15.3 \\
\hline $17451045+1600587$ & 4501194587422903936 & -26.5 & 0.20 & 0.00 & -4.210 & -4.210 & 0.10 & 27.2 \\
\hline $17452465+0613284$ & 4474168965270090368 & $\ldots$ & 5.11 & 0.33 & 2.179 & 2.179 & 1.56 & 112.9 \\
\hline $17452911+0549214$ & 4474076782390335616 & -111.6 & 2.00 & 0.07 & -3.599 & -3.599 & 0.62 & 414.2 \\
\hline $17460256+1657355$ & 4549397894441550848 & -13.3 & 0.94 & 0.02 & -0.624 & -0.624 & 0.38 & 14.0 \\
\hline $17465957+1519406$ & 4501077351995658496 & 16.2 & 3.32 & 0.15 & -13.038 & -13.038 & 1.22 & 203.0 \\
\hline $17470290-5058143$ & 5945699978662588416 & -16.9 & 0.17 & 0.00 & -59.345 & -59.345 & -0.01 & 34.2 \\
\hline $17471725-6723320$ & 5812356121877182080 & 451.0 & 4.92 & 0.34 & -0.375 & -0.375 & -1.58 & 480.6 \\
\hline
\end{tabular}


Table 4 (continued)

\begin{tabular}{|c|c|c|c|c|c|c|c|c|}
\hline $\begin{array}{c}\text { Star Name } \\
\text { (2MASS) }\end{array}$ & $\begin{array}{c}\text { Star Name } \\
\text { (Gaia EDR3) }\end{array}$ & $\begin{array}{c}V_{\mathrm{los}} \\
\left(\mathrm{km} \mathrm{s}^{-1}\right)\end{array}$ & $\begin{array}{l}d_{\text {helio }} \\
(\mathrm{kpc})\end{array}$ & $\begin{array}{c}\sigma_{d} \\
(\mathrm{kpc})\end{array}$ & 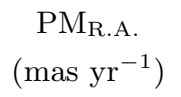 & $\begin{array}{c}\mathrm{PM}_{\text {Decl. }} \\
\left(\mathrm{mas} \mathrm{yr}^{-1}\right)\end{array}$ & $\begin{array}{l}Z_{\text {Gal }} \\
(\mathrm{kpc})\end{array}$ & $\begin{array}{c}V_{\mathrm{TAV}} \\
\left(\mathrm{km} \mathrm{s}^{-1}\right)\end{array}$ \\
\hline 17471835-0035363 & 4371256532336413056 & -19.3 & 0.13 & 0.00 & -18.048 & -18.048 & 0.06 & 22.9 \\
\hline $17472373+5750230$ & 1422587593292960640 & -270.0 & 7.75 & 0.65 & -3.173 & -3.173 & 4.03 & 291.6 \\
\hline $17473698+5229473$ & 1368840406910596992 & -98.7 & 2.43 & 0.06 & -3.127 & -3.127 & 1.27 & 136.9 \\
\hline 17485598-7415094 & 5803007691756413056 & 17.1 & 5.32 & 0.33 & -11.176 & -11.176 & -1.96 & 172.8 \\
\hline $17493006+1616540$ & 4501261550253801344 & -24.5 & 2.43 & 0.07 & 0.557 & 0.557 & 0.89 & 77.0 \\
\hline $17493192-5314272$ & 5921036111776622592 & 276.7 & 1.45 & 0.03 & -11.607 & -11.607 & -0.30 & 311.6 \\
\hline $17505960+1845110$ & 4551354475384137600 & -134.2 & 4.31 & 0.21 & -2.259 & -2.259 & 1.60 & 183.4 \\
\hline $17512033+4313226$ & 1346347658884884608 & -106.6 & 0.08 & 0.00 & -103.019 & -103.019 & 0.06 & 132.6 \\
\hline $17513368+0735474$ & 4475867611955393280 & 67.8 & 0.35 & 0.00 & 21.239 & 21.239 & 0.12 & 114.8 \\
\hline $17514375-8447409$ & 5767473713627782016 & -20.5 & 2.42 & 0.07 & -0.552 & -0.552 & -1.02 & 160.9 \\
\hline $17515635+0549550$ & 4473933497983061632 & $\ldots$ & 0.18 & 0.00 & -1.057 & -1.057 & 0.07 & 14.3 \\
\hline $17530760+0355502$ & 4472708298435677312 & -35.4 & 0.24 & 0.00 & 29.640 & 29.640 & 0.09 & 49.7 \\
\hline $17531820-5828173$ & 5918003040226875648 & 159.0 & 2.80 & 0.10 & -2.124 & -2.124 & -0.74 & 161.0 \\
\hline $17553087+1344549$ & 4499700449901916672 & -9.6 & 3.31 & 0.14 & 3.149 & 3.149 & 1.08 & 116.6 \\
\hline $17563856-6259025$ & 5911002106095737472 & -33.9 & 0.17 & 0.00 & 17.245 & 17.245 & -0.03 & 66.5 \\
\hline $17570985+6104567$ & 1435424254107968896 & -33.1 & 2.44 & 0.06 & -0.206 & -0.206 & 1.24 & 62.0 \\
\hline $17580876-5017025$ & 5947067839841987840 & $\ldots$ & 3.81 & 0.23 & -1.227 & -1.227 & -0.81 & 14.7 \\
\hline $17582442-5200036$ & 5945364387093681024 & 256.6 & 3.65 & 0.38 & -6.465 & -6.465 & -0.83 & 298.5 \\
\hline $17583367+0851134$ & 4476514330950969216 & -94.0 & 2.82 & 0.12 & 9.633 & 9.633 & 0.79 & 328.2 \\
\hline $17590214+0226114$ & 4468887736043966848 & $\ldots$ & 0.14 & 0.00 & -6.958 & -6.958 & 0.06 & 4.6 \\
\hline $17593067+0547081$ & 4474462912835838336 & -18.9 & 0.12 & 0.00 & 11.285 & 11.285 & 0.06 & 22.5 \\
\hline $17595240+0845349$ & 4476464986070993664 & 20.6 & 3.85 & 0.26 & 3.315 & 3.315 & 1.05 & 143.7 \\
\hline $17595579+0402394$ & 4469713473577956352 & 11.0 & 2.28 & 0.09 & -7.780 & -7.780 & 0.55 & 90.8 \\
\hline $18004880+4241389$ & 2113828593508701696 & 28.1 & 0.33 & 0.00 & -3.831 & -3.831 & 0.18 & 68.2 \\
\hline $18012521+1607266$ & 4501806912321934592 & 7.5 & 0.61 & 0.01 & -1.925 & -1.925 & 0.21 & 9.4 \\
\hline 18013011-6117020 & 6635006422507626880 & $\cdots$ & 0.26 & 0.00 & -14.125 & -14.125 & -0.05 & 53.3 \\
\hline $18015056-5628090$ & 6651913342230612608 & 10.3 & 0.28 & 0.00 & 12.413 & 12.413 & -0.05 & 14.5 \\
\hline $18025682-7550189$ & 6414540257944747008 & $\ldots$ & 0.52 & 0.00 & -6.146 & -6.146 & -0.18 & 10.7 \\
\hline $18033495+4211510$ & 2113756098755468288 & -132.3 & 2.83 & 0.08 & 13.460 & 13.460 & 1.28 & 298.9 \\
\hline $18042621-7447453$ & 6417661629719563264 & 169.9 & 3.17 & 0.10 & -3.183 & -3.183 & -1.22 & 176.4 \\
\hline $18042842-7051331$ & 6431507302910109952 & -38.7 & 3.41 & 0.11 & -6.868 & -6.868 & -1.24 & 225.3 \\
\hline $18045298-5543550$ & 6652206220339855104 & -55.9 & 5.36 & 0.39 & 0.375 & 0.375 & -1.45 & 347.0 \\
\hline $18045953+0826177$ & 4476206639494994944 & -131.7 & 1.82 & 0.05 & -7.197 & -7.197 & 0.47 & 164.0 \\
\hline $18052892+0754210$ & 4475421897432191616 & -166.7 & 3.57 & 0.23 & -3.849 & -3.849 & 0.88 & 285.4 \\
\hline $18062441-6457049$ & 6629637992566177664 & 50.3 & 0.13 & 0.00 & 37.876 & 37.876 & -0.02 & 111.8 \\
\hline $18063457+0547198$ & 4471868065390279680 & 111.1 & 1.09 & 0.03 & -17.779 & -17.779 & 0.26 & 346.4 \\
\hline $18072175+5952412$ & 2158636960809774336 & -38.1 & 0.14 & 0.00 & -7.218 & -7.218 & 0.09 & 69.6 \\
\hline $18073369+5849547$ & 2152484604840257280 & -16.0 & 0.14 & 0.00 & 35.332 & 35.332 & 0.09 & 45.4 \\
\hline $18082002-5104378$ & 6702907209758894848 & $\ldots$ & 0.60 & 0.01 & -5.741 & -5.741 & -0.12 & 37.4 \\
\hline $18083624-6750220$ & 6435985682492232320 & -0.5 & 0.22 & 0.01 & -32.418 & -32.418 & -0.05 & 19.0 \\
\hline $18085682-2106299$ & 4094063947258804608 & -18.6 & 0.82 & 0.01 & 0.257 & 0.257 & 0.02 & 27.3 \\
\hline 18095421-6630506 & 6437097494904121600 & 20.1 & 0.52 & 0.00 & -2.027 & -2.027 & -0.16 & 26.6 \\
\hline
\end{tabular}


Table 4 (continued)

\begin{tabular}{|c|c|c|c|c|c|c|c|c|}
\hline $\begin{array}{c}\text { Star Name } \\
\text { (2MASS) }\end{array}$ & $\begin{array}{c}\text { Star Name } \\
\text { (Gaia EDR3) }\end{array}$ & $\begin{array}{c}V_{\mathrm{los}} \\
\left(\mathrm{km} \mathrm{s}^{-1}\right)\end{array}$ & $\begin{array}{l}d_{\text {helio }} \\
(\mathrm{kpc})\end{array}$ & $\begin{array}{c}\sigma_{d} \\
(\mathrm{kpc})\end{array}$ & $\begin{array}{c}\mathrm{PM}_{\mathrm{R} . \mathrm{A}} \\
\left(\mathrm{mas} \mathrm{yr}^{-1}\right)\end{array}$ & $\begin{array}{c}\mathrm{PM}_{\text {Decl. }} \\
\left(\text { mas yr }^{-1}\right)\end{array}$ & $\begin{array}{l}Z_{\mathrm{Gal}} \\
(\mathrm{kpc})\end{array}$ & $\begin{array}{c}V_{\mathrm{TAV}} \\
\left(\mathrm{km} \mathrm{s}^{-1}\right)\end{array}$ \\
\hline $18100815-6731119$ & 6436045915114732672 & $\ldots$ & 0.36 & 0.00 & -13.760 & -13.760 & -0.11 & 19.1 \\
\hline 18102490-7119083 & 6431254307857238784 & -14.9 & 0.66 & 0.00 & 1.233 & 1.233 & -0.23 & 129.1 \\
\hline $18123519-6033510$ & 6635102251817906304 & -51.4 & $\ldots$ & $\ldots$ & $\ldots$ & $\ldots$ & 0.03 & 51.4 \\
\hline $18124977+5854462$ & 2152380494834185984 & -193.8 & 4.37 & 0.18 & -0.369 & -0.369 & 2.07 & 193.8 \\
\hline $18135644+4824196$ & 2122140905709563904 & -37.1 & 0.14 & 0.00 & 8.068 & 8.068 & 0.09 & 66.2 \\
\hline $18141754+1612114$ & 4498809506188122624 & .. & 0.74 & 0.01 & 1.879 & 1.879 & 0.22 & 30.2 \\
\hline $18145902+5852559$ & 2152342188021376384 & -69.6 & 0.19 & 0.00 & -30.116 & -30.116 & 0.11 & 85.8 \\
\hline $18150365-3751206$ & 6727766450449365248 & -80.6 & 3.74 & 0.20 & -12.805 & -12.805 & -0.61 & 218.5 \\
\hline $18162072-4725281$ & 6707603223921252608 & 174.6 & 2.46 & 0.11 & -15.213 & -15.213 & -0.57 & 254.2 \\
\hline $18165208+6146498$ & 2159095113561329024 & -33.0 & 4.83 & 0.21 & 5.399 & 5.399 & 2.27 & 193.4 \\
\hline $18171654+1311582$ & 4485396250300800768 & -13.1 & 0.34 & 0.00 & -2.444 & -2.444 & 0.10 & 22.3 \\
\hline $18190641-6824118$ & 6432812045255424128 & $\ldots$ & 2.80 & 0.61 & 5.285 & 5.285 & -1.03 & 140.7 \\
\hline 18191919-2029254 & 4091364130814956032 & $\ldots$ & 1.10 & 0.03 & 1.623 & 1.623 & -0.02 & 11.9 \\
\hline $18192007-5552473$ & 6649365494611393920 & -10.7 & 0.43 & 0.00 & -4.177 & -4.177 & -0.11 & 26.9 \\
\hline 18193127-3713134 & 6728130968571404800 & -179.4 & 2.26 & 0.09 & 0.705 & 0.705 & -0.38 & 274.4 \\
\hline 18194826-5310564 & 6653631805885843200 & 225.9 & 2.00 & 0.05 & -9.218 & -9.218 & -0.56 & 252.9 \\
\hline 18203066-2016012 & 4091455699522188416 & -16.2 & 0.13 & 0.00 & -11.030 & -11.030 & 0.02 & 18.2 \\
\hline 18204921-3419480 & 4044557100560972800 & -144.8 & 2.88 & 0.19 & -1.275 & -1.275 & -0.43 & 145.6 \\
\hline $18211549+5653298$ & 2150956936513301632 & -299.5 & 3.67 & 0.12 & -4.801 & -4.801 & 1.66 & 363.4 \\
\hline $18220774-7608090$ & 6413638383531665664 & $\ldots$ & 0.20 & 0.01 & -0.483 & -0.483 & -0.06 & 21.1 \\
\hline $18223327-5258169$ & 6653722004497397120 & 53.0 & 0.11 & 0.01 & -32.014 & -32.014 & -0.01 & 55.8 \\
\hline 18231998-3926193 & 6726604713316398336 & 184.6 & 4.55 & 0.33 & -12.030 & -12.030 & -0.91 & 485.7 \\
\hline $18232072-7312133$ & 6418085663251356416 & 21.7 & 0.25 & 0.00 & 0.782 & 0.782 & -0.08 & 30.9 \\
\hline $18273263-5638304$ & 6648380469631584128 & 5.4 & 0.61 & 0.01 & -49.945 & -49.945 & -0.18 & 393.4 \\
\hline $18274709+1732530$ & 4522680968780032128 & -34.1 & 2.79 & 0.11 & -6.930 & -6.930 & 0.65 & 200.9 \\
\hline 18285509-3410250 & 6734946570607247360 & -6.7 & 0.10 & 0.00 & 12.845 & 12.845 & 0.01 & 10.2 \\
\hline 18293868-2010483 & 4092688806190705152 & -68.0 & 0.30 & 0.00 & -13.222 & -13.222 & 0.00 & 71.8 \\
\hline 18301354-4555101 & 6708532208165979392 & 61.3 & 2.90 & 0.11 & 7.805 & 7.805 & -0.76 & 134.8 \\
\hline 18302241-3955233 & 6723716880376076928 & 206.6 & 3.47 & 0.17 & -0.126 & -0.126 & -0.77 & 268.6 \\
\hline $18305087-6953306$ & 6431994249123338240 & 2.0 & 0.47 & 0.00 & -9.323 & -9.323 & -0.16 & 49.8 \\
\hline $18311217+4103160$ & 2110452925308798080 & -143.6 & 5.53 & 0.33 & -7.560 & -7.560 & 2.01 & 230.9 \\
\hline $18315993-6920161$ & 6432068225640386432 & 309.6 & 3.11 & 0.15 & -12.102 & -12.102 & -1.22 & 327.9 \\
\hline $18321423-3829407$ & 6726896461847358848 & 1.1 & 5.99 & 0.64 & -0.559 & -0.559 & -1.33 & 34.3 \\
\hline $18324025-6202554$ & 6631161743881174400 & 139.1 & 2.54 & 0.08 & -10.368 & -10.368 & -0.91 & 182.5 \\
\hline $18334251+4115292$ & 2110471101610527616 & $\cdots$ & 0.22 & 0.00 & -24.160 & -24.160 & 0.10 & 41.2 \\
\hline $18340800-5627514$ & 6649667246135478144 & 8.4 & 0.14 & 0.00 & 3.095 & 3.095 & -0.02 & 26.0 \\
\hline 18352484-3639135 & 6733341416736455936 & $\ldots$ & 0.95 & 0.01 & -33.884 & -33.884 & -0.19 & 228.2 \\
\hline 18352710-4928306 & 6703851728906337152 & ... & 2.17 & 0.10 & -17.084 & -17.084 & -0.64 & 303.0 \\
\hline $18354018-3948416$ & 6723580609650147712 & 188.7 & 1.15 & 0.02 & -2.840 & -2.840 & -0.26 & 206.3 \\
\hline $18360814+1919587$ & 4524742759245230592 & -192.4 & 2.80 & 0.14 & -8.504 & -8.504 & 0.60 & 434.8 \\
\hline 18361214-7333443 & 6418205892271978624 & 41.9 & 2.76 & 0.11 & -7.782 & -7.782 & -1.13 & 53.3 \\
\hline 18371329-3141091 & 4046640705089371136 & $\ldots$ & 3.16 & 0.19 & -7.107 & -7.107 & -0.58 & 184.0 \\
\hline
\end{tabular}


Table 4 (continued)

\begin{tabular}{|c|c|c|c|c|c|c|c|c|}
\hline $\begin{array}{c}\text { Star Name } \\
\text { (2MASS) }\end{array}$ & $\begin{array}{c}\text { Star Name } \\
\text { (Gaia EDR3) }\end{array}$ & $\begin{array}{c}V_{\mathrm{los}} \\
\left(\mathrm{km} \mathrm{s}^{-1}\right)\end{array}$ & $\begin{array}{l}d_{\text {helio }} \\
(\mathrm{kpc})\end{array}$ & $\begin{array}{c}\sigma_{d} \\
(\mathrm{kpc})\end{array}$ & 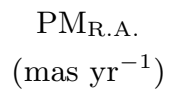 & $\begin{array}{c}\mathrm{PM}_{\text {Decl. }} \\
\left(\mathrm{mas} \mathrm{yr}^{-1}\right)\end{array}$ & $\begin{array}{l}Z_{\text {Gal }} \\
(\mathrm{kpc})\end{array}$ & $\begin{array}{c}V_{\mathrm{TAV}} \\
\left(\mathrm{km} \mathrm{s}^{-1}\right)\end{array}$ \\
\hline $18373068-5538076$ & 6649914292651257984 & 213.7 & 2.93 & 0.15 & -9.451 & -9.451 & -0.99 & 227.1 \\
\hline $18395804+4820336$ & 2119887903305583104 & -180.0 & 6.17 & 0.35 & -3.741 & -3.741 & 2.31 & 207.3 \\
\hline $18404278-5822181$ & 6636472453764175744 & 7.3 & 0.75 & 0.01 & -3.289 & -3.289 & -0.25 & 83.3 \\
\hline $18405632+5805144$ & 2154314260908589184 & -303.8 & 2.64 & 0.08 & -5.700 & -5.700 & 1.10 & 316.0 \\
\hline $18411282-6105584$ & 6632762873329420544 & 146.8 & 3.95 & 0.28 & -11.943 & -11.943 & -1.48 & 214.7 \\
\hline $18430593-7820300$ & 6364899129015765248 & 3.1 & 0.12 & 0.00 & -5.379 & -5.379 & -0.03 & 29.0 \\
\hline $18462350-4939426$ & 6656086710412617344 & -9.8 & 0.18 & 0.00 & -2.305 & -2.305 & -0.03 & 27.0 \\
\hline $18482959-4911163$ & 6656206965197014272 & 30.9 & 0.55 & 0.01 & -7.929 & -7.929 & -0.16 & 46.6 \\
\hline $18490576-5304063$ & 6651390073476420736 & $\ldots$ & 0.28 & 0.00 & 4.638 & 4.638 & -0.07 & 28.1 \\
\hline $18491122+4005588$ & 2098297480667292160 & -26.3 & 0.38 & 0.02 & 11.660 & 11.660 & 0.14 & 42.5 \\
\hline $18512136+5319111$ & 2146237458947371520 & -211.0 & 1.58 & 0.02 & 0.933 & 0.933 & 0.60 & 374.1 \\
\hline $18515139+7849432$ & 2293311669034242432 & 20.3 & 3.07 & 0.11 & -6.730 & -6.730 & 1.40 & 340.0 \\
\hline $18523585+4032042$ & 2103587162389240064 & -9.7 & 0.21 & 0.01 & 3.409 & 3.409 & 0.09 & 37.7 \\
\hline $18523981+4129288$ & 2104473093882735104 & $\ldots$ & 3.98 & 0.17 & -3.727 & -3.727 & 1.21 & 118.5 \\
\hline $19024429+5351114$ & 2134680487931467648 & -10.7 & 0.26 & 0.00 & 2.880 & 2.880 & 0.11 & 25.0 \\
\hline $19025533+4219090$ & 2104015387809994496 & -205.6 & 2.41 & 0.05 & -7.562 & -7.562 & 0.69 & 215.4 \\
\hline $19032297-4757304$ & 6662155460541591424 & 10.2 & 0.14 & 0.00 & -1.413 & -1.413 & -0.03 & 47.2 \\
\hline $19035178-5108429$ & 6657682239223060736 & 257.9 & 5.43 & 0.63 & -9.781 & -9.781 & -2.07 & 431.0 \\
\hline $19044854+5029122$ & 2133638842399952256 & $\ldots$ & 1.78 & 0.03 & -5.061 & -5.061 & 0.59 & 27.4 \\
\hline $19052503-7826440$ & 6364286563599950592 & -17.5 & 0.42 & 0.00 & -1.079 & -1.079 & -0.17 & 42.4 \\
\hline $19055032-5206090$ & 6656823172744784384 & -297.5 & 6.65 & 0.68 & -3.014 & -3.014 & -2.60 & 381.3 \\
\hline $19060062-5118110$ & 6657644374791106304 & $\ldots$ & 7.82 & 0.83 & 4.610 & 4.610 & -3.04 & 276.4 \\
\hline $19060226-6310122$ & 6438853483690577024 & -0.9 & 0.40 & 0.00 & 6.834 & 6.834 & -0.15 & 18.7 \\
\hline 19120066-6302562 & 6438895574370061568 & $\ldots$ & 5.98 & 0.45 & 1.986 & 1.986 & -2.62 & 545.6 \\
\hline $19131047+3313392$ & 2043394428389668992 & -89.5 & 4.30 & 0.18 & -10.875 & -10.875 & 0.80 & 221.9 \\
\hline $19155183-4922541$ & 6658254874322598016 & 293.8 & 1.86 & 0.05 & -28.380 & -28.380 & -0.73 & 336.2 \\
\hline $19155497+4038463$ & 2101342715562410240 & -212.9 & 3.64 & 0.12 & -14.158 & -14.158 & 0.84 & 284.2 \\
\hline $19160765+4246319$ & 2102705938181969408 & -297.3 & 2.21 & 0.06 & -1.378 & -1.378 & 0.56 & 297.8 \\
\hline $19184233+8121549$ & 2295494715010792576 & 7.3 & 0.60 & 0.00 & 19.794 & 19.794 & 0.29 & 30.2 \\
\hline 19214950-7947382 & 6361002631604746752 & $\ldots$ & 0.42 & 0.00 & 7.805 & 7.805 & -0.17 & 8.1 \\
\hline $19232375+4720104$ & 2129167334770344704 & -53.9 & 7.76 & 0.60 & -4.346 & -4.346 & 1.98 & 155.7 \\
\hline $19233833+4018284$ & 2101121026531480960 & $\cdots$ & 4.82 & 0.24 & 5.064 & 5.064 & 0.99 & 88.8 \\
\hline $19242686+8215294$ & 2295685828172114688 & $\cdots$ & 0.45 & 0.00 & 4.793 & 4.793 & 0.22 & 68.9 \\
\hline $19253278-2828581$ & 6764789893132461568 & $\ldots$ & 1.88 & 0.10 & 1.306 & 1.306 & -0.60 & 27.0 \\
\hline $19275678+3601156$ & 2049957756892384256 & -60.2 & 0.39 & 0.01 & 0.266 & 0.266 & 0.09 & 61.0 \\
\hline $19280042+4614069$ & 2126804793521418112 & $\ldots$ & 5.88 & 0.38 & 0.812 & 0.812 & 1.39 & 68.6 \\
\hline $19281201-2900223$ & 6764699423940862080 & 101.4 & 5.01 & 0.41 & -10.713 & -10.713 & -1.70 & 335.4 \\
\hline 19281988-6339344 & 6441410874720278272 & -108.3 & 3.03 & 0.12 & 4.836 & 4.836 & -1.40 & 175.9 \\
\hline $19333143+8023242$ & 2295227331824267136 & -24.3 & 0.71 & 0.00 & 10.229 & 10.229 & 0.33 & 27.7 \\
\hline $19352572+5114261$ & 2136022446171534848 & -20.1 & 0.12 & 0.00 & 20.497 & 20.497 & 0.06 & 29.1 \\
\hline $19375420+5750304$ & 2238305511775896064 & -30.1 & 0.20 & 0.01 & -38.550 & -38.550 & 0.08 & 37.7 \\
\hline $19383369+4309290$ & 2077979845822635648 & -22.4 & 0.31 & 0.01 & 5.657 & 5.657 & 0.08 & 23.5 \\
\hline
\end{tabular}


Table 4 (continued)

\begin{tabular}{|c|c|c|c|c|c|c|c|c|}
\hline $\begin{array}{l}\text { Star Name } \\
\text { (2MASS) }\end{array}$ & $\begin{array}{c}\text { Star Name } \\
\text { (Gaia EDR3) }\end{array}$ & $\begin{array}{c}V_{\mathrm{los}} \\
\left(\mathrm{km} \mathrm{s}^{-1}\right)\end{array}$ & $\begin{array}{l}d_{\text {helio }} \\
(\mathrm{kpc})\end{array}$ & $\begin{array}{c}\sigma_{d} \\
(\mathrm{kpc})\end{array}$ & $\begin{array}{c}\mathrm{PM}_{\mathrm{R} . \mathrm{A}} \\
\left(\mathrm{mas} \mathrm{yr}^{-1}\right)\end{array}$ & $\begin{array}{c}\mathrm{PM}_{\text {Decl. }} \\
\left(\mathrm{mas} \mathrm{yr}^{-1}\right)\end{array}$ & $\begin{array}{l}Z_{\mathrm{Gal}} \\
(\mathrm{kpc})\end{array}$ & $\begin{array}{c}V_{\mathrm{TAV}} \\
\left(\mathrm{km} \mathrm{s}^{-1}\right)\end{array}$ \\
\hline $19411203-4509560$ & 6684605636852993408 & -105.2 & 6.41 & 0.55 & 6.520 & 6.520 & -2.91 & 222.2 \\
\hline $19423665+0502288$ & 4290628386915732736 & -215.7 & 2.28 & 0.09 & 18.507 & 18.507 & -0.33 & 340.7 \\
\hline $19433935+7749001$ & 2291495585062080640 & -169.5 & 4.55 & 0.21 & -11.151 & -11.151 & 1.86 & 236.6 \\
\hline $19440238-4515172$ & 6684633743118584064 & $\cdots$ & 5.62 & 0.50 & -4.099 & -4.099 & -2.60 & 333.1 \\
\hline $19460015-4844202$ & 6671059481799424000 & 25.5 & 2.61 & 0.12 & 1.154 & 1.154 & -1.23 & 231.0 \\
\hline $19481329-4903448$ & 6670987390771270912 & 144.6 & 4.08 & 0.20 & -12.634 & -12.634 & -1.96 & 365.1 \\
\hline $19491264+0358174$ & 4242240117122573440 & -86.9 & 0.19 & 0.00 & 19.953 & 19.953 & -0.01 & 151.7 \\
\hline $19493291+1245526$ & 4305164750954492032 & -55.6 & 2.86 & 0.11 & -5.815 & -5.815 & -0.31 & 479.4 \\
\hline 19501371-7714505 & 6365989359218055680 & $\ldots$ & 0.73 & 0.01 & 11.073 & 11.073 & -0.33 & 54.9 \\
\hline $19512662-5013221$ & 6669955297247174784 & -54.9 & 1.94 & 0.05 & 4.358 & 4.358 & -0.94 & 308.3 \\
\hline $19523317+4918292$ & 2086984326351707008 & $\ldots$ & 0.17 & 0.00 & 11.316 & 11.316 & 0.06 & 8.6 \\
\hline $19533677-4832517$ & 6670382182636447104 & $\ldots$ & 4.05 & 0.25 & -4.931 & -4.931 & -2.00 & 365.2 \\
\hline $19545002+0803021$ & 4298283770977735168 & -219.1 & 3.96 & 0.20 & 2.476 & 2.476 & -0.67 & 365.3 \\
\hline $19550782+0003520$ & 4237149584437046784 & -59.9 & 0.43 & 0.00 & 15.455 & 15.455 & -0.08 & 71.1 \\
\hline $19552158-4613569$ & 6671915382881993472 & 155.1 & 3.99 & 0.29 & -7.273 & -7.273 & -1.97 & 353.4 \\
\hline $19580641-5217166$ & $\ldots$ & $\ldots$ & $\ldots$ & $\ldots$ & $\ldots$ & $\ldots$ & $\ldots$ & $\ldots$ \\
\hline $19583119+1403473$ & 1807178962362590336 & -189.4 & 3.27 & 0.16 & 4.246 & 4.246 & -0.43 & 291.1 \\
\hline 20000909-8240204 & 6347485957207236480 & 214.8 & 1.06 & 0.01 & 3.729 & 3.729 & -0.49 & 356.3 \\
\hline $20015470+1103254$ & 4300772996581672704 & -55.9 & 0.25 & 0.00 & 38.133 & 38.133 & -0.02 & 73.3 \\
\hline $20021812+0356003$ & 4247387510856965504 & $\ldots$ & 0.46 & 0.00 & 14.330 & 14.330 & -0.08 & 33.6 \\
\hline 20032253-1142028 & 4190620966764303488 & -52.1 & 2.40 & 0.10 & 7.440 & 7.440 & -0.84 & 539.3 \\
\hline $20035532-5028100$ & 6667107184173771648 & -316.7 & 4.89 & 0.53 & -8.823 & -8.823 & -2.55 & 625.6 \\
\hline 20042821-0634042 & 4219932228775714816 & -18.3 & 0.11 & 0.00 & 14.327 & 14.327 & -0.01 & 31.8 \\
\hline $20052878-5431260$ & 6473118900280458240 & -94.1 & 3.59 & 0.26 & -5.549 & -5.549 & -1.90 & 112.2 \\
\hline 20065053-0824044 & 4192486700559707136 & 160.3 & 3.13 & 0.13 & -13.408 & -13.408 & -1.07 & 382.8 \\
\hline 20065112-0135379 & 4235780417581602304 & -327.9 & 2.42 & 0.10 & 4.084 & 4.084 & -0.70 & 457.8 \\
\hline $20071356+0151191$ & 4243611826586899200 & $\cdots$ & 0.34 & 0.01 & 21.897 & 21.897 & -0.07 & 100.7 \\
\hline $20082836+1011584$ & 4299755432926365184 & -76.8 & 3.62 & 0.41 & -6.105 & -6.105 & -0.73 & 147.6 \\
\hline 20083729-1136333 & 6880822690942721024 & -17.5 & 0.08 & 0.00 & 0.887 & 0.887 & -0.01 & 27.0 \\
\hline 20111053-0042015 & 4236237642618470272 & 4.1 & 5.49 & 0.48 & -6.228 & -6.228 & -1.67 & 282.6 \\
\hline $20115949+0328555$ & 4244395262980430592 & 53.0 & 0.44 & 0.00 & 1.006 & 1.006 & -0.10 & 53.7 \\
\hline 20120663-1720171 & 6873767610163223552 & $\ldots$ & 0.12 & 0.00 & 99.213 & 99.213 & -0.02 & 54.5 \\
\hline 20121138-5616178 & 6471798631629401088 & -108.6 & 2.23 & 0.08 & 8.519 & 8.519 & -1.20 & 338.5 \\
\hline 20121853-1451042 & 6877707847518537088 & -73.0 & 3.79 & 0.19 & -5.285 & -5.285 & -1.54 & 167.9 \\
\hline $20124384+0008034$ & 4236447546259730816 & -25.1 & 0.15 & 0.00 & -5.759 & -5.759 & -0.02 & 26.6 \\
\hline $20125289+0124185$ & 4242787188566429184 & -80.6 & 3.67 & 0.19 & -10.891 & -10.891 & -1.07 & 346.4 \\
\hline 20125447-6143094 & 6442969093151242880 & -54.3 & 0.12 & 0.00 & 99.920 & 99.920 & -0.04 & 148.6 \\
\hline 20145497-0643013 & 4217089677680862080 & -209.0 & 4.83 & 0.32 & 0.049 & 0.049 & -1.75 & 325.0 \\
\hline 20152495-2220596 & 6852768686118280576 & 28.6 & 0.15 & 0.00 & 37.738 & 37.738 & -0.05 & 72.7 \\
\hline 20153131-5719468 & 6468635748993114112 & 118.9 & 4.03 & 0.33 & 2.363 & 2.363 & -2.22 & 383.7 \\
\hline 20160180-0523231 & 4217535808820767104 & -279.5 & 1.63 & 0.04 & 1.227 & 1.227 & -0.57 & 302.9 \\
\hline 20160744-0552554 & 4217300027997089280 & 21.9 & 0.96 & 0.43 & 1.519 & 1.519 & -0.33 & 46.2 \\
\hline
\end{tabular}


Table 4 (continued)

\begin{tabular}{|c|c|c|c|c|c|c|c|c|}
\hline $\begin{array}{c}\text { Star Name } \\
\text { (2MASS) }\end{array}$ & $\begin{array}{c}\text { Star Name } \\
\text { (Gaia EDR3) }\end{array}$ & $\begin{array}{c}V_{\mathrm{los}} \\
\left(\mathrm{km} \mathrm{s}^{-1}\right)\end{array}$ & $\begin{array}{l}d_{\text {helio }} \\
(\mathrm{kpc})\end{array}$ & $\begin{array}{c}\sigma_{d} \\
(\mathrm{kpc})\end{array}$ & 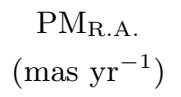 & $\begin{array}{c}\mathrm{PM}_{\text {Decl. }} \\
\left(\mathrm{mas} \mathrm{yr}^{-1}\right)\end{array}$ & $\begin{array}{l}Z_{\text {Gal }} \\
(\mathrm{kpc})\end{array}$ & $\begin{array}{c}V_{\mathrm{TAV}} \\
\left(\mathrm{km} \mathrm{s}^{-1}\right)\end{array}$ \\
\hline 20165001-1040388 & 6881177219022208512 & -36.4 & 0.09 & 0.00 & 29.287 & 29.287 & -0.01 & 59.4 \\
\hline $20165024+1403416$ & 1803616579035764992 & -10.1 & 5.12 & 0.31 & 6.241 & 6.241 & -1.02 & 181.0 \\
\hline 20174990-4403599 & 6673167103853359232 & 110.6 & 0.22 & 0.00 & 2.917 & 2.917 & -0.10 & 110.8 \\
\hline 20180356-1301215 & 6879502009977573504 & -198.8 & 1.83 & 0.05 & -13.991 & -13.991 & -0.75 & 279.2 \\
\hline $20185485-1428523$ & 6876208079299850752 & -165.1 & 3.33 & 0.17 & 8.554 & 8.554 & -1.42 & 258.2 \\
\hline 20192202-6130149 & 6430979984003489024 & -16.2 & 5.17 & 0.50 & -15.471 & -15.471 & -2.87 & 358.6 \\
\hline $20194097-2922268$ & 6797560699757276544 & -41.2 & 0.54 & 0.01 & 19.131 & 19.131 & -0.25 & 69.8 \\
\hline $20200915-4813436$ & 6668894268525757696 & 5.1 & 0.12 & 0.00 & 40.938 & 40.938 & -0.04 & 19.9 \\
\hline $20203472-2757112$ & 6846116468972086016 & 18.8 & 0.54 & 0.00 & 2.415 & 2.415 & -0.25 & 39.7 \\
\hline 20204659-1002234 & 6905253392633848960 & -216.1 & 6.34 & 0.57 & -4.527 & -4.527 & -2.59 & 268.0 \\
\hline 20205529-1340439 & 6876402624138104576 & 14.4 & 0.10 & 0.00 & -77.591 & -77.591 & -0.02 & 101.5 \\
\hline 20210974-6637116 & 6425821762641148800 & 80.5 & 3.92 & 0.16 & 8.616 & 8.616 & -2.14 & 337.5 \\
\hline $20214042-5350224$ & 6472730429078350976 & -10.7 & 0.50 & 0.15 & 26.342 & 26.342 & -0.26 & 136.0 \\
\hline 20214109-5550310 & 6469232749446813824 & 17.7 & 1.10 & 0.02 & -19.879 & -19.879 & -0.60 & 191.9 \\
\hline 20214764-1610513 & $\ldots$ & $\cdots$ & $\cdots$ & $\cdots$ & $\ldots$ & $\ldots$ & $\ldots$ & $\cdots$ \\
\hline 20214838-2917466 & 6797535960745546624 & $\ldots$ & 0.47 & 0.06 & 3.706 & 3.706 & -0.22 & 29.7 \\
\hline 20231318-0728503 & 4216093593161355776 & -131.3 & 2.60 & 0.13 & -5.448 & -5.448 & -1.02 & 170.0 \\
\hline 20231543-2104080 & 6859284155805054848 & 137.8 & 4.95 & 0.36 & -9.354 & -9.354 & -2.39 & 282.5 \\
\hline 20232260-0807452 & 6906102627927525760 & -16.5 & 5.28 & 0.65 & -7.422 & -7.422 & -2.13 & 332.8 \\
\hline 20233743-1659533 & 6873254241311424896 & -162.4 & 2.25 & 0.12 & -11.531 & -11.531 & -1.02 & 316.9 \\
\hline $20235225-1628512$ & 6873331791241472768 & -286.1 & 5.06 & 0.40 & -2.571 & -2.571 & -2.32 & 315.2 \\
\hline $20241045-6720324$ & 6425543307024121088 & 200.7 & 5.39 & 0.27 & 0.976 & 0.976 & -2.97 & 330.9 \\
\hline $20242459-2529550$ & 6848220487550880640 & 43.0 & 8.41 & 0.83 & 2.786 & 2.786 & -4.29 & 286.7 \\
\hline 20244286-2618599 & 6847828584672684416 & 12.9 & 0.34 & 0.00 & -3.064 & -3.064 & -0.15 & 69.2 \\
\hline 20244510-1605268 & 6874849529603646080 & -142.0 & 5.70 & 0.50 & 0.212 & 0.212 & -2.62 & 172.1 \\
\hline 20255659-4915524 & 6668166013871841536 & -3.1 & 0.12 & 0.00 & 43.894 & 43.894 & -0.05 & 20.8 \\
\hline $20263268-1025283$ & 6904467555355890304 & -41.5 & 0.19 & 0.00 & 18.179 & 18.179 & -0.06 & 44.6 \\
\hline 20271323-1658595 & 6862731949391812992 & -19.3 & 0.18 & 0.00 & 4.639 & 4.639 & -0.06 & 27.0 \\
\hline 20273786-1444546 & 6875659938390010240 & -57.1 & 0.21 & 0.01 & -32.700 & -32.700 & -0.07 & 69.5 \\
\hline 20273791-2627414 & 6847786940670450944 & -53.3 & 0.22 & 0.00 & -4.378 & -4.378 & -0.09 & 59.0 \\
\hline $20274485-4223567$ & 6679325159242871808 & -12.0 & 0.16 & 0.00 & -14.293 & -14.293 & -0.07 & 39.1 \\
\hline $20275301-5141137$ & 6475211236547281152 & -69.1 & 5.18 & 0.43 & 2.382 & 2.382 & -3.00 & 187.1 \\
\hline 20275840-1556595 & 6874956658968640512 & -36.6 & 0.10 & 0.00 & 15.071 & 15.071 & -0.02 & 42.0 \\
\hline $20281482-5351378$ & 6473996241838555392 & -13.8 & 2.18 & 0.06 & -12.257 & -12.257 & -1.25 & 201.0 \\
\hline 20284544-2638089 & 6847769863878031616 & -84.9 & 0.19 & 0.00 & 51.052 & 51.052 & -0.08 & 135.1 \\
\hline 20290062-2157354 & 6855992046192920960 & 33.8 & 0.57 & 0.00 & 3.303 & 3.303 & -0.27 & 35.0 \\
\hline $20290527-5059527$ & 6475303698603197824 & -37.1 & 0.82 & 0.01 & 3.918 & 3.918 & -0.46 & 39.1 \\
\hline 20292008-4513468 & 6675597235006194432 & -30.7 & 0.95 & 0.02 & 14.002 & 14.002 & -0.53 & 56.9 \\
\hline $20293622-3652218$ & 6694857444613732352 & 28.2 & 0.50 & 0.00 & -4.172 & -4.172 & -0.26 & 55.5 \\
\hline 20301912-2844399 & 6797804306004518400 & -1.7 & 0.25 & 0.00 & 13.967 & 13.967 & -0.11 & 16.8 \\
\hline 20313318-3054125 & 6796525780141266304 & 35.9 & 0.26 & 0.00 & -19.004 & -19.004 & -0.12 & 46.6 \\
\hline $20314518-5623277$ & 6469004910021548672 & 50.1 & 2.52 & 0.09 & 1.081 & 1.081 & -1.46 & 382.6 \\
\hline
\end{tabular}


Table 4 (continued)

\begin{tabular}{|c|c|c|c|c|c|c|c|c|}
\hline $\begin{array}{c}\text { Star Name } \\
\text { (2MASS) }\end{array}$ & $\begin{array}{c}\text { Star Name } \\
\text { (Gaia EDR3) }\end{array}$ & $\begin{array}{c}V_{\mathrm{los}} \\
\left(\mathrm{km} \mathrm{s}^{-1}\right)\end{array}$ & $\begin{array}{l}d_{\text {helio }} \\
(\mathrm{kpc})\end{array}$ & $\begin{array}{c}\sigma_{d} \\
(\mathrm{kpc})\end{array}$ & 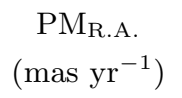 & 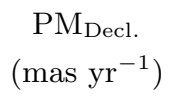 & $\begin{array}{l}Z_{\mathrm{Gal}} \\
(\mathrm{kpc})\end{array}$ & $\begin{array}{c}V_{\mathrm{TAV}} \\
\left(\mathrm{km} \mathrm{s}^{-1}\right)\end{array}$ \\
\hline $20331212-4200270$ & 6679615950005226240 & -309.0 & 2.01 & 0.09 & -2.346 & -2.346 & -1.16 & 410.6 \\
\hline 20331607-5047126 & 6475629909958997376 & -13.0 & 0.28 & 0.01 & 16.120 & 16.120 & -0.14 & 20.1 \\
\hline $20351407-4625056$ & 6674758921813508736 & -63.0 & 1.11 & 0.02 & 5.914 & 5.914 & -0.64 & 81.1 \\
\hline $20354257-0714557$ & 6907591641550947072 & -232.4 & 2.65 & 0.10 & -2.140 & -2.140 & -1.15 & 381.9 \\
\hline 20373334-3645452 & 6683227639544432512 & -9.6 & 0.16 & 0.00 & 20.808 & 20.808 & -0.07 & 18.8 \\
\hline 20375779-2518252 & 6800352497219577728 & & 0.70 & 0.05 & 6.036 & 6.036 & -0.37 & 33.3 \\
\hline 20381947-2750475 & 6798666258699212672 & 57.5 & 0.51 & 0.00 & -23.743 & -23.743 & -0.26 & 85.2 \\
\hline 20385050-2657542 & 6799133551141107712 & 50.2 & 0.78 & 0.01 & -6.996 & -6.996 & -0.42 & 72.6 \\
\hline 20404939-5843259 & 6455696764877344896 & 156.7 & 7.04 & 0.61 & -3.237 & -3.237 & -4.21 & 268.0 \\
\hline $20410627-3251350$ & 6792327226270824832 & 92.8 & 0.27 & 0.00 & -40.228 & -40.228 & -0.13 & 138.1 \\
\hline 20414841-5237274 & 6471501763492187520 & -18.9 & 6.50 & 0.53 & -9.421 & -9.421 & -3.96 & 249.0 \\
\hline 20430947-3600325 & 6779175490951538560 & 2.0 & 3.05 & 0.15 & -8.653 & -8.653 & -1.82 & 129.2 \\
\hline 20440973-2940093 & 6795239665071836160 & -45.4 & 4.68 & 0.33 & -4.318 & -4.318 & -2.74 & 185.0 \\
\hline 20442721-7405038 & 6369772538209241216 & $\ldots$ & 0.40 & 0.00 & 15.101 & 15.101 & -0.20 & 20.1 \\
\hline 20443065-2936534 & 6795263648167892992 & 34.2 & 0.12 & 0.00 & 43.907 & 43.907 & -0.05 & 40.6 \\
\hline 20445501-3732403 & 6778221802053181824 & -56.9 & 0.76 & 0.01 & 11.436 & 11.436 & -0.44 & 66.0 \\
\hline 20451053-2934207 & 6795077693263710080 & -140.7 & 1.29 & 0.03 & 26.855 & 26.855 & -0.74 & 357.6 \\
\hline $20460102+1506250$ & 1762690140608564480 & & 5.61 & 0.89 & -2.854 & -2.854 & -1.62 & 122.1 \\
\hline 20461039-3940468 & 6678691295087392640 & 84.3 & 3.48 & 0.17 & 9.996 & 9.996 & -2.13 & 251.0 \\
\hline 20461191-3833114 & 6681901765961577344 & 46.4 & 1.44 & 0.04 & -28.909 & -28.909 & -0.87 & 250.0 \\
\hline 20465470-3948150 & 6678675249089470464 & -245.4 & 1.92 & 0.07 & -1.853 & -1.853 & -1.17 & 246.1 \\
\hline 20474129-4949312 & 6480909150618081536 & 121.1 & 7.27 & 1.16 & 0.329 & 0.329 & -4.53 & 213.0 \\
\hline 20480642-3520259 & 6779625977182979328 & 36.7 & 0.14 & 0.00 & -37.638 & -37.638 & -0.06 & 46.3 \\
\hline 20485047-7341446 & 6370159673678547712 & 282.7 & 7.24 & 0.50 & 3.311 & 3.311 & -4.03 & 421.9 \\
\hline $20485505-4125356$ & 6678289698465087232 & -76.2 & 4.02 & 0.23 & 11.669 & 11.669 & -2.51 & 296.6 \\
\hline 20490816-2214501 & 6807184278357672576 & -17.2 & 5.28 & 0.43 & 14.831 & 14.831 & -3.02 & 421.2 \\
\hline 20492765-5124440 & 6477616903566840064 & 25.6 & 5.63 & 0.51 & -3.733 & -3.733 & -3.52 & 198.4 \\
\hline $20504877+1007551$ & 1751382561168000256 & -54.5 & 0.16 & 0.00 & 25.224 & 25.224 & -0.03 & 67.4 \\
\hline $20512785-4843325$ & 6481344866460154880 & 64.7 & 0.18 & 0.00 & 10.023 & 10.023 & -0.09 & 66.0 \\
\hline 20523629-5241033 & 6477303675894157824 & 147.0 & 9.99 & 1.35 & -2.983 & -2.983 & -6.32 & 244.6 \\
\hline 20530472-3836380 & 6774883405575766400 & -113.7 & 4.75 & 0.32 & -7.754 & -7.754 & -3.00 & 340.3 \\
\hline 20531334-4520139 & 6484187516335125760 & 28.8 & 0.13 & 0.00 & -16.089 & -16.089 & -0.06 & 30.1 \\
\hline $20531555+1147415$ & 1757847139781935616 & -321.2 & 5.40 & 0.37 & 0.053 & 0.053 & -1.86 & 394.4 \\
\hline 20541462-4811494 & 6481473440600905472 & -67.0 & 4.73 & 0.40 & 10.002 & 10.002 & -3.02 & 338.0 \\
\hline 20545308-4710289 & 6481731516596154368 & -41.9 & 4.51 & 0.42 & 5.293 & 5.293 & -2.88 & 171.1 \\
\hline 20555702-3912091 & 6774608463246378880 & -78.4 & 2.21 & 0.13 & 23.838 & 23.838 & -1.40 & 324.2 \\
\hline 20565365-5609461 & 6457897089506392064 & $\ldots$ & 2.64 & 0.09 & -10.744 & -10.744 & -1.65 & 138.2 \\
\hline $20571292-4958553$ & 6478163463924318208 & 22.7 & 4.40 & 0.31 & -4.550 & -4.550 & -2.82 & 267.5 \\
\hline 20574855-4154444 & 6677344182887180800 & 25.5 & 0.30 & 0.01 & -32.662 & -32.662 & -0.17 & 51.8 \\
\hline $20575772-5637258$ & 6457695677015968384 & -5.1 & 2.12 & 0.06 & 22.653 & 22.653 & -1.33 & 321.3 \\
\hline $20580267+1427040$ & 1761667216837966848 & -210.9 & 3.48 & 0.15 & 10.751 & 10.751 & -1.15 & 281.7 \\
\hline 20585673-4013142 & 6773745346616608000 & -39.0 & 0.13 & 0.00 & 138.889 & 138.889 & -0.06 & 75.8 \\
\hline
\end{tabular}


Table 4 (continued)

\begin{tabular}{|c|c|c|c|c|c|c|c|c|}
\hline $\begin{array}{c}\text { Star Name } \\
\text { (2MASS) }\end{array}$ & $\begin{array}{c}\text { Star Name } \\
\text { (Gaia EDR3) }\end{array}$ & $\begin{array}{c}V_{\mathrm{los}} \\
\left(\mathrm{km} \mathrm{s}^{-1}\right)\end{array}$ & $\begin{array}{l}d_{\text {helio }} \\
(\mathrm{kpc})\end{array}$ & $\begin{array}{c}\sigma_{d} \\
(\mathrm{kpc})\end{array}$ & $\begin{array}{c}\mathrm{PM}_{\mathrm{R} . \mathrm{A} .} \\
\left({\left.\text { mas } \mathrm{yr}^{-1}\right)}\right.\end{array}$ & $\begin{array}{c}\mathrm{PM}_{\text {Decl. }} \\
\left(\text { mas yr }^{-1}\right)\end{array}$ & $\begin{array}{l}Z_{\mathrm{Gal}} \\
(\mathrm{kpc})\end{array}$ & $\begin{array}{c}V_{\mathrm{TAV}} \\
\left(\mathrm{km} \mathrm{s}^{-1}\right)\end{array}$ \\
\hline $20590249+1847021$ & 1765354016765575680 & $\ldots$ & 0.33 & 0.00 & -3.330 & -3.330 & -0.07 & 15.0 \\
\hline 20592599-4724182 & 6483013375355411200 & -11.5 & 0.18 & 0.00 & -10.091 & -10.091 & -0.09 & 13.9 \\
\hline $20595162-4937337$ & 6478288460356702464 & 2.0 & 0.59 & 0.00 & 10.762 & 10.762 & -0.36 & 20.7 \\
\hline 21011036-4537322 & 6483420263376508032 & -79.8 & 3.05 & 0.13 & 10.480 & 10.480 & -1.98 & 177.3 \\
\hline 21011096-4148546 & 6581257453716372352 & 6.7 & 0.58 & 0.02 & 4.562 & 4.562 & -0.36 & 74.7 \\
\hline 21014399-4913588 & 6478323339786839168 & 8.7 & 0.10 & 0.00 & -28.197 & -28.197 & -0.04 & 12.6 \\
\hline $21032352-4211400$ & 6581180590980731520 & 42.8 & 0.11 & 0.00 & -34.963 & -34.963 & -0.05 & 44.8 \\
\hline 21032466-7254213 & 6370267975574142592 & & 6.62 & 0.49 & -5.177 & -5.177 & -3.80 & 249.8 \\
\hline 21032961-7321324 & 6370238112664426624 & 42.6 & 4.70 & 0.23 & 6.181 & 6.181 & -2.68 & 183.4 \\
\hline 21042794-4710017 & 6480078194704569088 & -76.9 & 4.91 & 0.33 & 9.149 & 9.149 & -3.25 & 276.2 \\
\hline 21051884-6831537 & 6376679571472808064 & 50.9 & 4.01 & 0.24 & 0.905 & 0.905 & -2.40 & 171.9 \\
\hline $21054066-4520565$ & 6483621714522704128 & -2.5 & 2.39 & 0.10 & 20.425 & 20.425 & -1.58 & 323.3 \\
\hline $21080234+1835409$ & 1788340995265967488 & $\ldots$ & 0.15 & 0.00 & 7.991 & 7.991 & -0.02 & 43.9 \\
\hline $21091442-4721520$ & 6480023214826621696 & -29.5 & 0.89 & 0.01 & 0.137 & 0.137 & -0.58 & 76.3 \\
\hline 21092218-4250491 & 6580163542725955840 & $\ldots$ & 3.89 & 0.30 & -8.990 & -8.990 & -2.62 & 161.0 \\
\hline 21094841-5600060 & 6463024322681098368 & $\ldots$ & 5.92 & 0.45 & 0.252 & 0.252 & -3.89 & 268.4 \\
\hline $21095801+1725439$ & 1788003032879354752 & -241.4 & 1.18 & 0.02 & -17.541 & -17.541 & -0.38 & 426.4 \\
\hline $21102133+3016061$ & 1852687405024593024 & -53.9 & 0.10 & 0.00 & -89.343 & -89.343 & 0.01 & 109.8 \\
\hline $21105535+2140380$ & 1790165875330692352 & -109.4 & 0.18 & 0.00 & -137.493 & -137.493 & -0.03 & 167.1 \\
\hline 21110533-4239222 & 6580263048527430400 & -159.7 & 4.78 & 0.34 & 2.635 & 2.635 & -3.24 & 384.1 \\
\hline $21111175-4126536$ & 6581456534040000128 & 74.5 & 1.97 & 0.06 & 6.832 & 6.832 & -1.32 & 361.6 \\
\hline 21114008-5138220 & 6476892256683323008 & $\ldots$ & 4.24 & 0.31 & -8.517 & -8.517 & -2.84 & 637.4 \\
\hline $21115127-5257071$ & 6464688708407259008 & 107.2 & 0.83 & 0.01 & -48.699 & -48.699 & -0.53 & 283.1 \\
\hline $21120163+2520001$ & 1841468911788506112 & -54.8 & 0.15 & 0.00 & -18.020 & -18.020 & -0.02 & 73.2 \\
\hline $21125173+2110327$ & 1790077841384173312 & -168.1 & 3.08 & 0.19 & 2.990 & 2.990 & -0.95 & 251.1 \\
\hline $21134390-6802355$ & 6399985335331112064 & -9.2 & 3.36 & 0.12 & 8.657 & 8.657 & -2.05 & 173.1 \\
\hline $21145602+2112242$ & 1791382721170477824 & -107.0 & 2.56 & 0.14 & 7.902 & 7.902 & -0.80 & 203.6 \\
\hline $21150824+2631245$ & 1847680160351457024 & 29.8 & 0.85 & 0.01 & 1.286 & 1.286 & -0.20 & 33.4 \\
\hline $21151790-4333404$ & 6579952677010742272 & 162.2 & 4.26 & 0.26 & 1.825 & 1.825 & -2.93 & 168.5 \\
\hline 21154971-6848520 & 6375872461218303488 & 57.7 & 4.46 & 0.23 & -6.409 & -6.409 & -2.72 & 80.2 \\
\hline $21171659-4115323$ & 6580773015762728704 & -37.9 & 0.41 & 0.00 & -11.043 & -11.043 & -0.26 & 43.3 \\
\hline $21190273+3318462$ & 1854629726683249024 & -154.8 & 2.53 & 0.08 & 0.581 & 0.581 & -0.47 & 218.1 \\
\hline 21192932-7715553 & 6356252535213950592 & -246.6 & 0.85 & 0.01 & 21.539 & 21.539 & -0.45 & 343.4 \\
\hline $21203573-5321426$ & 6463751271665659264 & 175.2 & 2.02 & 0.05 & -20.055 & -20.055 & -1.36 & 272.9 \\
\hline $21211669+2032551$ & 1790500809763786624 & -32.7 & 0.15 & 0.00 & 73.700 & 73.700 & -0.03 & 59.6 \\
\hline $21214670+1916532$ & 1785668323017152384 & $\ldots$ & 0.86 & 0.01 & -7.023 & -7.023 & -0.29 & 34.7 \\
\hline $21232828-5328287$ & 6463713682111820160 & 32.7 & 1.62 & 0.03 & 22.828 & 22.828 & -1.10 & 366.4 \\
\hline $21240060-5241520$ & 6465286018099447680 & -36.6 & 1.55 & 0.03 & 5.613 & 5.613 & -1.05 & 176.9 \\
\hline 21243758-6400120 & 6403279953204895360 & & 0.23 & 0.00 & -24.468 & -24.468 & -0.12 & 37.8 \\
\hline 21254398-6753045 & 6399850542078013568 & -34.0 & 0.07 & 0.00 & 182.652 & 182.652 & -0.02 & 47.3 \\
\hline $21262879+1749436$ & 1784515446419862656 & -10.8 & 0.60 & 0.00 & -3.355 & -3.355 & -0.21 & 22.5 \\
\hline $21263180+2031469$ & 1790358392944627712 & -178.9 & 4.66 & 0.60 & 8.481 & 8.481 & -1.66 & 283.2 \\
\hline
\end{tabular}


Table 4 (continued)

\begin{tabular}{|c|c|c|c|c|c|c|c|c|}
\hline $\begin{array}{l}\text { Star Name } \\
\text { (2MASS) }\end{array}$ & $\begin{array}{c}\text { Star Name } \\
\text { (Gaia EDR3) }\end{array}$ & $\begin{array}{c}V_{\mathrm{los}} \\
\left(\mathrm{km} \mathrm{s}^{-1}\right)\end{array}$ & $\begin{array}{l}d_{\text {helio }} \\
(\mathrm{kpc})\end{array}$ & $\begin{array}{c}\sigma_{d} \\
(\mathrm{kpc})\end{array}$ & $\begin{array}{c}\mathrm{PM}_{\mathrm{R} . \mathrm{A}} \\
\left(\mathrm{mas} \mathrm{yr}^{-1}\right)\end{array}$ & $\begin{array}{c}\mathrm{PM}_{\text {Decl. }} \\
\left(\mathrm{mas} \mathrm{yr}^{-1}\right)\end{array}$ & $\begin{array}{l}Z_{\mathrm{Gal}} \\
(\mathrm{kpc})\end{array}$ & $\begin{array}{c}V_{\mathrm{TAV}} \\
\left(\mathrm{km} \mathrm{s}^{-1}\right)\end{array}$ \\
\hline 21281884-6406460 & 6403090356168392192 & -55.1 & 5.78 & 0.35 & 5.585 & 5.585 & -3.76 & 87.2 \\
\hline $21292831-5558258$ & 6460174865281654144 & -0.5 & 0.17 & 0.00 & 14.964 & 14.964 & -0.10 & 25.9 \\
\hline 21313828-6858199 & 6396651616076109184 & $\ldots$ & 6.88 & 0.58 & 0.379 & 0.379 & -4.32 & 219.7 \\
\hline $21322880-6607539$ & 6400620298999247488 & 110.6 & 0.13 & 0.00 & -62.158 & -62.158 & -0.06 & 142.0 \\
\hline $21323054+1600097$ & 1772048878641647488 & -534.3 & 1.04 & 0.02 & 5.958 & 5.958 & -0.42 & 535.4 \\
\hline 21340283-6224209 & 6403844758583266688 & $\cdots$ & 2.91 & 0.11 & -2.144 & -2.144 & -1.93 & 364.5 \\
\hline $21343861+1538156$ & 17718329620458662656 & -8.6 & 0.18 & 0.01 & -31.693 & -31.693 & -0.05 & 68.4 \\
\hline $21351702-5533118$ & 6460110479427930624 & 62.7 & 2.47 & 0.08 & 22.093 & 22.093 & -1.72 & 356.9 \\
\hline 21373999-2446495 & 6814831136913490048 & 13.1 & 0.13 & 0.00 & -1.513 & -1.513 & -0.07 & 13.5 \\
\hline $21391225+3849406$ & 1953863502940549504 & -48.4 & 0.30 & 0.02 & -60.682 & -60.682 & -0.03 & 117.3 \\
\hline 21393394-5845548 & 6458363870847393792 & 280.4 & 5.78 & 0.37 & -0.460 & -0.460 & -4.01 & 282.4 \\
\hline 21414000-2854239 & 6809748884932883712 & -113.6 & 4.15 & 0.32 & -2.683 & -2.683 & -3.08 & 223.2 \\
\hline 21430664-6338509 & 6402785069892389760 & -0.6 & 0.15 & 0.00 & 11.138 & 11.138 & -0.07 & 4.2 \\
\hline $21440820+3813139$ & 1952857308060989312 & -221.1 & 1.91 & 0.04 & -10.150 & -10.150 & -0.35 & 273.2 \\
\hline $21445442-6319381$ & 64028185319807555584 & -16.0 & 0.51 & 0.00 & 11.290 & 11.290 & -0.32 & 97.1 \\
\hline $21453847+2351116$ & 1794596868534480128 & -62.8 & 0.91 & 0.01 & 31.373 & 31.373 & -0.32 & 182.3 \\
\hline 21461136-5420472 & 6461349457233919872 & -49.9 & 0.08 & 0.00 & 140.459 & 140.459 & -0.03 & 91.2 \\
\hline $21480606+4643071$ & 1974610531448247936 & -6.6 & 0.79 & 0.01 & 2.838 & 2.838 & -0.05 & 9.8 \\
\hline $21482057+2155222$ & 1793330952694172416 & $\ldots$ & 0.60 & 0.00 & 18.310 & 18.310 & -0.22 & 59.2 \\
\hline $21494865+1048431$ & 1765600930139450752 & -271.8 & 1.59 & 0.06 & -58.024 & -58.024 & -0.81 & 515.5 \\
\hline $21502426-6105576$ & 6409890217109702784 & $\ldots$ & 11.11 & 1.52 & 4.288 & 4.288 & -7.77 & 250.5 \\
\hline $21510307+3619543$ & 1949409175177462272 & -373.6 & 4.28 & 0.32 & 2.341 & 2.341 & -0.98 & 452.3 \\
\hline 21513710-7925459 & 6355014691279286400 & 1.2 & 1.14 & 0.01 & 8.019 & 8.019 & -0.62 & 10.0 \\
\hline $21515415+0537172$ & 2697022209558112768 & -87.3 & 2.65 & 0.10 & -6.058 & -6.058 & -1.52 & 270.4 \\
\hline $21525027-6604240$ & 6398934786330198784 & $\ldots$ & 0.55 & 0.00 & 13.740 & 13.740 & -0.34 & 47.2 \\
\hline $21555532+3501402$ & 1948312515407711104 & -313.6 & 2.54 & 0.08 & -3.190 & -3.190 & -0.64 & 368.6 \\
\hline 21573551-0308043 & 2670534149811033088 & 160.1 & 1.04 & 0.02 & 34.775 & 34.775 & -0.67 & 311.8 \\
\hline $21573761+3544196$ & 1948748437403259776 & -61.9 & 0.19 & 0.00 & 93.788 & 93.788 & -0.02 & 110.2 \\
\hline $21584417+0052490$ & 2681491607815613952 & -37.6 & 1.55 & 0.04 & -16.997 & -16.997 & -0.97 & 279.3 \\
\hline $21584491+0129524$ & 2681597607608087040 & -249.4 & 4.04 & 0.31 & 5.896 & 5.896 & -2.55 & 328.6 \\
\hline 22013669-6918312 & 6396019706127003520 & $\ldots$ & 5.70 & 0.36 & -2.812 & -2.812 & -3.73 & 241.5 \\
\hline $22032935-5635128$ & 6412646138709077888 & 110.1 & 4.67 & 0.22 & 4.486 & 4.486 & -3.46 & 170.3 \\
\hline $22040352+0016519$ & 2680470706974136192 & -134.3 & 5.88 & 0.62 & -3.459 & -3.459 & -3.86 & 388.8 \\
\hline $22043661-6044348$ & 6409307304149253248 & 90.3 & 1.89 & 0.06 & 11.331 & 11.331 & -1.34 & 283.8 \\
\hline $22044480+4148205$ & 1959828147361822848 & 4.0 & 0.28 & 0.00 & -3.684 & -3.684 & -0.03 & 19.4 \\
\hline $22050237+3107332$ & 1898564081014685184 & -31.7 & 0.30 & 0.01 & -19.982 & -19.982 & -0.08 & 56.7 \\
\hline $22082896-6607320$ & 6399063394830844544 & $\ldots$ & 3.89 & 0.18 & -0.161 & -0.161 & -2.66 & 145.3 \\
\hline 22104990-3947023 & 6573266443723504768 & -69.4 & 3.10 & 0.13 & -5.883 & -5.883 & -2.50 & 253.4 \\
\hline 22112456-3753100 & 6574080735161542784 & -3.9 & 2.42 & 0.10 & 29.374 & 29.374 & -1.95 & 327.8 \\
\hline $22115350-1209181$ & 2612971211403496832 & -119.9 & 3.30 & 0.25 & 4.789 & 4.789 & -2.50 & 255.7 \\
\hline $22120815-4215210$ & 6569918602615687808 & -15.0 & 5.29 & 0.41 & -4.489 & -4.489 & -4.27 & 307.3 \\
\hline 22125424-0235414 & 2675834650555144576 & -127.9 & 0.69 & 0.01 & 63.320 & 63.320 & -0.46 & 300.8 \\
\hline
\end{tabular}


Table 4 (continued)

\begin{tabular}{|c|c|c|c|c|c|c|c|c|}
\hline $\begin{array}{c}\text { Star Name } \\
\text { (2MASS) }\end{array}$ & $\begin{array}{c}\text { Star Name } \\
(\text { Gaia EDR3) }\end{array}$ & $\begin{array}{c}V_{\mathrm{los}} \\
\left(\mathrm{km} \mathrm{s}^{-1}\right)\end{array}$ & $\begin{array}{l}d_{\text {helio }} \\
(\mathrm{kpc})\end{array}$ & $\begin{array}{c}\sigma_{d} \\
(\mathrm{kpc})\end{array}$ & 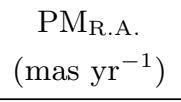 & 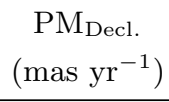 & $\begin{array}{l}Z_{\mathrm{Gal}} \\
(\mathrm{kpc})\end{array}$ & $\begin{array}{c}V_{\mathrm{TAV}} \\
\left(\mathrm{km} \mathrm{s}^{-1}\right)\end{array}$ \\
\hline 22125459-0408594 & 2627229884351014144 & -270.2 & 2.43 & 0.11 & -3.453 & -3.453 & -1.71 & 275.3 \\
\hline 22140658-0016065 & 2678634350759886336 & $\ldots$ & 0.09 & 0.00 & 15.430 & 15.430 & -0.04 & 10.9 \\
\hline $22165631-1154207$ & 2612857755547023232 & -9.7 & 0.14 & 0.00 & 20.176 & 20.176 & -0.08 & 24.2 \\
\hline 22211976-4111021 & 6569481855982624640 & -163.4 & 3.11 & 0.20 & 6.233 & 6.233 & -2.56 & 411.5 \\
\hline $22213981+3725402$ & 1906965002686762496 & $\ldots$ & 0.79 & 0.01 & -2.596 & -2.596 & -0.20 & 12.5 \\
\hline $22221878-7406283$ & 6357952517629300352 & 174.0 & 4.51 & 0.17 & -5.336 & -5.336 & -2.83 & 313.7 \\
\hline $22224538+3555471$ & 1905936405260048384 & -242.2 & 3.68 & 0.16 & -4.230 & -4.230 & -1.10 & 287.1 \\
\hline $22224859+2804264$ & 1881899328730042368 & -150.1 & 1.70 & 0.05 & 17.107 & 17.107 & -0.67 & 198.0 \\
\hline $22225487+2954084$ & 1894651090929156096 & 17.0 & 0.26 & 0.01 & 58.319 & 58.319 & -0.08 & 89.8 \\
\hline $22234372-8627533$ & 6342106630928779392 & 3.3 & 0.12 & 0.00 & 5.639 & 5.639 & -0.04 & 33.0 \\
\hline 22235096-6512086 & 6404249993041212928 & 8.7 & 0.17 & 0.00 & 166.503 & 166.503 & -0.10 & 86.1 \\
\hline $22241668+2548128$ & 1879125024671706624 & $\ldots$ & 1.19 & 0.02 & -5.619 & -5.619 & -0.50 & 57.2 \\
\hline $22241984+2430382$ & 1878702846566493440 & -18.8 & 0.47 & 0.00 & 27.285 & 27.285 & -0.19 & 60.6 \\
\hline $22244368+3236401$ & 1901779602770732032 & $\ldots$ & 0.28 & 0.00 & -27.414 & -27.414 & -0.08 & 42.2 \\
\hline $22251594+2911332$ & 1894379923873649536 & -89.6 & 2.09 & 0.10 & -7.684 & -7.684 & -0.81 & 272.8 \\
\hline $22280812+3546524$ & 1905786459361887488 & -39.4 & 0.11 & 0.00 & -6.598 & -6.598 & -0.01 & 41.7 \\
\hline $22284549-4124119$ & 6593293356532115712 & -25.2 & 0.20 & 0.00 & 69.372 & 69.372 & -0.14 & 55.1 \\
\hline $22290341+3954145$ & 1909092729485051776 & -23.6 & 1.17 & 0.03 & -11.627 & -11.627 & -0.28 & 75.1 \\
\hline $22293323+4332048$ & 1981952760850101376 & -278.4 & 1.64 & 0.04 & 4.300 & 4.300 & -0.32 & 292.6 \\
\hline 22294083-3305402 & 6600574876582217344 & 196.8 & 4.63 & 0.37 & 13.228 & 13.228 & -3.94 & 313.0 \\
\hline 22303946-1809055 & 2594309161890648320 & -6.0 & 0.07 & 0.00 & 94.353 & 94.353 & -0.04 & 45.3 \\
\hline $22310829+3023018$ & 1900531824573844608 & 2.7 & 0.25 & 0.00 & 9.700 & 9.700 & -0.08 & 15.3 \\
\hline $22311433-6656572$ & 6386075551166390144 & 58.3 & 2.74 & 0.13 & 8.504 & 8.504 & -1.91 & 255.9 \\
\hline $22315231+2320202$ & 1875411630307299456 & -15.6 & 0.99 & 0.05 & -7.264 & -7.264 & -0.46 & 50.6 \\
\hline $22342447+2739353$ & 1881261994239589888 & $\ldots$ & 0.77 & 0.01 & -1.907 & -1.907 & -0.31 & 27.9 \\
\hline $22345447-6605172$ & 6392134513070641408 & -54.9 & 2.55 & 0.10 & 9.874 & 9.874 & -1.80 & 171.7 \\
\hline 22354139-4305549 & 6520905878946665344 & 125.9 & 3.96 & 0.26 & -2.309 & -2.309 & -3.35 & 383.6 \\
\hline $22355721-2434108$ & 6623904314300239488 & -21.2 & 0.11 & 0.00 & 51.752 & 51.752 & -0.07 & 40.2 \\
\hline $22360602+3930413$ & 1908318669000212480 & -6.4 & 0.51 & 0.02 & -2.168 & -2.168 & -0.12 & 16.2 \\
\hline $22364074-7026524$ & 6384994623861077376 & 9.5 & 0.38 & 0.00 & 57.225 & 57.225 & -0.24 & 67.3 \\
\hline $22373316-4341181$ & 6520826714109323392 & -9.6 & 1.45 & 0.03 & 0.987 & 0.987 & -1.21 & 299.9 \\
\hline $22401067-3738259$ & 6595625729931613568 & -88.7 & 3.23 & 0.20 & 2.726 & 2.726 & -2.78 & 109.2 \\
\hline 22412632-3627304 & 6596002145160945664 & -68.9 & 3.88 & 0.25 & 8.280 & 8.280 & -3.37 & 348.1 \\
\hline $22413573+2931038$ & 1887539965117822464 & -4.2 & 0.12 & 0.01 & 70.505 & 70.505 & -0.03 & 37.9 \\
\hline $22432022-7547340$ & 6357547759911189120 & 20.4 & 0.27 & 0.00 & 17.523 & 17.523 & -0.14 & 22.2 \\
\hline $22451263+3532066$ & 1903656636619168768 & $\ldots$ & 4.73 & 0.24 & -3.712 & -3.712 & -1.64 & 100.3 \\
\hline $22453503+1301210$ & 2731609959149919360 & -506.8 & 4.58 & 0.31 & 10.187 & 10.187 & -2.89 & 564.2 \\
\hline $22454796+2826260$ & 1887186850086385152 & -11.3 & 0.19 & 0.00 & 6.210 & 6.210 & -0.06 & 19.9 \\
\hline $22471965-7201440$ & 6382433144021702656 & 5.6 & 0.45 & 0.00 & 36.835 & 36.835 & -0.27 & 35.6 \\
\hline $22472643+3532411$ & 1903562838830927744 & 10.7 & 0.23 & 0.00 & -22.706 & -22.706 & -0.06 & 35.1 \\
\hline $22473447-5511534$ & 6505905757201226368 & 2.5 & 0.40 & 0.04 & 13.755 & 13.755 & -0.30 & 19.0 \\
\hline $22474545+2517113$ & 1876790894628571264 & -1.2 & 0.19 & 0.00 & 13.293 & 13.293 & -0.07 & 11.0 \\
\hline
\end{tabular}


Table 4 (continued)

\begin{tabular}{|c|c|c|c|c|c|c|c|c|}
\hline $\begin{array}{c}\text { Star Name } \\
\text { (2MASS) }\end{array}$ & $\begin{array}{c}\text { Star Name } \\
\text { (Gaia EDR3) }\end{array}$ & $\begin{array}{c}V_{\mathrm{los}} \\
\left(\mathrm{km} \mathrm{s}^{-1}\right)\end{array}$ & $\begin{array}{l}d_{\text {helio }} \\
(\mathrm{kpc})\end{array}$ & $\begin{array}{c}\sigma_{d} \\
(\mathrm{kpc})\end{array}$ & 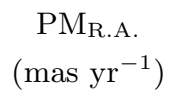 & $\begin{array}{c}\mathrm{PM}_{\text {Decl. }} \\
\left(\text { mas yr }^{-1}\right)\end{array}$ & $\begin{array}{l}Z_{\mathrm{Gal}} \\
(\mathrm{kpc})\end{array}$ & $\begin{array}{c}V_{\mathrm{TAV}} \\
\left(\mathrm{km} \mathrm{s}^{-1}\right)\end{array}$ \\
\hline 22481449-5703067 & 6504026829267642240 & -13.6 & 1.16 & 0.02 & 26.170 & 26.170 & -0.90 & 221.1 \\
\hline 22490353-5536242 & 6505879059684460032 & -29.8 & 0.10 & 0.00 & 185.005 & 185.005 & -0.06 & 86.5 \\
\hline 22492811-5717202 & 6503988105842414464 & 20.5 & 0.32 & 0.00 & 8.290 & 8.290 & -0.23 & 53.8 \\
\hline 22511876-3814381 & 6547694891340666752 & 196.7 & 1.56 & 0.04 & 12.905 & 12.905 & -1.36 & 344.4 \\
\hline 22512739-5049405 & 6513929237145370880 & -30.1 & 1.99 & 0.06 & 47.403 & 47.403 & -1.65 & 393.8 \\
\hline $22531950-6647163$ & 6391157150312476032 & 166.8 & 5.63 & 0.41 & 2.332 & 2.332 & -4.05 & 228.5 \\
\hline $22534476-5456485$ & 6506123701021637888 & -58.9 & 1.30 & 0.02 & 26.270 & 26.270 & -1.04 & 122.7 \\
\hline $22534538+7919586$ & 2285728337337892480 & -44.0 & 0.16 & 0.00 & -20.366 & -20.366 & 0.07 & 45.1 \\
\hline $22544750-3509160$ & 6554282379035795840 & 7.3 & 0.11 & 0.00 & 124.383 & 124.383 & -0.08 & 117.4 \\
\hline 22575548-5622538 & 6493611254400172416 & 188.5 & 6.07 & 0.43 & 5.754 & 5.754 & -4.91 & 416.8 \\
\hline $22591090-4829425$ & 6515325616912384256 & 16.9 & 3.92 & 0.21 & 7.412 & 7.412 & -3.35 & 140.5 \\
\hline 22594336-3819324 & 6546814427341069568 & 13.0 & 0.38 & 0.05 & 60.399 & 60.399 & -0.32 & 97.7 \\
\hline $23003637+2820257$ & 1885059776122105728 & -52.0 & 0.28 & 0.01 & -59.363 & -59.363 & -0.11 & 134.2 \\
\hline 23022817-4059099 & 6543252452048113152 & 53.9 & 2.86 & 0.13 & 17.250 & 17.250 & -2.53 & 213.1 \\
\hline 23035329-4139482 & 6543169129682052608 & $\ldots$ & 5.94 & 0.57 & -6.561 & -6.561 & -5.30 & 399.3 \\
\hline $23043022+0155166$ & 2652540916900514304 & -55.6 & 4.04 & 0.24 & 3.042 & 3.042 & -3.12 & 122.1 \\
\hline 23044868-4311029 & 6541868716664047744 & 54.5 & 0.12 & 0.00 & -87.102 & -87.102 & -0.08 & 65.6 \\
\hline 23055841-8636001 & 6341894558326196480 & -2.9 & 0.12 & 0.00 & 27.165 & 27.165 & -0.03 & 5.6 \\
\hline $23064708+2802027$ & 1884897937457948672 & -206.0 & 0.06 & 0.00 & 25.925 & 25.925 & -0.01 & 210.8 \\
\hline 23083044-7441319 & 6378503729982312576 & 135.1 & 4.19 & 0.22 & 19.992 & 19.992 & -2.70 & 196.7 \\
\hline $23085453-5226130$ & 6500818969734685440 & 0.0 & 0.11 & 0.00 & 50.188 & 50.188 & -0.07 & 17.8 \\
\hline 23093209-7132507 & 6381003327932886144 & 211.1 & 5.24 & 0.29 & -0.496 & -0.496 & -3.58 & 223.8 \\
\hline 23100319-7702165 & 6354182945092770176 & -53.9 & 3.44 & 0.17 & 17.144 & 17.144 & -2.12 & 182.0 \\
\hline $23123243-0240516$ & 2638139066923296128 & 11.3 & 0.14 & 0.00 & 17.529 & 17.529 & -0.09 & 16.7 \\
\hline $23124700+2701045$ & 2845306436489791232 & 0.5 & 0.11 & 0.00 & 168.657 & 168.657 & -0.03 & 81.8 \\
\hline 23130418-4332060 & 6541663554666433920 & 54.5 & 3.93 & 0.28 & 10.010 & 10.010 & -3.52 & 390.0 \\
\hline $23131220+5425552$ & 1996424051866554112 & -19.4 & 0.61 & 0.01 & 8.458 & 8.458 & -0.04 & 25.6 \\
\hline $23133742-5336585$ & 6500438435631474560 & -10.3 & 0.60 & 0.01 & 10.804 & 10.804 & -0.48 & 24.9 \\
\hline $23164530-4047253$ & 6548299386513340288 & 13.7 & 0.10 & 0.00 & -2.706 & -2.706 & -0.06 & 119.7 \\
\hline 23235454-4730233 & 6526777614634204672 & -39.8 & 0.96 & 0.01 & 31.476 & 31.476 & -0.83 & 127.9 \\
\hline $23273196+5438201$ & 1995911404570328192 & -1.5 & 0.27 & 0.00 & 10.051 & 10.051 & 0.00 & 13.4 \\
\hline $23293844+3337097$ & 2872688983306031616 & -184.0 & 2.04 & 0.11 & 21.475 & 21.475 & -0.88 & 261.7 \\
\hline $23321307+1950398$ & 2824757388800259840 & -83.7 & 0.07 & 0.00 & 134.382 & 134.382 & -0.02 & 107.5 \\
\hline $23341995+4703450$ & 1938499679067388288 & 19.5 & 0.67 & 0.02 & 13.922 & 13.922 & -0.13 & 42.5 \\
\hline $23362842-5537423$ & 6496310731541441152 & 29.8 & 2.24 & 0.08 & -4.864 & -4.864 & -1.88 & 256.8 \\
\hline $23371202+2100145$ & 2826450808506230016 & $\ldots$ & 0.09 & 0.00 & 209.668 & 209.668 & -0.03 & 88.9 \\
\hline $23400099+4959092$ & 1943744250753474944 & -244.4 & 2.01 & 0.04 & 26.689 & 26.689 & -0.37 & 298.8 \\
\hline $23421250-6346584$ & 6485737793369175680 & $\ldots$ & 0.19 & 0.02 & 0.139 & 0.139 & -0.12 & 17.8 \\
\hline $23430472-8200221$ & 6350719930141819520 & 5.3 & 0.12 & 0.00 & -122.785 & -122.785 & -0.04 & 12.2 \\
\hline $23433753+5008599$ & 1943761190104941824 & -85.2 & 1.77 & 0.04 & -2.690 & -2.690 & -0.32 & 87.1 \\
\hline $23450930+5538498$ & 1994885560221975552 & $\ldots$ & 0.63 & 0.00 & -6.569 & -6.569 & -0.04 & 19.5 \\
\hline $23465497+1201211$ & 2763718305727188480 & $\ldots$ & 0.19 & 0.00 & 77.406 & 77.406 & -0.12 & 70.3 \\
\hline
\end{tabular}


Table 4 (continued)

\begin{tabular}{|c|c|c|c|c|c|c|c|c|}
\hline $\begin{array}{c}\text { Star Name } \\
\text { (2MASS) }\end{array}$ & $\begin{array}{c}\text { Star Name } \\
\text { (Gaia EDR3) }\end{array}$ & $\begin{array}{c}V_{\text {los }} \\
\left(\mathrm{km} \mathrm{s}^{-1}\right)\end{array}$ & $\begin{array}{l}d_{\text {helio }} \\
(\mathrm{kpc})\end{array}$ & $\begin{array}{c}\sigma_{d} \\
(\mathrm{kpc})\end{array}$ & 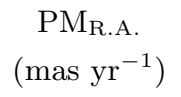 & 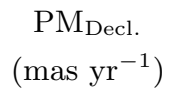 & $\begin{array}{l}Z_{\text {Gal }} \\
(\mathrm{kpc})\end{array}$ & $\begin{array}{c}V_{\mathrm{TAV}} \\
\left(\mathrm{km} \mathrm{s}^{-1}\right)\end{array}$ \\
\hline $23472384+4835469$ & 1940559103007605120 & -69.5 & 2.50 & 0.09 & 10.039 & 10.039 & -0.53 & 109.4 \\
\hline $23505349+1142348$ & 2766637307235175296 & -182.0 & 2.83 & 0.12 & 1.251 & 1.251 & -2.09 & 253.2 \\
\hline $23550005+1255039$ & 2766874664308017408 & 25.7 & 0.14 & 0.01 & 14.749 & 14.749 & -0.08 & 28.0 \\
\hline $23555398+5702462$ & 1998062118035485056 & -12.5 & 0.19 & 0.02 & 7.608 & 7.608 & 0.01 & 13.1 \\
\hline $23560245+1109200$ & 2765621221052354816 & -14.3 & 0.17 & 0.00 & 46.314 & 46.314 & -0.11 & 72.5 \\
\hline $23562635+0651168$ & 2746230337064331776 & -0.1 & 0.19 & 0.00 & 12.817 & 12.817 & -0.13 & 12.3 \\
\hline $23563722+4615436$ & 1927134439687586560 & -44.7 & 0.06 & 0.00 & -74.463 & -74.463 & 0.01 & 60.8 \\
\hline $23564530-4429484$ & 6532542491534291968 & 6.9 & 0.19 & 0.01 & -48.397 & -48.397 & -0.16 & 39.1 \\
\hline
\end{tabular}

